\title{
Rhenium and Osmium PNP Pincer Complexes for Nitrogen Fixation and Nitride Transfer
}

\author{
Dissertation \\ for the award of the degree \\ "DOCTOR RERUM NATURALIUM" \\ of the Georg-August-Universität Göttingen
}

within the doctoral program of the Georg-August University School of Science (GAUSS)

submitted by

Florian Wätjen, M. Sc.

from Papenburg, Germany

Göttingen, 2019 


\section{Thesis Committee}

Prof. Dr. Sven Schneider

Institut für Anorganische Chemie, Georg-August-Universität Göttingen

Prof. Dr. Franc Meyer

Institut für Anorganische Chemie, Georg-August-Universität Göttingen

\section{Members of Examination Board}

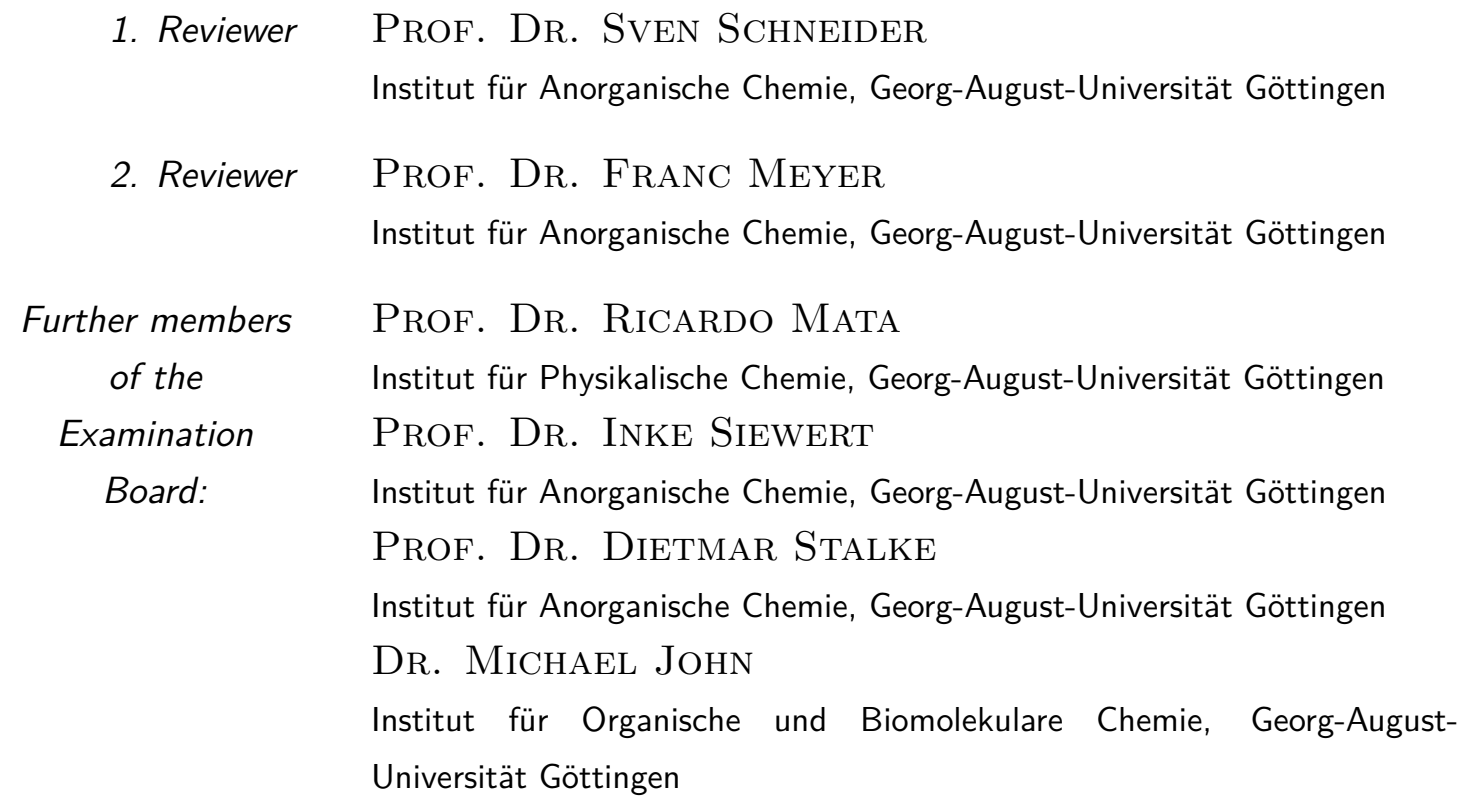

Date of oral examination: 27.09.2019 
In The Beginning There Was Silence And Darkness

All Across The Earth

Then Came The Wind And A Hole In The Sky

Thunder And Lightning Came Crashing Down

Hit The Earth And Split The Ground

Fire Burned High In The Sky

From Down Below Fire Melted The Stone

The Ground Shook And Started To Pound

The Gods Made Heavy Metal

And They Saw That It Was Good

- Manowar

"The Gods Made Heavy Metal" on "Louder Than Hell" 
First of all, I want to thank my supervisor Prof. DR. Sven Schneider for providing me with interesting and challenging scientific "nuts to crack" and the great degree of freedom but also of support I got during the last years. Your enthusiasm for our research is inspiring and infectious.

Furthermore I want to thank Prof. Dr. Franc Meyer for kindly assuming the part of my second supervisor and referee of this thesis.

My gratitude goes to Thomas Auth for the memorable time during his practical lab course as well as to my bachelor student Jan Christian Becker.

I thank Dr. Christian Würtele and Dr. Christian Volkmann (X-ray), Dr. Markus Finger and Prof. Dr. Vera Krewald (DFT, CASSCF), Dr. Milan Orlita (magnetic IR), as well as Dr. Michael John and Ralf Schöne (NMR) for their scientific contributions to this thesis as well as for the helpful and instructive discussions.

The analytical laboratories and the mass department of the our faculty for there efforts in measuring my samples.

I thank Mirko Paulikat and Stefan Ortgies for providing me with their LTEX templates, as well as Ricardo Langner who wrote the in here used CLEANTHESIS template. Additionally, I want to highlight the work of the numerous coders out there developing free software (like LINUX, ORCA, INKSCAPE, ATEX or VIM, just to name a few) which I used quite heavily.

A probably unusual peculiarity of this thesis are the incorporated quotes from heavy metal lyrics at the beginning of each chapter. These are chosen to highlight certain aspects of each chapter and are my personal homage to the many (heavy) metal bands, the music of which accompanied me throughout my entire $\mathrm{PhD}$ period (and if scientific papers can open with Monty Python quotes, a small non-scientific personal note in a thesis should be fine). ${ }^{[1]}$

My special thanks go to the whole AK Schneider group. I've spend some marvelous years with you in the lab and beyond and I will keep this (unfortunately limited) time in good memory. Especially Josh Abbenseth, Sarah Bete, Jan Gerkens, Sebastian Kopp, Felix Schneck and Richt van Alten made this time an unforgettable experience.

Last but not least I thank Lisa Wätjen, my wife and best friend. Your motivation carried me through the tougher times of my $\mathrm{PhD}$ and your wanderlust is the reason why I write these lines in the shadow of an olive tree in south France rather than in a stuffy office. For this and everything else, I love you! 


\section{Contents}

I Introduction $\quad 1$

1 Molecular dinitrogen, nitrogenase and the Haber-Bosch process 3

2 Binding of dinitrogen to transition metal complexes $\quad 7$

2.1 End-on bound $\mathrm{N}_{2} \ldots \ldots \ldots \ldots \ldots$

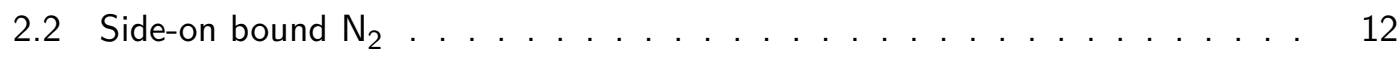

3 Reactions of dinitrogen complexes $\quad 15$

3.1 Ammonia formation . . . . . . . . . . . . . . . . . . . 15

3.2 Initial full dinitrogen cleavage . . . . . . . . . . . . . . . . . 18

3.2.1 Thermal $\mathrm{N}_{2}$ cleavage from end-on bound dimers . . . . . . . . . . 18

3.2.2 Thermal $\mathrm{N}_{2}$ cleavage from side-on bound dimers . . . . . . . 23

3.2.3 Photochemical $\mathrm{N}_{2}$ cleavage . . . . . . . . . . . . . . . 24

3.3 Nitrogen functionalization in side-on coordinated complexes . . . . . . . . . 27

4 Reactivity and functionalization of transition metal nitrides 29

4.1 General bonding considerations . . . . . . . . . . . . . . . . . . . 29

4.2 Reactivity of transition metal nitrides . . . . . . . . . . . . . . 31

4.2.1 Dinitrogen derived terminal nitrides and functionalization beyond ammonia formation . . . . . . . . . . . . . . . . 33

4.2.2 Low valent nitrido complexes from other nitrogen sources . . . . . 38

5 Scope of this work $\quad 43$

II Results and Discussion $\quad 45$

1 Low valent osmium nitride complexes with a PNP pincer ligand $\quad 47$

1.1 A square planar osmium(IV) nitride complex . . . . . . . . . . . . . 48

1.2 Functionalization of square planar osmium(IV) nitride $3 \ldots \ldots 50$

1.3 Redox chemistry of osmium(IV) and osmium(VI) nitrido complexes . . . . 56

1.4 Attempts of dinitrogen activation . . . . . . . . . . . . . . . 60

1.5 Summary . . . . . . . . . . . . . . . . . . . . . . . . 64

2 Extensions of the $\left[\operatorname{ReCl}_{2}\left(\mathrm{PNP}^{t \mathrm{Bu}}\right)\right]$ platform $\quad 65$

2.1 Characterization of $\mu-\mathrm{N}_{2}$ bridged Re dimer $\mathbf{X X} \ldots \ldots 66$ 
2.2 Intermolecular $\mathrm{C}-\mathrm{C}$ coupling of $\left[\operatorname{Re}\left(\mathrm{NCH}_{2}\right) \mathrm{Cl}\left(\mathrm{PNP}^{t \mathrm{Bu}}\right)\right]^{+}\left(\mathbf{1 8}^{+}\right) \ldots . . .69$

2.3 Effect of the backbone oxidation to the $\mathrm{P}=\mathrm{N}=\mathrm{P}$ platform . . . . . . . . 72

2.3.1 Synthesis and dinitrogen splitting . . . . . . . . . . . 72

2.3.2 Nitride functionalization . . . . . . . . . . . . . . . 75

2.4 Ligand exchange reactions from $\left[\operatorname{ReCl}_{2}\left(\mathrm{PNP}^{t \mathrm{Bu}}\right)\right] \ldots \ldots . \ldots . . \ldots 8$

2.5 Summary . . . . . . . . . . . . . . . . . . . . . . . . 81

3 Rhenium complexes of iso-propyl based PNP pincer ligands for dinitrogen activation

3.1 The starting platform $-\left[\operatorname{ReCl}_{3}\left(\mathrm{HPNP}^{i \mathrm{Pr}}\right)\right](29) \ldots \ldots \ldots . \ldots . \ldots 8$

3.1.1 Synthesis and characterization .............. 85

3.1 .2 The electronic structure of $29 \ldots \ldots \ldots$

3.2 Amide based pincer chemistry . . . . . . . . . . . . . . . . . . . . 91

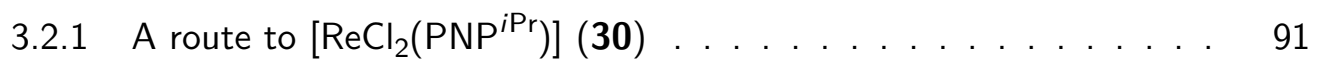

3.2.2 Dinitrogen chemistry with $\left[\mathrm{ReCl}_{2}\left(\mathrm{PNP}^{\mathrm{iPr}}\right)\right](30) \ldots . . \ldots 96$

3.2.3 Assessment of dinitrogen activation capabilities . . . . . . . . . 101

3.3 Amine based pincer chemistry . . . . . . . . . . . . . . . . . . 105

3.3.1 A thermally stable, $\mu-\mathrm{N}_{2}$ bridged $\mathrm{Re}(\mathrm{II})$ dimer and its photochemistry 105

3.3.2 An octahedral rhenium nitride complex and nitrogen centered, nucleophilic reactivity . . . . . . . . . . . . . . . . . . . . 111

3.3.3 Metal-ligand cooperative benzamide / benzonitrile formation . . . . 118

3.4 Summary . . . . . . . . . . . . . . . . . . . . . . . . . . . . . . . . . . . . . 124

4 Conclusion and outlook $\quad 127$

$\begin{array}{ll}\text { III Experimental details } & 135\end{array}$

1 Materials and Methods 137

1.1 General synthesis and materials . . . . . . . . . . . . . . . . . 137

1.2 Analytical methods . . . . . . . . . . . . . . . . . . 138

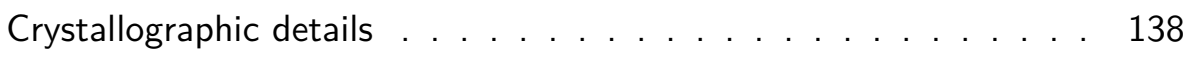

Cyclic voltammetry . . . . . . . . . . . . . . . . . . . 138

Elemental analysis . . . . . . . . . . . . . . . . . . . . . . . . . . . . . . . . . . . . . . . 139

Indophenolic titration . . . . . . . . . . . . . . . . . . 139

Irradiation and quantum yield determination . . . . . . . . . 140

Mass spectrometry . . . . . . . . . . . . . . . . . . . . . 142

Nuclear magnetic resonance . . . . . . . . . . . . . . . . . 142

SQUID measurement . . . . . . . . . . . . . . . . . . . . . 142

UV/vis spectroscopy . . . . . . . . . . . . . . . . . . . . . . . . . . . . . . . . . . . . . . . .

Vibrational spectroscopy . . . . . . . . . . . . . . . . 143

$\begin{array}{lll}2 \text { Synthesis } & 145\end{array}$ 
2.1 Osmium compounds . . . . . . . . . . . . . . . . . . . . . . . . . . . 145

$2.1 .1\left[\mathrm{Os}(\mathrm{H})(\mathrm{N})\left(\mathrm{PNP}^{t \mathrm{Bu}}\right)\right]^{\mathrm{X}}\left(\mathbf{2}^{\mathrm{X}}\right) \ldots \ldots \ldots \ldots \ldots \ldots \ldots \ldots$

$2.1 .2\left[\mathrm{Os}(\mathrm{N})\left(\mathrm{PNP}{ }^{t \mathrm{Bu}}\right)\right](3) \ldots \ldots \ldots \ldots$

$2.1 .3\left[\mathrm{Os}(\mathrm{H})(\mathrm{N}) \mathrm{Cl}\left(\mathrm{HPNP}^{t \mathrm{Bu}}\right)\right]^{\mathrm{Cl}}\left({ }^{\mathrm{Cl}}\right) \ldots \ldots \ldots \ldots \ldots \ldots$

$2.1 .4\left[\mathrm{Os}_{2}\left(\mathrm{NBAr}_{18}^{\mathrm{F}}\right)\left(\mathrm{PNP}{ }^{t \mathrm{Bu}}\right)\right](5) \ldots \ldots \ldots \ldots \ldots$

$2.1 .5\left[\mathrm{Os}\left(\mathrm{NSiMe}_{3}\right)\left(\mathrm{PNP}^{t \mathrm{Bu}}\right)\right]^{\mathrm{BAr}_{24}^{\mathrm{F}}\left(6^{\mathrm{BAr}}{ }_{24}^{\mathrm{F}}\right)} \ldots \ldots \ldots \ldots \ldots \ldots \ldots$

$2.1 .6\left[\mathrm{Os}\left(\mathrm{NPMe}_{3}\right)\left(\mathrm{PNP}^{t \mathrm{Bu}}\right)\right](\mathbf{7}) \ldots \ldots \ldots \ldots \ldots$

$2.1 .7\left[\mathrm{OsH}_{4}\left(\mathrm{HPNP}^{t \mathrm{Bu}}\right)\right](\mathbf{8}) \ldots \ldots \ldots \ldots \ldots \ldots$

$2.1 .8\left[\mathrm{OsH}_{3}\left(\mathrm{PNP}^{t \mathrm{Bu}}\right)\right](\mathbf{9}) \ldots \ldots \ldots \ldots \ldots \ldots \ldots \ldots$

2.1.9 $\left[\mathrm{Os}(\mathrm{N}) \mathrm{Cl}\left(\mathrm{PNP}^{t \mathrm{Bu}}\right)\right]^{\mathrm{BAr}{ }_{24}^{\mathrm{F}}}\left(\mathbf{1 0}^{\mathrm{BAr} \mathbf{r}_{24}^{\mathrm{F}}}\right)$ and $\left[\mathrm{Os}(\mathrm{N}) \mathrm{Cl}_{2}\left(\mathrm{PNP}^{t \mathrm{Bu}}\right)\right](\mathbf{1 0}-\mathbf{C l}) 149$

2.1.10 $\left.\mathrm{Os}\left(\mathrm{CN}^{\mathrm{t}} \mathrm{Bu}\right)_{2} \mathrm{Cl}\left\{\mathrm{N}\left(\mathrm{CH}_{2} \mathrm{CH}_{2} \mathrm{P}^{\mathrm{t}} \mathrm{Bu}_{2}\right)\left(=\mathrm{CHCH}_{2} \mathrm{P}\left({ }^{\mathrm{t}} \mathrm{Bu}\right)_{2}=\mathrm{NH}\right)\right\}\right]^{\mathrm{BAr}_{24}^{\mathrm{F}}}\left(\mathbf{1 1}^{\mathrm{BAr}_{24}^{\mathrm{F}}}\right.$

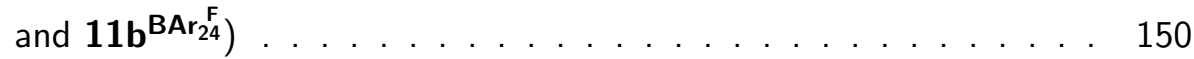

$2.1 .11\left[\mathrm{OsH}\left(\mathrm{N}_{2}\right)\left(\mathrm{PNP}^{t \mathrm{Bu}}\right)\right](\mathbf{1 3}) \ldots \ldots \ldots \ldots \ldots \ldots$

$2.1 .12\left[\mathrm{OsHCl}\left(\mathrm{PNP}^{t \mathrm{Bu}}\right)\right]^{\mathrm{BPh}_{4}}\left(\mathbf{1 2}^{\mathrm{BPh}_{4}}\right) \ldots \ldots \ldots \ldots \ldots . \ldots \ldots$

2.1.13 $\left[\mathrm{OsHCl}\left(\mathrm{CN}^{\mathrm{t}} \mathrm{Bu}\right)\left(\mathrm{PNP}^{t \mathrm{Bu}}\right)\right]^{\mathrm{BPh}_{4}}\left(\mathbf{1 4}^{\mathrm{BPh}_{4}}\right) \ldots \ldots \ldots \ldots \ldots \ldots$

$2.1 .14\left[\mathrm{OsCl}\left(\mathrm{CN}^{t} \mathrm{Bu}\right)\left(\mathrm{PNP}^{t \mathrm{Bu}}\right)\right](\mathbf{1 5}) \ldots \ldots \ldots \ldots \ldots \ldots \ldots$

2.2 Rhenium compounds based on the $\mathrm{PNP}^{t \mathrm{Bu}}$ ligand . . . . . . . . . . . 152

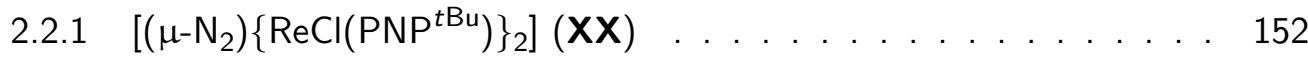

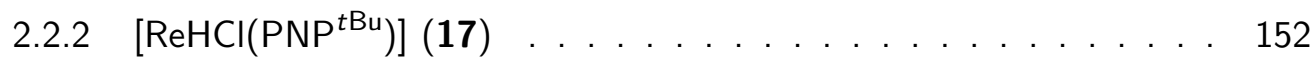

$2.2 .3\left[\left(N, N-\mathrm{C}_{2} \mathrm{H}_{4} \mathrm{~N}_{2}\right)\left\{\operatorname{ReCl}\left(\mathrm{PNP}^{t \mathrm{Bu}}\right)\right\}_{2}\right]^{(\mathrm{OTf})_{2}}\left(\mathbf{1 9}^{(\mathrm{OTf})_{2}}\right) \ldots \ldots \ldots .152$

$2.2 .4\left[\operatorname{Re}(\mathrm{O}) \mathrm{Cl}\left(\mathrm{PNP}^{t \mathrm{Bu}}\right)\right]^{\mathrm{BPh}_{4}}\left(\mathbf{2 0}^{\mathrm{BPh}_{4}}\right) \ldots \ldots \ldots \ldots \ldots \ldots \ldots \ldots$

$2.2 .5 \quad\left[\operatorname{ReCl}_{2}\left(\mathrm{P}=\mathrm{N}=\mathrm{P}^{t \mathrm{Bu}}\right)\right](\mathbf{2 1}) \ldots \ldots \ldots \ldots \ldots \ldots \ldots \ldots \ldots$

2.2.6 $\left[\operatorname{Re}(\mathrm{N}) \mathrm{Cl}\left(\mathrm{P}=\mathrm{N}=\mathrm{P}^{t \mathrm{Bu}}\right)\right](22) \ldots \ldots \ldots \ldots \ldots$

2.2.7 $\left[\operatorname{Re}(\mathrm{N}) \mathrm{Cl}\left(\mathrm{P}^{\mathrm{H}}=\mathrm{N}=\mathrm{P}^{t \mathrm{Bu}}\right)\right]^{\mathrm{OTf}}\left(\mathbf{2 3}^{\mathrm{OTf}}\right) \ldots \ldots \ldots \ldots \ldots \ldots \ldots \ldots$

$2.2 .8\left[\operatorname{Re}(\mathrm{NMe}) \mathrm{Cl}\left(\mathrm{P}=\mathrm{N}=\mathrm{P}^{t \mathrm{Bu}}\right)\right]^{\mathrm{OTf}}\left(24^{\mathrm{OTf}}\right) \ldots \ldots \ldots \ldots \ldots \ldots \ldots$

$2.2 .9\left[\operatorname{Re}\left(\mathrm{N}=\mathrm{CH}_{2}\right) \mathrm{Cl}\left(\mathrm{P}=\mathrm{N}=\mathrm{P}^{t \mathrm{Bu}}\right)\right](25) \ldots \ldots \ldots \ldots \ldots \ldots$

2.2.10 $\left[\operatorname{Re}(\mathrm{N}) \mathrm{Cl}\left(\mathrm{P}=\mathrm{N}=\mathrm{P}^{t \mathrm{Bu}}\right)\right]^{\mathrm{SbF}_{6}}\left(\mathbf{2 6}^{\mathrm{SbF}_{6}}\right) \ldots \ldots \ldots \ldots \ldots \ldots \ldots$

2.2.11 $\left[\operatorname{Re}(\text { bipy }) \mathrm{Cl}\left(\mathrm{PNP}^{t \mathrm{Bu}}\right)\right]^{\mathrm{BPh}_{4}}\left(27^{\mathrm{BPh}_{4}}\right) \ldots \ldots \ldots \ldots \ldots \ldots$

2.3 Rhenium compounds based on the $\mathrm{PNP}^{i \mathrm{Pr}}$ ligand . . . . . . . . . . . 158

2.3.1 $\left[\mathrm{ReCl}_{3}\left(\mathrm{HPNP}^{i \mathrm{Pr}}\right)\right](\mathbf{2 9}) \ldots \ldots \ldots \ldots \ldots \ldots \ldots \ldots$

2.3.2 $\left[\operatorname{ReCl}_{3}\left(\mathrm{PNP}^{i \mathrm{Pr}}\right)\right](31) \ldots \ldots \ldots \ldots \ldots \ldots \ldots$

2.3.3 $\left[\operatorname{ReCl}_{2}(\right.$ thf $\left.)\left(P N P^{i P r}\right)\right](32)$ and $\left[\operatorname{ReCl}_{2}\left(P N P^{i P r}\right)\right](30) \ldots \ldots . . .159$

2.3.4 $\left[\left\{\operatorname{ReCl}_{2, \text { cis }}\left(P N P^{i P r}\right)\right\}\left(\mu-N_{2}\right)\left\{\operatorname{ReCl}_{2, \text { trans }}\left(P N P^{i P r}\right)\right\}\right](33 a)$ and $\left[\left\{\operatorname{ReCl}_{2, \text { cis }}\right.\right.$

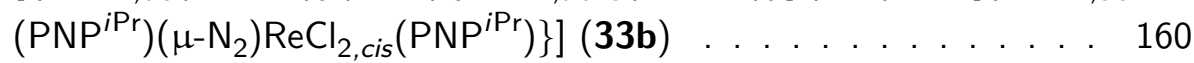

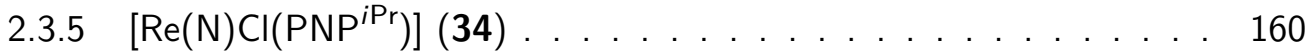

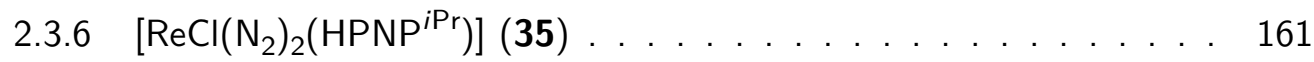

2.3.7 $\left[\left(\mu-\mathrm{N}_{2}\right)\left\{\mathrm{ReCl}_{2}\left(\mathrm{HPNP}^{\mathrm{iPr}}\right)\right\}_{2}\right](36) \ldots \ldots \ldots \ldots \ldots \ldots \ldots \ldots \ldots$

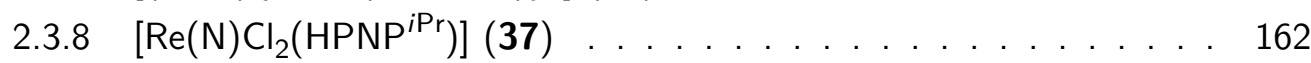

2.3.9 $\left.\operatorname{Re}(\mathrm{NH}) \mathrm{Cl}_{2}\left(\mathrm{HPNP}^{\mathrm{iPr}}\right)\right]^{\mathrm{OTf}}\left(\mathbf{3 8}^{\mathrm{OTf}}\right) \ldots \ldots \ldots \ldots \ldots \ldots \ldots$

2.3.10 $\left[\operatorname{Re}\left(\mathrm{NBAr}_{18}^{\mathrm{F}}\right) \mathrm{Cl}_{2}\left(\mathrm{HPNP}{ }^{i \mathrm{Pr}}\right)\right](39) \ldots \ldots \ldots \ldots . \ldots \ldots$

2.3.11 $\left[\operatorname{ReCl}_{3}\left(\mathrm{P}=\mathrm{NP}^{i \mathrm{Pr}}\right)\right](40) \ldots \ldots \ldots \ldots \ldots$ 
3.1 General procedure . . . . . . . . . . . . . . . . . . . . . 167

3.2 Model of $\left[\mathrm{Os}(\mathrm{N})\left(\mathrm{PNP}^{t \mathrm{Bu}}\right)\right]$ (3)and revised scan of the $\mathrm{N}-\mathrm{Os} \equiv \mathrm{N}$ bending mode168

3.3 Computational analysis of the reaction between 3 and $\mathrm{PMe}_{3}$ to form $7 \ldots 169$

3.3.1 XYZ-Coordinates . . . . . . . . . . . . . . . . . . 171

3.4 QDPT/NEVPT2/CASSCF $(14,10)$ on $29 \ldots \ldots \ldots . \ldots . \ldots . \ldots . \ldots$

3.4 .1 DFT-optimized geometries . . . . . . . . . . . . . . . 181

3.4 .2 CASSCF state composition . . . . . . . . . . . . . . . . . . 184

3.4 .3 QDPT state eigenvectors . . . . . . . . . . . . . . . . . 185

3.4.4 CASSCF, NEVPT2 and QDPT state energies . . . . . . . . . . . 187

3.4.5 Simulated influence of magnetic field on excitation between QDPT states $|0\rangle \rightarrow|4\rangle$ and $|0\rangle \rightarrow|5\rangle \ldots \ldots \ldots \ldots . \ldots . \ldots . \ldots . \ldots$

3.5 DFT and TDDFT calculations on the $\mu-\mathrm{N}_{2}$ bridged Re-dimer $36 \ldots 188$

3.6 Comparing the syn and anti isomers of $\left[\operatorname{Re}(\mathrm{N}) \mathrm{Cl}\left(\mathrm{HPNP}^{i \mathrm{Pr}}\right)\right]^{+} \ldots \ldots . . . .189$

3.6.1 DFT-optimized geometries . . . . . . . . . . . . . . . . 190

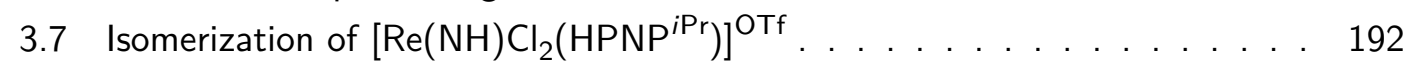

3.7 .1 Method and results . . . . . . . . . . . . . . . . . . . 192

3.7 .2 DFT-optimized geometries . . . . . . . . . . . . . . . . 193

$3.8 \mathrm{~N}_{2}$ splitting and functionalization by the $\left\{\operatorname{Re}\left({ }^{\mathrm{Me}} \mathrm{NPNP}{ }^{i \operatorname{Pr}}\right)\right\}$ platform . . . 197

3.8.1 DFT-optimized geometries . . . . . . . . . . . . . . . 198

$\begin{array}{ll}\text { IV Literature } & 205\end{array}$

$\begin{array}{ll}V \text { Appendix } & 227\end{array}$

$\begin{array}{lr}\text { A Crystal structures } & 229\end{array}$

A.1 $\left[\mathrm{Os}_{\left.\left(\mathrm{NBAr}_{18}{ }_{18}\right)\left(\mathrm{PNP}^{t \mathrm{Bu}}\right)\right](5) \ldots \ldots \ldots \ldots} 229\right.$

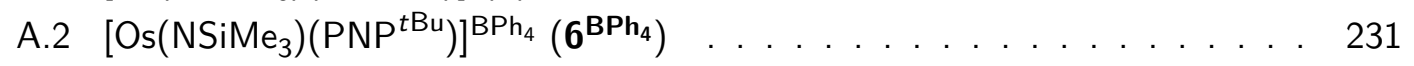

A.3 $\left[\mathrm{Os}\left(\mathrm{NPMe}_{3}\right)\left(\mathrm{PNP}^{t \mathrm{Bu}}\right)\right](7) \ldots \ldots \ldots \ldots . \ldots \ldots \ldots$

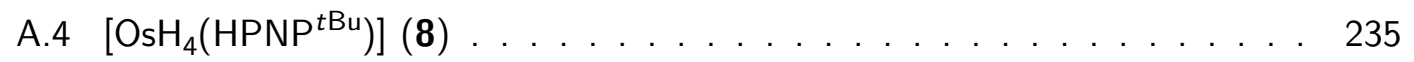

A.5 $\left[\mathrm{Os}\left(\mathrm{CN}^{\mathrm{t} B u}\right)_{2} \mathrm{Cl}\left\{\mathrm{N}\left(\mathrm{CH}_{2} \mathrm{CH}_{2} \mathrm{P}^{\mathrm{t}} \mathrm{Bu}_{2}\right)\left(=\mathrm{CHCH}_{2} \mathrm{P}\left({ }^{\mathrm{t}} \mathrm{Bu}\right)_{2}=\mathrm{NH}\right)\right\}\right]^{\mathrm{BAr}}{ }_{24}^{\mathrm{F}}\left(\mathbf{1 1}^{\mathrm{BAr}}{ }_{24}^{\mathrm{F}}\right) . \quad 237$

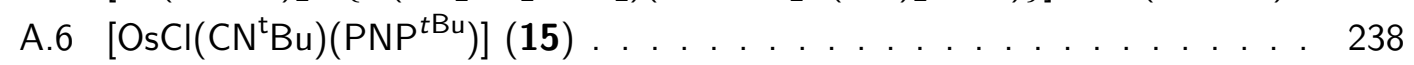

A.7 $\left[\left(\mu-\mathrm{N}_{2}\right)\left\{\operatorname{ReCl}\left(\mathrm{PNP}^{t \mathrm{Bu}}\right)\right\}_{2}\right](\mathbf{X X}) \ldots \ldots \ldots \ldots \ldots \ldots \ldots$

A.8 $\left[\mathrm{ReHCl}\left(\mathrm{PNP}^{t \mathrm{Bu}}\right)\right](\mathbf{1 7}) \ldots \ldots \ldots \ldots \ldots . \ldots \ldots . \ldots \ldots . \ldots \ldots$

A.9 $\left[\left(N, N-\mathrm{C}_{2} \mathrm{H}_{4} \mathrm{~N}_{2}\right)\left\{\mathrm{ReCl}\left(\mathrm{PNP}^{t \mathrm{Bu}}\right)\right\}_{2}\right]^{(\mathrm{OTf})_{2}}\left(\mathbf{1 9}^{(\mathrm{OTf})_{2}}\right) \ldots \ldots \ldots . \ldots . \ldots 243$

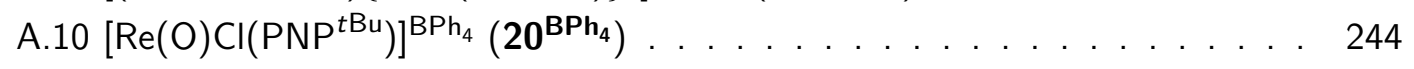

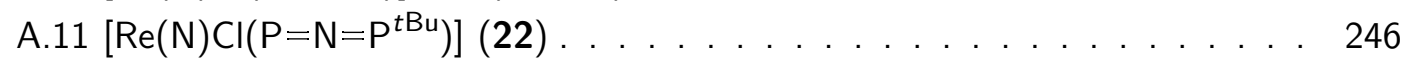

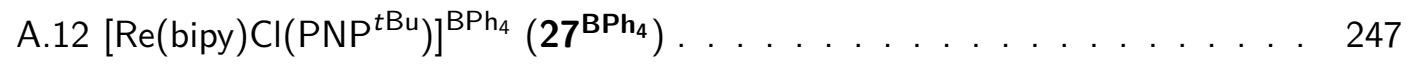

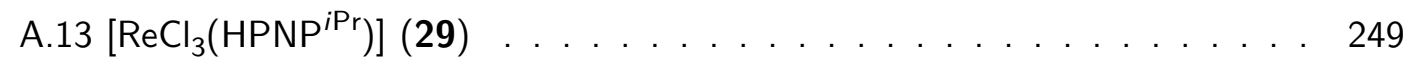

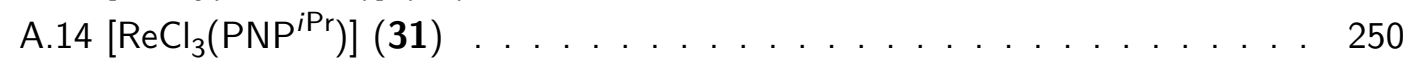

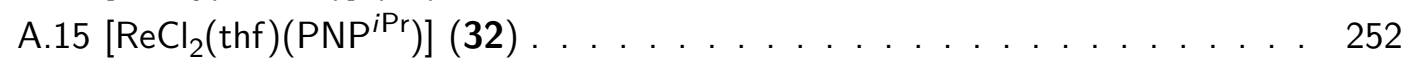




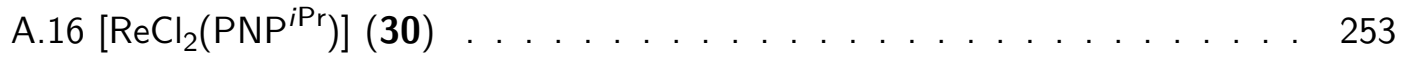

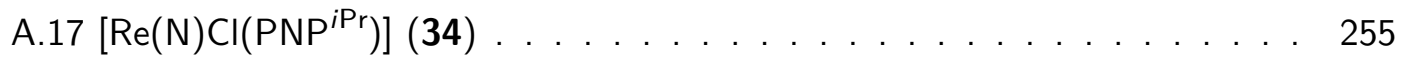

A.18 $\left[\mathrm{ReCl}\left(\mathrm{N}_{2}\right)_{2}\left(\mathrm{HPNP}^{i \mathrm{Pr}}\right)\right](35) \ldots \ldots \ldots \ldots \ldots \ldots . \ldots \ldots . \ldots \ldots$

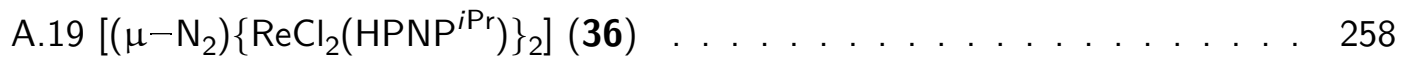

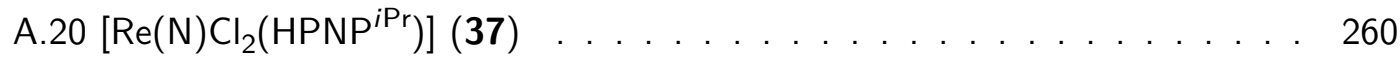

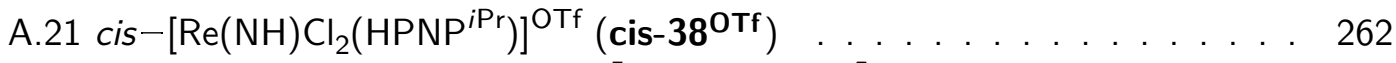

A.22 trans- $\left[\operatorname{Re}(\mathrm{NH}) \mathrm{Cl}_{2}\left(\mathrm{HPNP}^{i \mathrm{Pr}}\right)\right]^{\mathrm{BAr}_{24}^{\mathrm{F}}}\left(\right.$ trans-38 $\left.^{\mathrm{BAr}_{24}^{\mathrm{F}}}\right) \ldots \ldots \ldots \ldots . \ldots . \ldots 264$

A.23 $\left[\operatorname{Re}\left(\mathrm{NBAr}_{18}^{\mathrm{F}}\right) \mathrm{Cl}_{2}\left(\mathrm{HPNP}{ }^{\mathrm{iPr}}\right)\right](39) \ldots \ldots \ldots \ldots \ldots . \ldots \ldots . \ldots \ldots$

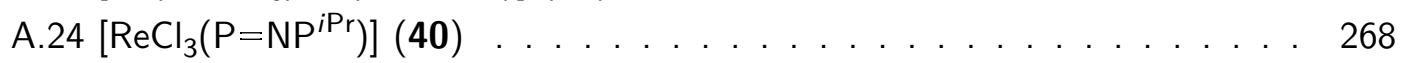

$\begin{array}{lll}\text { B List of chemical compounds } & 271\end{array}$

$\begin{array}{lll}\text { C List of abbreviations } & 275\end{array}$ 



\section{Part I}

\section{Introduction}

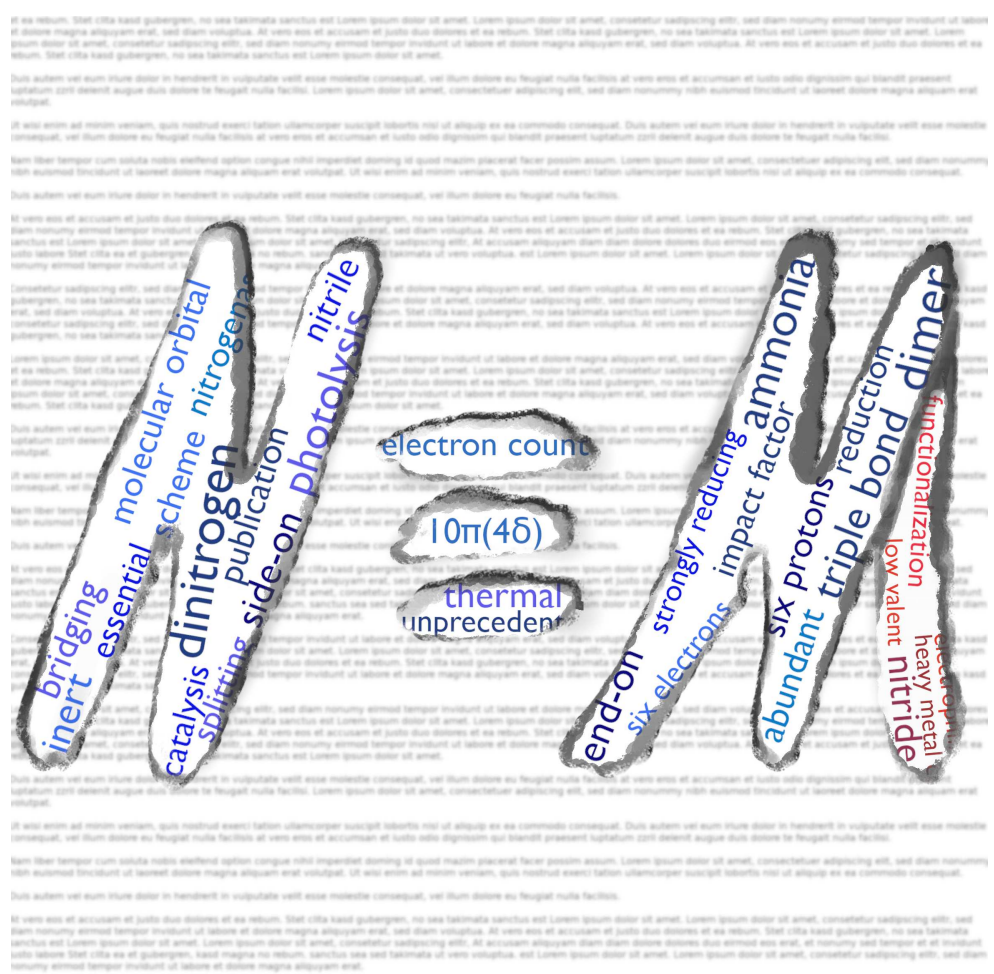





\title{
Molecular dinitrogen, nitrogenase and the Haber-Bosch process
}

And no one, just no one, no one will break this

\author{
- Heaven Shall Burn \\ "Endzeit" on "Iconoclast"
}

Nitrogen is one of the main elements in organic matter, next to hydrogen, carbon and oxygen, being incorporated in almost every bio-relevant molecule. Consequently, life on earth relies to a great extend on the availability of readily metabolizable nitrogen sources. Since nitrogen is continuously removed from the nutrient cycle by embedding in sediments or release of $\mathrm{N}_{2}$ by sequential nitrification and denitrification of ammonia, processes to make molecular dinitrogen accessible for living organisms are essential to life. ${ }^{[2]}$ Molecular nitrogen gas constitutes about $78 \%$ of earth's atmosphere, making it the most abundant unbound element available. However, dinitrogen features an exceptionally strong $\mathrm{N} \equiv \mathrm{N}$ triple bond, with a bond dissociation enthalpy (BDE) of $941 \mathrm{~kJ} \mathrm{~mol}^{-1}$ comparable to acetylene $\left(962 \mathrm{~kJ} \mathrm{~mol}^{-1}\right)$ or carbon monoxide $\left(1070 \mathrm{~kJ} \mathrm{~mol}^{-1}\right)$, the only diatomic molecule with a higher $\mathrm{BDE}$ than $\mathrm{N}_{2} \cdot{ }^{[3]}$ Additionally, cleaving the first bond already requires almost half of the entire BDE (i.e. $410 \mathrm{~kJ} \mathrm{~mol}^{-1}$ while for acetylene it is only $222 \mathrm{~kJ} \mathrm{~mol}^{-1}$, thus less than one quarter) and in contrast to $\mathrm{CO}, \mathrm{N}_{2}$ does not have a dipole moment. Both features render potential conversions highly demanding as they add an even higher kinetic barrier on top of the already challenging thermodynamic requirements. Many other properties like a proton affinity lower than that of methane $\left(\mathrm{N}_{2}: 493.8 \mathrm{~kJ} \mathrm{~mol}^{-1}, \mathrm{CH}_{4}: 543.5 \mathrm{~kJ} \mathrm{~mol}^{-1}\right)$ or an ionization potential comparable to that of argon $\left(\mathrm{N}_{2}: 15.58 \mathrm{eV}, \mathrm{Ar}: 15.75 \mathrm{eV}\right)$ and an extremely high energy gap between the highest occupied molecular orbital (HOMO) and the lowest unoccupied molecular orbital (LUMO) $(10.82 \mathrm{eV})$ make redox chemistry almost impossible and are in line with the inertness of dinitrogen. ${ }^{[4]}$

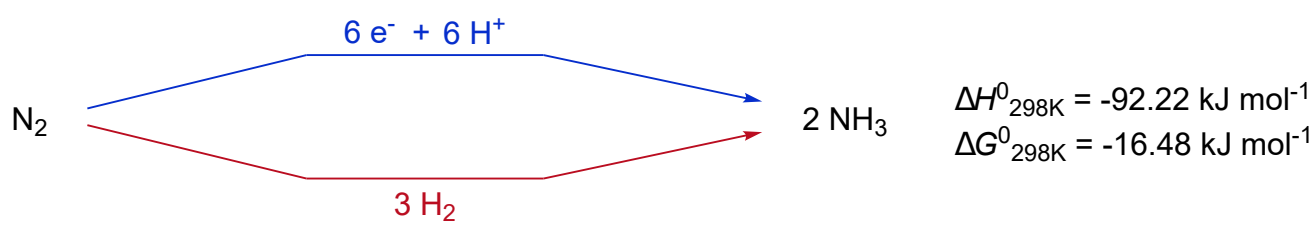

Scheme 1.1. Synthesis of ammonia via sequential proton and electron transfer (top) or direct reaction with $\mathrm{H}_{2}$ (bottom). 
While these properties highlight the difficulties connected to $\mathrm{N}_{2}$ fixation, there are actually some conversions which are at least thermodynamically feasible, with the conversion to ammonia being the most prominent example (see Scheme 1.1). In order to achieve nitrogen fixation in nature, sophisticated enzymes evolved, the so-called nitrogenases, found in bacteria, which form ammonia in approx. $2.5 \times 10^{11} \mathrm{~kg} /$ year. ${ }^{[5]}$ The catalytically active center in the most thoroughly investigated nitrogenase enzyme is a [7Fe-9S-Mo-C-homocitrate] cluster, the iron-molybdenum cofactor (FeMo-co), which was recently proven to contain an interstitial carbon atom. ${ }^{[6,7]}$ Additionally, nitrogenase systems that are based on a [VFe] or a pure $[\mathrm{Fe}]$ cofactor are known. ${ }^{[8]}$ Under optimal conditions, nitrogen fixation by these enzymes follows a stepwise $6 \mathrm{H}^{+} / 6 \mathrm{e}^{-}$reduction of $\mathrm{N}_{2}$ to ammonia according to the following reaction:

$$
\mathrm{N}_{2}+8 \mathrm{e}^{-}+16 \mathrm{MgATP}+8 \mathrm{H}^{+} \longrightarrow 2 \mathrm{NH}_{3}+\mathrm{H}_{2} \uparrow+16 \mathrm{MgADP}+16 \mathrm{P}_{\mathrm{i}}
$$

The "waste" of two $\mathrm{H}^{+}$and two $\mathrm{e}^{-}$to produce $\mathrm{H}_{2}$ from the so-called $E_{4}$ state has been proposed to be due to reductive activation of the FeMo-co necessary for initial $\mathrm{N}_{2}$ binding and $\mathrm{N}_{2} \mathrm{H}_{2}$ formation. ${ }^{[9]}$ Afterwards, protons and electrons are delivered in an alternating pathway (i.e. $\mathrm{NH}=\mathrm{NH} \underset{\mathrm{e}^{-}}{\stackrel{\mathrm{H}^{+}}{\longrightarrow}} \mathrm{NH}_{2}-\mathrm{NH} \underset{\mathrm{e}^{-}}{\stackrel{\mathrm{H}^{+}}{\longrightarrow}} \mathrm{NH}_{2}-\mathrm{NH}_{2} \underset{\mathrm{e}^{-}}{\stackrel{\mathrm{H}^{+}}{\longrightarrow}} \mathrm{NH}_{3} \uparrow+\mathrm{NH}_{2} \underset{\mathrm{e}^{-}}{\stackrel{\mathrm{H}^{+}}{\longrightarrow}}$ $\mathrm{NH}_{3} \uparrow$ ) to release two equivalents of ammonia (compare Scheme 3.1).

Until the early $20^{\text {th }}$ century, enzymes remained the main source of fixed nitrogen. This changed drastically with the development of laboratory scale ammonia production by Fritz Haber, patented 1908, and the subsequent upscaling of this process to a multi-ton ammonia plant by Carl Bosch. The resulting Haber-Bosch process utilizes heterogeneous iron or ruthenium, which are the most active catalysts next to highly expensive osmium or radioactive uranium. ${ }^{[10]}$ The mechanism of ammonia formation in this reaction is fundamentally different from the pathway in nitrogenase enzymes. Initially, the dihydrogen as well as dinitrogen molecules undergo full bond scission at the surface to form chemisorbed $\mathrm{H}$ and $\mathrm{N}$ atoms, which then react in a Langmuir-Hinshelwood type mechanism to form ammonia. By the formation of surface bound nitrides, the high energy demand for $\mathrm{N} \equiv \mathrm{N}$ bond cleavage is compensated and the reaction $\mathrm{N}_{2} \longrightarrow 2 \mathrm{~N}_{\text {ad }}$ is exothermic by $\Delta H^{0}=-17 \mathrm{~kJ} \mathrm{~mol}^{-1}$ (see Figure 1.1). ${ }^{[11]}$ In these plants, 300 to $500^{\circ} \mathrm{C}$ and pressures up to $300 \mathrm{~atm}$ are employed in order to drive the reaction, especially the rate determining $\mathrm{N}_{2}$ chemisorption. Including all industrially necessary steps, e.g. hydrogen production by steam reforming, purification or the process itself, synthesis of ammonia requires roughly $485 \mathrm{~kJ} \mathrm{~mol}^{-1}$ and at a world production of roughly $140 \mathrm{Mt}$ per year overall about $1.4 \%$ of the world energy production are consumed in this process. ${ }^{[10,12]}$

The high energy demand of this process as well as the related scientific challenges have triggered several decades of intense research efforts to the development of alternative, more environmentally friendly processes. Apart from homogeneous approaches, which will be 


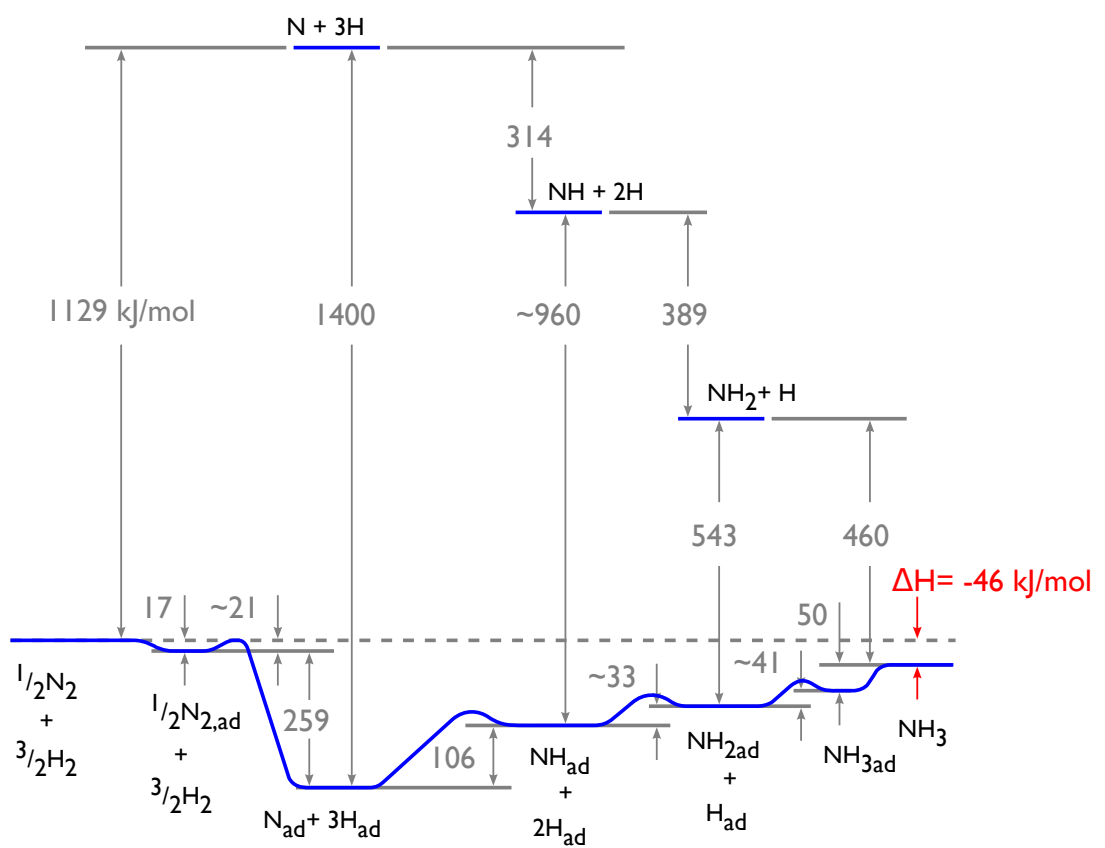

Fig. 1.1. Potential energy surface of ammonia formation in the iron catalyzed Haber-Bosch process. ${ }^{[11]}$ Energies are given in $\mathrm{kJ} \mathrm{mol}^{-1}$.

covered in Section 3.1, especially the field of heterogeneously catalyzed electrochemical ammonia synthesis from $\mathrm{N}_{2}$ and water has very recently gained a lot of attraction as an appealing approach where the necessary energy can potentially be derived from renewable resources. $[13,14]$

While the major part of industrially synthesized ammonia is used for production of fertilizers (approx. $88 \%$ ) $^{[12]}$ and therefore builds the foundation of modern agriculture, it also is the main source for the formation of industrially relevant organic molecules, building blocks and fine chemicals containing nitrogen atoms. In this context, the development of homogeneous catalysts which follow a Haber-Bosch type reaction, i.e. which undergo initial spitting of $\mathrm{N}_{2}$ into terminal nitrido complexes, provides a promising approach. Since a broad range of functionalization reactions is available for transition metal nitride complexes, ${ }^{[15]}$ such $\mathrm{N}_{2}$ derived nitrides open the possibility of directly incorporating $\mathrm{N}_{2}$ into higher value organic molecules, omitting the superfluous step of ammonia production. These functionalization reactions require well defined reaction centers and tuning of the active environment and therefore homogeneous transition metal complexes are predestined and can be assumed to perform with much better selectivity and efficiency than heterogeneous systems.

Consequently, homogeneous transition metal complexes involved in $\mathrm{N}_{2}$ fixation schemes as well as possibilities of $\mathrm{N}-\mathrm{X}$ bond formation and functionalization of nitrides in general will be the focus of this introduction. 



\title{
Binding of dinitrogen to transition metal complexes
}

\author{
Believe that together \\ We can change for the best
}

\section{- Architects}

"Untitled II" on "Lost Forever // Lost Together"

In general, dinitrogen is a rather poor ligand, acting as a weak $\sigma$-donor and an even weaker $\pi$-acceptor. However, ever since the discovery of the very first $N_{2}$ complex in 1965, i.e. $\left[\mathrm{Ru}\left(\mathrm{NH}_{3}\right)_{5} \mathrm{~N}_{2}\right]^{2+},{ }^{[17]}$ various binding modes of molecular dinitrogen to transition metal complexes have been reported in literature. Overall, $\mathrm{N}_{2}$ complexes can be classified in four main groups, i.e. (I) end-on terminal $\left(\eta^{1}-\mathrm{N}_{2}\right)$, (II) end-on bridging $\left(\mu-\eta^{1}: \eta^{1}-\mathrm{N}_{2}\right)$, (III) side-on bridging $\left(\mu-\eta^{2}: \eta^{2}-\mathrm{N}_{2}\right)$ and (IV) side-on-end-on bridging $\left(\mu-\eta^{2}: \eta^{1}-\mathrm{N}_{2}\right)$, of which (I) is the most dominant coordination mode observed (see Figure 2.1). In these complexes, the bond order between the two nitrogen atoms can vary drastically either due to partial reduction by the metal center(s) or after subsequent protonation and reduction to $\mathrm{N}_{2} \mathrm{H}_{\mathrm{X}}$ ligands. In order to judge the degree of activation in these complexes, the $\mathrm{N}-\mathrm{N}$ bond distances as well as

$$
M-N \equiv N \quad M-N \equiv N-M
$$

(I)
(II)<smiles>[M]1N=N1</smiles>

(III)<smiles></smiles>

(IV)

Fig. 2.1. Main classes of $\mathrm{N}_{2}$ binding modes in complexes found in literature.

Tab. 2.1. Bond lengths and stretching vibrations for free $\mathrm{N}_{2}$ and coordinated moieties in different reduction states. ${ }^{[16]}$

\begin{tabular}{c|cc}
\hline (coordinated) N-N moiety & bond lenght / $\AA$ & stretching vibration $/ \mathrm{cm}^{-1}$ \\
\hline free $\mathrm{N}_{2}$ & 1.10 & 2331 \\
$\mathrm{~N} \equiv \mathrm{N}$ & $1.10-1.20$ & $2331-1700$ \\
{$[\mathrm{~N} \equiv]^{-}$} & n.a. & n.a. \\
free $\mathrm{N}_{2} \mathrm{H}_{2}$ & 1.25 & $1583 / 1529$ \\
{$\left[\mathrm{~N}=\mathrm{N}^{2-}\right.$} & $1.20-1.35$ & $1700-1200$ \\
{$[\mathrm{~N}=\mathrm{N}]^{3-}$} & 1.40 & $1040-989$ \\
free $\mathrm{N}_{2} \mathrm{H}_{4}$ & 1.45 & 885 \\
{$[\mathrm{~N}-\mathrm{N}]^{4-}$} & $1.40-1.60$ & $1100-700$ \\
\hline
\end{tabular}


stretching vibrations are commonly employed as characteristic properties, ${ }^{[18,19]}$ which should be inversely correlated according to Badger's rule. ${ }^{20]}$ The typically observed parameters are summarized in Table 2.1. In general, the different degrees of activation are often restricted to the different binding motifs. For example, terminal $\mathrm{N}_{2}$ complexes are always rather unactivated with an intact $\mathrm{N} \equiv \mathrm{N}$ triple bond, whereas higher degrees of activation, i.e. $\mathrm{N}_{2}^{2-}$ or $\mathrm{N}_{2}^{4-}$ ligands, are found in dinuclear complexes (especially in side-on bridged complexes).

\subsection{End-on bound $\mathrm{N}_{2}$}

As stated above, the vast majority of transition metal complexes do form mononuclear, terminal, end-on bound $\mathrm{N}_{2}$ complexes, often with a $d^{6}$ electronic configuration of the metal center. ${ }^{[21]}$ The bonding in such complexes can be understood as a combination of $\sigma$ donation of the $\mathrm{N}_{2}$ lone pair into a suitable, empty $d$-orbital and a $\pi$ backdonation from filled $d$-orbitals into the $\mathrm{N}^{\pi^{*}} \mathrm{~N}$ orbitals, comparable to the situation found in $\mathrm{CO}$ (see Figure 2.2). However, due to the large HOMO-LUMO gap in $\mathrm{N}_{2}$ (see Section 1 ) the overlap is often rather poor and activation of the $\mathrm{N} \equiv \mathrm{N}$ bond occurs only to a minor degree.
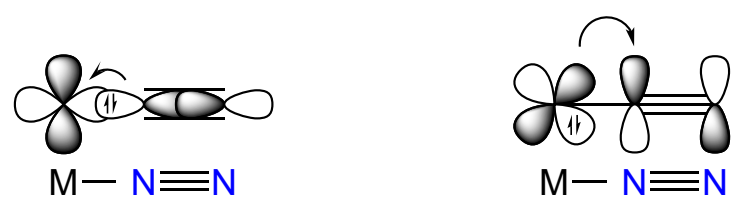

Fig. 2.2. Orbital interactions between the metal center and the $\mathrm{N}_{2}$ ligand in terminal end-on bound complexes.

A higher degree of activation is typically found in dinuclear complexes featuring an end-on bridging $\mathrm{N}_{2}$ ligand (i.e. coordination mode (II), see Figure 2.1). The very first complex with such a $\mu-\eta^{1}: \eta^{1}$ dinitrogen ligand, i.e. $\left[\left(\mu-\mathrm{N}_{2}\right)\left\{\mathrm{Ru}\left(\mathrm{NH}_{3}\right)_{5}\right\}_{2}\right]^{\left(\mathrm{BF}_{4}\right)_{4}}$, was discovered in 1968 by Taube and coworkers. ${ }^{[22]}$ Ever since, several such complexes were characterized. ${ }^{[16,18,19]}$ The interaction with two metal centers at the same time can lead to significant charge transfer of the metal atoms to the $\mathrm{N}_{2}$ bridge and thus to a decrease of the $\mathrm{N}-\mathrm{N}$ bond order. This was first rationalized by molecular orbital (MO) considerations regarding the $\{M N N M\}$ core by Gray and Chatt for complexes in an (idealized) fourfold symmetry. ${ }^{[23,24]}$ This picture was later adopted by Fryzuk and veryfied by means of semi-empirical calculations, while Bercaw and Cummins developed a closely related MO scheme for complexes in threefold symmetry. ${ }^{[25-27]}$ Combining the proposals and findings made in this work, a quite elaborate picture evolves (see Scheme 2.1). Assuming the $\{$ MNNM $\}$ core to be oriented along the z-axis, the frontier molecular orbitals (FMOs) of such dimers are constructed from the metal $d$ orbitals and nitrogen $p$ orbitals. The metal $d_{x z}$ and $d_{y z}$ as well as the nitrogen $p_{x}$ and $p_{y}$ orbitals are combined to form four sets of twofold degenerated, perpendicular $\pi$ MOs, i.e. $1 e_{\mathrm{u}}, 1 \mathrm{e}_{\mathrm{g}}, 2 \mathrm{e}_{\mathrm{u}}, 2 \mathrm{e}_{\mathrm{g}}$. The metal $d_{z^{2}}$ and nitrogen $p_{z}$ orbitals can be combined into a $\sigma$ 


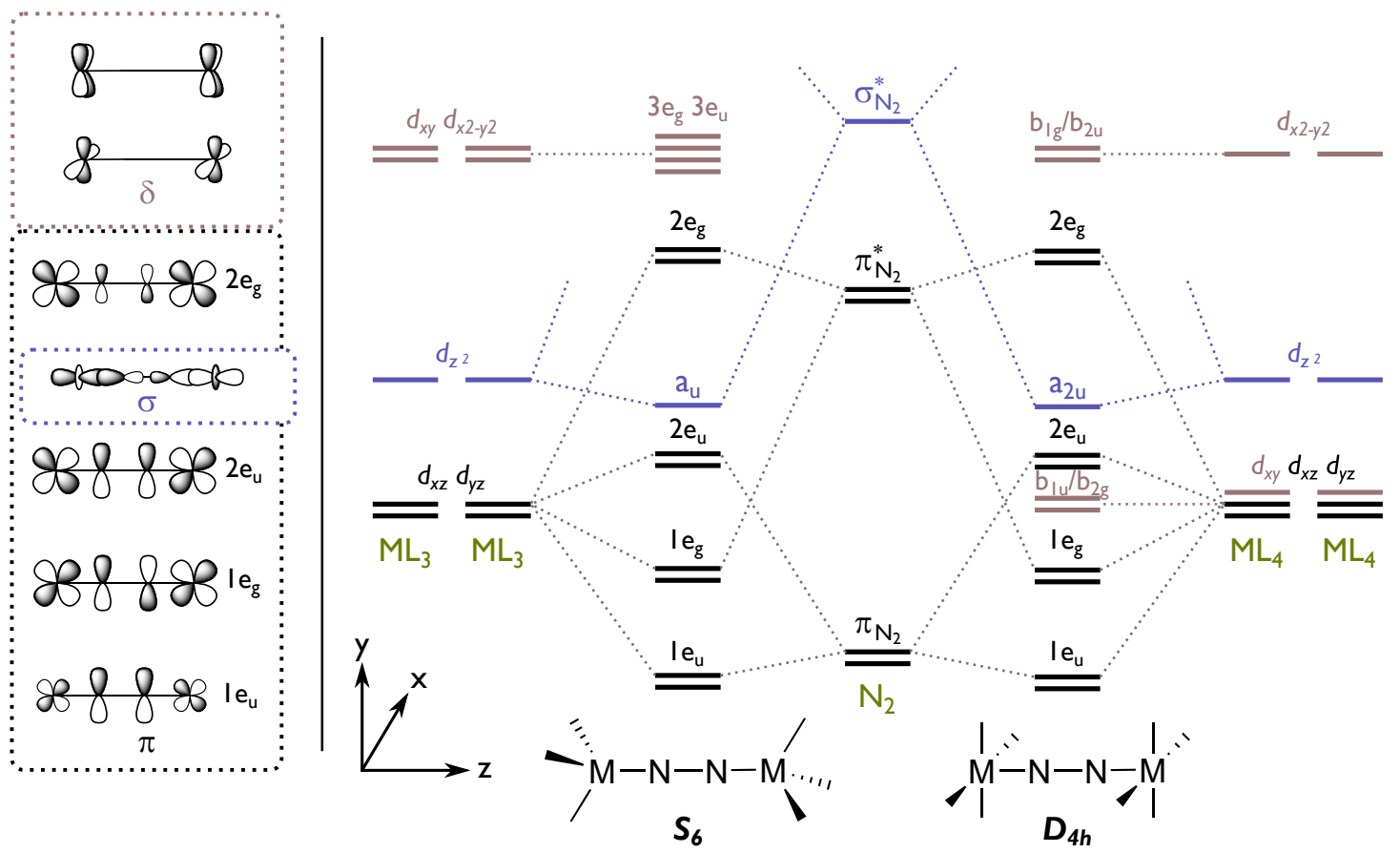

Scheme 2.1. The frontier molecular orbital scheme of end-on bridging dinuclear $\mathrm{N}_{2}$ complexes, comprised of $\sigma$ (blue), $\pi$ (black) and $\delta$ (red) symmetric molecular orbitals. ${ }^{[16]}$

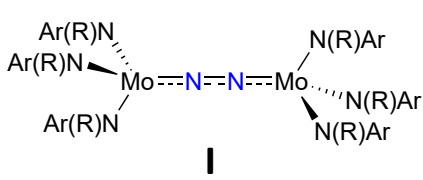

$$
\begin{gathered}
d_{\mathrm{NN}}=1.212(2) / 1.217(2) \AA \\
v_{\mathrm{NN}}=1630 \mathrm{~cm}^{-1} \\
\text { SQUID: } \mu_{\mathrm{eff}}=2.42 \mu_{\mathrm{B}} \\
10 \pi
\end{gathered}
$$

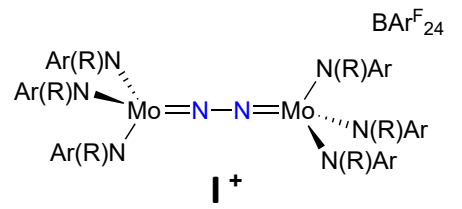

$$
\begin{gathered}
d_{\mathrm{NN}}=1.239(4) \AA \\
v_{\mathrm{NN}}=1503 \mathrm{~cm}^{-1} \\
\text { SQUID: } \mu_{\mathrm{eff}}=1.96 \mu_{\mathrm{B}} \\
9 \pi
\end{gathered}
$$

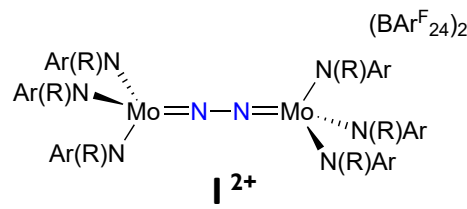

$d_{\mathrm{NN}}=1.265(5) \AA$

$v_{\mathrm{NN}}=1349 \mathrm{~cm}^{-1}$

SQUID: -- $(\mathrm{S}=0)$

$8 \pi$

Fig. 2.3. Redox series of $\mathbf{I}^{\mathbf{n}+}\left(\mathrm{n}=0-2, \mathrm{Ar}=3,5-\left(\mathrm{C}_{6} \mathrm{H}_{3}(\mathrm{Me})_{2}\right), \mathrm{R}=\mathrm{C}\left(\mathrm{CD}_{3}\right)_{2} \mathrm{CH}_{3}\right) \cdot{ }^{[27,28]}$

space, of which only the $\mathrm{M}^{-}-\mathrm{N}^{\sigma *}-\mathrm{N} \stackrel{\sigma}{-} \mathrm{M}$ orbital (of $\mathrm{a}_{\mathrm{u}}$ or $\mathrm{a}_{2 \mathrm{u}}$ symmetry, depending on the point group) is of relevance to the FMO space. Additionally, there are the $\delta$ symmetric MOs derived from the metal $d_{x y}$ and $d_{x^{2}-y^{2}}$ orbitals, which do vary quite significantly in the different dimers. In Cummins's $S_{6}$ symmetric dimer $\left[\left(\mu-\mathrm{N}_{2}\right)\left\{\mathrm{Mo}(\mathrm{N}(\mathrm{R}) \mathrm{Ar})_{3}\right\}_{2}\right](\mathrm{I}, \mathrm{Ar}=$ 3,5- $\left.\left(\mathrm{C}_{6} \mathrm{H}_{3}\left(\mathrm{CH}_{3}\right)_{2}\right), \mathrm{R}=\mathrm{C}\left(\mathrm{CD}_{3}\right)_{2} \mathrm{CH}_{3}\right)$, the amide donors interact strongly with the $\delta$ orbitals, raising them energetically high within the virtual orbital space. ${ }^{[27]}$ However, in $\mathrm{N}_{2}$ dimers with fourfold rotation symmetry, these orbitals are orthogonal to the ligand donor orbitals and therefore drop in energy, eventually becoming available for population.

As can be seen from the qualitative MO pictures in Scheme 2.1, the $\pi$ symmetric $e_{u}$ orbitals are mainly of $\mathrm{N}-\mathrm{N}$ bonding character, while the $e_{\mathrm{g}}$ orbitals are mainly antibonding with respect to the $\mathrm{N}_{2}$ bridge and their population should reduce the $\mathrm{N}-\mathrm{N}$ bond order. Due to rather poor orbital overlap, the $1 e_{u}$ orbital is predicted to be mainly located at the $\mathrm{N}_{2}$ bridge, while the $2 e_{\mathrm{g}}$ orbital is rather metal centered. Consequently, population of these orbitals 
contribute only to a minor degree to activation of the N-N bond. ${ }^{[29]}$ In contrast to this, the $1 \mathrm{e}_{\mathrm{g}}$ and $2 \mathrm{e}_{\mathrm{u}}$ orbitals are rather delocalized and Cummins and coworkers proved the influence of population of the latter by preparation of the redox series $\mathbf{I}^{\mathbf{n}+}(\mathbf{n}=0,1,2)$ (see Figure 2.3). ${ }^{[27,28]}$ In neutral $\mathbf{I}$, the constructed $\mathrm{MO}$ scheme in Scheme 2.1 is filled with a total of 10 electrons (two per nitrogen, three per formal Mo(III) center), ending up with a $10 \pi$ electronic configuration in the $\{M N N M\}$ core and both $2 e_{u}$ orbitals being singly occupied. This electronic structure is confirmed by superconducting quantum interference device (SQUID) measurements (see Figure 2.3). A comparison of the Raman shifts of the N-N stretching vibrations with the crystallographically determined $\mathrm{N}-\mathrm{N}$ bond distances shows a decrease of the $\mathrm{N}-\mathrm{N}$ bond order upon oxidation, in line with a stepwise depopulation of a $\mathrm{MO}$ with $\mathrm{N}-\mathrm{N}$ bonding character (i.e. $2 \mathrm{e}_{\mathrm{u}}$ ).

This picture is also consistent with the bond metrics of Taube's $D_{4 h}$ symmetric dimer

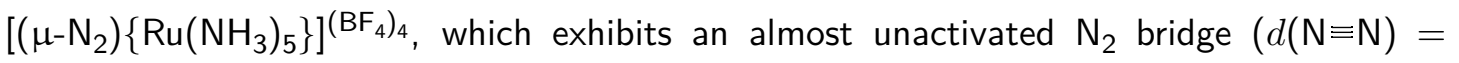
$\left.1.124(15) \AA, \nu_{\mathrm{N} \equiv \mathrm{N}}=2100 \mathrm{~cm}^{-1}\right)$, in line with the expected $12 \pi 4 \delta$ configuration and thus full population of the $2 \mathrm{e}_{\mathrm{u}}$ orbitals. ${ }^{[22,23,30]}$ Also, the $8 \pi 3 \delta$ electron complex $\left[\left\{\left(\mathrm{PMe}_{2} \mathrm{Ph}\right)_{4} \mathrm{ClRe}\right\}(\mu-\right.$ $\left.\left.\mathrm{N}_{2}\right)\left\{\mathrm{MoCl}_{4}(\mathrm{OMe})\right\}\right]$ shows a quite high degree of activation $\left(d(\mathrm{~N}=\mathrm{N})=1.21 \AA, \nu_{\mathrm{N} \equiv \mathrm{N}}=\right.$ $\left.1600 \mathrm{~cm}^{-1}\right)$.[31]

Another interesting redox series by Chirik and coworkers was published for $\left[\left(\mu-\mathrm{N}_{2}\right)\left\{\left({ }^{\mathrm{Ph}} \mathrm{Tpy}\right)\right.\right.$ $\left.\left(\mathrm{PPh}_{2} \mathrm{Me}\right)_{2} \mathrm{Mo}_{2}\right]^{\left(\mathrm{BAr}_{24}^{\mathrm{F}}\right)_{\mathrm{n}}}\left(\mathrm{II}^{\mathbf{n}+}, \mathrm{n}=0-4,{ }^{\mathrm{Ph}} \mathrm{Tpy}=44^{\prime}-\mathrm{Ph}-2,2^{\prime}, 6^{\prime} 2^{\prime \prime}\right.$-terpyridine $) .{ }^{[32]}$ Starting from the formal $\mathrm{Mo}(\mathrm{I}) / \mathrm{Mo}(\mathrm{I})$ dication, which should exhibit a $10 \pi 4 \delta$ electronic configuration, ${ }^{1}$ reversible one- and two-electron reductions and oxidations were possible. From investigation of all resulting complexes, the authors developed a closely related electronic structure, but pertubated by mixing with the terpyridine ligand $\pi$ system, which lifts the degeneracy of the $M^{\pi *} N^{\pi}-N^{\pi *} M$ orbitals and thus leads to a singlet ground state for the dication. Interestingly, oxidation (= depopulation of an N-N bonding orbital) as well as reduction (= population of an $\mathrm{N}-\mathrm{N}$ antibonding orbital) does lead to further activation of

${ }^{1}$ These are just formal oxidation states. A computationally study suggests the system to be best decribed as two $\mathrm{Mo}(\mathrm{II})$ centers bridged by an $\mathrm{N}_{2}^{2-}$ ligand.

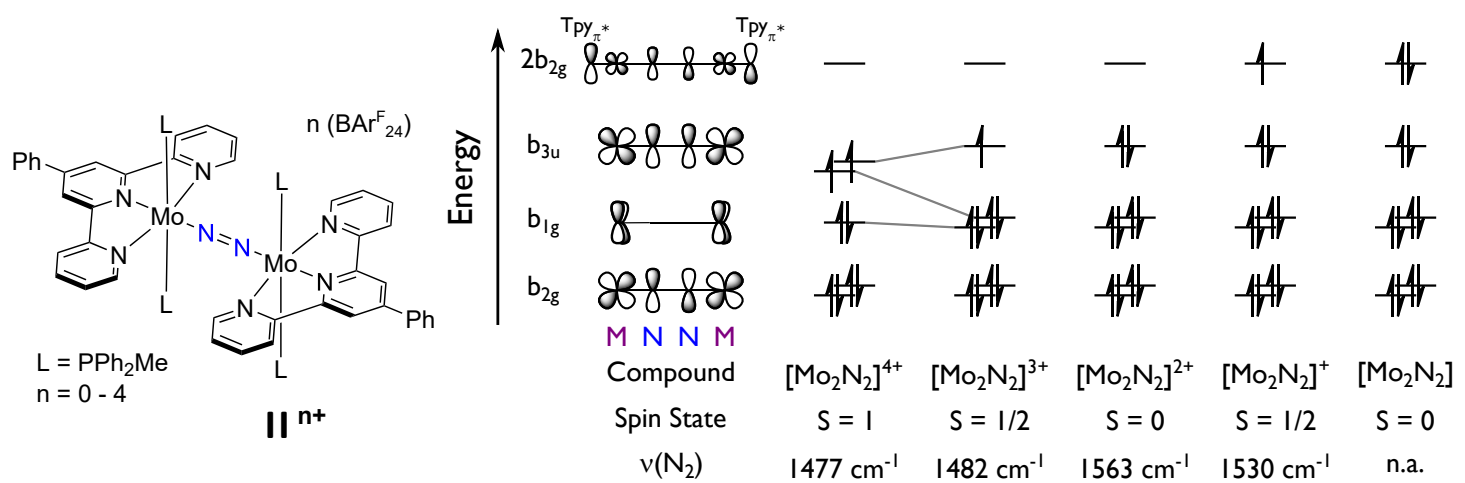

Fig. 2.4. Left: Chirik's molybdenum terpyridine complex redox series. Right: Qualitative FMO scheme of all five accessible redox states and the corresponding spin states and N-N stretching vibrations. 
the $\mathrm{N}_{2}$ bridge. In contrast to Cummins' system, no splitting of the $\mathrm{N}_{2}$ bridge was observed and no $\mathrm{N}$ centered functionalization reactions are reported. In recent, very sophisticated studies, the photophysics of this system were investigated and the terpyridine ligand was shown to act as a light harvesting moiety where excitation leads to an initial metal-ligand charge transfer (MLCT) to the terpyridine ligand followed by inter system crossing (ISC) which shifts electron density to the $\mathrm{N}_{2}$ bridge via Fermi-resonance coupling between the terpyrindine breathing and the $\mathrm{N}_{2}$ stretching vibrational modes. ${ }^{[33,34]}$ However the excited-state lifetimes are pretty short $(\tau=23-26 \mathrm{ps})$ rendering photoinduced bimolecular reactivity of this molecule unlikely.

The influence of different metals on the activation of $\mathrm{N}_{2}$ was investigated thoroughly by Sita and coworkers, who reported eight different transition metal complexes bridged by an $\mathrm{N}_{2}$ ligand with identical supporting ligands, i.e. $\left[\left(\mu-\mathrm{N}_{2}\right)\left\{\mathrm{M}\left(\mathrm{Cp}^{*}\right)(\mathrm{am})\right\}_{2}\right](\mathrm{M}=\mathrm{Ti}, \mathrm{Zr}, \mathrm{Hf}$, $\left.\mathrm{V}, \mathrm{Nb}, \mathrm{Ta}, \mathrm{Mo}, \mathrm{W} ; \mathrm{am}=\mathrm{N}\left({ }^{\mathrm{i}} \mathrm{Pr}\right) \mathrm{C}(\mathrm{Me}) \mathrm{N}\left({ }^{\mathrm{i}} \mathrm{Pr}\right)\right) \cdot{ }^{\left[{ }^{[3-38]}\right.}$ From all these complexes despite the $\mathrm{Zr}$ and $\mathrm{Hf}$ compounds, end-on $\mu-\eta^{1}: \eta^{1}-\mathrm{N}_{2}$ complexes could be obtained and structurally characterized (Me group of the am ligand is exchanged with $\mathrm{NMe}_{2}$ in the $\mathrm{Zr}$ and $\mathrm{Ph}$ in the $\mathrm{Nb}$ complex). The degree of activation of the dinitrogen bridge is expressed in the significantly deviating $\mathrm{N}-\mathrm{N}$ bond distances (see Table 2.2). The authors describe the $\mathrm{Ti}$ complex as formal $\mathrm{Ti}(\mathrm{III})$ centers with $d^{1}$ electron count, bridged by an $\mathrm{N}_{2}^{2-}$ ligand. The higher homologues, i.e. the $\mathrm{Zr}$ and $\mathrm{Hf}$ complexes are more easily oxidized and can reach the formal $\mathrm{M}(\mathrm{IV})$ oxidation state, yielding formally $\mathrm{N}_{2}^{4-}$ bridges and ending in a side-on $\mu-\eta^{2}: \eta^{2}$ coordination mode. ${ }^{[36]}$ The group 5 complexes initially all form end-on bridging $\mathrm{N}_{2}$ dimers, of which the $\mathrm{Nb}$ and $\mathrm{Ta}$ complexes are shown to undergo thermal rearrangement into bridging bis-nitrido complexes proposed to proceed via the $\mu-\eta^{2}: \eta^{2}$ coordination mode, which reflects the reduced reduction potential upon moving from group 4 to 5 . The remarkable thermal stability of the vanadium complex towards this reaction was explained by the authors as being due to a changes in the ground state electronic structure, comparable to the effects discussed by Mindiola for a related system. ${ }^{[39]}$ Moving further right to group 6, i.e. the Mo and $\mathrm{W}$ complexes, the $\mathrm{N}-\mathrm{N}$ bond distances again become shorter and formulation of the

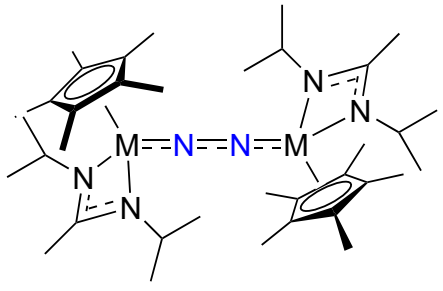

Fig. 2.5. Isostructural complexes of $\mathrm{Ti}, \mathrm{V}$, $\mathrm{Nb}, \mathrm{Ta}$, Mo and $\mathrm{W}$, prepared by Sita.
Tab. 2.2. Coordination modes and bond lengths in Sita's $\mathrm{M}\left(\mathrm{Cp}^{*}\right)(\mathrm{am}) \mathrm{N}_{2}$ complexes.

\begin{tabular}{l|cc}
\hline metal & coordination mode & $\mathrm{N}-\mathrm{N}$ bond distance / $\AA$ \\
\hline $\mathrm{Ti}^{[35]}$ & $\mu-\eta^{1}: \eta^{1}-\mathrm{N}_{2}$ & $1.270(2)$ \\
$\mathrm{Zr}[36]$ & $\mu-\eta^{2}: \eta^{2}-\mathrm{N}_{2}$ & $1.518(2)$ \\
$\mathrm{Hf}[36]$ & $\mu-\eta^{2}: \eta^{2}-\mathrm{N}_{2}$ & $1.611(4)$ \\
\hline $\mathrm{V}^{[37]}$ & $\mu-\eta^{1}: \eta^{1}-\mathrm{N}_{2}$ & $1.225(2)$ \\
$\mathrm{Nb}^{[37]}$ & $\mu-\eta^{1}: \eta^{1}-\mathrm{N}_{2}$ & $1.300(3)$ \\
$\mathrm{Ta}^{[38]}$ & $\mu-\eta^{1}: \eta^{1}-\mathrm{N}_{2}$ & $1.313(4)$ \\
\hline $\mathrm{Mo}^{[35]}$ & $\mu-\eta^{1}: \eta^{1}-\mathrm{N}_{2}$ & $1.267(2)$ \\
$\mathrm{W}^{[35]}$ & $\mu-\eta^{1}: \eta^{1}-\mathrm{N}_{2}$ & $1.277(8)$ \\
\hline
\end{tabular}


complexes as being comprised of M(IV) metal centers with a $d^{2}$ electron count, bridged by $\mathrm{N}_{2}^{2-}$ ligands were proposed. ${ }^{[35]}$

Consequently, slight changes in the systems employed to bind and activate $\mathrm{N}_{2}$ can have a large influence on the actual degree of activation, requiring careful design and fine-tuning to obtain the wanted results. This is also highlighted in Section 3.2.

\subsection{Side-on bound $\mathrm{N}_{2}$}

So far, no isolable mononuclear complex with a side-on bound $\mathrm{N}_{2}$ ligand has been reported. $\eta^{2}$ coordination of $\mathrm{N}_{2}$ to a single metal center has only been suggested based on electron paramagnetic resonance (EPR) spectroscopic results for the complex $\left[\mathrm{Zr}\left(\eta^{5}-\mathrm{C}_{5} \mathrm{H}_{4} \mathrm{R}^{\prime}\right)_{2}\left(\mathrm{~N}_{2}\right) \mathrm{R}\right](\mathrm{R}$ $=\mathrm{CH}\left(\mathrm{SiMe}_{3}\right)_{2}, \mathrm{R}^{\prime}=\mathrm{H}$ or $\left.\mathrm{Me}\right){ }_{,}{ }^{40]}$ based on infrared (IR) spectra of matrix isolated product of Co atoms with $\mathrm{N}_{2}$ at $10 \mathrm{~K},{ }^{[41]}$ based on the observation of intramolecular, non-dissociative end-to-end isomerization of $\left[\mathrm{Ru}\left(\mathrm{NH}_{3}\right)_{5}\left({ }^{14} \mathrm{~N}^{15} \mathrm{~N}\right)\right] \mathrm{Br}_{2}{ }^{[42]}$ and $\left[\operatorname{Re}(\mathrm{Cp})(\mathrm{CO})_{2}\left({ }^{14} \mathrm{~N}={ }^{15} \mathrm{~N}\right)\right],{ }^{[4,44]}$ and was eventually structurally characterized by $\mathrm{X}$-ray diffraction as meta-stable state after single-crystal to single-crystal irradiation of $\left[\mathrm{Os}\left(\mathrm{NH}_{3}\right)_{5}\left(\mathrm{~N}_{2}\right)\right]\left(\mathrm{PF}_{6}\right)_{2} \cdot{ }^{[45]}$ And even on bimetallic systems, until 1988, only two clusters with a tetrahedral, non-planar $\mathrm{Ni}_{2} \mathrm{~N}_{2}$ core supported by $\mathrm{Li}$ ions were reported. ${ }^{[46-48]}$

The first structurally characterized side-on $\mu-\eta^{2}: \eta^{2} \mathrm{~N}_{2}$ bridged complex with the dinitrogen ligand being coordinated in plane with the two metal centers, i.e. $\left[\left(\mu-\eta^{2}: \eta^{2}-N_{2}\right)\left\{\mathrm{Sm}\left(C p^{*}\right)_{2}\right\}_{2}\right]$ was published in 1988. [49] Only two years after, Fryzuk and coworkers were able to isolate and characterize the complex $\left[\left(\mu-\eta^{2}: \eta^{2}-\mathrm{N}_{2}\right)\left\{\mathrm{ZrCl}\left(\mathrm{N}\left(\mathrm{SiMe}_{2} \mathrm{CH}_{2} \mathrm{P}^{\mathrm{i}} \mathrm{Pr}_{2}\right)\right)\right\}_{2}\right]$ (III). [50] Based on this result and the finding that substitution of the chloride ligand in this complex with a $\mathrm{C} p$ ligand resulted in isomerization to the end-on bridged dimer IV, the authors developed

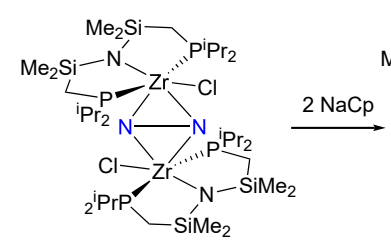

III

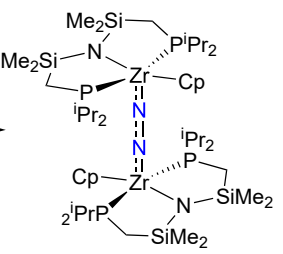

IV
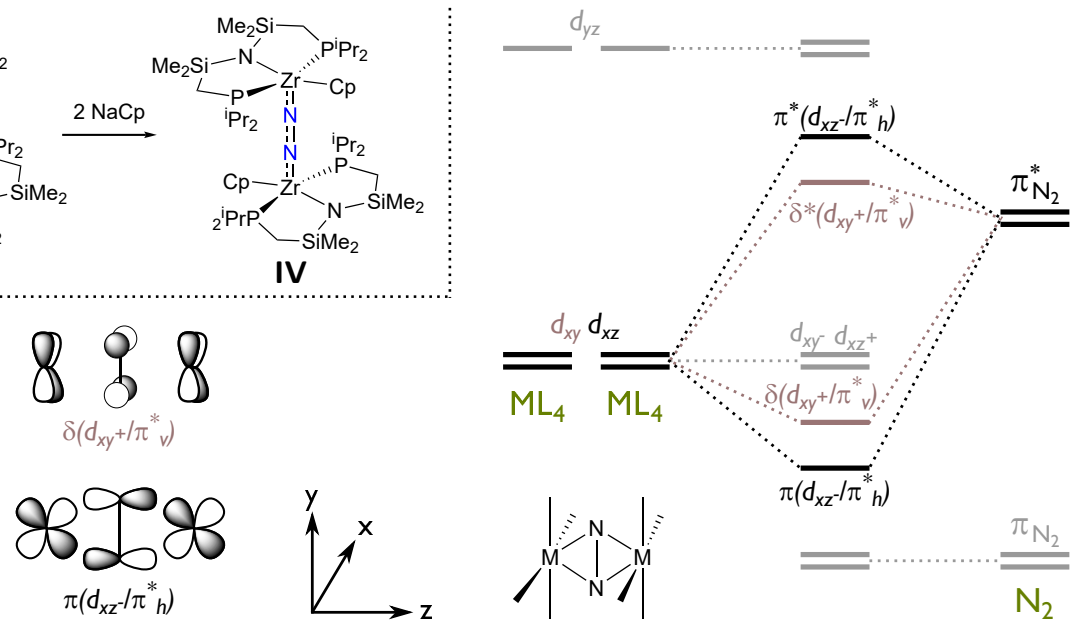

Fig. 2.6. Top left: Isomerization of Fryzuk's side-on bridged $\mathrm{N}_{2}$-dimer by exchange of the $\mathrm{Cl}^{-}$with a $\mathrm{Cp}^{-}$ligand. Main: FMO scheme of a side-on bridged $\mathrm{N}_{2}$-dimer. 
a bonding scheme for side-on bridged $\mathrm{N}_{2}$ dimers which allowed to rationalize the respective preferences of the $\mathrm{N}_{2}$ coordination mode.[25]

In general the more prevalent end-on bridging coordination mode is favorable, as here the metal centers and $\mathrm{N}_{2}$ bridge do form solely $\sigma$ - and $\pi$-interactions (compare Scheme 2.1), whereas in the side-on coordination mode, one $\pi$-interaction is replaced with a $\delta \mathrm{MO}$ combination, which can be regarded as unfavorable simply due to poorer orbital overlap, analog to considerations in metal-metal multiple bonding. ${ }^{[51]}$ However, if one of the $d$ orbitals needed for $\pi$ bonding is not available (e.g. due to interaction with strong field ligands), the $\delta$ bond can act as a "fallback" option to stabilize the system (see Figure 2.6). Two bonding orbitial combinations can be constructed, one $\pi$ symmetric combination of the $d_{x z}$ and the $\pi_{h}^{*} \mathrm{MO}$ of the ligand as well as a $\delta$ symmetric combination of the $d_{x y}$ orbitals and the $\pi_{v}^{*} \mathrm{MO}$ of the $\mathrm{N}_{2}$ moiety. In their specific case, Fryzuk and coworkers argued that the PNP amide $\pi$-donor and chloride $\sigma$-donor in III interact too strongly with the $d_{y z}$ orbitals, raising them in energy and making them unavailable for $\pi$-bonding with the $\mathrm{N}_{2}$ bridge. When the chloride is exchanged with a $\mathrm{Cp}^{-}$ligand, this interacts considerably with the $d_{x y}$ orbital required for the $\delta$ bond, and the end-on bridging mode becomes more favorable again. This is also expressed in an exceptionally long $\mathrm{Zr}-\mathrm{N}_{\text {amide }}$ bond length in IV $(d(\mathrm{Zr}-\mathrm{N})=2.303(3)$ and 2.306(3) $\AA$ ), indicating a reduced bond order and thus weaker interaction with the $d_{y z}$ orbital. This picture was later qualitatively confirmed by density functional theory (DFT) calculations. ${ }^{[52]}$ Despite these electronic influence, sterics were also shown to be of considerable importance in zirconocene complexes (see Scheme 2.2). A series of $\operatorname{Zr}\left(\mathrm{Cp}^{\mathrm{X}}\right)_{2}-\mathrm{N}_{2}$ complexes was made by Bercaw $\left(\left[\left(\mu-\eta^{1}: \eta^{1}-\mathrm{N}_{2}\right)\left\{\operatorname{Zr}\left(\mathrm{C}_{5} \mathrm{Me}_{5}\right)_{2}\left(\mathrm{~N}_{2}\right)\right\} 2\right](\mathbf{V})\right)^{[53]}$ and Chirik $\left(\left[\left(\mu-\eta^{1}: \eta^{1}-\right.\right.\right.$ $\left.\left.\left.\mathrm{N}_{2}\right)\left\{\mathrm{Zr}\left(\mathrm{C}_{5} \mathrm{Me}_{5}\right)\left(\mathrm{C}_{5} \mathrm{Me}_{4} \mathrm{H}\right)\left(\mathrm{N}_{2}\right)\right\} 2\right](\mathbf{V I}),\left[\left(\mu-\eta^{2}: \eta^{2}-\mathrm{N}_{2}\right)\left\{\mathrm{Zr}\left(\mathrm{C}_{5} \mathrm{Me}_{4} \mathrm{H}\right)_{2}\right\} 2\right](\mathbf{V I I})\right)^{[54,55]}$ of which only the latter, sterically least crowded complex forms a side-on bridged $\mathrm{N}_{2}$ dimer.

One of the main differences between end-on and side-on coordinated dinuclear dimers is their reactivity. While the only reported $\mathrm{N}_{2}$ centered reactivity of the former is their cleavage into nitrides (see Section 3.2.1), side-on bridged $\mathrm{N}_{2}$ ligands often exhibit a much higher degree of activation and can be reactive towards $\mathrm{N}-\mathrm{X}$ bond formation $(\mathrm{X}=\mathrm{H}, \mathrm{B}, \mathrm{C}, \mathrm{Si}$; see Section 3.3). [56]

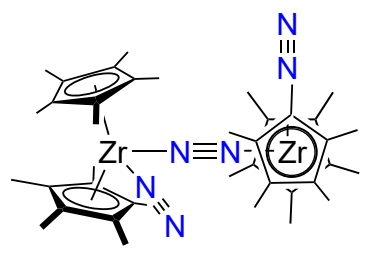

V

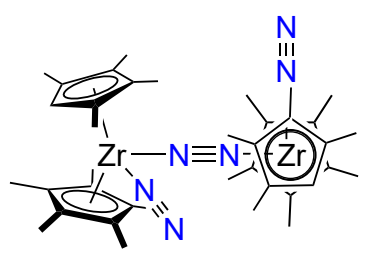

VI

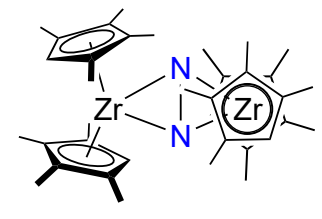

VII

Scheme 2.2. Different coordination modes of $\mathrm{N}_{2}$ in zirconocene complexes controlled by the steric bulk of the Cp-ligands. 



\section{Reactions of dinitrogen complexes}

You've got to cut the cord

Cut the cord and wake up!

\section{- The Agonist}

"Disconnect Me" on "Eye of Providence"

\subsection{Ammonia formation}

In light of the unmatched importance of industrial ammonia production by the Haber-Bosch process, the discovery of molecular dinitrogen complexes naturally sparked hopes for the development of homogeneous catalysts capable of the same reaction. By now, this goal has been matched by a number of systems (even though with much less efficiency) and has been thoroughly reviewed in several recent publications. ${ }^{[56-59]}$ However, for most of the time (1972 - 2003), only stochiometric conversion to ammonia was known, pioneered by the work of Chatt and coworkers, who reported protonation of $\left[\mathrm{M}\left(\mathrm{N}_{2}\right)_{2}(\mathrm{dppe})_{2}\right](\mathrm{M}=\mathrm{Mo}, \mathrm{W}$; dppe $=\mathrm{Ph}_{2} \mathrm{PCH}_{2} \mathrm{CH}_{2} \mathrm{PPh}_{2}$ ) with $\mathrm{HCl}$ to form an $\mathrm{N}_{2} \mathrm{H}_{2}$ ligand, ${ }^{[60]}$ followed by the report on ammonia formation after protonation of $\left[\mathrm{M}\left(\mathrm{N}_{2}\right)_{2}\left(\mathrm{PR}_{3}\right)_{4}\right]\left(\mathrm{M}=\mathrm{Mo}, \mathrm{W} ; \mathrm{PR}_{3}=\mathrm{PMePh}_{2}\right.$ or $\left.\mathrm{PMe}_{2} \mathrm{Ph}\right){ }^{[61]}$ This discovery and intensive follow-up research led to the formulation of the Chatt cycle for ammonia formation from terminally bound $\mathrm{N}_{2}$ which is depicted in Scheme 3.1. ${ }^{[62,63]}$ This cycle, which is also discussed as the most likely mechanism of ammonia formation by the nitrogenase enzymes, starts with stepwise protonation and reduction of the terminal nitrogen atom (for which this is also called the distal pathway) until reaching

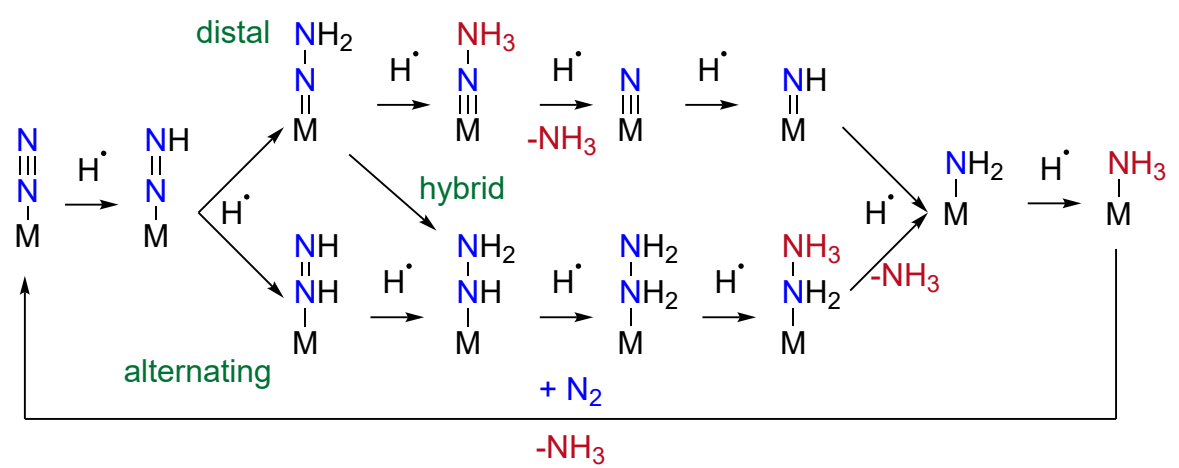

Scheme 3.1. Discussed mechanisms for ammonia formation from terminal bound $\mathrm{N}_{2}$ by transfer of $\mathrm{H}^{+} / \mathrm{e}^{-}\left(\right.$marked as $\left.\mathrm{H}^{*}\right)$. 
the $\left[\mathrm{M} \equiv \mathrm{N}-\mathrm{NH}_{3}\right]$ state, from which the first equivalent of ammonia is released. Only then, the remaining metal nitride is stepwise protonated and reduced until a second equivalent of ammonia is released. Such a cycle can only produce ammonia, while for formation of hydrazine, an alternating pathway is required. In addition to this achievement, the authors were furthermore able to perform the first $\mathrm{N}-\mathrm{C}$ bond formation from $\mathrm{N}_{2}$ by reaction with acetyl chlorides. ${ }^{[64,65]}$

The next major breakthrough after these finding was marked by the first report of a welldefined molecular system with a single metal center which is able to catalyze ammonia formation, i.e. Schrock's $\left[\mathrm{Mo}\left(\mathrm{N}_{2}\right)\left(\mathrm{HIPTN}{ }_{3} \mathrm{~N}\right)\right]\left(\mathrm{VIII}, \mathrm{HIPTN}_{3} \mathrm{~N}^{3-}=\left[\left\{3,5-\left(2,4,6-{ }^{\mathrm{i}} \mathrm{Pr}_{3} \mathrm{C}_{6} \mathrm{H}_{2}\right)_{2}\right.\right.\right.$ $\left.\left.\mathrm{C}_{6} \mathrm{H}_{3} \mathrm{NCH}_{2} \mathrm{CH}_{2}\right\}_{3} \mathrm{~N}\right]^{3-}$ ), which does react with $\mathrm{Co}\left(\mathrm{Cp}^{*}\right)_{2}$ and $\operatorname{LuH}\left(\mathrm{BAr}_{24}^{\mathrm{F}}\right)$ and is capable of forming up to 8 equivalents of ammonia with respect to Mo. ${ }^{[66]}$ The reaction most likely follows a reduction scheme which is comparable to the Chatt cycle, which is backed up by a number of independently synthesized potential intermediates which are also catalytically active as well as by DFT calculations. ${ }^{[63,67-70]}$ This large availability of characterized intermediates renders Schrock's catalyst the best understood systems and even though much better performing catalysts are known by now, there are still ongoing efforts to aquire data on even the most unstable intermediates. ${ }^{[71]}$ Notably, this work includes one of only two literature known parent imido complexes derived from dinitrogen, i.e. $\left[\mathrm{Mo}(\mathrm{NH})\left(\mathrm{HIPTN}_{3} \mathrm{~N}\right)\right]^{0 /+} .{ }^{[67]}$

A slightly different situation might be at hand in the iron catalyzed ammonia formation reported by Peters and coworkers, who prepared a series of trigonal pyramidal complexes $\left[\mathrm{Fe}\left(\mathrm{N}_{2}\right)\left(\mathrm{XP}_{3}{ }^{i \mathrm{Pr} r}\right)\right]^{-}\left(\mathbf{I X}, \mathrm{XP}_{3}{ }^{i P r}=\mathrm{X}\left(2-i \mathrm{Pr}_{2} \mathrm{P}_{-} \mathrm{C}_{6} \mathrm{H}_{4}\right)_{3}, \mathrm{X}=\mathrm{B}, \mathrm{C}, \mathrm{Si}\right)$, of which the first two are catalytically active. ${ }^{[2-74]}$ In this system, a hybrid mechanism between the distal and the alternating pathway (i.e. alternating protonation of both $\mathrm{N}$ atoms) has been proposed based on the disproportionation of $\left[\mathrm{Fe}=\mathrm{N}-\mathrm{NH}_{2}\right]$ and $\left[\mathrm{Fe}=\mathrm{N}-\mathrm{NH}_{2}\right]^{+}$to $\left[\mathrm{Fe}-\mathrm{N}_{2}\right]$ and $\left[\mathrm{Fe}-\mathrm{NH}_{2}-\mathrm{NH}_{2}\right]^{+}$, where after formation of the $\left[\mathrm{M}=\mathrm{N}-\mathrm{NH}_{2}\right]$ moiety, the metal bound ni-

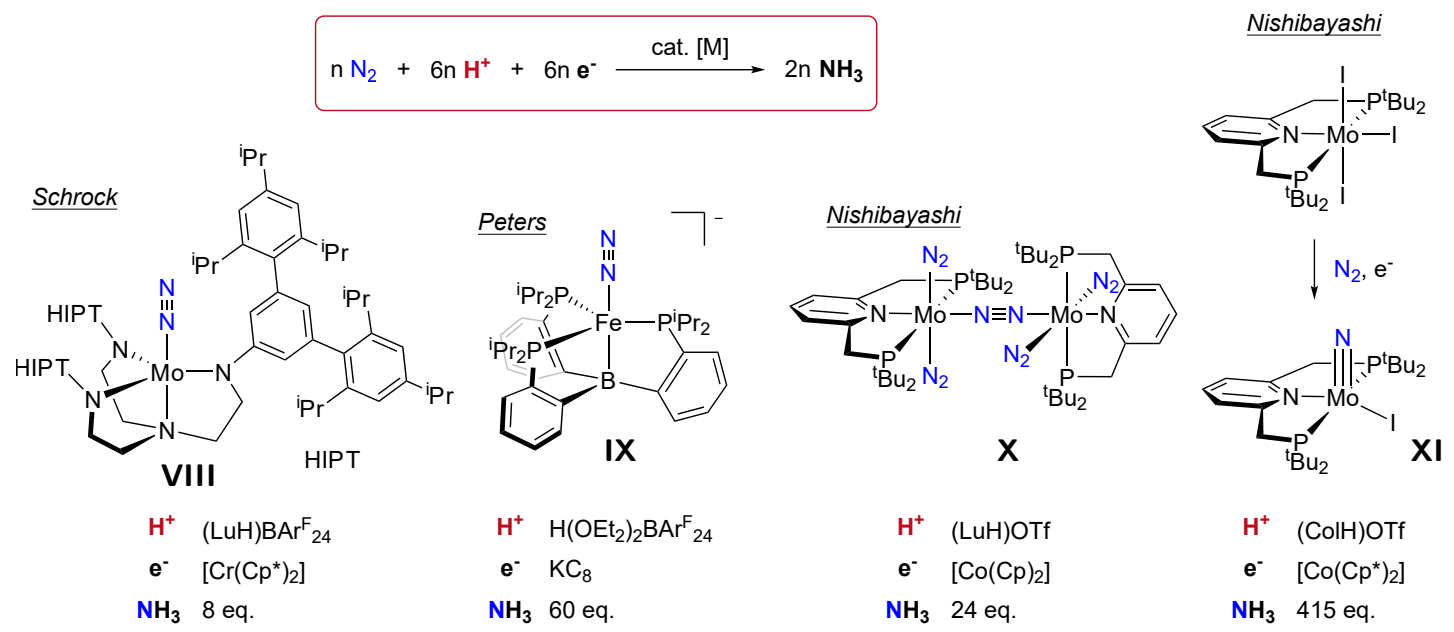

Fig. 3.1. Pioneering molecular catalysts mediating protonation/reduction cycles for ammonia production from $\mathrm{N}_{2}$. 
trogen is protonated before the terminal one. However, later studies contradicted these results, as the formation of a terminal nitrido complex $\left[\mathrm{Fe}(\mathrm{N})\left(\mathrm{BP}_{3}^{i \mathrm{Pr}}\right)\right]^{+}$was observed to form from threefold protonation of $\left[\mathrm{Fe}\left(\mathrm{N}_{2}\right)\left(\mathrm{BP}_{3}^{i \mathrm{Pr}}\right)\right]^{2-}$, accompanied by the release of equimolar amounts of $\mathrm{NH}_{3} \cdot{ }^{[75]}$ Furthermore, Peters could show that the commonly used reduction / protonation agent pair, $\mathrm{Co}\left(\mathrm{Cp}_{2}^{*}\right)$ and lutidinium salts $\left(\mathrm{LuH}^{+}\right)$, might actually act as a proton coupled electron transfer (PCET) reagent in the catalyses, where instead of stepwise protonation and reduction of the $\mathrm{N}_{2}$ complexes one $\mathrm{Cp}^{*}$ ring is first protonated, followed by an $\mathrm{H}$ atom transfer to the substrate. ${ }^{[76]}$

A very interesting observation regarding the pathway of ammonia formation was reported by Nishibayashi and coworkers. They developed the second ever reported catalytic system for ammonia production, $\left[\left(\mu-\mathrm{N}_{2}\right)\left\{\mathrm{Mo}\left(\mathrm{N}_{2}\right)_{2}(\mathrm{PNP})\right\}_{2}\right]\left(\mathbf{X}, \mathrm{PNP}=2,6-\left(\mathrm{CH}_{2} \mathrm{P}^{t} \mathrm{Bu}_{2}\right)_{2} \mathrm{NC}_{5} \mathrm{H}_{5}\right)$, which is obtained by reduction of the trichloro-complex $\left[\mathrm{MoCl}_{3}(\mathrm{PNP})\right]$ in the presence of dinitrogen. ${ }^{[77]}$ Treatment with $\mathrm{Co}(\mathrm{Cp})_{2}$ and $\mathrm{LuH}^{+}$under $\mathrm{N}_{2}$ atmosphere resulted in formation of 12 equivalents $\mathrm{NH}_{3}$ per Mo center. This reaction is supposed to undergo a distal pathway protonation and reduction scheme at one of the terminally bound $\mathrm{N}_{2}$ ligands. Variation of the para substituent of the pyridine ligand as well as computational investigation of the reaction led to the conclusion, that the initial protonation to $\mathrm{M}-\mathrm{N}=\mathrm{NH}$ is rate determining and intermetallic charge transfer from the second Mo in the dimer was proposed to be crucial for stabilizing this intermediate. ${ }^{[58,78]}$ When the pyridine moiety was exchanged with an $\mathrm{N}$-heterocyclic carbene $(\mathrm{NHC})$, i.e. in the complexes $\left[\left(\mu-\mathrm{N}_{2}\right)\left\{\mathrm{Mo}\left(\mathrm{N}_{2}\right)_{2}(\mathrm{PCP})\right\}_{2}\right]$ (PCP $=1$,3-bis((di-tert-butylphosphino)methyl)benzimidazol-2-ylidene or 1,3-bis(2-(di-tertbutylphosphino)ethyl)imidazol-2-ylidene), especially the former, i.e. the benzimidazole based complex performed remarkably well in catalysis with 115 turnovers per Mo center. ${ }^{[79]}$ Based on DFT calculations, this was attributed to the NHC acting not only as $\sigma$ donor but also as $\pi$ acceptor, which was considered beneficial for the reaction. However, when in the pyridine complex the chloride ligands of the precursor are exchanged with iodide, a significantly different mechanism comes into play. Upon reduction under $\mathrm{N}_{2}$, the complex undergoes initial $\mathrm{N} \equiv \mathrm{N}$ bond scission to form the terminal nitrido complex [Mo(N)I(PNP)] (XI), presumably via an $\mu-\eta^{1}: \eta^{1}$ bridged dimer. ${ }^{[80]}$ The system still is catalytic but stepwise protonation and reduction to ammonia takes place only after the $\mathrm{N}_{2}$ splitting step, raising the question on whether such initial cleavage reactions might also play a role in the previously reported systems. In addition to these mechanistic considerations, in their latest contribution, Nishibayashi and coworkers were also able to significantly tweak the turn-over-numbers of their catalysts by using $\mathrm{Sml}_{2} / \mathrm{H}_{2} \mathrm{O}$ as PCET reagents, obtaining up to 2175 equivalents $\mathrm{NH}_{3}$ per Mo center. ${ }^{[81]}$ 


\subsection{Initial full dinitrogen cleavage}

\subsubsection{Thermal $\mathrm{N}_{2}$ cleavage from end-on bound dimers}

The initial full cleavage of the $\mathrm{N} \equiv \mathrm{N}$ triple bond reported by Nishibayashi for his iodide complex $\mathbf{X I}$ is unique for systems which catalyze the production of ammonia. However, it is by far not the first example of an initial $\mathrm{N}_{2}$ cleavage by dinuclear transition metal catalysts. In fact, the very first report on such a reaction dates back to 1995, when Cummins and coworkers reported their dimolybdenum complex I (see Section 2.1) to undergo selective $\mathrm{N} \equiv \mathrm{N}$ bond cleavage to form the corresponding nitrido complex $\left[\mathrm{Mo}(\mathrm{N})(\mathrm{N}(\mathrm{R}) \mathrm{Ar})_{3}\right](\mathbf{X I I})$ at room temperature. ${ }^{[27,82]}$ This reaction was thoroughly investigated and can be rationalized based on the MO scheme developed earlier for these end-on bridged dimers (see Section 2.1, Scheme 2.1).

Comparison of the FMO scheme of the dimer and the monomer reveals that while the dimer has an electronic structure with $10 \pi$ and $6 \sigma$ electrons in the $\{$ MNNM $\}$ core, the formation of two monomers requires a total of $8 \pi$ and $8 \sigma$ electrons $\left(\mathrm{M}^{-}-\mathrm{N}\right.$ and the lonepairs at the two nitride ligands). Consequently, the former $\mathrm{a}_{\mathrm{u}}$ orbital with $\mathrm{M}-\stackrel{\sigma}{-} \mathrm{N}^{\sigma *}-\stackrel{\sigma}{-} \mathrm{M}$ character in the dimer drops dramatically in energy and becomes an occupied, bonding, $1 b_{u}$ symmetric $\mathrm{M}^{\sigma}-\mathrm{N}$ orbital in the nitride complexes. In order to achieve this and to obtain electron transfer from the $\pi$ orbital space into a formally orthogonal $\sigma$ orbital, the splitting needs to proceed via a zig-zag transition state, which breaks the symmetry, lifts the degeneracy of the orbital sets and allows for the required mixing of the orbitals (i.e. $3 b_{u}$ and $2 b_{u}$, see Scheme 3.2). These symmetry considerations were later reproduced by DFT calculations on a truncated model (i.e. $\left[\left(\mu-\mathrm{N}_{2}\right)\left\{\mathrm{Mo}\left(\mathrm{NH}_{2}\right)_{3}\right\}_{2}\right){ }^{[83]}$

This electronic requirements can also explain why the mono- and dicationic complexes $\mathbf{I}^{+}$ and $\mathbf{I}^{2+}$ do not undergo splitting into terminal nitrides, even though the $\mathrm{N}_{2}$ ligand is stronger activated (vida supra). ${ }^{[27]}$ From these dimers, no closed shell nitrides can be obtained, which is energetically unfavorable and thus, the $\mathrm{N}_{2}$ cleavage becomes thermodynamically uphill.

Variations of this system have led to some valuable insights regarding the requirements for $\mathrm{N}_{2}$ activation. The effect of the ligands on the electronic structure is well exemplified by comparing Cummins' dimeric complex with those prepared by Schrock (i.e. [( $\mu$ $\left.\left.\left.\mathrm{N}_{2}\right)\left\{\mathrm{Mo}\left(\mathrm{N}\left(\mathrm{CH}_{2} \mathrm{CH}_{2} \mathrm{NR}\right)_{3}\right)\right\}_{2}\right]\left(\mathbf{X I I I}, \mathrm{R}=\mathrm{SiMe}_{3}\right)\right)$ and Floriani $\left(\left[\left(\mu-\mathrm{N}_{2}\right)\left\{\mathrm{MoMes}_{3}\right\}_{2}\right], \mathbf{X I V}\right) .{ }^{[84-86]}$ Both dimers feature a $\mu-\eta^{1}: \eta^{1}$ bridging $\mathrm{N}_{2}$ ligand, just as in Cummins' complex. Nevertheless, both systems are thermally stable and do not split into the corresponding nitrides, even though they do formally feature the same oxidation state of the metal and exhibit threefold rotation symmetry, rendering them isoelectronic to $\mathbf{I}$.

Schrock's dimeric molybdenum complex also features three amide donor ligands. However, they are connected via an amine, which acts as an additional $\sigma$ donor in trans position with respect to the $\mathrm{N}_{2}$ bridge. This should on the one hand destabilize $\sigma$-bonding in the 


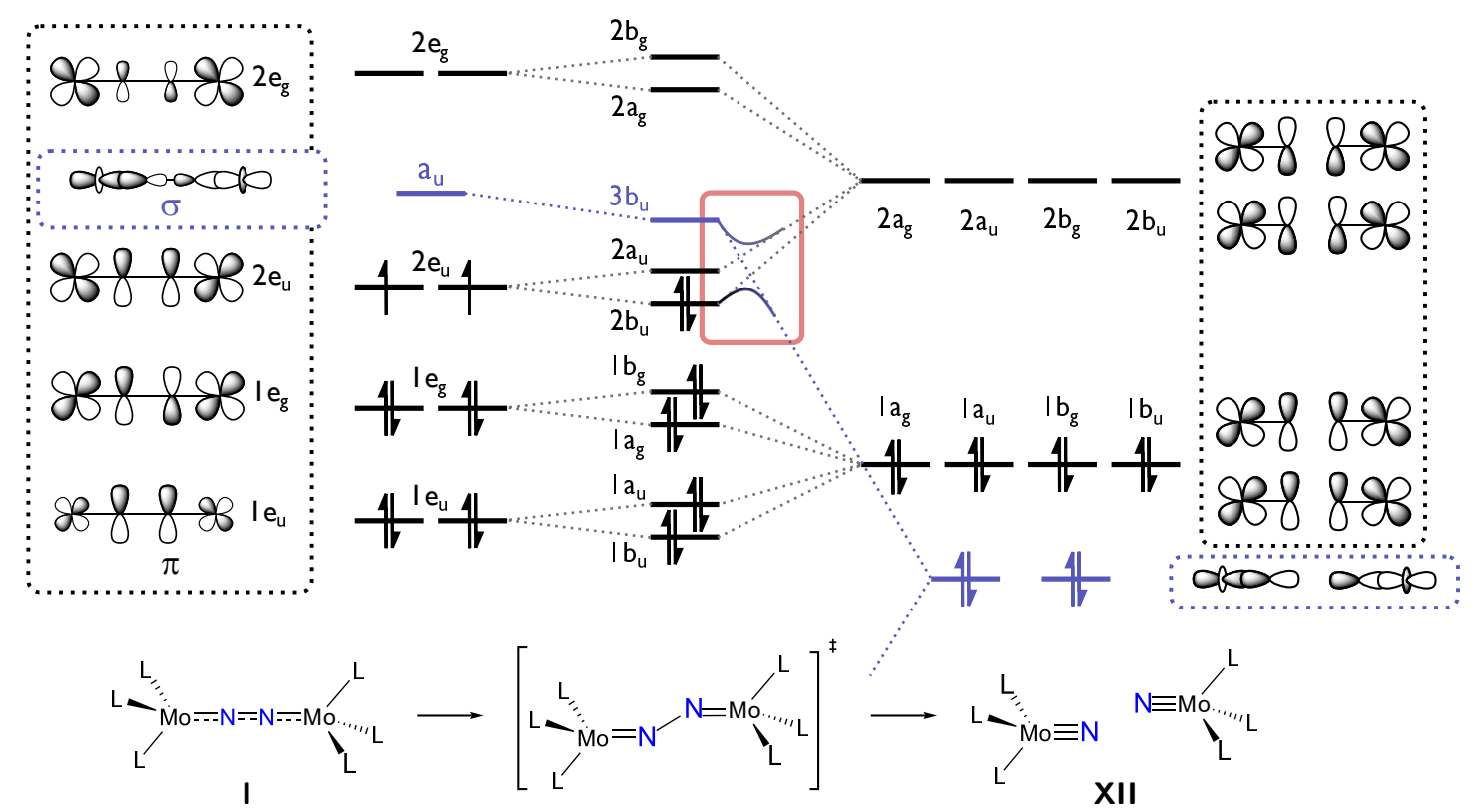

Fig. 3.2. FMO scheme the the thermal splitting of formal Mo(III) derived dimer $\mathbf{I}$ into terminal nitrido complexes $\mathbf{X I I}$.
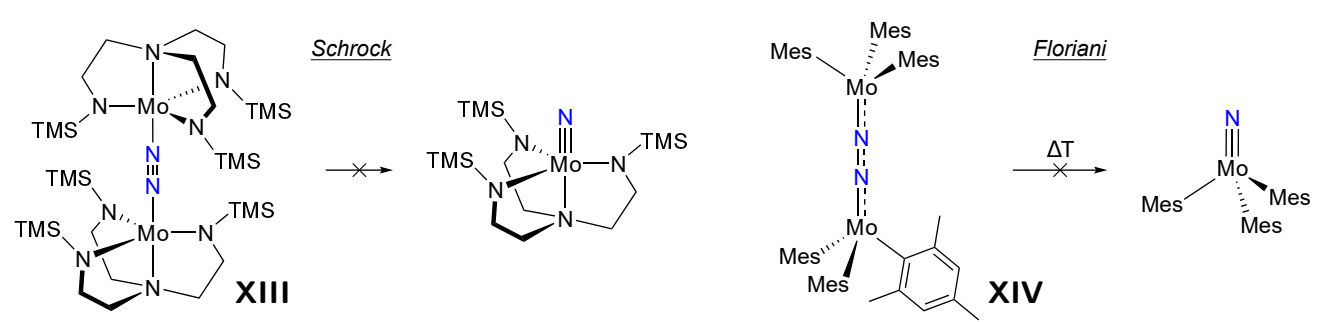

Fig. 3.3. Mo(III) based $\mathrm{N}_{2}$ dimers by Schrock and Floriani, which are isoelectronic to Cummins' dimer but do not undergo thermal $\mathrm{N}_{2}$ splitting.

nitride complex and on the other raise the $a_{u}$ orbital energetically, therefore hampering the required orbital mixing in the transition state. Additionally, extensive theoretical studies on a truncated model by Stranger initially indicated amide rotation to be of importance for the $\mathrm{N}_{2}$ scission, which would obviously be prevented by the amide link to the amine. ${ }^{[87]}$ Later studies on the full model revealed this rotation is largely suppressed in the complexes with the bulky amides and therefore might not have a significant effect anyways. ${ }^{[88]}$

In Floriani's dimer XIV, the most prominent difference is the replacement of $\pi$-donating amide with pure $\sigma$-donating aryl ligands. ${ }^{[86]}$ While no detailed electronic structure considerations were addressed, a possible explanation for the thermal stability of the dimer might be the reduced $\pi$ and $\sigma$ donor strength of the aryl groups compared to the amide ligands in Cummins' system. This should lowerthe $2 \mathrm{e}_{\mathrm{u}}$ in energy and increase the energetic gap to the $a_{u}$ orbitals (see Scheme 2.1) which prevents mixing of the two orbitals and electron transfer in the transition state as discussed above. However, the complex is reported to undergo photochemical splitting of $\mathrm{N}_{2}$, which is discussed in Section 3.2.3. 
A strong influence on the product formation was found for the steric bulk of the amide ligands in Cummins' starting platform $\left[\mathrm{Mo}\left(\mathrm{N}\left({ }^{\mathrm{t}} \mathrm{Bu}\right) \mathrm{Ar}\right)_{3}\right](\mathbf{X V})$. When small lignads like $\mathrm{NMe}_{2}^{-}$, $\mathrm{NMeEt}^{-}$or $\mathrm{NEt}_{2}^{-}$are employed, the corresponding $\mathrm{Mo}(\mathrm{III})$ trisamide complexes undergo dimerization reactions forming $\mathrm{Mo} \equiv$ Mo triple bonds, which prevents any further reactivity towards dinitrogen. ${ }^{[89]}$ When the tert-butyl groups in $\mathbf{X V}$ are replaced with iso-propyl groups, the initially formed complex is $\left[\mathrm{Mo}(\mathrm{H})\left(\eta^{2}-\mathrm{Me}_{2} \mathrm{C}=\mathrm{NAr}\right)\left(\mathrm{N}\left({ }^{\mathrm{i}} \mathrm{Pr}\right) \mathrm{Ar}\right)_{2}\right](\mathbf{X V I I})$, where one ${ }^{i} \operatorname{Pr}$ group is activated by the metal forming a hydride species (see Scheme 3.2 top). ${ }^{[90]}$ This $\mathrm{C}-\mathrm{H}$ activation was shown to be fully reversible, and under a dinitrogen atmosphere, a nitrido-bridged dimer $\left[(\mu-\mathrm{N})\left\{\mathrm{Mo}\left(\mathrm{N}\left({ }^{\mathrm{i}} \mathrm{Pr}\right) \mathrm{Ar}_{3}\right\}_{2}\right]\right.$ (XVIII) could be isolated as main product, which is believed to form by initial $N_{2}$ cleavage into the corresponding nitrido complexes and fast follow-up reaction with excess starting complex. DFT calculations on the system confirmed that such a dimerization is kinetically and thermodynamically favorable for the smaller ligand, but unfavorable for the more bulky tert-butyl based ligand. Increasing the steric shielding even further (i.e. using $N(A d) A r, A d$ = adamantyl) fully shuts down the observable $\mathrm{N}_{2}$ chemistry at the $\mathrm{Mo}(\mathrm{III})$ stage and $\left[\mathrm{Mo}(\mathrm{N}(\mathrm{Ad}) \mathrm{Ar})_{3}\right]$ was reported to be stable indefinitely under $\mathrm{N}_{2}$ atmosphere. ${ }^{[91]}$ This series clearly shows how minor changes in ligand size can significantly impact the chemistry of the entire system and are therefore a highly important parameter in the design of new systems.

In order to elucidate the mechanism not only of the splitting but also of the dimer assembly in Cummins' dimer, intermediates of the reaction were trapped experimentally. ${ }^{[91]}$ While the starting complexd $\mathbf{X V}$ is stable towards $\mathrm{NaHg}$ under $\mathrm{Ar}$, a new species $\mathrm{Na}(\mathrm{THF})_{\times}\left[\mathrm{Mo}\left(\mathrm{N}_{2}\right)\right.$ $\left.\left(\mathrm{N}\left({ }^{\mathrm{t}} \mathrm{Bu}\right) \mathrm{Ar}\right)_{3}\right](\mathbf{X V I})$ is readily formed under a $\mathrm{N}_{2}$ atmosphere, suggesting the unobserved neutral mononuclear $\mathrm{N}_{2}$ complex to be the electron accepting complex (see Scheme 3.2 bottom). This anionic complex could then be further functionalized by addition of MeOTs, $\mathrm{SiMe}_{3} \mathrm{Cl}$ or $\mathrm{PhCOCl}$, undergoing $\mathrm{N}-\mathrm{C}$ or $\mathrm{N}-\mathrm{Si}$ coupling at the terminal nitrogen atom. Interestingly, the dimer-formation was shown to be redox-catalyzed by reductants, and the
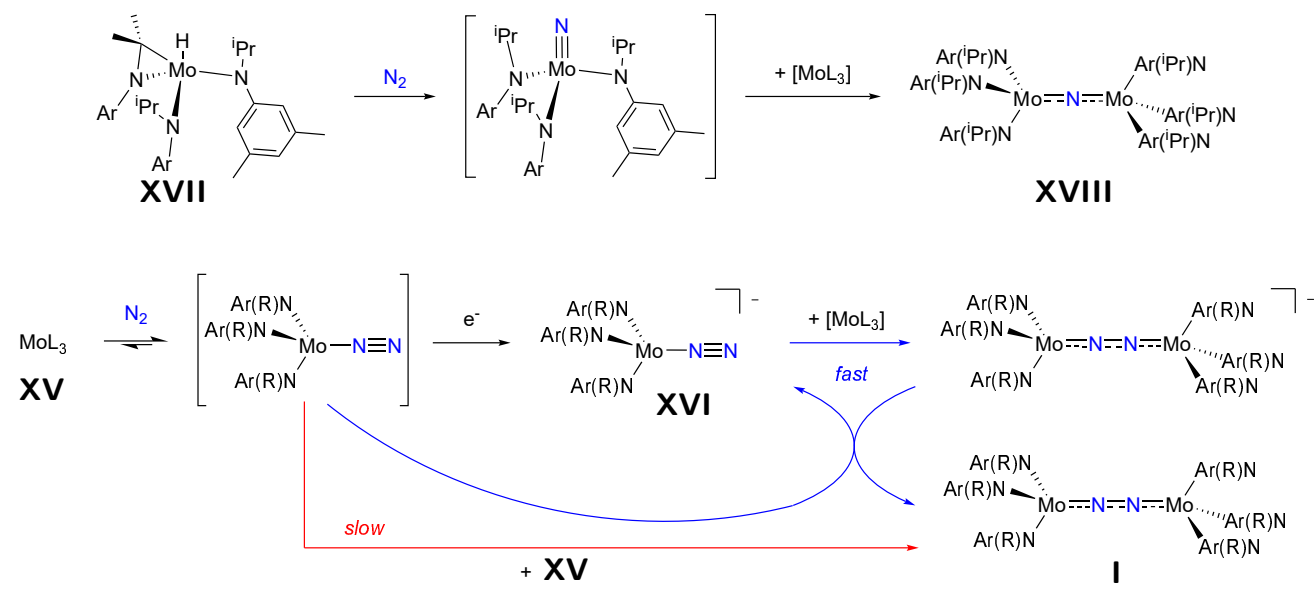

Scheme 3.2. Top: Reducing the steric bulk in Cummins' original Mo complex leads to exclusive formation of a $\mu-\mathrm{N}$ bridged dimer from a hydride resting state. Bottom: Small amounts of reducing agents were found to redox-catalyze the formation of the neutral dimer $\mathbf{I}$. 
authors proposed that binding of a second metal fragment $\mathbf{X V}$ to the anionic $\mathrm{N}_{2}$ complex $\mathbf{X V I}$ would be much faster than in the neutral case, followed by reduction of a neutral $\mathrm{N}_{2}$ adduct by the formed anionic dimer (see Scheme 3.2 bottom). ${ }^{[91]}$ It is also reported that the slow formation of the dimer can be significantly enhanced not only by reductant, but also by the addition of $\mathrm{N}$-heterocyclic bases. ${ }^{\left[{ }^{92}\right]}$ The authors ascribed this to a spin state change from quartet to doublet upon coordination of the base to the $\left[\mathrm{Mo}\left(\mathrm{N}\left({ }^{\mathrm{t}} \mathrm{Bu}\right) \mathrm{Ar}\right)_{3}\right]$ precursor and subsequent fast $\mathrm{N}_{2}$ binding. This is also in line with the very recent publication on $\mathrm{N}_{2}$ splitting by $\left[\mathrm{Mo}\left(\mathrm{OSi}\left(\mathrm{O}^{t} \mathrm{Bu}\right)_{3}\right)_{3}\right]$ reported by Copéret and coworkers. ${ }^{[93]}$ Here a corresponding end-on $\mu-\mathrm{N}_{2}$ bridged dimer forms within $30 \mathrm{~s}$ and in the product, one silylether ligand arm acts as additional ligand, coordinating trans to the $\mathrm{N}_{2}$ ligand and consequently might have a comparable effect to external ligands in Cummins' system.

The very first (and to the present day exclusive) example of $\mathrm{N}_{2}$ bond cleavage by a group 7 transition metal complex was published by Schneider and coworkers in 2014. The Re(III) complex $\left[\mathrm{ReCl}_{2}\left(\mathrm{PNP}^{t \mathrm{Bu}}\right)\right](\mathbf{X I X})$ was shown to split dinitrogen upon one-electron reduction under a dinitrogen atmosphere to yield $\operatorname{Re}(\mathrm{V})$ nitrido complex $\left[\operatorname{Re}(\mathrm{N}) \mathrm{Cl}\left(\mathrm{PNP}^{t \mathrm{Bu}}\right)\right]$ $(\mathbf{X X I}){ }^{[94]}$ This splitting was initially proposed to proceed via the dimeric complex $[(\mu-$ $\left.\left.\mathrm{N}_{2}\right)\left\{\operatorname{ReCl}\left(\mathrm{PNP}^{t \mathrm{Bu}}\right)\right\}_{2}\right](\mathbf{X X})$, which exhibits a $10 \pi 4 \delta$ electron count in the $\{\mathrm{MNNM}\}$ core, fully consistent with the above discussed mechanism. ${ }^{1}$ An in-depth follow-up investigation of the reaction, especially by means of cyclic voltammetry, allowed for the construction of a mechanistic proposal for the dimer formation and splitting. ${ }^{2}$ According to this model, after initial reduction to an anionic $\operatorname{Re}(\mathrm{II})$ complex, $\mathrm{N}_{2}$ is bound, followed by chloride loss and a second reduction step to formal $\operatorname{Re}(\mathrm{I})$ complex $\left[\operatorname{Re}\left(\mathrm{N}_{2}\right) \mathrm{Cl}\left(\mathrm{PNP}^{t \mathrm{Bu}}\right)\right]^{-}$. ${ }^{[95]}$ This species then binds to a $\operatorname{Re}(\mathrm{III})$ fragment and comproportionats to yield the dimeric complex $\mathbf{X X}$, which renders the formation an overall $\mathrm{EC}^{\mathrm{Cl}} \mathrm{C}^{\mathrm{N}_{2}} \mathrm{EC}[\mathrm{Re}]$ mechanism. Importantly, it is shown that $\mathrm{N}_{2}$ is bound and activated at the $\operatorname{Re}(\mathrm{II})$ stage. While the two systems are not entirely comparable due to different reaction conditions (especially with respect to addition of reductant), the role of "overreduced" $\operatorname{Re}(\mathrm{I})$ and Mo(II) species in Schneider's system and Cummins' reported redox-catalysis for promoting dimer formation is strikingly similar.

${ }^{1}$ The dimer $\mathbf{X X}$ is actually characterized and proven to be an intermediate in the formation of $\mathbf{X X \mathbf { I }}$ within this thesis (see Part II Section 2.1).

${ }^{2}$ The characterization of the thermal splitting of $\mathbf{X X}$ to $\mathbf{X X I}$ is part of this thesis and will therefore be discussed in Part II Section 2.1.

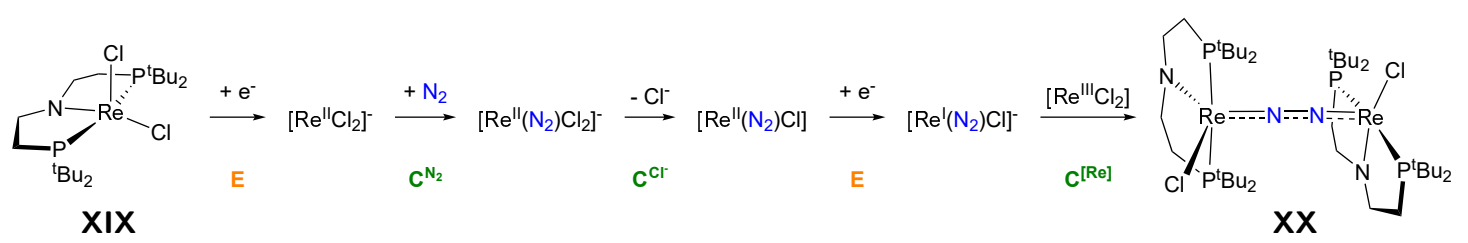

Scheme 3.3. Formation of the $\operatorname{Re} \mu-N_{2}$ dimer $\mathbf{X X}$ via an $\mathrm{EC}^{\mathrm{Cl}} \mathrm{C}^{\mathrm{N}_{2}} \mathrm{EC}[\mathrm{Re}]$ mechanism. 

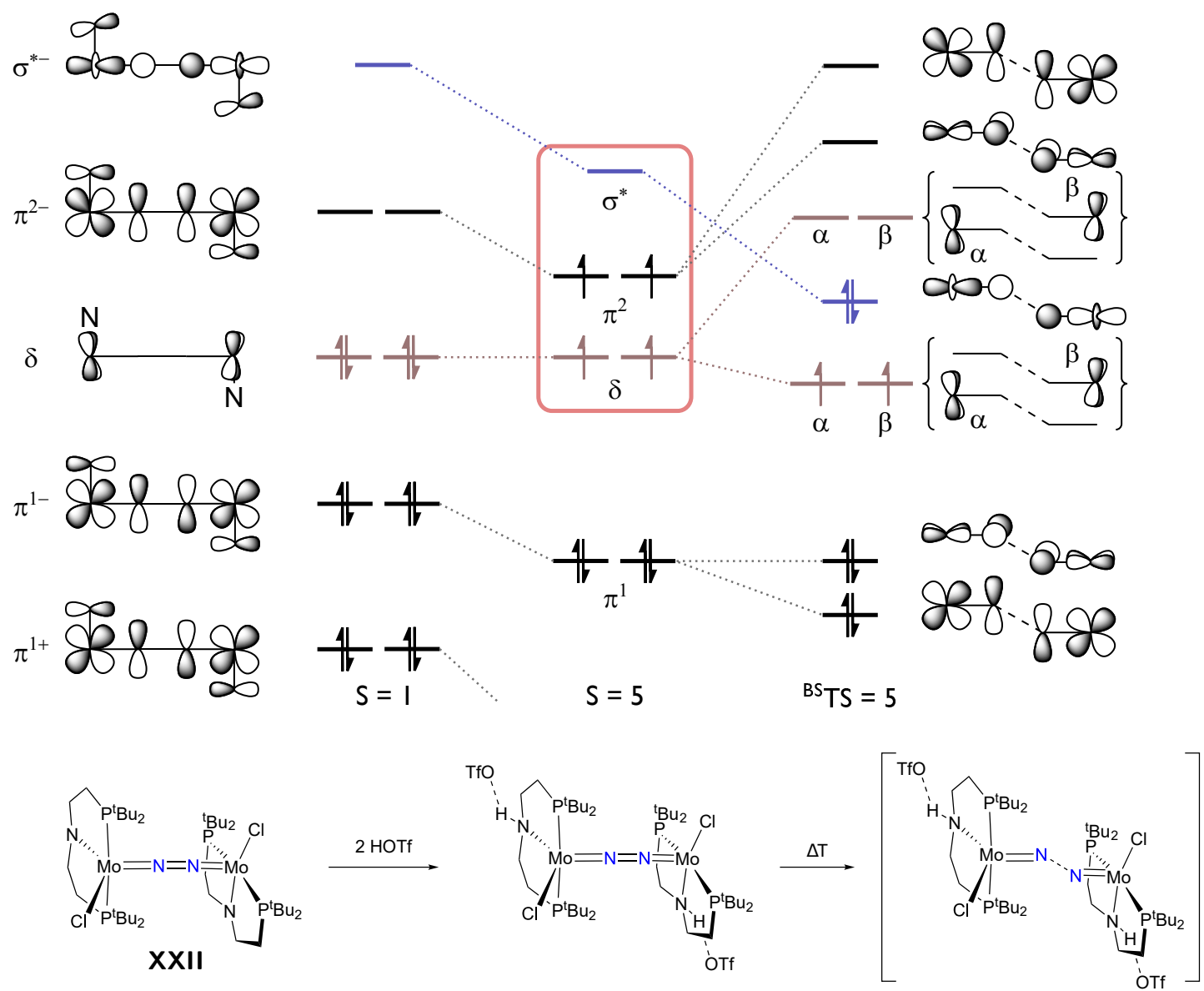

Fig. 3.4. Protonation coupled dinitrogen splitting via spin state change in a Mo dimer.

In 2017, Schneider and coworkers further reported $\mathrm{N}_{2}$ splitting from the formal $\mathrm{Mo}$ (II) dinitrogen bridged dimer $\left[\left(\mu-\mathrm{N}_{2}\right)\left\{\mathrm{MoCl}\left(\mathrm{PNP}^{t \mathrm{Bu}}\right)\right\}_{2}\right]\left(\mathbf{X X I I}, \mathrm{PNP}^{t \mathrm{Bu}}=\mathrm{N}\left(\mathrm{CH}_{2} \mathrm{CH}_{2} \mathrm{P}^{t} \mathrm{Bu}_{2}\right)_{2}^{-}\right) \cdot{ }^{[96]}$ Due to the idealized fourfold rotational symmetry, that dimer exhibits a $8 \pi 4 \delta$ electronic configuration in the $\{M N N M\}$ core, which disqualifies it for splitting according to the considerations made before. And indeed, no thermal splitting was observed. However, twofold protonation of the PNP amide moiety results in splitting into the corresponding terminal nitrido complexes, which is counter intuitive at first glance since protonation should remove electron density from the metal centers, which are already short by one electron each for splitting. The authors identified a spin-state change from singlet to quintet due to quenched $\pi$-donation of the ligand upon protonation (identified by Evan's measurement and computations), which led to population of the " $2 \mathrm{e}_{\mathrm{u}}$ " orbitals and significantly reduced the energy gap of the new HOMO to the " $a_{2 u}$ " orbital, enabeling thermal splitting (see Scheme 3.4).

Another example illustrating both the subtle influence of ligand variation as well as the complexity of some splitting mechanisms is provided by the group of Holland. They prepared a series of iron $\beta$-diketiminate complexes $[\mathrm{FeCl}(\mathrm{Ln})]_{1 \text { or2 }}\left(\mathbf{X X I I I}{ }^{\mathbf{L n}}, \mathrm{Ln}=\mathrm{RC}\left(\mathrm{C}\left(\mathrm{R}^{\prime}\right) \mathrm{N}(2,6\right.\right.$ $\left.\left.\mathrm{R}_{2}{ }_{2} \mathrm{C}_{6} \mathrm{H}_{3}\right)\right)_{2}$, with L1: $\mathrm{R}=\mathrm{H}, \mathrm{R}^{\prime}={ }^{\mathrm{t}} \mathrm{Bu}, \mathrm{R}^{\prime \prime}={ }^{\mathrm{i}} \mathrm{Pr}$; L2: $\mathrm{R}=\mathrm{H}, \mathrm{R}^{\prime}=\mathrm{Me}, \mathrm{R}$ " $={ }^{\mathrm{i}} \operatorname{Pr}$; L3: R $\left.=\mathrm{R}^{\prime}=\mathrm{R}^{\prime \prime}=\mathrm{Me}\right)$, of which the $\mathrm{L} 1$ and $\mathrm{L} 2$ complexes do form end-on, dinitrogen bridged 


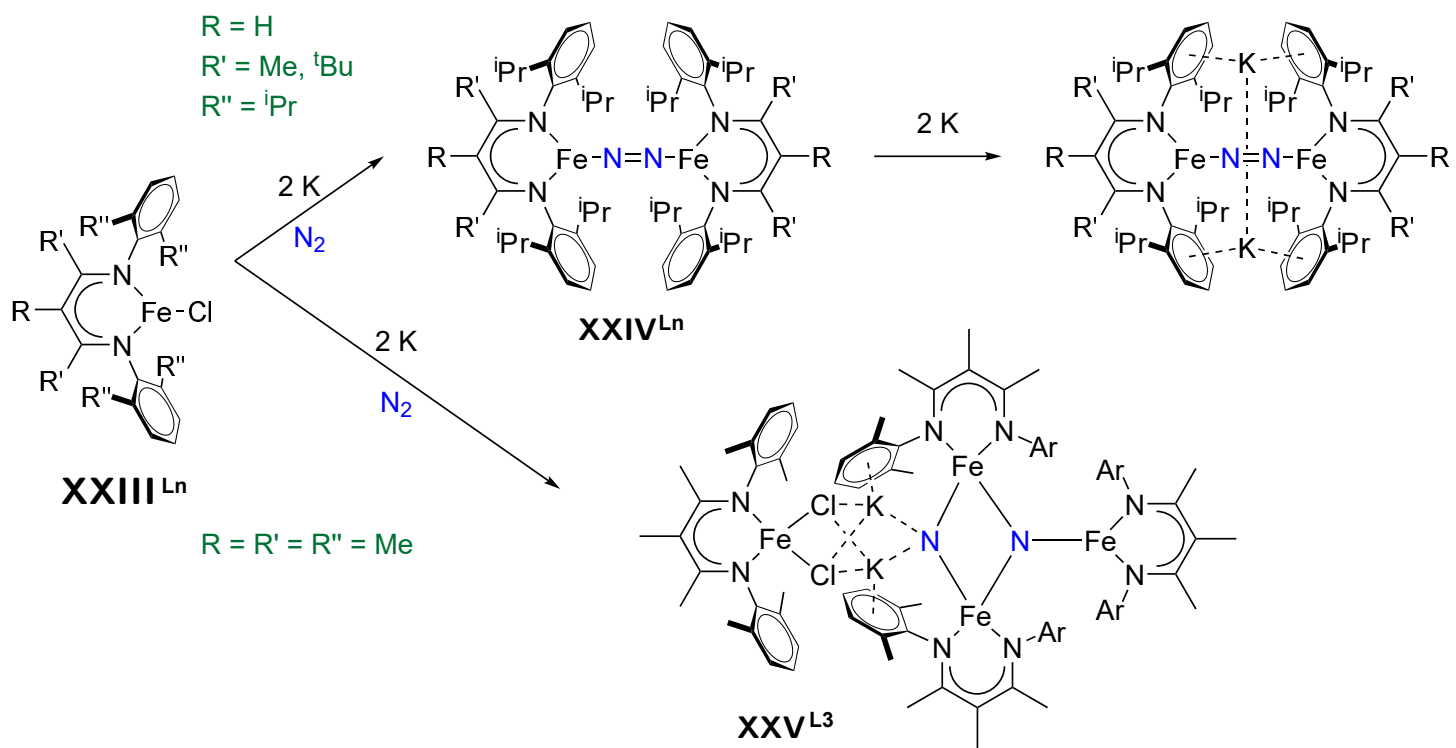

Scheme 3.4. Steric control by ligand bulk of multinuclear $\mathrm{N}_{2}$ splitting by an Fe complex.

dimers upon reduction with $\mathrm{K}$, i.e. $\left[\left(\mu-\mathrm{N}_{2}\right)\{\mathrm{Fe}(\mathrm{L})\}_{2}\right]\left(\mathbf{X} \mathbf{X I V} \mathbf{V}^{\mathbf{L n}}\right)$, which can be reduced further by two electrons with the potassium cations being intercorporated into the molecular structure by coordination to the aryl substituents (see Scheme 3.4). ${ }^{[97,98]}$ In contrast to this, utilizing the least bulky ligand L3 resulted in full cleavage of the $\mathrm{N}_{2}$ ligand into bridging nitrides by formation of a $\left[\mathrm{Fe}_{4}\left(\mathrm{~L}_{3}\right)_{4}(\mu-N)_{2} \mathrm{Cl}_{2} \mathrm{~K}_{2}\right]$ cluster $\left(\mathbf{X X V} \mathbf{V}^{\mathbf{L}}\right)$, from which ammonia could be released by protonolysis. ${ }^{[99]}$ DFT calculations indicate initial formation of an endon dimer analog to the systems utilizing $\mathrm{L} 1$ and $\mathrm{L} 2$, followed by side-on coordination of a third $\mathrm{Fe}(\mathrm{L} 3)$ fragment to the $\mathrm{N}_{2}$ ligand. ${ }^{[100]}$ The potassium cations are found to act as promotors in the reaction and to stabilize the splitting by $\sim 10 \mathrm{kcal} \mathrm{mol}^{-1}$, rendering this interaction indispensable to achieve splitting. However, potassium is not unique in this behavior and sodium and rubidium also resulted in $\mathrm{N}_{2}$ splitting, while caesium yielded a cyclic $\left[\mathrm{Fe}_{3}\left(\mu-\mathrm{N}_{2}\right)_{3}\right]$ cluster with intact $\mathrm{N}_{2}$ ligands. Interestingly, the $\mathrm{N}_{2}$ splitting was found to be reversible as addition of $\mathrm{CO}$ gas to $\mathbf{X} \mathbf{X} \mathbf{V}^{\mathbf{L} 3}$ resulted in formation of 4 eq $\left[\mathrm{Fe}(\mathrm{CO})_{3}\left(\mathrm{~L}_{3}\right)\right]$ accompanied by release of $\mathrm{N}_{2} \cdot{ }^{[101]}$

\subsubsection{Thermal $\mathrm{N}_{2}$ cleavage from side-on bound dimers}

Apart from the splitting of end-on bridged dinitrogen complexes into terminal nitrides, initial splitting in side-on bridged dimers to yield two bridging nitrides has also been reported, but is investigated in less detail. In side-on bridging $\mathrm{N}_{2}$ complexes, typically a much higher degree of activation can be observed (vide supra) and the transition into bridging nitrides is almost fluent. The first example reported on full $\mathrm{N} \equiv \mathrm{N}$ cleavage was published by Cloke and coworkers and occurred upon one-electron reduction of [VCl $\left.\left(\mathrm{N}^{\prime} \mathrm{N}_{2}\right)\right]\left(\mathrm{N}^{\prime} \mathrm{N}_{2}\right.$ $\left.=\mathrm{Me}_{3} \mathrm{SiN}\left(\mathrm{CH}_{2} \mathrm{CH}_{2} \mathrm{NSiMe}_{3}\right)_{2}{ }^{2-}\right)$ resulting in $\left[\mathrm{V}(\mu-\mathrm{N})\left(\mathrm{N}^{\prime} \mathrm{N}_{2}\right)\right]_{2} \cdot{ }^{[102]}$ This complex features an intranuclear $\mathrm{N}-\mathrm{N}$ distance of $d(\mathrm{~N} \cdots \mathrm{N})=2.50(2) \AA$, thus proving full dinitrogen splitting 
and the origin of the nitrides from $\mathrm{N}_{2}$ was unequivocally confirmed by labeling with ${ }^{15} \mathrm{~N}_{2}$. Detailed spectroscopic and theoretical investigations were performed in order to understand the electronic structure of the resulting nitrido complex. ${ }^{[103]}$

Additionally, the reaction pathway which leads to splitting was analyzed by DFT methods. ${ }^{104]}$ They confirmed initial side-on coordination to a single $\mathrm{V}(\mathrm{II})\left[\mathrm{V}\left(\mathrm{N}^{\prime} \mathrm{N}_{2}\right)\right]$ fragment to be favorable over an end-on coordination mode. This was predicted to be required for electron transfer from the metal into the $\mathrm{N}^{\sigma^{*}} \mathrm{~N}$ orbital. Again, a $10 \pi$ electron count in the $\{\mathrm{MNNM}\}$ core seems to be required for achieving the splitting.

\subsubsection{Photochemical $\mathrm{N}_{2}$ cleavage}

Almost 25 years past since Cummins' discovery of thermal $\mathrm{N}_{2}$ splitting by molecular transition metal complexes and an impressive number of related systems undergoing thermal $\mathrm{N} \equiv \mathrm{N}$ bond cleavage has been published ever since. ${ }^{[16,56]}$ In contrast to these achievements, the prospect of using (visible) light as an energy source to achieve dinitrogen splitting as been addressed significantly less. However, photoinduced cleavage can potentially provide access to nitrides whose generation is thermally uphill and which might therefore exhibit increased reactivity. This would be highly desirable for achieving follow-up $\mathrm{N}$-functionalization as most nitrides obtained from $\mathrm{N}_{2}$ splitting are quite stable and unreactive (see Section 4.2.1). Probably the first discussion of the potential of photochemistry in transition metal mediated $\mathrm{N}_{2}$ fixation can be found in a contribution from Fischler and Gustorf from 1975. [105] They suggested that photoexcitation in an $\mathrm{Fe}-\mathrm{N} \equiv \mathrm{N}$ complex should in principle be dominated by two transitions: a) a $d-d$ transition from a $\mathrm{Fe}^{-}-\mathrm{N} \equiv \mathrm{N}$ into a $\mathrm{Fe}-\frac{\sigma^{*}}{-} \mathrm{N} \equiv \mathrm{N}$ orbital, sigificantly weakening the $\mathrm{M} \cdots \mathrm{N}_{2}$ bond strength and $b$ ) a MLCT transition from the $\mathrm{Fe}-\stackrel{\pi}{-} \mathrm{N} \equiv \mathrm{N}$ into a $\mathrm{Fe}-\mathrm{N} \stackrel{\pi^{*}}{=} \mathrm{N}$ orbital, transfering electron density into an antibonding ligand orbital and thus potentially increasing the ligand centered reactivity, e.g. towards protonation. Along these lines it is interesting to note, that Peters and coworkers indeed reported enhanced turnover rates under irradiation with an $\mathrm{Hg}$ lamp for their Fe-based ammonia formation catalysts $\left[\mathrm{Fe}\left(\mathrm{N}_{2}\right)\left(\mathrm{BP}_{3}{ }^{i \operatorname{Pr}}\right)\right]^{-}(\mathbf{I X}$, see Section 3.1), even though the authors assigned this effect to photochemical regeneration of a $\mathrm{H}_{2}$-poisoned iron dihydride complex by reducitve elimination of $\mathrm{H}_{2} \cdot{ }^{[106]}$

Until today, only five examples of photochemical $\mathrm{N}_{2}$ cleavage are known, which are summarized in Scheme 3.5. The very first example was reported by Floriani and coworkers in $2001 .{ }^{[86]}$ As discussed above, their end-on bridging $\mathrm{N}_{2}$ dimer $\left[\left(\mu-\mathrm{N}_{2}\right)\left\{\mathrm{MoMes}_{3}\right\}_{2}\right]$ (XIV) is thermally stable in refluxing benzene (see Section 3.2.1). However, when the complex was irradiated with ultraviolet (UV) light $(\lambda=365 \mathrm{~nm})$, selective formation of a $\mu-N$ bridged dimer $\left[(\mu-\mathrm{N})\left\{\mathrm{MoMes}_{3}\right\}_{2}\right](\mathbf{X X V I})$ was formed. The authors proposed photochemical cleavage into the terminal nitrides, followed by formation of an $[\mathrm{Mo}=\mathrm{N}-\mathrm{Mo}-\mathrm{N}=\mathrm{N}-\mathrm{Mo}-\mathrm{N}=\mathrm{Mo}]$ chain (with $\mathrm{Mo}=\mathrm{MoMes}_{3}$ ) by adduction fromation of two nitrides with one dimer, from which the centeral $\mathrm{N}_{2}$ moiety is extruded. 


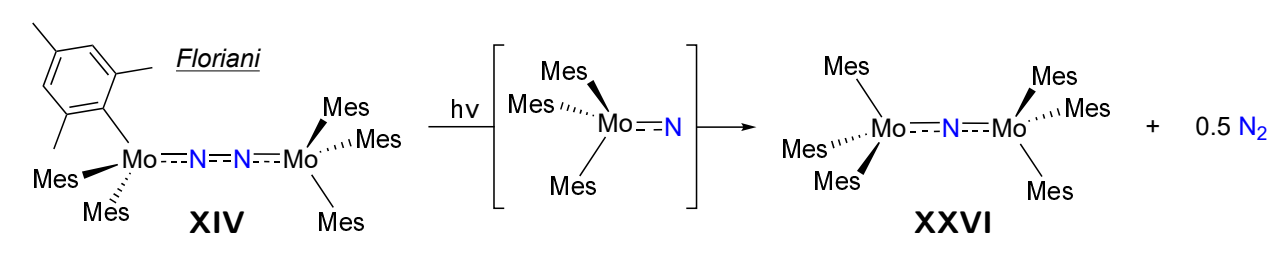

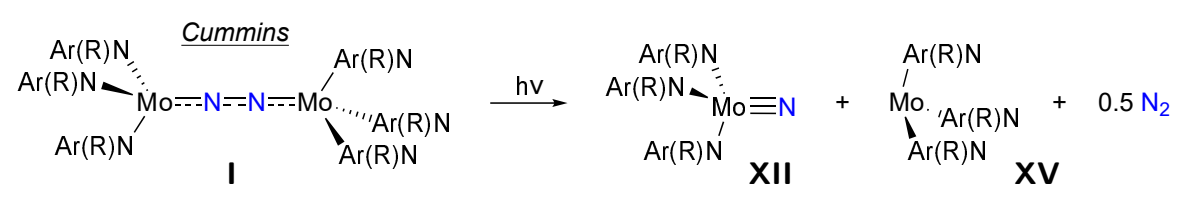

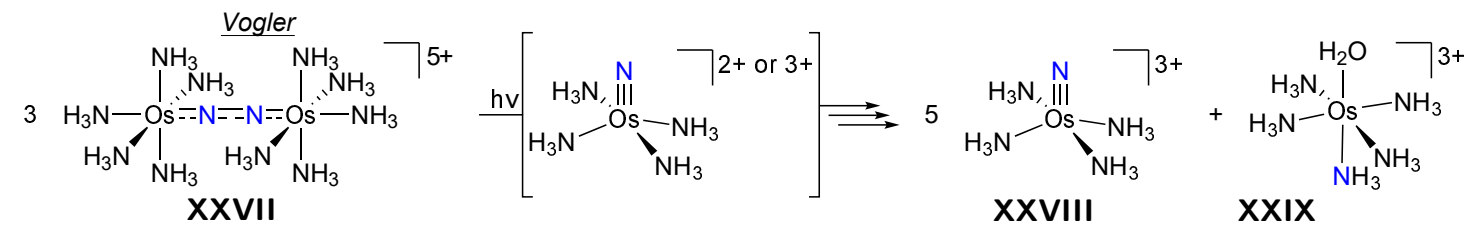
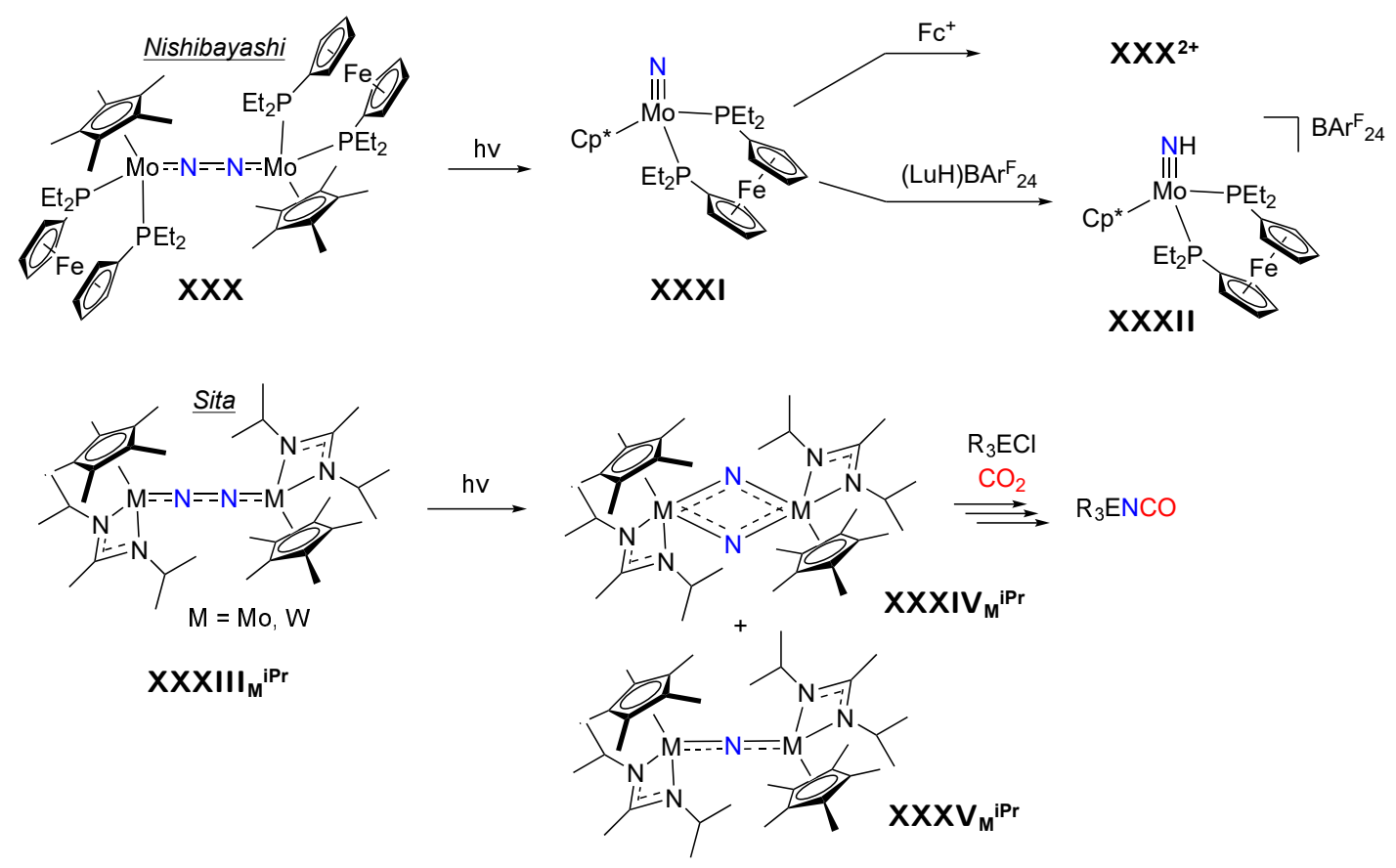

Scheme 3.5. Summary of all five literature known examples of photochemical dinitrogen cleavage.

In 2008, Cummins and coworkers also reported the photolysis of their dimer $\mathbf{I}$ at $-78^{\circ} \mathrm{C}$ (at which the dimer is thermally stable) by irradiating a pronounced absorption band at $544 \mathrm{~nm}$ to produce an equimolar mixture of the nitride $\left[\mathrm{MoN}\left(\mathrm{N}\left({ }^{\mathrm{t}} \mathrm{Bu}\right) \mathrm{Ar}\right)_{3}\right](\mathrm{XII})$ and the starting complex $\left[\mathrm{Mo}\left(\mathrm{N}\left({ }^{\mathrm{t}} \mathrm{Bu}\right) \mathrm{Ar}\right)_{3}\right](\mathbf{X V}) .{ }^{[28]}$ Competitive $\mathrm{N} \equiv \mathrm{N}$ and $\mathrm{Mo}-\mathrm{N}_{2}$ bond cleavage analog to the proposal by Fischler and Gustorf ${ }^{[105]}$ was proposed as a possible mechanism and the authors suggested that a similar situation might also be the case for Floriani's isoelectronic system. The non-occurance of a bridging nitride complex in Cummins' system can rationlized by the large ligand sterics rendering such a dimerization unfavorable. ${ }^{107]}$ This is the only photochemical reaction available which was studied in detail using time-resolved electronic absorption pump-probe experiments. ${ }^{[108]}$ The study revealed initial excitation into 
an exited triplet state followed by full relaxation into the ground state within $300 \mathrm{fs}$ by internal conversion (IC), excluding excited state photochemistry. Instead, $\mathrm{N} \equiv \mathrm{N}$ or $\mathrm{Mo}-\mathrm{N}_{2}$ bond dissociation occur from a vibrationally hot ground state after IC and have to compete with vibrational energy relaxation (VER), resulting in a low quantum-yield of $\sim 2 \%$ for each splitting channel. Since formation of the nitride and the $\mathrm{N}_{2}$ extrusion product will have different activation barriers, their equimolar formation is explained by a nonstatistical energy distribution after IC.

In 2010, Kunkely and Vogler reported on the photolysis of the mixed valent Os(II)/Os(III) complex $\left[\left(\mu-\mathrm{N}_{2}\right)\left\{\mathrm{Os}\left(\mathrm{NH}_{3}\right)_{5}\right\}_{2}\right]^{5+}(\mathbf{X X V I I})$ in aqueous solution, a complex which is strongly related to the very first bridging $\mathrm{N}_{2}$ complex by Taube (see Section 2.1). ${ }^{[22,109]}$ Solely based on ultraviolet/visible (UV/vis) absorption data, the authors claim an initial photodissociatiaon into an $\mathrm{Os}(\mathrm{VI})(\mathbf{X X V I I I})$ and an Os(v) tetraamine nitrido complex, accompanied by the release of two equivalents of ammonia ligands. The $\mathrm{Os}(\mathrm{v})$ complex is supposed to be unstable and undergo further disproportionation into the Os(VI) and an Os(IV) nitride, of which the latter disproportionates again and forms $\mathbf{X X V I I I}$ and an $\left[\mathrm{Os}^{\mathrm{III}}\left(\mathrm{NH}_{3}\right)_{5}\left(\mathrm{H}_{2} \mathrm{O}\right)\right]^{3+}$ complex (XXIX) where one of the amine ligands is obtained from the nitride and protons, leading to an overall conversion of $\mathrm{N}_{2}$ to ammonia in a maximum of $17 \%$. Interestingly, the photolysis of one-electron oxidized Os(III)/Os(III) dimer is reported to undergo quantitative $\mathrm{N}_{2}$ release rather than $\mathrm{N} \equiv \mathrm{N}$ bond scission, again showing both processes to be feasible under photochemical conditions. ${ }^{[109]}$ As an extension to this work, the authors also reported the dimer photolysis in the presence of $\mathrm{ClO}_{2}$ to yield $\left[\mathrm{Os}^{\mathrm{II}}\left(\mathrm{NH}_{3}\right)_{5}(\mathrm{NO})\right]^{3+}$, as well as the photolysis of monomeric $\left[\mathrm{Os}\left(\mathrm{NH}_{3}\right)_{5}\left(\mathrm{~N}_{2}\right)\right]^{2+}$ under $\mathrm{O}_{2}$ and $\mathrm{H}_{2} \mathrm{O}$ to yield $\left[\mathrm{Os}^{\mathrm{VI}} \mathrm{N}\left(\mathrm{NH}_{3}\right)_{4}\right]^{3+}+$ $\mathrm{NO}_{2}^{-}$and $\left[\mathrm{Os}^{\mathrm{VI}} \mathrm{N}\left(\mathrm{NH}_{3}\right)_{4}\right]^{3+}+\mathrm{N}_{2} \mathrm{H}_{4}+\mathrm{OH}^{-}$, respectively. ${ }^{[110-112]}$ Despite the characterization of these reactions being based a lot on experience and chemical intuition, this system was investigated in a sophisticated theoretical study by Krewald and González using multireference CASPT2/CASSCF methods. ${ }^{[113]}$ Splitting of the $\mathrm{N}_{2}$ bridge was simulated by stepwise increase of the $\mathrm{N} \equiv \mathrm{N}$ bond length of $\mathbf{X X V I I}$ in a linear fashion (neglecting the proposed zig-zag structure, vide supra) and evolution of the electronic structure was analyzed, revealing the $\mathrm{M}^{-}-\mathrm{N}^{\sigma}-\mathrm{N}-\stackrel{\sigma}{-} \mathrm{M}$ orbital to dramatically decrease in energy along the reaction coordinate becoming first singly, then doubly occupied, in agreement with the qualitative picture developed by Cummins (see Figure 3.2).

An important step for utilizing photochemical dinitrogen splitting for $\mathrm{N}_{2}$ fixation was made in 2014, when Nishibayashi and coworkers reported a Mo $\mathrm{N}_{2}$ dimer which does undergo photolytical $\mathrm{N}_{2}$ cleavage into terminal nitrides which could subsequently be functionalized further. ${ }^{[114]}$ The thermally stable, formal $\mathrm{Mo}(\mathrm{I})$ based dimer $\left[\left(\mu-\mathrm{N}_{2}\right)\left\{\mathrm{Mo}\left(\mathrm{Cp}^{*}\right)(\operatorname{depf})\right\}_{2}\right]$ (XXX, depf $=1,1^{\prime}$-bis(diethylphosphino)ferrocene), which exhibits a $10 \pi 4 \delta$ electronic configuration in the $\{M N N M\}$ core, does cleave the $\mathrm{N} \equiv \mathrm{N}$ bond to yield the corresponding terminal nitride complex in $33 \%$ yield. Wavelength selective irradiation indicated a pronounced absorption band at $748 \mathrm{~nm}$ to be inactive in the splitting process, whereas irradiation with 
$\lambda>400 \mathrm{~nm}$ led to conversion. Using time-dependent density functional theory (TDDFT) calculations, a transition between 400 and $450 \mathrm{~nm}$ with significant electron loss between the nitrogen atoms was identified which might be relevant for the photolysis. When the obtained nitride $\left[\mathrm{Mo}(\mathrm{N})\left(\mathrm{Cp}^{*}\right)(\mathrm{depf})\right](\mathbf{X X X I})$ is oxidized by one electron, $\mathrm{N}-\mathrm{N}$ coupling to the dicationic $\mu-\mathrm{N}_{2}$ bridged dimer is observed, in line with Cummins findings that splitting of a $8 \pi$ system would be uphill (see Section 3.2.1). Importantly, the neutral nitride can be protonated at the nitride using $(\mathrm{LuH}) \mathrm{BAr}_{24}^{\mathrm{F}}$ to yield the only other reported parent imido complex derived from $\mathrm{N}_{2}(\mathbf{X X X I I )}$ ) despite the one by Schrock (see Section 3.1), accompanied by partial $\mathrm{H}_{2}$ generation and formation of the dicationic dimer. Treating the nitride with an excess of $\mathrm{Co}\left(\mathrm{Cp}^{*}\right)_{2}$ and $(\mathrm{LuH}) \mathrm{BAr}_{24}^{\mathrm{F}}$ gave ammonia in $37 \%$ yield based on Mo, indicating photogenerated nitrido complexes to have a potential in $\mathrm{N}_{2}$ fixation. ${ }^{[115]}$

A even more versatile example was provided in 2015 by Sita and coworkers, who could

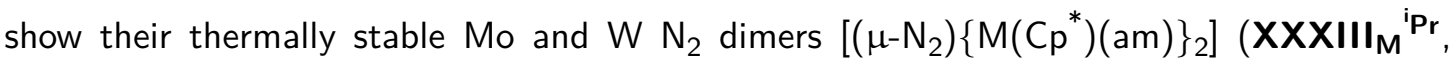
am $=N\left({ }^{i} \operatorname{Pr}\right) C(M e) N\left({ }^{i} \operatorname{Pr}\right), M=M o$ or $\left.W\right)$ to be photolyzed into a 1:1 mixture of the bis-nitride and mono-nitride bridged dimers $\left[(\mu-N){ }_{2}\left\{\mathrm{M}\left(\mathrm{Cp}^{*}\right)(\mathrm{am})\right\}_{2}\right]\left(\mathbf{X X X X}_{\mathbf{X}} \mathbf{V}_{\mathbf{M}}{ }^{\mathrm{i} \mathbf{P r}}\right)$ and $[(\mu-$ N) $\left.\left\{\mathrm{M}\left(\mathrm{Cp}^{*}\right)(\mathrm{am})\right\}_{2}\right]\left(\mathbf{X X X X V}_{\mathbf{M}}{ }^{i} \mathbf{P r}\right){ }^{[116]}$ The occurance of the mono- $\mu-\mathrm{N}$ bridged dimer is reminiscent of the observations by Floriani and Cummins, whereas the bis- $\mu-\mathrm{N}$ bridged dimer was shown to be formed as well by reductive dimerization of $\left[\mathrm{M}(\mathrm{N})\left(\mathrm{Cp}^{*}\right) \mathrm{Cl}(\mathrm{am})\right]$, suggesting terminal nitrides to be the initial photoproduct. Irradiation in the presence of $\mathrm{R}_{3} \mathrm{ECl}$ $(\mathrm{R}=\mathrm{Me}, \mathrm{Ph} ; \mathrm{E}=\mathrm{C}, \mathrm{Si}, \mathrm{Ge})$ resulted in concomitant formation of $\left[\mathrm{MCl}_{2}\left(\mathrm{Cp}^{*}\right)(\mathrm{am})\right]$ and $\left[\mathrm{M}\left(\mathrm{N}=\mathrm{ER}_{3}\right)\left(\mathrm{Cp}^{*}\right)(\mathrm{am})\right]$. The imido complex was then shown to react with $\mathrm{CO}_{2}$ to form isocyanide derivatives $\mathrm{R}_{3} \mathrm{ENCO}$ and the oxo complexes $\left[\mathrm{M}(\mathrm{O})\left(\mathrm{Cp}^{*}\right)(\mathrm{am})\right]$ and detailed reactivity studies resulted in the development of a three-step synthetic cylce for the generation of isocyanides from $\mathrm{N}_{2}, \mathrm{CO}_{2}$ and $\mathrm{R}_{3} \mathrm{ECl}$. This work marked the first and so far only example of direct incorporation of photochemically obtained nitrides into organic molecules and thus underpins the potential of this approach especially in generating reactive nitrido complexes which are thermally hard to produce.

\subsection{Nitrogen functionalization in side-on coordinated complexes}

While splitting into terminal nitrides is the only $\mathrm{N}_{2}$ functionalization reaction known for the end-on $\mu-\eta^{1}: \eta^{1}$ coordination mode, dinuclear complexes with a $\mu-\eta^{2}: \eta^{2}$ side-on coordinated $\mathrm{N}_{2}$ bridge are also known to undergo N-E bond formation reactions. ${ }^{[16,56]}$ In particular, the groups of Chirik and Fryzuk reported remarkable reactivity of side-on bound dizirconium complexes (see Figure 3.5). Fryzuk's complex $\left[\left(\mu-\eta^{2}: \eta^{2}-\mathrm{N}_{2}\right)\left\{\operatorname{Zr}\left(\mathrm{P}_{2} \mathrm{~N}_{2}\right)\right\}_{2}\right]\left(\mathbf{X X X V I}, \mathrm{P}_{2} \mathrm{~N}_{2}=\right.$ $\left.\mathrm{PhP}\left(\mathrm{CH}_{2} \mathrm{SiMe}_{2} \mathrm{NSiMe}_{2} \mathrm{CH}_{2}\right)_{2} \mathrm{PPh}\right)$ was reported to undergo heterolytic $\mathrm{H}_{2}$ cleavage to form one $\mathrm{N}-\mathrm{H}$ bond and a bridging zirconium hydride. ${ }^{117]}$ Additionally, the same complex also 
cleaves the related $\mathrm{Si}-\mathrm{H}$ bond in ${ }^{\mathrm{n}} \mathrm{BuSiH}_{3}$ to yield a $\mathrm{N}-\mathrm{Si}$ bond and again a bridging hydride. Later, also reaction with arylalkynes were reported, leading to $\mathrm{N}-\mathrm{C}$ bond formation. ${ }^{[118]}$ Chirik and coworkers achieved a quite comparable reaction with their $\left[\left(\mu-\eta^{2}: \eta^{2}-\mathrm{N}_{2}\right)\left\{\operatorname{Zr}\left(\mathrm{Cp}^{\prime}\right)_{2}\right\}_{2}\right]$ complex ( VII, Cp' $=\mathrm{C}_{5} \mathrm{HMe}_{4}$, see Section 2.2), however in this complex two equivalents of $\mathrm{H}_{2}$ are added forming $\left[\left(\left(\mu-\eta^{2}: \eta^{2}-\mathrm{NH}\right)_{2}\right)\left\{\mathrm{Zr}(\mathrm{H})\left(\mathrm{Cp}^{\prime}\right)_{2}\right\}_{2}\right]\left(\mathbf{X X X V I I ) .}{ }^{[55]}\right.$ Heating this complex under a $\mathrm{H}_{2}$ atmosphere to $85^{\circ} \mathrm{C}$ eventually resulted in release of $10-15 \%$ ammonia, marking the first and still exclusive example of full $\mathrm{N}_{2}$ hydrogenolysis to $\mathrm{NH}_{3}$ mediated by a molecular transition metal complex. The $\mathrm{Hf}$ analog furthermore reacts with $\mathrm{CO}_{2}$ to undergo twofold $\mathrm{N}-\mathrm{C}$ bond formation at one $\mathrm{N}$ atom and subsequent workup with excess $\mathrm{Me}_{3} \mathrm{Sil}$ results in release of the hydrazine derivative $\left(\mathrm{Me}_{3} \mathrm{Si}\right)_{2} \mathrm{~N}-\mathrm{N}\left(\mathrm{CO}_{2} \mathrm{SiMe}_{3}\right)_{2}{ }^{[119]}$ Similar reactions with $\mathrm{CO}$ or $\mathrm{CO}_{2}$ were reported on closely related systems, suggesting the use of these gases to promote further reactivity as a general strategy. ${ }^{[56]}$ Comparable reactivity was also obtained by Sita and coworkers on their zirconium and hafnium complexes $\left[\left(\mu-\eta^{2}: \eta^{2}-\mathrm{N}_{2}\right)\left\{\mathrm{M}\left(\mathrm{Cp}^{*}\right)(\mathrm{am})\right\}_{2}\right]$ complex (see Section 2.2, including $\mathrm{H}_{2}$ clevage, silylation with $\mathrm{PhSiH}_{3}$ and alkylation with $\mathrm{EtBr}$ (the latter only in case of $\mathrm{Hf}$ ). [36]

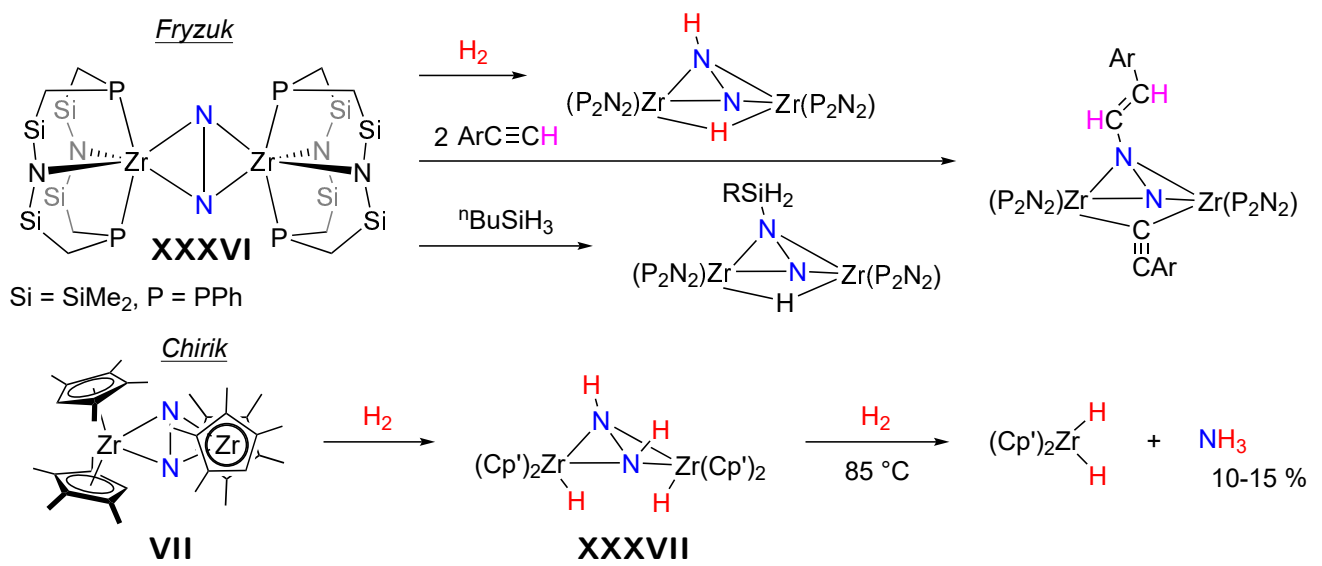

Fig. 3.5. Examples of direct functionaization of side-on bound $\mathrm{N}_{2}$ by Fryzuk and Chirik. 


\title{
Reactivity and functionalization of transition metal nitrides
}

Get me out of here!

I've been lost in the dark!

Get me out of here!

Now!

\author{
- Gojira \\ "The Cell" on "Magma"
}

\subsection{General bonding considerations}

Terminal transition metal nitride complexes have been known for more than a century, with the first example, i.e. $\mathrm{K}\left[\mathrm{OsO}_{3} \mathrm{~N}\right]$, dating back to $1847 .{ }^{[120]}$ Thus, this field of chemistry is much older than transition metal dinitrogen chemistry and an overwhelming number of examples is known. ${ }^{[15]}$ However, the vast majority of these nitrides is derived from nitrogen sources other than dinitrogen itself, typically via ammonia deprotonation and decomposition of azide or hydrazine and its derivatives. ${ }^{[15,121]}$ Metal-nitride bonds are generally considered triple bonds consisting of one $\sigma$ and two $\pi$ bonds and the nitride is both a strong $\sigma$ - and strong $\pi$-donor.

An elaborated picture for the electronic structure of such complexes with metal-ligand multiple bonds was developed by Gray and Ballhausen for an octahedral vanadyl oxo ion, i.e. $\left[\mathrm{V}(\mathrm{O})\left(\mathrm{H}_{2} \mathrm{O}\right)_{5}\right]^{2+}$, and can be adopted for octahedral nitride complexes as well. ${ }^{[122]}$ The strong donor properties of the nitrido complex lead to an energetic increase of all metal $d$ orbitals with a $z$ component, resulting in the MO splitting displayed in Figure 4.1 center. According to this scheme, all but the $d_{x y}$ orbital are antibonding and population of these leads to reactive, unstable compounds. As an immediate consequence, only transition metal nitrides with a $d^{0}-d^{2}$ electron count should be able to form stable octahedral transition metal nitrides, which is the reason for the scarce occurance of late transition metal nitrido complexes. ${ }^{[123-125]}$ This electronic limitation is exemplified by the report on the first terminal iron nitride, obtained after laser photolysis of an iron azide supported by a phorphyrine ligand at $30 \mathrm{~K}$ and characterized by resonance Raman measurements. ${ }^{[126]}$ The produced $\mathrm{Fe}(\mathrm{V}) d^{3}$ nitrido species is unstable and local heating afforded the N-N coupling product. ${ }^{[127]}$ Similar results were obtained by Wieghardt and Neese for cyclam supported iron azide complexes 
and in depth spectroscopic and theoretical analysis of the formed $\mathrm{Fe}(\mathrm{V})$ nitrides indeed indicated a doublet ground state with a $\left(d_{x y}\right)^{2}\left(d_{x z}, d_{y z}\right)^{1}$ electronic configuration. ${ }^{[128,129]}$ Later, a high valent octahedral $\mathrm{Fe}(\mathrm{VI})$ nitride was synthesized which was stable indefinitely at $77 \mathrm{~K}$ and marks the second ever reported $\mathrm{Fe}(\mathrm{VI})$ complex besides $\left[\mathrm{FeO}_{4}\right]^{2-}{ }^{[}[130]$

The formation of stable nitrides with a higher $d$ electron count can be achieved by removal of ligands and symmetry reduction, either to a tetrahedral geometry with a threefold rotation axis or a square planar geometry with twofold rotation symmetry. In threefold geometry, the $d_{x^{2}-y^{2}}$ as well as the $d_{z^{2}}$ orbitals drop in energy, but only the former drops enough to become non-bonding and thus available for population (see Figure 4.1 left). Consequently, most transition metal nitrides with a $d^{4}$ electron count (in particular iron complexes) adopt a tetrahedral geometry (see Figure 4.2 left). ${ }^{[75,131-134]}$ The first example, i.e. $\left[\mathrm{Fe}(\mathrm{N})\left(\mathrm{PhBP}_{3}{ }^{i \mathrm{Pr}}\right)\right]$ $\left(\mathbf{X X X V I I I}{ }^{\mathrm{iPr}}, \mathrm{PhBP}_{3}{ }^{i \mathrm{Pr}-}=\mathrm{PhB}\left(\mathrm{CH}_{2} \mathrm{CH}_{2} \mathrm{P}^{\mathrm{i}} \mathrm{Pr}_{2}\right)_{3}^{-}\right)$, was reported by Peters and coworkers and exhibits a singlet ground state and an electronic configuration evaluted by DFT calculations in full agreement with the proposed FMO scheme in Figure 4.1.[131] Later Smith and Meyer independently reported on closely related carbene stabilized $\mathrm{Fe}(\mathrm{IV})$ nitrido complexes ( $\mathbf{X X X I X ~ a n d ~} \mathbf{X L}$ ), both of which exhibited electronic configurations in agreement with the observations made by Peters. ${ }^{[132,133]}$ These complexes exhibit unusually short $\mathrm{Fe} \equiv \mathrm{N}$ bond

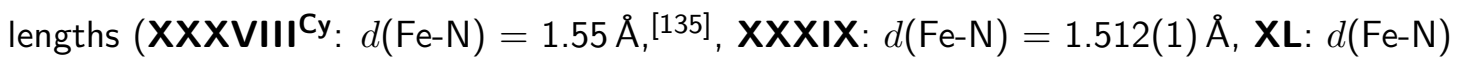
$=1.526(2) \AA)$, indicative of a rather high covalency of the iron-nitrogen triple bond.

In a square-planar coordination geometry, where the $\mathrm{M} \equiv \mathrm{N}$ bond is oriented along the $\mathrm{x}$-axis, the $d_{z^{2}}$ decreases drastically in energy and becomes non-bonding, allowing for taking up two

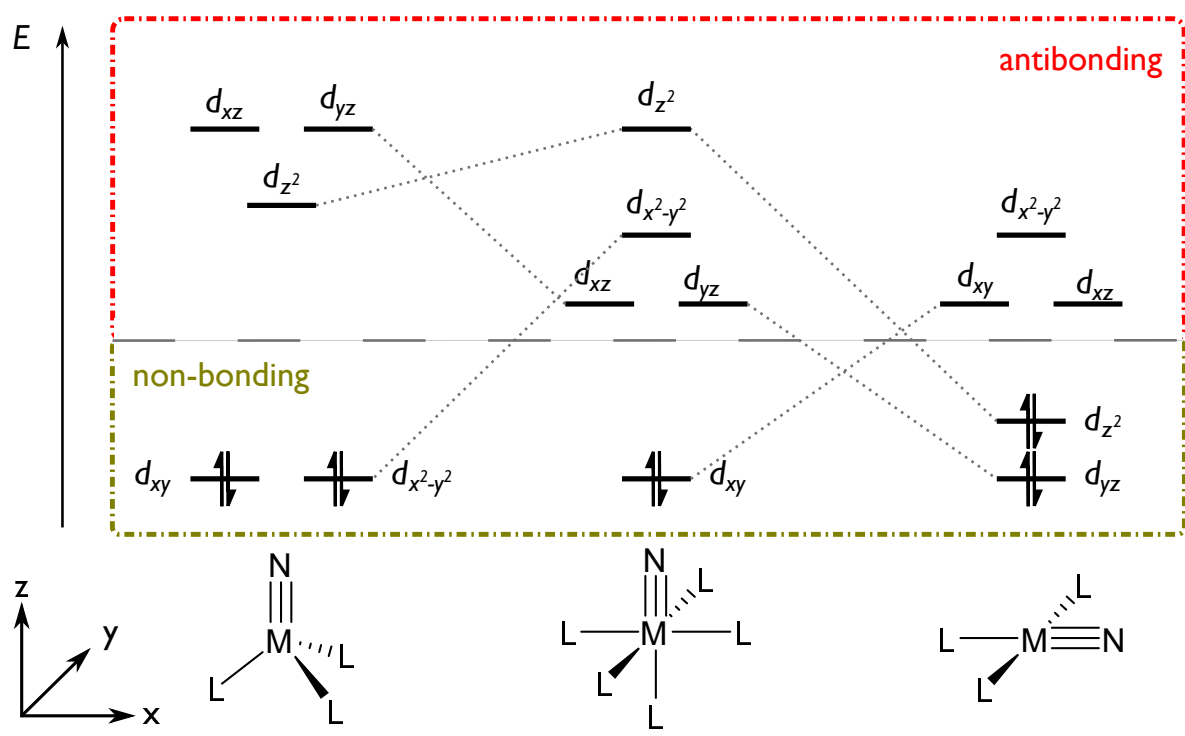

Fig. 4.1. Qualitative FMO schemes of transition metal nitrido complexes in octahedral (center) as well as tetrahedral (right) and square-planar (right) coordination geometries, allowing for an $d$ electron count up to $d^{2}$ and $d^{4}$, respectively. 


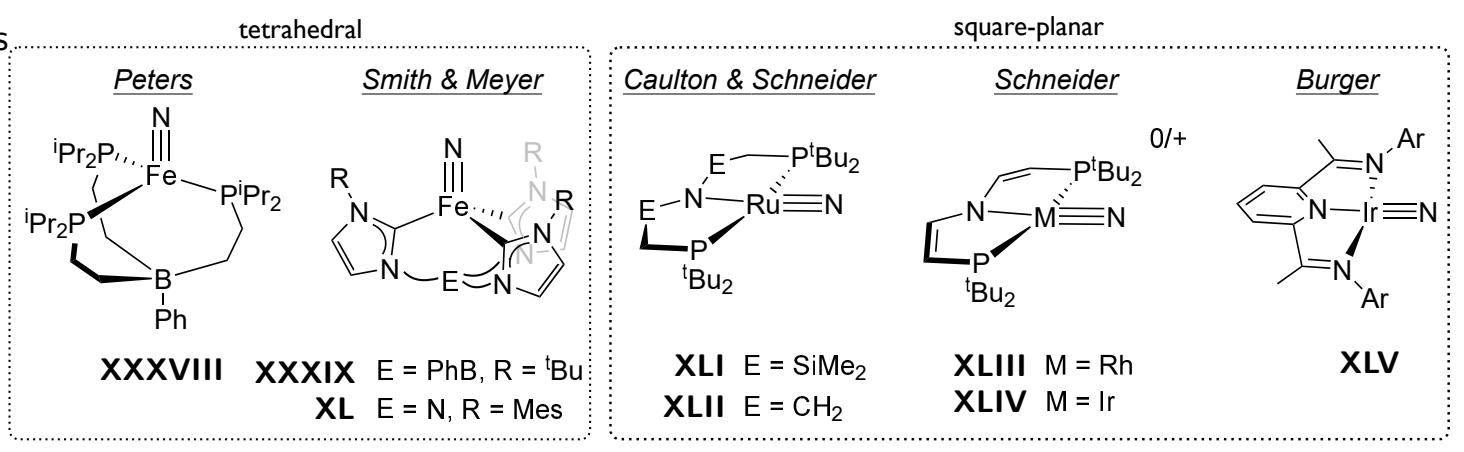

Fig. 4.2. Summary of important $d^{4}-d^{6}$ transition metal nitrido complexes which adopt either a tetrahedral (left) or square-planar coordination geometry (right).

additional electrons and thus for a $d^{4}$ electron count (see Figure 4.2 right). As can be seen in Figure 4.1, the $d_{z^{2}}$ orbital in such a complex becomes the HOMO, while the LUMO and LUMO +1 are still dominated by antibonding $\pi$ combinations of the $d_{x z}$ and $d_{y z}$ orbitals with the nitride ligand. Consequently, the FMOs of such as system exhibit similar energies, occupation number and symmetry as in a $d^{2}$ octahedral nitrido complex, rendering it isolobal to the latter. ${ }^{[136]}$ The first realization of this strategy was reported by Caulton and coworkers in 2005 who were able to synthesize the stable $\mathrm{Ru}(\mathrm{IV})$ nitrido complex $\left[\mathrm{Ru}(\mathrm{N})\left(\mathrm{PNP}_{\mathrm{Si}}^{t \mathrm{Bu}}\right)\right]$ $\left(\mathbf{X L I}, \mathrm{PNP}_{\mathrm{Si}}^{t \mathrm{Bu}}=\mathrm{N}\left(\mathrm{SiMe}_{2} \mathrm{CH}_{2} \mathrm{P}^{t} \mathrm{Bu}_{2}\right)_{2}^{-}\right)$from an azide. ${ }^{[137]}$ The most striking feature in this complex is the small $\mathrm{N}-\mathrm{Ru} \equiv \mathrm{N}$ angle $\left(\measuredangle(\mathrm{N}-\mathrm{Ru}-\mathrm{N})=155.86(13)^{\circ}\right)$, which was assigned to the pincer amide and the nitride both being strong $\pi$ donors which are thus interacting with the same set of orbitals. An analog Ru complex with a slightly modified ligand, i.e. $\left[\mathrm{Ru}(\mathrm{N})\left(\mathrm{PNP}^{t \mathrm{Bu}}\right)\right]\left(\mathbf{X L I I}, \mathrm{PNP}^{t \mathrm{Bu}-}=\mathrm{N}\left(\mathrm{CH}_{2} \mathrm{CH}_{2} \mathrm{P}^{t} \mathrm{Bu}_{2}\right)_{2}^{-}\right)$was later reported by Schneider and coworkers, featuring a similarly bent $\mathrm{Ru} \equiv \mathrm{N}$ moiety $\left(\measuredangle(\mathrm{N}-\mathrm{Ru}-\mathrm{N})=147.3(2)^{\circ}\right){ }^{[138]} \mathrm{Re}-$ markably, this coordination geometry even allowed for the synthesis of (meta)stable group 9 nitrido species with $d^{4}$ and even $d^{5}$ electron count, including $\left[\mathrm{M}(\mathrm{N})\left(\mathrm{P}=\mathrm{N}=\mathrm{P}^{t \mathrm{Bu}}\right)\right]^{0 /+}$ $\left.\left(\mathrm{P}=\mathrm{N}=\mathrm{P}^{t \mathrm{Bu}-}=\mathrm{N}\left(\mathrm{CHCHP}^{\mathrm{t}} \mathrm{Bu}_{2}\right)_{2}^{-} ; \mathrm{M}=\mathrm{Rh}(\mathbf{X L I I I}), \operatorname{Ir}(\mathbf{X L I V})\right), \operatorname{Ir}(\mathrm{N})(\mathrm{PDI})\right](\mathbf{X L V}, \mathrm{PDI}=$ pyridinediimino ${ }^{[139-141]}$

\subsection{Reactivity of transition metal nitrides}

In general, transition metal nitrides can be classified by their principal reactivity towards external reagents, being either nucleophilic or electrophilic. In a simple bonding picture, the difference can originate from the relative energies of the metal and nitrogen orbitals involved in forming the $\mathrm{M} \equiv \mathrm{N}$ bond (see Figure 4.3). ${ }^{[142]}$ If the metal $d$ orbitals are rather high in energy compared to the nitrogen $p$ orbitals, the resulting $\pi$ bonding orbital, is more nitrogen centered, and the nitride is consequently expected to be nucleophilic. On the other hand, if the metal $d$ orbitals are lower in energy, the $\pi^{*}$ orbital becomes nitrogen centered and the nitride can be expected to be rather electrophilic. Based on these very simple considerations, early transition metal complexes can be expected to form rather nucleophilic nitrides, while 


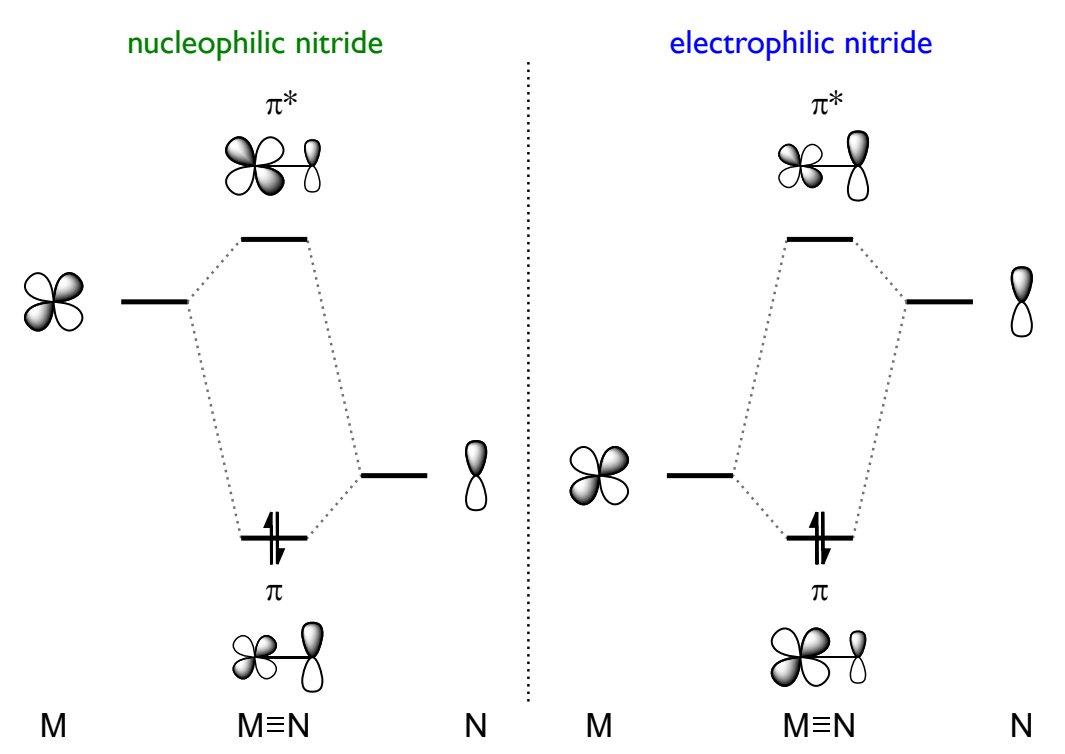

Fig. 4.3. Simplified MO scheme rationalizing the difference between an nucleophilic nitride (left) and an electrophilic nitride complex (right).

moving to the right along the periodic table should increase the electrophilicity of $M \equiv N$ complexes. This trend was qualitatively exemplified by means of DFT calculations connected with population analyses on $\left[\mathrm{M}(\mathrm{N}) \mathrm{Cl}_{4}\right]^{2-}$ and $[\mathrm{M}(\mathrm{N})($ salen $)]$ complexes $(\mathrm{M}=\mathrm{V}, \mathrm{Cr}, \mathrm{Mn}, \mathrm{Fe})$, which found decreasing negative charges at the nitride ligand upon moving to late transition metals. ${ }^{[143]}$ This bonding picture is of course insufficient to describe all effects influencing a nitride's reactivity like ligand influences, but can give a very simplified explanation for observed trends. A quite instructive example in this regard was published by Mayer and coworkers. They reported on the complexes $\left[\mathrm{Os}(\mathrm{N}) \mathrm{X}_{2}(\mathrm{Tp})\right]$ (XLVI, Tp = trispyrazolylborate, $\mathrm{X}=\mathrm{Cl}$ or $\mathrm{Ph}$ ), of which the chloro complex is electrophilic and reacts with Grignard reagents to form $\mathrm{N}-\mathrm{C}$ bonds and coordinated $\mathrm{HNR}^{-}$amide ligands after aqueous workup. ${ }^{[144]}$ When reacted with triarylboranes, the chloro complex does undergo electrophilic insertion into the $\mathrm{B}-\mathrm{C}$ bond and $\left[\mathrm{Os}\left(\mathrm{N}(\mathrm{Ar}) \mathrm{BAr}_{2}\right) \mathrm{Cl}_{2}(\mathrm{Tp})\right]$ is formed. ${ }^{[145]}$ This is in stark contrast to the second complex, where the chloride ligands are exchanged with phenyl ligands. Reaction of $\left[\mathrm{Os}(\mathrm{N}) \mathrm{Ph}_{2}(\mathrm{Tp})\right]$ with Grignard compounds occurs only very slowly and with the borane, a simple Lewis adduct rather than the $\mathrm{B}-\mathrm{C}$ insertion product is formed, indicating a more nucleophilic behavior. ${ }^{[146]}$ DFT calculations on model complexes show the near-degenerate
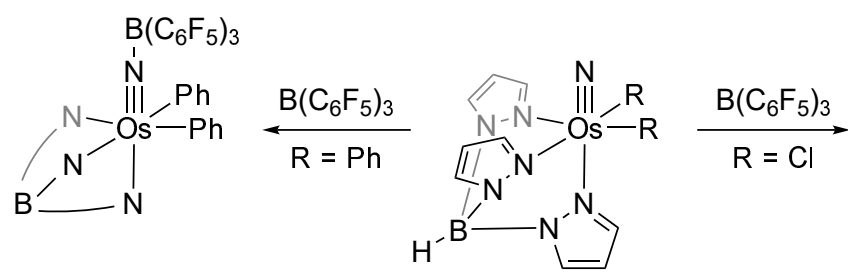

XLVI

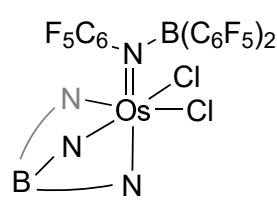

Scheme 4.1. Switching between nucleophilic (left) and electrophilic (right) reactivity of an Os( $v i)$ nitride through ligand exchange. 
LUMO and $L U M O+1$ to be the antibonding $\mathrm{M}^{\pi^{*}}-\mathrm{N}$ orbitals, which are rather low in energy and are significantly $\mathrm{N}$-centered in the chloro complex. Upon exchanging the chlorides with more $\sigma$-donating phenyl groups, these orbtials rise in energy, explaining the reduced electrophilicity in the resulting complex.

This example clearly shows on the one hand that especially group 8 transition metal nitrides are just on the border between electrophilic and nucleophilic behavior and on the other, that even subtle changes of the supporting ligand framework can have a substantial influence on the obtained reactivty.

\subsubsection{Dinitrogen derived terminal nitrides and functionalization beyond ammonia formation}

All terminal nitrido complexes derived from full $\mathrm{N}_{2}$ bond cleavage are nucleophilic, which is partly due to the overwhelming majority of them being based on early to mid transition metals. ${ }^{[59]}$ Indeed, the only reports of $\mathrm{N}_{2}$ derived terminal nitrides beyond group 7 are Kunkeley and Vogler's $\left[\mathrm{Os}(\mathrm{N})\left(\mathrm{NH}_{3}\right)_{4}\right]^{3+}(\mathbf{X X V I I I}$, see Section 3.2.3) and Peters' iron complex $\left[\mathrm{Fe}(\mathrm{N})\left(\mathrm{BP}_{3}{ }^{i \mathrm{Pr}}\right)\right]^{+}$(see Section 3.1), of which only the former is proposed to be obtained after initial full bond cleavage. ${ }^{[16]}$ Consequently, so far only electrophiles have been employed in functionalization of these complexes, and in most cases, quite strong reactants were needed to obtain any nitride centered reactivity at all.

Interestingly, the most obvious nucleophilic reactivity, i.e. acting as a Brønsted base, is hardly observed, with the examples of Schrock and Nishibayashi being the only so far reported parent imido complexes obtained after $\mathrm{N}_{2}$ splitting (see Section 3.1 and 3.2.3). ${ }^{[67,114]}$ In contrast to this, the reaction with other electrophiles is much more common and the ubiquitous example of Cummins' Mo nitride XII is a good representative for this type of chemistry. Various adducts with Lewis acids have been reported, especially with $\mathrm{EX}_{3}(\mathrm{X}=\mathrm{F}$ ; $\mathrm{E}=\mathrm{B} / \mathrm{X}=\mathrm{Cl} ; \mathrm{E}=\mathrm{B}, \mathrm{Al}, \mathrm{Ga}, \mathrm{In} / \mathrm{X}=\mathrm{Br} ; \mathrm{E}=\mathrm{Al} / \mathrm{X}=\mathrm{I} ; \mathrm{E}=\mathrm{Al})$ and $\mathrm{ECl}_{2}(\mathrm{E}=\mathrm{Ge}$, $\mathrm{Sn}) .{ }^{[147]}$ However, in these reactions hardly any influence on the $\mathrm{Mo} \equiv \mathrm{N}$ bond distance was found and the resulting complexes often decomposed back to the starting materials within $3 \mathrm{~h}$ at room temperature (RT). In contrast to this, using Mel, $\mathrm{Me}_{3} \mathrm{SiOTf}$ or $\mathrm{Ph}(\mathrm{CO}) \mathrm{OTf}$ resulted in formation of stable imido complexes $\left[\mathrm{Mo}(\mathrm{NR})\left(\mathrm{N}\left({ }^{\mathrm{t}} \mathrm{Bu}\right) \mathrm{Ar}\right)_{3}\right]^{\mathrm{X}}(\mathbf{X L V I I})$ with a linear $\mathrm{Mo}=\mathrm{N}-\mathrm{E}$ angle $(\mathrm{E}=\mathrm{C}, \mathrm{Si})$ and a significantly increase $\mathrm{Mo}=\mathrm{N}$ bond length (XII: $d$ (Mo$\mathrm{N})=1.651(4) \AA, \mathbf{X L V I I} \mathbf{R}_{\mathbf{R}} \mathrm{SiMe}_{3}: d(\mathrm{Mo}-\mathrm{N})=1.715(6) \AA$.). ${ }^{[28,147]}$ The methylated complex could be further deprotonated to yield a linear ketimido complex $\left[\mathrm{Mo}\left(\mathrm{NCH}_{2}\right)\left(\mathrm{N}\left({ }^{\mathrm{t}} \mathrm{Bu}\right) \mathrm{Ar}\right)_{3}\right]$ $(\mathrm{XLVIII})$, with a long $\mathrm{Mo}=\mathrm{N}$ bond $(d(\mathrm{Mo}-\mathrm{N})=1.777(4) \AA)$, ranging between a single and a double bond, and reaction with an additional equivalent of Mel yielded the ethylimdo complex.

In a follow up study, the authors further reported on full conversion of the nitride to a cyanide ligand. ${ }^{[148]}$ Initial reaction with $\mathrm{MeOCH}_{2} \mathrm{Cl}$ and ${ }^{\mathrm{i}} \mathrm{Pr}_{3} \mathrm{SiOTf}$ afforts $\left[\mathrm{Mo}\left(\equiv \mathrm{N}-\mathrm{CH}_{2} \mathrm{OMe}\right)\left(\mathrm{N}\left({ }^{\mathrm{t}} \mathrm{Bu}\right)\right.\right.$ $\left.\mathrm{Ar})_{3}\right]^{+}(\mathbf{X L I X a})$ and deprotonation yielded the imido complex $\left[\mathrm{Mo}(-\mathrm{N}=\mathrm{CHOMe})\left(\mathrm{N}\left({ }^{\mathrm{t}} \mathrm{Bu}\right) \mathrm{Ar}\right)_{3}\right]$ 


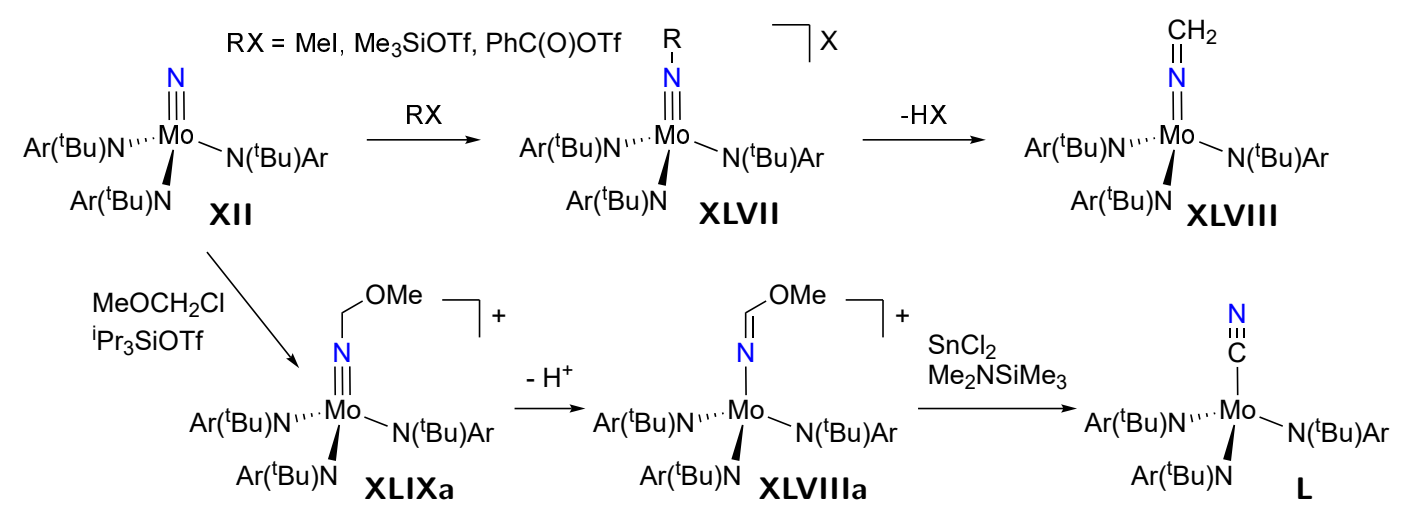

Scheme 4.2. Functionalization schemes of the $\mathrm{N}_{2}$ derived nitride complex $\mathbf{X I I}$.

(XLVIIIa). Subsequent reaction with $\mathrm{SnCl}_{2}$ and $\mathrm{Me}_{2} \mathrm{NSiMe}_{3}$ results in formation of the cyanide complex $\left[\mathrm{Mo}(\mathrm{CN})\left(\mathrm{N}\left({ }^{\mathrm{t}} \mathrm{Bu}\right) \mathrm{Ar}\right)_{3}\right](\mathbf{L})$. Importantly, this process includes reduction of the Mo(VI) nitrido to an Mo(IV) cyanide complex with the reduction equivalents steming from the ligand $\mathrm{C}-\mathrm{H}$ bonds rather than an external reduction source, an approach which was later also used by Schneider and coworkers (vide infra).

The first report of the incorporation of $\mathrm{N}_{2}$ derived nitrogen into an organic molecule came from de Vries and coworkers, who reported the direct release of trifluoroacetamide from reaction of Cummins' nitrido complex XII with trifluoroacetic acid, accompanied by partial degradation of the amide ligands to an imide, releasing isobutene and protons from one of the tert-butyl groups (see Scheme 4.3, top). ${ }^{[149]}$

In 2016, Mézailles and coworkers published a paper on splitting of $\mathrm{N}_{2}$ upon two-electron reduction of $\left[\mathrm{MoCl}_{3}(\mathrm{PPP})\right]\left(\mathrm{LI}, \mathrm{PPP}=\mathrm{PhP}\left(\mathrm{CH}_{2} \mathrm{CH}_{2} \mathrm{PCy}_{2}\right)_{2}\right)$ into the corresponding nitride complexes, which was proposed to proceed via an unobserved $\mu-\eta^{1}: \eta^{1} \mathrm{~N}_{2}$ bridged dimer LII (in fact, a related dimer, i.e. $\left[\left(\mu-\mathrm{N}_{2}\right)\left\{\mathrm{Mo}\left(\mathrm{N}_{2}\right)_{2}(\mathrm{PPP})\right\}\right]$ was previously observed and shown to be an active catalyst in the generation of silylamines from $\mathrm{N}_{2}$ ) (see Scheme 4.3, bottom). ${ }^{[150,151]}$ After successful dinitrogen cleavage into [Mo(N)I(PPP)] (LIII) (in the presence of $\mathrm{Nal}$ ), the obtained nitride could be reacted with substituted silanes to form Mo-H and $\mathrm{N}$-Si bonds. Importantly, the reaction with $\mathrm{HSiMe}_{2}\left(\mathrm{CH}_{2} \mathrm{CH}_{2}\right) \mathrm{Me}_{2} \mathrm{SiH}$ resulted in inital 1,2 -addition of one $\mathrm{Si}-\mathrm{H}$ group to the $\mathrm{Mo} \equiv \mathrm{N}$ moiety and heating to $80^{\circ} \mathrm{C}$ released the bis(silyl)amine after intramolecular reaction with the second $\mathrm{Si}-\mathrm{H}$ moiety. The suspected concomitant formation of $[\mathrm{Mo}(\mathrm{H}) \mathrm{I}(\mathrm{PPP})]$ (LIV) could not be directly proven, but a $\mathrm{PMe}_{3}$ adduct was isolated from the reaction mixture after addition of the phosphine, proving rereduction of the metal center to $\mathrm{Mo}(\mathrm{II})$, with the reduction equivalents originating from the reactant itself rather than from an external electron source. Rather recently, the authors reported a closely related reaction, in which the $\mathrm{N}_{2}$ derived nitride was functionalized by $\mathrm{HBPin}$ to give triborylamine $\mathrm{N}(\mathrm{BPin})_{3}$ in high yields, again accompanied by formation of Mo hydride complexes. ${ }^{[152]}$ Unfortunately, closing the cycle has not been achieved so far in neither of the two reactions. 


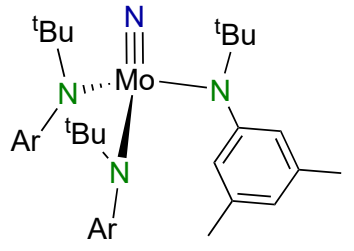

XII<smiles>O=C(OC(=O)C(F)(F)F)C(F)(F)F</smiles>

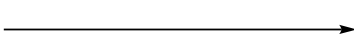

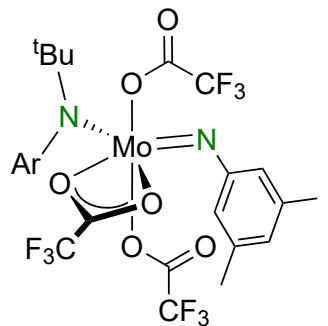

$2 \prod$<smiles>NC(=O)C(F)(F)C(F)(F)F</smiles>

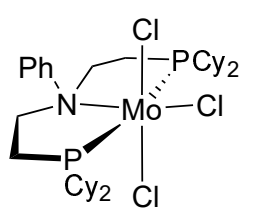

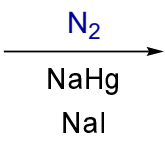

LI

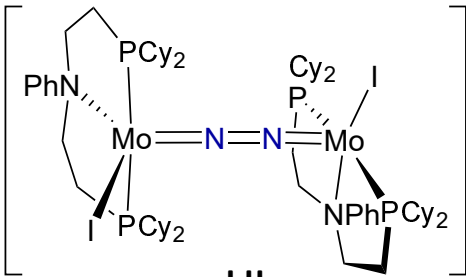

LII

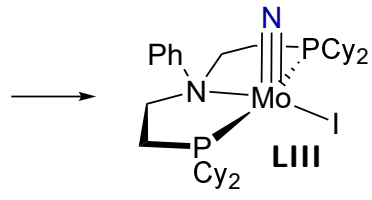

$80^{\circ} \mathrm{C} \downarrow-\overbrace{\mathrm{Hi}}^{\mathrm{Si}} \mathrm{H}^{\prime}$

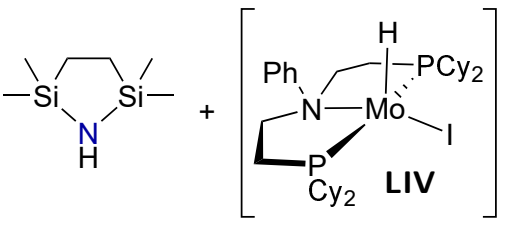

Scheme 4.3. Top: Release of an amide from Cummins' nitrido complex, accompanied by ligand degradation. Bottom: Conversion of a Mo nitride to a bissilylamine with simultaneous rereduction of the metal center.

Cummins went a step further as he not only achieved incorporation of the nitrido ligand into an organic molecule, but at the same time was able to regenerate the starting Mo complex, thus closing a synthetic cycle (see Scheme 4.4). After acylation of the nitride XII with in situ generated acyl triflates, the obtained imido complexes can be reduced using $\mathrm{Mg}$ in the presence of $\mathrm{Me}_{3} \mathrm{SiOTf}$ to affort the ketimido complexes $\left[\mathrm{Mo}\left(\mathrm{N}=\mathrm{C}(\mathrm{R}) \mathrm{OSiMe}_{3}\right)\left(\mathrm{N}\left({ }^{\mathrm{t}} \mathrm{Bu}\right) \mathrm{Ar}\right)_{3}\right]$ $\left(\mathbf{L V} ; \mathrm{R}=\mathrm{Ph},{ }^{\mathrm{t}} \mathrm{Bu}, \mathrm{Me}\right)$. Subsequent reaction with $\mathrm{SnCl}_{2}$ or $\mathrm{ZnCl}_{2}$ results in clean formation of the corresponding nitrile compounds $\mathrm{R}-\mathrm{C} \equiv \mathrm{N}$ and $\left[\mathrm{MoCl}\left(\mathrm{N}\left({ }^{\mathrm{t}} \mathrm{Bu}\right) \mathrm{Ar}\right)_{3}\right](\mathbf{X V})$ of which the later could be cleanly rereduced to the Mo(III) starting complex $\mathbf{X V}$. This way, a synthetic cylce for the synthesis of nitrogen containing organic molecules directly from $\mathrm{N}_{2}$ was realized for the first time, even though it is already apparent that the required reactants (acyl triflates, $\mathrm{Mg}(0))$ are not compatible with each other and thus prevent catalytic turnover.

A slightly different approach was used by the group of Schneider and coworkers, who reported the formation of acetonitrile from $\mathrm{N}_{2}$ in the coordination sphere of a rhenium complex (see Scheme 4.5). The $\operatorname{Re}(\mathrm{v})$ nitrido complex $\left[\operatorname{Re}(\mathrm{N}) \mathrm{Cl}\left(\mathrm{PNP}^{t \mathrm{Bu}}\right)\right](\mathbf{X X I})$ obtained from splitting of $\mathrm{N}_{2}$ (see Section 3.2.1 and Scheme 3.3) was initially reported to undergo nucleophilic N-C bond formation with MeOTf. ${ }^{[94]}$ In a subsequent study, the authors published the ethylation with in situ prepared EtOTf yielding $\left[\mathrm{Re}(\mathrm{NEt}) \mathrm{Cl}\left(\mathrm{PNP}^{t \mathrm{Bu}}\right]^{\mathrm{OTf}}(\mathbf{L V I})\right.$ followed by stepwise deprotonation ultimately leading to the release of acetonitrile. ${ }^{[153]}$ Initial deprotonation yields 
the ketimido complex $\left[\operatorname{Re}(\mathrm{N}=\mathrm{CHMe}) \mathrm{Cl}\left(\mathrm{PNP}^{t \mathrm{Bu}}\right)\right](\mathbf{L V I I})$, in which bond distances and angle $\left(d(\operatorname{Re}-\mathrm{N})=1.822(4) \AA, d(\mathrm{~N}=\mathrm{C})=1.273(7) \AA, \measuredangle(\mathrm{Re}=\mathrm{N}=\mathrm{C})=174.3(5)^{\circ}\right)$ suggest a pronounced heterocummulene character. A second deprotonation in the presence of 2 equivalents of $\mathrm{CN}^{\mathrm{t}} \mathrm{Bu}$ resulted in almost quantitative release of acetonitrile accompanied by the formation of $\left[\operatorname{Re}\left(\mathrm{CN}^{t} \mathrm{Bu}\right)_{2}\left(\mathrm{PNP}^{t \mathrm{Bu}}\right)\right]$ (LVIII), a formal $\operatorname{Re}(\mathrm{I})$ complex. This is remarkable in that respect that the reduction equivalents necessary for this $\operatorname{Re}(\mathrm{V}) \rightarrow \operatorname{Re}(\mathrm{I})$ conversion are obtained from the $\mathrm{C}-\mathrm{H}$ bonds $\left(2 \mathrm{e}^{-}\right.$each) of the ethyl group rather than from an external reductant. Alternatively, external oxidants can be utilized to circumvent the need of $\pi$-accepting ligands to stabilize the $\operatorname{Re}(\mathrm{I})$ species. Using a combination of $\mathrm{Ag}^{+}$and 2,4,6-tris-tert-butylphenoxy radical (TTBP) to formally remove $2 \mathrm{e}^{-} / \mathrm{H}^{+}$from LVIII results in the vinylimide complex $\left[\operatorname{Re}\left(\mathrm{NCHCH}_{2}\right) \mathrm{Cl}\left(\mathrm{PNP}^{t \mathrm{Bu}}\right)\right]^{+}(\mathbf{L I X})$, which is a tautomer of the corresponding acetonitrile complex. Addition of a chloride source could trigger acetonitrile release and reformation of starting complex XIX. Alternatively, direct conversion of LVII could be achieved with $N$-chlorosuccinimide (NCS). To test, whether this latter, one-step conversion necessarily proceeds via the vinyl imido tautomer, the reaction was also performed after benzylation. ${ }^{[154]}$ The benzyl imido analog to LVII, which is inherently unable to undergo such a tautomerization gives similar yields of benzonitrile upon reaction with NCS, rendering this not a prerequisit for the observed reactivity.

Interestingly, in a very recent study, $\mathbf{X X I}$ was oxidized at the ligand backbone with 3chloroperbenzoic acid and subsequently at the metal center to form the nitroxide $\operatorname{Re}(\mathrm{VI})$

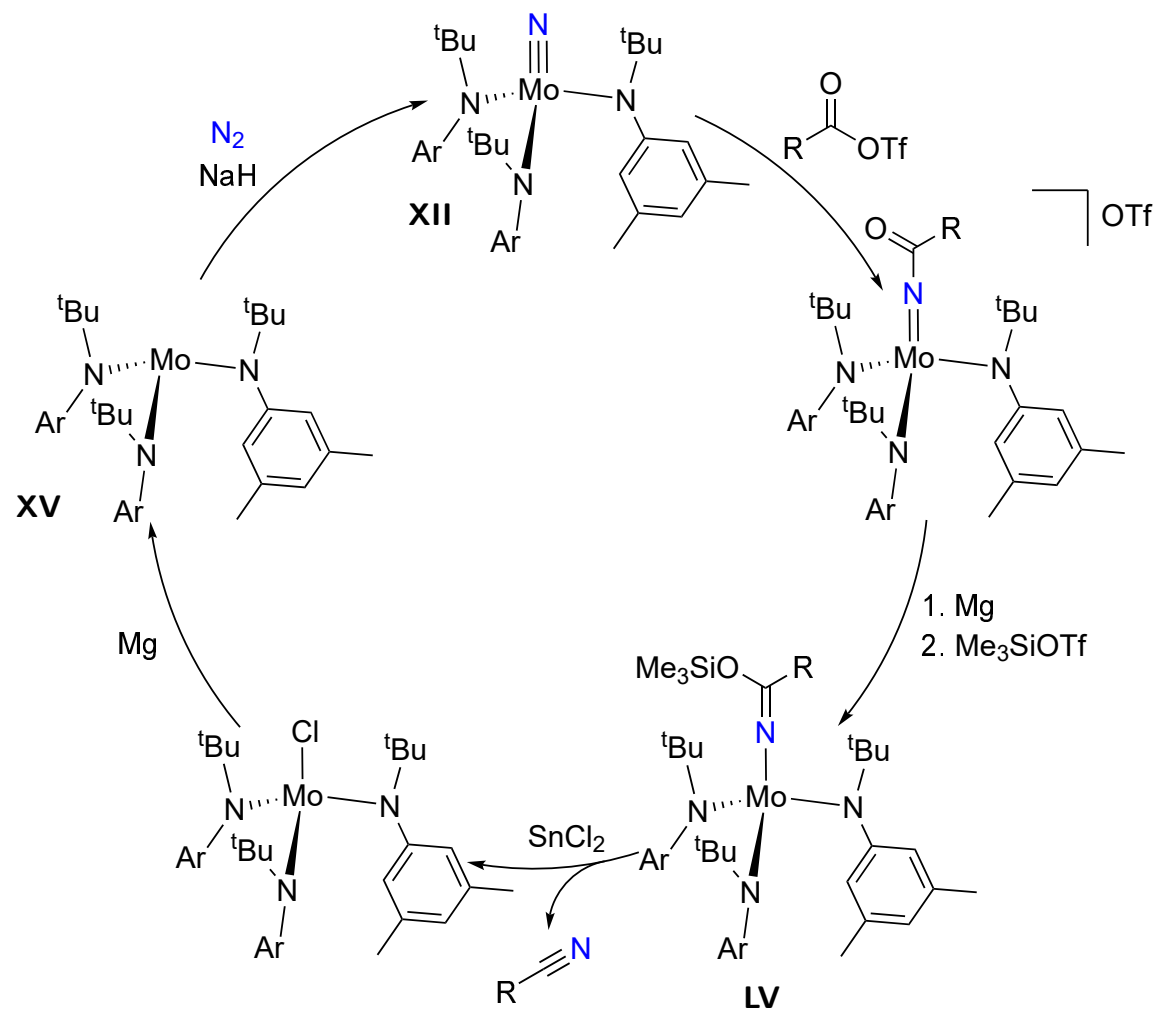

Scheme 4.4. Synthetic cylce for the conversion of dinitrogen into nitriles developed by Cummins and coworkers. 


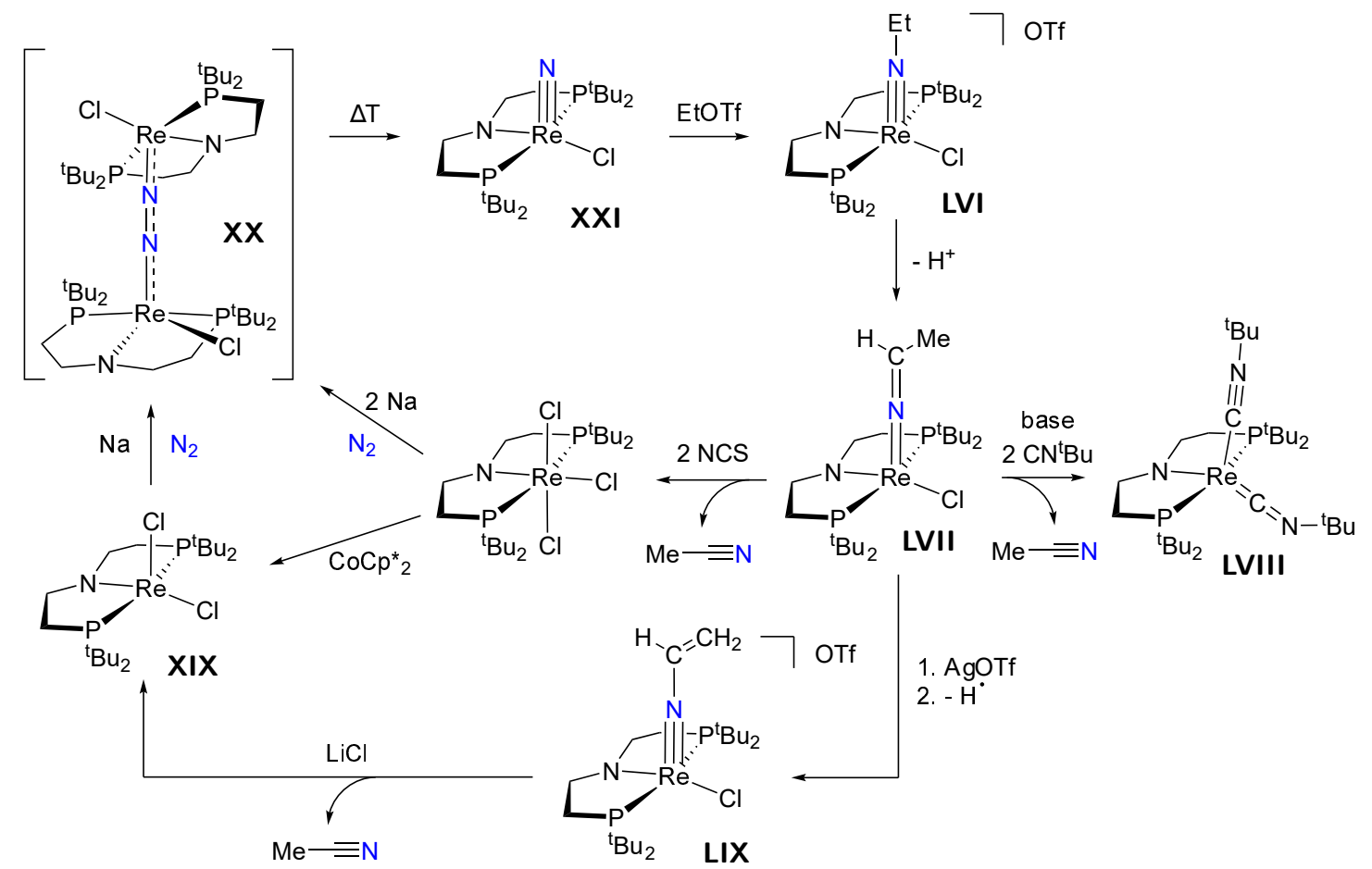

Scheme 4.5. Synthetic cycle for acetonitrile formation from $\mathrm{N}_{2}$ as employed by Schneider and coworkers.

complex $\left[\operatorname{ReNCl}\left(\mathrm{P}^{\mathrm{O}} \mathrm{NP}^{t \mathrm{Bu}}\right)\right]^{\mathrm{PF} 6}{ }^{[155]}$ These oxidations now lead to an umpolung of the nitride reactivity, which does form (unstable) phosphoraneiminato complexes with various phosphines, rendering it electrophilic.

The probably most efficient synthetic cycle for $\mathrm{N}_{2}$ functionalization to organic molecules was reported by Sita and coworkers (see Scheme 4.6). The Mo and W end-on bound dimeric complexes $\mathbf{X X X I I I} \mathbf{I o}^{{ }^{i}}{ }^{\operatorname{Pr}}(\mathrm{M}=\mathrm{Mo}, \mathrm{W})$ were already discussed for their ability to undergo photochemical $\mathrm{N}_{2}$ cleavage (see Section 3.2.3). By reducing the steric strain of the ligand (replacing the iso-propyl groups with ethyl groups) the authors were able to switch from a photochemical to a thermal splitting pathway. ${ }^{[156]}$ Again, bis- $\mu$-nitrido bridged complexes are obtained and the Mo congener is shown to react with $\mathrm{Me}_{3} \mathrm{SiCl}$ to form the silylimido complexes $\left[\mathrm{M}\left(=\mathrm{NSiMe}_{3}\right)\left(\mathrm{Cp}^{*}\right)(\mathrm{am})\right]\left(\mathbf{L X}_{\mathbf{M o}}{ }^{\mathrm{Et}}\right)$ and the starting complex $\mathbf{L X I}_{\mathbf{M o}}{ }^{\mathrm{Et}}$. In a later study, the imido complex was reported to undergo further reaction with alcohols and $\mathrm{Me}_{3} \mathrm{SiCl}$

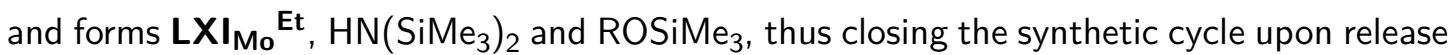
of disilylamine. ${ }^{[157]}$ It is exciting to note that the used chemicals (i.e. $\mathrm{Me}_{3} \mathrm{SiCl}$ and $\mathrm{ROH}$ )

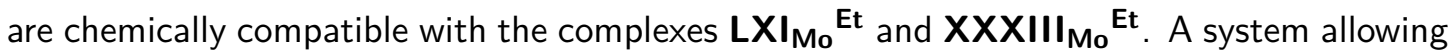
for turnover therefore seems to depend only on finding a suitable reductant.

From the above discussed examples of synthetic cycles for $\mathrm{N}_{2}$ functionalization, some important aspects and strategies can be derived:

An inherent problem of transition metal complexes which are capable of splitting the $N \equiv N$ triple bond is their tendency to form very strong $\mathrm{M} \equiv \mathrm{N}$ triple bonds in the corresponding 


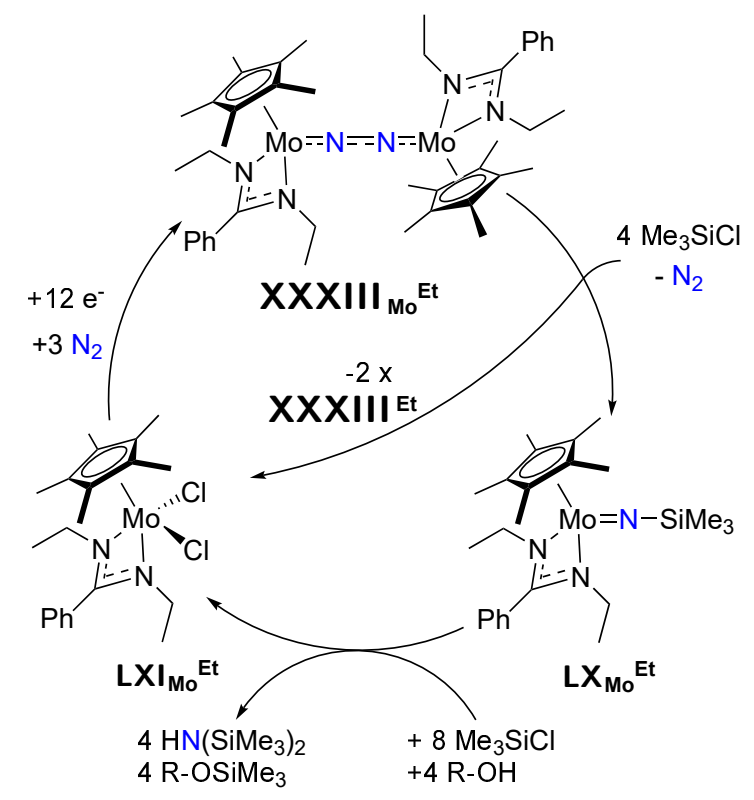

Scheme 4.6. Synthetic cycle for bis-silylamine generation from a $\mathrm{Mo}\left(\mathrm{Cp}^{*}\right)(\mathrm{am})$ complex developed by Sita.

nitrides, which drives the reaction in the first place. This problem was accurately expressed by Fryzuk and Shaver: 'The problem with developing a viable process coupling nitride formation with the production of useful nitrogen-containing materials is the inherent stability of the metal nitrides generated. Reactions of nitrides employ harsh reagents to effect moderate changes.' [158]

This stability can in principle be met by two strategies. One the one hand, forming products with strong $\mathrm{N}-\mathrm{X}$ bonds, like nitriles containing a $\mathrm{N} \equiv \mathrm{C}$ triple bond can account at least partially for the energy-demand of cleaving the strong $\mathrm{M} \equiv \mathrm{N}$ triple bond and can thus facilitate the incorporation of dinitrogen into organic products. On the other hand, it seems desirable to find transition metal complexes which do undergo $\mathrm{N} \equiv \mathrm{N}$ bond cleavage but result in less stabilized compounds which are therefore more easily functionalized. As will be shown in the next section, especially nitrido complexes of late transition metals are often very reactive and do undergo quite rich transformations. Developing compounds based on these metals for $\mathrm{N}_{2}$ fixation therefore seems a promising approach.

\subsubsection{Low valent nitrido complexes from other nitrogen sources}

As discussed in Section 4.2, most early to mid transition metal complexes exhibit nucleophilic reactivity at the nitride. This behavior changes quite significantly for the nitrido complexes formed by late transition metals (i.e. group 8 or 9 ). Unfortunately, these compounds are so far not available by direct activation of dinitrogen but rather from other sources (mostly by decomposition of azides). Nevertheless, their reactivity is versatile.

The only reported $\mathrm{Fe}(\mathrm{VI})$ nitrido complex prepared by Wieghardt is only stable at $77 \mathrm{~K}$ and yields an $\mathrm{Fe}(\mathrm{III})$ species upon warming, so no defined reactivity is known for this complex. ${ }^{[130]}$ 
a)
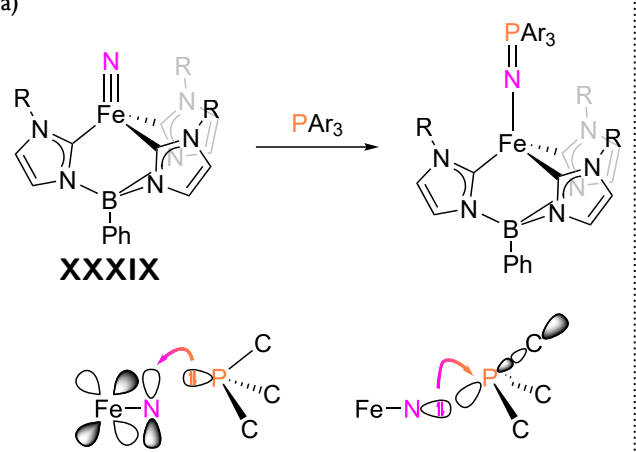

$\pi^{*}(\mathrm{Fe} \equiv \mathrm{N}) \leftarrow \sigma_{\mathrm{P}}(\mathrm{LP})$

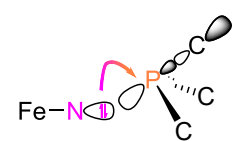

$\sigma_{\mathrm{N}}(\mathrm{LP}) \rightarrow \sigma^{*}(\mathrm{P}-\mathrm{C})$ b)

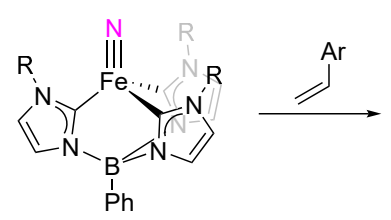

$\operatorname{XXXIX}$

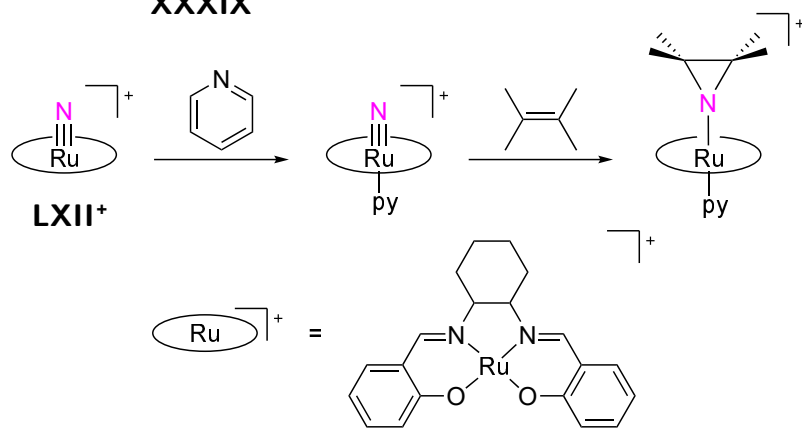

Scheme 4.7. a) Reaction of $\mathbf{X X X I X}$ with $\mathrm{PAr}_{3}$ determined by two mutual donor-acceptor interactions. b) Alkene aziridination mediated by $\mathbf{X X X I X ~ ( t o p ) ~ a n d ~ L X I I ~ ( b o t t o m ) . ~}$

This is different in the case of tetrahedral Fe(IV) nitrido complexes. The complexes presented in Section 4.1 are described as being rather electrophilic. In particular, Peters as well as

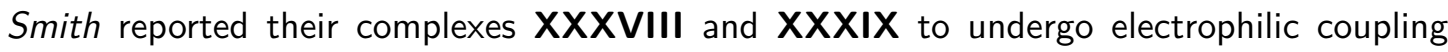
with phosphines to phosphoraneiminato ligands. ${ }^{[131,133]}$ However, this reaction, which is often reported to be archtypical for an electrophilic nitride was later investigated in detail by Smith and coworkers. By following the kinetics of the reaction with substituted triphenylphosphines, a Hammett analysis revealed the counter intuitive trend that electron-poorer phosphines do react faster than electron richer congeners. ${ }^{[159]}$ This could be explained by DFT calculations which revealed a dual nature of the reaction transition state in which on the one hand the phosphine centered lone pair donates electron density into the $\mathrm{Fe}-{ }^{\pi^{*}} \mathrm{~N}$ orbital, but on the other hand, the nitride centered lone pair donates also into a $\mathrm{P}^{\sigma^{*}} \mathrm{C}$ bond of the phosphine (see Scheme 4.7 a). This ambiphilicity was also proposed to be important for the reported $\mathrm{N}-\mathrm{C}$ bond formation with $\mathrm{CO}$ and $\mathrm{CN}^{t} \mathrm{Bu} .{ }^{[160]}$ Furthermore, reaction with 1-hydroxyl-2,2,6,6tetramethylpiperidine (TEMPOH) was reported to yield ammonia and the $\mathrm{Fe}$ (II) complex $\left[\mathrm{Fe}(\right.$ tempo $\left.)\left(\mathrm{PhB}(\mathrm{Mes} / \mathrm{m})_{3}\right)\right]$.

In a rather recent work, Smith and coworkers further reported their iron complex to be potent of alkene aziridination. ${ }^{[161]}$ Reaction of $\mathbf{X X X I X}$ with various styrenes results in equilibrium mixtures of the nitride and the $\mathrm{Fe}(\mathrm{II})$ aziridino complexes and combined theoretical and mechanistical investigations pointed towards an asynchronous radical addition as the most likely pathway (see Scheme 4.7 b, top). ${ }^{[162]}$

The only other example of direct alkene aziridination by a terminal nitride stems from an $\mathrm{Ru}(\mathrm{VI})$ salen complex, i.e. $\left[\mathrm{Ru}(\mathrm{N})(\text { salchda) }]^{\mathrm{PF}_{6}}\right.$ (LXII, salchda $=N, N^{\prime}$-bis(salicylidene)-ocyclohexyldiamine dianion) reported by $L a u$ and coworkers (see Scheme 4.7 b, bottom). [163] Aziridination of alkenes only takes place in the presence of pyridine or 1-methylimidazole and kinetic measurements indicated coordination of the base to the Ru to be the initial step of 

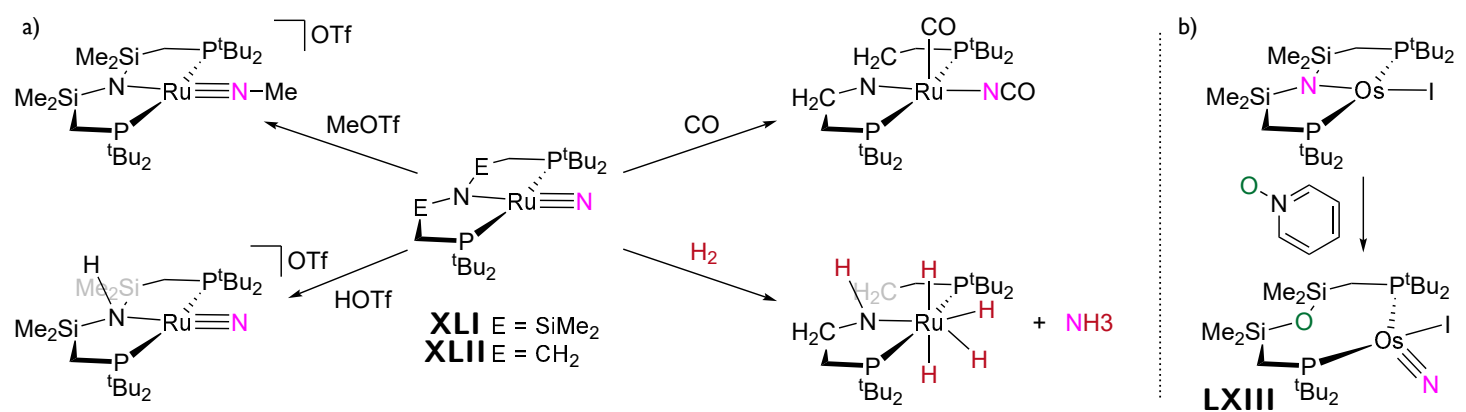

Scheme 4.8. a) Nucleophilic Ru(IV) nitrido complexes $\mathbf{X L I}$ and XLII. b) Synthesis of the only literature known Os(IV) nitrido complex LXIII.

the reaction. This example shows quite clearly how the variation of a trans ligand can alter the reactivity of a nitride. In comparison, $\mathrm{Os}(\mathrm{VI})$ nitrido complex cis-[Os $(\mathrm{N}) \mathrm{Cl}_{2}($ terpy $\left.)\right]^{+}$has been reported to insert into the $\mathrm{C}=\mathrm{C}$ bond of stilbene, yielding $\eta^{2}$ coordinated azaallenium complexes. ${ }^{[164,165]}$

In line with this example ruthenium(VI) nitrido complexes are in general electrophilic. ${ }^{[15,121,125]}$ This changes quite drastically with change of the oxidation states. The two $\mathrm{Ru}(\mathrm{IV}) \mathrm{com}$ plexes reported by Caulton and Schneider, i.e. XLI and XLII, both show defined nucleophilic reactivity (see Scheme 4.8 a). Reaction of $\mathbf{X L I}$ with HOTf and MeOTf is proposed to yield $\left[\mathrm{Ru}(\mathrm{N})\left(\mathrm{HPNP}_{\mathrm{Si}}^{\mathrm{tBu}}\right)\right]^{\mathrm{OTf}}$ and $\left[\mathrm{Ru}(\mathrm{NMe})\left(\mathrm{PNP}_{\mathrm{Si}}^{t \mathrm{Bu}}\right)\right]^{\mathrm{OTf}}$, respectively, based on 1D-nuclear magnetic resonance (NMR) data and hints from an unrefined $X$-ray diffraction study. ${ }^{[166]}$ Furthermore, reaction with two equivalents of $\mathrm{NO}$ resulted in the formation of $\left[\mathrm{Ru}(\mathrm{NO})\left(\mathrm{PNP}_{\mathrm{Si}}^{t \mathrm{Bu}}\right)\right]$ and $\mathrm{N}_{2} \mathrm{O}$, where the former nitride is transferred to $\mathrm{N}_{2} \mathrm{O}$, as indicated by ${ }^{15} \mathrm{~N}$ labeling. ${ }^{[167]}$ Schneider's related complex $\mathbf{X L I I}$ does undergo N-C coupling with $\mathrm{CO}$ to yield $[\mathrm{Ru}(\mathrm{NCO})(\mathrm{CO})$ $\left.\left(\mathrm{PNP}^{t \mathrm{Bu}}\right)\right]$, which is indicated by preliminary computational results to result from a direct electrophilic attack of $\mathrm{CO}$ at the nitride (see Scheme 4.8 a). ${ }^{[138]}$ Importantly, the complex is also reactive towards $\mathrm{H}_{2}$ and does undergo full hydrogenolysis to give ammonia in $80 \%$ yield and $\left[\mathrm{Ru}(\mathrm{H})_{4}\left(\mathrm{HPNP}^{t \mathrm{tBu}}\right)\right]$, the latter of which could be reconverted into the starting complex $\left[\mathrm{RuCl}_{2}\left(\mathrm{HPNP}^{\mathrm{tBu}}\right)\right]$ by application of vacuum, addition of $\mathrm{HCl}$ and a second evacuation. The mechanism of the hydrogenation reaction was investigated quite thoroughly by computational calculations and the minimum energy pathway revealed metal-ligand cooperative heterolytic $\mathrm{H}_{2}$ splitting over the $\mathrm{Ru}-\mathrm{N}_{\text {backbone }}$ bond to be a key step in this reaction.

The sole low-valent osmium nitrido complex ever reported is Caulton's Os(IV) complex $\left[\mathrm{Os}(\mathrm{N}) \mathrm{I}\left(\mathrm{POP}_{\mathrm{Si}}^{t \mathrm{Bu}}\right)\right]\left(\mathbf{L X I I I}, \mathrm{POP}_{\mathrm{Si}}^{t \mathrm{Bu}}=\mathrm{O}\left(\mathrm{SiMe}_{2} \mathrm{CH}_{2} \mathrm{P}^{\mathrm{t}} \mathrm{Bu}_{2}\right)_{2}\right)$ which was obtained rather coincidental by reaction of [Osl( $\mathrm{PNP}_{\mathrm{Si}}^{\mathrm{tBu}}$ )] with pyridine- $N$-oxide (see Scheme $4.8 b$ ). ${ }^{[168]} \mathrm{N} / \mathrm{O}$ exchange at the oxophilic position in the ligand backbone resulted in formation of this distorted tetrahedrally coordinated complex $\left(\tau_{4}^{\prime}=0.674 ;{ }^{1} d(\right.$ Os $\cdots O)=3.14 \AA$, thus the ligand oxygen is not bound). However, no follow-up reactivity of this complex is reported.

\footnotetext{
${ }^{1}$ Calculated according to Ref [169] and the structure data deposited at The Cambridge Crystallographic Data Center, CCDC-778422.
} 
The above described reaction of a nitrido ligand with $\mathrm{H}_{2}$ is very rare. Among the only other reports is Burger's complex $\mathbf{X L V}$, one of the few examples of isolated group 9 nitrido complexes, which reacts with $\mathrm{H}_{2}$ to yield $\left[\operatorname{Ir}\left(\mathrm{NH}_{2}\right)(\mathrm{PDI})\right]$ (or analogously with $\mathrm{R}_{3} \mathrm{SiH}$ to give the Ir $-\mathrm{N}\left(\mathrm{SiR}_{3}\right) \mathrm{H}$ product $) .{ }^{[139,170,171]}$ Despite this reaction, the complex exhibits electrophilic reactivity, as indicated by the formation of an $\mathrm{NO}$ ligand with $\mathrm{Me}_{3} \mathrm{NO}$ as single electron donor. Thermolysis of the complex for extended time resulted in intramolecular $\mathrm{C}-\mathrm{H}$ activation, whereas attempts to isolate the rhodium congener of the complex (LXIV) always resulted in a twofold ligand activation (see Scheme 4.9 a). ${ }^{[172]}$ While no final explanation for this difference could be provided, the authors suggested $\mathbf{X L V}$ to directly insert into the $\mathrm{C}-\mathrm{H}$ bond, while the $\mathrm{Rh}$ complex might first abstract an $\mathrm{H}$ atom, generating a biradical, which can then rearrange so that the second iso-propyl group can be activated as well.

Similarly, the group of Schneider reported on the synthesis of $\operatorname{Ir}\left(\mathbf{X L I V}^{\mathbf{0} /+}\right)$ and $\mathrm{Rh}(\mathbf{X} \mathbf{L I I I})$ nitrido species (see Scheme 4.9 b). ${ }^{[140,141]}$ The $d^{4}$ complex $\mathbf{X L I V}{ }^{+}$is thermally stable and can be isolated. It is reported to react with $\mathrm{Me}_{3} \mathrm{NO}$ to yield the corresponding nitrosyl complex $\left[\operatorname{lr}(\mathrm{NO})\left(\mathrm{P}=\mathrm{N}=\mathrm{P}^{t \mathrm{Bu}}\right)\right]^{+}$, hence it is electrophilic as well. ${ }^{[140]}$ However, the neutral, formal $d^{5}$ complex XLIV can only be characterized at low temperatures in frozen toluene. The complex exhibits a singly occupied molecular orbital (SOMO) and spin density which resemble the $\operatorname{lr}_{-}^{\pi^{*}} \mathrm{~N}$ orbital, with almost equal spin populations on the metal and the nitrido complex. Upon warming, the complex is shown to undergo $\mathrm{N}-\mathrm{N}$ coupling to form a dimeric, $\mu-\eta^{1}: \eta^{1} \mathbf{N}_{2}$ bridged complex $\mathbf{L X V}$, a reaction which is the microscopic reverse of the above discussed $\mathrm{N}_{2}$ splitting reactions. In a follow-up study, the authors further prepared the mono and dicationic dimers $\left[\left(\mu-\mathrm{N}_{2}\right)\left\{\operatorname{lr}\left(\mathrm{P}=\mathrm{N}=\mathrm{P}^{t \mathrm{Bu}}\right)\right\}_{2}\right]^{+/ 2+}\left(\mathbf{L X V}^{\mathbf{n}+}, \mathbf{n}=1-2\right)$, which were shown to be thermally stable as well. ${ }^{[173]}$ DFT calculations revealed the cationic complex $\mathbf{X L I V}^{+}$ to be stabilized only kinetically and coupling to $\mathbf{L} \mathbf{X} \mathbf{V}^{\mathbf{2}+}$ should in theory be downhill for this complex as well. Considering the isolobal relation between square-planar $d^{n}$ and octahedral $d^{n-2}$ compounds and thus the formal $10 \pi$ electronic configuration of the $\{\mathrm{MNNM}\}$ core of

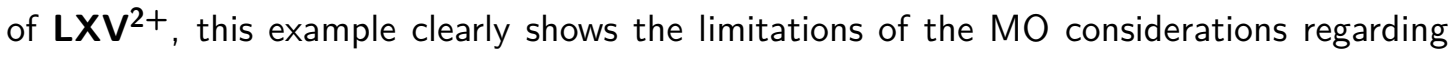
the prerequisites for $\mathrm{N}_{2}$ cleavage made in Section 3.2.1. The low stability of late transition metal nitrides can render splitting unfeasible, even though the formally required electronic configuration is provided. Similar behavior is also observed for the Rh congener, however
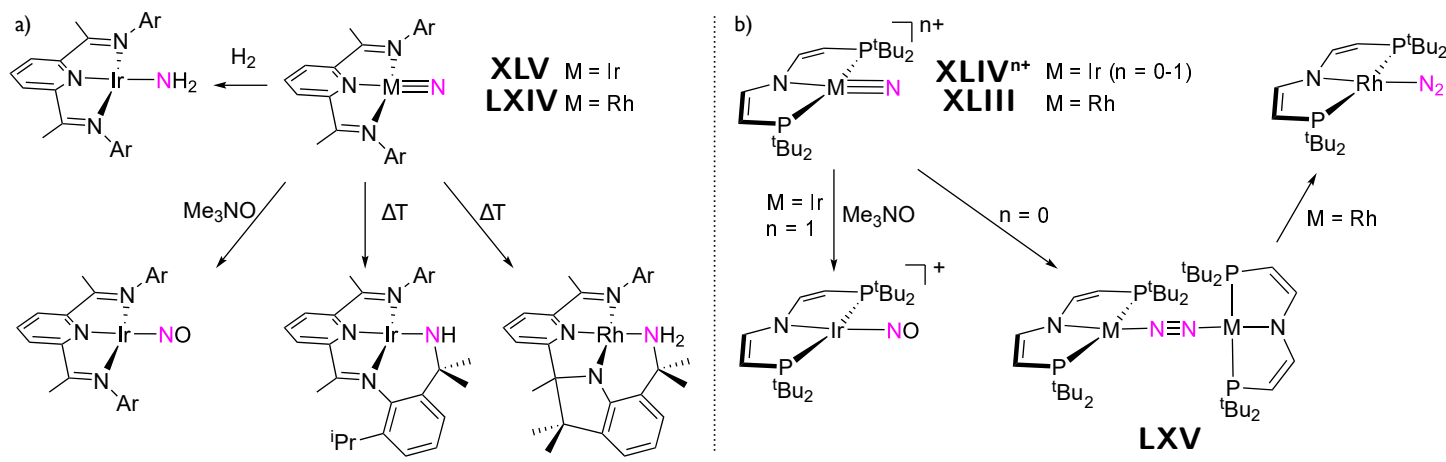

Scheme 4.9. Electrophilic group 9 transition metal nitrido complexes reported by Burger (a) and Schneider (b). 
here no nitride could be isolated and the coupled dimer even reacts further with atmospheric $\mathrm{N}_{2}$ (from azide decomposition) to form $\left[\mathrm{Rh}\left(\mathrm{N}_{2}\right)\left(\mathrm{P}=\mathrm{N}=\mathrm{P}^{t \mathrm{Bu}}\right)\right] .{ }^{[141]}$ 


\section{Scope of this work}

Terminal nitrides have been shown to undergo various nitrogen functionalization reactions, leading to a large zoo of available N-E bond formation schemes as well as the possibility to incorporate metal bound nitrides into organic molecules. Especially low-valent nitrides of late transition metal complexes were proven to exhibit remarkable reactivity, often even towards comparably unactivated substrates.

With the advent of full dinitrogen bond cleavage by transition metal complexes into terminal nitrides, these reactions become a promising toolbox to directly utilize $\mathrm{N}_{2}$ for $\mathrm{N}$ functionalization of relevant molecules, ultimately omitting the generation of ammonia in the Haber-Bosch process. However, the currently available systems suffer from the formation of extremely strong $\mathrm{M} \equiv \mathrm{N}$ bonds, hampering $\mathrm{N}$-centered reactivity.

This thesis falls within the intersection of both areas of research:

- Supported by a PNP pincer ligand, the previously synthesized, unprecedented squareplanar Os(IV) nitrido complex $\left[\mathrm{Os}(\mathrm{N})\left(\mathrm{PNP}^{t \mathrm{Bu}}\right)\right](3)$ is investigated in terms of reactivity. As group 8 transition metal nitrides have previously been reported to exhibit reactivity just on the borderline between electrophilicity and nucleophilicity, distinguishing these two principal reactivities will be emphasized.

- Due to their increased reactivity with respect to early transition metal nitrides, $\mathrm{O} s \equiv \mathrm{N}$ compounds derived from $\mathrm{N}_{2}$ are promising yet elusive candidates for dinitrogen functionalization. By molecular orbital considerations, $d^{2}$ and $d^{4}$ compounds can be imagined as possible splitting products and thus $\mathrm{Os}(\mathrm{VI})$ nitrido complexes related to 3 will be prepared. The prospects of accessing these target molecules by $\mathrm{N}_{2}$ cleavage will be evaluated. Especially, reaction conditions aiming for a formal $10 \pi$ electron count in the $\{M N N M\}$ core of a potential dimeric intermediate will be tested.

- The previously reported dinitrogen splitting by $\operatorname{Re}$ complex $\left[\operatorname{ReCl}_{2}\left(\mathrm{PNP}^{t \mathrm{Bu}}\right)\right](\mathbf{X X I})$ will be explored further in terms of the actual splitting reaction as well follow-up functionalization of the obtained nitride. In particular, observing and identifying potential intermediates of the $\mathbf{N}_{\mathbf{2}}$ splitting reaction of the original system will be attempted in order to gain further insight into the process and to provide experimental evidence for the theoretical predictions made in the original paper about the nature of the intermediary $\mu-\mathrm{N}_{2}$ bridged dimer. 
- In light of the oxidative conditions needed for acetonitrile release in this Re system, the pincer ligand will be modified to meet these conditions. Established procedures for ligand backbone oxidation to the vinylamido based $\mathbf{P}=\mathbf{N}=\mathbf{P}$ pincer will be employed and the resulting complex will be tested towards $\mathrm{N}_{2}$ activation and functionalization.

- The prevalent problem of nitride overstabilization after $\mathrm{N}_{2}$ splitting will be addressed. Theoretical results indicate reduction of the steric bulk of the pincer ligand to be a promising approach to obtaining a more reactive nitride. Therefore, a $\operatorname{Re}($ III) platform with the iso-propyl substituted PNP pincer ligand will be synthesized from which the ability of this complex in $\mathbf{N}_{\mathbf{2}}$ coordination, activation and functionalization will be investigated in an attempt to optimize the previously developed synthetic cycle and approaching real, catalytic turnover. 


\section{Part II}

Results and Discussion

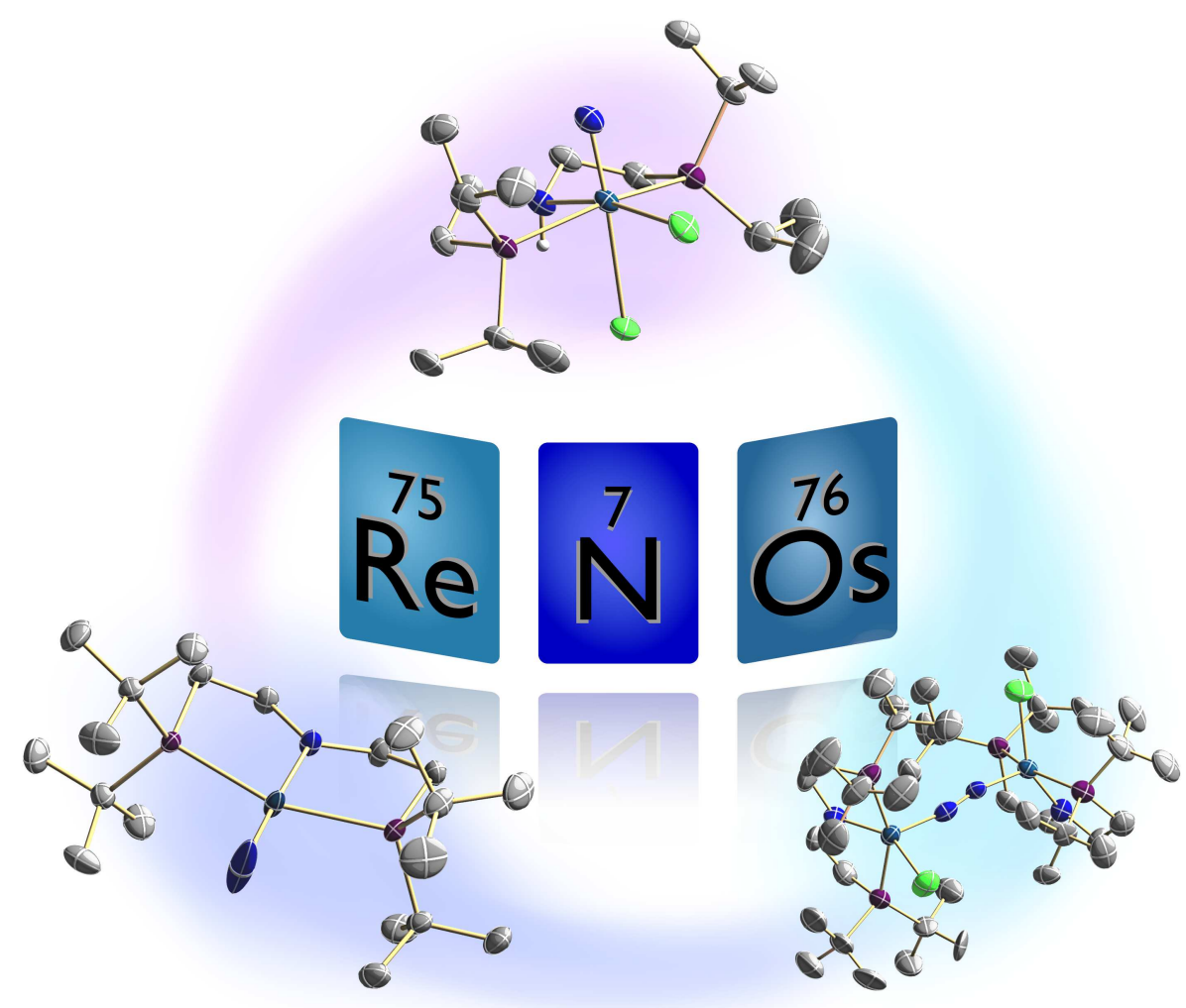





\title{
Low valent osmium nitride complexes with a PNP pincer ligand
}

You build me up, you break me down

Until I'm falling to pieces.

The price I pay to live this way,

And the fantasy stays alive.

- Dream Theater

"Build Me Up, Break Me Down" on "A Dramatic

Turn of Events"

\author{
Parts of this chapter (especially Section 1.1 as well as 1.2) have been published in: F. Schendzielorz, M. \\ Finger, C. Volkmann, C. Würtele, S. Schneider, "A Terminal Osmium(IV) Nitride: Ammonia Formation and \\ Ambiphilic Reactivity", Angew. Chem. Int. Ed. 2016, 55, 11417-11420.
}

The successful synthesis of low-valent nitride complexes of late transition metals and their interesting and sometimes unprecedented reactivity motivated the attempt to get access to the first osmium complex in this group (compare Part I Section 4.2.2).

The synthesis and characterization of the first square-planar Os(IV) nitrido complex [OsN $\left.\left(\mathrm{PNP}^{\mathrm{tBu}}\right)\right]$ (3) was already established in the master's thesis of Florian Schendzielorz and is summarized and extended in Section 1.1, including a synthetic route to the analytically pure complex as well as improved understanding of the involved species.

The unexplored reactivity of this unique compound is investigated in Section 1.2, with the main emphasis on the distinction of $\mathbf{3}$ being rather nucleophilic or electrophilic. Nitride centered nucleophilic reactivity towards classic Lewis acids as well as a reaction with ambiphilic $\mathrm{PMe}_{3}$ are found and theoretical investigation of the latter lead to the description of $\mathbf{3}$ as an ambiphile. Additionally, it is shown that hydrogenolysis leads to full cleavage of the $\mathrm{Os} \equiv \mathrm{N}$ bond and concomitant formation of ammonia.

An exploration of the redox chemistry of $\mathbf{3}$ and related compounds is presented in Section 1.3. Attempts to access a hypothetical Os(v) nitride by oxidation of the Os(IV) complex or by reduction of corresponding $\mathrm{Os}(\mathrm{VI})$ nitrides were not successful, pointing to an inherent instability of the target molecule. 
Finally, Section 1.4 summarizes the largely unsuccessful efforts to bind, activate or even split dinitrogen in the coordination sphere of the previously developed platform. Two stable Os(II) complexes evolve from this investigation, one of which at least bears an $\mathrm{N}_{2}$ ligand.

\subsection{A square planar osmium(Iv) nitride complex}

Large parts of the synthesis and characterization of the square planar osmium(IV) nitrido complex 3 have already been reported in the master's thesis of Florian Schendzielorz and will therefore be described only briefly. ${ }^{[174]}$ The synthesis starts with initial formation of an unidentified complex from HPNP ${ }^{t B u}$ and $\mathrm{OsCl}_{2}\left(\mathrm{PPh}_{3}\right)_{3}$, which converts over time to the $\mathrm{Os}(\mathrm{IV})$ complex $\left[\mathrm{OsHCl}_{2}\left(\mathrm{PNP}^{t \mathrm{tBu}}\right)\right]$ (1) by net oxidative addition of the pincer $\mathrm{NH}$ moiety to the Os center. Reaction of $\mathbf{1}$ with $\mathrm{NaN}_{3}$ leads to formation of an intermediate hydride complex, which was proposed to be $\left[\mathrm{OsHCIN}\left(\mathrm{PNP}^{t \mathrm{Bu}}\right)\right]\left(\boldsymbol{2}_{\text {old }}\right)$ in the thesis. In situ deprotonation with potassium tert-butoxide $\left(\mathrm{KO}^{t} \mathrm{Bu}\right)$ yields the square planar $\mathrm{Os}(\mathrm{IV})$ complex $\left[\mathrm{Os}(\mathrm{N})\left(\mathrm{PNP}^{\mathrm{tBu}}\right)\right](3)$ (see Scheme 1.1).

Subsequent investigations revealed the proposal of $\boldsymbol{2}_{\text {old }}$ being an uncharged complex to be rather unlikely. Based on DFT calculations, it was assumed that after chloride dissociation the coordination site trans to the hydride is vacant and consequently the nitride also resides in this position in $\mathbf{2}_{\text {old }}$. However, performing the synthesis of $\mathbf{2}$ in the presence of $\mathrm{KPF}_{6}$ gave identical NMR signatures, even though $\mathrm{PF}_{6}^{-}$clearly served as a counteranion according to the NMR data. Hence, the complex is most likely cationic, i.e. $\left[\mathrm{Os}(\mathrm{N})(\mathrm{H})\left(\mathrm{PNP}^{t \mathrm{Bu}}\right)\right]^{\mathrm{Cl}}$ $\left(2^{\mathrm{Cl}}\right)$ or $\left[\mathrm{Os}(\mathrm{H})(\mathrm{N})\left(\mathrm{PNP}^{t \mathrm{Bu}}\right)\right]^{\mathrm{PF} 6}\left(2^{\mathrm{PF}_{6}}\right)$, depending on whether $\mathrm{KPF}_{6}$ is added during the synthesis or not. The use of the $\mathrm{PF}_{6}^{-}$salt also allowed for isolation of analytically pure $\mathbf{2}^{\mathrm{PF}_{6}}$, as well as the synthesis of analytically pure 3 by deprotonation with $\mathrm{KO}^{t} \mathrm{Bu}$, both of which was not possible previously due to partial complex decomposition. Complex $\mathbf{2}^{+}$features a well isolated $\mathrm{Os}-\mathrm{H}$ stretching vibration in the IR spectrum at $\tilde{\nu}_{\nu(\mathrm{Os}-\mathrm{H})}=2056 \mathrm{~cm}^{-1}$, which is significantly shifted to lower wavenumbers compared to $1\left(1: \tilde{\nu}_{\nu(\mathrm{Os}-\mathrm{H})}=2216 \mathrm{~cm}^{-1}\right)$. Unfortunately, no single crystals suitable for $\mathrm{X}$-ray diffraction measurements could be obtained.

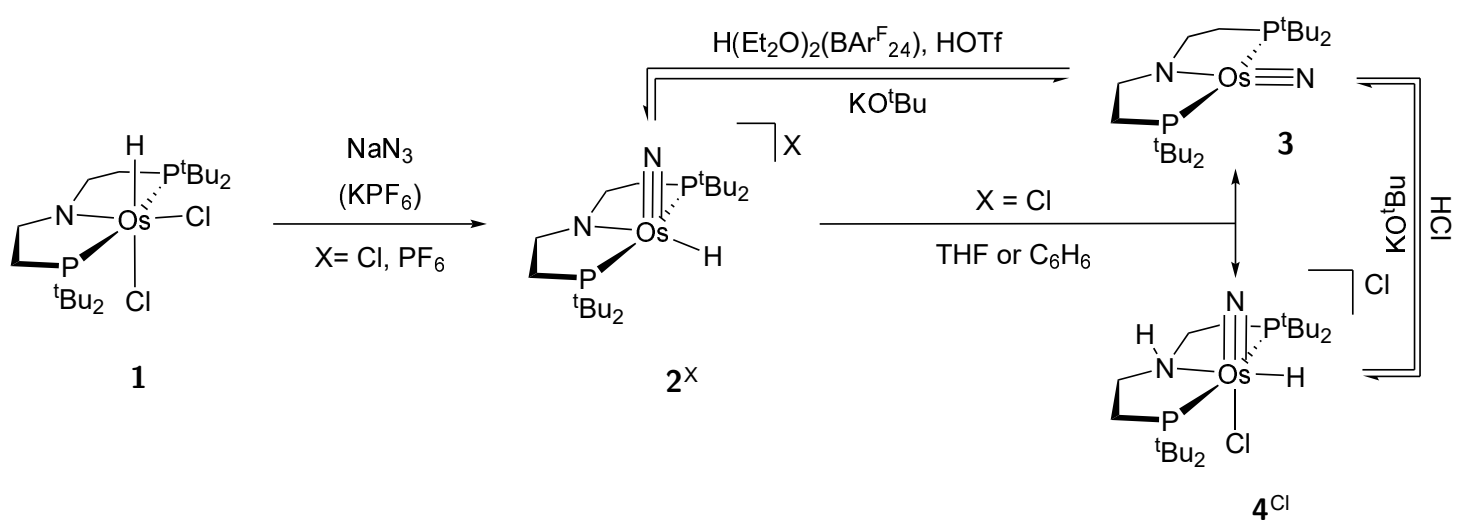

Scheme 1.1. Revised scheme for the synthesis of 3 . If $\mathrm{KPF}_{6}$ is added to the reaction of $\mathbf{1}$ with $\mathrm{NaN}_{3}, 2^{\mathrm{PF}_{6}}$ is formed, otherwise $2^{\mathrm{Cl}}$ is obtained. 


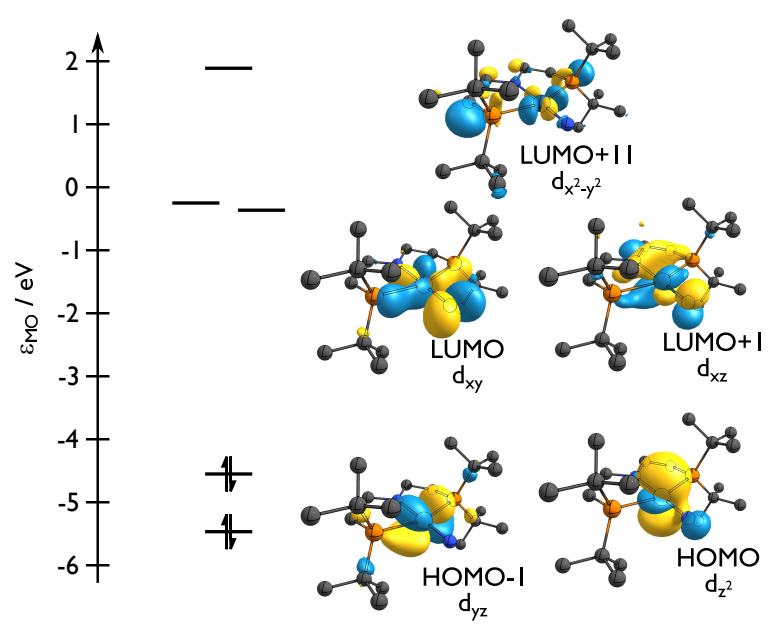

Fig. 1.1. FMO scheme of 3 reproducing the qualitative scheme in Part I Figure 4.1.

It is noteworthy that $\mathbf{2}^{+}$is the first reported osmium complex bearing a hydride as well as a nitride at the same metal center. Such a species might be of interest as a possible intermediate in the hydrogenolysis under basic conditions of an $\mathrm{Os}(\mathrm{VI})$ nitrido complex ( $\mathrm{HBPB}) \mathrm{Os}(\mathrm{N}) \mathrm{Cl}_{2}\left(\mathrm{HBPB}^{-}=2,6\right.$-bis(benzimidazolyl)pyridine) with $\mathrm{H}_{2}$ in presence of $\mathrm{KOH}_{\mathrm{aq}}$ reported by Hashiguchi and coworkers. ${ }^{[175]}$

Additionally, the instability of $2^{\mathrm{Cl}}$ and the product(s) formed from it have to be revised. While a dichloromethane (DCM) solution of $2^{\mathrm{Cl}}$ was stable for at least some days, the observation was made that in benzene or tetrahydrofurane (THF), spontaneous decomposition into the square planar nitride $\mathbf{3}$ and one additional species $\mathbf{4}$ takes place, of which the latter can be converted into $\mathbf{3}$ by addition of base. This process (i.e. $\mathbf{2}^{\mathrm{Cl}} \rightarrow \mathbf{3}+\mathbf{4}$ ) can also be induced in DCM by irradiation with a $\mathrm{Hg}(\mathrm{Xe})$ arc lamp equipped with a long wave pass filter for $\lambda>305 \mathrm{~nm}$ and the formation of $\mathbf{4}$ was assigned to an isomerization of $\boldsymbol{2}_{\text {old }}$ in the original thesis. However, the suggested structure of that isomer was just as unlikely as the initial proposal for $\mathbf{2}$ itself. Instead, it was found that the full reprotonation of $\mathbf{3}$ with $\mathrm{HCl}$ in THF leads to formation of 4 and requires addition of 2 equivalents (eqs) for full conversion, pointing to the reaction of 2 being a dissociation of $2^{\mathrm{Cl}}$ rather than an isomerization. ${ }^{1} \mathrm{H}$ NMR data revealed signals corresponding to an $\mathrm{NH}$ proton $\left(\delta_{1_{\mathrm{H}}}=6.35 \mathrm{ppm}\right)$ as well as an osmium hydride $\left(\delta_{1_{\mathrm{H}}}=-2.52 \mathrm{ppm}\right)$. These two signals, the formation by disproportionation of $2^{\mathrm{Cl}}$ and the low solubility of the compound it benzene or even THF all point towards it being $\left[\mathrm{OsH}(\mathrm{N}) \mathrm{Cl}\left(\mathrm{HPNP}^{t \mathrm{Bu}}\right)\right]^{\mathrm{Cl}}\left(4^{\mathrm{Cl}}\right)$.

If acids with non-coordinating anions like $\mathrm{H}\left(\mathrm{Et}_{2} \mathrm{O}\right)_{2}\left(\mathrm{BAr}_{24}^{\mathrm{F}}\right)\left(\mathrm{BAr}_{24}^{\mathrm{F}}=\right.$ tetrakis $\{3,5-$ (trifluoromethyl)phenyl\}borate) or HOTf are used for protonation of $3,2^{\mathbf{X}}\left(\mathrm{X}=\mathrm{BAr}_{24}^{\mathrm{F}}, \mathrm{OTf}\right)$ is reobtained. This is in contrast to the protonation of the related ruthenium complex $\left[\mathrm{Ru}(\mathrm{N})\left(\mathrm{PNP}_{\mathrm{Si}}^{t \mathrm{Bu}}\right)\right]$ (see Part I Section 4.2.2) ${ }^{[138]}$ which is protonated at the pincer amide and thus reflects the higher metal basicity of osmium versus (vs.) ruthenium. This matches the proposed FMO scheme derived from the Gray-Ballhausen scheme (see Part I Section 4.1), which predicts the $d_{z^{2}}$ orbital to be the HOMO and thus the most basic side in the 
complex. Indeed, inspecting the FMOs of a DFT derived model, ${ }^{1}$ the expected $d$-orbital splitting is reproduced accordingly (see Figure 1.1).

\subsection{Functionalization of square planar osmium(Iv)}

\section{nitride 3}

$\left[\mathrm{Os}(\mathrm{N})\left(\mathrm{PNP}^{t \mathrm{Bu}}\right)\right](3)$ represents the first reported square planar Os(IV) nitride complex and the second Os(IV) nitride at all. The only other example published by Caulton and coworkers is tetrahedrally coordinated $\left[\mathrm{Os}(\mathrm{N}) \mathrm{I}\left(\mathrm{PNP}_{\mathrm{Si}}^{t \mathrm{Bu}}\right)\right]($ LXIII, see Part I Section 4.2.2) was obtained rather coincidentally and no further studies of this product were performed. Consequently, the general reactivity of complex $\mathbf{L X I I I}$ and especially its behavior towards nucleophilic and electrophilic reagents was of fundamental interest.

Protonation of the metal to form $2^{+}$already identified the most nucleophilic side of the complex (see Section 1.1). When the steric bulk of the used Lewis acid is increased, the steric bulk of the pincer ligand prevents reactions at the metal center and the nitride becomes the reactive side as examplified by the reaction with tris- $\{3,5-($ trifluoromethyl)phenyl $\}$ borane $\left(\mathrm{BAr}_{18}^{\mathrm{F}}\right.$ ) or $\mathrm{Me}_{3} \mathrm{SiBr}$ (see Figure 1.2). Mixing 3 with $\mathrm{BAr}_{18}^{\mathrm{F}}$ led to an immediate color change to yellow and clean conversion to $\left[\mathrm{Os}\left(\mathrm{NBAr}_{18}^{\mathrm{F}}\right)\left(\mathrm{PNP}^{t \mathrm{tBu}}\right)\right](5)$. A single resonance in the ${ }^{31} \mathrm{P}\left\{{ }^{1} \mathrm{H}\right\}$ NMR spectrum at $\delta^{31} \mathrm{P}=98.2 \mathrm{ppm}$ and retained $C_{2 v}$ symmetry according to the ${ }^{1} \mathrm{H}$ NMR spectrum provided good evidence for the successful and clean formation of $\mathbf{5}$. Single crystals grown from benzene allowed for the determination of the molecular structure by $\mathrm{X}$-ray diffraction measurements and confirmed the connectivity as a nitride-borane adduct. The osmium center is coordinated in a square planar geometry $\left(\tau_{4}^{\prime}=0.06\right)^{[169]}$ The $\mathrm{Os} \equiv \mathrm{N}$ bond is significantly elongated with respect to parent $3(\mathbf{5}$ : $d($ Os1-N2) $=1.7257(17) \AA ; 3$ : $d($ Os1-N2) $=1.683(2) \AA)$, while the bond to the ligand amide is shortened (5: $d($ Os1-N1) $=1.9958(18) \AA ; 3: d($ Os1-N1) $=2.0573(17) \AA)$, reflecting the reduced trans influence. The rather linear coordination of the nitride $\left(\measuredangle(\mathrm{Os} 1-\mathrm{N} 2-\mathrm{B} 1)=167.77(15)^{\circ}\right)$ points to a retention of a significant triple bond character of the $\mathrm{Os} \equiv \mathrm{N}$ bond.

Analogously, 3 can also be reacted with $\mathrm{Me}_{3} \mathrm{SiOTf}$ or $\mathrm{Me}_{3} \mathrm{SiBr}$, whereas $\mathrm{SiMe}_{3} \mathrm{Cl}$ did not lead to any reaction. While both former reagents initially gave the same product, slow decomposition was observed over time. However, starting with a solution of 3 and $\mathrm{Na}\left(\mathrm{BAr}_{24}^{\mathrm{F}}\right)$, slow addition of $\mathrm{Me}_{3} \mathrm{SiBr}$ gave clean and persistent formation of analytically pure $\left[\mathrm{Os}\left(\mathrm{NSiMe}_{3}\right)\right.$ $\left.\left(\mathrm{PNP}^{t \mathrm{Bu}}\right)\right]^{\mathrm{BAr}_{24}^{\mathrm{F}}}\left(\mathbf{6}^{\mathrm{BAr}_{24}^{\mathrm{F}}}\right)$. Similar to $5,6^{\mathrm{BAr}_{24}^{\mathrm{F}}}$ exhibits $C_{2 v}$ symmetry on the NMR timescale, as indicated by a single resonance for the tert-butyl groups in the ${ }^{1} \mathrm{H}$ NMR spectrum at $\delta_{{ }_{1}}$ $=1.55 \mathrm{ppm}$ as well as a singlet in ${ }^{31} \mathrm{P}\left\{{ }^{1} \mathrm{H}\right\}$ NMR spectrum at $\delta^{31} \mathrm{P}=106.9 \mathrm{ppm}$. Nitride centered reactivity and a resulting square planar coordination geometry is confirmed by ${ }^{1} \mathrm{H}$ nuclear Overhauser effect spectroscopy (NOESY) giving cross peaks in the resulting spectrum between the $\mathrm{Me}_{3} \mathrm{Si}$ and the tert-butyl groups, as well as by the molecular structure

${ }^{1} \mathrm{PBE} 0 / \mathrm{D} 3 \mathrm{BJ} / \mathrm{RIJCOSX} / \mathrm{def} 2-\mathrm{TZVPP}$ 
a)
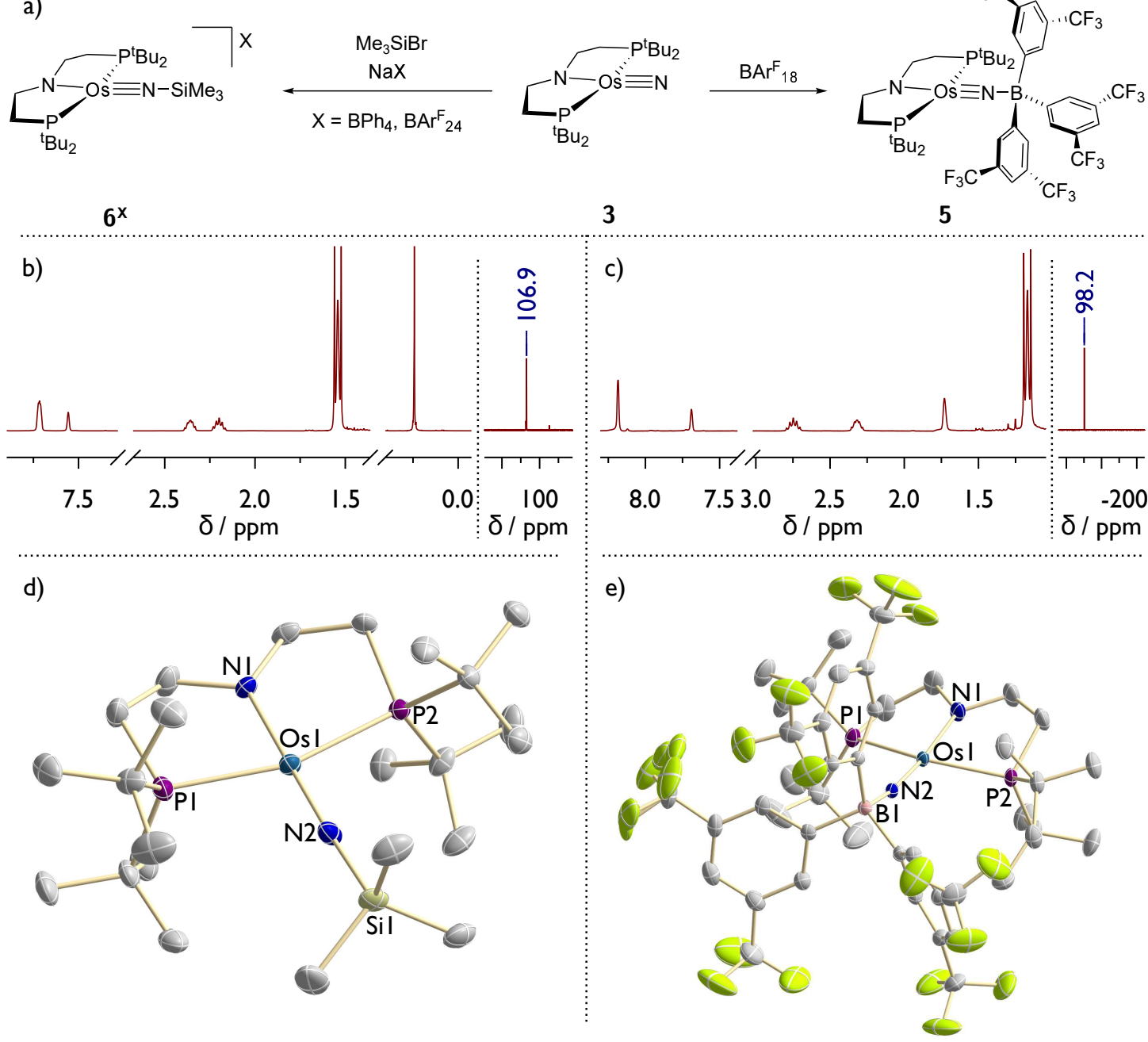

Fig. 1.2. a) Synthesis of $\mathbf{5}$ (right) and $\mathbf{6}^{\mathbf{X}}$ (left) from 3. Middle: ${ }^{1} \mathrm{H}$ (left) and ${ }^{31} \mathrm{P}\left\{{ }^{1} \mathrm{H}\right\}$ (right) NMR spectra of $\mathbf{6}^{\mathrm{BAr}_{24}^{\mathrm{F}}}(b)$ and $\mathbf{5}(c)$. Bottom: Molecular structure of $\mathbf{6}^{\mathrm{BAr}_{24}^{\mathrm{F}}}(d)$ and $\mathbf{5}$ (e) obtained by single crystal X-ray diffraction measurements. All $\mathrm{H}$ atoms, the anion and a cocrystallized DCM molecule in $6^{\mathrm{BAr}_{24}^{\mathrm{F}}}$ are omitted for clarity. Anisotropic displacement

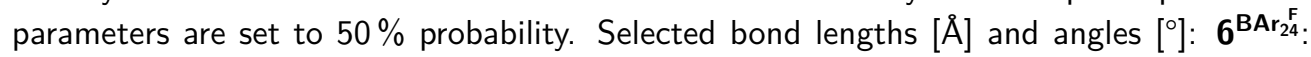
Os1-N1 1.988(3), Os1-N2 1.733(4), N2-Si1 1.797(4), P1-Os1-P2 160.80(4), N1-Os1-N2 177.78(17), Os1-N2-Si1 176.0(3). 5: Os1-N1 1.9958(18), Os1-N2 1.7257(17), N2-B1 1.567(3), P1-Os1-P2 153.67(6), N1-Os1-N2 169.75(8), Os1-N2-B1 167.77(15).

derived by X-ray diffraction measurements on single crystals. The osmium center is coordinated in a square planar coordination environment $\left(\tau_{4}^{\prime}=0.07\right)^{[169]}$ and again the nitride is coordinated almost linearly $\left(\measuredangle(\mathrm{Os} 1-\mathrm{N} 2-\mathrm{Si} 1)=177.78(17)^{\circ}\right)$ and exhibits an elongated Os $\equiv \mathrm{N}$ distance $(d(\mathrm{Os} 1-\mathrm{N} 2)=1.733(4) \AA)$, in line with an activated triple bond.

The only other group of Os(IV) hydrocarbon based imido complexes prepared by Schrock and coworkers exhibit similar structural parameters, i.e. a linear coordiantion of the imido ligand, ${ }^{[176,177]}$ whereas azidoimido and cyanoimido complexes by the group of T. J. Meyer were considerably bend. ${ }^{[178,179]}$

In contrast to this, using intermediate sized electrophiles like methyl triflate or methyl iodide did not give selective reactions, but rather complex mixtures of different compounds, of 
which only $2^{+}$could be identified spectroscopically. However the occurrence of a triplet in the ${ }^{1} \mathrm{H}$ NMR spectrum at $\delta_{1_{\mathrm{H}}}=2.61 \mathrm{ppm}\left({ }^{3} J_{\mathrm{HP}}=4.2 \mathrm{~Hz}\right.$, becomes a singlet in ${ }^{1} \mathrm{H}\left\{{ }^{31} \mathrm{P}\right\}$ NMR) suggests at least partial methylation at the osmium center, as it is reminiscent of e.g. the spectrum of $\left[\operatorname{Re}(\mathrm{N}) \mathrm{Me}\left(\mathrm{PNP}^{t \mathrm{Bu}}\right)\right]$ prepared by Isabel Klopsch. ${ }^{[180]}$ Apparently, the steric shielding of the pincer is insufficient to prevent such reactivity. Further increasing the ligand bulk might improve the selectivity in this respect (e.g. using the adamantyl based pincer).

Also other tested electrophiles like acetyl chloride or trifluoroacetic anhydride led to conversion of 3, even though these reactions were not selective and the products were not identified. No reactions were observed with pure nucleophiles like 1-hexen, 1-hexyne, $\mathrm{MeMgCl}$ or even with bulky ambiphiles like $\mathrm{PPh}_{3}{ }^{2}$ Therefore the principal reactivity of $\mathbf{3}$ must be considered nucleophilic. However, when 3 was reacted with $\mathrm{PMe}_{3}$, slow reaction to a new product 7 was observed. Two singlets in the ${ }^{31} \mathrm{P}\left\{{ }^{1} \mathrm{H}\right\}$ NMR spectrum at $\delta^{31} \mathrm{P}=43.9$ and $22.1 \mathrm{ppm}$ with a 2:1 ratio and retained $C_{2 v}$ symmetry according to the ${ }^{1} \mathrm{H}$ NMR spectrum suggested formation of a phosphoraneiminato complex $\left[\mathrm{Os}\left(\mathrm{NPMe}_{3}\right)\left(\mathrm{PNP}^{t \mathrm{Bu}}\right)\right]$. Spatial proximity between the new $\mathrm{N}=\mathrm{PMe}_{3}$ moiety and the tert-butyl groups of the ligand was confirmed by a ${ }^{1} \mathrm{H}$ NOESY spectrum. This was further substantiated by starting from 3 with $50 \%{ }^{15} \mathrm{~N}$ labeled nitride. In this reaction, the signal intensity of the singlet at $\delta_{31} \mathrm{P}=22.1 \mathrm{ppm}$ dropped by $50 \%$ and a new doublet arose at $\delta^{31} \mathrm{P}=22.1 \mathrm{ppm}$ with a coupling constant of ${ }^{1} J_{\mathrm{P}^{15} \mathrm{~N}}$ $=22.7 \mathrm{~Hz}$. Additionally, a new doublet was detected in the ${ }^{15} \mathrm{~N}\left\{{ }^{1} \mathrm{H}\right\} \mathrm{NMR}$ spectrum at $\delta_{15} \mathrm{~N}=-126.5 \mathrm{ppm}$, exhibiting an identical coupling constant. The reaction did not go to completion as indicated by NMR spectroscopy which showed the formation of $\mathbf{7}$ to plateau at an equilibrium concentration with an estimated equilibrium constant of $K_{c}=150 \mathrm{M}^{-1}$ $\left(\Delta G^{0}=-12.4 \mathrm{~kJ} \mathrm{~mol}^{-1}\right)$. Indeed, removal of all volatiles and subsequent redissolving of the obtained complex led to reformation of 3 and liberation of free $\mathrm{PMe}_{3}$, proving the reaction to be fully reversible. This reverse reaction represents a rather unique way of nitride formation, exemplified only once by the group of $A b r a m$ reporting the reaction of $\left[\mathrm{Tc}(\mathrm{O}) \mathrm{Cl}_{4}\right]^{-}$with $\mathrm{Me}_{3} \mathrm{SiNPPh}_{3}$ to form $\left[\mathrm{Tc}(\mathrm{N}) \mathrm{Cl}_{2}\left(\mathrm{PPh}_{3} \mathrm{NH}\right)_{2}\right] .{ }^{[181]}$ Attempts to drive the reaction to completion by addition of a large excess of the phosphine resulted in partial decomposition of the complexes. Consequently, 7 could not be isolated as a pure compound. Nevertheless, single crystals obtained from the reaction mixture allowed determination of the molecular structure by $\mathrm{X}$-ray diffraction measurements and thus for the unequivocal confirmation of the structural assignment made above. The osmium center is still coordinated in a square planar coordination environment $\left(\tau_{4}^{\prime}=0.02\right) .{ }^{[169]}$ In contrast to $\mathbf{5}$ and $\mathbf{6}^{\mathbf{B A r}_{24}^{\mathrm{F}}}$, the osmium-nitride bond is significantly elongated $(d(\mathrm{Os} 1-\mathrm{N} 2)=1.968(2) \AA)$. In combination with the short $\mathrm{N}=\mathrm{P}$ bond length of the newly formed ligand $(d(\mathrm{~N} 2-\mathrm{P} 3)=1.532(2) \AA)$ in line with a double bond, this confirms formation of a phosphoraneiminato ligand.

While $\mathrm{PMe}_{3}$ is often described as a nucleophile in literature, Smith and coworkers showed by Hammett analysis of the adduct formation of a tetrahedral tris(carbene)borate $\mathrm{Fe}(\mathrm{IV}$ ) nitride with triarylphosphines that these can also also act as electron acceptors by backbonding into

\footnotetext{
${ }^{2}$ For a discussion of the ambiphilicity of phosphines see below as well as Part I Section 4.2.2.
} 
a)

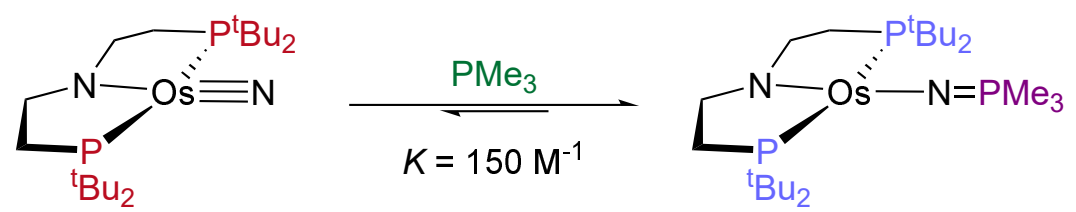

3 7
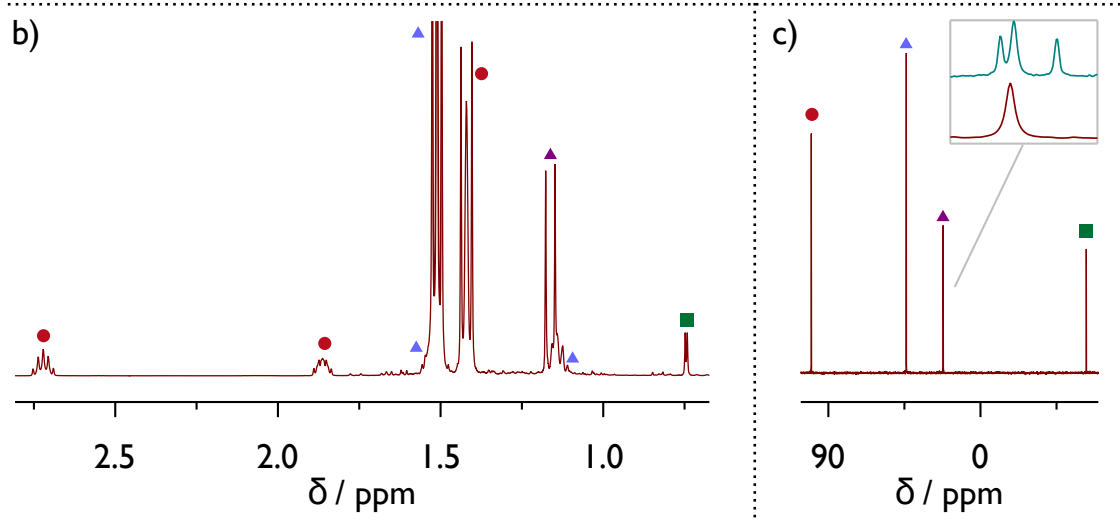

d)

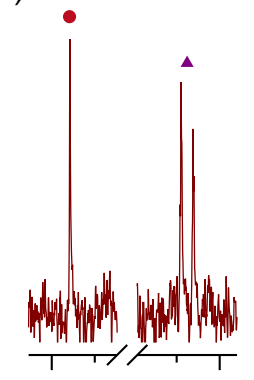

392 $\delta / \mathrm{ppm}$

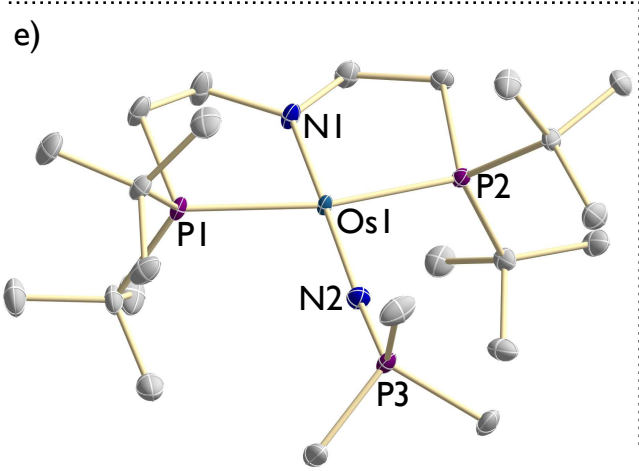

f)

(1)

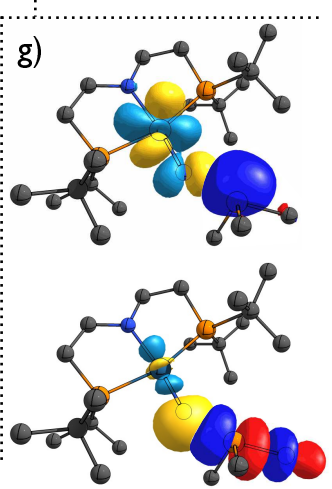

Fig. 1.3. a) Equilibrium reaction between $3+\mathrm{PMe}_{3}$ and 7. Middle: $\mathrm{NMR}$ spectra of the equilibrium mixture with color coded compounds: 3 (red circle), free $\mathrm{PMe}_{3}$ (green square) and $\mathbf{7}$ with independently marked PNP pincer (violet triangle) and phoshphoraneiminato ligand (pink triangle). Shown are $b$ ) the $\left.{ }^{1} \mathrm{H}, c\right)$ the ${ }^{31} \mathrm{P}\left\{{ }^{1} \mathrm{H}\right\}$ (with comparison of the phosphoraneiminato signal resulting from $\left[\mathrm{Os}\left({ }^{14} \mathrm{~N}\right)\left(\mathrm{PNP}^{t \mathrm{Bu}}\right)\right]$ (bottom) and $\left[\mathrm{Os}\left({ }^{14 / 15} \mathrm{~N}\right)\left(\mathrm{PNP}^{t \mathrm{Bu}}\right)\right]($ top $)$ in the inset) and $d$ ) the ${ }^{15} \mathrm{~N}\left\{{ }^{1} \mathrm{H}\right\}$ NMR spectra from the reaction with $\left[\mathrm{Os}\left({ }^{14 / 15} \mathrm{~N}\right)\left(\mathrm{PNP} \mathrm{Pu}^{t \mathrm{Bu}}\right)\right]$. e) Molecular structure of $\mathbf{7}$ obtained by single crystal X-ray diffraction measurements. All $\mathrm{H}$ atoms are omitted for clarity. Anisotropic displacement parameters are set to $50 \%$ probability. Selected bond lengths $[\AA]$ and angles $\left[^{\circ}\right]$ : Os1-N1 1.920(2), Os1-N2 1.968(2), N2-P3 1.532(2), P1-Os1-P2 167.09(2), N1-Os1-N2 178.63(11), Os1-N2-P3 173.57(17). $f)$ DFT derived geometry of the transition state for the reaction of 3 with $\mathrm{PMe}_{3}$. All $\mathrm{H}$ atoms are omitted for clarity. Selected bond lengths $[\AA]$ and angles $\left[{ }^{\circ}\right]$ : Os1-N2 1.755, N2-P3 2.104, P3-C21 1.914, P3-C22 1.852, P3-C33 1.853, Os1-N2-P3 146.92, N2-P3-C21 164.66, N2-P3-C22 92.47, N2-P3-C23 92.65. g) Donor-acceptor interactions dominating the bond formation between the phosphine and the nitride according to a natural bonding orbital (NBO) analysis. Top: Dominant donation for the phosphine lone pair into a Os $\pi^{*} \mathrm{~N}$ MO. Bottom: Backdonation of the nitride centered lone pair into a $\mathrm{P}^{\sigma^{*}} \mathrm{C} \mathrm{MO}$.

$\mathrm{P}^{\sigma^{*}} \mathrm{C}$ MOs (see Part I Section 4.2.2). [159] This raised the question of the nature of the reaction between 3 and $\mathrm{PMe}_{3}$. Since $\mathrm{PPh}_{3}$ was unreactive, a Hammett plot was impossible in the present case. Therefore, a computational analysis of the reaction was performed to shed some light on it (see Part III Section 3.3 for details). The almost thermoneutral reaction was confirmed $\left(\Delta G^{0}=-13.2 \mathrm{~kJ} \mathrm{~mol}^{-1}\right)$ and a rather high activation barrier was found $\left(\Delta G^{\ddagger}\right.$ 
$\left.=60.2 \mathrm{~kJ} \mathrm{~mol}^{-1}\right)$, which is in agreement with the long timescale of reaction equilibration. Closer analysis of the transition state revealed an interesting picture reminiscent of the findings by Smith. The Os-N...P angle strongly deviates from linearity $\left(\measuredangle(\right.$ Os1-N2-P3 $\left.)=147^{\circ}\right)$, indicating an nucleophilic attack of the phosphine lone pair at the LUMO of 3. Additionally, the phosphine is strongly tilded with one N...P-C angle approaching linearity ( $\angle$ (N2-P3-C21) $\left.=164.7^{\circ}\right)$ resulting in a disphenoidal phosphine coordination. The corresponding $\mathrm{P}-\mathrm{C}$ bond is elongated $(d(\mathrm{P} 3-\mathrm{C} 21)=1.914 \AA, d(\mathrm{P} 3-\mathrm{C} 22)=1.852 \AA, d(\mathrm{P} 3-\mathrm{C} 23)=1.853 \AA)$, as a result from backdonation of the nitride lone pair into the $\mathrm{P}^{\sigma^{*}} \mathrm{C} \mathrm{MO}$. Both donor-acceptor interactions could also be identified in an NBO analysis, rendering a clear identification of an electrophilic vs. nucleophilic nature of the reaction non-trivial. Comparison of the natural population analysis (NPA) of both the reactants as well as the transition state revealed a net charge transfer of $0.3 \mathrm{e}^{-}$from the phosphine to the nitride, making the electrophilic interaction of the nitride the dominant contribution to the reaction. Hence, $\mathbf{3}$ is not only a nucleophile but can exhibit electrophilic reactivity under certain circumstances, rendering it an ambiphile.

In addition to this ambiphilicity, 3 could also be shown to cleanly react with $\mathrm{H}_{2}$ gas, when heated to $100^{\circ} \mathrm{C}$ over several hours. As the immediate reaction product, [OsH $\left.{ }_{4}\left(\mathrm{HPNP}^{t \mathrm{Bu}}\right)\right]$ (8) could be identified and isolated in $74 \%$ yield (see Figure 1.4). A broad singlet in the ${ }^{1} \mathrm{H}$ NMR spectrum at $\delta_{1_{\mathrm{H}}}=4.88 \mathrm{ppm}$ as well as three broad signals between $\delta_{1_{\mathrm{H}}}=-6.00$ - $-14.33 \mathrm{ppm}$ which split into four distinct and sharp signals at $-50^{\circ} \mathrm{C}$ correspond to the $\mathrm{NH}$ atom and the four hydrides. In the IR spectrum, the $\mathrm{NH}$ as well as the OsH stretching vibrations were found at $\tilde{\nu}=3294.7 \mathrm{~cm}^{-1}\left(\nu_{\mathrm{N}-\mathrm{H}}\right), 2013.6 \mathrm{~cm}^{-1}, 2071.5 \mathrm{~cm}^{-1}, 2044.0 \mathrm{~cm}^{-1}$ and $1852.6 \mathrm{~cm}^{-1}\left(\nu_{\mathrm{Os}-\mathrm{H}}\right)$ (see Figure $1.4 \mathrm{f}$ ). $\mathrm{H}_{2}$ was unambiguously confirmed as source of these $\mathrm{H}$ atoms when the reaction was performed with $\mathrm{D}_{2}$. All corresponding signals vanished from the ${ }^{1} \mathrm{H}$ NMR spectrum, the ND stretching vibration was shifted to $\tilde{\nu}=2455.5 \mathrm{~cm}^{-1}$ and the $\mathrm{OsD}$ stretching vibrations were shifted into the fingerprint region and could therefore not be identified unequivocally. Single crystals suitable for $\mathrm{X}$-ray diffraction measurements gave a molecular structure of $\mathbf{8}$ confirming the NMR and IR based assignments (see Figure 1.4 e). All four $\mathrm{OsH}$ atoms are found from the residual density map and the protonation of the backbone $\mathrm{N}$ is clearly evident by the pyramidal coordination $\left(\Sigma_{\measuredangle}(\mathrm{N} 1)=350.5^{\circ}\right)$. Two long $(d(\mathrm{H} 1-\mathrm{H} 2)=2.11(7) \AA)$ and one short $\left(d\left(\mathrm{H} 2-\mathrm{H} 2{ }^{\prime}\right)=1.70(9) \AA\right)$ intramolecular distances between the $\mathrm{H}$-atoms underpinned the formulation as hydrides with one compressed dihydride moiety. This assignment is also in line with the proposal by Gusev and coworkers on the analogous complex $\left[\mathrm{OsH}_{4}\left(\mathrm{HPNP}^{i \mathrm{Pr}}\right)\right]$ or the related complex $\left[\mathrm{OsH}_{4}\left(\mathrm{PNP}^{t \mathrm{Bu}}\right)\right](\mathrm{PNP}=$ $\left.\kappa^{3}-\mathrm{C}_{5} \mathrm{NH}_{3} 2,6-\left(\mathrm{CH}_{2} \mathrm{PR}_{2}\right)_{2}\right)$ prepared by Goldman and coworkers. ${ }^{[182,183]}$

The fate of the nitride ligand was elucidated by closer analysis of the ${ }^{1} \mathrm{H}$ NMR spectrum of the reaction mixture. A broadened triplet at $\delta_{1_{\mathrm{H}}}=0.33 \mathrm{ppm}\left({ }^{1}{J_{1}}_{\mathrm{H}^{14} \mathrm{~N}}=42.8 \mathrm{~Hz}\right)$ could be identifed which gives a cross peak in the ${ }^{1} \mathrm{H}-{ }^{14} \mathrm{~N}-\mathrm{HMQC}$ spectrum at $\delta_{14} \mathrm{~N}=-388.0 \mathrm{ppm}$, characteristic for ammonia (see Figure $1.4 \mathrm{~d}$ ). If ${ }^{14 / 15} \mathrm{~N}-3$ with $50 \%{ }^{15} \mathrm{~N}$ labeled nitride was used, the ${ }^{1} \mathrm{H}$ NMR resonance became very broad at RT, due to overlapping ${ }^{14} \mathrm{NH}_{3}$ and 
${ }^{15} \mathrm{NH}_{3}$ signals, but gave sharp signals at $-50{ }^{\circ} \mathrm{C}$ with a cleanly identifiable ${ }^{15} \mathrm{~N}-{ }^{1} \mathrm{H}$ doublet $\left({ }^{1} J_{1^{15} \mathrm{~N}}=61.2 \mathrm{~Hz}\right)$, fully in agreement with the expected ratio of $J$-coupling constants $\left(\gamma_{15} \mathrm{~N} / \gamma_{14} \mathrm{~N}=-1.41\right)$ (see Figure $1.4 \mathrm{c}$ ). Indophenolic titration according to a literature procedure allowed for quantification of the formed ammonia to $79.4 \%$, matching the yield of 8 (see also resulting spectra in Part III, Section 1.2, Figure 1.1). [184]

While full hydrogenolysis of a nitride ligand to ammonia is rare, it is not unprecedented. The ruthenium complex analogous to 3, i.e. $\left[R u(N)\left(P_{N P}^{t B u}\right)\right](X L I I)$, prepared by Schneider and coworkers, was also described to undergo full hydrogenolysis to form $\mathrm{NH}_{3}$ (see Part I, Section 4.2.2). ${ }^{[138]}$ Furthermore, a variation of 3 , i.e. the congener with fully oxidized ligand backbone $\left[\mathrm{OsN}\left(\mathrm{P}=\mathrm{N}=\mathrm{P}^{t \mathrm{Bu}}\right)\right]$, was developed by the Schneider group as a follow up of the in here presented results, which also reacts with $\mathrm{H}_{2}$ to give $\mathrm{NH}_{3}$ and 8. ${ }^{[185]}$ Additionally, Hashiguchi

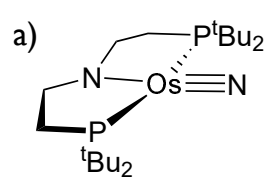

3

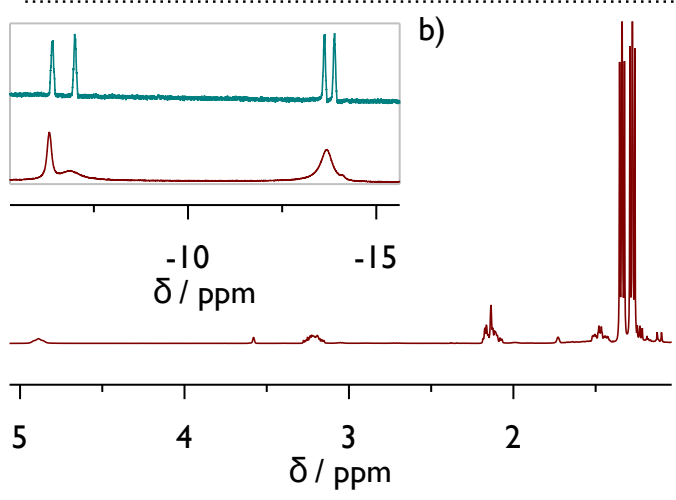

e)

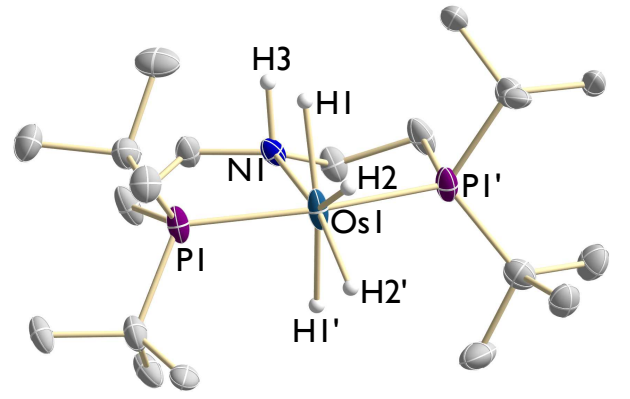

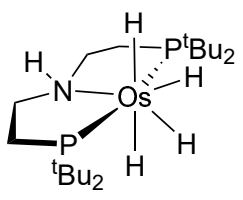

8
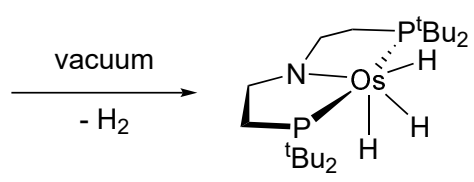

9
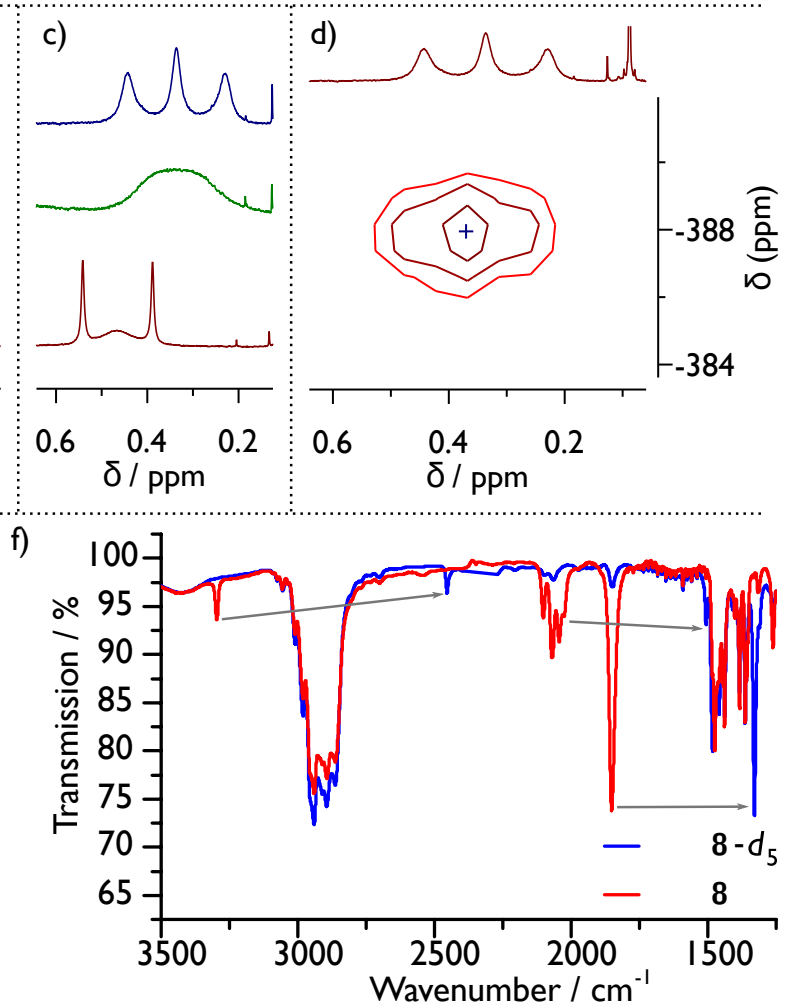

Fig. 1.4. a) Hydrogenation of $\mathbf{3}$ to form ammonia and $\mathbf{8}$. Applying vacuum to a solid sample leads to $\mathrm{H}_{2}$ loss and formation of $\mathbf{9}$. b) ${ }^{1} \mathrm{H}$ NMR spectrum of $\mathbf{8}$. Inset: Hydride region at RT (bottom) and $-50{ }^{\circ} \mathrm{C}$ (top). c) ${ }^{1} \mathrm{H}$ NMR region of the $\mathrm{NH}_{3}$ signal of the reaction mixture (top) and of a reaction starting from ${ }^{14 / 15} \mathrm{~N}-3$ at RT (middle) and at $-50^{\circ} \mathrm{C}$ (bottom). d) ${ }^{1} \mathrm{H}-{ }^{14} \mathrm{~N}$ HMQC NMR of the reaction mixture. e) Molecular structure of $\mathbf{8}$ obtained by single crystal $\mathrm{X}$-ray diffraction measurements. All carbon bound $\mathrm{H}$ atoms are omitted for clarity. Anisotropic displacement parameters are set to $50 \%$ probability. Selected bond lengths $[\AA]$ : Os1-N1 2.210(5), Os1-H1 1.78(5), Os1-H2 1.77(5), H1-H2 2.11(7), H2-H2' $1.70(9)$. $f$ ) IR spectrum of $\mathbf{8}($ red $)$ and $\mathbf{8}-d_{5}$ obtained from the reaction with $\mathrm{D}_{2}$ (blue). 
and coworkers reported increased formation of $\mathrm{NH}_{3}$ upon base accelerated hydrolysis of an Os(VI) nitride when the Ar atmosphere was replaced with $\mathrm{H}_{2} \cdot{ }^{[175]}$ The actual mechanism of the hydrogenation of $\mathbf{3}$ is unknown, but can be expected to be quite comparable to XLII, for which a DFT derived mechanism is available. This indicated the presence of potentially highly reactive intermediates like e.g. $\left[\mathrm{Ru}(\mathrm{NH})\left(\mathrm{HPNP}^{t \mathrm{Bu}}\right)\right]$, the product of the first metalligand cooperative $\mathrm{H}_{2}$ addition. In a first attempt to trap some intermediates, the reaction was performed in the presence of 10 eq styrene. However, rather than projected aziridination or amination of the olefin, simple and quantitative hydrogenation to ethylbenzene was observed. This could indicate either $\mathbf{3}$ or $\mathbf{8}$ to be quite potent hydrogenation catalysts. In fact, the iso-propyl substituted analog to $\mathbf{8},\left[\mathrm{OsH}_{4}\left(\mathrm{HPNP}^{i \mathrm{Pr}}\right)\right]$, was reported to be an efficient catalyst for transfer hydrogenation and dehydrogenative coupling of alcohols. ${ }^{[182]}$

Finally, 8 could be shown to undergo reversible $\mathrm{H}_{2}$ loss by applying vacuum to the solid sample, just as it was reported for $\left[\mathrm{Ru}(\mathrm{N})\left(\mathrm{PNP}^{t \mathrm{Bu}}\right)\right] \cdot{ }^{[138]}$ While the product was not fully characterized, the ${ }^{1} \mathrm{H}$ NMR spectrum pointing to a $C_{2 v}$ symmetry and the appearance of a single hydride peak integrating to three protons relative to the tert-butyl groups at $\delta_{1_{\mathrm{H}}}=$ $-13.64 \mathrm{ppm}$ is fully inline with loss of one $\mathrm{H}_{2}$ molecule and formation of [Os $\left.\mathrm{O}_{3}\left(\mathrm{PNP}^{t \mathrm{tBu}}\right)\right]$ (9), analog to the reported Ru compound and Gusevs iso-propyl based complex.

\subsection{Redox chemistry of osmium(IV) and osmium(VI) nitrido complexes}

The cyclic voltammetry $(\mathrm{CV})$ data of $\left[\mathrm{Os}(\mathrm{N})\left(\mathrm{PNP}^{t \mathrm{Bu}}\right)\right](3)$, which were previously reported, ${ }^{174]}$ revealed a quasi-reversible oxidation at $E_{1 / 2}=-0.75 \mathrm{~V}$ vs. $\mathrm{Fc} / \mathrm{Fc}^{+}$. Concentration dependent measurements indicated a reduced reversibility at higher concentrations of $\mathbf{3}$, especially at low scan rates, suggesting a bimolecular follow-up reaction. This could indicate a potential coupling of the cationic nitride complexes to form a dimer, in line with previous reports by Taube and Meyer. ${ }^{[186-188]}$ Considering the isolobal relationship between squareplanar $d^{4}$ and octahedral $d^{2}$ nitrido complexes, such a coupling would be analogous to the observation by Nishibayashi of his complex $\left[\mathrm{Mo}(\mathrm{N})\left(C \mathrm{p}^{*}\right)(\mathrm{depf})\right](\mathbf{X X X I})$ coupling to the dimer $\left[\left(\mu-\mathrm{N}_{2}\right)\left\{\mathrm{Mo}\left(\mathrm{Cp}^{*}\right)(\mathrm{depf})\right\}_{2}\right](\mathbf{X X X})$ upon one-electron oxidation (see Part I, Section 3.2.3). ${ }^{[114]}$ Furthermore, $\mathrm{N} \cdots \mathrm{N}$ coupling reactions from $\mathrm{Os}(\mathrm{V})$ nitrido complexes are well known in the literature. ${ }^{[42,189,190]}$ Consequently, the chemical oxidation of $\mathbf{3}$ was investigated to elucidate potential formation of a dimeric compounds.

Initial attempts using $\left[\mathrm{Fe}(\mathrm{Cp})_{2}\right] \mathrm{PF}_{6}$ resulted in the formation of little $\left[\mathrm{Os}(\mathrm{N}) \mathrm{H}\left(\mathrm{PNP}^{t \mathrm{Bu}}\right)\right]^{+}$ $\left(2^{+}\right)$, along the formation of a doublet in ${ }^{31} \mathrm{P}\left\{{ }^{1} \mathrm{H}\right\}$ NMR spectroscopy at $\delta_{31} \mathrm{P}=96.5 \mathrm{ppm}$ $\left(J_{\mathrm{PF}}=20.5 \mathrm{~Hz}\right)$, tentatively attributed to activation of the $\mathrm{PF}_{6}^{-}$anion. Replacing the anion with $\mathrm{BAr}_{24}^{\mathrm{F}}$ indeed gave no sign of such species. Instead, almost equimolar amounts of hydride species $2^{+}$and an unidentified $C_{S}$ symmetric compound with a resonance singlet at $\delta_{31} \mathrm{P}=98.1 \mathrm{ppm}$ in the ${ }^{31} \mathrm{P}\left\{{ }^{1} \mathrm{H}\right\}$ NMR spectrum were obtained. However, the nature of that 
a)

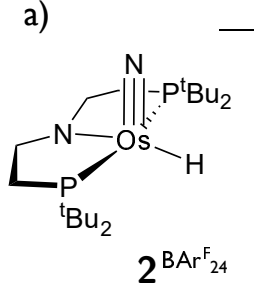

$\mathrm{BAr}_{24}$

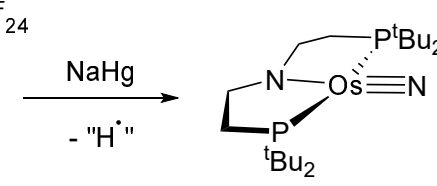

3 b)

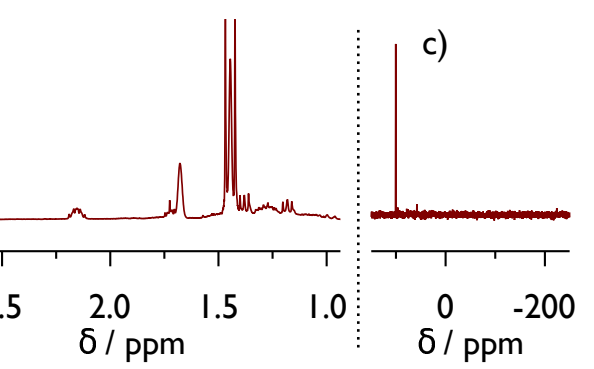

Fig. 1.5. a) Reduction of $2^{\mathrm{BAr}_{24}^{\mathrm{F}}}$ with 1 eq NaHg. b) ${ }^{1} \mathrm{H}$ and c) ${ }^{31} \mathrm{P}\left\{{ }^{1} \mathrm{H}\right\}$ NMR spectra indicate clean formation of 3 .

species currently remains unclear as well. Another attempt using the trityl cation as oxidant resulted in almost quantitative formation of $2^{+}$. While non of these reactions yielded an expected $C_{2 v}$ symmetric complex according to ${ }^{1} \mathrm{H}$ NMR spectroscopy, it is noteworthy that also no sign of any paramagnetic complexes was observed. The initially formed open-shell species can therefore be assumed to be highly reactive. Attempts with mild oxidants and non-coordinating anions, like $\left[\mathrm{Fe}\left(\mathrm{Cp}^{*}\right)_{2}\right]\left(\mathrm{BAr}_{24}^{\mathrm{F}}\right)$ were not performed but might be promising candidates for selective reactions. A second approach aiming for an osmium(v) nitride which could undergo coupling reactions was the one-electron reduction of $2^{\mathrm{BAr}_{24}^{\mathrm{F}}}$. If that osmium(VI) nitride complex was mixed with $1 \mathrm{eq} \mathrm{NaHg}$, surprisingly quantitative and clean formation of $\mathbf{3}$ was observed, suggesting formal $\mathrm{H}$-atom loss from the $\mathrm{Os}(\mathrm{v})$ stage, even though no $\mathrm{H}_{2}$ could be detected in the NMR (see Figure 1.5).

When 3 was reacted with 1.0 eq NCS, which can act as one or two-electron oxidant, a more desired reaction was observed. Mixing the two reactants resulted in immediate and selective formation of a new, diamagnetic, $C_{S}$ symmetric compound, which slowly decomposed at RT over several hours. This suggested a two-electron oxidation and therefore indicated the potential formation of a succinimide anion. Consequently, addition of a proton source after the successful reaction resulted in selective formation of analytically pure Os(VI) nitride $\left[\mathrm{Os}(\mathrm{N}) \mathrm{Cl}\left(\mathrm{PNP}^{t \mathrm{Bu}}\right)\right]^{\mathrm{X}}\left(\mathbf{1 0}^{\mathrm{X}}(\mathrm{X}=\right.$ anion of the used acid $)$. The complex resonates at $\delta_{31} \mathrm{P}=$ $96.2 \mathrm{ppm}$ in the ${ }^{31} \mathrm{P}\left\{{ }^{1} \mathrm{H}\right\}$ NMR spectrum, is $C_{S}$ symmetric on the NMR timescale and bears no other proton containing group but the PNP pincer according to the ${ }^{1} \mathrm{H}$ NMR spectrum. It is interesting to note that the recorded spectra are slightly different when $\mathrm{HCl}$ was used as acid, instead of those with non-coordinating anions, suggesting the formation of an octahedrally coordinated nitride $\left[\mathrm{Os}(\mathrm{N}) \mathrm{Cl}_{2}\left(\mathrm{PNP}^{t \mathrm{Bu}}\right)\right](\mathbf{1 0}-\mathrm{Cl})$ (see Figure 1.6).

Since $10^{+}$does not bear a hydride ligand anymore, reduction of this complex can not lead to $\mathrm{H}^{*}$ loss and might therefore be more suitable to initiate a potential coupling reaction. However, the reaction of $\mathbf{1 0}^{\mathrm{BAr}}{ }_{24}^{\mathrm{F}}$ with $\mathrm{Co}\left(\mathrm{Cp}^{*}\right)_{2}$ at $\mathrm{RT}$ leads now to the formation of equimolar amounts of $\mathbf{3}$ and $\mathbf{1 0}-\mathbf{C l}$, which is the result of a formal disproportionation reaction of the formed Os(v) nitride (see Scheme 1.2).

Due to the higher oxidation state of $10^{\mathrm{BAr}_{24}^{\mathrm{F}}}$ the complex can be assumed to be rather electrophilic and its behavior towards styrene was tested. However, no reaction with up to 


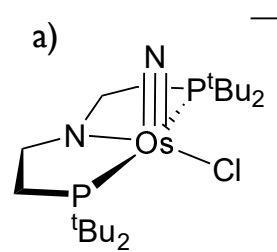

$10^{\mathrm{BAr}_{24}}$ $\frac{\text { 1) NCS }}{\text { 2) } H\left(\text { BArF }_{24}\right)}$

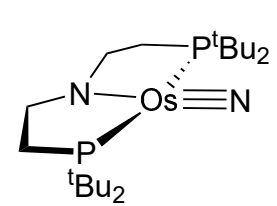

1) NCS

2) $\mathrm{HCl}$

3

b)
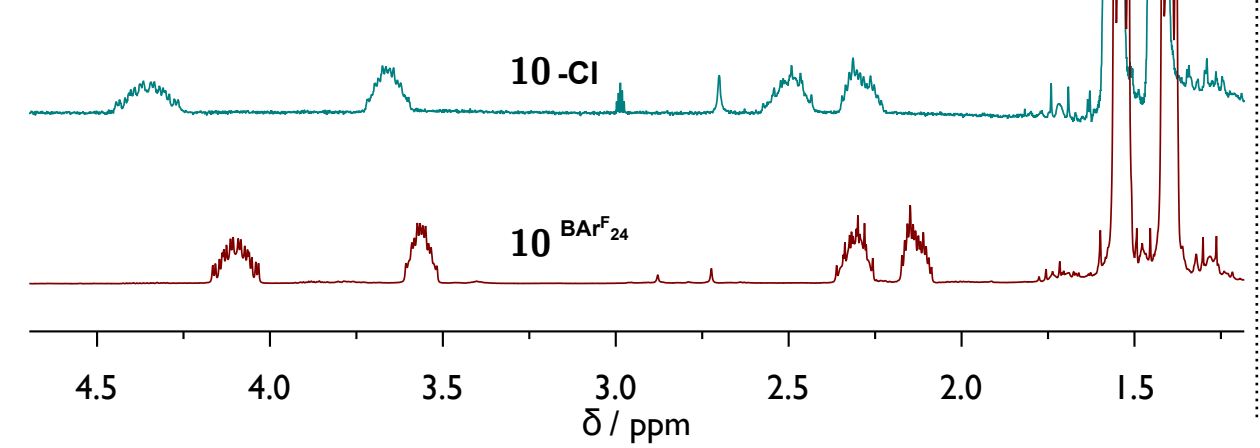

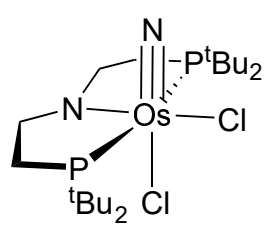

$10-\mathrm{Cl}$

Fig. 1.6. a) Syntheses of $\mathbf{1 0 - C l}$ and $10^{\mathrm{BA} \mathbf{r}_{24}^{\mathrm{F}}}$ from 3 and NCS. The ${ }^{1} \mathrm{H}(b)$ and ${ }^{31} \mathrm{P}\left\{{ }^{1} \mathrm{H}\right\}$ (c) NMR spectra show clearly distinguishable compounds, suggesting chloride coordination in $\mathbf{1 0}-\mathbf{C l}$.

10 eq was observed. One way to increase nitride centered reactivity was published by Lau and cowokers who observed alkene aziridination reaction by $\left[\mathrm{Ru}(\mathrm{N})(\text { salchda) }]^{\mathrm{PF}_{6}}\right.$ (LXII), initiated by coordination of a ligand (pyridine) in trans position to the nitride (see Part I, Section 4.2.2). ${ }^{[163]}$ Adopting this strategy, $\mathbf{1 0}^{\mathrm{BAr}_{24}^{\mathrm{F}}}$ and 10 eq styrene were mixed with pyridine as well as the strong $\sigma$-donor ligand $\mathrm{CN}^{t} \mathrm{Bu}$. While the former reagent is also unreactive, mixing $10^{\mathrm{BAr}_{24}^{\mathrm{F}}}$ with one equivalent $\mathrm{CN}^{\mathrm{t}} \mathrm{Bu}$, resulted in only $50 \%$ conversion. Furthermore, the reaction was observed to be fully independent of styrene being present or not. Full conversion was achieved upon addition of another equivalent or by starting the reaction with an excess of $\mathrm{CN}^{\mathrm{t}} \mathrm{Bu}$ right away. Moreover, ${ }^{31} \mathrm{P}\left\{{ }^{1} \mathrm{H}\right\} \mathrm{NMR}$ spectroscopy revealed the formation of two new complexes 11a and 11b in an estimated ratio of 1:0.2, each with inequivalent $\mathrm{P}$ atoms and very small coupling constants $\left(J_{\mathrm{PP}}=11.6\right.$ and $\left.6.7 \mathrm{~Hz}\right)$. While the two products were not isolated from each other and no clean samples were obtained, ${ }^{1} \mathrm{H}$ NMR measurements suggest the species to be $C_{S}$ symmetric with the pincer being in plane with the mirror plane. Additionally, a doublet at $\delta_{1_{\mathrm{H}}}=7.92 \mathrm{ppm}\left(J_{\mathrm{HP}}=17.1 \mathrm{~Hz}\right)$ can be identified, which collapses to a singlet in the ${ }^{1} \mathrm{H}\left\{{ }^{31} \mathrm{P}\right\}$ NMR spectrum. Full NMR characterization of the complexes is still pending. Nevertheless, single crystals suitable for

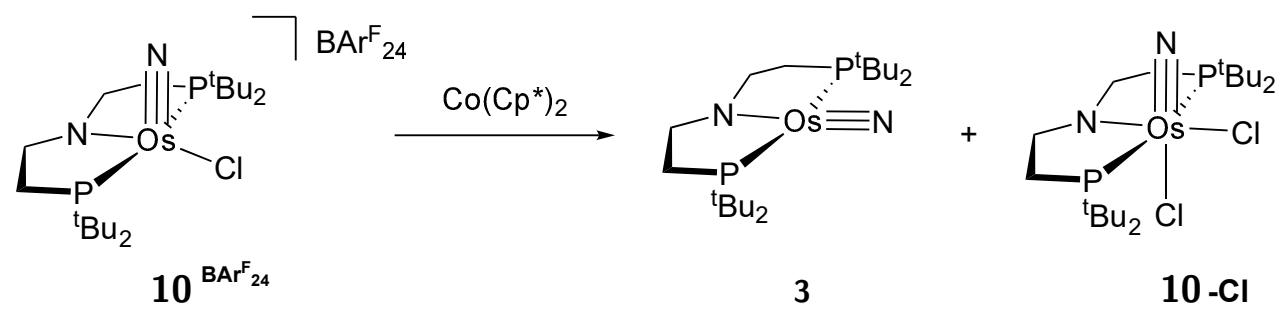

Scheme 1.2. Disproportionation of $10^{\mathrm{BAr}_{24}^{\mathrm{F}}}$ upon reduction. 
a)

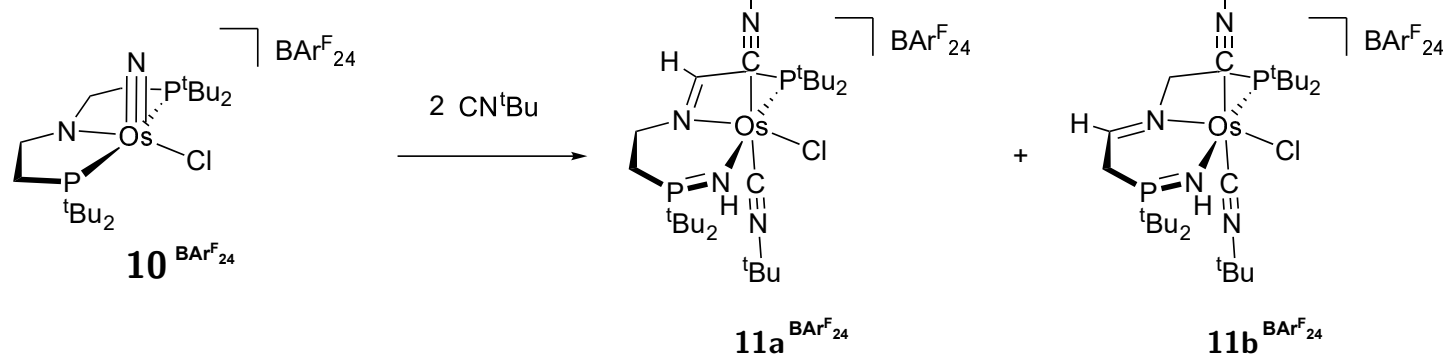

b)

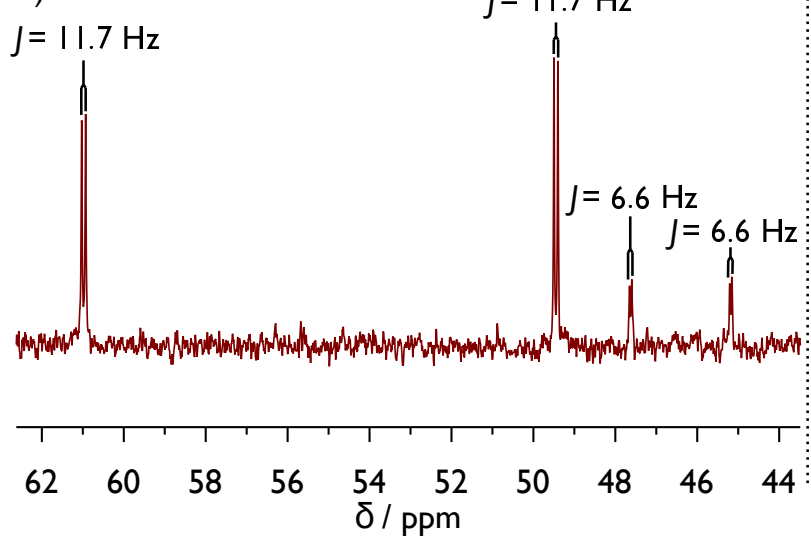

c)

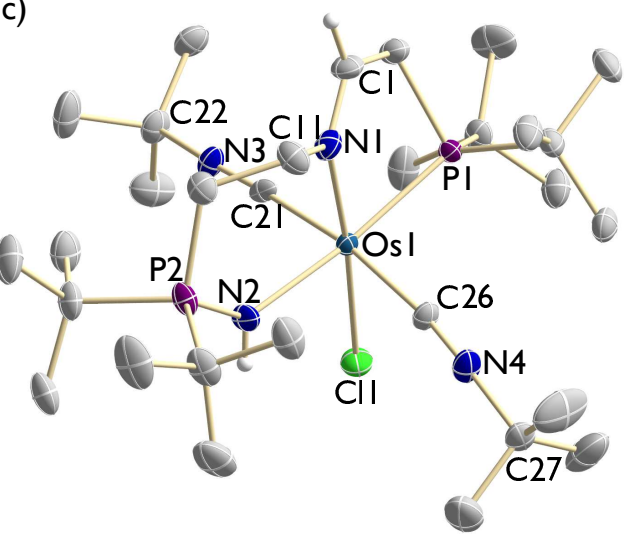

Fig. 1.7. a) Reaction of $10^{\mathrm{BAr}_{24}^{\mathrm{F}}}$ with 2 eq $C N^{t} \mathrm{Bu}$ and the most likely formed products $11 \mathrm{a}^{\mathrm{BAr}}{ }_{24}^{\mathrm{F}}$ and $11 \mathbf{b}^{\mathrm{BAr}_{24}^{\mathrm{F}}}$. b) ${ }^{31} \mathrm{P}\left\{{ }^{1} \mathrm{H}\right\}$ NMR spectrum of $11 \mathbf{a}^{\mathrm{BAr}{ }_{24}^{\mathrm{F}}}$ and $11 \mathbf{b}^{\mathrm{BA} r_{24}^{\mathrm{F}}}$. c) Molecular structure of one of the two isomers obtained by single crystal $\mathrm{X}$-ray diffraction measurements. All $\mathrm{H}$ atoms but the $\mathrm{NH}$ and imine $\mathrm{CH}$ atoms, as well as the anion are omitted for clarity. Anisotropic displacement parameters are set to $50 \%$ probability. Selected bond lengths $[\AA]$ and angles [']: Os1-N1 2.058(2), Os1-N2 2.175(2), P2-N2 1.592(2), N1-C1 1.308(4), N1C11 1.447(4), C21-N3 1.177(4), C26-N4 1.146(3), Os1-N2-P2 132.15(15), C26-N4-C27 170.0(3), C21-N3-C22 171.8(3), N1-Os1-Cl1 175.19(7), P1-Os1-N2 171.64(7), C21-Os1C26 170.68(11).

X-ray diffraction measurements could be obtained and revealed one of the species to be $\left[\mathrm{Os}\left(\mathrm{CN}^{\mathrm{t}} \mathrm{Bu}\right)_{2} \mathrm{Cl}\left\{\mathrm{N}\left(\mathrm{CH}_{2} \mathrm{CH}_{2} \mathrm{P}^{\mathrm{t}} \mathrm{Bu}_{2}\right)\left(=\mathrm{CHCH}_{2} \mathrm{P}\left({ }^{\mathrm{t}} \mathrm{Bu}\right)_{2}=\mathrm{NH}\right)\right\}\right]^{\mathrm{BAr}}{ }_{24}^{\mathrm{F}}$, i.e. the product of nitride insertion into the $\mathrm{Os}-\mathrm{P}$ bond, accompanied by $\mathrm{CH}$ activation of the backbone and imine formation. The osmium center is coordinated octahedrally by the newly formed $\mathrm{PNN}^{\mathrm{H}}$ pincer, the chloride and two isocyanide ligands in trans position to each other, leading to the overall observed $C_{S}$ symmetry. Both isocyanides are almost linear $(\measuredangle(\mathrm{Os}-\mathrm{C}-\mathrm{N})=170.0(3)$ and $\left.171.8(3)^{\circ}\right)$ and feature similar $\mathrm{C} \equiv \mathrm{N}$ bond length $(d(\mathrm{C}-\mathrm{N})=1.177(4)$ and $1.146(3) \AA)$, indicating a modest degree of backbonding. The newly formed imino phosphorane features a $\mathrm{P}=\mathrm{N}$ bond length of $d(\mathrm{P} 2-\mathrm{N} 2)=1.592(2) \AA$, in line with a double bond. This imino phosphorane moiety formation also perfectly matches the small coupling constants in ${ }^{31} \mathrm{P}\left\{{ }^{1} \mathrm{H}\right\}$ NMR, as these are now ${ }^{3} J$ coupling constants. The NH proton was found from the residual density map and most likely corresponds to the doublet at $\delta_{1_{\mathrm{H}}}=7.92 \mathrm{ppm}$ in the ${ }^{1} \mathrm{H}$ NMR spectrum. The two $\mathrm{N}-\mathrm{C}$ bond distances in the backbone strongly differ ( $d(\mathrm{~N} 1-\mathrm{C} 1)$ $=1.308(4) \AA, d(\mathrm{~N} 1-\mathrm{C} 11)=1.447(4) \AA)$, which is in line with an intramolecular $\mathrm{CH}$ bond activation and imine formation in the pincer backbone. 
Similar insertion reactions into metal-ligand bonds are rare and have been reported for Fe, ${ }^{[191]}$ $\mathrm{Co}^{[192,193]}$ and $\mathrm{Ni}^{\left[{ }^{194]}\right.}$ In all these cases, the insertion products were obtained by thermal or photolytic decomposition of complexes featuring nitride precursors (azides or dbabh $=$ 2,3:5,6-dibenzo-7-azabicyclo[2.2.1] hepta-2,5-diene) and the formation of transient nitrido species remained only hypothetical, with initial insertion of the precursor and subsequent loss of the leaving groups standing to reason. In the present case no defined mechanism is available, and several possible pathways could play a role, including intramolecular $\mathrm{CH}$ activation directly by the nitride and subsequent insertion into the $\mathrm{Os}-\mathrm{P}$ bond; initial insertion, followed by $\beta$-hydride elimination and shift to the $\mathrm{P}=\mathrm{N}$ ligand, or even initial insertion followed by intermolecular $\mathrm{CH}$ activation. Slightly different barriers for the formation of the syn or anti isomers $11 \mathbf{a}^{\mathrm{BAr}_{24}^{\mathrm{F}}}$ and $\mathbf{1 1 b}^{\mathrm{BAr}} \mathbf{r}_{24}^{\mathrm{F}}$ with respect to the newly formed imine in the backbone and the phosphoraneiminino ligand might than explain the appearance of the two different signal sets as observed by ${ }^{31} \mathrm{P}\left\{{ }^{1} \mathrm{H}\right\}$ NMR.

Even though this reaction is not full characterized yet, its occurance is promising and further trapping experiments could open access to a rather unique way of nitride functionalization reactions. Especially if at some point $\mathrm{N}_{2}$ activation should become feasible at this platform, closer investigation of the reaction at hand might become of greater interest.

\subsection{Attempts of dinitrogen activation}

The $\left\{\mathrm{Os}\left(\mathrm{PNP}^{t \mathrm{Bu}}\right)\right\}$ platform might be a promising candidate for $\mathrm{N}_{2}$ activation and functionalization, as pointed out in Part I, Section 5. Therefore, the potential of $\mathbf{1}$ and derived compounds with respect to $\mathrm{N}_{2}$ activation was thoroughly investigated and will be discussed in this section. Three different targets of hypothetical $\mathrm{N}_{2}$ splitting pathways were identified and independently synthesized from azide source, i.e. $\mathbf{2}^{\mathbf{X}}, \mathbf{3}$ and $\mathbf{1 0}^{\mathbf{X}}$ (see Section 1.1 and 1.3 for syntheses and characterization of these complexes). Starting from 1 and the thereof derived monocationic complex $\left[\mathrm{Os} \mathrm{ClH}\left(\mathrm{PNP}^{t \mathrm{Bu}}\right)\right]^{\mathrm{X}}\left(\mathbf{1 2}^{\mathrm{X}}\right)$, overall five different strategies were developed for how the target molecules might be obtained from $\mathrm{N}_{2}$ (see Figure 1.3 and Table 1.1). According to the considerations expounded in Part I, Section 3.2.1, a $10 \pi(4 \delta)$ electronic configuration of a $\{M N N M\}$ core should be necessary for efficient splitting of $\mathrm{N}_{2}$ into terminal nitrides. Consequently, an Os(III) dinitrogen bridged dimer in an idealized $D_{4 h}$ geometry which is isoelectronic to the formal $\operatorname{Re}(\mathrm{II})$ compound $\mathbf{X X}$ should be an ideal candidate for splitting into the $\mathrm{Os}(\mathrm{VI})$ nitride complexes $\mathbf{2}^{+}$and $\mathbf{1 0}^{+}$. This was attempted by one-electron reduction of the starting complexes under a dinitrogen atmosphere $(\mathrm{I})$, by in situ deprotonation and subsequent one-electron oxidation (III $b$ ) or by single hydrogen atom abstraction with TTBP under a dinitrogen atmosphere (III a).

Despite this MO rationalized approach, splitting attempts to directly obtain Os(IV) nitrido complex 3 were followed as an alternative pathway, taking into account the isolobal relationship between octahedral $d^{n}$ and square-planar $d^{n+2}$ complexes. In related Ir complexes, splitting was calculated to be thermodynamically uphill, but was assumed to potentially 


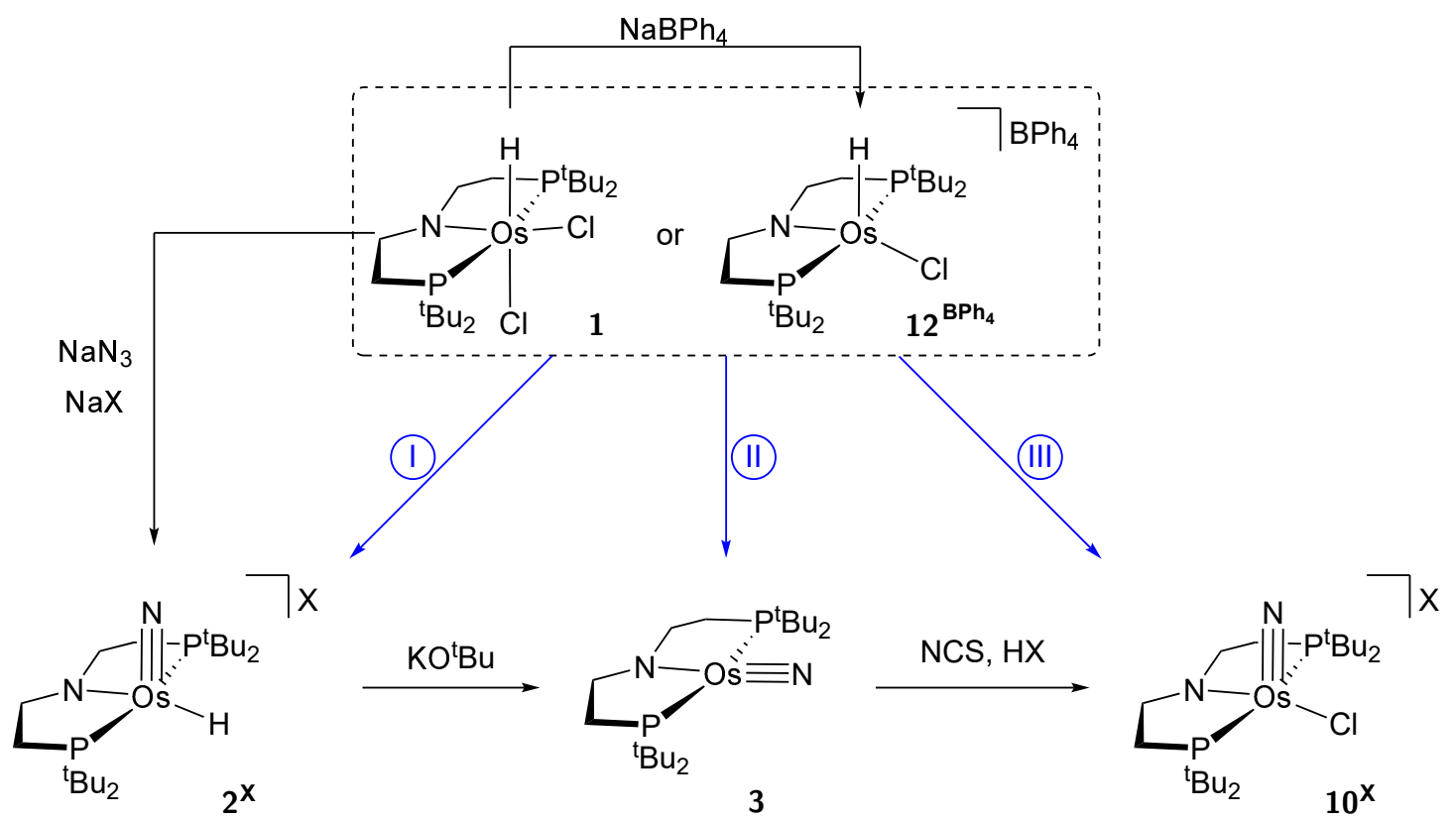

Scheme 1.3. Summary of the reaction strategies I, II or III detailed in Table 1.1, which were envisioned to potentially lead to $\mathrm{N}_{2}$ splitting with the osmium platform.

Tab. 1.1. Explanation of strategies I, II or III depicted in Scheme 1.3 and the list of tested reagents. Most reactions were carried out under various conditions (solvent, temperature).

\begin{tabular}{c|cl}
\hline I & $+\mathrm{e}^{-}+\mathrm{N}_{2}$ & Reductants: $\mathrm{NaHg}, \mathrm{CoCp}_{2}, \mathrm{Co}\left(\mathrm{Cp}^{*}\right)_{2}$ \\
II $a$ & $+3 \mathrm{e}^{-}+\mathrm{N}_{2}$ & Reductants: $\mathrm{NaHg}, \mathrm{KC}_{8}$ \\
II $b$ & + base $+\mathrm{e}^{-}+\mathrm{N}_{2}$ & Bases: $\mathrm{KO}^{t} \mathrm{Bu}, \mathrm{KHMDS}, \mathrm{NEt}_{3}, \mathrm{DBU}$. Reductants: $\mathrm{NaHg}, \mathrm{KC}_{8}$ \\
III $a$ & $-\mathrm{H}^{+}+\mathrm{N}_{2}$ & Oxidants: TTBP, 1,4-benzoquinone \\
III $b$ & + base $-\mathrm{e}^{-}+\mathrm{N}_{2}$ & Bases: $\mathrm{KO}^{t} \mathrm{Bu}, \mathrm{KHMDS}$. Oxidants: $\mathrm{Fc}^{+}, \mathrm{Ac} \mathrm{Fc}^{+}, \mathrm{Ag}^{+}$, magic blue \\
\hline
\end{tabular}

result in an almost thermoneutral splitting product. For this, the starting complexes were either reacted with an excess of $\mathrm{KC}_{8}$ in order to reduce all the way down to an Os(I) fragment (II a), or treated in situ with a base and then with $\mathrm{KC}_{8}$, all under dinitrogen atmosphere (III b).

Most of these reactions gave mixtures of several, unidentifiable species and will therefore not be discussed here in detail. Most importantly, in more than 60 different reactions, no sign for any of the three different target compounds could be identified, suggesting an inherent problem of the chosen system. In many reactions, especially in those which involved bases, $C_{1}$ symmetric species bearing hydride ligands and signals between $5-8 \mathrm{ppm}$ in the ${ }^{1} \mathrm{H}$ NMR spectrum could be identified as part of the product mixtures, suggesting $\beta$-hydride elimination from the ligand. This points to a rather low stability of low coordinated Os(II) compounds towards intramolecular $\mathrm{CH}$ activation as one major issue, which is in line with the few literature reports on square-planar Os(II) complexes. ${ }^{[168,195]}$ Yet it is noteworthy that reaction of 1 with $\mathrm{KO}^{t} \mathrm{Bu}$ at $-80^{\circ} \mathrm{C}$ resulted in a defined color change from green to violet, indicating an initial clean reaction product persistent at low temperatures. Warming these reaction to $T>-50^{\circ} \mathrm{C}$ already leads to color change to brown. Unfortunately, no NMR data on this violet species are available. 
It was possible to gain access to two stable Os(II) complexes when additional ligands were introduced via two different routes (see Figure $1.8 \mathrm{a}$ ). On the one hand, reaction of $\mathbf{1}$ with 3 eq $\mathrm{KC}_{8}$ under a dinitrogen atmosphere in THF gives selective reaction to a new complex 13. The ${ }^{1} \mathrm{H}$ NMR spectrum reveals a hydride signal at $\delta_{1_{\mathrm{H}}}=-26.88 \mathrm{ppm}$ and $C_{s}$ symmetry on the NMR timescale (see Figure 1.8 b). A single resonance in the ${ }^{31} \mathrm{P}\left\{{ }^{1} \mathrm{H}\right\} N M R$ at $\delta^{31} \mathrm{P}$ $=85.9 \mathrm{ppm}$ is split into a doublet $\left({ }^{2} J_{\mathrm{HP}}=8.7 \mathrm{~Hz}\right)$, attributed to insufficient decoupling of the hydride signal (see Figure $1.8 \mathrm{c}$ ). Two bands in the attenuated total reflection infrared (ATR-IR) spectrum at $\tilde{\nu}=2114.5 \mathrm{~cm}^{-1}\left(\nu_{\mathrm{Os}-\mathrm{H}}\right)$ and $2007.7 \mathrm{~cm}^{-1}\left(\nu_{\mathrm{N} \equiv \mathrm{N}}\right)$ support 13 to be the $\mathrm{Os}(\mathrm{II})$ hydride dinitrogen complex $\left[\mathrm{OsH}\left(\mathrm{N}_{2}\right)\left(\mathrm{PNP}^{t \mathrm{Bu}}\right)\right]$ (see Figure $1.8 d$ ).

The identification of this complex explained why the strategy II a was unsuccessful. Apparently, 13 can not be reduced any further, not even with $\mathrm{KC}_{8}$. Unfortunately, oxidation of 13 by one electron also gave no signal of the $\mathrm{Os}(\mathrm{VI})$ hydride nitride complex $\mathbf{2}^{+}$, which is why the complex was not further investigated.

Stabilization of an Os(II) complex by addition of an electron-acceptor ligand appears to be an attractive option to prevent unwanted backbone activation. However the presence of a hydride in any hypothetical splitting product can be considered undesirable, as for example $2^{\mathrm{Cl}}$ is known to decompose into 3 and $\mathbf{4}^{\mathrm{Cl}}$ (see Section 1.1). Consequently, a similar route to stabilize the deprotonation product of $\mathbf{1}$ was investigated. A sequence of chloride abstraction using $\mathrm{NaBPh}_{4}$, tert-butylisocyanide coordination and deprotonation was finally developed, yielding $\left[\mathrm{OsHCl}\left(\mathrm{PNP}^{t \mathrm{Bu}}\right)\right]^{\mathrm{BPh}_{4}}\left(\mathbf{1 2}^{\mathrm{BPh}_{4}}\right),\left[\mathrm{OsHCl}\left(\mathrm{CN}^{\mathrm{t}} \mathrm{Bu}\right)\left(\mathrm{PNP}^{t \mathrm{Bu}}\right)\right]^{\mathrm{BPh}_{4}}\left(\mathbf{1 4}^{\mathrm{BPh}_{4}}\right)$ and $\left[\mathrm{OsCl}\left(\mathrm{CN}^{\mathrm{t}} \mathrm{Bu}\right)\left(\mathrm{PNP}^{t \mathrm{Bu}}\right)\right](\mathbf{1 5})$ (see Figure $1.8 \mathrm{a}$ ). Successful chloride abstraction in the first step is most evident by the shift of the hydride signal from $\delta_{1_{H}}=-4.48 \mathrm{ppm}$ to $-20.8 \mathrm{ppm}$ and the fact, that $12^{\mathrm{BPh}_{4}}$ becomes unstable towards $\mathrm{DCM}$, which is different from parent $\mathbf{1}$. Overall, the signals of $\mathbf{1 2}^{\mathrm{BPh}_{4}}$ are often quite broad, in ${ }^{1} \mathrm{H}$ as well as ${ }^{31} \mathrm{P}\left\{{ }^{1} \mathrm{H}\right\}$ NMR spectroscopy. Successful coordination of $\mathrm{CN}^{t} \mathrm{Bu}$ to form $14^{\mathrm{BPh}_{4}}$ again becomes evident from the ${ }^{1} \mathrm{H}$ NMR spectrum, showing a backshifting of the hydride signal to $\delta_{1_{\mathrm{H}}}=0.30 \mathrm{ppm}$ as well as the appearance of a new singlet integrating to nine protons at $\delta_{1_{\mathrm{H}}}=1.47 \mathrm{ppm}$, overlapping with the resonance of one of the tert-butyl groups. Importantly, at this stage the stochiometry of the isocyanide ligand needed to be controlled with care, because already a minor excess leads to formation of side products which could not be separated from the main complex. Finally, deprotonation using $\mathrm{KO}^{t} \mathrm{Bu}$ resulted in formation of $\mathbf{1 5}$, which exhibits no signal corresponding to a hydride or the anion anymore but still contains a single $\mathrm{CN}^{\mathrm{t}} \mathrm{Bu}$ group. Further spectroscopic measurements on these compounds are not available at this point. However, all three complexes were confirmed by liquid injection field desorption ionization (LIFDI) mass spectrometry and single crystals suitable for X-ray diffraction measurements allowed to determine the molecular structure of the final complex 15, suggesting a successful synthetic route (see Figure $1.8 \mathrm{~g}$ ). The osmium center is coordinated in a square pyramidal coordination geometry $\left(\tau_{5}=0.06\right)^{[196]}$ with the isocyanide ligand adopting the apical position. The isocyanide moiety is strongly bent $\left(\measuredangle\left(\right.\right.$ Os1-C21-N2) $=170.1(2)^{\circ}$, $\left.\measuredangle(\mathrm{C} 21-\mathrm{N} 2-\mathrm{C} 22)=141.3(3)^{\circ}\right)$, indicating a significant amount of backbonding. Such highly bent isocyanides were observed before for isoelectronic $\mathrm{W}(0)$ and $\operatorname{Re}(\mathrm{I})$ complexes. ${ }^{[153,197]}$ 


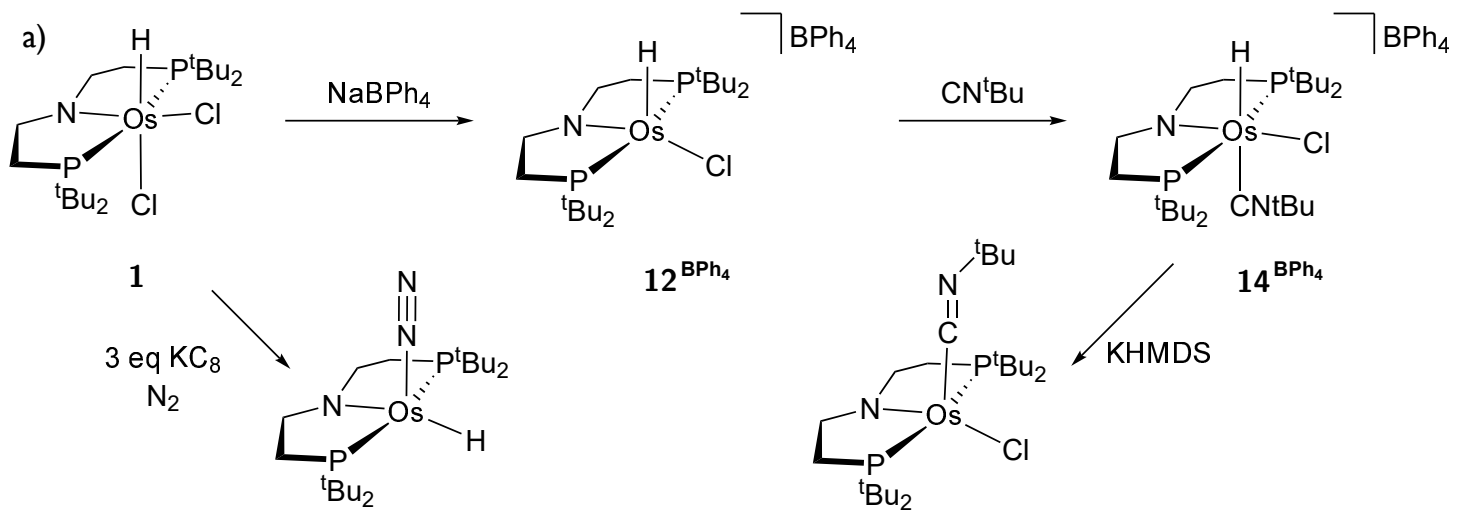

13

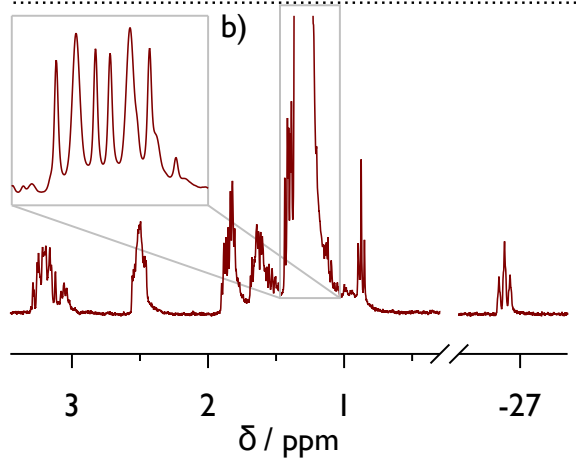

c) $\quad \vdots d)$

15
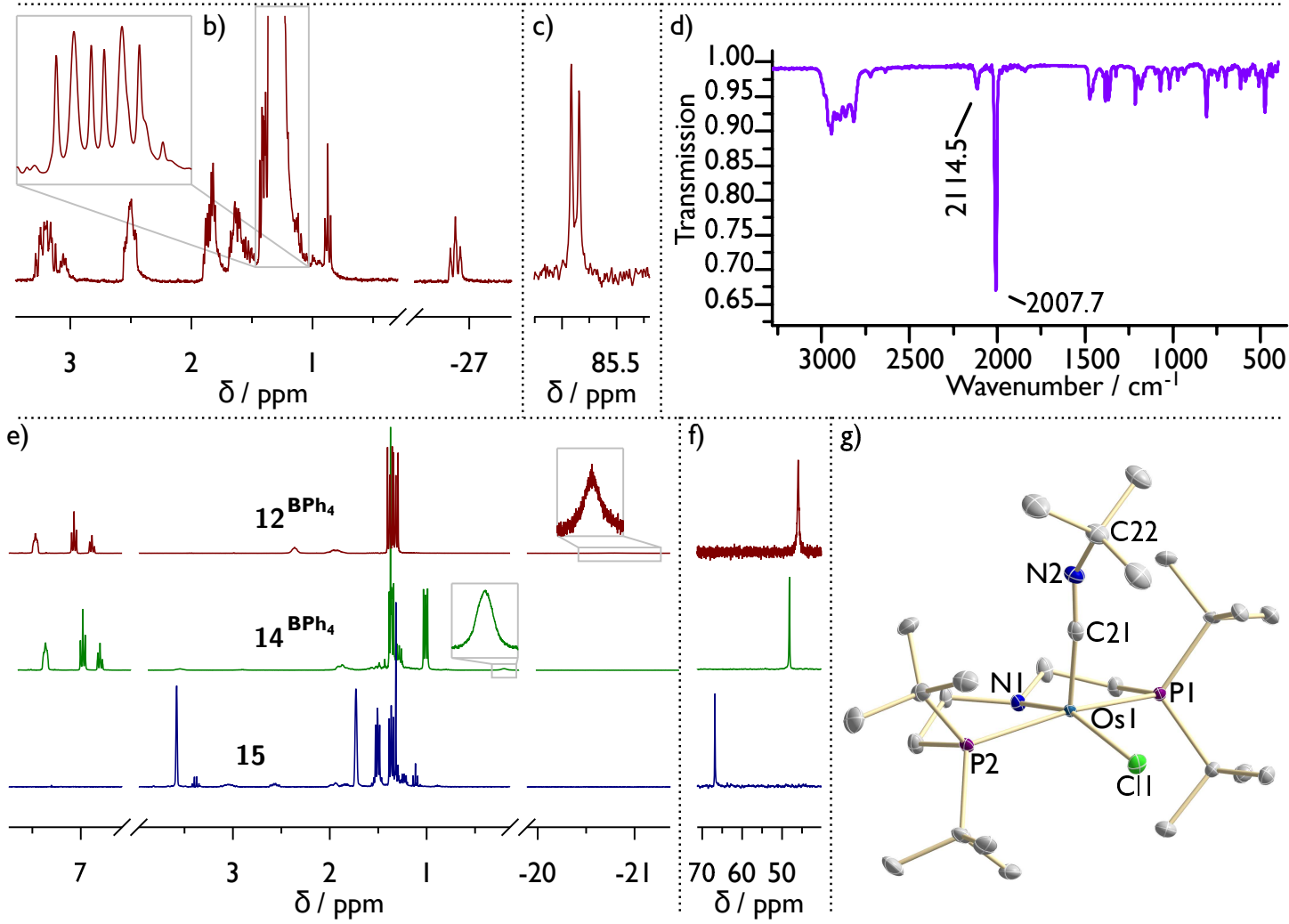

Fig. 1.8. a) Synthesis scheme describing the syntheses of Os(II) complexes $\mathbf{1 3}$ and $\mathbf{1 5}$. Middle: ) ${ }^{1} \mathrm{H}$ (b) and ${ }^{31} \mathrm{P}\left\{{ }^{1} \mathrm{H}\right\}(c)$ NMR spectrum as well as IR spectrum $(d)$ of 13. Bottom: ${ }^{1} \mathrm{H}(e)$ and ${ }^{31} \mathrm{P}\left\{{ }^{1} \mathrm{H}\right\}(f)$ NMR spectra of $\mathbf{1 2}^{\mathrm{BPh}_{4}}$ (red), $\mathbf{1 4} \mathbf{B P h}^{\mathrm{BPh}_{4}}$ (green) and $\mathbf{1 5}$ (blue). g) Molecular structure of $\mathbf{1 5}$ obtained by single crystal X-ray diffraction measurements. All $\mathrm{H}$ atoms are omitted for clarity. Anisotropic displacement parameters are set to $50 \%$ probability. Selected bond lengths $[\AA \AA]$ and angles $\left[{ }^{\circ}\right]$ : Os1-N1 1.972(2), Os1-Cl1 2.4639(7), Os1-C21 1.838(3), C21-N2 1.206(4), P1-Os1-P2 161.66(3), N1-Os1-Cl1 157.95(7), Os1-C21-N2 170.1(2), C21-N2-C22 141.3(3).

This is in line with a slight elongation of the $\mathrm{N} \equiv \mathrm{C}$ bond in that moiety $(d(\mathrm{C} 21-\mathrm{N} 2)=$ $1.206(4) \AA$ ) compared to unactivated isocyanides like $\mathbf{1 1}^{\mathrm{BAr}_{24}^{\mathrm{F}}}$ (see Section 1.3).

Preliminary reactivity studies suggest an instability of $\mathbf{1 5}$ in the presence of sodium salts with different outcomes under $\mathrm{Ar}$ or $\mathrm{N}_{2}$. However, these reaction were hardly selective and lacked reproducibility. The appearance of many different signals in the high field region of 
the ${ }^{1} \mathrm{H}$ NMR spectrum suggested hydride formation and thus backbone activation under these conditions. Thus, also this complex was not investigated further.

\subsection{Summary}

At the beginning of this thesis, it was known how the square-planar Os(IV) nitride $\mathbf{3}$ could be synthesized, including most of the characterization. However, the nature of the intermediates and involved species was only discussed on a rather speculative basis. Continued research provided a refined picture, especially on the nature of the initially formed Os(VI) nitrido complex $\mathbf{2}^{+}$, and its disproportionation if chloride was the counteranion. Eventually, these results allowed for improving the synthesis of 3 .

Exploration of the principal reactivity of $\mathbf{3}$ showed nitride centered reactivity towards large Lewis acids like $\mathrm{Me}_{3} \mathrm{Si}^{+}$or $\mathrm{BAr}_{18}^{\mathrm{F}}$, whereas exposure to smaller electrophiles result in metal centered reactivity, highlighting the importance of the steric shielding by the tert-butyl groups. Contrary to this, $\mathbf{3}$ is inert towards most nucleophiles. However, upon mixing with $\mathrm{PMe}_{3}$, the reversible formation of a phosphoraneiminato ligand is observed. Theoretical investigations by DFT calculations and NBO analysis proved the reaction to feature a dual nature push pull interaction between both reactants, with the electrophilic properties of $\mathbf{3}$ dominating, rendering it an ambiphile.

Additionally, full hydrogenolysis of $\mathbf{3}$ was proven to yield $\mathrm{NH}_{3}$ and polyhydride complex $\mathbf{8}$.

Attempts to synthesize an $\operatorname{Os}(\mathrm{V})$ nitride species by either oxidation of $\mathbf{3}$ or reduction of $2^{+}$or $\mathbf{1 0}^{+}$gave either non-selective reactions in the former or full reduction down to 3 in the latter case. In this context, $\mathbf{1 0}^{+}$was found to react with two equivalents of $\mathrm{CN}^{\mathrm{t}} \mathrm{Bu}$ undergoing backbone $\mathrm{CH}$ activation and insertion of the nitride into an $\mathrm{Os}-\mathrm{P}$ bond, forming an phosphoraneimine moiety. This reaction shows how the nitride centered reactivity can be increased through addition of trans ligands and might be exploited in further functionalization schemes.

Finally, extensive investigation of potential $\mathrm{N}_{2}$ activation schemes gave no sign of nitride formation at any stage, pointing to some inherent problems of the system at hand. Backbone $\mathrm{CH}$ activation seemed to be a major problem. In the cause of this research, two Os(II) complexes 13 and $\mathbf{1 5}$ could be obtained and were tested towards $N_{2}$ splitting as well, yet unsuccessful. 


\section{Extensions of the $\left[\operatorname{ReCl}_{2}\left(\mathrm{PNP}^{\mathrm{tBu}}\right)\right]$ platform}

The extension of existence to a further level of Being

$$
\begin{aligned}
& \text { - Vader } \\
& \text { "Xeper" on "Litany" }
\end{aligned}
$$

In previous work, the rhenium PNP pincer complex $\left[\operatorname{ReCl}_{2}\left(\mathrm{PNP}^{t \mathrm{Bu}}\right)\right](\mathbf{X I X})$ was shown to be a potent platform for $\mathrm{N}_{2}$ activation and functionalization (see Part I Section 4.2.1). Notably, the reductive splitting into nitrido complex $\left[\operatorname{ReCl}(N)\left(\mathrm{PNP}^{t \mathrm{Bu}}\right)\right](\mathbf{X X I})$ and the subsequent functionalization with alkyl triflates resulted in synthetic cycles for nitrile formation. ${ }^{94,153]}$

The nature of the proposed intermediate of the dinitrogen splitting process, i.e. [ $\left(\mu-N_{2}\right)\{\operatorname{Re}$ $\left.\left.\mathrm{Cl}\left(\mathrm{PNP}^{t \mathrm{Bu}}\right)\right\}_{2}\right](\mathbf{X X})$, was previously only predicted from DFT calculations. However, during the reduction of $\mathbf{X I X}$ a color change to deep red was observed which vanished rapdily at $\mathrm{RT}$, indicating a short-lived, yet observable intermediate. Investigations which led to the spectroscopic, structural and thermodynamic characterization of this dimer $\mathbf{X X}$ are described in Section 2.1.

The above mentioned nitrile formation was achieved after ethylation and benzylation of the nitrido complex XXI. Interestingly, previous work indicated that following a similar route with the methylated complex $\left[\mathrm{Re}(\mathrm{NMe}) \mathrm{Cl}\left(\mathrm{PNP}^{t \mathrm{Bu}}\right)\right]^{+}$(16) led to intermolecular $\mathrm{C}-\mathrm{C}$ coupling instead of cyanide formation. ${ }^{[180]}$ This reaction was investigated further and is described in Section 2.2.

The synthetic cycles which lead to nitrile formation require strong reductants for the initial $\operatorname{Re}(\mathrm{III} / \mathrm{II})$ reduction to enable $\mathrm{N}_{2}$ activation on the one hand, and oxidants for the subsequent functionalization on the other. In the strive for developing catalytic systems, electrochemistry provides a promising alternative to chemical reactants which would enable to apply reductive and oxidative conditions to a bulk solution simultaneously. However, any potential system for such a scenario should be inert to oxidative stress, i.e. side reactions leading to decomposition should be avoided. Previous work in our group identified the oxidation of the ligand backbone, forming $\mathrm{N}\left(\mathrm{CHCHPR}_{2}\right)_{2}^{-}\left(\mathrm{P}=\mathrm{N}=\mathrm{P}^{-}\right)$, as strategy to easily access a versatile platform which is less sensitive to oxidative decomposition. Therefore, the preparation of 
complex $\left[\operatorname{ReCl}_{2}\left(\mathrm{P}=\mathrm{N}=\mathrm{P}^{t \mathrm{Bu}}\right)\right](\mathbf{2 1})$ as well as its application in $\mathrm{N}_{2}$ activation, splitting, and functionalization is described in Section 2.3.

\subsection{Characterization of $\mu-\mathrm{N}_{2}$ bridged Re dimer $\mathbf{X X}$}

Part of this section has been published in: B. M. Lindley, R. S. van Alten, M. Finger, F. Schendzielorz, C. Würtele, A. J. M. Miller, I. Siewert, S. Schneider, "Mechanism of Chemical and Electrochemical $\mathrm{N}_{2}$ Spitting by a Rhenium Pincer Complex", J. Am. Chem. Soc. 2018, 140, 7922-7935.

The splitting of $N_{2}$ by $\mathbf{X I X}$ under reductive conditions was proposed to proceed via dinuclear, $\mu-\mathrm{N}_{2}$ bridged dimer $\mathbf{X X}$, the structure of which was predicted by DFT calculations. ${ }^{[94]}$ These computations also pointed to a significant activation barrier for the actual splitting step and thus suggested a considerable lifetime of the dimer, giving rise to the question whether it could be at least spectroscopically observable.

A first evidence of a short-lived, yet observable, intermediate had already been reported in terms of a color change during the reaction from violet (XIX), via deep red (intermediate $\mathbf{X X})$ to yellow $(\mathbf{X X I})$ within minutes at RT. Reduction of $\mathbf{X I X}$ under $\mathrm{N}_{2}$ atmosphere at $-40{ }^{\circ} \mathrm{C}$ preserved the red color and low temperature NMR measurements revealed, despite several minor side products, two new doublets in the ${ }^{31} \mathrm{P}\left\{{ }^{1} \mathrm{H}\right\}$-NMR spectrum at $\delta^{31} \mathrm{P}=16.5$ and $-115.3 \mathrm{ppm}$ which featured a mutual coupling constant of ${ }^{2} J_{\mathrm{PP}}=234 \mathrm{~Hz}$ (see NMR spectra in Figure 2.1). Warming the sample to RT and monitoring the process showed clean conversion of the newly formed species into $\mathbf{X X I}$ proving it to be an actual intermediate in the $\mathrm{N}_{2}$ splitting process. In the ${ }^{1} \mathrm{H}-\mathrm{NMR}$ spectrum, a new set of paramagnetically shifted, yet sharp signals with resolved $J$-coupling constants was observed. A total of eight signals for backbone protons between $\delta_{{ }^{H}}=12.2$ and $-16.7 \mathrm{ppm}$ could be identified, while an identification of the tert-butyl groups was not fully possible as they became very broad at low temperatures (probably due to dynamic effects). These data are in agreement with a single new complex $\mathbf{X X}$ exhibiting a $C_{2}$ symmetric dimer structure resembled by the DFT-predicted structure as well as the analogous Mo complex $\left[\left(\mu-\mathrm{N}_{2}\right)\left\{\mathrm{MoCl}\left(\mathrm{PNP}^{t \mathrm{Bu}}\right)\right\}_{2}\right]\left(\mathbf{X X I I )} .^{[96]} \mathrm{How}-\right.$ ever, an attempt to further substantiate the dimeric nature of $\mathbf{X X}$ by ${ }^{31} \mathrm{P}\left\{{ }^{1} \mathrm{H}\right\}$-diffusion ordered spectroscopy (DOSY) was not successful. The $T_{1}$ relaxation time of these doublets

2

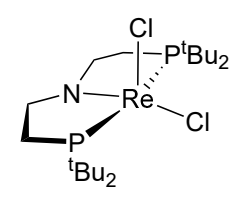

XIX
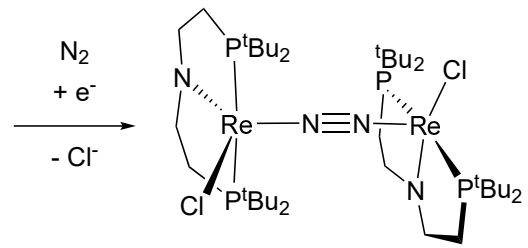

$\mathbf{X X}$

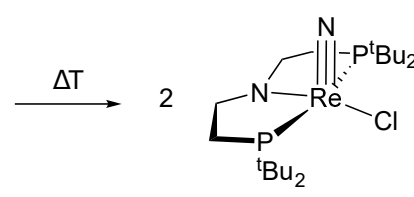

$\mathbf{X X I}$

Scheme 2.1. Thermal dinitrogen splitting after reduction of $\mathbf{X I X}$ proceeds via dimeric, $\mu-\mathrm{N}_{2}$ bridged complex $\mathbf{X X}$ to yield terminal nitrido complex $\mathbf{X X \mathbf { I }}$. 


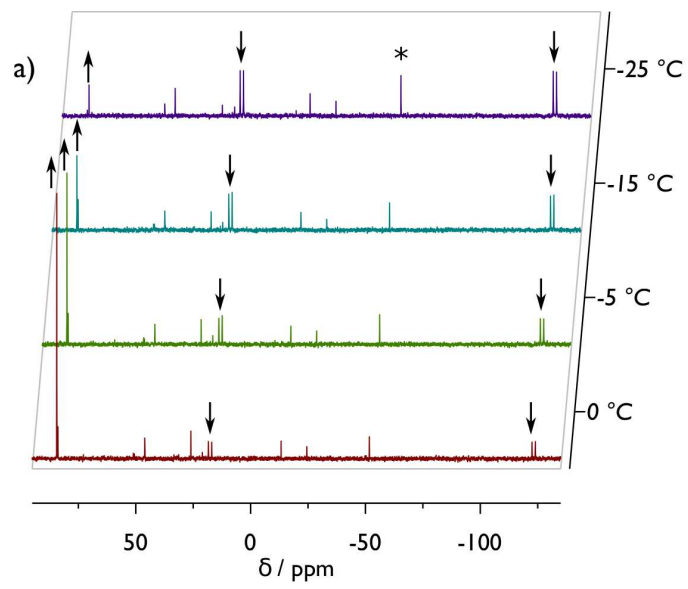

b)
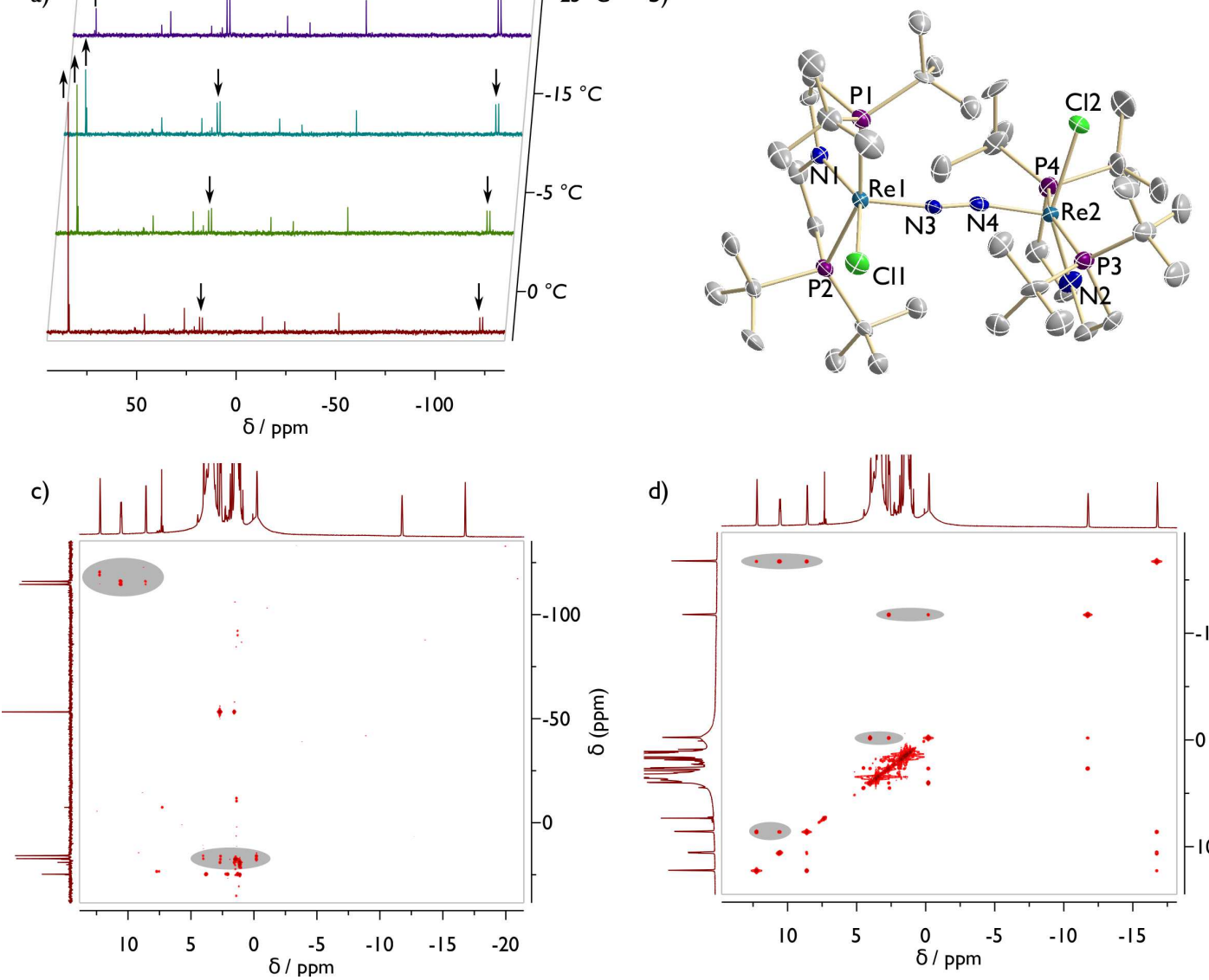

d)

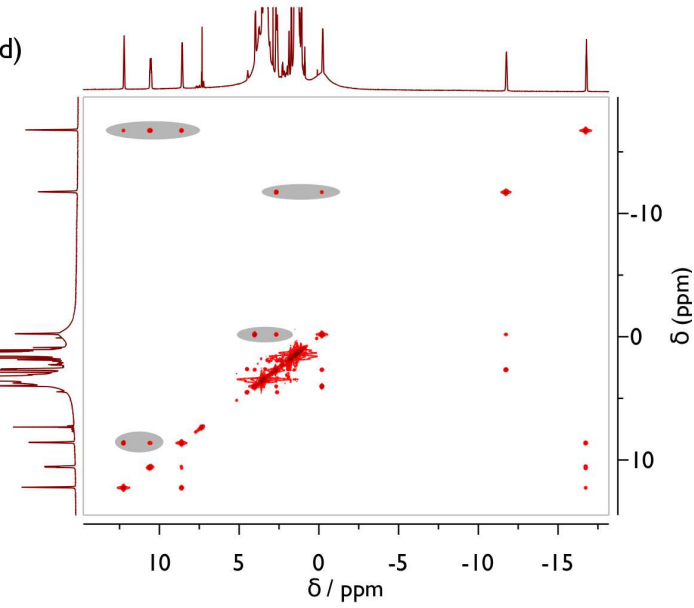

Fig. 2.1. Characterization of $N_{2}$ bridged dimer $\mathbf{X X}$ : a) Reaction monitoring of an in situ prepared solution of $\mathbf{X X}\left(\delta_{31} \mathrm{p}=16.5\right.$ and $\left.-115.2 \mathrm{ppm}\right)$ to $\mathbf{X X I}\left(\delta_{31} \mathrm{p}=84.4 \mathrm{ppm}\right)$ by warming from $-25^{\circ} \mathrm{C}$ to $0{ }^{\circ} \mathrm{C}$. Signals corresponding to the dimer decrease (arrow down) while the peak of the nitride rises (arrow up). The peak marked with an asterisk corresponds to residual $\mathbf{X I X}$. b) Molecular structure of $\mathbf{X X}$ obtained by single crystal $\mathbf{X}$-ray diffraction measurements. $\mathrm{H}$-atoms as well as co-crystallized pentane, $\mathbf{X I X}$ and $\mathbf{X X \mathbf { I }}$ are omitted for clarity. Anisotropic displacement parameters are set to $50 \%$ probability. Selected bond lengths $[\AA]$ ] and angles [ ${ }^{\circ}$ ]: Re1-N1 1.937(7), Re1-N3 1.861(8), Re1-P1 2.406(3), Re1-P2 2.405(3), Re1-Cl1 2.463(3), Re2-N2 1.949(8), Re2-N4 1.886(8), Re2-P3 2.409(3), Re2-P4 2.406(3), Re2-Cl2 2.455(3), N3-N4 1.202(10), N1-Re1-N3 118.0(3), P1-Re1-P2 157.38(9), N1-Re1-Cl1 146.3(2), N2-Re2-N4 119.3(3), P3-Re2-P4 156.28(9), N2-Re2-Cl2 146.6(3), Cl1-Re1-Re2-Cl2 112.06. c) ${ }^{1} \mathrm{H}^{3}{ }^{31} \mathrm{P}-\mathrm{HMBC}$ NMR spectrum. The cross peaks corresponding to $\mathbf{X X}$ are highlighted in gray. d) ${ }^{1} \mathrm{H}_{-}{ }^{1} \mathrm{H}-\mathrm{COSY}$ NMR spectrum of $\mathbf{X X}$ showing cross peaks for all eight paramagnetically shifted backbone signals (highlighted in gray) (both $2 \mathrm{D}-\mathrm{NMR}$ spectra were recorded at $-50^{\circ} \mathrm{C}$ ).

was estimated by inversion recovery to $15 \mathrm{~ms}$, which was too fast for the available DOSY measurements. The unusually strongly shifted ${ }^{31} \mathrm{P}$ - and ${ }^{1} \mathrm{H}-\mathrm{NMR}$ signals are most likely a result of significant spin-orbit coupling (SOC) of low-lying, yet isolated excited states into the ground state and the resulting temperature independent paramagnetism (TIP), analogous to the effects discussed in Section 3.1.2.

Even though the thermal instability of $\mathbf{X X}$ rendered its isolation impossible, after many attempts to crystallize the compound, single crystals suitable for $\mathrm{X}$-ray diffraction measure- 
ments could be obtained from diffusing pentane into a THF solution at $-40{ }^{\circ} \mathrm{C}$ (see Figure 2.1).

The two independent Re centers are coordinated in a distorted square pyramidal coordination environment $\left(\tau_{5}(\operatorname{Re} 1)=0.185 \text { and } \tau_{5}(\operatorname{Re} 2)=0.161\right)^{[196]}$ with rather long $\operatorname{Re}-\mathrm{N}_{2}$ bonds (1.861(8) and 1.886(8) $\AA$ ). The $N \equiv N$ bond (1.202(10) $\AA$ ) indicates slight activation of the dinitrogen moiety and the two $\{$ PNPReCl $\}$ fragments are twisted with respect to each other by $112.06^{\circ}$, perfectly mirroring the structural features of the predicted structure in the first publication on this complex. The molecule co-crystallized with superimposed 0.5 molecules of $\mathbf{X I X}$ and $\mathbf{X X I}$ each, reflecting the presence of both in the in situ prepared solution, as indicated by ${ }^{31} \mathrm{P}\left\{{ }^{1} \mathrm{H}\right\}$-NMR spectroscopy (see Figure 2.1 a).

Despite dimeric complex $\mathbf{X X}$ is not being formed exclusively, once its formation has finished it seems to cleanly react to $\mathbf{X X I}$ (the byproducts of the dimer formation remain basically unchanged during formation of $\mathbf{X X I}$, see Figure $2.1 \mathrm{a}$ ). Encouraged by this finding, a first effort was made to monitor the kinetics of this reaction by variable temperature nuclear magnetic resonance (VT-NMR) spectroscopy. Initial results indicated the reaction to proceed via a first-order reaction. Later, this analysis was finalized by Richt van Alten, confirming the reaction order and leading to values of $\Delta H^{\ddagger}=100.4 \pm 4.1 \mathrm{~kJ} \mathrm{~mol}^{-1}$ and $\Delta S^{\ddagger}=59 \pm$ $13 \mathrm{~J} \mathrm{~mol}^{-1} \mathrm{~K}^{-1}$.[95]

Interestingly, it was noted before that in the synthesis of $\mathbf{X X}$, using $\mathrm{NaHg}$ as a reductant gave much higher yields than $\mathrm{Co}\left(\mathrm{Cp}^{*}\right)_{2}$, even though the reduction potential of the latter should be sufficient for quantitative reduction $\left(E_{1 / 2}\left(\mathrm{Co}(\mathrm{Cp})_{2}\right)=-1.84 \mathrm{~V}\right.$ vs. $\mathrm{Fc} / \mathrm{Fc}^{+}, E_{1 / 2}(\mathbf{X I X})$ $=-1.90 \mathrm{~V}$. A close analysis of the NMR spectra after reduction revealed a side product (17) corresponding to a ${ }^{31} \mathrm{P}\left\{{ }^{1} \mathrm{H}\right\}-\mathrm{NMR}$ signal at $\delta_{31 \mathrm{P}}=29.8 \mathrm{ppm}$ which forms exclusively when $\mathrm{Co}\left(\mathrm{Cp}^{*}\right)_{2}$ is used as a reductant but not with alkali metals. A higher yield in $\mathbf{1 7}$ can be obtained, if an excess of $\mathrm{Co}\left(\mathrm{Cp}^{*}\right)_{2}$ is used and ${ }^{1} \mathrm{H}$-NMR spectroscopy indicated a $C_{S^{-}}$ symmetric species bearing a hydride $\left(\delta_{1_{\mathrm{H}}}=-9.28 \mathrm{ppm}, \mathrm{t},{ }^{2} J_{\mathrm{HP}}=12.9 \mathrm{~Hz}\right)$. Furthermore, 17 is formed also in the absence of $\mathrm{N}_{2}$ and requires two equivalents of reductant for quantitative conversion. All these observations are in agreement with the formation of the $\mathrm{Re}-\mathrm{H}$ species $\left[\mathrm{ReHCl}\left(\mathrm{PNP}^{t \mathrm{Bu}}\right)\right]$ (17) (see Figure $2.2 \mathrm{a}$ ). The origin of the hydride can be explained considering the reported PCET reactivity of $\mathrm{Co}\left(\mathrm{Cp}^{*}\right)_{2}$ forming $\left[\mathrm{Co}\left(\mathrm{Cp}^{*}\right)\left(\mathrm{C}_{5} \mathrm{Me}_{4}\left(\mathrm{CH}_{2}\right)\right)\right]$, even though the latter complex was not found in the NMR spectra. ${ }^{[198]} 17$ exhibits only limited stability, as it showed significant decomposition if stored for extended time (days) in solution. Nevertheless, X-ray structure determination was possible from single crystals grown from cold pentane. 17 exhibits a square-pyramidal coordination geometry $\left(\tau_{5}=0.022\right)^{[196]}$ with the hydride in apical position. The $\mathrm{Re}-\mathrm{H}$ bond distance is in agreement with a hydride ligand $(d(\mathrm{Re}-\mathrm{H})=1.70(2) \AA)$ and planar coordination of the backbone nitrogen indicates an intact amide ligand. Overall, the formation of $\mathbf{1 7}$ can explain the reduced yield of dinitrogen splitting utilizing $\mathrm{Co}\left(\mathrm{Cp}^{*}\right)_{2}$, as it is the product of an off-pathway consuming two equivalents of reductant. 


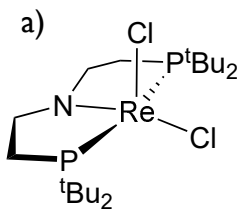

$\mathrm{XIX}$

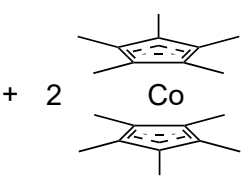

1

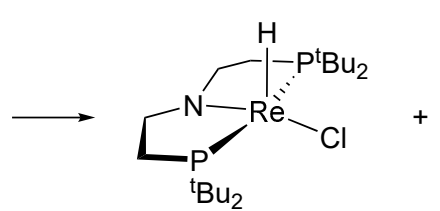

17 b)

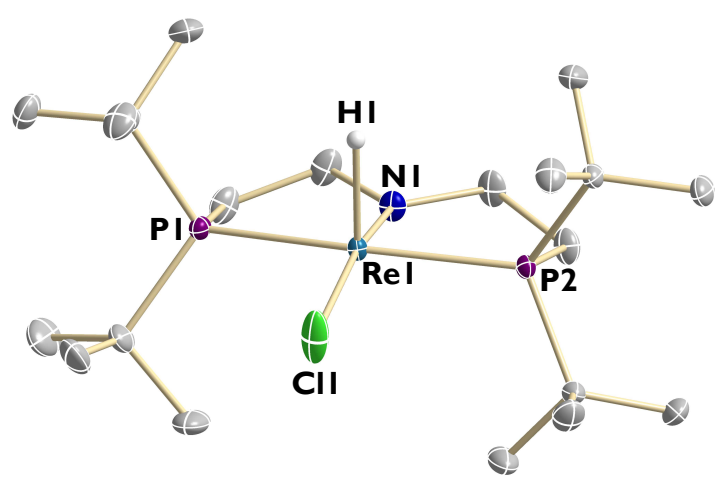

c)

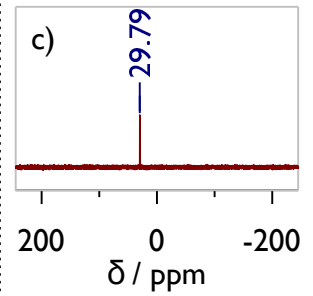

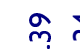

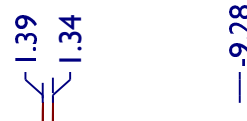

$\stackrel{\infty}{\sim}$

i
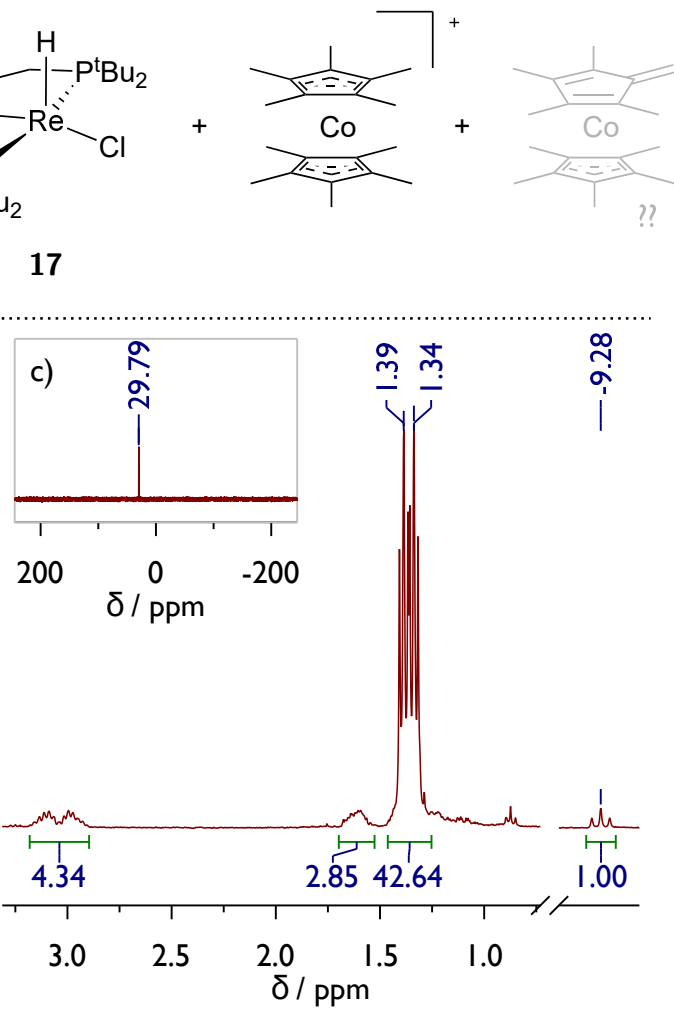

Fig. 2.2. a) Synthesis scheme for the formation of $\mathbf{1 7}$ by PCET reaction of $\mathbf{X I X}$ and $\left.\operatorname{Co}\left(\mathrm{Cp}^{*}\right)_{2} . \quad b\right)$ Molecular structure of $\mathbf{1 7}$ obtained by single crystal X-ray diffraction measurements. All $\mathrm{H}$-atoms despite the $\mathrm{Re}-\mathrm{H}$ are omitted for clarity. Anisotropic displacement parameters are set to $50 \%$ probability. The isotropically refined $\mathrm{Re}-\mathrm{H}$ was found from the residual density map. Selected bond lengths $[\AA]$ and angles [ $\left.{ }^{\circ}\right]$ : Re1-N1 1.9107(18), Re1-P1 2.3520(5), Re1-P2 2.3641(5), Re1-Cl1 2.3995(6), Re1-H1 1.70(2), P1-Re1-P2 165.963(18), N1-Re1$\mathrm{Cl} 1$ 167.33(6), Cl1-Re1-H1 93.6(8). c) ${ }^{1} \mathrm{H}-\mathrm{NMR}$ spectrum of $\mathbf{1 7}$ showing the hydride resonance signal at $-9.28 \mathrm{ppm}$. Inset: ${ }^{31} \mathrm{P}\left\{{ }^{1} \mathrm{H}\right\}-\mathrm{NMR}$ of $\mathbf{1 7}$.

\subsection{Intermolecular $\mathrm{C}-\mathrm{C}$ coupling of $\left[\mathrm{Re}\left(\mathrm{NCH}_{2}\right) \mathrm{Cl}\left(\mathrm{PNP}^{\mathrm{tBu}}\right)\right]^{+}\left(18^{+}\right)$}

The first report of functionalization of $\mathbf{X X I}$ described methylation at the nitride with MeOTf yielding $\left[\mathrm{Re}(\mathrm{NMe}) \mathrm{Cl}\left(\mathrm{PNP}^{t \mathrm{Bu}}\right)\right]^{\text {OTf }}\left(\mathbf{1 6}^{\mathrm{OTf}}\right) \cdot{ }^{[94]}$ In analogy to later reported nitrile formation after ethylation by sequential deprotonation and oxidation, ${ }^{[153]}$ the release of cyanide from $16^{\mathrm{OTf}}$ could be imagined. However, it was found that after deprotonation to $\left[\operatorname{Re}\left(\mathrm{NCH}_{2}\right) \mathrm{Cl}\right.$ $\left.\left(\mathrm{PNP}^{t \mathrm{Bu}}\right)\right](\mathbf{1 8})$, oxidation with $\mathrm{AgOTf}$ results in various compounds with the main product most likely being the dicationic product from intermolecular $\mathrm{C}-\mathrm{C}$ coupling of of the ketimido $\mathrm{CH}_{2}$ moieties, $\left[\left(\mathrm{N}, \mathrm{N}-\mathrm{C}_{2} \mathrm{H}_{4} \mathrm{~N}_{2}\right)\left\{\mathrm{ReCl}\left(\mathrm{PNP}^{t \mathrm{Bu}}\right)\right\}_{2}\right]^{(\mathrm{OTf})_{2}}\left(\mathbf{1 9}^{\left.(\mathrm{OTf})_{2}\right)}\right)^{[180]}$ The appearance of several byproducts was attributed to chloride abstraction from 18 rather than mere oxidation. Thus, a much cleaner reaction was obtained after exchanging the chloride ligand in $\mathbf{1 8}$ with a methyl group to form $\mathbf{1 8}^{\mathrm{Me}}$ and subsequent oxidation (see Scheme 2.2). Via this route single crystals suitable for molecular structure determination were obtained, confirming the

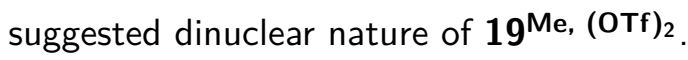




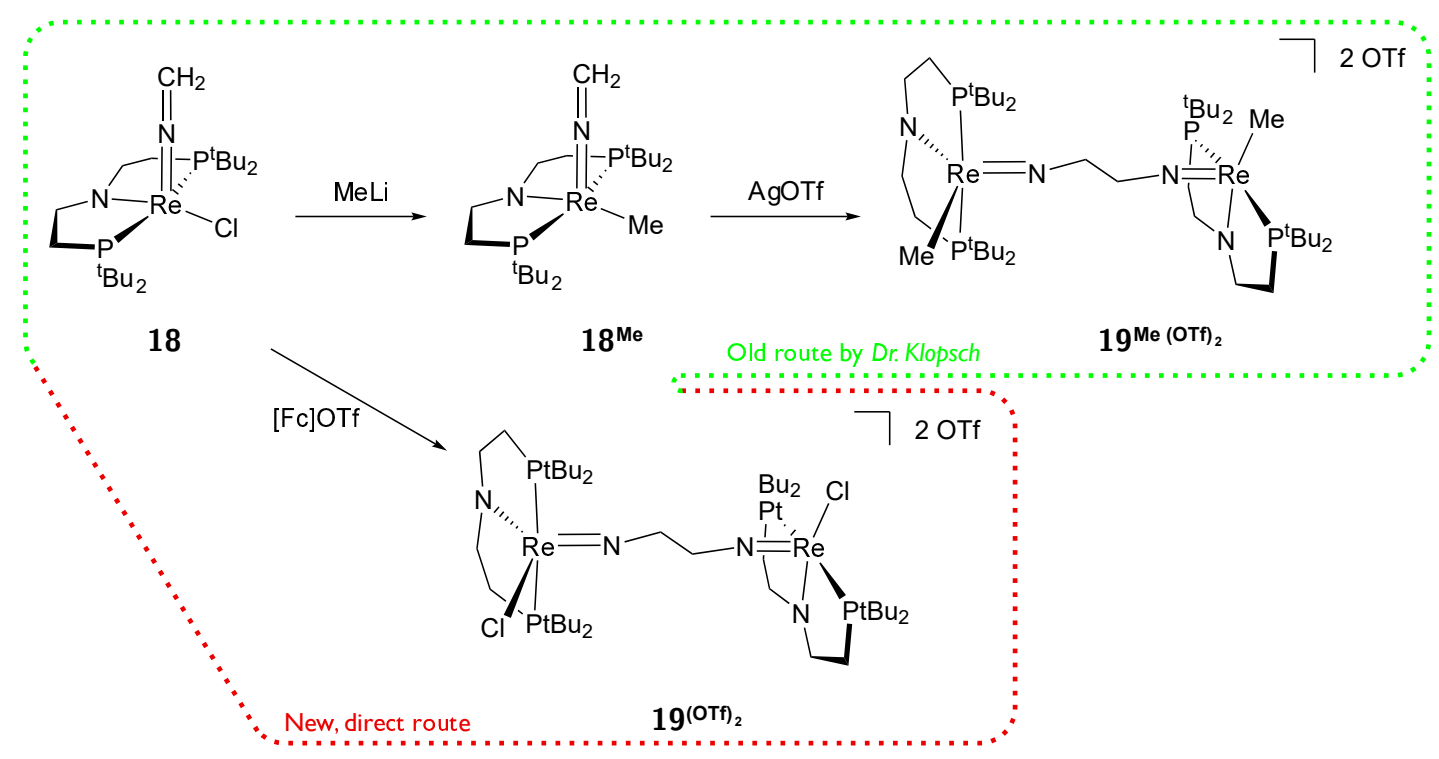

Scheme 2.2. Previously reported oxidative intermolecular coupling of ketimide complexes $\mathbf{1 8}^{\mathrm{Me}}$ with $\mathrm{AgOTf}$ and newly developed route directly from 18 with $\left[\mathrm{Fe}(\mathrm{Cp})_{2}\right]^{\mathrm{OTf}}$.

This radical coupling provides an interesting entry into new, $\mathrm{N}_{2}$ derived organic molecules. To omit unnecessary steps, the initially explored reaction starting from chloro complex $\mathbf{1 8}$ was reinvestigated. Previous work suggested that the precipitation of $\mathrm{AgCl}$ likeley is a problem, therefore alternative pathways to the above described procedure were considered. Unfortunately, the most appealing approach, i.e. direct $\mathrm{H}$ atom abstraction from $\mathbf{1 6}^{+}$rather than stepwise deprotonation and oxidation, did not give the wanted result (16 stayed fully intact upon mixing with equimolar amounts of TTBP, even after gentle heating). Therefore, the use of oxidants other than silver salts was tried. CV data of $\mathbf{1 8}$ show a reversible oxidation wave at $-0.50 \mathrm{~V}$ vs. $\mathrm{Fc}^{+} / \mathrm{Fc}_{\mathrm{c}}{ }^{[180]}$ thus rendering ferrocenium salts suitable oxidants with much reduced tendency for salt exchange.

Oxidation with $\left[\mathrm{Fe}(\mathrm{Cp})_{2}\right] \mathrm{PF}_{6}$ in DCM showed already cleaner conversion to one major species which exhibits $C_{s}$ symmetry on the NMR time scale and displays a singlet in ${ }^{31} \mathrm{P}\left\{{ }^{1} \mathrm{H}\right\}-\mathrm{NMR}$ at $\delta_{31} \mathrm{P}=90.2 \mathrm{ppm}$ as well as in ${ }^{1} \mathrm{H}-\mathrm{NMR}$ spectroscopy for the $\mathrm{N}-\mathrm{CH}_{2}$ group $\left(\delta_{1_{\mathrm{H}}}=3.31 \mathrm{ppm}\right.$, analog to $\left.19^{\mathrm{Me}}(\mathrm{OTf})_{2}\right)$. However, full conversion is not achieved and remarkably, $\mathbf{1 6}^{+}$is obtained as side product, hinting to either an $\mathrm{H}^{+}$source prior to oxidation or the presence of an $\mathrm{H}$ atom donor. Changing the anion from $\mathrm{PF}_{6}^{-}$to OTf and replacing $\mathrm{DCM}$ with $\mathrm{Na} / \mathrm{K}$ dried THF finally resulted in analytically pure coupling product $\left.\mathbf{1 9}^{(\mathrm{OTf})}\right)_{2}$ in $61 \%$ isolated yield (see Section 2.2.3). Single crystals suitable for X-ray diffraction were obtained from layering a concentrated toluene solution with hexamethyldisiloxane (HMDSO) (see Figure 2.3).

The two independent Re centers are found in a distorted square pyramidal coordination environnment $\left(\tau_{5}(\operatorname{Re} 1)=0.295\right.$ and $\left.\tau_{5}(\operatorname{Re} 2)=0.1475\right){ }^{[196]}$ The $\mathrm{Re}-\mathrm{N}_{\text {imide }}$ bonds are significantly shorter than in the parent ketimide complex $18(d(\operatorname{Re}-\mathrm{N})=1.723(2) \AA, 1.720(2) \AA$ vs. $1.8365(16) \AA$ in 18) while the corresponding $\mathrm{N}-\mathrm{C}$ bonds became much longer $(d(\mathrm{~N}-\mathrm{C})$ $=1.448(3) \AA, 1.441(3) \AA$ vs. $1.248(3) \AA$ in 18). Combined with a now bent $\mathrm{Re}-\mathrm{N}-\mathrm{C}$ moi- 


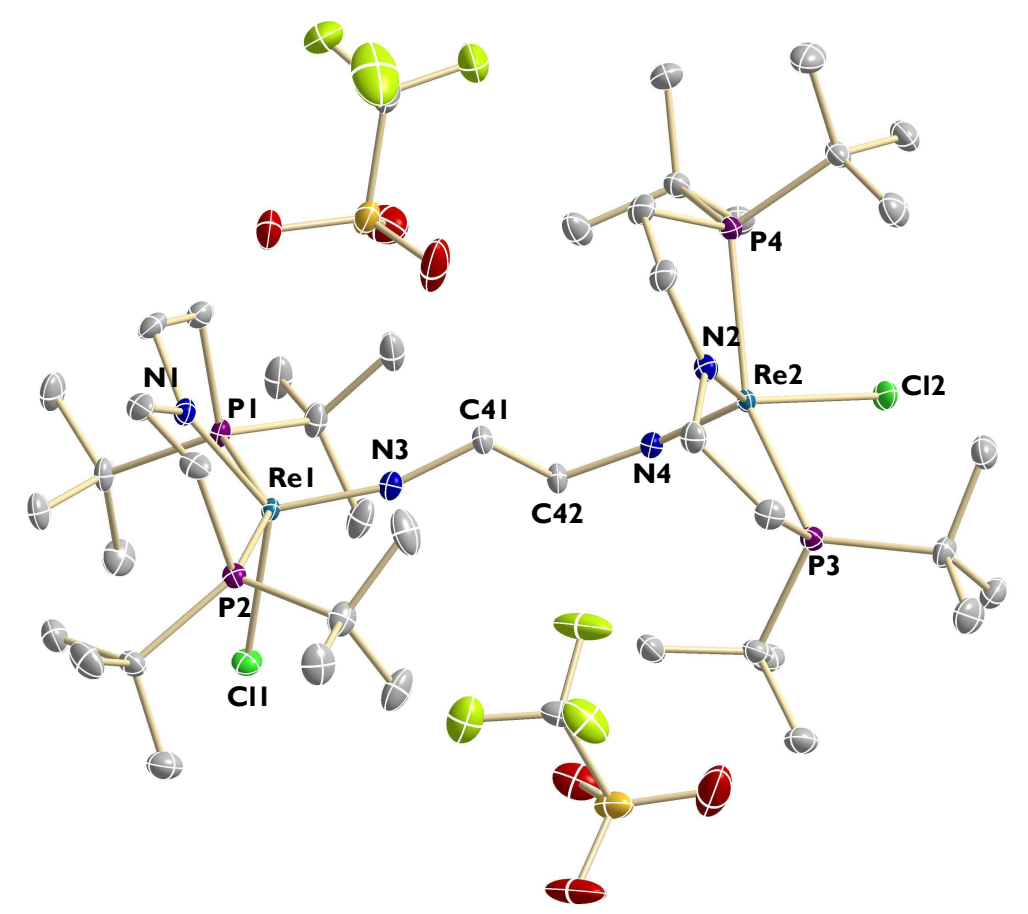

Fig. 2.3. Molecular structure of $\mathbf{1 9}^{(\mathrm{OTf})_{2}}$ obtained by single crystal X-ray diffraction measurements. $\mathrm{H}$-atoms are omitted for clarity. Anisotropic displacement parameters are set to $50 \%$ probability. Selected bond lengths $[\AA]$ and angles $\left[^{\circ}\right]$ : Re1-N1 1.942(2), Re1-N3 1.723(2), N3-C41 1.448(3), N1-Re1-N3 117.37(9), N1-Re1-Cl1 133.66(6), P1-Re1-P2 151.36(2), Re1-N3-C41 163.63(18), Re2-N2 1.9298(19), Re2-N4 1.720(2), N4-C42 1.441(3), N2-Re2N4 110.31(9), N2-Re2-Cl2 140.61(6), P3-Re2-P4 149.46(2), Re2-N4-C42 160.24(17).

ety $\left(\measuredangle(\operatorname{Re}-\mathrm{N}-\mathrm{C})=163.63(18)^{\circ}, 160.24(17)^{\circ}\right.$ vs. $172.56(16)^{\circ}$ in 18$)$, all these parameters are in agreement with the formation of an imido ligand.

Adding stochiometric amounts of water to $19^{(\mathrm{OTf})_{2}}$ did not result in a hydrolysis to the oxo complex $\left[\operatorname{Re}(\mathrm{O}) \mathrm{Cl}\left(\mathrm{PNP}^{t \mathrm{Bu}}\right)\right]^{+}\left(\mathbf{2 0}^{+}\right)$as confirmed by comparision with an authentic sample prepared by reaction of $\mathbf{X I X}$ with $\mathrm{NaBPh}_{4}$ and $\mathrm{Me}_{3} \mathrm{NO}$ (see Section 2.2.4). Instead, no reaction was observed, not even upon heating. On the other hand, reaction with base, especially with potassium bis(trimethylsilyl)amide (KHMDS), led to conversion. First, two new signals in ${ }^{31} \mathrm{P}\left\{{ }^{1} \mathrm{H}\right\}$-NMR were observed at $\delta^{31} \mathrm{P}=69.5 \mathrm{ppm}$ and $69.5 \mathrm{ppm}$, which were converted to a singlet at $84.2 \mathrm{ppm}$ upon addition of more equivalents of base. However, the exact stochiometry of this reaction as well as the identity of the product are unknown.

While a new route to $\mathbf{1 9}^{(\mathrm{OTf})_{2}}$ has been developed and the complex is now synthetically accessible, further investigations concerning its follow-up chemistry and potential release of nitrogen containing organic molecules are still pending. 


\subsection{Effect of the backbone oxidation to the $\mathrm{P}=\mathrm{N}=\mathrm{P}$ platform}

Part of this section has been published in: J. Becker, "Rhenium-vermittelte $\mathrm{N}_{2}$-Funktionalisierung", Bachelor thesis, Georg-August-Universität Göttingen, 2017.

\subsubsection{Synthesis and dinitrogen splitting}

The cycle for nitrile generation from $\mathrm{N}_{2}$, mediated by $\mathbf{X I X}$, requires a sequence of reductive (for $\mathrm{N}_{2}$ splitting) and oxidative (for $\mathrm{N}_{2}$ functionalization) steps. Thus, its realization can be envisioned by means of electrochemistry, where both conditions can be applied simultaneously without the redox agents reacting with each other. However, such an approach would require the $\{\operatorname{Re}(P N P)\}$ fragment to be rugged towards reductive and oxidative conditions. While no $\{P N P\}$ decomposition has been observed even with very harsh reductants such as $\mathrm{KC}_{8}$, previous experiments indicated ligand backbone oxidation to be a viable side reaction under certain conditions. Hence, the backbone oxidized complex $\left[\operatorname{ReCl}_{2}\left(\mathrm{P}=\mathrm{N}=\mathrm{P}^{t \mathrm{tBu}}\right)\right]$ (21) $\left(\mathrm{P}=\mathrm{N}=\mathrm{P}^{t \mathrm{Bu}}=\left\{\mathrm{N}(\mathrm{CHCHPtBu})_{2}\right\}^{-}\right)$was synthesized according to previous developed procedures ${ }^{[180]}$ as an attempt to harden $\mathbf{X I X}$ towards oxidative conditions, and its behavior in $\mathrm{N}_{2}$ activation and functionalization was investigated.

Stirring XIX with excess TTBP at $50{ }^{\circ} \mathrm{C}$ in benzene resulted in formation of 21 . A paramagnetic side product which most likely results from over-oxidation to $\operatorname{Re}(\mathrm{IV})$ center could be converted to the desired product by addition of small quantities of $\mathrm{Co}(\mathrm{Cp})_{2} . \mathbf{2 1}$ could be isolated in analytical purity after sublimation of the formed phenol and excess $\mathrm{Co}(\mathrm{Cp})_{2}$ in $63 \%$ isolated yield (see Scheme 2.3).

NMR spectroscopic investigation show that 21 exhibits a $C_{2 v}$ symmetry on the NMR timescale with a backbone signal pattern characteristic for the fully oxidized ligand. ${ }^{[195,199,200]}$ Especially the downfield shift of the $\mathrm{CH}$ group next to the amide as well as the peak separation $\Delta \delta>2 \mathrm{ppm}$ of the $\alpha$ - and $\beta$-protons are typical of vinylamines ${ }^{[201]}$ and characteristic

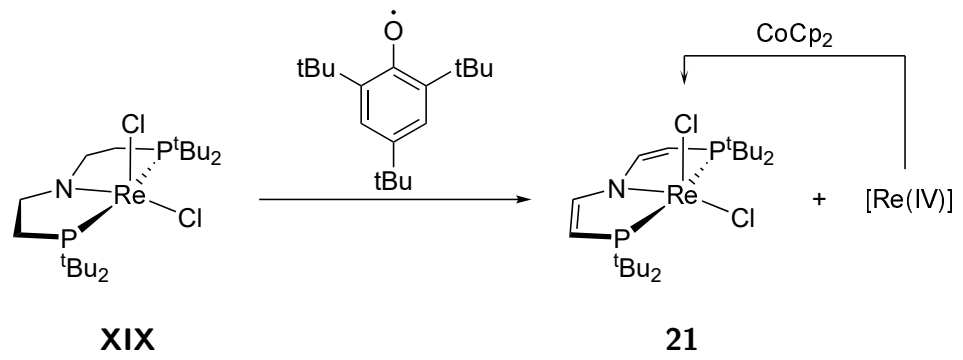

Scheme 2.3. Synthesis of $\left[\operatorname{ReCl}_{2}\left(P=N=P^{t B u}\right)\right]$ (21). 

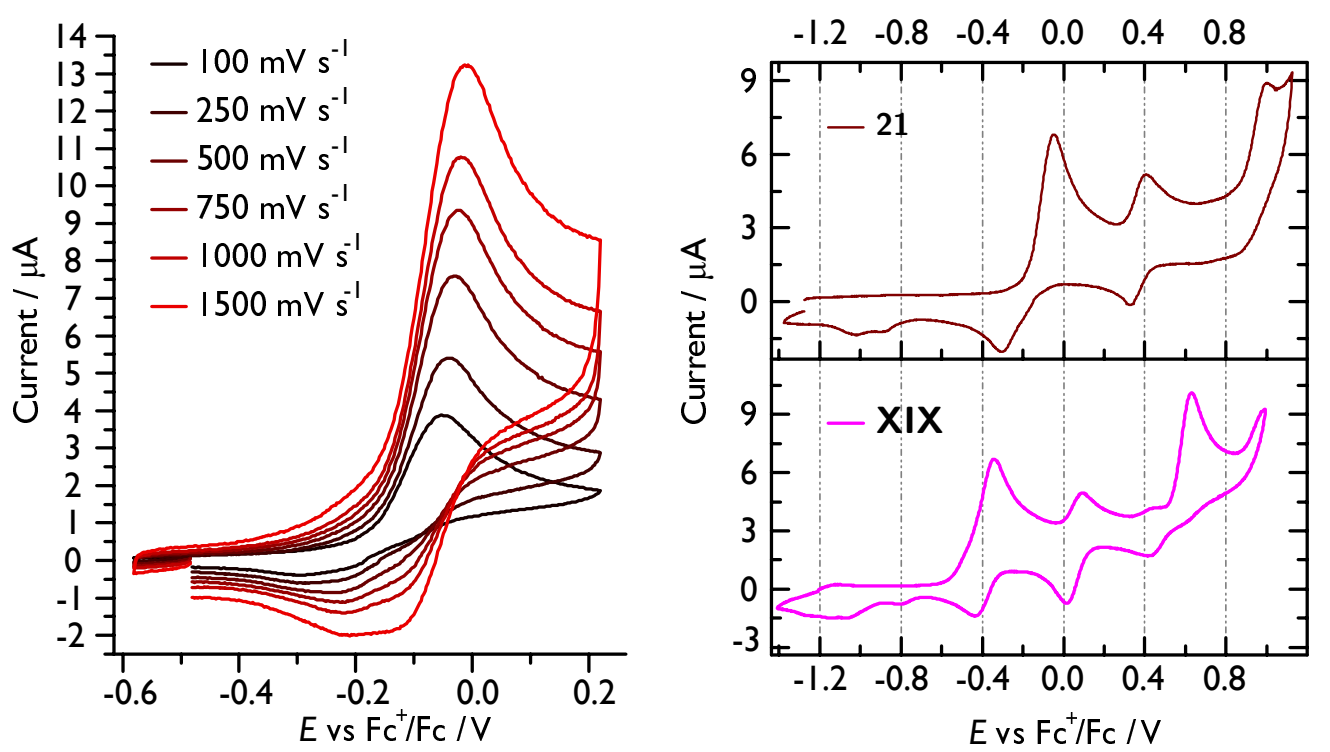

Fig. 2.4. Left: $\mathrm{CV}$ measurements of $\operatorname{Re}(\mathrm{IV} / \mathrm{III})$ oxidation of $\mathbf{2 1}$ at various scan rates. Right: Comparison of the anodic region of $\mathbf{X I X}$ (bottom) and $\mathbf{2 1}$ (top) at $100 \mathrm{mV} \mathrm{s}^{-1}$. All spectra are recorded in THF with $10^{-3} \mathrm{M}$ complex, $0.1 \mathrm{M} \mathrm{N}^{\mathrm{n}} \mathrm{Bu}_{4} \mathrm{PF}_{6}$, working electrode (WE): glassy carbon $(\mathrm{GC})$, reference electrode (RE): Ag-wire, counter electrode (CE): Pt-wire.

for this ligand. Notably, the ${ }^{31} \mathrm{P}\left\{{ }^{1} \mathrm{H}\right\}-N M R$ spectrum shows a tremendous high field shift of the resonance peak to $\delta_{31} \mathrm{P}=-275.6 \mathrm{ppm}$. Again, this shift is most likely a result TIP (see Section 3.1.2 for a detailed discussion). Unfortunately, no single crystals suitable for X-ray structure determination could be obtained.

Closer investigation of the CV data of compounds $\mathbf{X I X}$ and $\mathbf{2 1}$ reveals very similar traces (see Figure 2.4 right). The first oxidation event is shifted anodically by almost $300 \mathrm{mV}$ (XIX: $i_{p, a}=-0.34 \mathrm{~V}$ vs. $\mathrm{Fc}^{+} / \mathrm{Fc} ; 21: i_{p, a}=-0.05 \mathrm{~V}$; both at $100 \mathrm{mV} \mathrm{s}^{-1}$ ), in line with the reduced amide donor strength. Also the other two oxidation events are shifted in the same direction. However, while these results suggest increased stability of the complex towards oxidative conditions, it has to be noted that for $\mathbf{X I X}$, the first oxidation wave is fully reversible, as long as the second event is not scanned as well. In contrast to this, the first oxidation event of $\mathbf{2 1}$ is irreversible even at higher scan rates, at least in the absence of an external chloride source (see Figure 2.4, left). So even though the potential of the first oxidation is indeed shifted anodically, in terms of chemical stability $21^{+}$must be considered less stable than $\mathbf{X I X ^ { + }}$, at least under the conditions of the CV experiments.

Nevertheless, investigations concerning the $\mathrm{N}_{2}$ cleavage and functionalization capabilities of 21 were investigated to see if the modified ligand backbone has a significant influence in this field. Chemical reduction with $\mathrm{Co}\left(\mathrm{Cp}^{*}\right)_{2}$ under $\mathrm{N}_{2}$ atmosphere leads to formation of a mixture of products, as indicated by ${ }^{31} \mathrm{P}\left\{{ }^{1} \mathrm{H}\right\}$ and ${ }^{1} \mathrm{H}-\mathrm{NMR}$ spectroscopy. In this mixture, the $\operatorname{Re}(\mathrm{v})$ nitrido species $\left[\operatorname{Re}(\mathrm{N}) \mathrm{Cl}\left(\mathrm{P}=\mathrm{N}=\mathrm{P}^{t \mathrm{Bu}}\right)\right]$ (22) could be identified in approx. $60 \%$ spectroscopic yield, based on comparison with an authentic sample prepared in analytical purity by the reaction of $\mathbf{2 1}$ with 5 eq $\left(\mathrm{Me}_{3} \mathrm{Si}\right) \mathrm{N}_{3}$ in $72 \%$ yield (see Figure 2.5 a). Furthermore, LIFDI mass spectra of the reaction mixture confirmed formation of nitrido complex 22. 22 
resonates at $\delta_{31} \mathrm{P}=71.8 \mathrm{ppm}$ in the ${ }^{31} \mathrm{P}\left\{{ }^{1} \mathrm{H}\right\}$-NMR spectrum and exhibits $C_{S}$ symmetry in the ${ }^{1} \mathrm{H}$ NMR.

Single crystals suitable for $\mathrm{X}$-ray diffraction measurements were obtained (see Figure $2.5 \mathrm{c}$ ). The $\operatorname{Re}(\mathrm{v})$ center is coordinated in a slightly distorted square-pyramidal geometry with the nitrido ligand in the apical position $\left(\tau_{5}=0.15\right) .{ }^{[196]}$ The $\mathrm{Re} \equiv \mathrm{N}$ bond length $(d(\mathrm{Re}-\mathrm{N} 2)=$ $1.647(18) \AA$ ) lies in a typical range of a metal-nitrogen triple bonds and is in very good agreement with the DFT-optimized structure of parent $\left[\operatorname{Re}(\mathrm{N}) \mathrm{Cl}\left(\mathrm{PNP}^{t \mathrm{Bu}}\right)\right](\mathbf{X X I})\left(d(\operatorname{Re}-\mathrm{N})_{\mathrm{DFT}}\right.$ $=1.65 \AA$; unfortunately no $\mathrm{X}$-ray structure of that complex is available). Additionally, the flat ligand backbone and short $\mathrm{C}-\mathrm{C}$ distances $(d(\mathrm{C} 1-\mathrm{C} 2)=1.35(2) \AA)$ show the vinyl moiety to be still intact.

While the occurance of dinitrogen splitting from $\mathbf{2 1}$ is not too surprising, given its similarity with $\mathbf{X I X}$, it still is only the second example ever reported of a rhenium complex capable of this reaction.

The observed anodic shift of redox events in $\mathbf{2 1}$ attributed to the less donating ligand motivated an investigation of the electrochemical properties of 22 to see if this effect also makes the $\operatorname{Re}(\mathrm{V} / \mathrm{IV})$ redox couple accessible (see Figure 2.6). CV measurements revealed a reversible $\mathrm{Re}(\mathrm{V} / \mathrm{VI})$ oxidation at $E_{1 / 2}=+0.21 \mathrm{~V}$ vs. $\mathrm{Fc} / \mathrm{Fc}^{+}$which is significantly anodically

a)

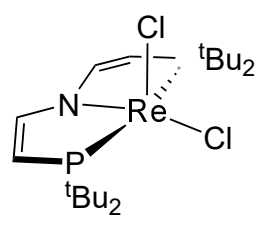

21

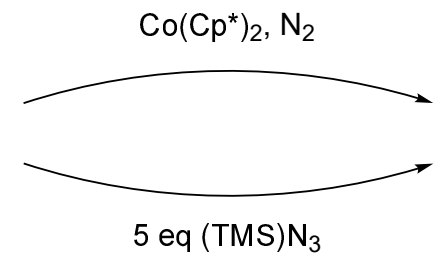

5 eq $(\mathrm{TMS}) \mathrm{N}_{3}$

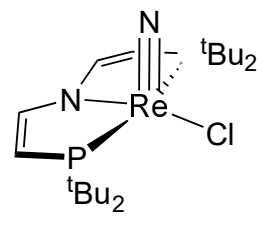

22

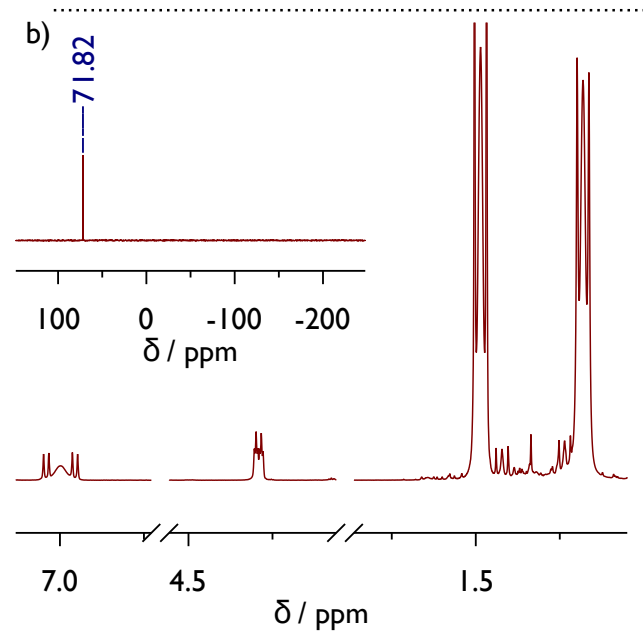

c)

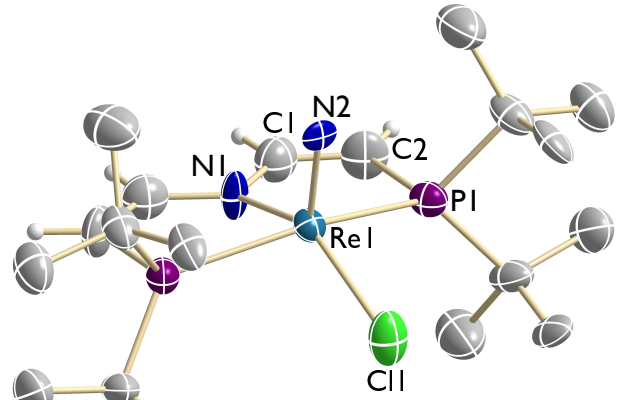

Fig. 2.5. a) Synthesis scheme for 22 via $\mathrm{N}_{2}$ splitting or TMS azide as nitrogen source. b) ${ }^{1} \mathrm{H}-\mathrm{NMR}$ spectrum of 22 (inset: ${ }^{31} \mathrm{P}\left\{{ }^{1} \mathrm{H}\right\}-N M R$ spectrum). c) Molecular structure of 22 obtained by single crystal $\mathrm{X}$-ray diffraction measurements. Selected $\mathrm{H}$ atoms are omitted for clarity. Anisotropic displacement parameters are set to $50 \%$ probability. Selected bond lengths $[\AA]$ and angles [ ${ }^{\circ}$ : Re1-N1 2.106(13), Re1-N2 1.647(18), Re1-Cl1 2.395(7), C1-C2 1.35(2), N1-Re1-N2 109.2(5), P1-Re1-P1\# 155.11(13), N1-Re1-Cl1 145.9(2). 


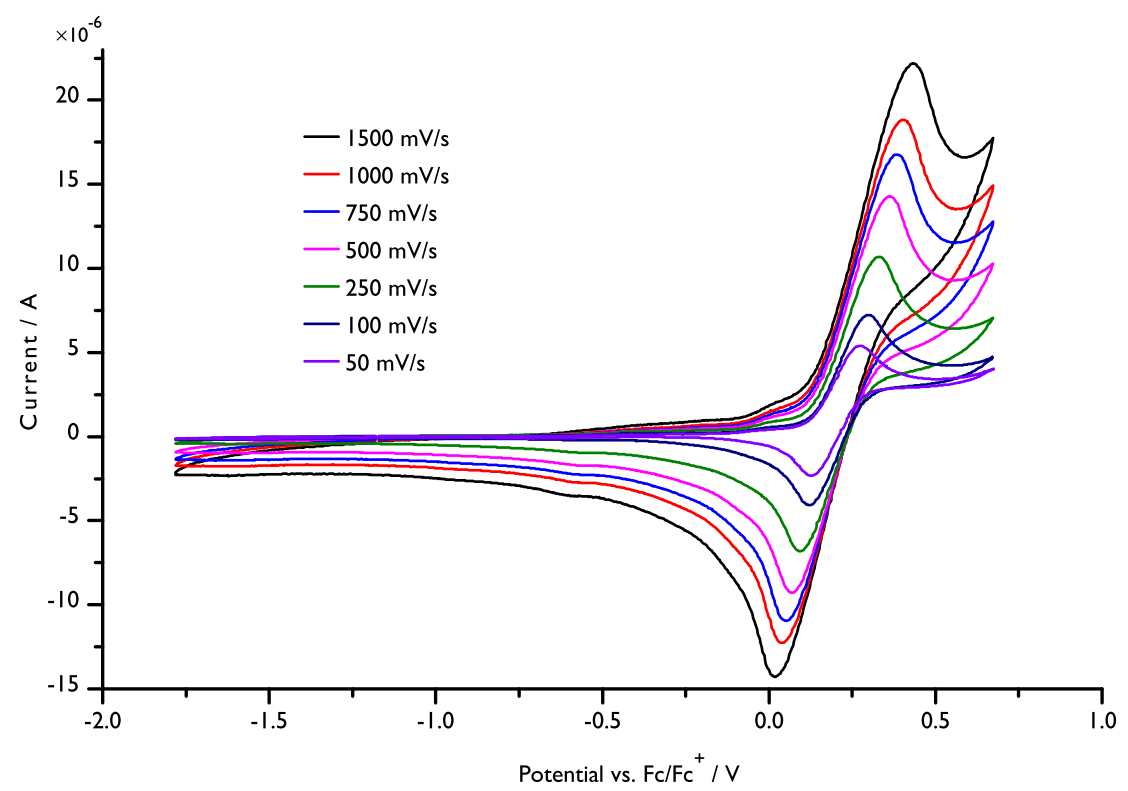

Fig. 2.6. $\mathrm{CV}$ measurements of 22 in THF at different scan rates $\left(10^{-3} \mathrm{M} 22,0.1 \mathrm{M} \mathrm{N}^{\mathrm{n}} \mathrm{Bu}_{4} \mathrm{PF}_{6}, W E\right.$ : GC, RE: Ag-wire, CE: Pt-wire).

shifted with regard to the parent nitride $\left(E_{1 / 2}(\mathbf{X X I})=-0.09 \mathrm{~V}\right){ }^{[180]}$ Unfortunately, no reduction event occurs until potentials as negative as $E_{p, c}=-3.3 \mathrm{~V}$. Thus, the reduced $\pi$-donation of the ligand does not provide and entry into reductive nitride functionalization.

\subsubsection{Nitride functionalization}

In order to increase the electrophilic character of $\mathbf{2 2}$, its reactivity towards acids was investigated. Introducing a cationic charge should facilitate reduction of the nitrido complex and could therefore open up a route to ammonia synthesis following well known stepwise protonation and reduction schemes. Reacting nitride complex 22 with 1 eq of HOTf or $\mathrm{HBAr}_{24}^{\mathrm{F}}\left(\mathrm{HBAr}_{24}^{\mathrm{F}}=\mathrm{H}\left(\mathrm{OEt}_{2}\right)_{2}\left[\mathrm{~B}\left\{{ }_{4,5}-\left(\mathrm{CF}_{3}\right)_{2} \mathrm{C}_{6} \mathrm{H}_{3}\right\}_{4}\right]\right)$ leads to clean and selective formation of the monoprotonated complex $\left[\operatorname{Re}(\mathrm{N}) \mathrm{Cl}\left(\mathrm{P}^{\mathrm{H}}=\mathrm{N}=\mathrm{P}^{t \mathrm{Bu}}\right)\right]^{\mathrm{X}}\left(23^{\mathrm{X}}\right)$ with $\mathbf{X}$ being the resulting anions from the acids $\left(\mathrm{P}^{\mathrm{H}}=\mathrm{N}=\mathrm{P}^{t \mathrm{Bu}}=\left\{{ }^{-} \mathrm{N}\left(\mathrm{CHCH}_{2} \mathrm{PtBu} 2\right)\left(\mathrm{CHCHPtBu} \mathrm{Bu}_{2}\right)\right\}\right)$. The protonation of one vinylidene moiety of the pincer breaks the symmetry of the complex, leading to the formation of two doublets in ${ }^{31} \mathrm{P}\left\{{ }^{1} \mathrm{H}\right\}-\mathrm{NMR}$ at $\delta^{31} \mathrm{P}=70.0$ and $73.0 \mathrm{ppm}$, respectively, with a mutual coupling constant of ${ }^{2} J_{\mathrm{PP}}=148.1 \mathrm{~Hz}$ (see Figure 2.7). ${ }^{1} \mathrm{H}$ NMR shows five resonances of $\mathrm{CH}$ protons between $\delta_{{ }_{1}}=9.5$ and $3.5 \mathrm{ppm}$, with a large coupling constant of ${ }^{2} J_{\mathrm{HH}}=21.3 \mathrm{~Hz}$ between the protons of the $\mathrm{CH}_{2}$ group. In $\mathrm{CV}$ measurements, 23 again exhibits a reversible oxidation wave at $E_{1 / 2}=+0.24 \mathrm{~V}$ vs. $\mathrm{Fc} / \mathrm{Fc}^{+}$, suggesting that the oxidation is metal centered and thus not affected by the ligand centered protonation. At potentials below $-2.0 \mathrm{~V}$, a new reduction feature appears. However, the slope of the wave is rather shallow and resembles the appearance of proton reduction waves of organic 
a)

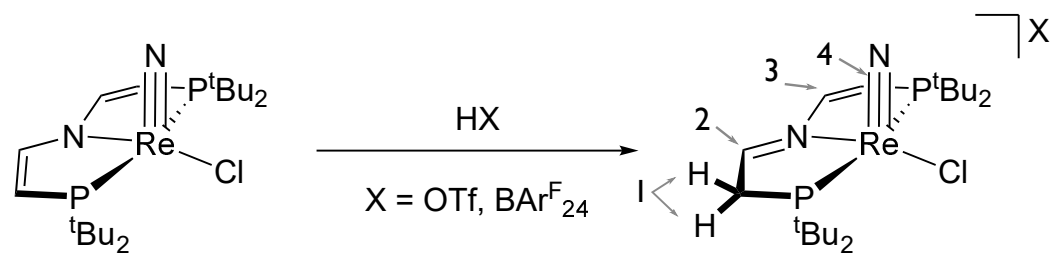

22

$23^{x}$
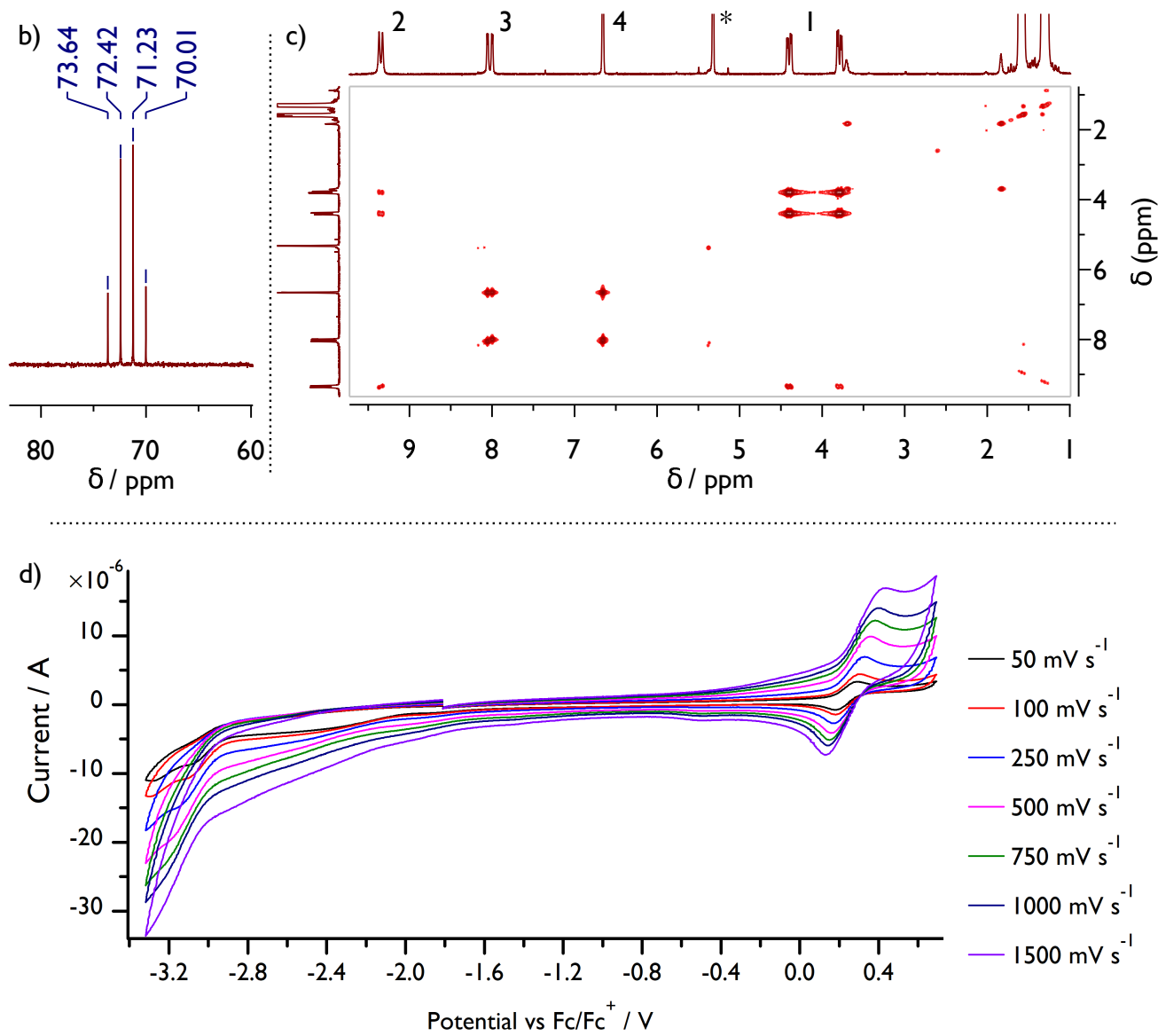

Fig. 2.7. a) Synthesis scheme for $23^{\text {OTf }}$ via protonation of 22 . b) ${ }^{31} \mathrm{P}\left\{{ }^{1} \mathrm{H}\right\}$ NMR spectrum of $23^{\text {OTf }}$. c) ${ }^{1} \mathrm{H}-{ }^{1} \mathrm{H}$ COSY showing the coupling between the five backbone protons and allow for full assignment (in combination with other 2D-techniques). Solvent signals are marked with an asterisk. d) CV measurements of $23^{\mathrm{OTf}}$ in THF at different scan rates $\left(10^{-3} \mathrm{M} \mathbf{2 3}^{\mathrm{OTf}}\right.$, $0.1 \mathrm{M} \mathrm{N}^{\mathrm{n}} \mathrm{Bu}_{4} \mathrm{PF}_{6}, \mathrm{WE}$ : GC, RE: Ag-wire, CE: Pt-wire).

acids. Therefore, protonation to $23^{X}$ did not yield the desired effects and the approach was abandoned.

As can be suggested from the electrochemical investigations, the very negative potential for reduction of $\mathbf{2 2}$ is reflected in its inertness towards electron donating reagents. All attempted reactions with nucleophiles like $\mathrm{Me}_{3} \mathrm{NO}, \mathrm{PMe}_{3}$, alkenes, as well as $\mathrm{CO}$ gave no detectable conversion, not even at elevated temperatures. Again, this behavior is completely analogous to parent nitride $\mathbf{X X I}$.

Following this similarity, $\mathrm{C}-\mathrm{N}$ bond formation on $\mathbf{2 2}$ was achieved by methylation with 
strongly electrophilic MeOTf. Stirring in chlorobenzene at $60^{\circ} \mathrm{C}$ gave analytically pure $\left[\operatorname{Re}(\mathrm{NMe}) \mathrm{Cl}\left(\mathrm{P}=\mathrm{N}=\mathrm{P}^{t \mathrm{Bu}}\right)\right]^{\mathrm{OTf}}\left(\mathbf{2 4}{ }^{\mathrm{OTf}}\right)$ in $80.5 \%$ yield. 24 retains a $C_{S}$ symmetry on the NMR timescale and resonates at $\delta_{31} \mathrm{P}=88.8 \mathrm{ppm}$ in ${ }^{31} \mathrm{P}\left\{{ }^{1} \mathrm{H}\right\}$-NMR spectroscopy. $\quad \mathrm{N}$ Methylation was confirmed by ${ }^{1} \mathrm{H}-{ }^{1} \mathrm{H}$ NOESY where a cross peak appeared solely between the methyl group (singlet at $\delta_{1_{\mathrm{H}}}=2.70 \mathrm{ppm}$ ) and one tert-butyl group, but not with the backbone protons (see Figure $2.8 \mathrm{c}$ ). Unfortunately, no single crystals suitable for an X-ray structure determination could be obtained, despite several attempts.

Deprotonation of $\mathbf{2 4}^{\mathrm{OTf}}$ with $\mathrm{NEt}_{3}$ gave spectroscopically almost pure ketimido complex $\left[\operatorname{Re}\left(\mathrm{N}=\mathrm{CH}_{2}\right) \mathrm{Cl}\left(\mathrm{P}=\mathrm{N}=\mathrm{P}^{t \mathrm{Bu}}\right)\right](25)$ in approximately $50 \%$ yield after workup. Attempts with stronger bases like $\mathrm{KO}^{t} \mathrm{Bu}$ or $\mathrm{KHMDS}$ gave either decomposition in the former, or formation of non-separable side products in the latter case. 25 exhibits a single resonance in ${ }^{31} \mathrm{P}\left\{{ }^{1} \mathrm{H}\right\}$ NMR and displays $C_{S}$ symmetry in the ${ }^{1} \mathrm{H}-\mathrm{NMR}$ spectrum. The two ketimido protons could be identified by ${ }^{1} \mathrm{H}_{-}{ }^{13} \mathrm{C}$ heteronuclear single quantum coherence (HSQC) spectroscopy as two broad singlets at $\delta_{{ }_{\mathrm{H}}}=1.74$ and $1.63 \mathrm{ppm}$, indicating the rotation around the $\mathrm{Re}=\mathrm{N}=\mathrm{C}$ moiety to be hindered at RT (see Figure 2.8).

Interestingly, in both complexes $24^{\mathrm{OTf}}$ and $\mathbf{2 5}$, the more highfield shifted ${ }^{1} \mathrm{H}$ NMR signal belonging to a tert-butyl group is significantly broadened (see Figure $2.8 b\left(\delta_{1_{\mathrm{H}}}=1.29 \mathrm{ppm}\right)$ for $24^{\mathrm{OTf}}$ and $d\left(\delta_{1_{\mathrm{H}}}=1.25 \mathrm{ppm}\right)$ for 25$)$. VT-NMR spectra indicate this effect to be due to dynamic behavior in solution, however, the nature of these dynamics is not yet understood and will need further investigation. Given the so far observed similarity between the alkyl and vinyl based pincer systems, no further investigation of $\mathbf{2 5}$ or its follow-up reactivity was conducted. Nitrile release as observed after alkylation of $\mathbf{X X I}$ can be considered rather likely and would only be of interest if this reaction could be achieved electrochemically (following the previously proposed scheme of fully electrochemially driven dinitrogen splitting and functionalization).

Apart from the described $\mathrm{C}-\mathrm{N}$ bond formation, the well defined and apparently reversible oxidation of 22 to a $\operatorname{Re}(\mathrm{VI})$ stage rendered redox chemistry a promising approach to further functionalize the $\mathrm{N}_{2}$ derived nitride. Oxidation could in theory alter the nitride centered reactivity from nucleophilic (but only towards very harsh reagents) to become rather electrophilic. Initial DFT calculations of neutral $\mathbf{2 2}$ and its monooxidized analogue $\mathbf{2 6}^{+}$revealed very little change in structural parameters (root-mean-square deviation (of atomic positions) (RMSD) $=0.042$, also: see Figure $2.9 \mathrm{c}) .{ }^{1}$ Furthermore, an NBO analysis indicated a slightly decreased population at the nitride $\left(22: 7.40 \mathrm{e}^{-}, \mathbf{2 6}^{+}: 7.32 \mathrm{e}^{-}\right)$and a reduced Wiberg bond order (22: $2.52, \mathbf{2 6}^{+}:$: 2.47). These results encouraged the actual synthesis of the monooxidized nitride complex.

Mixing of 22 with 1 eq $\mathrm{AgSbF}_{6}$ in THF results in immediate color change to deep red. Full conversion of the starting material, clearly evident by ${ }^{31} \mathrm{P}\left\{{ }^{1} \mathrm{H}\right\}$ NMR spectroscopy, is accompanied by the formation of a very broad, paramagnetic peak in ${ }^{1} \mathrm{H}$ NMR spectrum between $\delta_{1_{\mathrm{H}}}=7-1 \mathrm{ppm}$, indicating the formation of $S=1 / 2$ compound $\left[\operatorname{Re}(\mathrm{N}) \mathrm{Cl}\left(\mathrm{P}=\mathrm{N}=\mathrm{P}^{t B u}\right)\right]^{\mathrm{SbF}_{6}}$

\footnotetext{
${ }^{1}$ The RMSD value was calculated by using the CHEMCRAFT software package.
} 
a)

22

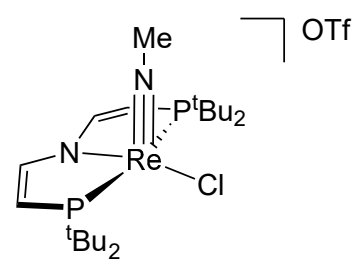

$24^{\text {OTf }}$

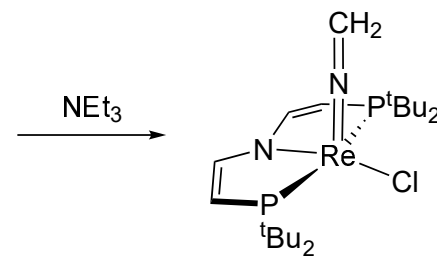

25

b)
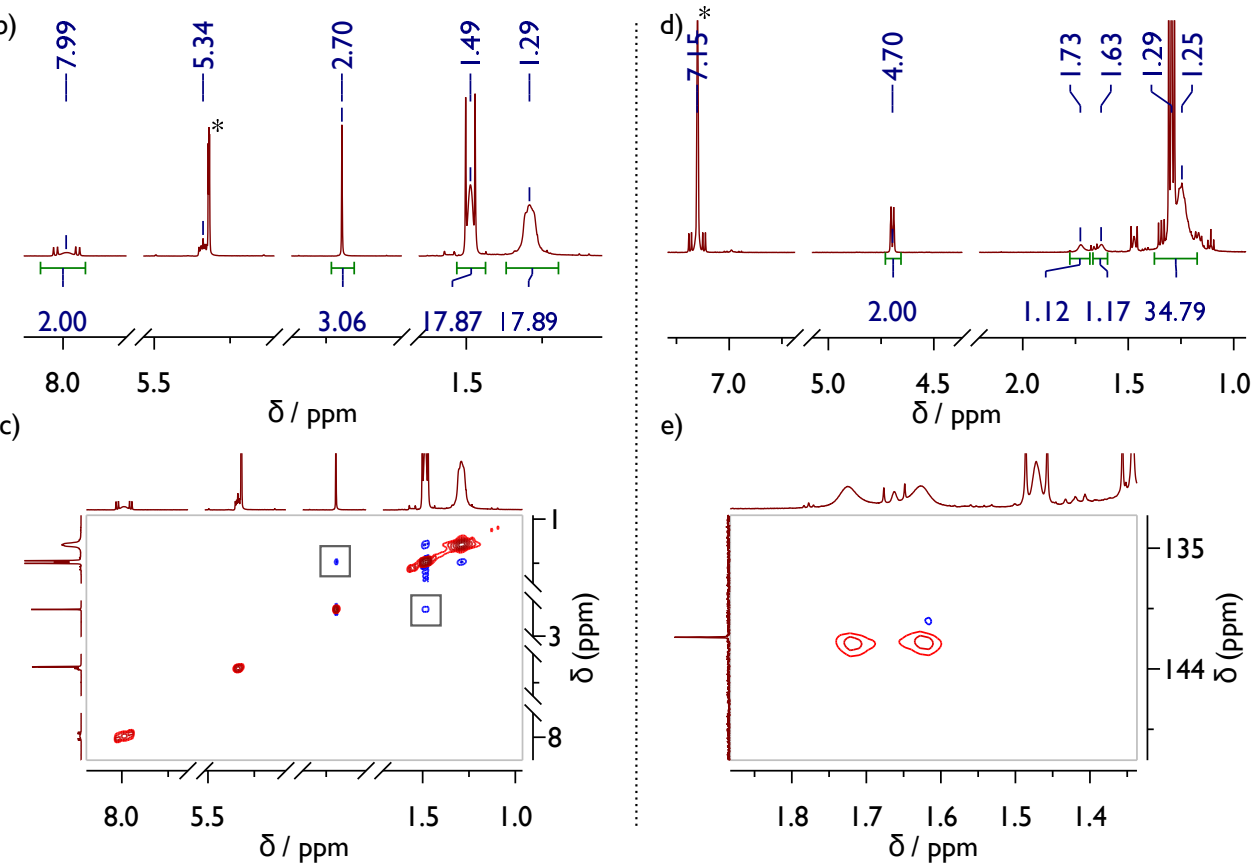

Fig. 2.8. a) Synthesis scheme for alkylation of 22 with MeOTf to $24^{\text {OTf }}$ and subsequent deprotonation to 25 . b) ${ }^{1} \mathrm{H}-\mathrm{NMR}$ spectrum of $24^{\mathrm{OTf}}$ in $\mathrm{CD}_{2} \mathrm{Cl}_{2}$ (solvent signal - marked with an asterisk - is overlapping with one backbone signal). c) ${ }^{1} \mathrm{H}-{ }^{1} \mathrm{H}$ NOESY of $24^{\text {OTf }}$ showing cross-peaks between the methyl group with only one tert-butyl group. d) ${ }^{1} \mathrm{H}$ NMR spectrum of 25 (solvent signal - marked with an asterisk - is overlapping with one backbone signal). For readability, spectral regions without compound signals are not shown. $d$ ) ${ }^{1} \mathrm{H}_{-}{ }^{13} \mathrm{C}$ HSQC spectrum of 25 identifying the two broadened protons of the $\mathrm{CH}_{2}$ group.

$\left(26^{\mathrm{SbF}_{6}}\right)$. Unfortunately, minor amounts of monoprotonated nitride $\mathbf{2 3}^{+}$are formed as a side-product which could not be separated from the product due to very similar solubility behavior. This probably indicates impurities in the oxidant which thus requires further purification before usage. While further analytics are still pending and the protic impurity $\mathbf{2 3}^{+}$ prevented detailed studies of the reactivity of $\mathbf{2 6}^{\mathbf{S b F}_{\mathbf{6}}}$ towards nucleophiles, the formation of the target molecule is rather likely and the stability of the solution was confirmed by ${ }^{1} \mathrm{H} N M R$ spectroscopy over several days, substantiating the previous findings from CV measurements. Hence, future investigation of this pathway could be of interest for modifying and extending the range of possible functionalization schemes for dinitrogen derived rhenium nitrides.

Overall, it could be shown, that the vinyl based pincer ligand exhibits basically identical reactivity compared with its parent alkyl counterpart. Reductive $\mathrm{N}_{2}$ activation and splitting with chemical reductants seems to proceed with comparable efficiency for PNP based XIX 

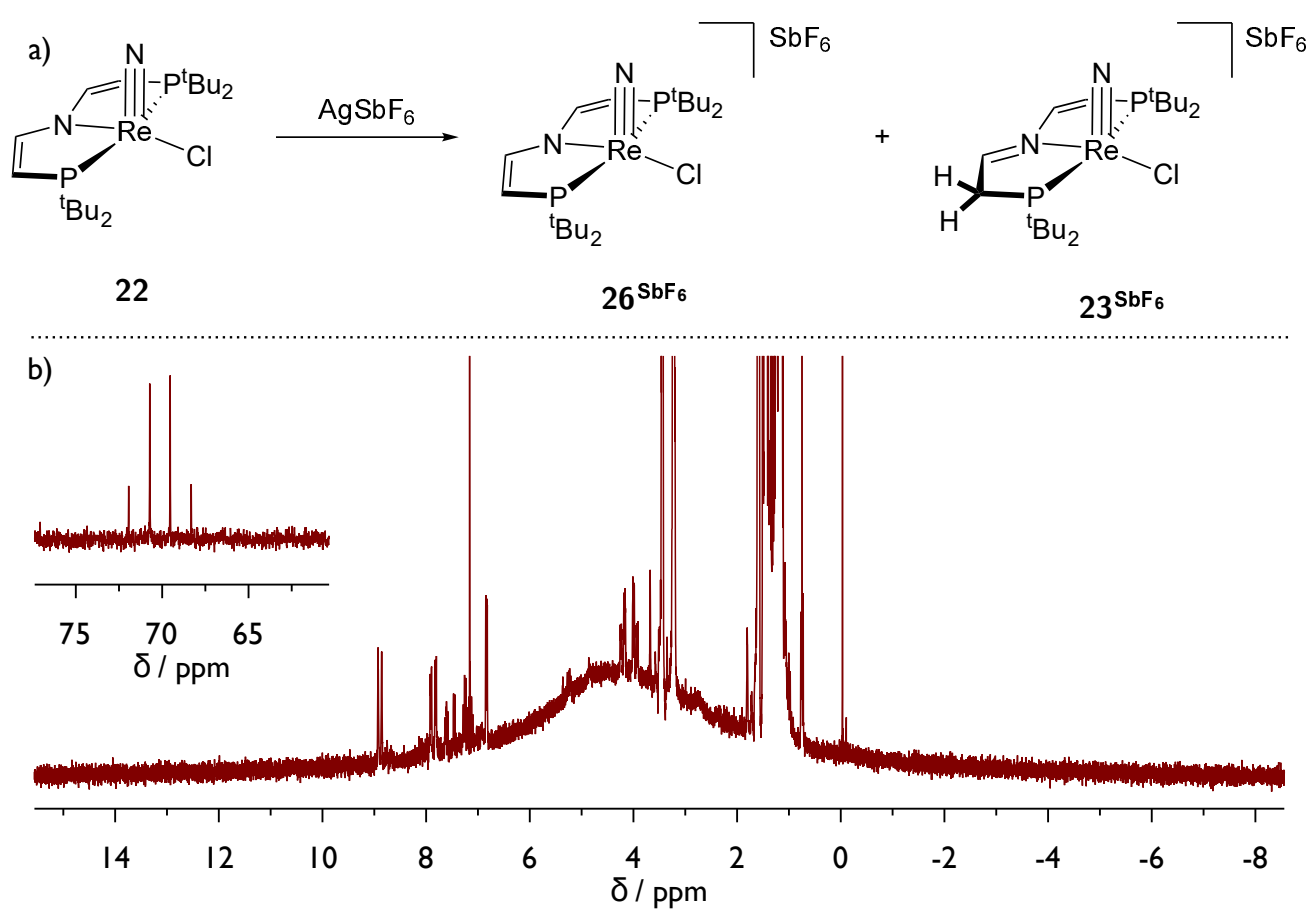

。
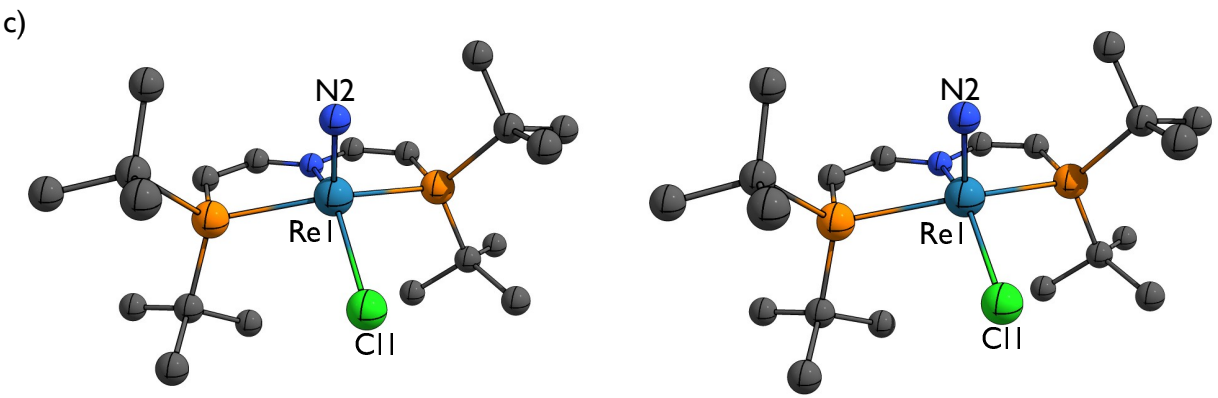

Fig. 2.9. a) Synthesis of $26^{\mathrm{SbF}_{6}}$ with minor formation of $23^{\mathrm{SbF}_{6}}$. $\quad b^{1} \mathrm{H}$ NMR spectrum of $2 \mathbf{6}^{\mathrm{SbF}_{6}}$. Inset: ${ }^{31} \mathrm{P}\left\{{ }^{1} \mathrm{H}\right\}$ NMR showing the signal of ${ }^{23} \mathrm{SbF}_{6}$. c) DFT (RI-PBE|D3BJ|def2-SVP) optimized gas phase structures of 22 (left) and $26^{+}$(right) with the following structural parameters: 22: Re1-N2 1.664 $\AA, \operatorname{Re} 1-\mathrm{Cl} 12.434 \AA$, $\tau_{5}$ 0.15. 26: Re1-N2 1.661 $\mathrm{A}, \operatorname{Re} 1-\mathrm{Cl} 1$ $2.321 \AA, \tau_{5} 0.06$.

and $\mathrm{P}=\mathrm{N}=\mathrm{P}$ based 21, although the former was shown to be more efficient with alkali metals, whereas the latter works better with $\mathrm{Co}\left(\mathrm{Cp}^{*}\right)_{2}$. The hypothesis of increased electrophilic reactivity of the resulting nitride 22 due to reduced electron donations of the pincer amide could not be confirmed. Rather, the only successful functionalization at the nitride was achieved by using highly reactive MeOTf, just as it was known for parent $\mathbf{X X \mathbf { I }}$, while protonation occured at the pincer backbone. Subsequent deprotonation of the methylated complex to form the ketimido complex 25 provided access to a platform, from which electrochemical oxidative nitrile release might be explored. However, investigation of such reactions will need to be part of a following project. Finally, first experimental evidence on a fully reversible chemical oxidation of 22 to its monocationic $\operatorname{Re}(\mathrm{VI})$ stage were gathered, pointing to stable and potentially isolable complex $26^{+}$, which could open interesting alternatives in the accessible nitride functionalization schemes. 
a)<smiles>[R16][Pb]1(Cl)[Pb]N2CC[R]1(Cl)C2</smiles><smiles>c1ccc(-c2ccccn2)nc1</smiles>

$\mathrm{XIX}$

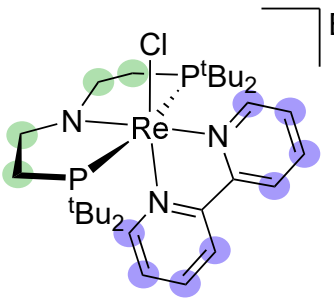

$27^{\mathrm{BPh}_{4}}$

b)

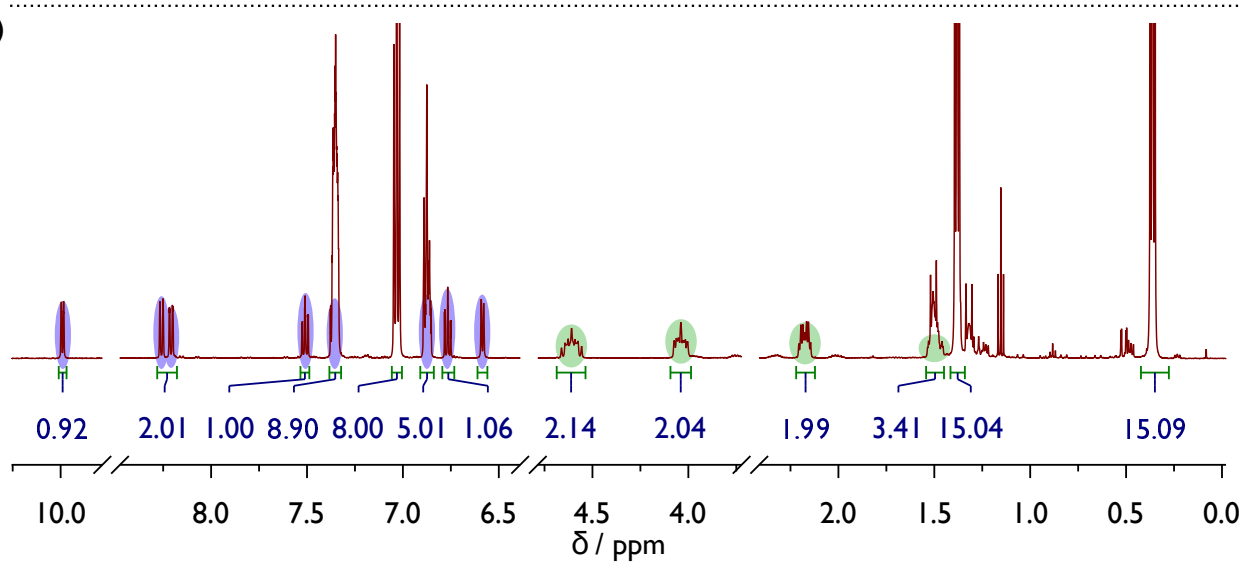

c)

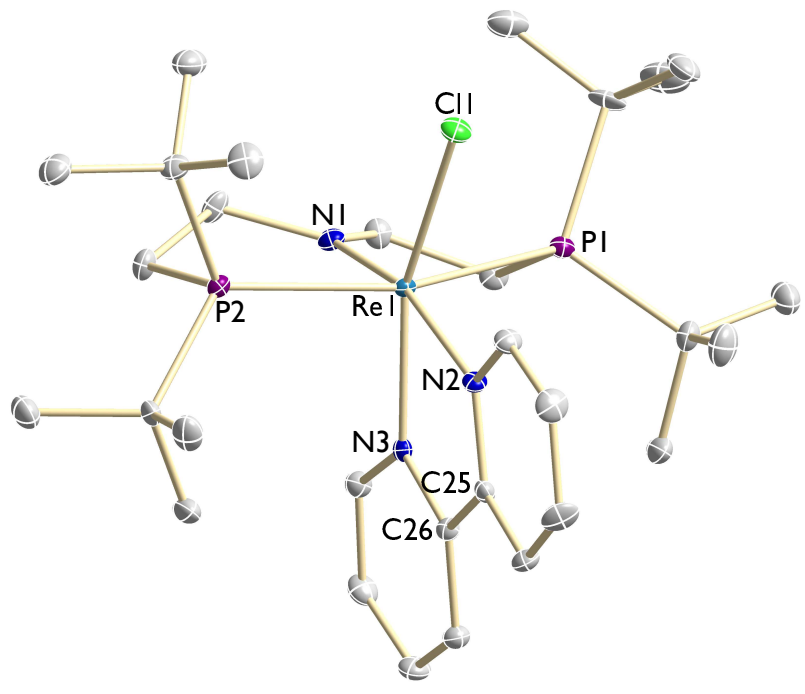

Fig. 2.10. a) Synthesis of 2,2'-bipyridine (bipy) complex $27^{\mathrm{BPh}_{4}}$. b) ${ }^{1} \mathrm{H}$ NMR spectrum of $27^{\mathrm{BPh}_{4}}$ in $\mathrm{CD}_{2} \mathrm{Cl}_{2}$. Aromatic signals belonging to the bipy ligand are marked in violet (partially overlapping with those of the anion), signals of the pincer backbone are marked in green. c) Molecular structure of $27^{\mathrm{BPh}_{4}}$ obtained by single crystal X-ray diffraction measurements. $\mathrm{H}$ atoms, a cocrystallized solvent molecule and the anion are omitted for clarity. Anisotropic displacement parameters are set to $50 \%$ probability. Selected bond lengths $[\AA]$ and angles $\left[^{\circ}\right]$ : Re1-N1 1.911(3), Re1-N2 2.185(3), Re1-N3 2.096(3), Re1-Cl1 2.3781, P1-Re1-P2 161.21(3), Cl1-Re1-N3 159.14(7), N1-Re1-N2 165.54(10), N2-C25-C26-N3 4.1(4). 


\subsection{Ligand exchange reactions from $\left[\mathrm{ReCl}_{2}\left(\mathrm{PNP}^{t \mathrm{Bu}}\right)\right]$}

The non-reducability of the rhenium nitride complexes with both the saturated and the unsaturated ligand backbone ( $\mathbf{X X I}$ and $\mathbf{2 2}$ respectively) necessitated the incorporation of other strategies to facilitate electron-storage on these complexes. A well-known approach to circumvent this problem is the usage of redox non-innocent ligands which can serve as an electron-reservoir upon reduction. A possible reaction could include reduction of the complex with the non-innocent ligand being the electron acceptor side, subsequent protonation and formation of a parent imide in an overall PCET type reaction. bipy is a workhorse in this type of chemistry and previously been successfully employed in our group by Josh Abbenseth. Stirring of $\left[\mathrm{ReCl}_{2}\left(\mathrm{PNP}^{t \mathrm{Bu}}\right)\right](\mathbf{X I X})$ with 1 eq bipy and $\mathrm{Na}\left(\mathrm{BPh}_{4}\right)$ in $\mathrm{THF}$ at $60^{\circ} \mathrm{C}$ overnight resulted in formation of a new, deep red complex which exhibits $C_{S}$ symmetry on the NMR timescale and does not dissolve in pentane or $\mathrm{Et}_{2} \mathrm{O}$ anymore, unlike the starting complex, indicating the formation of a cationic complex. Full NMR analysis as well as LIFDI mass spectrometry identified the new complex to be $\left[\operatorname{Re}(\text { bipy }) \mathrm{Cl}\left(\mathrm{PNP}^{\mathrm{tBu}}\right)\right]^{\mathrm{BPh}_{4}}\left(\mathbf{2 7}^{\mathrm{BPh}_{4}}\right)$ (see Figure 2.10). A characteristic feature is a significant high-field shift of one of the tertbutyl groups in the complex, due to the aromatic ring current in the bipy moiety $\left(\delta_{1} \mathrm{H}=\right.$ $0.36 \mathrm{ppm}$, see Figure 2.10). Crystals suitable for $\mathrm{X}$-ray structure analysis could be obtained from $\mathrm{THF} / \mathrm{Et}_{2} \mathrm{O}$ diffusion. The Re center is coordinated octahedrally by the two nitrogen donors of the bipy ligand, the PNP pincer and the chloride. The amide nature of the pincer nitrogen donor can be verified by its planar coordination $\mathrm{G}\left(\Sigma\left(\measuredangle_{\mathrm{N} 1}\right)=359.9^{\circ}\right)$ and the bipy ligand is coordinated perpendicular to the pincer with both aromatic rings being coplanar $\left(\phi_{\mathrm{N} 2-\mathrm{C} 25-\mathrm{C} 26-\mathrm{N} 3}=-4.1(4)^{\circ}\right)$.

Unfortunately, attempts to reduce the complex with $\mathrm{NaHg}$ under $\mathrm{N}_{2}$ atmosphere did not lead to the selective formation of one new compound, as would be expected from dinitrogen splitting. Instead, two new diamagnetic species are formed, as indicated by ${ }^{31} \mathrm{P}\left\{{ }^{1} \mathrm{H}\right\}-\mathrm{NMR}$ signals at $\delta_{31} \mathrm{P}=49.9 \mathrm{ppm}$ (singlet) and $32.7 / 26.5 \mathrm{ppm}$ (two doublets, ${ }^{2} J^{\mathrm{PP}}=206.3 \mathrm{~Hz}$ ). Reduction of $27^{\mathrm{BPh}_{4}}$ under Ar gave rather selectively one diamagnetic product, potentially bearing a hydride ligand as indicated by a triplet in the ${ }^{1} \mathrm{H}-\mathrm{NMR}$ spectrum at $\delta_{1_{\mathrm{H}}}=4.56 \mathrm{ppm}$ $\left({ }^{2} J_{\mathrm{HP}}=17.9 \mathrm{~Hz}\right)$. These two distinctly different results indicate $\mathrm{N}_{2}$ to take part in the reaction. No further investigation of the reaction was performed during this thesis, but if splitting is actually possible, the platform could offer an entry into reductive functionalization of a $\operatorname{Re}(\mathrm{V})$ nitride.

\subsection{Summary}

In previous work the rhenium complex $\left[\mathrm{ReCl}_{2}\left(\mathrm{PNP}^{t \mathrm{Bu}}\right)\right](\mathbf{X I X})$ was shown to be a versatile platform for $\mathrm{N}_{2}$ activation and functionalization reactions. Based on this finding, several 
variations and extensions of the known research were added, in order to broaden the understanding as well as the applications of this system.

Importantly, the dimeric, $\mu-N_{2}$ bridged Re-complex $\mathbf{X X}$ resulting from reduction of $\mathbf{X I X}$ under $\mathrm{N}_{2}$ atmosphere could be characterized spectroscopically to large extends as well as by $\mathbf{X}$-ray structure analysis. The clean thermal conversion of $\mathbf{X X}$ to nitrido complex $\left[\operatorname{Re}(\mathrm{N}) \mathrm{Cl}\left(\mathrm{PNP}^{t \mathrm{Bu}}\right)\right](\mathbf{X X \mathbf { I }})$ was proven by means of NMR spectroscopy and following this process at various temperatures allowed for a fist estimation of activation parameters to $\Delta H^{\ddagger} \approx 110 \mathrm{~kJ} \mathrm{~mol}^{-1}$, which is in reasonable agreement with the originally published DFT calculated barrier of $\Delta H^{\ddagger}=84.4 \mathrm{~kJ} \mathrm{~mol}^{-1}$. ${ }^{[94]}$

Additionally the hydride complex $\left[\mathrm{ReHCl}\left(\mathrm{PNP}^{\mathrm{tBu}}\right)\right](\mathbf{1 7})$ could be identified and characterized as a side product of the reduction of $\mathbf{X I X}$ with $\mathrm{Co}\left(\mathrm{Cp}^{*}\right)_{2}$, serving as an explanation for the experimentally observed reduced yield of $\mathbf{X X \mathbf { I }}$ when using this reductant.

Furthermore, the oxidative $\mathrm{C}-\mathrm{C}$ coupling of ketimido complex $\left[\mathrm{Re}\left(\mathrm{NCH}_{2}\right) \mathrm{Cl}\left(\mathrm{PNP}{ }^{t \mathrm{Bu}}\right)\right](\mathbf{1 8})$ which was proposed by Dr. Isabel Klopsch was developed into a clean and reproducable reaction and the product could be isolated and fully characterized. Even though no continuative reactions were investigated, this coupling opens a route to new, nitrogen containing organic molecules as well as odd-electron nitride functionalization schemes and should thus be followed more thoroughly in the future.

With this latter reaction as well as the known, oxidative nitrile release reactions from $\left[\operatorname{Re}(\mathrm{NR}) \mathrm{Cl}\left(\mathrm{PNP}^{t \mathrm{Bu}}\right)\right]^{+}(\mathrm{R}=\mathrm{Et}, \mathrm{Bn})$ in mind, the vinylamido congener of $\mathbf{X I X}$, i.e. $\left[\mathrm{ReCl}_{2}\right.$ $\left(\mathrm{P}=\mathrm{N}=\mathrm{P}^{t \mathrm{Bu}}\right)$ ] (21) was synthesized and investigated towards $\mathrm{N}_{2}$ functionalization reactions. CV data suggest a only slightly increased stability of $\mathbf{2 1}$ in comparison with parent $\mathbf{X I X}$, but successful $\mathrm{N}_{2}$ splitting encouraged further investigation of its functionalization capabilities. Overall, the vinylamido PNP pincer based system turned out to exhibit very similar reactivity to the alkyl based pincer, with hardly any differences in redox chemistry, stability, $\mathrm{N}_{2}$ activation and splitting behavior or nucleophilicity of the corresponding nitrido complexes. Thus, the products of protonation (with $\mathrm{H}\left(\mathrm{OEt}_{2}\right)_{2}\left(\mathrm{BAr}_{24}^{\mathrm{F}}\right)$ ), methylation (with MeOTf) and oxidation (with $\mathrm{Ag}\left(\mathrm{SbF}_{6}\right)$ ) were investigated and the resulting products were characterized (despite the oxidized nitride $\mathbf{2 6}^{\mathbf{S b F}_{6}}$ ). By doing so, a promising starting point for further investigation of potential electrochemical functionalization pathways, where oxidative and reductive conditions can be applied simultaneously to a reaction mixture, was developed.

Finally, the non-reducability of the $\operatorname{Re}(\mathrm{v})$ nitrido complexes $\mathbf{X X I}$ and 22 was addressed by an attempt to introduce the potentially redox non-innocent ligand 2,2'-bipyridine. The

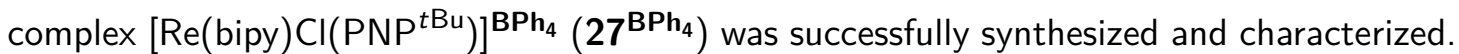
Preliminary experiments revealed it to reaction to different products upon reduction under Ar or $\mathrm{N}_{2}$. Investigations concerning the identification of the resulting products are still pending. 


\title{
Rhenium complexes of iso-propyl based PNP pincer ligands for dinitrogen activation
}

\author{
All we need is lightning, \\ With power and might
}

\author{
- Lordi \\ "Hard Rock Halleluja" on "The Arockalypse"
}

\begin{abstract}
Parts of this chapter (Section 3.1.1 and well as major parts of Section 3.3) have been published in: F. Schendzielorz, M. Finger, J. Abbenseth, C. Würtele, V. Krewald, S. Schneider, "Metal-Ligand Cooperative Synthesis of Benzonitrile by Electrochemical Reduction and Photolytic Splitting of Dinitrogen", Angew. Chem. Int. Ed. 2019, 58, 830-834.
\end{abstract}

Successful $\left[\operatorname{ReCl}_{2}\left(\mathrm{PNP}^{t \mathrm{Bu}}\right)\right](\mathbf{X I X})$ mediated synthesis of organic nitriles from dinitrogen (see Part I Scheme 4.5 and corresponding section) inspired further research to improve the system. One of the major drawbacks of this conversion is the need to work with strong, alkali metal based reductants as well as strong oxidants like Ag salts or NCS. Additionally, highly reactive electrophiles are necessary (i.e. alkyl triflates) to obtain any nitride centered functionalization. This implies non-compatible reaction conditions, preventing one pot mixtures and therefore rendering catalytic turnover of such a system very unlikely.

Consequently, new ways to tune the properties responsible for these reaction requirements are needed. One option to potentially increase the nucleophilicity of the nitride and thus enhance its reactivity towards weaker electrophiles is indicated by DFT calculations (see Figure 3.1). If the splitting of the $\mathrm{N}_{2}$ bridged dimer $\left[\left(\mu-\mathrm{N}_{2}\right)\left\{\operatorname{ReCl}\left(\mathrm{PNP}^{t \mathrm{Bu}}\right)\right\}_{2}\right](\mathbf{X X})$ is computed, it is found to proceed via a modest transition state (which rendered the spectroscopic observation of this dimer possible, see Section 2.1) and results in the strongly exergonic splitting product $\left[\operatorname{Re}(\mathrm{N}) \mathrm{Cl}\left(\mathrm{PNP}^{t \mathrm{Bu}}\right)\right](\mathbf{X X I})\left(\Delta G^{0}=-98.3 \mathrm{~kJ} \mathrm{~mol}^{-1}\right)$. In this reaction the very strong $\mathrm{N} \equiv \mathrm{N}$ triple bond is exchanged with two very strong $\operatorname{Re} \equiv \mathrm{N}$ triple bonds, eventually leading to an overstabilization of the reaction product. From this thermodynamic sink, harsh reaction conditions are needed to obtain nitride centered reactivity, which is also commonly observed in other systems and can therefore be regarded as a general problem. ${ }^{[16]}$ However, if the steric bulk of the ligand is reduced in silico by exchanging the tert-butyl residues with methyl groups, this has a major impact on the calculated thermodynamics of the reaction. 


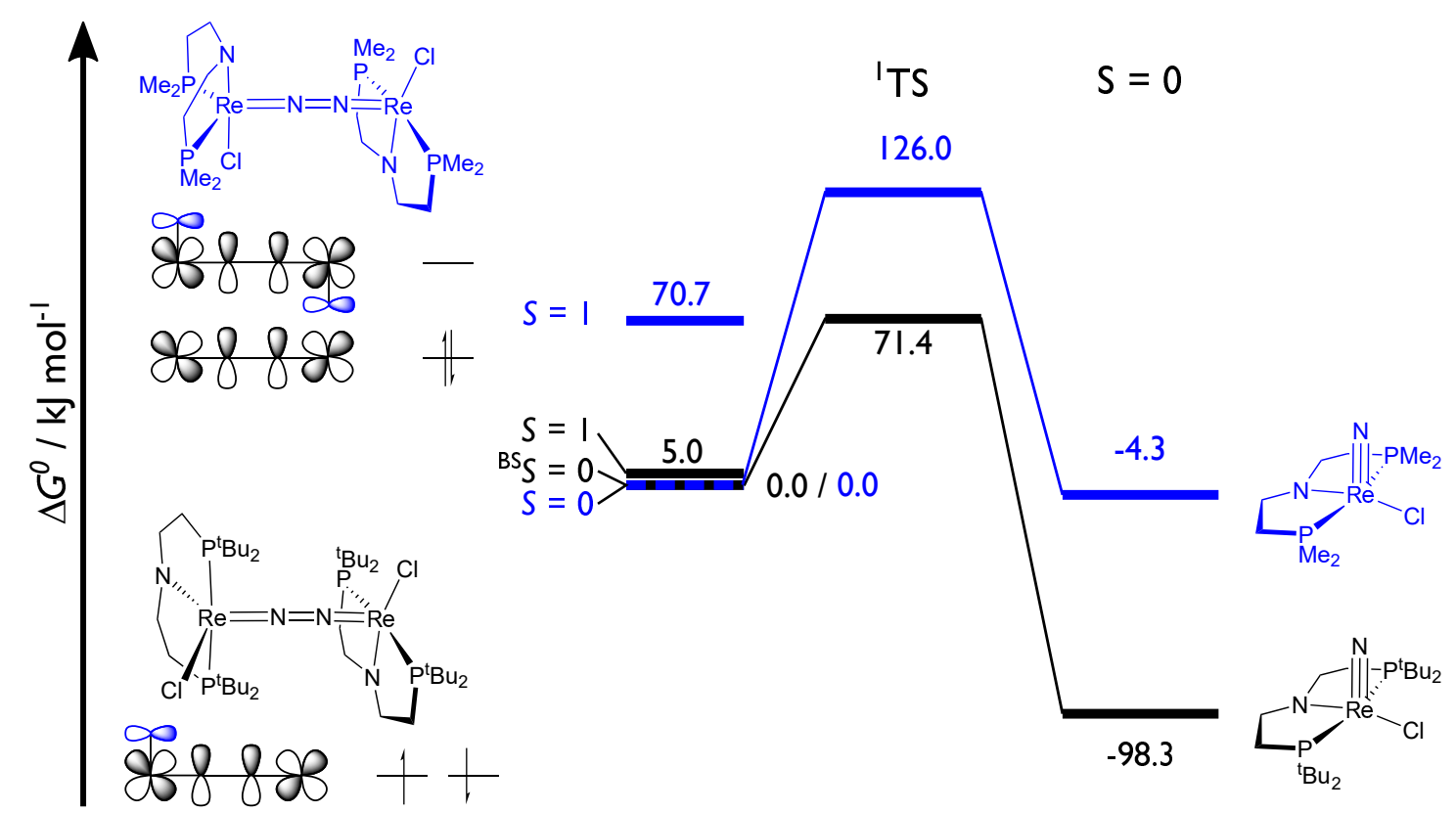

Fig. 3.1. Comparison of the DFT computed thermal dinitrogen splitting from $\mathbf{X X}$ (black) with in silico simulated 28 (blue) into the corresponding nitrido complexes (PBE/D3BJ/RI/def2TZVP ||$P B E / D 3 B J / R I / d e f 2-S V(P)) .{ }^{1}$

On the one hand, in the ground state geometry one $\left\{\operatorname{ReCl}\left(\mathrm{PNP}^{\mathrm{Me}}\right)\right\}$ fragment is found to be rotated by $90^{\circ}$ with respect to the tert-butyl analog, accompanied by a change from a broken symmetry open shell singlet ${ }^{\mathrm{BS}} S=0$ in $\mathbf{X X}$ to a closed shell singlet $S=0$ in the methyl-PNP based dimer $\left[\left(\mu-\mathrm{N}_{2}\right)\left\{\operatorname{ReCl}\left(\mathrm{PNPMe}^{\mathrm{Me}}\right)\right\}_{2}\right](\mathbf{2 8})$. This change in electronic structure can be understood considering the MO scheme of $\mathbf{X X}$ (also see Scheme 2.1). The rotation of the pincer results in the amido donor interacting solely with one of the orthogonal sets of $\pi$ orbitals, thereby lifting their degeneracy and stabilizing the $S=0$ state as ground state. On the other hand, splitting now results in an almost thermoneutral nitride $\left(\Delta G^{0}=\right.$ $-4.3 \mathrm{~kJ} \mathrm{~mol}^{-1}$ ) which can be expected to be more reactive than in the tert-butyl substituted system. At the same time, the kinetic barrier for the splitting is computed to become much higher, which should lead to a more stable, potentially isolable dimer.

Based on these results, reducing the steric bulk of the pincer ligand seemed to be a promising approach to increase the reactivity of the $\mathrm{N}_{2}$ derived nitride complex. For this, changing to the iso-propyl based pincer HPNP ${ }^{i P r}$ was favored over HPNPMe, because it is already significantly smaller than the tert-butyl based ligand but unlike the latter can still shield the metal center to some degree, preventing potentially unwanted side-reactions (Tolman cone angles: $\left.\Theta_{\mathrm{P}\left({ }^{\mathrm{t} B u}\right)_{2} \mathrm{Et}}=165^{\circ}, \Theta_{\mathrm{P}\left({ }^{i} \mathrm{Pr}\right)_{2} \mathrm{Et}}=151^{\circ}, \Theta_{\mathrm{P}(\mathrm{Me})_{2} \mathrm{Et}}=123^{\circ}\right) .{ }^{[202]}$

In this chapter, the synthesis and chemistry of rhenium complexes with the iso-propyl based PNP pincer ligand will be described. To this end, Section 3.1 details the synthesis and

\footnotetext{
${ }^{1}$ The DFT calculations presented in Figure 3.1 were performed by Dr. Markus Finger. The data on the tert-butyl system were published previously, ${ }^{[94]}$ while the data on the Me based system are unpublished.
} 
properties of starting complex $\left[\mathrm{ReCl}_{3}\left(\mathrm{HPNP}^{i \mathrm{Pr}}\right)\right](\mathbf{2 9})$ and discusses its remarkable electronic structure and spectroscopic features.

A route to the formal deprotonation product $\left[\mathrm{ReCl}_{2}\left(\mathrm{PNP}^{i \mathrm{Pr}}\right)\right](\mathbf{3 0})$ via $\mathrm{H}$ atom abstraction to form $\left[\mathrm{ReCl}_{3}\left(\mathrm{PNP}^{i \mathrm{Pr}}\right)\right](31)$ and subsequent reduction is reported in Section 3.2, as well as the resulting $\mathrm{N}_{2}$ coordination/activation chemistry of $\mathbf{3 0}$ and the attempts to split dinitrogen with this platform.

Section 3.3 finally describes the $\mathrm{N}_{2}$ activation with the amine based pincer, i.e. the formation of $\left[\left(\mu-\mathrm{N}_{2}\right)\left\{\mathrm{ReCl}_{2}\left(\mathrm{HPNP}^{i \mathrm{Pr}}\right)\right\}_{2}\right]$ (36) and its photolysis into the corresponding nitride complexes $\left[\operatorname{Re}(\mathrm{N}) \mathrm{Cl}_{2}\left(\mathrm{HPNP}^{i \mathrm{Pr}}\right)\right]$ (37). The nitride exhibits defined nucleophilic reactivity towards Lewis acids as exemplified by the reaction with $\mathrm{HOTf}$ or $\operatorname{BAr}_{18}^{\mathrm{F}}\left(\mathrm{BAr}_{18}^{\mathrm{F}}=\right.$ tris $\{3,5$-bis(trifluoromethyl)phenyl\}borane). Imporantly, the reaction with benzoyl chloride results in a metal-ligand cooperative cleavage of the $\mathrm{Re} \equiv \mathrm{N}$ bond, formation of a benzamide/benzonitrile mixture and $\left[\operatorname{ReCl}_{3}\left(\mathrm{P}=\mathrm{NP}{ }^{i \mathrm{Pr}}\right)\right](\mathbf{4 0})$. The latter can be regenerated to reform 29 via electrolysis and thus a synthetic cycle for $\mathrm{N}_{2}$ fixation is obtained.

\subsection{The starting platform - $\left[\mathrm{ReCl}_{3}\left(\mathrm{HPNP}^{i \mathrm{Pr}}\right)\right](29)$}

\subsubsection{Synthesis and characterization}

Initial attempts to synthesize the amide complex $\left[\operatorname{ReCl}_{2}\left(\mathrm{PNP}^{i \mathrm{Pr}}\right)\right](\mathbf{3 0})$, analog to the tert-butyl system were not successful. Reactions of precursor $\left[\mathrm{ReCl}_{3}\left(\mathrm{PPh}_{3}\right)_{2}(\mathrm{MeCN})\right]$ with $\mathrm{HPNP}^{i \mathrm{Pr}}$ in the presence of the bases $\mathrm{NEt}_{3}, \mathrm{KO} t \mathrm{Bu}$ or KHMDS did not lead to the formation of an isolable product, but rather to an inseparable mixture of different compounds (for further information, see Section 3.2.1). However, when $\left[\mathrm{ReCl}_{3}\left(\mathrm{PPh}_{3}\right)_{2}(\mathrm{MeCN})\right]$ and $\mathrm{HPNP}^{\mathrm{iPr}}$ were refluxed overnight in toluene without base, clean formation of the new complex $\left[\mathrm{ReCl}_{3}\left(\mathrm{HPNP}^{i \mathrm{Pr}}\right)\right](\mathbf{2 9})$ was observed in $70 \%$ isolated yield. Apparently, 29 is less acidic than the tert-butyl counterpart and therefore, partial deprotonation of the complex by non-coordinated HPNP ${ }^{i P r}$ does not take place before full complexation.

29 displays uniquely strongly shifted NMR signals (see Figure $3.2 b$ and c), e.g. the $\mathrm{NH}$ signal resonates at $\delta_{1_{\mathrm{H}}}=154.45 \mathrm{ppm}$, the backbone $\mathrm{CH}_{2}$ protons between $\delta_{1_{\mathrm{H}}}=8.31$ and $-10.44 \mathrm{ppm}$, the ${ }^{15} \mathrm{~N}$ signal of the backbone amine, identified by ${ }^{1} \mathrm{H}-{ }^{15} \mathrm{~N} \mathrm{HSQC}$, exhibits a signal at $\delta_{15} \mathrm{~N}=-1226.5 \mathrm{ppm}$ and the ${ }^{31} \mathrm{P}\left\{{ }^{1} \mathrm{H}\right\}$ NMR shows a singlet at $\delta_{31 \mathrm{P}}=-1525.9 \mathrm{ppm}$, which is likely to be the strongest highfield shift for a trialkylphosphine ligand ever reported in literature. Despite these shifts into spectral regions which are typical for paramagnetic compounds, all signals exhibit well resolved $J_{\mathrm{HH}}$ and $J_{\mathrm{HP}}$-coupling, pointing to a non-trivial electronic structure, which is discussed in detail in Section 3.1.2. The molecular structure 

$\left[\mathrm{ReCl}_{3}\left(\mathrm{PPh}_{3}\right)_{2}(\mathrm{MeCN})\right]+{ }^{\mathrm{i}} \mathrm{Pr}_{2} \mathrm{P}{ }_{\text {toluene }}^{9{ }^{\circ} \mathrm{C}, 16 \mathrm{~h}}$

29
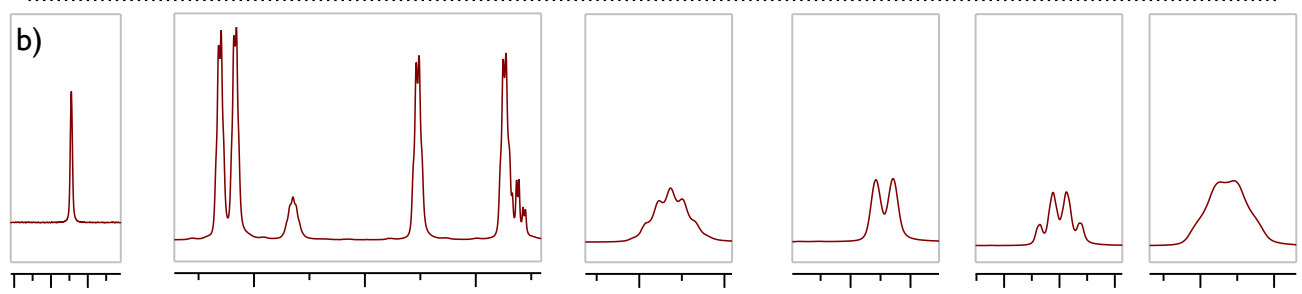

154 9.5

8.5

$5.5 \quad 5.4$

$\begin{array}{llll}0.6 & 0.5 & -5.0 & -5.2\end{array}$

$-10.5$
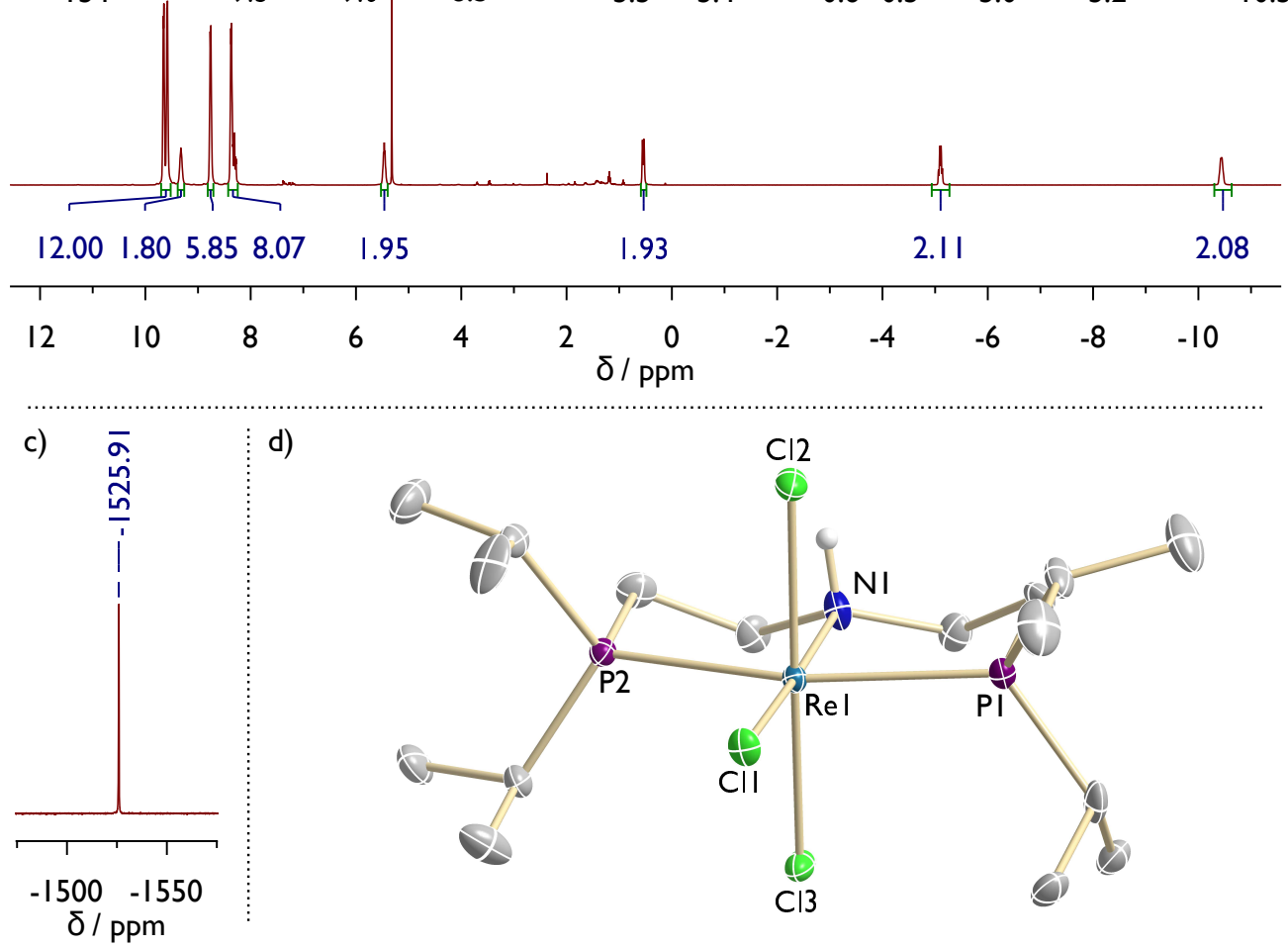

d)

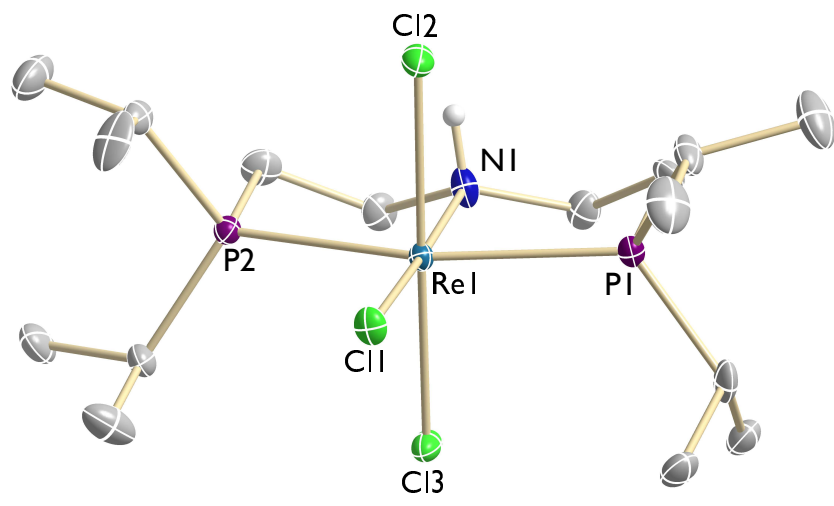

Fig. 3.2. a) Synthesis of 29. b) ${ }^{1} \mathrm{H}$ NMR spectrum of 29. Insets: Magnification of the individual signals. c) ${ }^{31} \mathrm{P}\left\{{ }^{1} \mathrm{H}\right\}$ NMR spectrum of 29. d) Molecular structure of $\mathbf{2 9}$ obtained by single crystal X-ray diffraction measurements. All $\mathrm{H}$ atoms but the $\mathrm{NH}$ proton are omitted for clarity. Anisotropic displacement parameters are set to $50 \%$ probability. Selected bond lengths $[\AA]$ and angles $\left[^{\circ}\right]$ : Re1-N1 2.158(6), Re1-Cl1 2.4117(18), Re1-Cl2 2.3791(18), Re1Cl3 2.3725(17), P1-Re1-P2 161.58(6), N1-Re1-Cl1 177.47(18), Cl2-Re1-Cl3 174.73(7).

of 29 could be determined by X-ray diffraction measurements of single crystals (see Figure $3.2 d$ ). The Re center is found to be octahedrally coordinated by the PNP pincer and three chloride ligands. The backbone nitrogen displays defined pyramidal coordination $\left(\Sigma\left(\measuredangle_{\mathrm{N} 1}\right)\right.$ $\left.=342.1^{\circ}\right)$ and the $\mathrm{NH}$ proton was found from the residual density map and isotropically refined.

In cyclic voltammetry measurements, 29 shows a $\operatorname{Re}(\mathrm{IV} / \mathrm{III})$ oxidation wave at $E_{1 / 2}=$ $-0.24 \mathrm{~V}$ vs. $\mathrm{Fc}^{+} / \mathrm{Fc}$ as well as a quasi-reversible wave with an estimated reduction potential 
a)

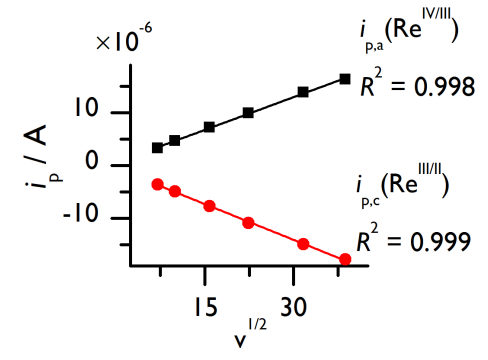

c)

\begin{tabular}{|c|c|c|}
\hline$v / \mathrm{mVs}^{-1}$ & $\begin{array}{l}\operatorname{Re}^{I V / I I I} \\
i_{p, c} / i_{p, a}\end{array}$ & $\begin{array}{l}\operatorname{Re}{ }^{I I / / I I} \\
i_{p, a} / i_{p, c}\end{array}$ \\
\hline 50 & 1.0641 & 0.602 \\
\hline 100 & 1.0313 & 0.5803 \\
\hline 250 & 1.0072 & 0.6906 \\
\hline 500 & 1.0275 & 0.7882 \\
\hline 1000 & 1.0268 & 0.8328 \\
\hline 1500 & 1.0301 & 0.8569 \\
\hline
\end{tabular}

b)

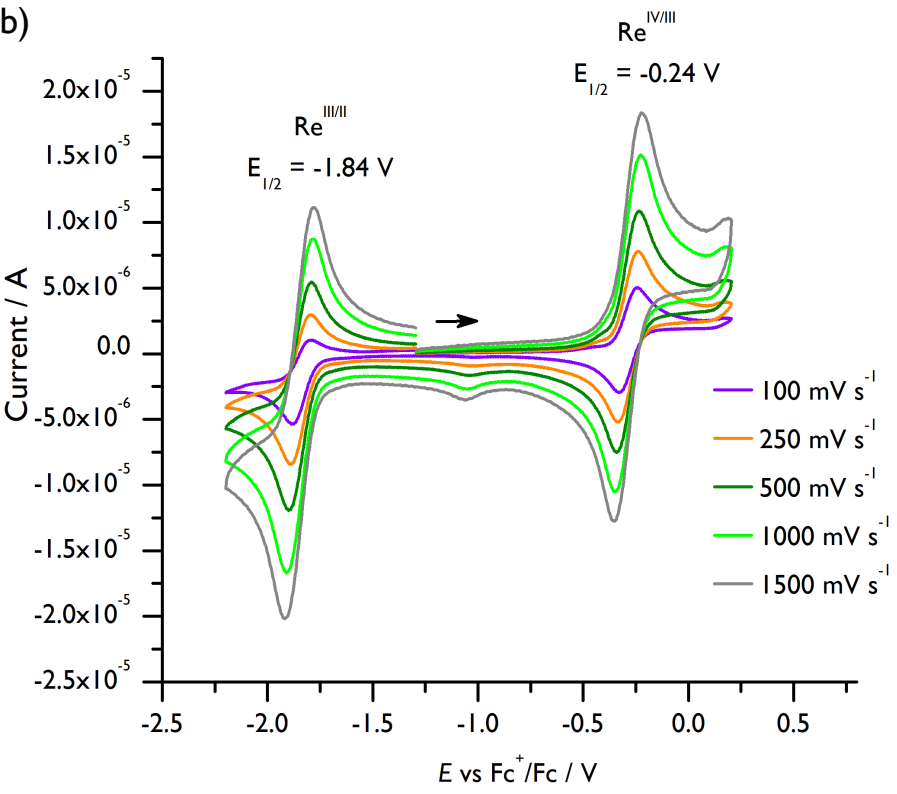

Fig. 3.3. a) Plot and linear fit of $i_{p}$ vs. $v^{1 / 2}$ ( $i_{p, a}$ for the $\operatorname{Re}(\mathrm{IV} / \mathrm{III})$ couple and $i_{p, c}$ for the $\operatorname{Re}(\mathrm{III} / \mathrm{II})$ couple.) b) CV measurements of first oxidation and reduction of $\mathbf{2 9}$ in THF at different scan rates $\left(10^{-3} \mathrm{M} 29,0.1 \mathrm{M} \mathrm{N}^{\mathrm{n}} \mathrm{Bu}_{4} \mathrm{PF}_{6}, \mathrm{WE}\right.$ : GC, RE: Ag-wire, CE: Pt-wire). c) Scan rate dependent ratio between forward and backward peak current of both waves.

of $E_{1 / 2}=-1.84 \mathrm{~V}$. Plotting the anodic peak current $i_{p, a}$ vs. $v^{1 / 2}$ for the oxidation and the cathodic peak current $i_{p, c}$ vs. $v^{1 / 2}$ for the reduction according to the Randles-Sevcik equation (see equation (eq.) 1.1 in Section 1.2), electrochemical reversibility and fast electron transfer to freely diffusing complexes can be assumed for both redox processes. A constant, scan-rate independent ratio of the forward and backward peak current close to unity proves chemical reversibility of the oxidation, while a pronounced decrease of that ratio with slower scan-rates shows the reduction to be irreversible. This irreversibility is tentatively ascribed to chloride loss after the reduction as observed for $\left[\mathrm{ReCl}_{2}\left(\mathrm{PNP}^{t \mathrm{Bu}}\right)\right](\mathbf{X I X}) \cdot{ }^{[95]}$ However, a $\mathrm{CV}$ series with varying chloride concentrations would be necessary to prove this assumption. Reactivity which follows from the reduction of $\mathbf{2 9}$ is described in Sections 3.2.3 and 3.3.1.

\subsubsection{The electronic structure of 29}

The strongly shifted NMR signals of 29 described in Section 3.1.1, in particular the presence of a ${ }^{31} \mathrm{P}$ resonance at $\delta_{31} \mathrm{P}=-1525.9 \mathrm{ppm}$, are reminiscent of previously published work on the osmium(II) PNP pincer complex $\left[\mathrm{OsCl}\left(\mathrm{P}=\mathrm{N}=\mathrm{P}^{t \mathrm{Bu}}\right)\right]$. $^{[195]}$ This complex exhibits a peak in ${ }^{31} \mathrm{P}\left\{{ }^{1} \mathrm{H}\right\}$ NMR spectroscopy at $\delta_{{ }^{31} \mathrm{P}}=-978.2 \mathrm{ppm}$ and a TIP, as indicated by a linear behaviour in the $\mathcal{X}_{M} T$ vs. $T$ plot derived from SQUID magnetometry, with a constant susceptibility of $\mathcal{X}_{M}(\mathrm{TIP})=1030 \cdot 10^{-6} \mathrm{~cm}^{3} \mathrm{~mol}^{-1}$. Such behavior has been ascribed to the second-order Zeeman effect which arises from mixing of the thermally isolated ground state (i.e. $k_{\mathrm{B}} T<<\Delta E$, with $\Delta E$ describing the energy difference between the ground state and 

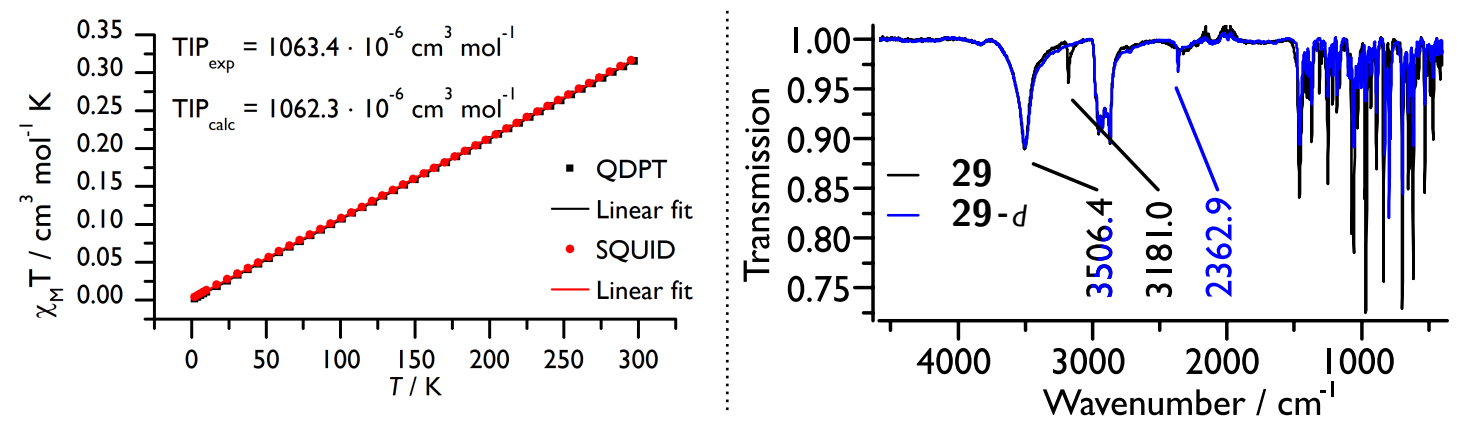

Fig. 3.4. Left: Temperature-dependent molar susceptibility data of $\mathbf{2 9}$ determined experimentally by SQUID magnetometry (red) as well as computationally by QDPT/NEVPT2/CASSCF calculations (black). Right: ATR-IR spectrum of $\mathbf{2 9}$ (black), compared with the spectrum of 29-d (blue).

the first excited state) with excited states in the presence of a magnetic field. ${ }^{203]}$ Indeed, multireference calculations on the Os(II) complex indicated the complex to exhibit rather strong SOC effects. A similar situation for $\mathbf{2 9}$ seems very likely, as TIP is a known phenomenon especially for octahedrally coordinated heavy metal complexes with a $d^{4}$ electron configuration like $\operatorname{Re}(\mathrm{III}){ }^{[204]}$

In order to gain insight into the electronic structure of the system at hand, its magnetic behavior was evaluted by means of SQUID magnetometry (see Figure 3.4). Over a temperature range of 2-295 K, complex 29 exhibits a strong TIP as indicated by a linearly increasing $\mathcal{X}_{M} T$ vs. $T$ curve, corresponding to a molar susceptibility of $\mathcal{X}_{M}(\mathrm{TIP})=1063.4 \cdot 10^{-6} \mathrm{~cm}^{3} \mathrm{~mol}^{-1}$, which is only marginally different from the value obtained for $\left[\mathrm{OsCl}\left(\mathrm{P}=\mathrm{N}=\mathrm{P}^{t \mathrm{Bu}}\right)\right]$. Therefore, it seems not to be possible to draw a simple, linear correlation between experimentally determinable TIP and ${ }^{31} \mathrm{P}$ NMR shifts which one might be tempted to assume.

Nevertheless, 29 obviously features strong SOC effects. To back up these data, theoretical calculations were performed (see Part III, Section 3.4 for further details on the exact methods and settings used). To this end, the X-ray structure of $\mathbf{2 9}$ was used as a starting point and optimized for both the $S=0$ and $S=1$ state without any constraints. The derived PBE0/RIJCOSX/D3BJ/def2-TZVP||PBE/RI/D3BJ/def2-SVP energies indicated the $S=1$ state to be favorable by $\Delta G=-36.5 \mathrm{~kJ} \mathrm{~mol}^{-1}$, which obviously does not reflect the experimental findings. Hence, higher level calculations were needed. The DFT-predicted minimal energy structure was the geometry used for all further calculations.

The SQUID data show $\mathbf{2 9}$ to exhibit electronic states which are rather close in energy to the ground state. Such quasi-degenerate systems are poorly described by single-reference methods like DFT or coupled cluster (CC). Therefore, complete active space self consistent field (CASSCF) calculations were performed on this system, as they are capable of describing strongly statically correlated systems and serve as starting point for multi-reference methods like N-electron valence state perturbation theory (NEVPT2) or quasi-degenerate perturbation 
theory (QDPT). Starting in the SVP basis, a $\operatorname{CASSCF}(14,10)$ calculation $^{2}$ was set up which included the five metal-centered $d$-orbitals as well as the five ligand based MOs balancing the active space (i.e. the bonding orbitals corresponding to the antibonding $d$-space, see Figure 3.5). Inclusion of further, chloride centered orbitals, which are dominated by $\mathrm{Cl} p$ orbitals and are basically non-bonding, did not lead to significant variation of the results (no improvement of the overall energy, negligible influence on excited state energies). Preliminar results based on a reduced $\operatorname{CASSCF}(4,5)$ active space including only the $d$ orbitals indicate that the overall picture is only slightly affected by the ligand based orbitals. However, having a balanced active space was considered good practice and since the $(14,10)$ space was computationally perfectly feasible, this was used.

The final set of orbitals was obtained after reconverging the $\operatorname{CASSCF}(14,10)$ calculation in the TZVP basis averaging over a total of 50 singlet, 45 triplet and 5 quintet states (i.e. over all possible excitations within the $d$ orbitals) and the obtained energies were corrected for dynamic correlation by means of NEVPT2. The triplet ground state is confirmed with

${ }^{2}$ Throughout this thesis, for the active space of the performed calculations the following notation will be employed: $\operatorname{method}\left(n_{\text {active electrons }}, n_{\text {active orbitals }}\right)$.

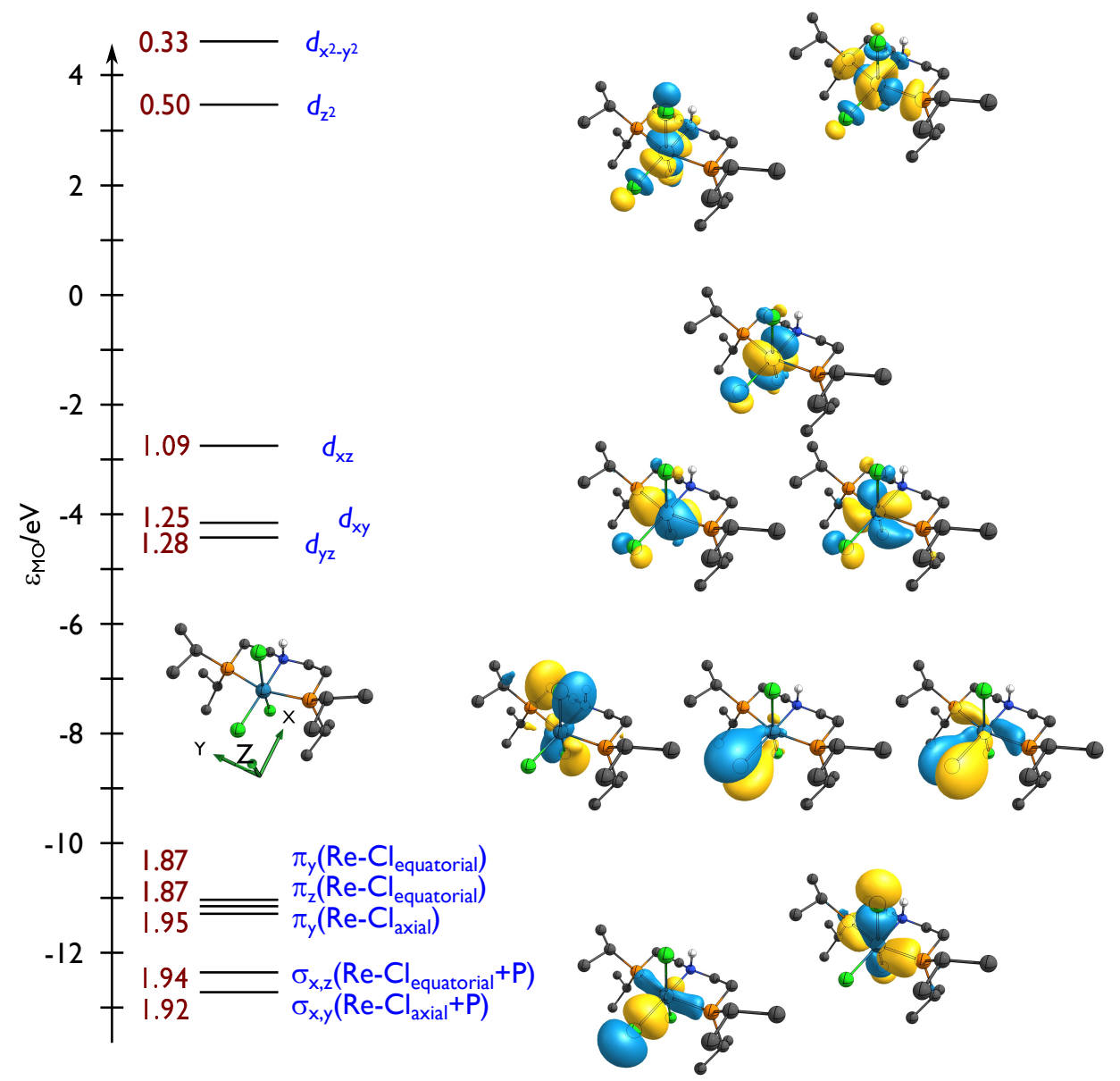

Fig. 3.5. MO scheme of 29 from a stage-averaged $\operatorname{CASSCF}(14,10)$ calculation in the triple- $\zeta$ basis averaging over 50 singlet, 45 triplet and 5 quintet states. Occupation numbers (red) orbital labels (blue) and orbital plots at an isovalue of 0.04 are given as well. 


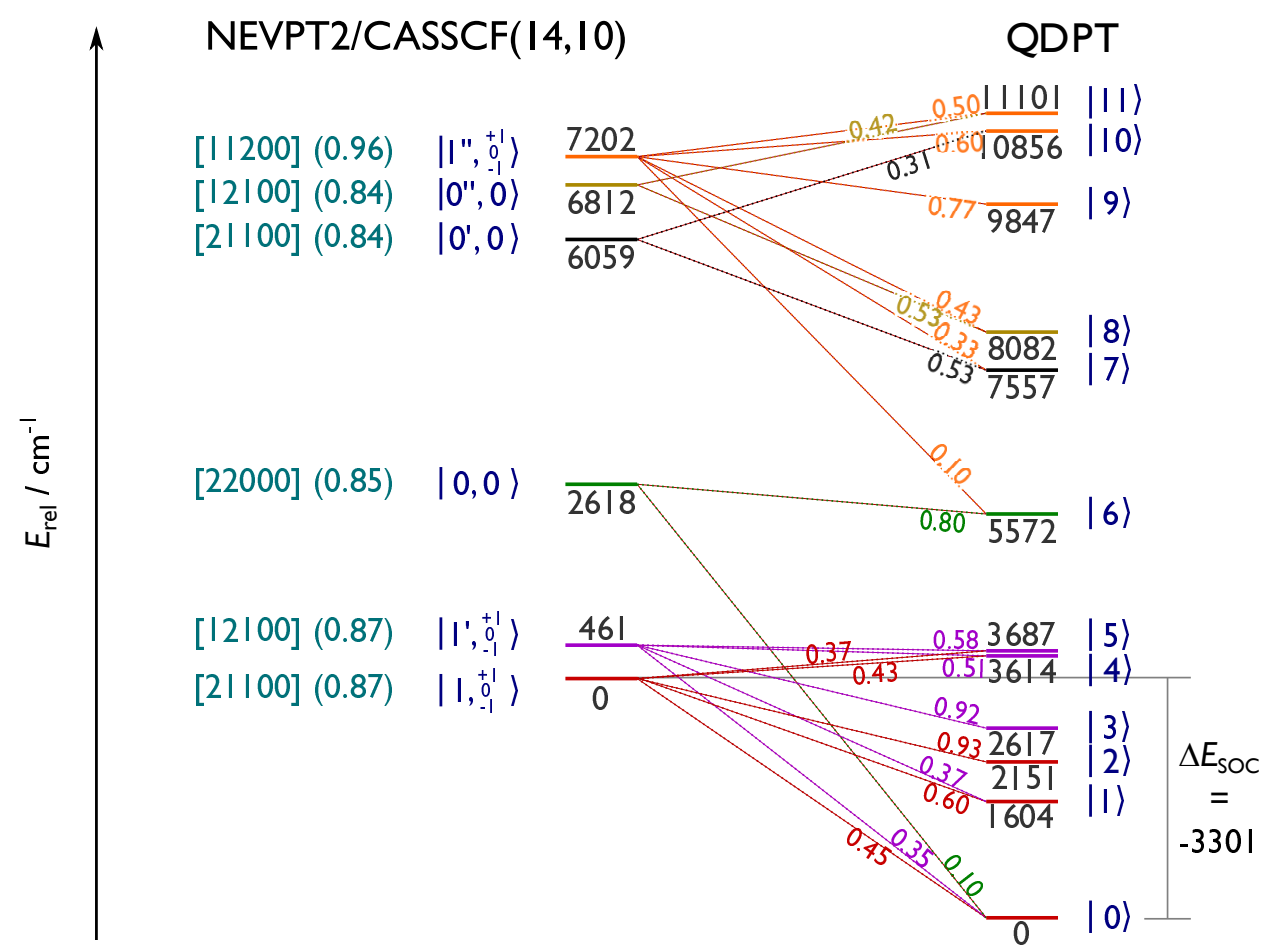

Fig. 3.6. State-energy diagramm of 29 based on a NEVPT2/CASSCF $(14,10)$ calculation. Nonrelativistic states $\left|S, M_{S}\right\rangle$ (blue) and their dominant configuration [XXXXX] with the respective weights in brackets (turquoise) are given (left), where each $\mathrm{X}$ corresponds to the occupation of the $d$-orbitals in energetically increasing order (the ligand orbitals are fullly occupied in all configurations and are thus omitted). Spin-orbit states derived from QDPT treatment (right) are color-coded according to the dominant spin-free state and individual contributions are given (weights $\geq 10 \%$, on the dashed lines). State energies are given in $\mathrm{cm}^{-1}$ respective to the ground states.

a $\left(d_{y z}\right)^{2},\left(d_{x y}\right)^{1},\left(d_{x z}\right)^{1}$ electron configuration (87\% weight), while the first exited triplet, which lies only $5.5 \mathrm{~kJ} \mathrm{~mol}^{-1}$ above the ground state is mainly composed of the corresponding $\left(d_{y z}\right)^{1},\left(d_{x y}\right)^{2},\left(d_{x z}\right)^{1}$ (87\% weight), reflecting the near-degeneracy of the $d_{x y}$ and $d_{y z}$ orbitals. However, if these states were subjected to a SOC calculation by means of a QDPT treatment, the degeneracy of each state is lifted. The lowest eigenstate is stabilized by $\Delta E_{\mathrm{SOC}}=-3301 \mathrm{~cm}^{-1}$ and features considerable multireference character, i.e. it is mainly composed from $|1\rangle$ (45\% weight), $\left|1^{\prime}\right\rangle$ (35\% weight) and 0$\rangle$ (10\% weight) (see Figure 3.6).

The strong SOC effect, especially on the ground state are in good agreement with the observed TIP from magnetometry (see Figure 3.4) and simulated SQUID data from the QDPT calculation reproduce the TIP value exactly $\left(\mathcal{X}_{M} T_{\mathrm{QDPT}}=1062.3 \cdot 10^{-6} \mathrm{~cm}^{3} \mathrm{~mol}^{-1}\right)$. The computed $\mathrm{SOC}$ stabilization energy is comparable to that of $\left[\mathrm{OsCl}\left(\mathrm{P}=\mathrm{N}=\mathrm{P}^{t \mathrm{Bu}}\right)\right]\left(\Delta E_{\mathrm{SOC}}=\right.$ $\left.-9.2 \mathrm{kcal} \mathrm{mol}^{-1} \approx-3200 \mathrm{~cm}^{-1}\right)$ and would therefore support a linear correlation of $\Delta E_{\mathrm{SOC}}$ and $\mathcal{X}_{M}$ (TIP). However, further data would be needed to underpin this suggestion.

Interestingly, the SOC calculation predicted significant oscillator strengths for the $|0\rangle \rightarrow|4\rangle$ and $|0\rangle \rightarrow|5\rangle$ electronic excitations, with very low excitation energies close to the near 
infrared (NIR)/mid infrared (MIR) limit $\left(3614 \mathrm{~cm}^{-1}\right.$ and $3687 \mathrm{~cm}^{-1}$, respectively) (see Part III, Table 3.12). And indeed, ATR-IR on a solid sample as well as regular transmission IR measurements in Nujol revealed a very prominent and broad peak at $3507 \mathrm{~cm}^{-1}$ with a small shoulder at slightly lower energies, which is in very good agreement with the calculations. While the band shape and central frequency strongly reminds of water, this result was verified with different batches, each of which were pure by elemental analysis and showed no sign for water in the NMR spectra, excluding this to be the source of the signal. Additionally, and $\mathrm{H} / \mathrm{D}$ exchange at the backbone amine by stirring with $\mathrm{D}_{2} \mathrm{O}^{3}$ proved the smaller and sharper peak at $\tilde{\nu}=3181 \mathrm{~cm}^{-1}$ to be the $\mathrm{N}-\mathrm{H}$ stretching vibration $\left(\nu_{\mathrm{N}-\mathrm{D}}: \tilde{\nu}=2363 \mathrm{~cm}^{-1}\right.$; $\left.\Delta \tilde{\nu}_{\text {harm. osz. }}=858 \mathrm{~cm}^{-1}\right)$.

IR measurements with an applied magnetic field and plotting of the obtained data as $T_{B} / T_{B=0}$ allowed to unambiguously identify the observed spectroscopic feature as an electronic absorption, detectable with a regular MIR spectrometer (see Figure 3.7, left). All vibrational absorptions, which by nature are not influenced by an magnetic field, are canceled out in this spectrum and only electronic excitations which are perturbed by the field become visible. However, the shift is so small that a quantification of the effect is difficult, which is in line with the simulations of the effect by QDPT calculations, where by applying a field along the $y$-axis a maximum shift of $\leq 1 \mathrm{~cm}^{-1}$ at $10 \mathrm{~T}$ is predicted (see Figure 3.7 right and Part III Section 3.4.5).
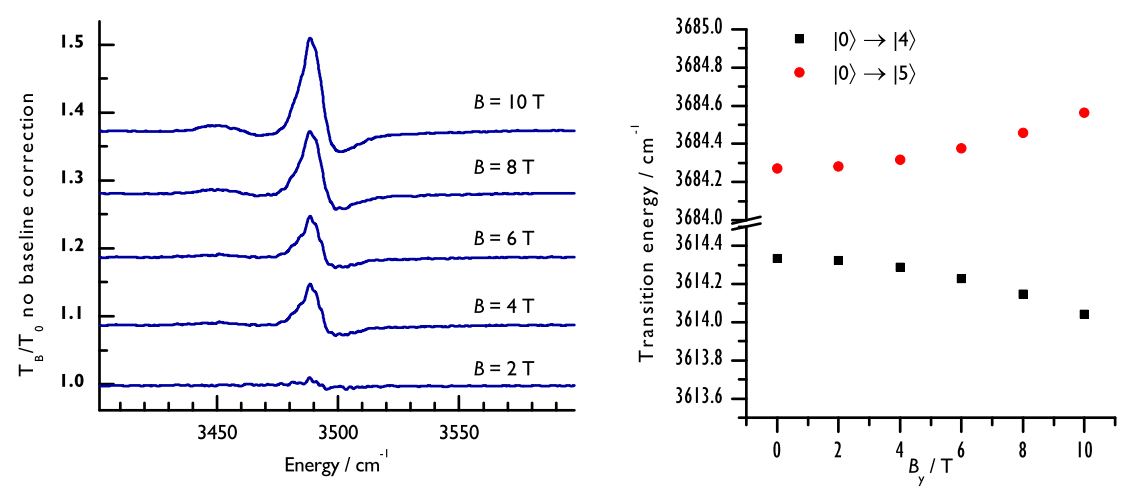

Fig. 3.7. Left: Relative change of IR transmission in applied field, plotted as $T_{B} / T_{B=0}$. The original $y$-intercept (1) of each spectrum is manipulated so that the spectra appear stacked. Right: QDPT simulated influence of a magnetic field on the energies of the states $|4\rangle$ and $|5\rangle$.

\subsection{Amide based pincer chemistry}

\subsubsection{A route to $\left[\mathrm{ReCl}_{2}\left(\mathrm{PNP}^{\mathrm{iPr}}\right)\right](30)$}

As discussed in Section 3.1.1, the synthesis of $\left[\operatorname{ReCl}_{2}\left(P N P^{i P r}\right)\right](30)$ was not possible using the same procedure as for the related tert-butyl substituted derivative XIX. Similarly,

\footnotetext{
${ }^{3}$ See Part III Section 2.3.1 for details.
} 
a)

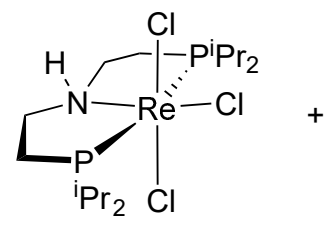<smiles>CC(C)(C)c1cc(Br)c([O])c(Br)c1</smiles>

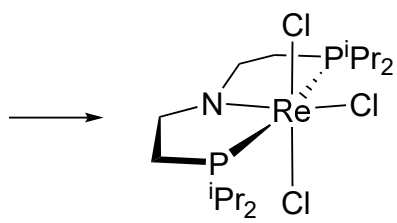

29

31
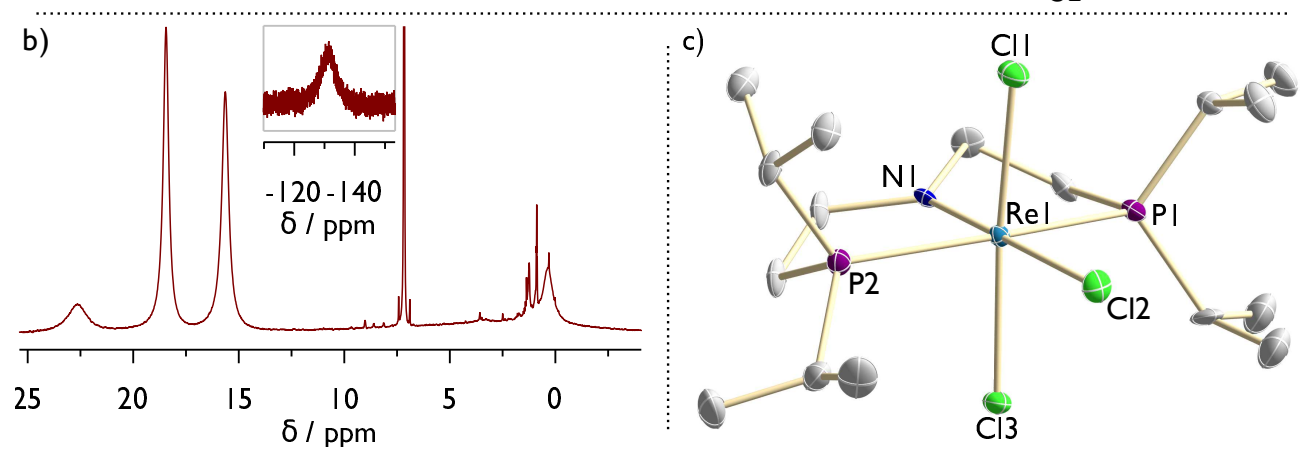

Fig. 3.8. a) Synthesis of complex 31. b) ${ }^{1} \mathrm{H}$ NMR spectrum of 31 in $\mathrm{C}_{6} \mathrm{D}_{6}$. c) Molecular structure of $\mathbf{3 1}$ obtained by single crystal X-ray diffraction measurements. $\mathrm{H}$ atoms are omitted for clarity. Anisotropic displacement parameters are set to $50 \%$ probability. Selected bond lengths $[\AA]$ and angles $\left[{ }^{\circ}\right]$ : Re1-N1 1.902(7), Re1-Cl1 2.379(2), Re1-Cl2 2.429(2), Re1-Cl3 2.369(2), N1-Re1-Cl2 178.7(2), P1-Re1-P2 163.48(8), Cl1-Re1-Cl3 174.01(8).

reactions of $\mathbf{2 9}$ with bases did not lead to an isolable complex in most cases. The only seemingly selective reaction was observed when KHMDS was used, however that reaction did not proceed clean enough to allow for isolation of the new complex. This is most likely due to the strong tendency of $\mathbf{3 0}$ to bind a sixth ligand whenever possible, as will be discussed later in this section. As an alternative to deprotonation of $\mathbf{2 9}$, the synthesis of $\mathbf{3 0}$ can be envisioned by stepwise $\mathrm{H}$-atom abstraction (from the $\mathrm{NH}$ moiety of the ligand) and subsequent reduction. Stirring $\mathbf{2 9}$ with 1 eq of TTBP in benzene resulted in a color change to deep red. $\left[\mathrm{ReCl}_{3}\left(\mathrm{PNP}^{i \mathrm{Pr}}\right)\right]$ (31) could be isolated as the sole product of the reaction in $63 \%$ yield. The ${ }^{1} \mathrm{H}$ NMR spectrum shows broad, paramagnetically shifted signals, while the ${ }^{31} \mathrm{P}\left\{{ }^{1} \mathrm{H}\right\}$ NMR spectrum shows no resonance. The expected $S=1 / 2$ spin state is supported by Evans' method, ${ }^{[205]}$ which yielded an effective magnetic moment of $\mu_{\text {eff }}=1.37$ $\pm 0.14 \mu_{\mathrm{B}}$ in $\mathrm{CD}_{2} \mathrm{Cl}_{2}$ at $\mathrm{RT}$, which is in reasonable agreement with the expected spin-only value of $\mu_{\text {eff }}=1.73 \mu_{\mathrm{B}}$, thus confirming the formal oxidation of the metal center to $\operatorname{Re}(\mathrm{IV})$. For the $\mathrm{CH}_{3}$ groups of the molecule, two large signals integrating to 12 protons each could be identified, pointing to a $C_{2 v}$ symmetry on the NMR timescale. In the molecular structure derived from X-ray diffraction measurements the Re atom is coordinated octahedrally by the PNP pincer and the $\mathrm{Cl}$ ligands. Successful $\mathrm{H}$-atom abstraction from the amine moiety is confirmed by planar coordination of the pincer nitrogen atom $\left(\Sigma\left(\measuredangle_{\mathrm{N} 1}\right)=359.8^{\circ}\right)$, accompanied by a shortening of the $\operatorname{Re}-\mathrm{N}$ distance $(31: d(\operatorname{Re} 1-N 1)=1.902(7) \AA ;$; 29: 2.158(6) $\AA$ ), supporting the amide nature of the ligand.

Since $\mathbf{3 1}$ was prepared as precursor for the synthesis of $\operatorname{Re}(\mathrm{III})$ compound $\mathbf{3 0}$, its electrochemical properties were investigated by means of CV measurements. A quasi-reversible reduction was found at $E_{1 / 2}=-1.06 \mathrm{~V}$ vs. $\mathrm{Fc}^{+} / \mathrm{Fc}$. Analysis of the scan rate dependence 

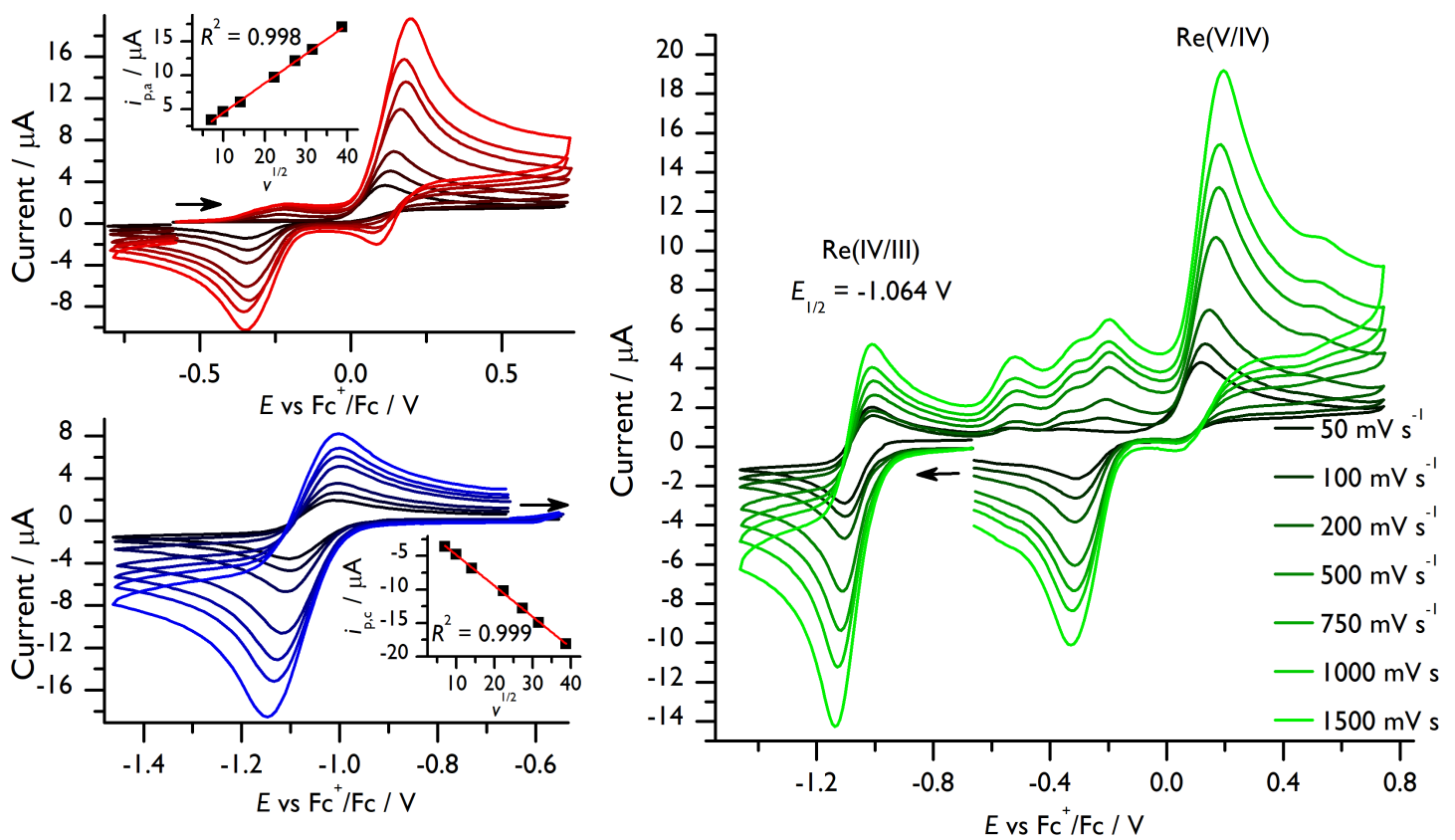

Fig. 3.9. Green: $C V$ measurements of 31 in $T H F$ at different scan rates $\left(10^{-3} \mathrm{M} 31,0.1 \mathrm{M} \mathrm{N}^{\mathrm{n}} \mathrm{Bu}_{4} \mathrm{PF}_{6}\right.$, WE: GC, RE: Ag-wire, CE: Pt-wire). Red: $\operatorname{Re}(\mathrm{v} / \mathrm{IV})$ oxidation. Blue: $\operatorname{Re}(\mathrm{IV} / \mathrm{III})$ reduction. Insets: Plot and linear fit of $i_{p}$ vs. $v^{1 / 2}$.

Tab. 3.1. Peak analysis of reduction and oxidation of 31. $\Delta E_{\text {sample }} / \Delta E_{\text {ref }}$ is the peak separation as a ratio to the peak separation of the internally added decamethylferrocene standard. All potentials are reported against $\mathrm{Fc}^{+} / \mathrm{Fc}$.

\begin{tabular}{c|ccc|cc}
\hline & \multicolumn{3}{|c|}{$\operatorname{Re}(\mathrm{IV} / \mathrm{III})$} & \multicolumn{2}{c}{$\operatorname{Re}(\mathrm{V} / \mathrm{IV})$} \\
\hline$v / \mathrm{mV} \mathrm{s}^{-1}$ & $E_{1 / 2} / \mathrm{V}$ & $i_{p, a n} / i_{p, c a t}$ & $\Delta E_{\text {sample }} / \Delta E_{\text {ref }}$ & $E_{\text {an }} / \mathrm{V}$ & $i_{p, a n} / \mu \mathrm{A}$ \\
\hline 50 & -1.057 & 0.81 & 1.00 & 0.113 & 3.43 \\
100 & -1.057 & 0.78 & 1.00 & 0.131 & 4.63 \\
200 & -1.057 & 0.75 & 1.00 & 0.140 & 6.05 \\
500 & -1.062 & 0.73 & 1.11 & 0.163 & 9.66 \\
750 & -1.066 & 0.70 & 1.00 & 0.181 & 12.10 \\
1000 & -1.071 & 0.65 & 1.10 & 0.176 & 13.78 \\
1500 & -1.075 & 0.66 & 1.00 & 0.194 & 17.07 \\
\hline
\end{tabular}

of $i_{p, c}$ according to the Randles-Sevcik equation (see Part III, Section 1.2, eq. 1.1) gives a linear relationship between $i_{p, c}$ and $v^{1 / 2}$, indicating electrochemically reversible electron transfer to a freely diffusing analyte. The ratio of $\frac{i_{p, a}}{i_{p, c}}$ decreases upon increasing scan rate $v$, indicating a first-order reversible chemical follow-up reaction after electrochemically reversible electron transfer in comparable timescale to the CV experiment. ${ }^{[206]}$ Assuming the immediate reaction to be chloride loss, this reaction would therefore be much slower than in the case of the tert-butyl substituted congener $\left[\operatorname{ReCl}_{3}\left(\mathrm{PNP}^{\mathrm{tBu}}\right)\right]$, where no reverse peak could be observed even in the presence of 50 eq chloride ions at high scan rates. ${ }^{[207]}$ The oxidation is also proven to be electrochemically reversible according to the linear relationship between $i_{p, a}$ and $v^{1 / 2}$. However, a reverse-peak appeared only at $v=1500 \mathrm{mV} \mathrm{s}^{-1}$, and a 
new reduction feature around $-0.4 \mathrm{~V}$ indicates a rather selective $\mathrm{EC}$ mechanism after initial oxidation to a new product.

While these results suggest $\mathrm{CoCp}_{2}$ to be a proper reductant for simple $\operatorname{Re}(\mathrm{IV} / \mathrm{III})$ reduction, actually using this reagent led to basically blank in situ NMR spectra and the formation of a precipitate which was unsoluble in THF. A possible explanation would be the formation of insoluble $\left[\mathrm{ReCl}_{3}\left(\mathrm{PNP}^{i \mathrm{Pr}}\right)\right]\left[\mathrm{Co}(\mathrm{Cp})_{2}\right]$ rather than precipitation of $\left[\mathrm{Co}(\mathrm{Cp})_{2}\right] \mathrm{Cl}$. However, this is only a working hypothesis and needs further investigation. Therefore, $\mathrm{Na}(\mathrm{Hg})$ was used as a reductant. Reduction of $\mathbf{3 1}$ with $\mathrm{Na}(\mathrm{Hg})$ did give selective conversion of the starting material. Interestingly, two different complexes of $C_{2 v}$ symmetry on the NMR timescale were obtained depending on whether the reaction was conducted in THF $\left(\delta_{31 \mathrm{P}}=-14.5 \mathrm{ppm}\right.$, deep orange) (32) or in non-coordinating solvents like benzene, toluene or 1,4 -dioxane $\left(\delta_{31} \mathrm{P}=\right.$ 9.9 ppm, deep violet) (30) (see Figure $3.10 \mathrm{~b}$ ). The first complex is largely uncharacterized, despite the molecular structure obtained from $\mathrm{X}$-ray diffraction measurements on single crystals grown from $\mathrm{THF} / \mathrm{Et}_{2} \mathrm{O}$ (see Figure $3.10 \mathrm{~d}$ ). Complex 32 is found to be octahedrally coordinated $\left[\mathrm{ReCl}_{2}(\mathrm{thf})\left(\mathrm{PNP}{ }^{i \mathrm{Pr}}\right)\right]$ where a THF molecule binds at the vacant coordination side trans to the amide, resulting from chloride dissociation after reduction. The planar coordination of the PNP amide ligand $\left(\Sigma\left(\measuredangle_{\mathrm{N} 1}\right)=360.0^{\circ}\right)$ and the presence of only two chloride ligands confirm successful reduction to a $\operatorname{Re}(\mathrm{III})$ species. Nevertheless, applying vacuum to the complex caused release of that ligand and (rather clean, but not exclusive) formation of $\mathbf{3 0}$, which prevented isolation or further characterization of $\mathbf{3 2}$.

If $\mathbf{3 1}$ is reduced in toluene (or other non-coordinating solvents), the reaction takes longer (several hours, attributed to reduced activity of the amalgam in low polarity solvents), but yields a clean complex identified as $\left[\mathrm{ReCl}_{2}\left(\mathrm{PNP}{ }^{i \mathrm{Pr}}\right)\right](\mathbf{3 0})$. NMR analysis indicates spectroscopically clean formation of a $C_{2 v}$ symmetric complex. The deep violet color resembles that of tert-butyl substituted complex XIX and the LIFDI mass is in agreement with the proposed structure. Single crystals grown by slow evaporation of a toluene solution allowed for molecular structure determination by X-ray diffraction measurements. The Re center in 30 is five-fold coordinated and adopts a distorted trigonal bipyramidal coordination geometry with the two phosphorous atoms in axial position $\left(\tau_{5}=0.677\right) .{ }^{[196]}$ The short $\mathrm{Re}-\mathrm{N}$ distance $\left(d_{\mathrm{Re} 1-\mathrm{N} 1}=1.873(4) \AA\right)$ and planar coordination of the nitrogen atom $\left(\Sigma\left(\measuredangle_{\mathrm{N} 1}\right)=\right.$ $\left.360.0^{\circ}\right)$ confirm the amide nature of the PNP ligand.

At this point, it interesting to note that all compounds within the steric variation series of $\left[\operatorname{ReCl}{ }_{2}\left(\mathrm{PNP}^{i \mathrm{Pr}}\right)\right]\left(\mathbf{3 0}\right.$, this work), $\left[\operatorname{ReCl}_{2}\left(\mathrm{PNP}^{t \mathrm{Bu}}\right)\right]\left(\mathbf{X I X}\right.$, reference [94]) and $\left[\operatorname{ReCl}_{2}\left(\mathrm{PNP}^{\mathrm{Ad}}\right)\right]$ (30-Ad, unpublished work by Lukas Alig) ${ }^{4}$ are coordinated by closely related ligands and could thus be expected to exhibit similar electronic structures (the variation of the trialkylphosphine donor strength can be assumed to be neglectible upon interchanging $\mathrm{R}=$ $i \mathrm{Pr}, t \mathrm{Bu}$ and $\mathrm{Ad}) .{ }^{[208]}$ Contrary to this assumption, the three complexes resonate at highly

\footnotetext{
${ }^{4}$ I appreciate Lukas' permission to include the ${ }^{31} \mathrm{P}$ NMR shift as well as the $\tau_{5}$ value of his complex $\mathbf{3 0}$-Ad in this discussion. Further information on this complex can be expected to be found in his thesis.
} 
different ${ }^{31} \mathrm{P}$ NMR shifts $\left(\delta_{31} \mathrm{P}=10.0 \mathrm{ppm}(\mathbf{3 0}),-51.5 \mathrm{ppm}(\mathbf{X I X})\right.$ and $-412.5 \mathrm{ppm}(\mathbf{3 0}$ Ad)). One might be tempted to assume this to be due to increasing influence of spin orbit coupling and a resulting TIP. However, the comparsionn of $\left[\mathrm{OsCl}\left(\mathrm{P}=\mathrm{N}=\mathrm{P}^{t \mathrm{Bu}}\right)\right]$ and $2 \mathbf{9}$ did already indicate that a direct linear correlation between ${ }^{31} \mathrm{P}$ NMR shifts and TIP is not likely (see Section 3.1.2 for further details). A striking difference within the series is the change in coordination geometry from distorted trigonal bipyramidal in $30\left(\tau_{5}=0.677\right)$ to distorted

a)

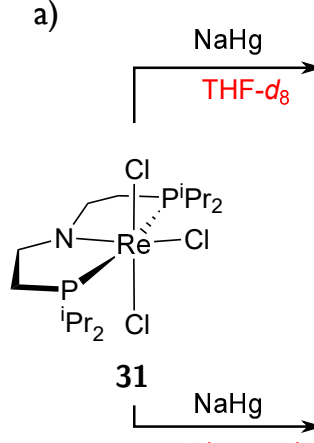

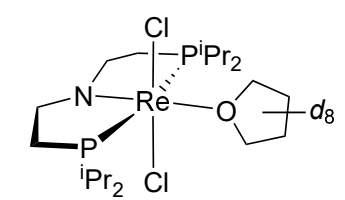

N 32
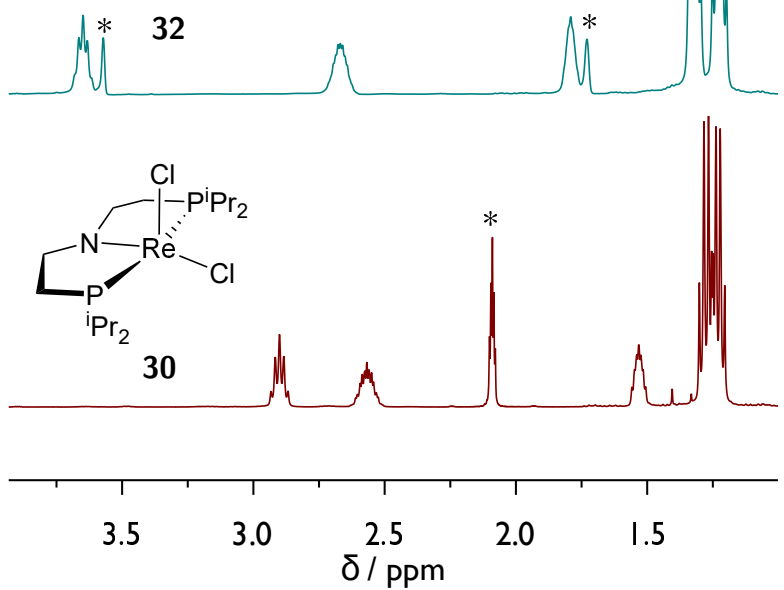

b)

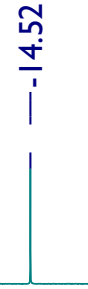

๙

$\ddot{1}$

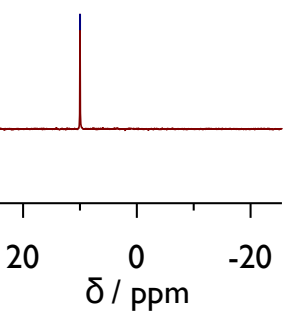

c)

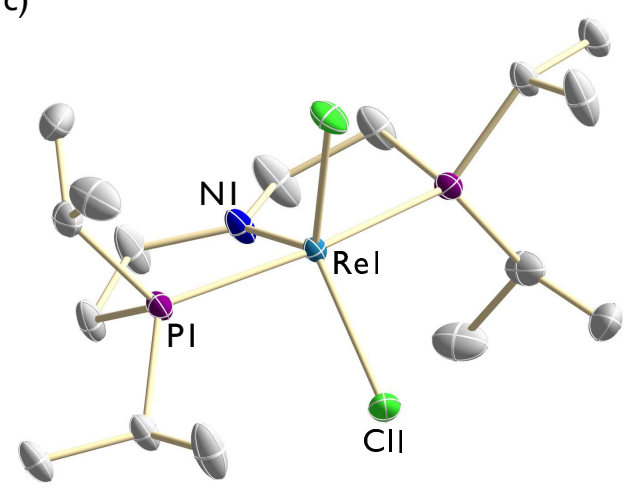

d)

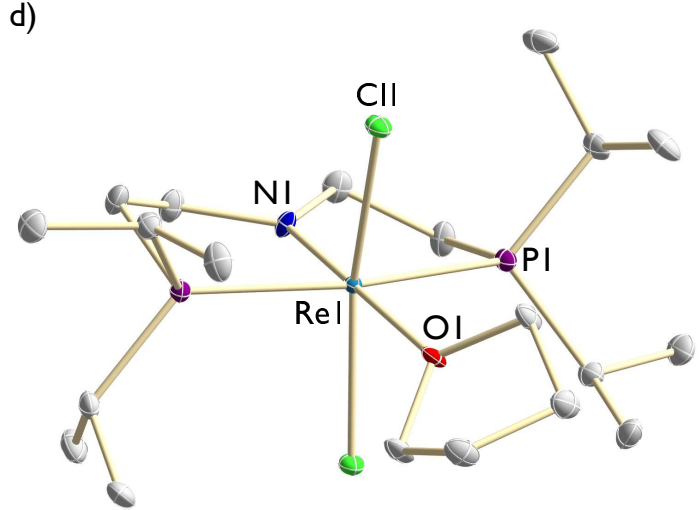

Fig. 3.10. a) Reaction scheme and ${ }^{1} \mathrm{H}$ NMR spectra of the reduction of 31 in THF and toluene leading to the formation of $\mathbf{3 2}$ and $\mathbf{3 0}$, respectively. Residual solvent signals are marked with asterisks. b) ${ }^{31} \mathrm{P}\left\{{ }^{1} \mathrm{H}\right\}$ NMR spectra of $\mathbf{3 2}$ (top) and $\mathbf{3 0}$ (bottom) in THF- $d_{8}$ and toluene- $d_{8}$, respectively. c) Molecular structure of $\mathbf{3 0}$ obtained by single crystal X-ray diffraction measurements. The asymmetric unit contains a half molecule. $\mathrm{H}$ atoms are omitted for clarity. Anisotropic displacement parameters are set to $50 \%$ probability. Selected bond lengths $[\AA]$ and angles $\left[^{\circ}\right]$ : Re1-N1 1.873(4), Re1-Cl1 .3272(7), Re1-P1 2.3731(7), Cl1-Re1-Cl\#1 126.07(4), P1-Re1-P\#1 166.66(4), N1-Re1-Cl1 116.97(2). d) Molecular structure of $\mathbf{3 2}$ obtained by single crystal X-ray diffraction measurements. The asymmetric unit contains a half molecule. $\mathrm{H}$ atoms are omitted for clarity. Anisotropic displacement parameters are set to $50 \%$ probability. Selected bond lengths $[\AA]$ and angles $\left[^{\circ}\right]$ : Re1-N1 1.900(3), Re1-O1 2.264(2), Re1-P1 2.3725(6), Re1-Cl1 2.4097(5), N1-Re1O1 180.0, P1-Re1-P\#1 166.07(3), Cl1-Re1-Cl\#1 164.22(3). 
a)

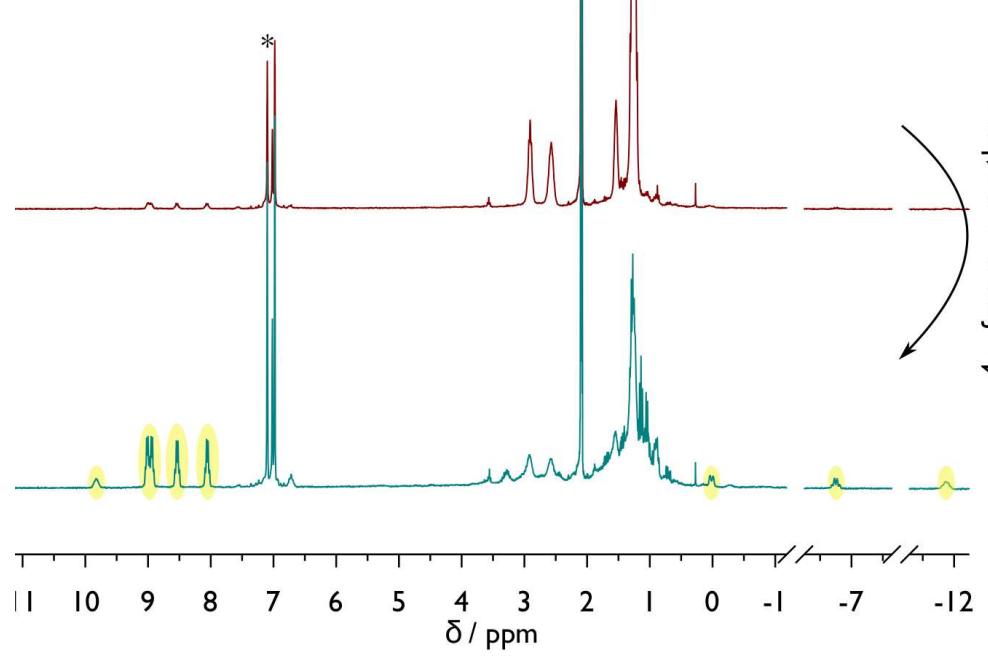

b)

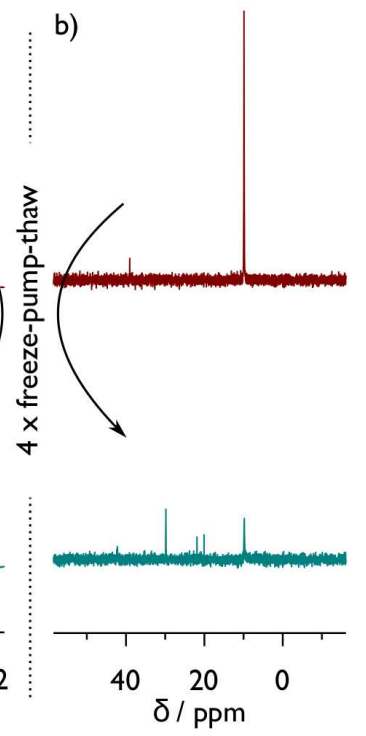

Fig. 3.11. Changes in the ${ }^{1} \mathrm{H}(a)$ and ${ }^{31} \mathrm{P}\left\{{ }^{1} \mathrm{H}\right\}(b)$ NMR spectra of a solution of 30 in toluene- $d_{8}$ after four freeze-pump-thaw cycles, demonstrating the instability of the complex to even these conditions. Residual solvent signals are marked with asterisks. The yellow shaded ${ }^{1} \mathrm{H}$ NMR signals can be assigned to the starting complex $\mathbf{2 9}$, although the origin of its formation is unclear.

square pyramidal ( $\tau_{5}=0.371$ and 0.261 in $\mathbf{X I X}$ and 30-Ad, respectively). This change in geometry should directly influence the $d$-orbital splitting and thus have a defined influence on the electronic structure. However, detailed (theoretical) investigation of the underlying effects is necessary to draw founded conclusions.

Nevertheless, $\mathbf{3 0}$ can be considered to be the exact, sterically less encumbered analog to $\mathrm{PNP}^{t \mathrm{tBu}}$ complex $\mathbf{X I X}$ and thus the proper candidate to test the proposed effects of reduced steric shielding on $\mathrm{N}_{2}$ activation as outlined at the beginning of this chapter (3). However, despite several attempts, clean isolation of $\mathbf{3 0}$ could not be achieved. The main reason for this can be found in a remarkable instability of the complex towards almost any conditions. While a toluene solution was stable for at least several days, irreversible decomposition of $\mathbf{3 0}$ was observed upon solvent removal or even simple freezing and thawing of the solution (see Figure 3.11). Therefore, only in situ prepared samples were used for all further investigations, which could be separated from $\mathrm{Hg}$ and precipitated $\mathrm{NaCl}$ by simple filtration. This was proven to be a viable approach by measuring elemental analysis of a filtered and lyophilized sample. Even though lyophilization induced partial complex decomposition as proven by NMR spectroscopy, the fitting analysis combined with clean spectroscopy prior to removal of the solvent point to a clean solution containing only $\mathbf{3 0}$ after filtration.

\subsubsection{Dinitrogen chemistry with $\left[\mathrm{ReCl}_{2}\left(\mathrm{PNP}^{i \mathrm{Pr}}\right)\right](30)$}

30 shows a high tendency to bind a sixth ligand, as exemplified by the formation of a THF complex or the non-trivial synthesis of the penta-coordinated complex 30 . This can be 
attributed to the reduced steric shielding of the metal center and is in line with previous results on the corresponding $\left[\mathrm{RuCl}_{2}\left(\mathrm{HPNP}^{\mathrm{R}}\right)\right]$ complexes $(\mathrm{R}=i \mathrm{Pr}, t \mathrm{Bu}) \cdot{ }^{209,210}$ Accordingly, when 30 is exposed to an $\mathrm{N}_{2}$ atmosphere, the color changes immediately from deep violet to brown-orange, indicating fast reaction with dinitrogen at the $\operatorname{Re}(\mathrm{III})$ oxidation state. The NMR spectra reveal largely broadened signals, with the ${ }^{31} \mathrm{P}\left\{{ }^{1} \mathrm{H}\right\}$ NMR signal vanishing almost in the spectral noise, at a similar shift as pure $\mathbf{3 0}$. However, if the sample is cooled $\left(-15--50^{\circ} \mathrm{C}\right.$ ), a new set of signals forms (see Figure $3.12 \mathrm{a}$ ). Warming the sample back to $\mathrm{RT}$ results in reformation of the previous, broad signals and when the sample is heated (up to $75^{\circ} \mathrm{C}$ ) or when $\mathrm{Ar}$ is bubbled through the solution, $\mathbf{3 0}$ becomes the main species again. Hence, the reaction with $\mathrm{N}_{2}$ is dynamic, fully reversible and thus most likely mere coordination chemistry. The following discussion therefore focuses on the identity of the species which are present at low temperatures under an $\mathrm{N}_{2}$ atmosphere.

The ${ }^{31} \mathrm{P}\left\{{ }^{1} \mathrm{H}\right\}$ NMR spectrum reveals five new signals, i.e. two doublets at $\delta^{31} \mathrm{P}=1.3$

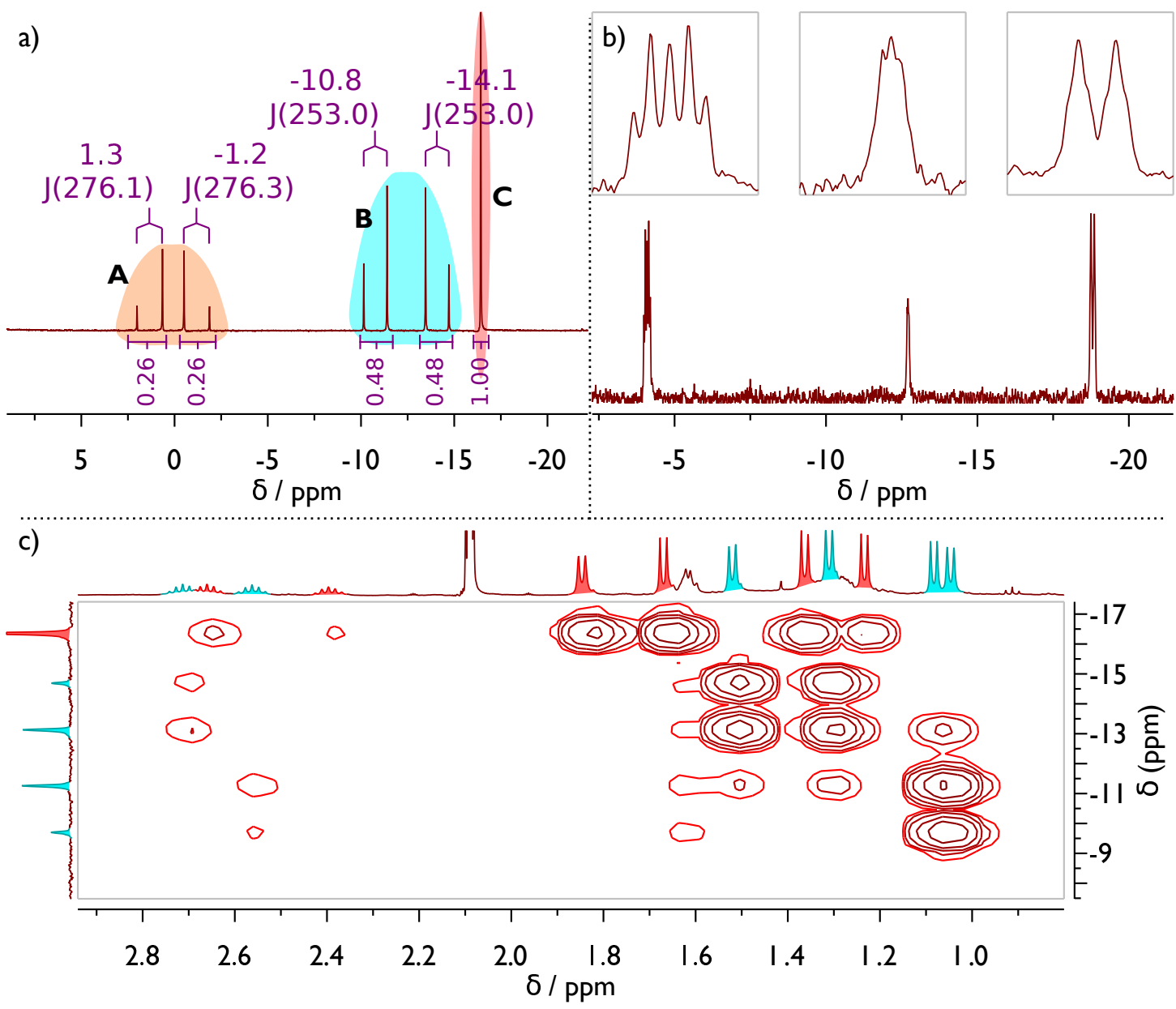

Fig. 3.12. Low temperature NMR spectroscopy of the reaction of $\mathbf{3 0}$ with $\mathrm{N}_{2}$. a) ${ }^{31} \mathrm{P}\left\{{ }^{1} \mathrm{H}\right\} \mathrm{NMR}$ spectrum, showing three pincer fragments. Coupling constants are given in $\mathrm{Hz} . \quad b)$ ${ }^{15} \mathrm{~N}\left\{{ }^{1} \mathrm{H}\right\}$ NMR spectrum, recorded inverse gated. c) ${ }^{1} \mathrm{H}^{31}{ }^{31} \mathrm{P}$ heteronuclear multiple bond correlation (HMBC) revealing both, fragment $\mathbf{B}$ (turquoise) and $\mathbf{C}($ red) to feature a mirror plane within and orthogonal to the pincer plane, respectively. For better readability, the ${ }^{1} \mathrm{H}$ NMR trace was substituted with the ${ }^{1} \mathrm{H}\left\{{ }^{31} \mathrm{P}\right\}$ NMR spectrum. 
and $-1.2 \mathrm{ppm}$ with a mutual coupling ${ }^{2} J_{\mathrm{PP}}=276.2 \mathrm{~Hz}$ (denoted $\mathbf{A}$ in the following), two doublets at $\delta_{31}=-10.8$ and $-14.1 \mathrm{ppm}$ with a mutual coupling of ${ }^{2} J_{\mathrm{PP}}=253.0 \mathrm{~Hz}$ (denoted B) and a singlet at $\delta_{31} \mathrm{P}=-16.4 \mathrm{ppm}$ (denoted C), indicating three different PNP-pincer ligands, two with inequivalent phosphorous atoms as well as one with equivalent phosphorous atoms. Importantly, the ratio $\mathbf{B}: \mathbf{C}$ was found to be close to $1: 1$ in every single spectrum that was measured, while the ratio $\mathbf{A}: \mathbf{B} / \mathbf{C}$ changed between the experiments. The coupling constants are typical for trans coupling of the pincer ligand in an asymmetric environment, whereas the constant 1:1 ratio of $\mathbf{B}$ and $\mathbf{C}$ indicates either some kind of connectivity between these fragments or a dissociation of one intermediate into two products. The origin of these varying ratios was not investigated, yet it is noteworthy that $\mathbf{A}$ was the minor product in all measurements and most of the times the signals were less intense than in the exemplary spectrum in Figure $3.12 \mathrm{a}$.

Therefore the focus of further investigations was on the nature of $\mathbf{B}$ and $\mathbf{C}$, which was further elucidated using 2D-NMR spectroscopy. By ${ }^{1} \mathrm{H}_{-}{ }^{31} \mathrm{P}$ HMBC spectroscopy, coupling of B and $\mathbf{C}$ to two inequivalent iso-propyl groups (one $\mathrm{CH}$ and two $\mathrm{CH}_{3}$ signal) each could be detected. Therefore, both pincer ligands are coordinated in a $C_{S}$ symmetric environment, but with orthogonal mirror planes (i.e. once within the ligand plane $(\mathbf{B})$ and once perpendicular to it $(\mathbf{C})$. An identification of the $i \operatorname{Pr}$ signals belonging to the ${ }^{31} \mathrm{P}$ signal $\mathbf{A}$ was not possible, due to the low concentration. If $\mathbf{3 0}$ was placed under an atmosphere of ${ }^{15} \mathrm{~N}_{2}$ gas, at low temperatures three signals were identified by ${ }^{15} \mathrm{~N}\left\{{ }^{1} \mathrm{H}\right\}$ NMR spectroscopy at $\delta_{15} \mathrm{~N}=-4.1 \mathrm{ppm}$ (dt, $J=5.5$ and $2.6 \mathrm{~Hz}$ ), $-12.7 \mathrm{ppm}(\mathrm{s})$ and $-18.8 \mathrm{ppm}(\mathrm{d}, J=5.7 \mathrm{~Hz}$ with non-resolved shoulders which can be estimated to a coupling constant of $\approx 1.5 \mathrm{~Hz}$ ) (see Figure $3.12 \mathrm{~b}$ ). Again, the signals at $\delta_{15} \mathrm{~N}=-4.1$ and $-18.8 \mathrm{ppm}$ integrate in a $1: 1$ ratio, while the signal at $\delta_{15} \mathrm{~N}=-12.7 \mathrm{ppm}$ integrates to 0.4 . This matches the ratio between $\mathbf{B} / \mathbf{C}$ vs. A determined by ${ }^{31} \mathrm{P}\left\{{ }^{1} \mathrm{H}\right\}$ NMR spectroscopy. The coupling constants indicates the resonances at $\delta_{15} \mathrm{~N}=$ -4.1 and $-18.8 \mathrm{ppm}$ to belong to a single, asymmetrically coordinated $\mathrm{N}_{2}$ ligand, while the signal at $-12.7 \mathrm{ppm}$ must belong to an $\mathrm{N}_{2}$ ligand in a symmetric coordination environment. Notably, under ${ }^{15} \mathrm{~N}_{2}$, in the ${ }^{31} \mathrm{P}\left\{{ }^{1} \mathrm{H}\right\}$ NMR spectrum the resonance at $\delta_{31} \mathrm{P}=-16.4 \mathrm{ppm}$ splits into a doublet with $J_{\mathrm{PN}}=2.6 \mathrm{~Hz}$, matching the small coupling of the signal at $\delta_{15} \mathrm{~N}$ $=-4.1 \mathrm{ppm}$ and thereby proving binding of the $\mathrm{N}_{2}$ ligand to fragment $\mathbf{C}$.

Finally, the sample was subjected to an ${ }^{1} \mathrm{H}\left\{{ }^{31} \mathrm{P}\right\}$ NOESY as well as a ${ }^{31} \mathrm{P}\left\{{ }^{1} \mathrm{H}\right\}$ DOSY measurement (see Figure 3.13). The NOESY revealed small cross peaks between $i \operatorname{Pr}$ groups belonging to $\mathbf{B}$ and $\mathbf{C}$, indicating both fragments to actually belong to a single compound 33a. This was confirmed by the low temperature ${ }^{31} \mathrm{P}$-DOSY measurement, which was performed first at $-30^{\circ} \mathrm{C}$ on a sample of 30 in toluene under Ar. Then $\mathrm{N}_{2}$ gas was bubbled through the solution and the sample was remeasured under $\mathrm{N}_{2}$ at the same temperature. The data shows all three pincer moieties to have the same diffusion coefficient of $D=$ $1.89 \cdot 10^{-6} \mathrm{~cm}^{2} \mathrm{~s}^{-1}$, while pure 30 has a coefficient of $D=2.61 \cdot 10^{-6} \mathrm{~cm}^{2} \mathrm{~s}^{-1}$. Since these values are derived in toluene- $d_{8}$ at $-30^{\circ} \mathrm{C}$, for comparision with the previously published diffusion constant of tert-butyl substituted complex $\mathbf{X I X}\left(D=9.1 \cdot 10^{-6} \mathrm{~cm}^{2} \mathrm{~s}^{-1}\right.$ in THF- $d_{8}$ 

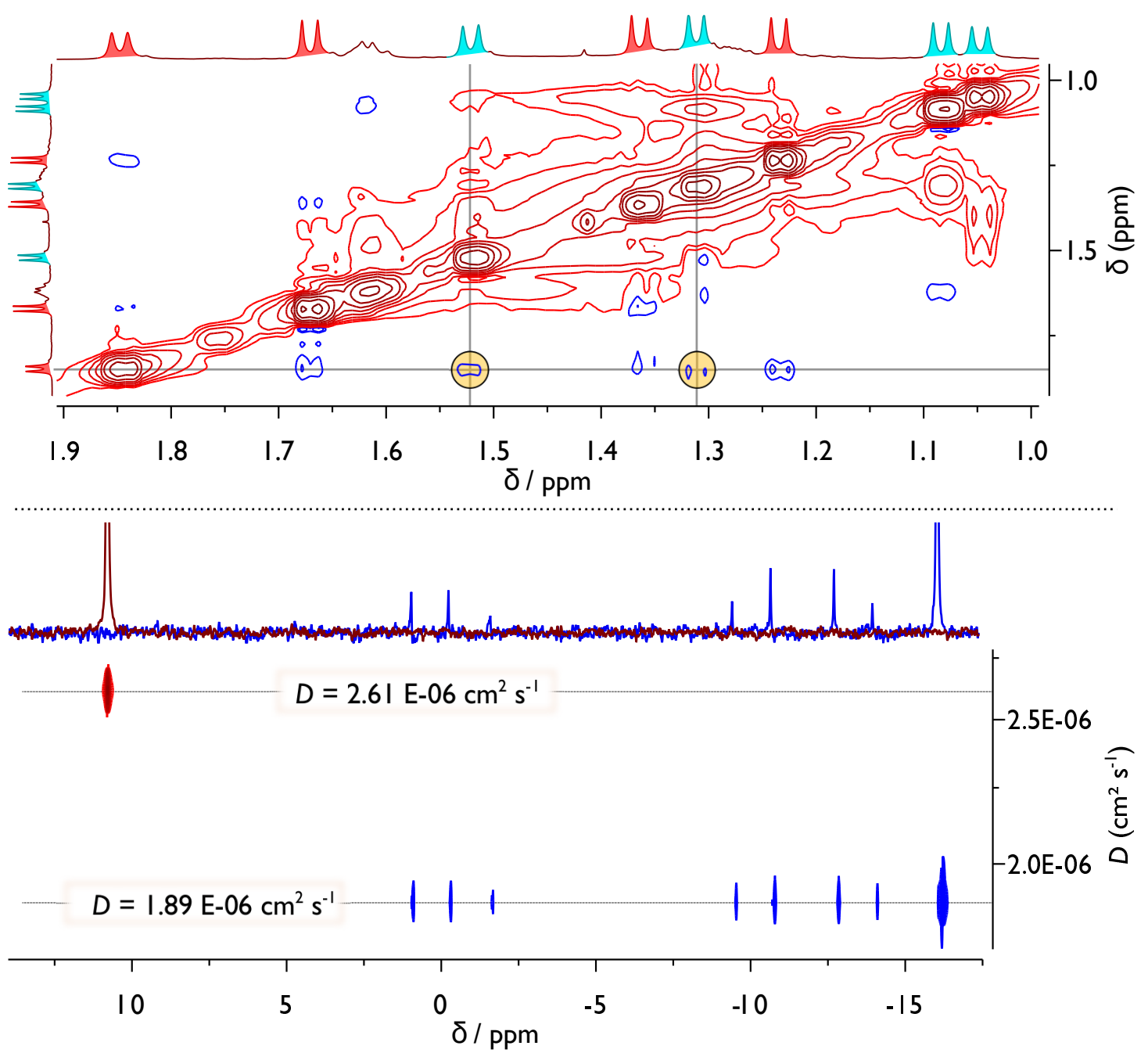

Fig. 3.13. Low temperature ${ }^{1} \mathrm{H}-\mathrm{NOESY}$ (top) and ${ }^{31} \mathrm{P}$-DOSY (bottom) of the reaction of $\mathbf{3 0}$ with $\mathrm{N}_{2}$ in toluene- $d_{8}$. The NOESY shows cross peaks between $i \operatorname{Pr}$ groups belonging to the fragments $\mathbf{B}$ and $\mathbf{C}$ while the DOSY shows smaller diffusion coefficients for $\mathbf{A}, \mathbf{B}$ and $\mathbf{C}$ (blue), compared to 30 (red) (all measured at $-30{ }^{\circ} \mathrm{C}$ ).

at RT ${ }^{[95]}$ the Stokes radii for all three diffusion constants were calculated according to the Stokes-Einstein equation:

$$
D=\frac{k_{\mathrm{B}} T}{6 \pi \eta r}
$$

where $D$ is the diffusion constant, $k_{\mathrm{B}}$ is Boltzmann's constant, $T$ is the temperature, $\eta$ is the dynamic viscosity of the solvent at that temperature and $r$ is the Stokes radius, i.e. the radius a spherical particle with that $D$ would have. The restriction of this equation to spherical particals limits its use for quantitative radii determination, however since the molecules discussed here do not deviate too much from that geometry, eq. 3.1 can be used in reasonable approximation of qualitative analysis. With $\eta_{\text {toluene, }-30^{\circ} \mathrm{C}}=1.39 \mathrm{mPs}$ and $\eta_{\mathrm{THF}, 25^{\circ} \mathrm{C}}=0.456 \mathrm{mPs},{ }^{211,212}$ the following Stokes radii could be calculated: $r(\mathbf{X I X})=$ $4.95 \AA, r(30)=4.48 \AA$ and $r(33 a)=6.69 \AA$. 


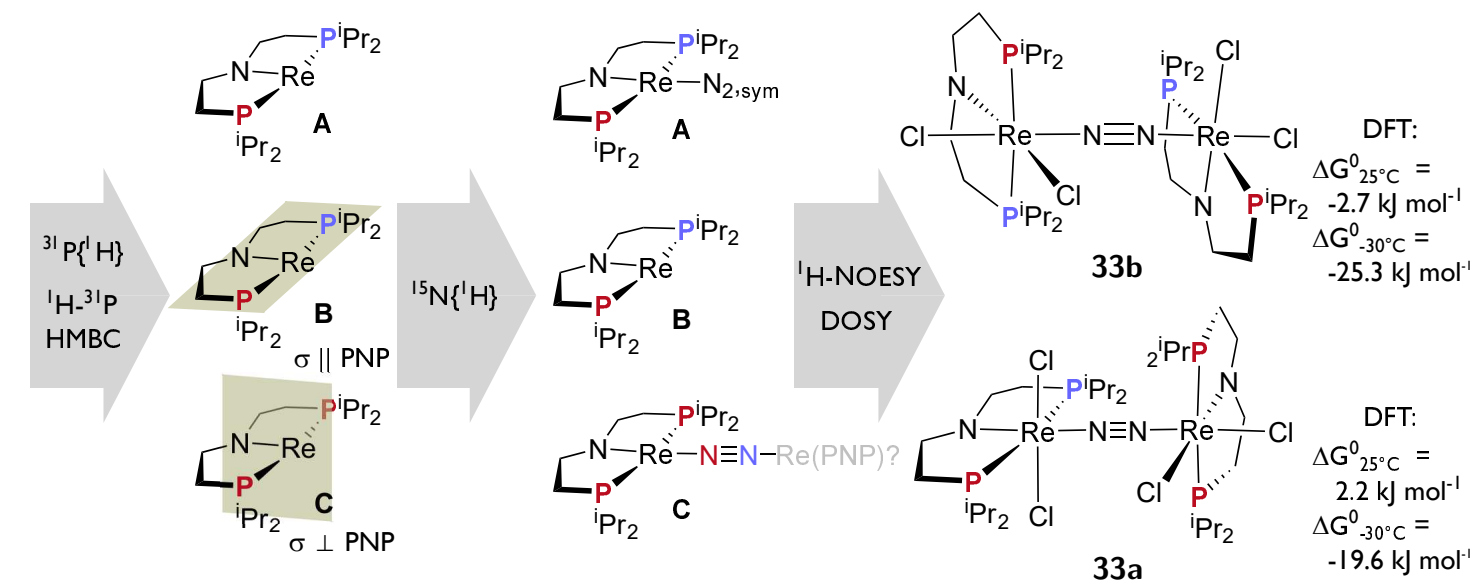

Fig. 3.14. Summary of structure determination of 33a and 33b by low temperature NMR spectroscopy and computational analysis of the thermochemistry of the formation of 33a and 33b at different temperatures.

All these data form a coherent picture of the reaction of $\mathbf{3 0}$ with $\mathrm{N}_{2}$, which is summerized in Figure 3.14:

- The reversible nature of the reaction tendentially indicates simple coordination of $\mathrm{N}_{2}$. The formation of the product(s) only at low temperatures points to an entropic penalty of the reaction, in line with a dimerization process.

- ${ }^{31} \mathrm{P}$ NMR as well as ${ }^{1} \mathrm{H}_{-}{ }^{31} \mathrm{P}$ HMBC spectra allow for identification of three pincer fragments and their symmetry planes.

- ${ }^{15} \mathrm{~N}$ NMR spectroscopy proves $\mathrm{N}_{2}$ binding to fragment $\mathbf{C}$, while binding of the same ligand to $\mathbf{B}$ is suggested by a triplet-like shoulders of the second signal of similar intensity. If $\mathbf{A}$ coordinates $\mathrm{N}_{2}$ it has to be bound symmetrically.

- NOESY and ${ }^{31}$ P-DOSY reveal all three fragments to belong to dimers, with $\mathbf{B}$ and $\mathbf{C}$ actually being two sides of the same dimer.

Consequently, A most likely corresponds to $\left[\left(\mu-\mathrm{N}_{2}\right)\left\{\operatorname{ReCl}_{2, \text { cis }}\left(\mathrm{PNP}^{i \mathrm{Pr}}\right)\right\}_{2}\right](\mathbf{3} \mathbf{3 b})$, a symmetric analog of the previously observed tert-butly substituted analog $\mathbf{X X}$ (see Section 2.1) but with additional chloride ligands trans to the $\mathrm{N}_{2}$ bridge. On the other hand, $\mathbf{B}$ and $\mathbf{C}$ are the two sides of an asymmetrical dimer $\left[\left\{\operatorname{ReCl}_{2, \text { cis }}\left(\mathrm{PNP}^{i \mathrm{Pr} r}\right)\right\}\left(\mu-\mathrm{N}_{2}\right)\left\{\mathrm{ReCl}_{2, \text { trans }}\left(\mathrm{PNP}^{i \mathrm{Pr}}\right)\right\}\right]$ (33a) as depicted in Figure 3.14. These suggestions are supported by DFT calculations, ${ }^{5}$ which reveal the binding of $\mathrm{N}_{2}$ to form dimer $33 \mathbf{a}$ and $33 \mathbf{b}$ to be favorable at $-30^{\circ} \mathrm{C}$ by $\Delta G^{0}=-19.6$ and $-25.3 \mathrm{~kJ} \mathrm{~mol}^{-1}$, respectively. At $25^{\circ} \mathrm{C}$, the reaction is computed to be almost thermoneutral $\left(\Delta G^{0}=2.2\right.$ and $\left.-2.7 \mathrm{~kJ} \mathrm{~mol}^{-1}\right)$, fully consistent with the experimental observation. Coordination of $\mathrm{N}_{2}$ to $\mathbf{3 0}$ either cis or trans to the amido ligand was calculated to be uphill in all cases. Unfortunately, neither 33a nor 33b could be crystallized to allow for X-ray diffraction, despite various attempts. Hence, no molecular structures could be determined experimentally.

${ }^{5}$ PBE0/D3BJ/RIJCOSX/def2-TZVP/CPCM(toluene)||PBE/D3BJ/RI/def2-SVP 


\subsubsection{Assessment of dinitrogen activation capabilities}

The fact that dinitrogen binding and dimer formation already occurs straightforward at the $\operatorname{Re}(\mathrm{III})$ oxidation state encouraged a thorough investigation of the abilities of $\mathbf{3 0}$ to activate and/or split $\mathrm{N}_{2}$ under reductive conditions.

In order to be able to evaluate the success or failure of a reaction, the expected product of successful splitting, $\left[\operatorname{Re}(\mathrm{N}) \mathrm{Cl}\left(\mathrm{PNP}^{i \mathrm{Pr}}\right)\right]$ (34) was synthesized by deprotonation of $\left[\mathrm{Re}(\mathrm{N}) \mathrm{Cl}_{2}\left(\mathrm{HPNP}^{i \mathrm{Pr}}\right)\right](37) .{ }^{6}$ Stirring 37 with 1 eq $\mathrm{KO}^{t} \mathrm{Bu}$ in benzene resulted in clean formation of a new, diamagnetic, species with a single resonance in the ${ }^{31} \mathrm{P}\left\{{ }^{1} \mathrm{H}\right\}$ NMR spectrum at $\delta_{31} \mathrm{P}=74.7 \mathrm{ppm}$ (see Figure 3.15). ${ }^{1} \mathrm{H}$ NMR spectroscopy revealed 34 to exhibit $C_{S}$ symmetry in solution and the lack of a signal corresponding to an $\mathrm{NH}$ proton indicated successful deprotonation. LIFDI mass spectrometry confirmed the proposed molecular composition and finally single crystals obtained from chlorobenzene/pentane diffusion allowed for a X-ray diffraction measurement to determine the molecular structure of $\mathbf{3 4}$. The $\operatorname{Re}$ center is coordinated in a distorted square pyramidal coordination geometry with the nitride ligand in apical position $\left(\tau_{5}=0.30\right)^{[196]}$. The $\mathrm{Re} \equiv \mathrm{N}$ bond length $(d(\operatorname{Re} 1-\mathrm{N} 2)=1.666(2) \AA)$ is in full agreement with a nitride ligand and does indicate only moderate bond elongation as compared to $d(\operatorname{Re} \equiv \mathrm{N})=1.647(18) \AA$ in compound 22 (see Section 2.3.1) or $d(\operatorname{Re} \equiv \mathrm{N}$ ) $=1.643(6) \AA$ in XXI ${ }^{[155]}$ most likely due to the distorted geometry. The short distance to the backbone nitrogen donor $(d(\mathrm{Re} 1-\mathrm{N} 1)=2.0086(18) \AA)$ and the planar coordination of the nitrogen atom $\left(\Sigma\left(\measuredangle_{\mathrm{N} 1}\right)=352.6^{\circ}\right)$ support the formulation as an amide.

With this target molecule in hand, different routes to potential $\mathrm{N}_{2}$ splitting were investigated. The $\operatorname{Re}(\mathrm{III}) / \operatorname{Re}(\mathrm{III})$ dimer(s) 33b/33a were treated with chloride abstracting agents ( $\mathrm{NaX}$, with $\left.\mathrm{X}=\mathrm{BPh}_{4}^{-}, \mathrm{OTf}^{-}\right)$to stabilize the dimers analog to literature known $8 \pi$ electron systems. Additionally, acids ( $\mathrm{HOTf}, \mathrm{H}\left(\mathrm{OEt}_{2}\right)_{2}\left(\mathrm{BAr}_{24}{ }^{\mathrm{F}}\right)$ ) were added, aiming to split analog to the Mo system reported in the Schneider group ${ }^{[96]}$. Both approaches did not lead to any selective reaction (see Section 3.2.1 for a discussion of these strategies). Thus those reactions were focused were solutions of $\mathbf{3 0}$ under $\mathrm{N}_{2}$ were treated with various reductants $\left(\mathrm{Co}\left(\mathrm{Cp}{ }^{*}\right)_{2}\right.$, $\mathrm{NaHg}, \mathrm{KC}_{8}, \mathrm{Na}$ (NAP) (NAP = naphthalenide)). The necessity of in situ preparation of the Re complex meant a uncertainty in these reactions, since the stochiometry of the reductant had to be chosen based on assuming quantitative formation of 30. Nevertheless, a consistent picture evolved during these attempts: reduction of $\mathbf{3 0}$ under $\mathrm{N}_{2}$ resulted in complex decomposition and formation of various unknown species under all tested conditions (including initial dimer formation at low temperatures and subsequent addition of a reductant). This also applies to reactions starting from $\mathbf{3 1}$ with two equivalents of the reductant. In all of these mixtures, no signal was found which could indicate the formation of the desired nitride complex 34. Instead, the actual composition of products changed between the reactions, and hardly any similarities in the product distribution was observed by comparing ${ }^{31} \mathrm{P}\left\{{ }^{1} \mathrm{H}\right\}$ NMR spectra of different runs.

\footnotetext{
${ }^{6}$ See Section 3.3.2 for further information on the synthesis and properties of this complex.
} 
a)

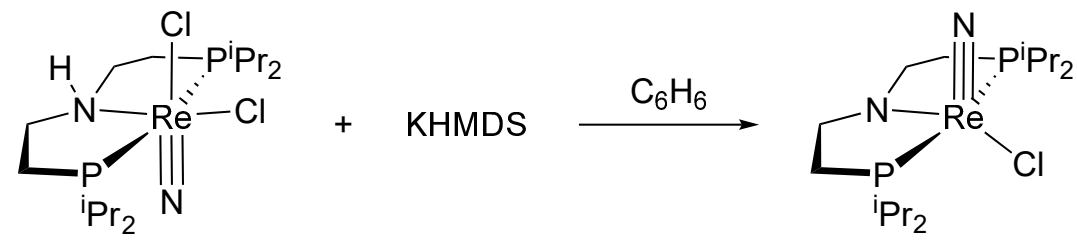

37

34

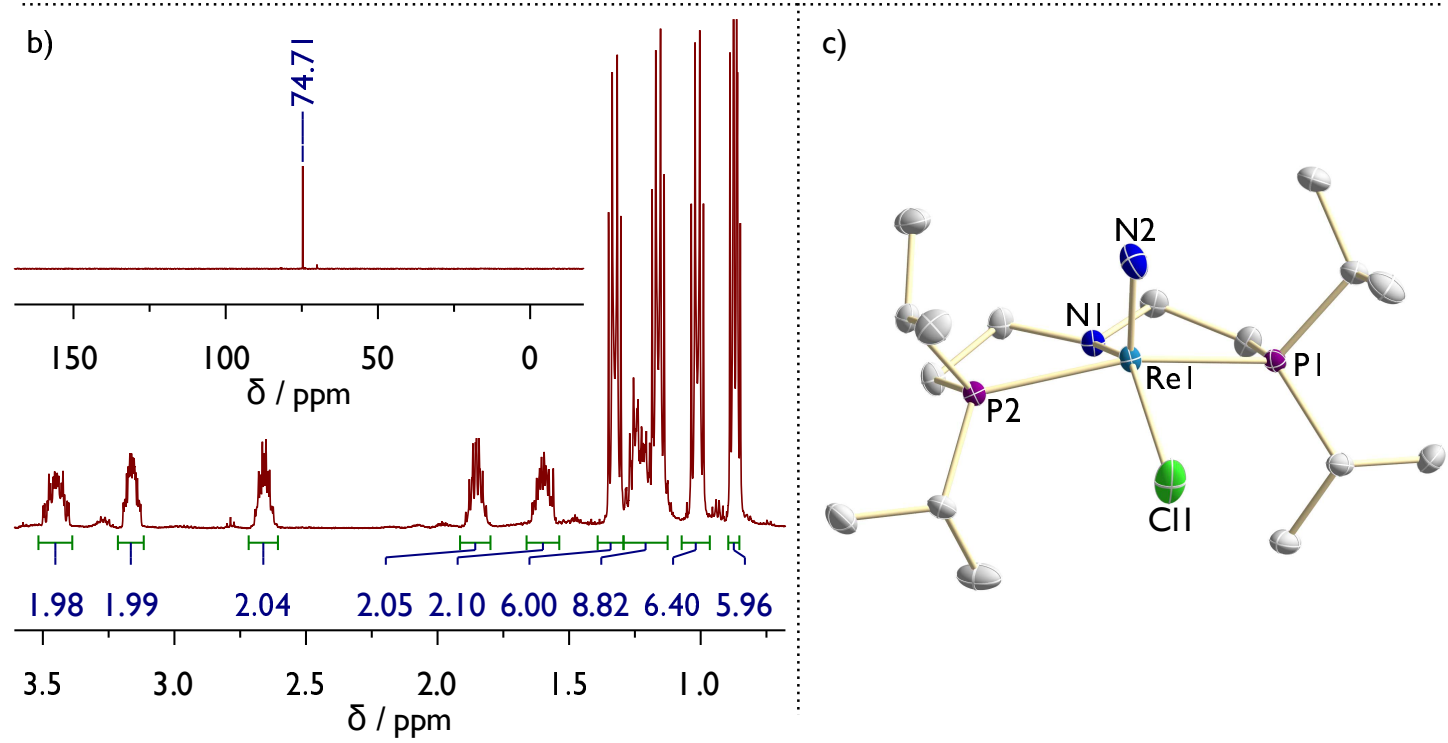

Fig. 3.15. a) Synthesis of 34 by deprotonation of 37 . b) ${ }^{1} \mathrm{H}$ NMR spectrum of 34 in $\mathrm{C}_{6} \mathrm{D}_{6}$. Inset: the corresponding ${ }^{31} \mathrm{P}\left\{{ }^{1} \mathrm{H}\right\}$ NMR spectrum. c Molecular structure of 34 obtained by single crystal $\mathrm{X}$-ray diffraction measurement. A cocrystallized chlorobenzene molecule and all $\mathrm{H}$ atoms are omitted for clarity. Anisotropic displacement parameters are set to $50 \%$ probability. Selected bond lengths $[\AA]$ and angles [ $\left.{ }^{\circ}\right]$ : Re1-N1 2.0086(18), Re1-N2 1.666(2), Re1-Cl1 2.4248(6), N1-Re1-Cl1 137.43(6), P1-Re1-P2 155.43(2), N1-Re1-N2 $111.37(9)$.

The sole exception was a broad peak at $\delta_{31} \mathrm{p} \approx 33 \mathrm{ppm}$ which appeared in many spectra in significant amounts (although never as an exclusive product). Single crystals could be isolated from one of the reaction mixtures, which revealed the $\operatorname{Re}(\mathrm{I})$ bis- $\mathrm{N}_{2}$ complex $\left[\operatorname{ReCl}\left(\mathrm{N}_{2}\right)_{2}\left(\mathrm{HPNP}^{i \mathrm{Pr}}\right)\right](35)$ to be one of the reaction products by $\mathrm{X}$-ray diffraction. While the origin of the $\mathrm{NH}$ proton (or hydrogen atom) is unclear so far, the presence of a $\operatorname{Re}(\mathrm{I})$ species in the mixture is of particular interest with regard to the findings that the $t \mathrm{Bu}$ based $\mu-\mathrm{N}_{2}$ bridged dimer $\mathbf{X X}$ is most likely formed via an ECCEC mechanism (E denoting an electron transfer step, $C$ denoting a chemical step), consisting of inital $\operatorname{Re}(\mathrm{III} / \mathrm{II})$ reduction $(\mathrm{E}), \mathrm{N}_{2}$ binding (C), chloride loss (C), $\operatorname{Re}(\mathrm{II} / \mathrm{I})$ reduction (E) and final binding of another $\operatorname{Re}(\mathrm{III})$ species and comproportionation (C) to the dimer. ${ }^{[95]}$

Targeted synthesis of $\mathbf{3 5}$ by twofold reduction of amine trichloride precursor $\mathbf{2 9}$ with $\mathrm{NaHg}$ in THF under a dinitrogen atmosphere resulted in a single resonance in the ${ }^{31} \mathrm{P}\left\{{ }^{1} \mathrm{H}\right\} \mathrm{NMR}$ spectrum at $\delta_{31} \mathrm{P}=34.5 \mathrm{ppm}$ in benzene, thus confirmed the broad peak observed in almost all reduction attempts of $\mathbf{3 0}$ to correspond to $\mathbf{3 5}$ (in THF, the ${ }^{31} \mathrm{P}$ resonance occasionally showed significant broadening, especially in the presence of side products). The ${ }^{1} \mathrm{H} N M R$ spectrum shows a $C_{S}$ symmetric complex with the $\mathrm{NH}$ proton resonating at $\delta_{{ }_{1} \mathrm{H}}=2.90 \mathrm{ppm}$. 
The $\nu(\mathrm{N}-\mathrm{H})$ as well as two $\nu(\mathrm{N} \equiv \mathrm{N})$ stretching vibrations were found in the IR spectrum at $\tilde{\nu}=3152.6$ and $2038.8 / 1936.1 \mathrm{~cm}^{-1}$, respectively. Compared to the stretching vibration of free $\mathrm{N}_{2}\left(\nu(\mathrm{N} \equiv \mathrm{N})=2359 \mathrm{~cm}^{-1}\right)^{[213]}$ these are rather typical stretching vibrations for $\operatorname{Re}(\mathrm{I})$ complexes with end-on bound $\mathrm{N}_{2}$ ligands and indicate quite a signifcant amount of $\pi$-backbonding. ${ }^{214]}$ This is also reflected in a surprisingly cathodic reversible oxidation potential of $-0.23 \mathrm{~V}$ vs. $\mathrm{Fc}^{+} / \mathrm{Fc}$ corresponding to the $\operatorname{Re}(\mathrm{I} / \mathrm{II})$ couple and deviating only marginally from the $\operatorname{Re}(\mathrm{III} / \mathrm{IV})$ potential in $29\left(E_{1 / 2}=-0.24 \mathrm{~V}\right.$ v, $\mathrm{Fc}^{+} / \mathrm{Fc}$, see Section 3.1.1). Nevertheless, the CV included no reduction wave except for an irreversible wave as

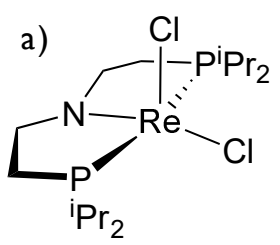

30

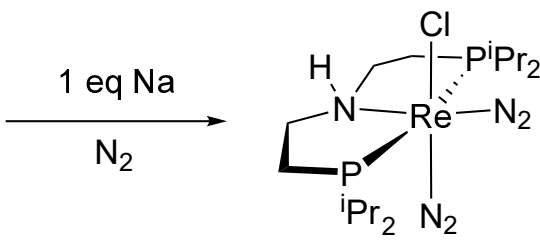

35
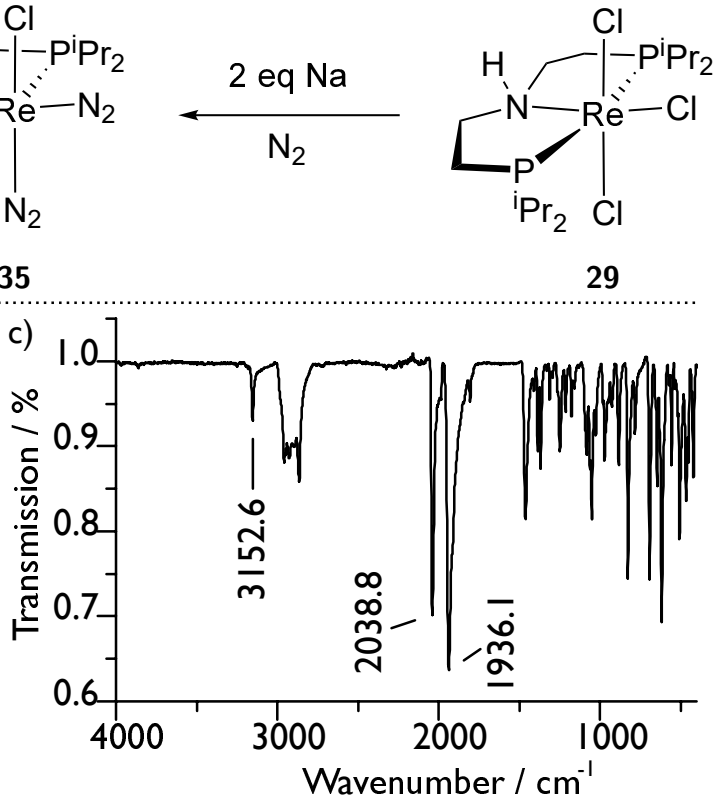

29
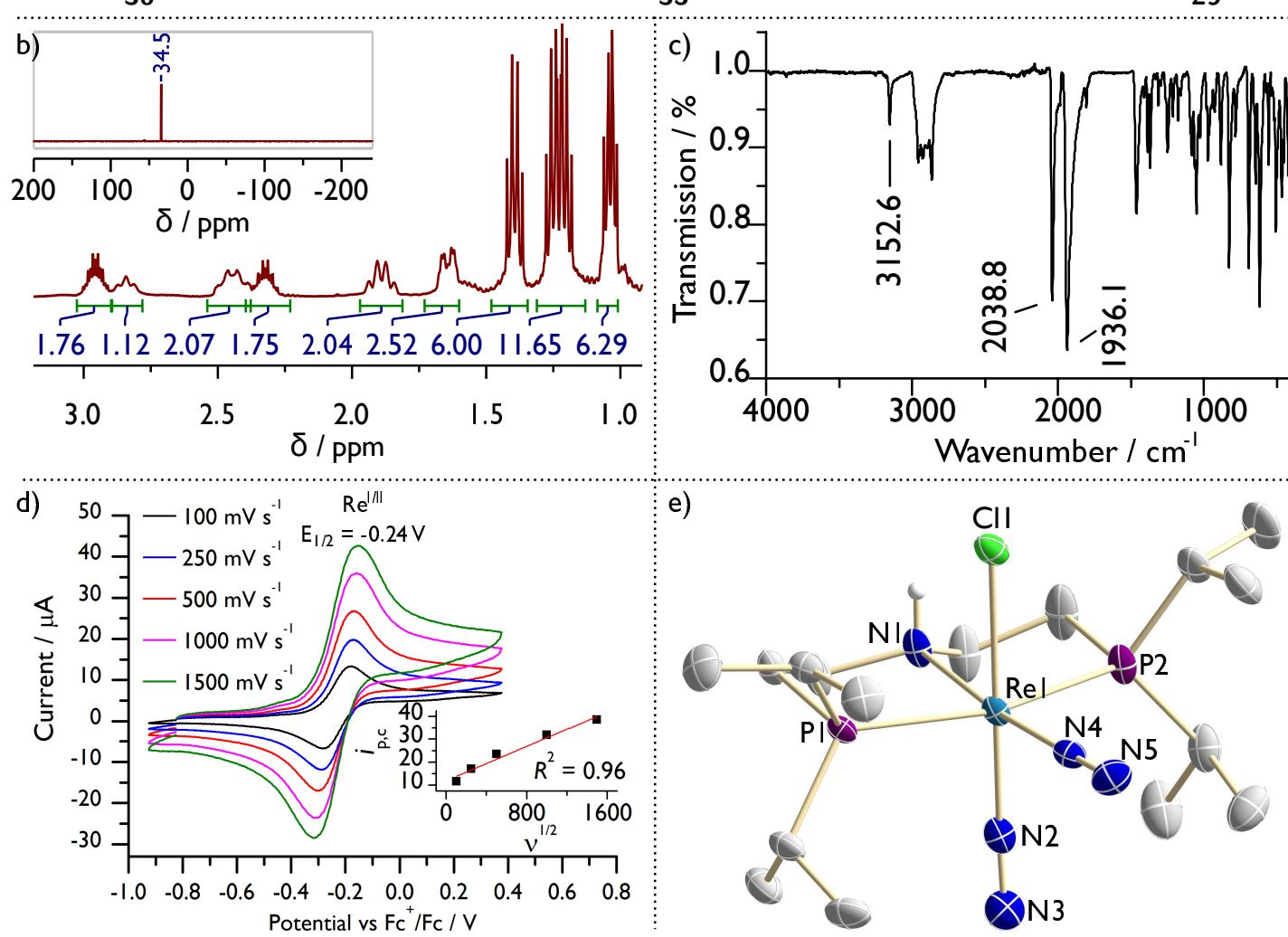

Fig. 3.16. a) Synthesis of $\mathbf{3 5}$ either by reducing $\mathbf{3 0}$ (formally by one electron, product is obtained among others) or by twoelectron reduction of 29 , both under $\mathrm{N}_{2}$ atmosphere. b) ${ }^{1} \mathrm{H}$ and ${ }^{31} \mathrm{P}\left\{{ }^{1} \mathrm{H}\right\}$ (inset) NMR spectra of complex 35 in $\mathrm{C}_{6} \mathrm{D}_{6}$. c) ATR-IR spectrum of 35 with marked $\mathrm{NH}$ and $\mathrm{N} \equiv \mathrm{N}$ stretching vibrations. d) $\mathrm{CV}$ measurements of $\operatorname{Re}(\mathrm{II} / \mathrm{I})$ oxidation of 35 in THF at different scan rates $\left(10^{-3} \mathrm{M}\right.$ in 35, $0.1 \mathrm{M}$ in $\mathrm{N}^{\mathrm{n}} \mathrm{Bu}_{4} \mathrm{PF}_{6}, \mathrm{WE}$ : GC, RE: Ag-wire, CE: Pt-wire). Inset: Plot and linear fit of $i_{p, c}$ vs. $v^{1 / 2}$. e) Molecular structure of 35 obtained by single crystal $\mathrm{X}$-ray diffraction measurements. Carbon bonded $\mathrm{H}$ atoms are omitted for clarity. Anisotropic displacement parameters are set to $50 \%$ probability. Selected bond lengths $[\AA]$ and angles $\left[^{\circ}\right]$ : Re1-N1 2.188(3), Re1-N2 1.909(3), N2-N3 1.124(4), Re1-N4 1.969(3), N4-N5 1.090(4), Re1-Cl1 2.4994(8), Re1-N2-N3 175.13(12), Re1-N4-N5 179.0(3), N1-Re1-N4 173.86(11), Cl1-Re1-N2 178.57, P1-Re1-P2 161.23(3). 
cathodic as $-3.34 \mathrm{~V}$. The molecular structure of $\mathbf{3 5}$ shows the Re center to be coordinated octahedrally, with two $\mathrm{N}_{2}$ ligands binding end-on and cis to each other. The $\mathrm{N} \equiv \mathrm{N}$ bond distances $(d(\mathrm{~N} 2-\mathrm{N} 3)=1.124(4) \AA$ and $d(\mathrm{~N} 4-\mathrm{N} 5)=1.090(4) \AA)$ as well as the $\operatorname{Re}-\mathrm{N} \equiv \mathrm{N}$ binding angles $\left(\measuredangle(\operatorname{Re} 1-\mathrm{N} 2-\mathrm{N} 3)=175.2(3)^{\circ}, \measuredangle(\operatorname{Re} 1-\mathrm{N} 4-\mathrm{N} 5)=179.0(3)^{\circ}\right)$ are slightly different, reflecting the different amounts of backbonding indicated by the IR absorptions. The amine nature of the PNP pincer is shown by a rather long Re-N bond distance $(d(\operatorname{Re} 1-\mathrm{N} 1)=$ $2.188(3) \AA)$ and a pyramidal coordination of the pincer nitrogen atom $\left(\Sigma\left(\measuredangle_{\mathrm{N} 1}\right)=341.4^{\circ}\right)$. The formation of this product clearly indicates that overreduction could be an issue in the present system. One possible explanation for it's formation could be the following scenario: The initial reduction of a $\operatorname{Re}(\mathrm{III})$ complex is followed by chloride loss, leading to a $\operatorname{Re}(\mathrm{II})$ complex with an open coordination side. Due to the high tendency of the iso-propyl substituted pincer complex to be coordinated octahedrally, this complex can be expected to bind a second $\mathrm{N}_{2}$ ligand, which leads to an anodic shift of it's reduction potential, as it is observed for 35 resulting in a potential inversion and thus in overreduction. Nevertheless, $\operatorname{Re}(\mathrm{I}) \mathrm{N}_{2}$ complex represents an interesting compound to investigate the $\operatorname{Re}(\mathrm{I})-\operatorname{Re}(\mathrm{III})$ comproportionation proposed for the parent tert-butyl based system (see Part I Section 3.2.1). [95] Especially in combination with the high ligand affinity of the $\operatorname{Re}(\mathrm{III})$ dichloro complex $\mathbf{3 0}$, the formation of a mixed valent $\operatorname{Re}(\mathrm{I}) / \operatorname{Re}(\mathrm{III})$ dimer appears quite likely. As a matter of facts, this very dimer could be crystallized from the reaction mixture of an splitting attempt, i.e. from a mixture in which $\mathbf{3 0}$ is the starting material and $\mathbf{3 5}$ is known to be formed in significant amounts (see Figure 3.17). Unfortunately, the X-ray data were insufficient for unambiguous bond-length and angle determination, however the basic connectivity within the molecule can be treated as given. In line with the preceding discussion, the more activated, i.e. more electron-rich, $\mathrm{N}_{2}$ ligand in $\mathbf{3 5}$ becomes the $\mu$-bridging ligand in the dimer. Notably, the pincer and ligand orientation in this complex resembles the proposed structure

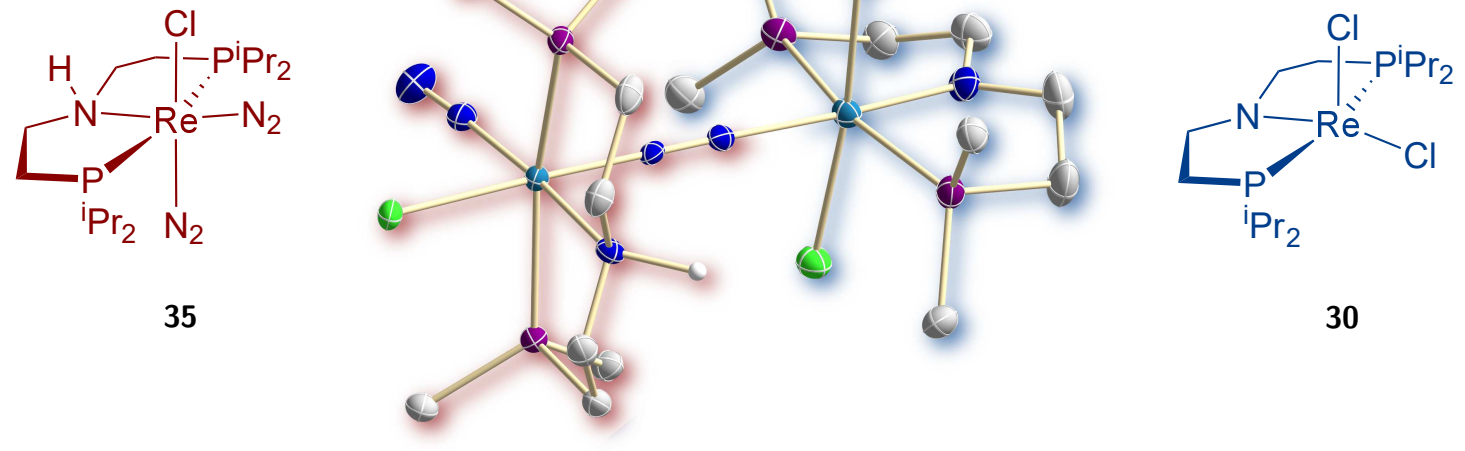

Fig. 3.17. Molecular structure obtained by X-ray diffraction measurements of a mixed valent dimer composed of $\operatorname{Re}(\mathrm{I})$ fragment $\mathbf{3 5}$ and $\operatorname{Re}(\mathrm{III})$ fragment $\mathbf{3 0}$ crystallized from a reaction mixture. The $\mathrm{X}$-ray diffraction data quality does not allow for a detailed bond lengths and angle determination. $\mathrm{CH}_{3}$ groups and all carbon bonded $\mathrm{H}$ atoms are omitted for clarity. 
of the asymmetric $\operatorname{Re}(\mathrm{III}) / \operatorname{Re}(\mathrm{III})$ dimer 33a, there based solely on NMR data. To date, neither further analysis or targeted synthesis of the mixed-valent dimer is available, nor is it known whether such a dimer would undergo dinitrogen splitting reactivity, e.g. under basic conditions. However, as synthetic routes to both building blocks are known, this would be an interesting problem to be solved in the future.

\subsection{Amine based pincer chemistry}

\subsubsection{A thermally stable, $\mu-\mathrm{N}_{2}$ bridged $\mathrm{Re}(\mathrm{II})$ dimer and its photochemistry}

Theoretical computations presented in this section were performed by Dr. Markus Finger (DFT) and Prof. Dr. Vera Krewald (TDDFT).

The overreduction of the $\mathrm{N}_{2}$ dimers $33 \mathrm{~b} / \mathbf{3 3 a}$ to $\operatorname{Re}(\mathrm{I})$ complex 35 can potentially occur due to the reaction proceeding through an coordinationally unsaturated $\operatorname{Re}(\mathrm{II})$ complex, as discussed above. Consequently, the direct reduction of octahedrally coordinated amine complex 29 was attempted, because here reduction and subsequent chloride loss would allow for only one $\mathrm{N}_{2}$ molecule to bind to the metal center. When $\left[\operatorname{ReCl}\left(\mathrm{N}_{2}\right)_{2}\left(\mathrm{HPNP}{ }^{i \operatorname{Pr}}\right)\right](35)$ was formed by twofold reduction of $\mathbf{2 9}$, the observation was made that this proceeds via a deeply blue colored intermediate, before the actual, orange complex is formed. This corresponds to the reduction wave around $-1.84 \mathrm{~V}$ vs. $\mathrm{Fc} / \mathrm{Fc}^{+}$in the $\mathrm{CV}$ of $\mathbf{2 9}$ and therefore to a single electron reduction (see Figure 3.3). Purposeful synthesis of the blue intermediate by reaction with 1 eq $\mathrm{Co}\left(\mathrm{Cp}^{*}\right)_{2}$ under an $\mathrm{N}_{2}$ atmosphere resulted in clean formation of a new complex, which again featured paramagnetically shifted but sharp NMR signals with resolved $J$ couplings, similar to those observed for 29 . Two mutually coupling doublets in the ${ }^{31} \mathrm{P}\left\{{ }^{1} \mathrm{H}\right\} \mathrm{NMR}$ spectrum $\left(\delta_{31 \mathrm{P}}=-370.6\right.$ and $\left.-380.4 \mathrm{ppm},{ }^{2} J_{\mathrm{PP}}=237.0 \mathrm{~Hz}\right)$, eight signals for inequivalent methyl groups, and a mole peak of 1152.2 determined by LIFDI spectrometry are all in agreement with this species being the $\mu-\mathrm{N}_{2}$ bridged dimer $\left[\left(\mu-\mathrm{N}_{2}\right)\left\{\operatorname{ReCl}_{2}\left(\mathrm{HPNP}^{i \mathrm{Pr}}\right)\right\}_{2}\right](36)$, an amine pincer based analog of $\mathbf{X X}$ with a corresponding $10 \pi 4 \delta$ electron configuration. If the reaction is carried out under fully labeled ${ }^{15} \mathrm{~N}_{2}$, a singlet can be detected in ${ }^{15} \mathrm{~N}\left\{{ }^{1} \mathrm{H}\right\} \mathrm{NMR}$

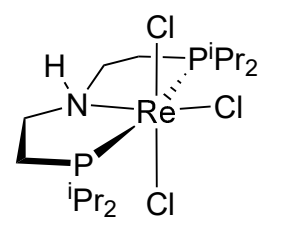

29
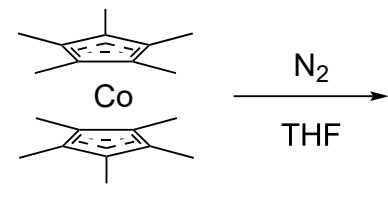

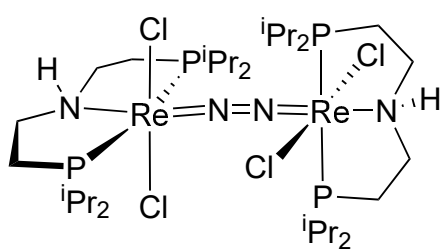

36

Scheme 3.1. Synthesis of $\mathbf{3 6}$ by reduction of $\mathbf{2 9}$ under an $\mathrm{N}_{2}$ atmosphere. 
spectroscopy with a strongly highfield shifted resonance at $\delta_{15} \mathrm{~N}=-1113.0 \mathrm{ppm}$. While the IR spectrum of $\mathbf{3 6}$ does not show any sign for an $\mathrm{N}_{2}$ band, the resonance Raman spectrum $\left(\lambda_{\text {exc. }}=632.8 \mathrm{~nm}\right)$ shows a very prominent band at $\tilde{\nu}=1733.3 \mathrm{~cm}^{-1}$, which shifts to $\tilde{\nu}=$ $1675.2 \mathrm{~cm}^{-1}$ in the ${ }^{15} \mathrm{~N}_{2}$ labeled complex, in perfect agreement with the harmonic oscillator approximation for an $\mathrm{N}_{2}$ stretching vibration, which predicts a shift of $\Delta \tilde{\nu}\left({ }^{14} \mathrm{~N}_{2}{ }^{15} \mathrm{~N}_{2}\right)$ $=58.7 \mathrm{~cm}^{-1}$. Since the complex is intensely colored, the UV/vis absorption spectrum was recorded. A strong band at $\lambda=578 \mathrm{~nm}\left(\epsilon=12294 \mathrm{M}^{-1} \mathrm{~cm}^{-1}\right)$, assigned to a $\pi \rightarrow \pi^{*}$ transition in the $\mu-\mathrm{N}_{2}$ ligand by TDDFT calculations (vide infra), as well as an even stronger band peak at $\lambda=336 \mathrm{~nm}\left(\epsilon=38027 \mathrm{~m}^{-1} \mathrm{~cm}^{-1}\right)$ were found as characteristic features of 36. X-ray diffraction data on single crystals confirmed the molecular structure of $\mathbf{3 6}$ as a dimer with the two Re centers being bridged by a $\mu-\mathrm{N}_{2}$ ligand (see Figure $3.18 \mathrm{c}$ ). Two
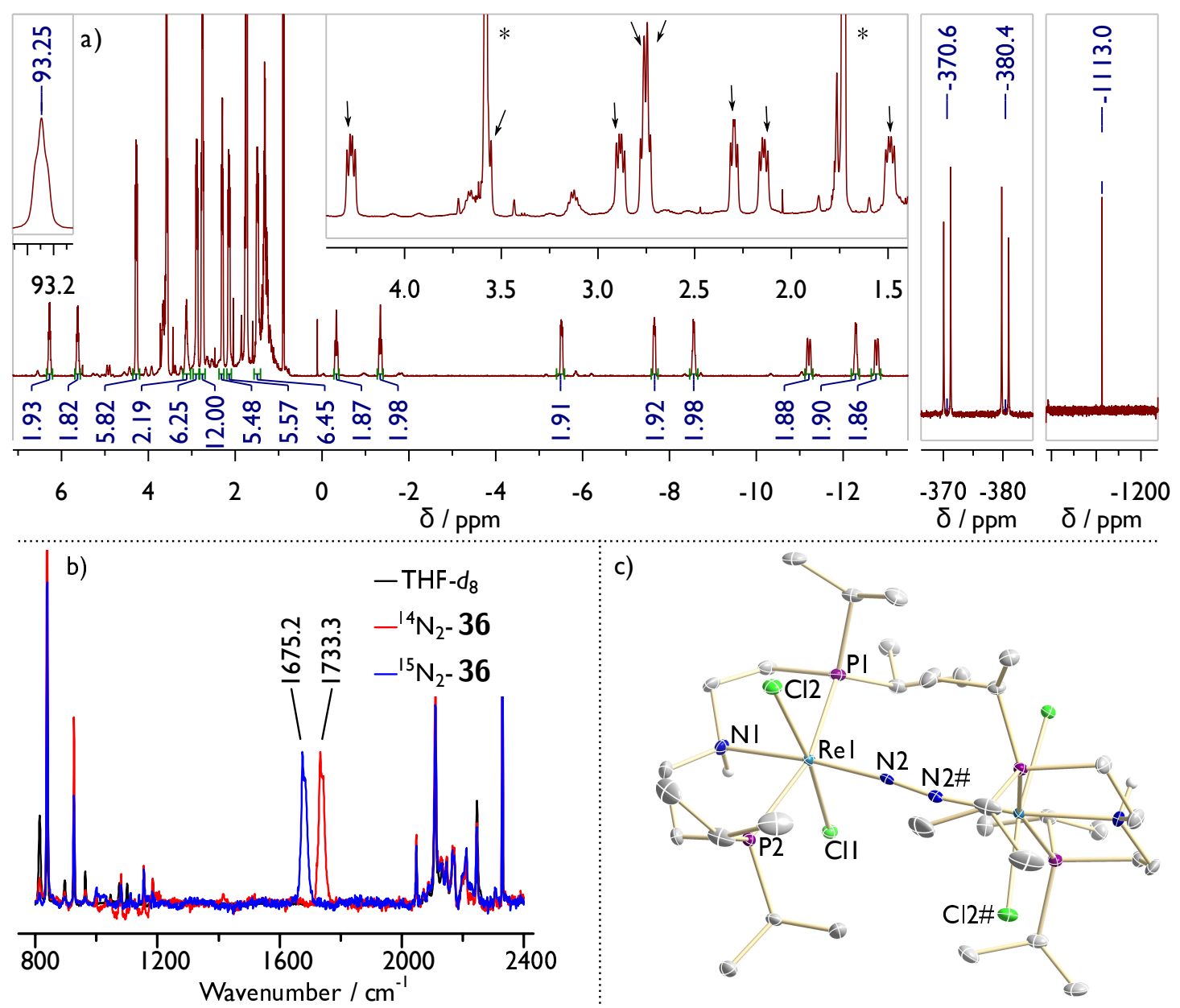

Fig. 3.18. a) NMR spectra of 36: left $={ }^{1} \mathrm{H}-\mathrm{NMR}$, arrows in the inset mark the eight $i \operatorname{Pr}$ groups; middle $={ }^{31} \mathrm{P}\left\{{ }^{1} \mathrm{H}\right\}$-NMR spectrum; right $={ }^{15} \mathrm{~N}\left\{{ }^{1} \mathrm{H}\right\}$-NMR spectrum of 36, synthesized under ${ }^{15} \mathrm{~N}_{2}$ atmosphere. b) Resonance raman spectrum $\left(\lambda_{\text {exc. }}=632.8 \mathrm{~nm}\right)$ of $\mathbf{3 6}$ (red) and ${ }^{15} \mathrm{~N}_{2}-\mathbf{3 6}$ (blue). c) Molecular structure of $\mathbf{3 6}$ obtained by single crystal X-ray diffraction measurements. All $\mathrm{H}$ atoms but the $\mathrm{NH}$ proton are omitted for clarity. Anisotropic displacement parameters are set to $50 \%$ probability. Selected bond lengths $[\AA]$ and angles []: Re1-Cl1 2.4530(7), Re1-Cl2 2.4271(8), Re1-N1 2.212(3), Re1-N2 1.906(2), N2-N2\# 1.169(5), Cl1-Re1-Cl2 170.72(3), P1-Re1-P2 160.33(3), N1-Re1-N2 171.84(10), Re1-N2N2\# 171.5(3), Cl1-Re1-Re1\#-Cl2\# 75.5. 
chloride ligands and the HPNP ligand complete the octahedral coordination environment of both metal centers. The $\mathrm{N}=\mathrm{N}$ bond distance of $d(\mathrm{~N} 2-\mathrm{N} 2 \#)=1.169(5) \AA$ is slightly shorter than in the tert-butyl pincer based dimer $\mathbf{X X}(d(\mathrm{~N} 3-\mathrm{N} 4)=1.202(10) \AA$, see Section 2.1), indicating a moderate activation of the ligand, which is also reflected in the stretching vibrations. As a striking difference to the tert-butyl pincer based dimer $\mathbf{X X}$, the pincer nitrogen atom is coordinated trans to the $\mathrm{N}_{2}$ bridge. Since the pincer $\left\{\mathrm{ReCl}_{2}\left(\mathrm{HPNP}^{\mathrm{iPr}}\right)\right\}$ fragments are twisted towards each other by $75.5^{\circ}$, the $\mathrm{N}-\mathrm{H}$ moieties are the reason for the observed $C_{2}$, breaking the otherwise obtainable $C_{2 v}$ symmetry. Consequently, the rotation of the two fragments against each other seems to still be slow on the NMR timescale, indepedent of the reduced ligand bulkiness. Evaluation of the electrochemical properties of $\mathbf{3 6}$ by means of CV measurements show the dimer to undergo three redox events, a irreversible reduction and two fully reversible oxdiations (see Figure 3.19). All events show fast electron transfer to freely diffusing reactants according to the analysis of the forward peak current $i_{p}$ vs. $v^{1 / 2}$ (using the Randles-Sevcik equation, see Equation 1.1). Chemical reversibility for both oxidations is further shown by a scan-rate independent ratio of the forward and backward peaks close to unity, while the ratio of $i_{p, c}$ of the reduction vs. $i_{p, a}$ of the first oxidation suggests more than one electrons are passed to the analyte (the decrease of that ratio with higher scan-rates could indicate an ECE mechanism, were both E steps have idenctical or even inverted potentials - however, closer analysis would be needed for a precise statement).

In contrast to XX, 36 exhibits a remarkable thermal stability, i.e. heating overnight to $60^{\circ} \mathrm{C}$ did not lead to any sample decomposition detectable by NMR spectroscopy. To rationalize this, the thermal splitting was investigated computationally by Dr. Markus Finger. The shifted NMR signals of $\mathbf{3 6}$ are reminiscent of those of $\mathbf{2 9}$, hence it can be assumed that TIP, induced by SOC, might play a role in describing the electronic structure of the dimer at hand. While basically DFT is inherently unable to accurately describe such compounds, the size of the system renders application of higher-level calculations extremely challenging. Therefore $\mathrm{DFT}^{7}$ remained the method of choice for this study, keeping in mind that the results have to be treated with some care.

DFT resonably well reproduced the X-ray geometry of the dimer $\left(\mathrm{RMSD}_{\mathrm{CI}, \mathrm{N}, \mathrm{P}, \mathrm{Re}}=0.139\right)$ and similar to 29, the triplet ground state was found to be favorable over the open shell singlet state by $\Delta G^{0}=-34 \mathrm{~kJ} \mathrm{~mol}^{-1}$. Splitting by simply increasing the $\mathrm{N}=\mathrm{N}$ bond distance proceeds via the well-known zig-zag transition state (see Part I Section 3.2.1 for further details) with an estimated barrier of $\Delta G^{\ddagger}=175 \mathrm{~kJ} \mathrm{~mol}^{-1}$ and results in an dichloro nitride complex $\left(37^{\text {trans }}\right)$ where the nitrido ligand is coordinated trans to the pincer amine donor. This product is still uphill by $\Delta G^{0}=+9 \mathrm{~kJ} \mathrm{~mol}^{-1}$ and features an extremely long $\mathrm{Re}-\mathrm{NH}$ bond distance of $2.53 \AA$, resulting from the strong trans influence of the nitride. Isomerization of $37^{\text {trans }}$ to either $37^{\text {cis- } 1}$ with the $\mathrm{Re} \equiv \mathrm{N}$ bond being syn to the $\mathrm{N}-\mathrm{H}$ bond or to $37^{\text {cis-2 }}$ with the $\mathrm{Re}-\mathrm{Cl}$ bond being syn to the $\mathrm{N}-\mathrm{H}$ bond results in product stabilization of $\Delta G^{0}$

\footnotetext{
${ }^{7}$ M06/def2TZVP/SMD(THF)||PBE/D3BJ/RI/def2SVP(def2TZVP for Re, P, N, Cl).
} 


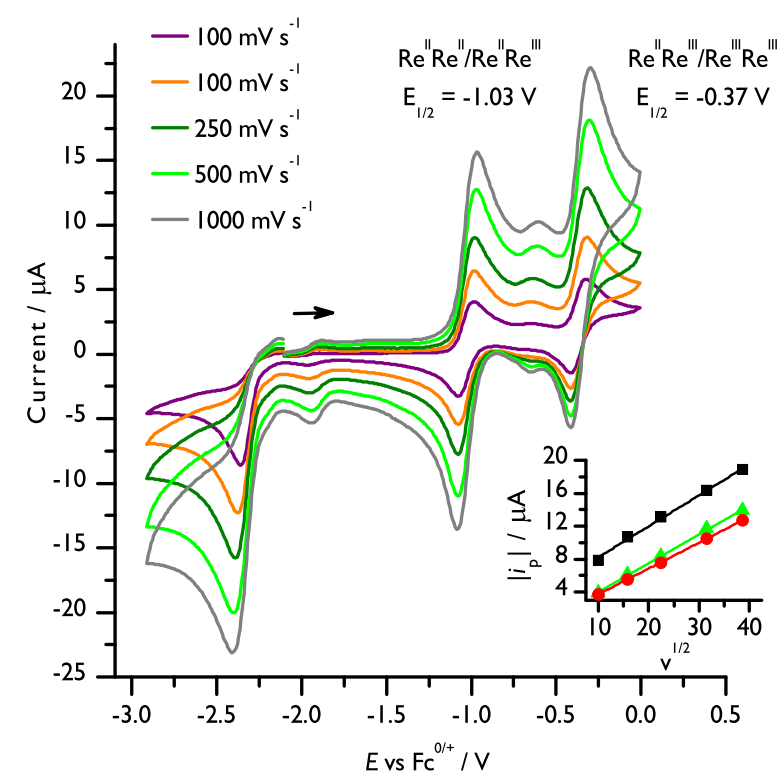

Fig. 3.19. CV measurements of two reversible oxidations and one irreversible reduction of 36 in THF at different scan rates $\left(10^{-3} \mathrm{M} 36\right.$, $0.1 \mathrm{M} \mathrm{N}^{\mathrm{n}} \mathrm{Bu}_{4} \mathrm{PF}_{6}$, WE: GC, RE: Ag-wire, CE: Pt-wire). Inset: Plot and linear fit of $i_{p}$ vs. $v^{1 / 2}\left(i_{p, a}\right.$ for first oxidation in green, $R^{2}=0.999 ; i_{p, a}$ for second oxidation in red, $R^{2}=1.000 ; i_{p, c}$ for first reduction in black, $\left.R^{2}=0.995\right)$.
Tab. 3.2. Peak current analysis of the forwards peak of the first and second oxidation as well as the first reduction.

\begin{tabular}{c|c}
\hline$v / \mathrm{mV} \mathrm{s}^{-1}$ & $\frac{i_{p, c}}{i_{p, a}}$ (first ox.) \\
\hline 100 & 1.006 \\
250 & 0.989 \\
500 & 1.005 \\
1000 & 1.056 \\
1500 & 1.102 \\
\hline & $\frac{i_{p, c}}{i_{p, a}}($ second ox. $)$ \\
\hline 100 & 1.039 \\
250 & 1.059 \\
500 & 1.076 \\
1000 & 1.002 \\
1500 & 1.006 \\
\hline & $\frac{i_{p, c}}{i_{p, a} \text { (rstox }}$ (red.) \\
\hline 100 & 2.015 \\
250 & 1.757 \\
500 & 1.558 \\
1000 & 1.398 \\
1500 & 1.353 \\
\hline
\end{tabular}

$=-46$ and $-89 \mathrm{~kJ} \mathrm{~mol}^{-1}$, respectively. This can easily be rationalized on the one hand since isomerization reduces the trans repulsion to the nitride ligand, as chloride is a much weaker donor than an amine and on the other hand because in $37^{\text {cis-2 }}$, the coordination of the chloride ligand trans to the nitride can additionally be stabilized by an intra-molecular hydrogen bond to the $\mathrm{N}-\mathrm{H}$ proton. Overall, the DFT derived scheme can explain the thermal stability by a significantly increased activation barrier, as compared to $\mathbf{X X}\left(\Delta G^{\ddagger}\right.$ $=113 \mathrm{~kJ} \mathrm{~mol}^{-1}$ ). This increased activation barrier can be rationalized, as the amine ligand trans to the $\mathrm{N}_{2}$ bridge in 36 raises the energy of the $\mathrm{a}_{2 \mathrm{u}}$ symmetric $\mathrm{M}^{-}-\mathrm{N}^{\sigma}-\mathrm{N}^{*}-\mathrm{M}$ orbital and thereby reduces its mixing with orbitals of the $\pi$ system in the transition state, which is required for splitting (see Part I Figure 3.2). Additionally, no $\pi$ donor is interacting with the $\pi$-MO space anymore which lowers the energy of the frontier orbitals and thus even further increases the MO energy gap. The influence of the trans ligand might also serve as an explanation for the comparison of Cummins' $\mathrm{N}_{2}$ dimer complex I (see Part I, Section 2.1 and 3.2.1 $)^{[27,28,82]}$ with Schrock's $\left[\left(\mu-\mathrm{N}_{2}\right)\left\{\mathrm{Mo}\left(\mathrm{N}\left(\mathrm{CH}_{2} \mathrm{CH}_{2} \mathrm{NR}\right)_{3}\right)\right\}_{2}\right]$ complex $(\mathrm{R}$ $\left.=\mathrm{C}_{6} \mathrm{~F}_{5}\right)^{[84,85]}$ of which only the former splits $\mathrm{N}_{2}$, while the latter, which features a trans amine ligand, does not.

In a nutshell, thermal population of the $\mathrm{a}_{2 \mathrm{u}}$ symmetric $\mathrm{M}^{\sigma}-\mathrm{N}^{\sigma}-\mathrm{N}-\stackrel{\sigma}{-} \mathrm{M}$ orbital through mixing with the HOMOs in a zig-zag transition state is not possible in $\mathbf{3 6}$. Therefore alternative 


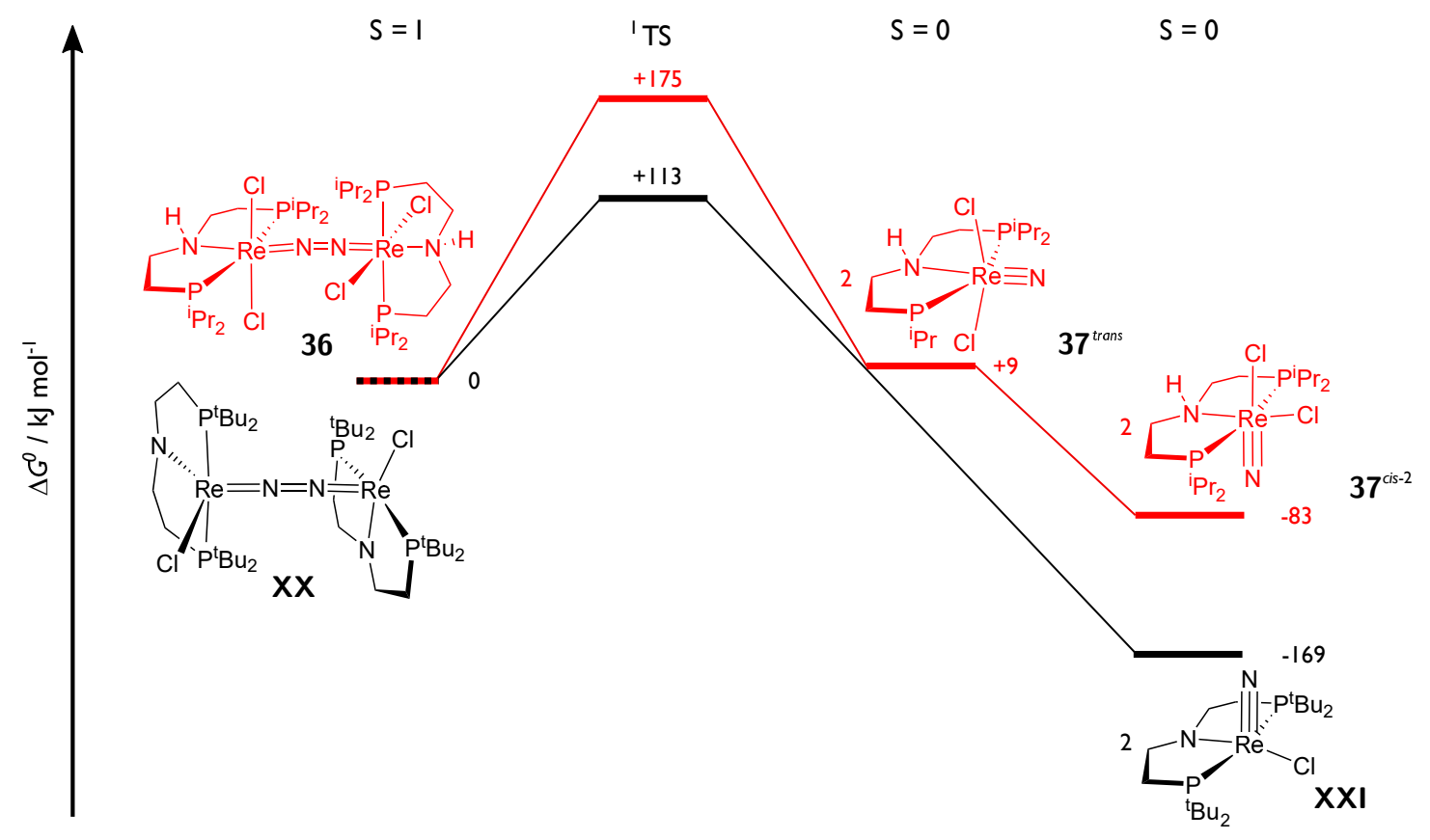

Fig. 3.20. DFT calculated thermodynamic scheme for the splitting of $\mathbf{X X}$ and $\mathbf{3 6}$ into the corresponding nitrido species.

ways for intramolecular electron transfer into the $\mathrm{N}-\mathrm{N}$ antibonding $\mathrm{MO}$ space are needed. A well established possibility for achieving this is electronic excitation by absorption of photons. Consequently, a sample of $\mathbf{3 6}$ in THF was irradiated with a $\mathrm{Hg}(\mathrm{Xe})$ arc lamp equipped with a long wave pass filter for $\lambda>305 \mathrm{~nm}$, leading to a gradual color change from blue to orange. In the UV/vis spectrum, a decay of all bands could be observed while NMR measurements indicated the formation of a single, diamagnetic, $C_{S}$ symmetric species in $95 \%$ spectroscopic yield, determined against an internal standard. Further spectroscopic characterization as well as preparation of an authentic sample via the reaction of $\mathbf{2 9}$ with an azide source revealed this species to be the splitting product $\left[\operatorname{Re}(N) \mathrm{Cl}_{2}\left(\mathrm{HPNP}^{\mathrm{i} P r}\right)\right](37)$, where the two chloride ligands are oriented cis to each other and the $\mathrm{N}-\mathrm{H}$ in anti orientation towards the $\mathrm{Re} \equiv \mathrm{N}$ bond, which was also predicted by DFT to be the thermodynamically favored isomer (vide supra). This is a quite rare finding, as so far only five other system have been reported, which also do undergo photolytic splitting of dinitrogen, non of which bears a rhenium atom (see Part I Section 3.2.3). ${ }^{[28,86,109,114,116]}$ The high selectivity of the photochemical dinitrogen splitting is an unique feature among these systems, especially because it seems to not suffer from $\mathrm{M}-\mathrm{N}_{2}$ bond cleavage which is an often observed or hypothesized side reaction.

To identify the productive range for this process, wavelength-dependent irradiations were performed. When a long wave pass filter for $\lambda>420 \mathrm{~nm}$ was used, no conversion could be observed, excluding the pronounced absorption at $578 \mathrm{~nm}$ to be relevant for the photolysis. Rather, irradiation with a $390 \mathrm{~nm}$ LED (FWHM $=11 \mathrm{~nm}$ ) leads to successful conversion of 36 to 37 , indicating the small shoulder around $\lambda \approx 400 \mathrm{~nm}$ to contain the transitions which are relevant for the reaction. The quantum yield of this reaction was determined to be very low $(0.36 \%)$ (see Section 1.2 for further details on the determination). 
At this point, only little can be said about the excited state dynamics, by which the reaction proceeds. Based on the above hypothesized influence of the trans ligand on the thermal splitting pathway, photochemically induced chloride dissociation and subsequent thermal splitting was standing to reason. This could be excluded in so far as chloride concentration dependent photolysis did not lead to any distinguishable differences in the reaction kinetics. Therefore chloride dissociation (which likely has to occur at some point on the route to 37) must take place only after the rate determining step of the reaction, which is believed to be the actual splitting step. However, other pathways can be envisioned to play a role during this process (e.g. phosphine dissociation, metal-nitrogen bond cleavage) and detailed analysis of the process (i.e. by transient spectroscopy) will be necessary to elucidate the actual mechanism. A first hint on the underlying processes can be derived from TDDFT calculations which were performed to gain inside into the nature of the relevant transitions in the experimentally identified, productive region (see Figure 3.21). The calculations reasonably well reproduced the spectroscopic features of the experimental electronic excitation spectrum. The excitation energies are all slightly overestimated, leading to a blueshift in the calculated spectrum, which is quite regularly observed in TDDFT calculations. ${ }^{[215]}$ As mentioned above, the prominent absorption at $578 \mathrm{~nm}$ is identified as an excitation within the

a)

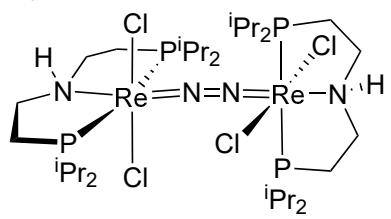

36

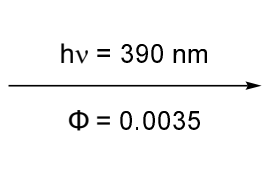

Energy / eV

c)
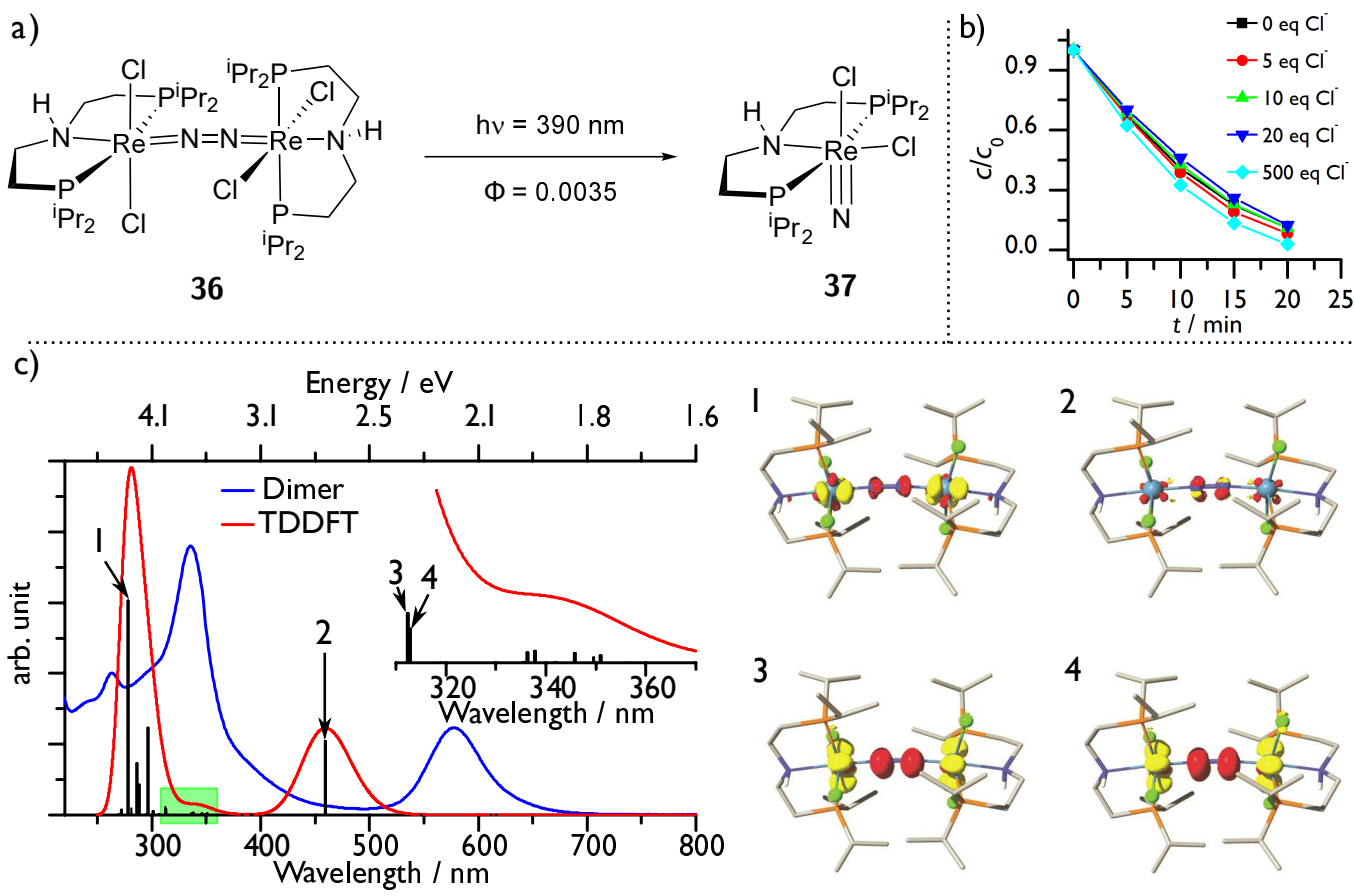

2

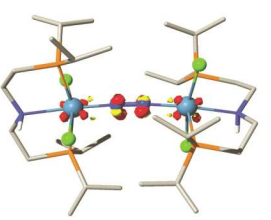

3

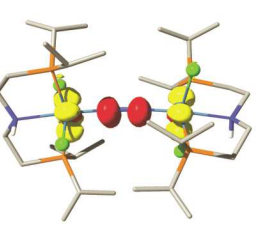

4

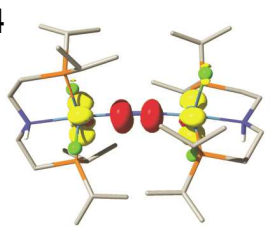

Fig. 3.21. a) Photolytic splitting of $\mathbf{3 6}$ into the corresponding nitride species $\mathbf{3 7}$ with a $390 \mathrm{~nm}$ LED, proceeding with a quantum yield of $0.36 \%$. b) Time-dependent concentration decay of 36 by photolysis $(\lambda>305 \mathrm{~nm})$ in the presence of various concentrations of $\left.\mathrm{NHex}_{4} \mathrm{Cl} . c\right)$ Experimental (blue) vs. TDDFT calculated (red) electronic absorption spectrum of 36. Bottom right: The electron difference densities of the marked excitations, with 1 and 2 being mainly located within the $\mathrm{Re}-\mathrm{N}-\mathrm{N}-\mathrm{Re}$ moiety, while 3 and 4 are of considerable charge transfer from the metal centered $\delta$ orbitals into the $\operatorname{Re}-\mathrm{N}-\mathrm{N}-\operatorname{Re} \pi^{*}-\pi^{*}-\pi^{*}$ orbital (see Figure 3.4 in Section 3.5 for the corresponding natural transition orbitals). Electron depletion is colored yellow, electcron gain in red. 
$\operatorname{Re}-\mathrm{N}-\mathrm{N}-\operatorname{Re} \pi$ manifold $\left(\operatorname{Re}{ }^{-}-\mathrm{N}^{\pi^{*}}-\mathrm{N} \stackrel{\pi}{-} \operatorname{Re} \rightarrow \operatorname{Re}^{\pi^{*}} \mathrm{~N}^{-}-\mathrm{N}^{\pi^{*}}-\mathrm{Re}\right)$. Closer investigation of the photolytically active region allowed for identification of two weak absorptions ( $\left.f_{\text {osc }} \leq 0.02\right)$ with a pronounced MLCT character from the metal-centered, nonbonding $\delta$ orbitals into the $\operatorname{Re} \stackrel{\pi^{*}}{-}{ }^{\pi^{*}}-N \stackrel{\pi^{*}}{-}$ Re orbital. As a hypothetical effect from such an MLCT, increased flexibility of the $\mathrm{M}-\mathrm{N}-\mathrm{N}-\mathrm{M}$ core could facilitate the splitting process. Additionally, the induced charge separation already resembles the situation in the final products, which might also be beneficial. Interestingly, the complex $\left[\left(\mu-\mathrm{N}_{2}\right)\left\{\mathrm{Mo}\left(\mathrm{Cp}^{*}\right)(\mathrm{PP})\right\}_{2}\right]\left(\mathrm{PP}=\mathrm{Fe}\left(1-\mathrm{PEt}_{2}-\mathrm{C}_{5} \mathrm{H}_{4}\right)_{2}\right)$ prepared by Nishibayashi and coworkers, which also does undergo photolytic splitting of the bridging dinitrogen ligand, exhibits very similar UV/vis spectroscopic features with a pronounced absorption at $748 \mathrm{~nm}$ located within the N-N fragment and a shoulder at $394 \mathrm{~nm}$, which is associated with a loss of electron density between the two nitrogen atoms and is speculated to be the productive transition. ${ }^{[114]}$

It is noteworthy, that of those systems which are reported to undergo photoinduced $\mathrm{N}_{2}$ bond scission, only two exhibit a $10 \pi(0 \delta)$ electronic configuration of the $\{$ MNNM $\}$ core (i.e. the examples by Cummins and Floriani, see Part I Section 3.2.3), ${ }^{[28,86]}$ while all others, including the in here presented complex 36, have filled $\delta$ orbitals. The conclusion of Cummins from transient spectroscopy that the vibrationally hot ground state after IC rather than an excited state is relevant to $\mathrm{N}_{2}$ splitting in his and probably also in Floriani's dimer thus raises the question whether filled $\delta$ symmetric orbitals at the metal center might be a general requirement for photoinduced dinitrogen splitting. ${ }^{[216]}$ However, the current availability of data on this subject is scarce, and more detailed investigation of the underlying excited state dynamics in these systems will be needed.

\subsubsection{An octahedral rhenium nitride complex and nitrogen centered, nucleophilic reactivity}

Complex $\left[\operatorname{Re}(\mathrm{N}) \mathrm{Cl}_{2}\left(\mathrm{HPNP}^{i \mathrm{Pr}}\right)\right]$ (37) exhibits a single resonance in ${ }^{31} \mathrm{P}\left\{{ }^{1} \mathrm{H}\right\}$ NMR spectra at $\delta_{31} \mathrm{P}=36.1 \mathrm{ppm}$ (see Figure $3.22, c$ ). ${ }^{1} \mathrm{H}-\mathrm{NMR}$ reveals it to be a $C_{S}$ symmetric compound with a prominant peak corresponding to the $\mathrm{NH}$ moiety at $\delta_{1_{\mathrm{H}}}=4.93 \mathrm{ppm}$ (see Figure 3.22 b). When a labeled sample ${ }^{15} \mathrm{~N}_{2}-36$ is irradiated, in the ${ }^{15} \mathrm{~N}\left\{{ }^{1} \mathrm{H}\right\} \mathrm{NMR}$ spectrum of the product a singlet is detectable at $\delta_{15} \mathrm{~N}=383.8 \mathrm{ppm}$, confirming 37 to be a nitride species. Single crystals grown from $\mathrm{DCM} / \mathrm{Et}_{2} \mathrm{O}$ allowed for molecular structure determination by $\mathrm{X}$ ray diffraction measurements (see Figure $3.22 \mathrm{a}$ ). The Re center is found to be coordinated in an octahedral coordination sphere. The nitride ligand occupies an apical position in anti orientation to the $\mathrm{N}-\mathrm{H}$ group and the chloride, which is bound trans to it exhibits a significantly elongated $\mathrm{Re}-\mathrm{Cl}$ bond distance when compared with the second chloride $(d(\operatorname{Re} 1-\mathrm{Cl} 4)=2.6712(7) \AA, d(\operatorname{Re} 1-\mathrm{Cl} 3)=2.4309(7) \AA)$. The small angle between the pincer backbone, the metal center and the chloride $\left(\measuredangle(\mathrm{N} 3-\mathrm{Re} 2-\mathrm{Cl} 4)=78.68(7)^{\circ}\right)$, as well as the rather short distance to the backbone $\mathrm{N}-H$ atom $(d(\mathrm{Cl} 4-\mathrm{H} 112)=2.5596(1) \AA)$ suggest some 

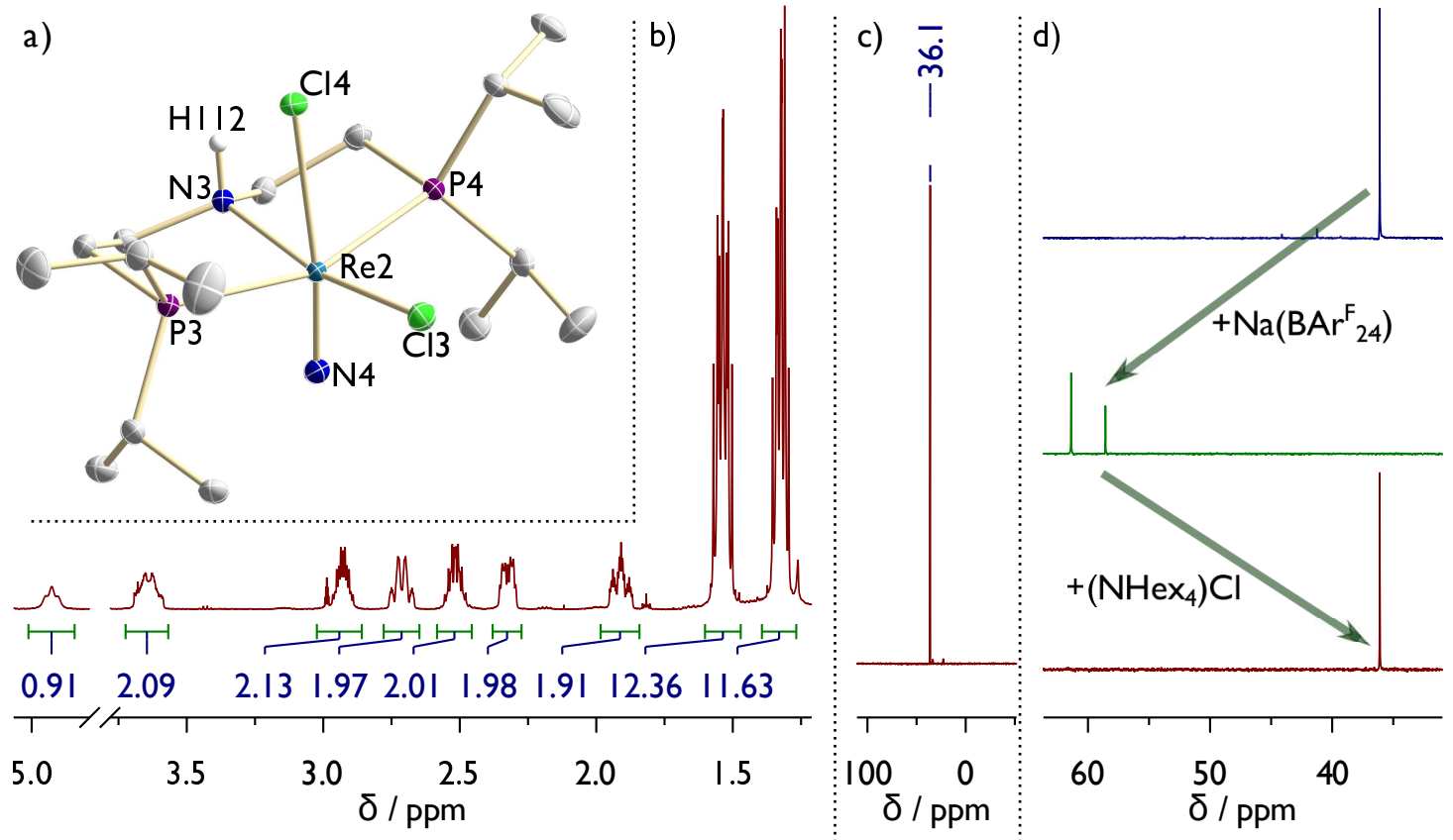

Fig. 3.22. a) Molecular structure of $\mathbf{3 7}$ obtained by single crystal X-ray diffraction measurements. All $\mathrm{H}$ atoms but the $\mathrm{NH}$ proton, a second, disordered nitride molecule, a DCM and a $\mathrm{Et}_{2} \mathrm{O}$ molecule which all cocrystallized in the unit cell are omitted for clarity. Anisotropic displacement parameters are set to $50 \%$ probability. Selected bond lengths $[\AA]$ and angles []: Re2-N3 2.159(2), Re2-N4 1.669(2), Re2-Cl3 2.4309(7), Re2-Cl4 2.6712(7), Cl4-H112 2.5596(1), N3-Re2-N4 92.65(11), P3-Re2-P4 159.92(2), Cl3-Re2-Cl4 88.23(2), N3-Re2$\mathrm{Cl} 478.68(7)$. b) ${ }^{1} \mathrm{H}$ NMR spectrum of 37 in $\mathrm{CD}_{2} \mathrm{Cl}_{2}$. c) ${ }^{31} \mathrm{P}\left\{{ }^{1} \mathrm{H}\right\}$ NMR spectrum of 37 in $\mathrm{CD}_{2} \mathrm{Cl}_{2}$. d) ${ }^{31} \mathrm{P}\left\{{ }^{1} \mathrm{H}\right\}$ NMR spectra of 37 (blue), 37 after addition of 1 eq of $\mathrm{Na}\left(\mathrm{BAr}_{24}^{\mathrm{F}}\right)\left(\mathrm{BAr}_{24}^{\mathrm{F}}=\right.$ tetrakis $\{3,5-($ trifluoromethyl)phenyl $\}$ borate) (green) and after subsequent addition of $\left(\mathrm{NHex}_{4}\right) \mathrm{Cl}($ red $)$.

degree of intramolecular hydrogen bonding to stabilize the coordination of the chloride. This is in stark contrast to the analog tert-butly based complex $\left[\operatorname{Re}(\mathrm{N}) \mathrm{Cl}\left(\mathrm{HPNP}^{t \mathrm{Bu}}\right)\right]^{\mathrm{Cl}}$, prepared by protonation of $\mathbf{X X I}$ with $\mathrm{HCl}$, where the chloride was found to be non-coordinating in the $\mathrm{X}$-ray structure. ${ }^{\left[{ }^{94]}\right.}$ The influence of the additional trans ligand in $\mathbf{3 7}$ is also reflected by a slightly elongated $\mathrm{Re} \equiv \mathrm{N}$ bond distance as compared to the tert-butyl system $(d(\mathrm{Re} \equiv \mathrm{N})$ $=1.669(2) \AA$ vs. $1.642(4) \AA$ in $\left.\left[\operatorname{ReNCl}\left(\mathrm{HPNP}^{t \mathrm{Bu}}\right)\right]^{\mathrm{Cl}}\right)$, which suggests a higher degree of activation of the nitride. To exclude the coordination of the second chloride to be solely due to packing effects in the solid state, chloride dependent NMR spectra of $\mathbf{3 7}$ were recorded (see Figure $3.22 d$ ). After addition of 1 eq of $\mathrm{Na}\left(\mathrm{BAr}_{24}^{\mathrm{F}}\right)$ as a chloride abstraction agent, 37 was cleanly converted into two new species as indicated by two singlets in the ${ }^{31} \mathrm{P}\left\{{ }^{1} \mathrm{H}\right\}$ NMR spectrum $\left(\delta_{31} \mathrm{P}=58.6\right.$ and $\left.61.4 \mathrm{ppm}\right)$, which are also matched in ${ }^{1} \mathrm{H}$ NMR spectra. Although the new species are not fully characterized, they are tentatively assigned to the formation of two isomers of the cation obtained from chloride abstraction, with the $\mathrm{N}-\mathrm{H}$ bond being oriented either syn or anti to the $\mathrm{Re} \equiv \mathrm{N}$ moiety. In fact, the two isomers are found to be almost thermoneutral according to DFT calculations $\left(\Delta G^{0}=3.7 \mathrm{~kJ} \mathrm{~mol}^{-1}\right.$, see Section 3.6 for further details), as the intramolecular hydrogen bond to the trans chloride is removed, which stabilizes the anti isomer in $\mathbf{3 7}$ (compare Section 3.3.1). Subsequent 


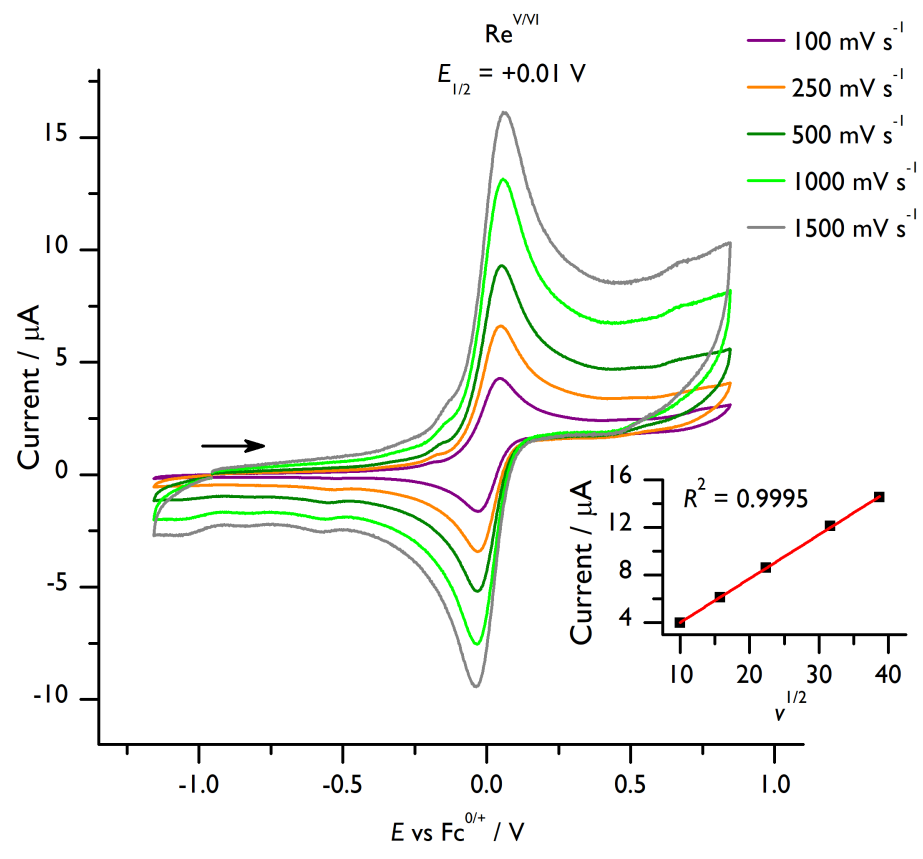

Fig. 3.23. $\mathrm{CV}$ measurements of $\operatorname{Re}(\mathrm{V} / \mathrm{VI})$ oxidation 37 in $\mathrm{THF}$ at different scan rates $\left(10^{-3} \mathrm{M} 37,0.1 \mathrm{M} \mathrm{N}^{\mathrm{n}} \mathrm{Bu}_{4} \mathrm{PF}_{6}\right.$, WE: GC, RE: Ag-wire, CE: Pt-wire). Inset: Plot and linear fit of $i_{p, a}$ vs. $v^{1 / 2}$.
Tab. 3.3. Full peak analysis of the $\operatorname{Re}(\mathrm{V} / \mathrm{VI})$ oxidation wave. $v$ is the scan rate in $\mathrm{mVs}^{-1}, i_{p, a}$ the anodic peak current in $\mu \mathrm{A}$ and $\Delta E$ is the peak separation given as ratio to peak separation of the internal standard.

\begin{tabular}{c|ccc}
\hline$v$ & $i_{p, a}$ & $\left|\frac{i_{p, c}}{i_{p, a}}\right|$ & $\Delta E$ \\
\hline 100 & 3.98 & 0.81 & 1.28 \\
250 & 6.13 & 0.74 & 1.27 \\
500 & 8.61 & 0.78 & 1.22 \\
1000 & 1.21 & 0.76 & 1.24 \\
1500 & 1.45 & 0.76 & 1.15 \\
\hline
\end{tabular}

addition of 1.1 eq of $\left(\mathrm{NHex}_{4}\right) \mathrm{Cl}$ quantitatively restored 37 , proving the chloride abstraction to be reversible.

Recording the electrochemical response by $\mathrm{CV}$ measurements revealed a single oxidation as the only redox event. Linear behavior of the forward peak current with $v^{1 / 2}$ indicate fast electron transfer to freely diffusing complexes, while an constant peak current ratio and a peak separation comparable to that of the internal standard suggest chemical reversibility of the reaction. The fact that the forward peak current is slightly higher than the backward peak current is assigned to a small impurity identified by a preshoulder of the forwards peak, which leads to a (constant) offset of the current. Consequently, the reduced donation of amine pincer ligand in $\mathbf{3 7}$ as compared to the amide ligand in the tert-butyl analog $\mathbf{X X I}$ does not lead to any significant changes in the redox behavior of the complex and initial reduction to a $\operatorname{Re}(\mathrm{IV})$ nitride is not possible.

The absence of any reduction wave in the CV of $\mathbf{3 7}$ rendered an electrophilic reactivity of this complex rather unlikely and also treatment with hydrogen or carbon monoxide gas did not lead to any reactions, not even at elevated temperatures $\left(60^{\circ} \mathrm{C}\right.$, overnight). Therefore, the reactivity studies of the nitride focused on its nucleophilic behavior. Inital attempts to protonate 37 with acids like $(\mathrm{LuH}) \mathrm{OTf}\left(\mathrm{p} K_{\mathrm{a}}{ }^{\mathrm{THF}}=7.2\right)$ or $\left(\mathrm{PhNH}(\mathrm{Me})_{2}\right) \mathrm{OTf}\left(\mathrm{p} K_{\mathrm{a}}{ }^{\mathrm{THF}}\right.$ $=4.9)^{[217]}$ did also not lead to any reaction, showing the nitride to be a fairly weak base. However, reaction with strong Lewis acids like HOTf $\left(\mathrm{p} K_{\mathrm{a}}{ }^{\mathrm{MeCN}}=2.6\right)^{[77]}$ or $\mathrm{BAr}_{18}^{\mathrm{F}}\left(\mathrm{BAr}_{18}^{\mathrm{F}}\right.$ $=\operatorname{tris}\{3,5$-bis(trifluoromethyl)phenyl $\}$ borane) do lead to immediate conversion to new com- 
plexes as indicated by new singlets in the ${ }^{31} \mathrm{P}\left\{{ }^{1} \mathrm{H}\right\}$-NMR spectrum at $\delta_{31} \mathrm{P}=30.2 \mathrm{ppm}$ and $23.9 \mathrm{ppm}$, respectively.

In the reaction with HOTf, the NMR signals were found to be very broad in many cases, independent of whether the samples were measured in $\mathrm{CD}_{2} \mathrm{Cl}_{2}$ or THF- $d_{8}$. Nevertheless, full assignment of all NMR signal was possible, by cooling the sample to $-35^{\circ} \mathrm{C}$. Despite the expected signals belonging to the iso-propyl groups and ligand backbone, a broadened triplet at $4.96 \mathrm{ppm}$ and a broad singlet at $14.24 \mathrm{ppm}$ were found in the ${ }^{1} \mathrm{H}$ NMR spectrum which integrated to one proton each. The ${ }^{1} \mathrm{H}_{-}{ }^{15} \mathrm{~N}-\mathrm{HSQC}$ spectrum contains two cross peaks, one for the pincer backbone $\mathrm{NH}$ at $\delta_{1_{\mathrm{H}}}=4.96 \mathrm{ppm} / \delta_{{ }_{15} \mathrm{~N}}=-330.0 \mathrm{ppm}$ and one for a $\mathrm{Re} \equiv \mathrm{N}-\mathrm{H}$ proton at $\delta_{1_{\mathrm{H}}}=14.24 \mathrm{ppm} / \delta_{15} \mathrm{~N}=18.1 \mathrm{ppm}$, proving successful nitride centered protona-

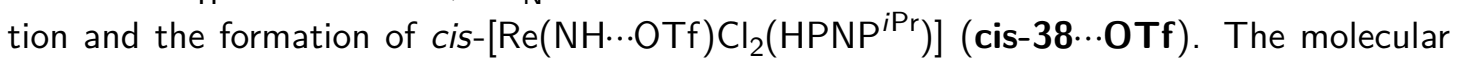
structure of cis-38...OTf derived from $X$-ray diffraction measurements of single crystals confirms the compound to be an octahedrally coordinated parent imido complex with the triflate anion forming a hydrogen bond to the corresponding proton. The $\mathrm{Re} \equiv \mathrm{NH}$ bond distance $\left(d\left(\operatorname{Re}_{1}-\mathrm{N}_{2}\right)=1.692(5) \AA\right)$ is only slightly elongated in comparison to nitrido complex $37(\Delta d(\operatorname{Re} \equiv \mathrm{N})=0.02 \AA)$, but the deviation of the $\mathrm{Re} \equiv \mathrm{N}-\mathrm{H}$ angle $(\measuredangle(\operatorname{Re} 1-\mathrm{N} 2-\mathrm{H} 112)$ $\left.=167(5)^{\circ}\right)$ from linearity and the significantly shortened $\mathrm{Re}-\mathrm{Cl}$ bond length of the trans

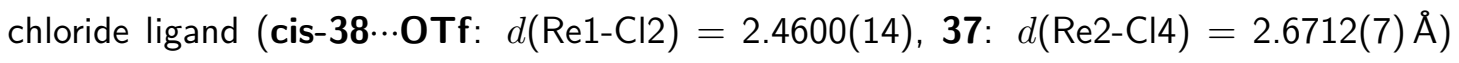
still substantiate the imido nature of the $\mathrm{NH}$ ligand.

The formation of cis-38... OTf highlights the advantage of the reduced ligand sterics which allow for binding of a sixth ligand trans to the nitride. Since the Re center of $\mathbf{3 7}$ is coordinated octahedrally and the backbone nitrogen is already protonated, the nitride remains the only basic side of the molecule, rendering imido formation most favorable. Additionally, the fact that both chlorides are coordinating omits the formation of a dication, which would have been necessary in the case of the tert-butyl complex $\left[\operatorname{Re}(N) C l\left(\mathrm{HPNP}^{t \mathrm{Bu}}\right)\right]^{\mathrm{Cl}}$. This is further underpinned by the finding that the two isomers formed from chloride abstraction from 37 are unreactive even towards $\mathrm{H}\left(\mathrm{Et}_{2} \mathrm{O}\right)_{2}\left(\mathrm{BAr}_{24}^{\mathrm{F}}\right)$.

While parent imido compounds are not unprecedented (see ref. [218] and references [12-35] therein), to date only three other examples in the coordination sphere of rhenium were reported, with $\mathrm{Re} \equiv \mathrm{NH}$ bond distances of 1.664(3)-1.712(8) $\AA{ }^{.}{ }^{[219-221]}$ Additionally, there are only two literature examples of stable parent imido complexes derived from dinitrogen splitting, even though they are regularly discussed as intermediates in transition metal catalyzed dinitrogen fixation to ammonia. ${ }^{[68,114]}$

The reason for the broadend NMR signals is unclear at this point, but might be due to a fast equilibrium between different isomers of $\mathbf{3 8}^{\mathrm{OTf}}$. Indeed, in the ${ }^{31} \mathrm{P}\left\{{ }^{1} \mathrm{H}\right\}$ NMR spectrum of an analytically pure sample at $-40^{\circ} \mathrm{C}$, a small, similarly broadened singlet at $44.9 \mathrm{ppm}$ can be found, which might correspond to such an isomer (see inset in Figure $3.22 \mathrm{c}$ ). Computational evaluation ${ }^{8}$ of different isomers also points to this possibility (see Part III Section 3.7 for

\footnotetext{
${ }^{8}$ DFT: PBE0/D3BJ/RIJCOSX/def2-TZVPP or PBE/D3BJ/RI/def2-TZVPP with CPCM(THF) or $\mathrm{CPCM}(\mathrm{DCM}) \| \mathrm{PBE} / \mathrm{D} 3 \mathrm{BJ} / \mathrm{RI} /$ def2-SVP.
} 
a)
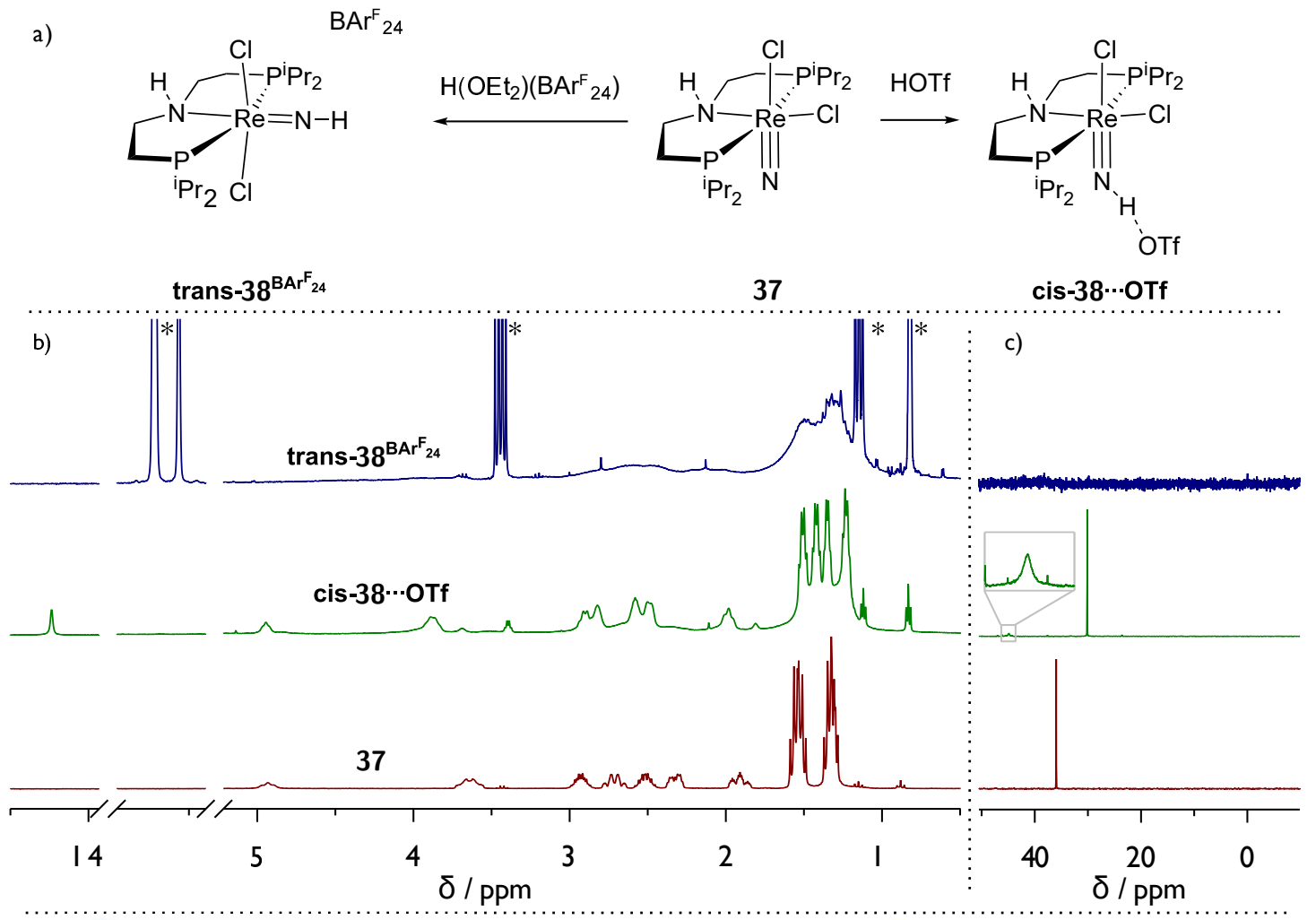

d)
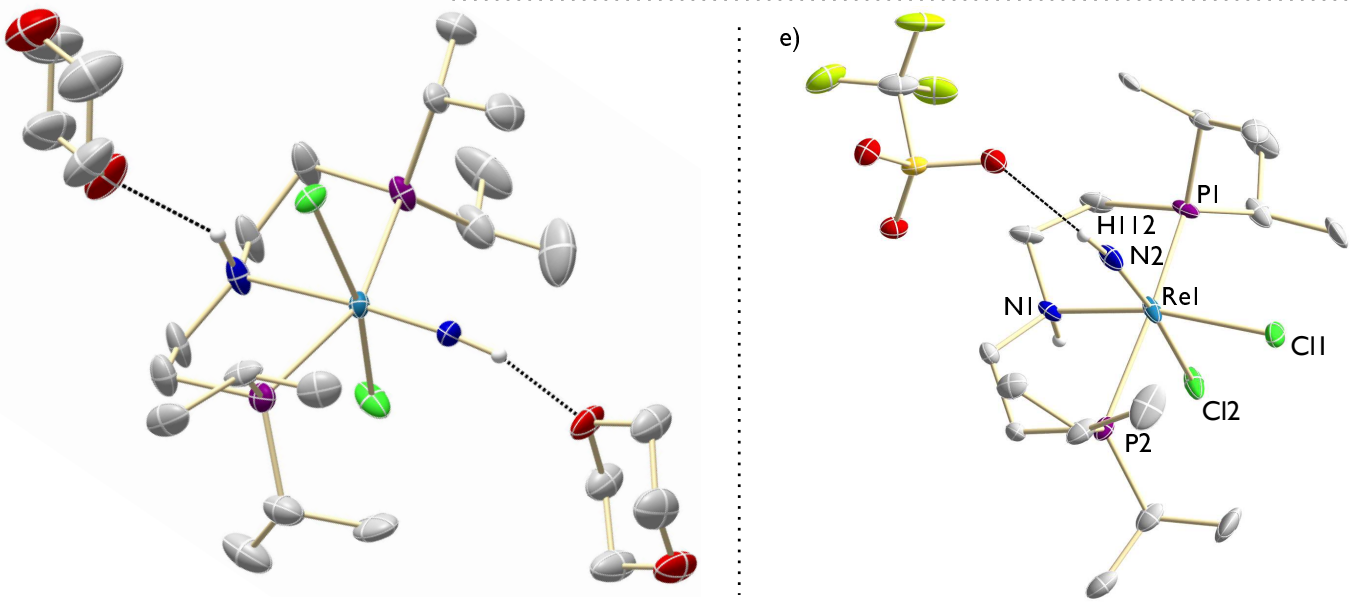

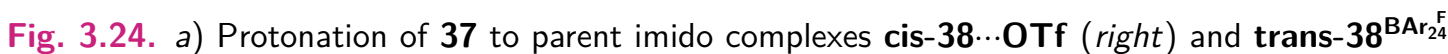
(left) by HOTf and $\mathrm{H}\left(\mathrm{OEt}_{2}\right)\left(\mathrm{BAr}_{24}^{\mathrm{F}}\right.$ ), respectively. b) ${ }^{1} \mathrm{H}-\mathrm{NMR}$ spectra (cis-38...0Tf was measured at $-35^{\circ} \mathrm{C}$; signals marked with asterisks in the spectrum of trans-38 ${ }^{\mathrm{BAr}_{24}{ }^{\mathrm{F}}}$ correspond to the anion, $\mathrm{Et}_{2} \mathrm{O}$ from the acid and $\mathrm{PMe}_{3}$ which was added as internal standard in a capillary). c) ${ }^{31} \mathrm{P}\left\{{ }^{1} \mathrm{H}\right\}-N M R$ spectra (cis-38... OTf was measured at $-35^{\circ} \mathrm{C}$ ). $d+e$ ) Molecular structures of trans-38 ${ }^{\mathrm{BAr}_{24}{ }^{\mathrm{F}}}(d)$ and cis-38...0Tf $(e)$ obtained by single crystal X-ray diffraction measurements. All $\mathrm{H}$ atoms but the $\mathrm{NH}$ protons as well as disordered atoms, the $\mathrm{BAr}_{24}^{\mathrm{F}}$ anion and a cocrystallized solvent molecules are omitted for clarity. Anisotropic displacement parameters are set to $50 \%$ probability. Selected bond lengths $[\AA]$ and angles $\left[^{\circ}\right]$ : trans-38 ${ }^{\mathrm{BAr}}{ }_{24}^{\mathrm{F}}$ : Re1-N1 2.298(5), Re1-N2 1.706(5), Re1-Cl1 2.3929(13), Re1-Cl2 2.4094(15), N1-Re1-N2 175.4(2), Cl1-Re1-Cl2 163.27(5), Re1-N2H112 170(5), P1-Re1-P2 156.41(6). cis-38‥OTf: Re1-N1 2.164(5), Re1-N2 1.692(5), Re1-Cl1 2.473(12), Re1-Cl2 2.4600(14), N1-Re1-N2 90.8(2), Cl1-Re1-Cl2 87.7(3), Re1N2-H112 167(5), P1-Re1-P2 164.3(3). 
details). Investigated were the imido complex isomers with cis- and trans-chlorides, each of

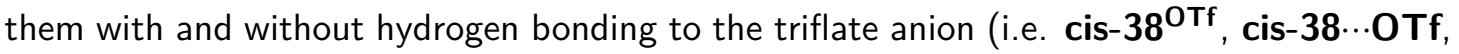
trans-38 ${ }^{\text {TTf }}$ and trans-38...OTf). For the reaction of $\mathbf{3 7}$ with HOTf, the crystallized isomer

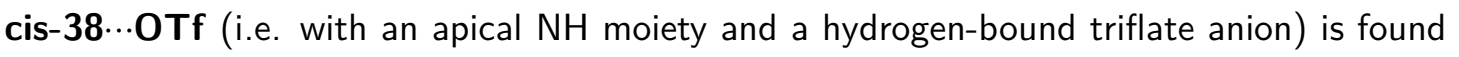
to be very close in energy with the cationic trans-chloro analog with a non-coordinating anion trans-38 ${ }^{\mathrm{OTf}}$. Actually, when a DCM solvent model is used, trans-38 ${ }^{\mathrm{OTf}}$ is even favored $\left(\Delta G^{0}=-1.9 \mathrm{~kJ} \mathrm{~mol}^{-1}\right.$ (PBE), $-6.5 \mathrm{~kJ} \mathrm{~mol}^{-1}$ (PBE0)), while with the THF model

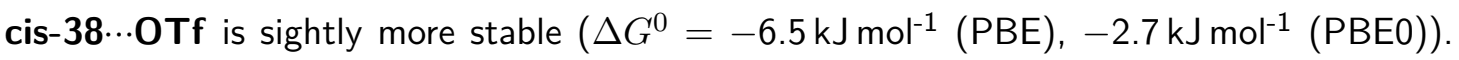
Interestingly, a hydrogen bond between the imido hydrogen atom in the trans isomer and the triflate anion (i.e. isomer trans-38...OTf) is thermodynamically unfavorable in all calculations, and in these structures the proton is found to be localized at the triflate anion, rather than at the nitride. Furthermore, when only the isomers cis-38 ${ }^{\circ T f}$ and trans-38 OTf are compared (i.e. neglecting the possibility of hydrogen bond formation), trans-380Tf is predicted to be the more stable anion in all cases.

These results would also be in line with the observation that the peak broadening strongly depends on the reaction conditions and the acid. To test these computational results, protonation of $\mathbf{3 7}$ was repeated with the strong acid $\mathrm{H}\left(\mathrm{OEt}_{2}\right)_{2}\left(\mathrm{BAr}_{24}^{\mathrm{F}}\right)$, which features a noncoordinating anion. Unfortunately, the NMR spectra in these reactions were almost not interpretable due to extreme line broadening. Selectivity of the reaction is nevertheless proven by subsequent treatment of the reaction mixture with $\mathrm{NEt}_{3}$, which leads to regeneration of starting complex $\mathbf{3 7}$ by more than $90 \%$ and single crystals allowed for molecular structure determination by $\mathrm{X}$-ray diffraction. Indeed, the non-coordinating anion results in isomerization to the imido complex trans-38 ${ }^{\mathrm{BAr}}{ }_{24}^{\mathrm{F}}$, with the two chloride ligands being oriented trans to each other and the imido group being coordinated trans to the pincer $\mathrm{NH}$ functionality. The $\mathrm{Re} \equiv \mathrm{N}$ bond is slightly elongated in comparison with cis-38...0Tf $(d(\operatorname{Re} 1$ $\mathrm{N} 2)=1.706(5) \AA$ ) as a direct influence of the stronger trans donor ligand, also expressed

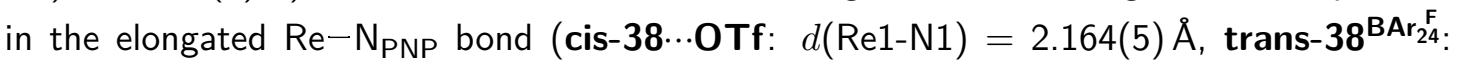
$d(\operatorname{Re} 1-\mathrm{N} 1)=2.298(5) \AA)$. The imido moiety is tendentially linear, but the angle must not be interpreted to much due to the large error of that specific value $(\measuredangle(\operatorname{Re} 1-\mathrm{N} 2-\mathrm{H} 112)=$ $\left.170(5)^{\circ}\right)$. Consequently, the choice of the anion has a significant influence on the molecular structure. The fact that the crystal structure of trans-38 $8^{\mathrm{BAr}_{24}}$ contains hydrogen bonded dioxane molecules, while the structure of cis-38...OTf features a THF molecule which does not form any hydrogen bond further highlights how sensitive this effect is to small changes.

An indication on whether this cis-trans isomerization occurs after protonation or if $\mathbf{3 7}$ itself might already exhibit such an equilibrium (only strongly on the cis side) is obtained from the reaction with $\mathrm{BAr}_{18}^{\mathrm{F}}$. A new signal in the ${ }^{31} \mathrm{P}$ NMR spectrum at $\delta_{31} \mathrm{P}=23.9 \mathrm{ppm}$ as well as the ${ }^{1} \mathrm{H}$ NMR spectrum show clean conversion to a new $C_{S}$ symmetric complex with an intact $\mathrm{NH}$ proton at $\delta_{1_{\mathrm{H}}}=4.98 \mathrm{ppm}$ and a slight shift of those signals corresponding to the borane, indicating coordination. This complex can be assumed to be the cis chloro complex $\left[\operatorname{Re}\left(\mathrm{NBAr}_{18}^{\mathrm{F}}\right) \mathrm{Cl}_{2}\left(\mathrm{HPNP}^{\mathrm{iPr}}\right)\right]$ (cis-39) analog to $\mathbf{3 8}^{\mathrm{OTf}}$. Surprisingly, the complex turned out 
a)

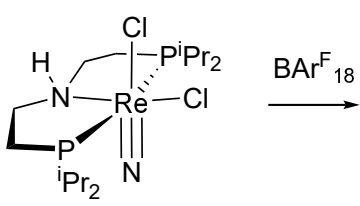

37

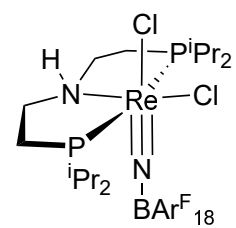

cis-39

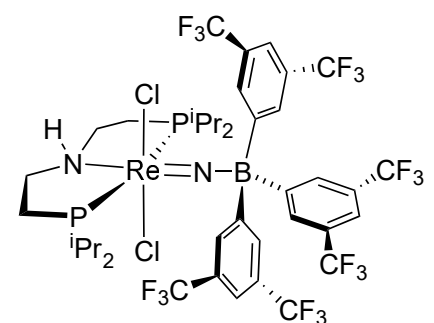

trans-39

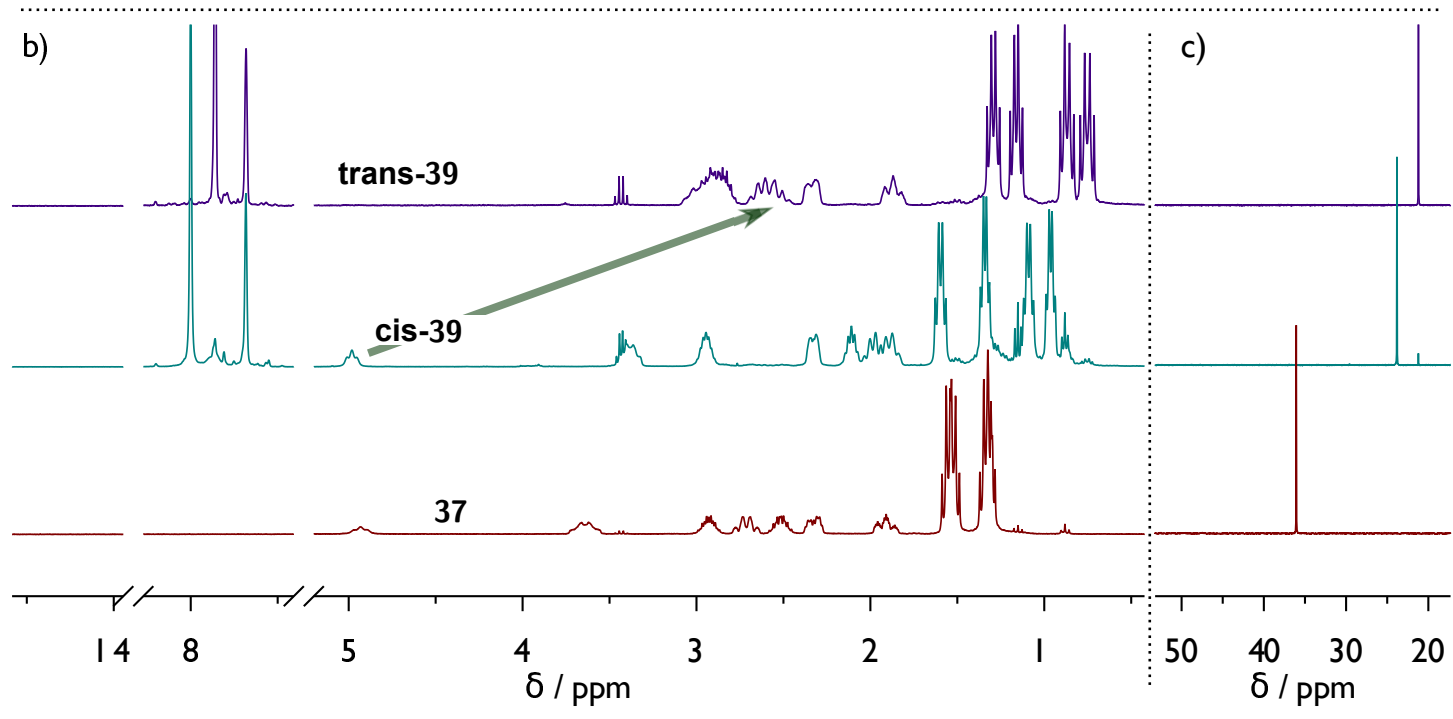

e)

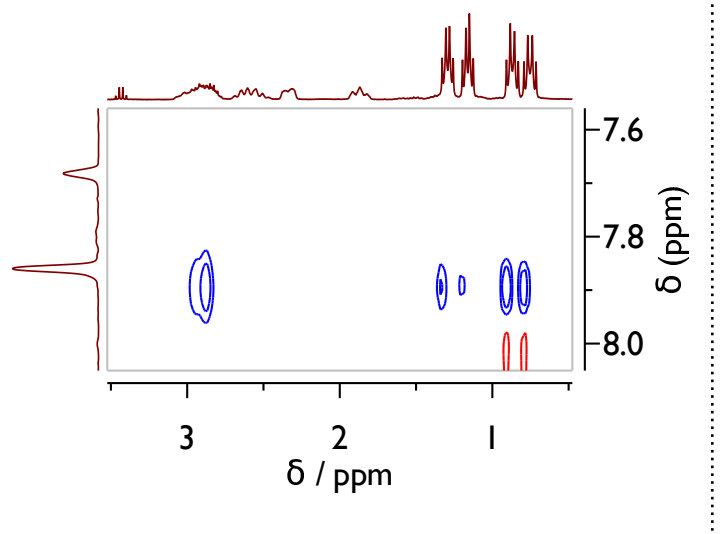

f)

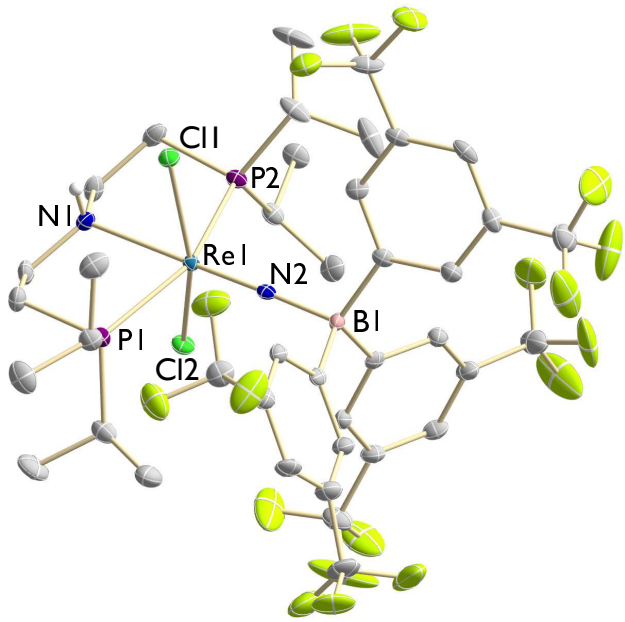

Fig. 3.25. a) Synthesis of cis-39 and isomerization to trans-39. b) ${ }^{1} \mathrm{H}-\mathrm{NMR}$ spectra. The green arrow highlights the sift of the pincer $\mathrm{NH}$ proton upon isomerization. $c$ ) ${ }^{31} \mathrm{P}\left\{{ }^{1} \mathrm{H}\right\}-\mathrm{NMR}$ spectra. d) NOESY spectrum of trans-39 showing cross peaks between the aromatic protons and all iso-propyl groups. e) Molecular structure of trans-39 obtained by single crystal X-ray diffraction measurements. All $\mathrm{H}$ atoms but the $\mathrm{NH}$ proton as well as disordered atoms are omitted for clarity. Anisotropic displacement parameters are set to $50 \%$ probability. Selected bond lengths $[\AA]$ and angles [ $\left.{ }^{\circ}\right]$ : Re1-N1 2.341(3), Re1-N2 1.705(3), Re1-Cl1 2.4311(9), Re1-Cl2 2.3788(9), N2-B1 1.568(5), N1-Re1-N2 176.93(13), Cl1-Re1Cl2 159.55(3), Re1-N2-B1 175.5(3), P1-Re1-P2 156.22(3). 
to be unstable at RT, undergoing a selective follow-up reaction overnight to a new species with a resonance at $\delta_{31} \mathrm{P}=21.2 \mathrm{ppm}$. The ${ }^{1} \mathrm{H}$ NMR spectrum also revealed all signals to be shifted of which the $\mathrm{NH}$ proton exhibits the largest shift to $\delta_{1_{\mathrm{H}}}=2.51 \mathrm{ppm}$. According to LIFDI mass spectrometry, the new complex still is a simple borane-nitride adduct, so the chemical composition did not change but rather the complex isomerized. Indeed, cross peaks in the NOESY spectrum indicate spacial proximity between the aromatic protons of the borane and all four signals belonging to the iso-propyl groups, indicating an isomerization to the trans-chloro complex $\left[\operatorname{Re}\left(\mathrm{NBAr}_{18}^{\mathrm{F}}\right) \mathrm{Cl}_{2}\left(\mathrm{HPNP}^{\mathrm{iPr}}\right)\right]$ (trans-39), resembling the structure

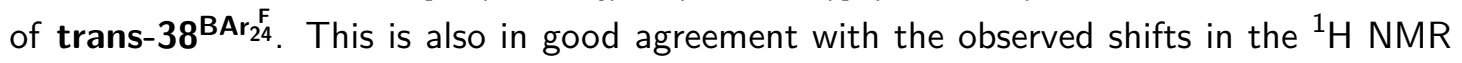
spectrum, since the effect on the pincer $\mathrm{NH}$ proton can be assumed to be strongest. This assignment was confirmed by $\mathrm{X}$-ray diffraction analysis of single crystals of trans-39. The Re center was indeed found in an octahedral coordination environment, again with the two chloride ligands oriented trans to each other and the $\mathrm{N}-\mathrm{BAr}_{18}^{\mathrm{F}}$ moiety in trans position to the pincer amine donor. The bond lengths and angles are very similar to those of trans-38 ${ }^{\mathbf{B A r}_{24}^{\mathrm{F}}}$ $\left(d(\operatorname{Re} 1-\mathrm{N} 1)=2.341(3) \AA\right.$ and $d(\operatorname{Re} 1-\mathrm{N} 2)=1.705(3) \AA$, 38 $\left.{ }^{\mathrm{OTf}}: d(\operatorname{Re} 1-\mathrm{N} 1)=2.164(5) \AA\right)$, reflecting the mutual trans influence of two strong donors, and the $\mathrm{Re}=\mathrm{N}-\mathrm{B}$ angle is again closer to linearity than in cis-38 $\cdots$ OTf $\left(\measuredangle(\operatorname{Re} 1-N 2-B 1)=175.5(3)^{\circ}\right)$.

No reactivity studies have been performed on trans- or cis-39. In contrast, very preliminary reactions of cis-38...0Tf with excess of $\mathrm{Co}(\mathrm{Cp})_{2}$ or $\mathrm{Co}\left(\mathrm{Cp}^{*}\right)_{2}(4 \mathrm{eq})$ and $\left(\mathrm{Ph}_{2} \mathrm{NH}_{2}\right)$ OTf (5 eq) were executed, in an attempt to form ammonia. While the reaction with $\mathrm{Co}(\mathrm{Cp})_{2}$ seemed to yield rather selectively an unknown, paramagnetic species, the reaction with $\mathrm{Co}\left(\mathrm{Cp}^{*}\right)_{2}$ resulted in a ${ }^{1} \mathrm{H}$ NMR spectrum which appeared to be identical with $\left[\mathrm{ReCl}_{3}\right.$ $\left.\left(\mathrm{HPNP}^{i \mathrm{Pr}}\right)\right]$ (29). However, since no additional chloride source was added, the reaction is unbalanced and rather formation of a cationic analog with (non)coordinating triflate might have occured. In the end, even though these results do look quite promising with regard to potential ammonia formation, only NMR data are available on those reactions and no attempts to detect ammonia were made, leaving a lot of room for further research before any conclusions can be drawn.

\subsubsection{Metal-ligand cooperative benzamide / benzonitrile formation}

This nitride centered reactivity raised the question in how far $\mathbf{3 7}$ exhibits improved reactivity towards carbon centered electrophiles. One of the main motivations for using the iso-propyl ligand was the hope to access a more reactive nitride than with the tert-butyl ligand. Therefore the reactivity of $\mathbf{3 7}$ towards acyl chlorides was tested, which are less reactive than previously utilized alkyl triflates. In fact, heating $\mathbf{3 7}$ with benzoyl chloride or acetyl chloride in 1,4-dioxane to $90^{\circ} \mathrm{C}$ overnight does lead to conversion to main one new complex. While the reaction seems to proceed analogous in with both reagents (identical spectroscopic signature for the obtained Re complex in the ${ }^{1} \mathrm{H}$ and ${ }^{31} \mathrm{P}\left\{{ }^{1} \mathrm{H}\right\}$ NMR spectra), the reaction with 
a)

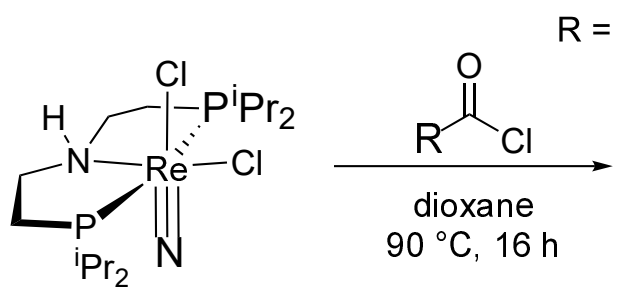

37

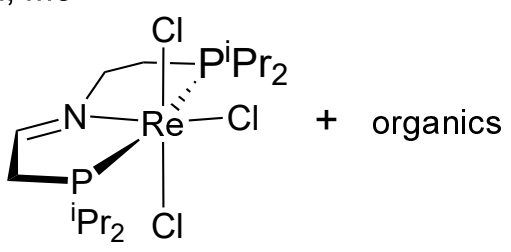

40

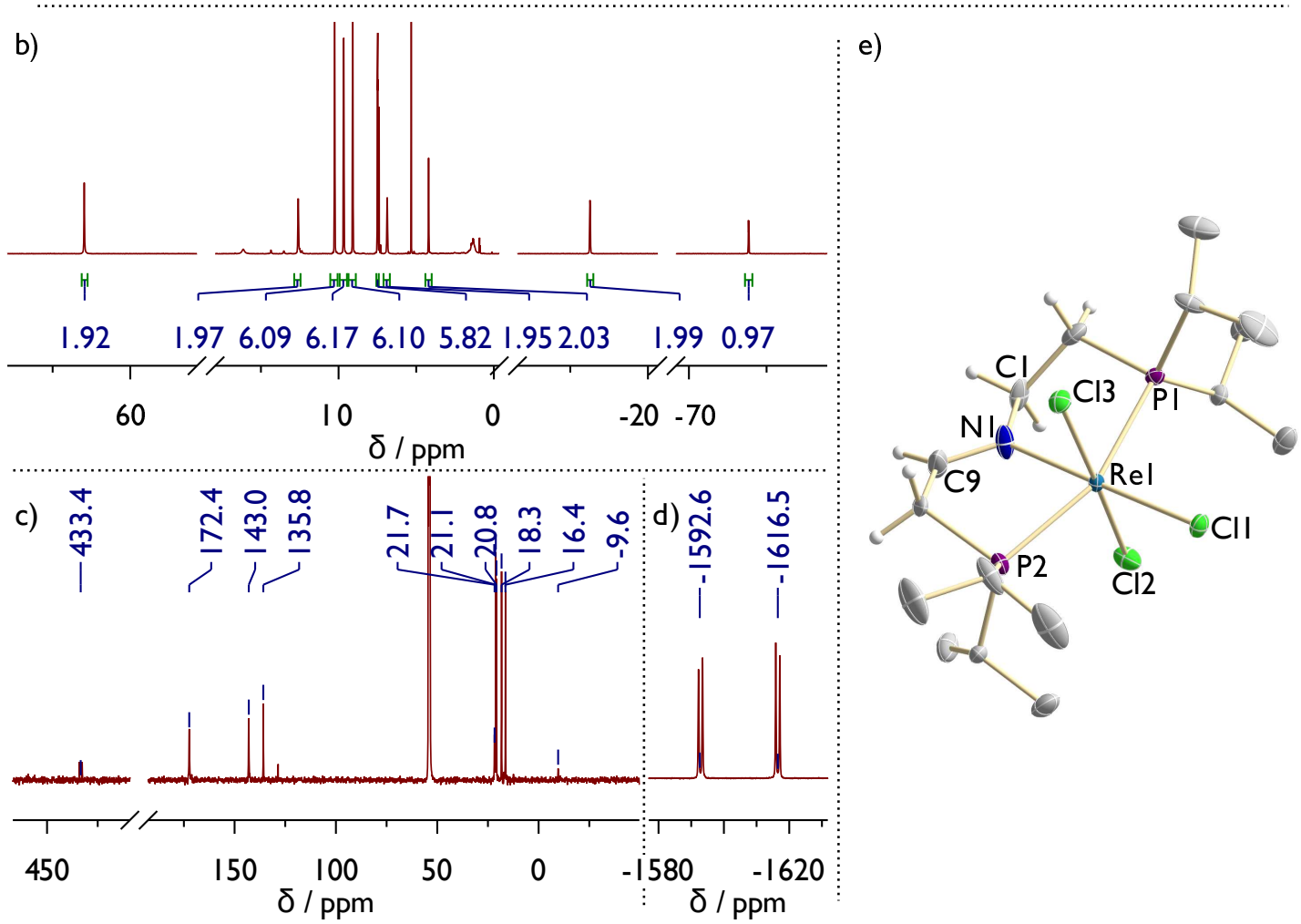

Fig. 3.26. a) Reaction of $\mathbf{3 7}$ acetyl chlorides to $\mathbf{4 0}$ and organic compouds. b) ${ }^{1} \mathrm{H}$ NMR spectrum of 40. c) ${ }^{13} \mathrm{C}\left\{{ }^{1} \mathrm{H}\right\}$ NMR spectrum of $\mathbf{4 0}$. d) ${ }^{31} \mathrm{P}\left\{{ }^{1} \mathrm{H}\right\}$ NMR of 40. e) Molecular structure of $\mathbf{4 0}$ obtained by single crystal $\mathrm{X}$-ray diffraction measurements. All $\mathrm{H}$ atoms but the backbone protons are omitted for clarity. Anisotropic displacement parameters are set to $50 \%$ probability. Selected bond lengths $[\AA]$ and angles [ $\left.{ }^{\circ}\right]$ : Re1-N1 2.109(5), Re1-Cl1 2.3970(13), Re1-Cl2 2.3686(13), Re1-Cl3 2.3958(13), N1-C1 1.460(7), N1-C9 1.299(7), P1-Re1-P2 159.78(5), N1-Re1-Cl1 177.88(14), Cl2-Re1-Cl3 176.41(5).

acetyl chloride was not further investigated and all data reported here refer to the reaction with benzoyl chloride. The new Re complex features two new doublets in ${ }^{31} \mathrm{P}\left\{{ }^{1} \mathrm{H}\right\}$ NMR at $\delta_{31} \mathrm{P}=-1592.6$ and $-1616.5 \mathrm{ppm}$ with a mutual coupling constant of ${ }^{2} J_{\mathrm{PP}}=248 \mathrm{~Hz}$. In the ${ }^{1} \mathrm{H}$ NMR spectrum, four signals corresponding to iso-propyl groups (i.e. two inequivalent groups) identify the complex to exhibit $C_{S}$ symmetry on the NMR timescale with the mirror plane being coplanar with the PNP pincer ligand. Additionally, four signals arising from the pincer backbone with a ratio of 2:2:2:1 resonate in a range of $63--74 \mathrm{ppm}$ and no $\mathrm{NH}$ proton could be identified, suggesting an oxidation of the pincer backbone to an imine (i.e. $\mathrm{N}\left(=\mathrm{CHCH}_{2} \mathrm{PiPr}_{2}\right)\left(\mathrm{CH}_{2} \mathrm{CH}_{2} \mathrm{PiPr}\right)\left(\mathrm{P}=\mathrm{NP} \mathrm{Pr}^{i \mathrm{Pr}}\right)$. Despite being strongly shifted, all signals are very sharp and exhibit resolved $J$-couplings, reminiscent of the spectra of $\operatorname{Re}(\mathrm{III})$ 


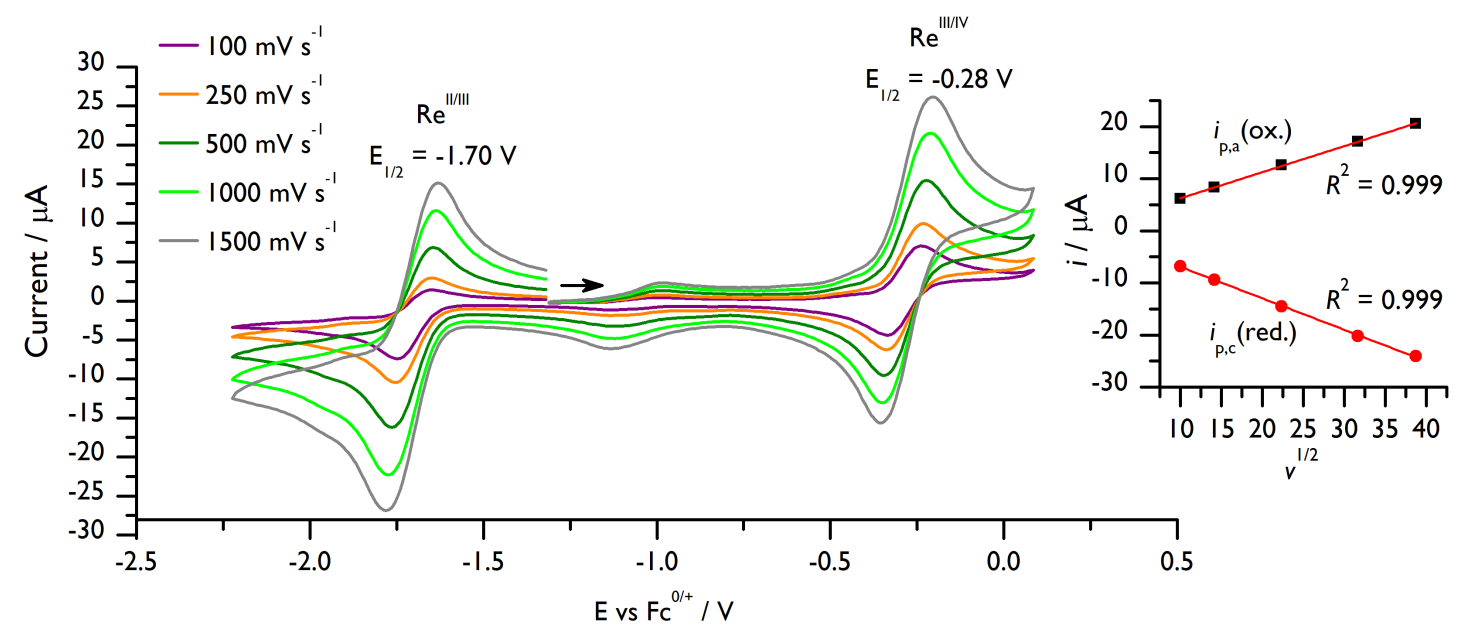

Fig. 3.27. CV measurements of reversible oxidation and quasi-reversible reduction of $\mathbf{4 0}$ in THF at different scan rates $\left(10^{-3} \mathrm{M}\right.$ 40,0.1 $\mathrm{M} \mathrm{N}^{\mathrm{n}} \mathrm{Bu}_{4} \mathrm{PF}_{6}, \mathrm{WE}$ : GC, RE: Ag-wire, CE: Pt-wire). Inset: Plot and linear fit of $i_{p, a}$ (oxidation) and $i_{p, c}\left(\right.$ reduction) vs. $v^{1 / 2}$.

Tab. 3.4. Peak analysis for the oxidation and reduction of 40. $\Delta E$ denotes the peak separation of the oxidation wave as ratio of the peak separation of subsequent added ferrocene.

\begin{tabular}{c|cc|cc|c}
\hline & \multicolumn{2}{|c|}{ oxidation } & \multicolumn{2}{|c|}{ reduction } & \\
\hline$v / \mathrm{mV} \mathrm{s}^{-1}$ & $i_{p, a} / \mu \mathrm{A}$ & $\left|\frac{i_{p, c}}{i_{p, a}}\right|$ & $i_{p, c} / \mu \mathrm{A}$ & $\left|\frac{i_{p, a}}{i_{p, c}}\right|$ & $\Delta E$ \\
\hline 100 & 6.13 & 1.051 & -6.83 & 0.507 & 1.000 \\
200 & 8.30 & 1.077 & -9.38 & 0.564 & 1.083 \\
500 & 12.64 & 1.087 & -14.47 & 0.726 & 1.154 \\
1000 & 17.11 & 1.098 & -20.17 & 0.809 & 1.067 \\
1500 & 20.53 & 1.076 & -24.08 & 0.857 & 1.125 \\
\hline
\end{tabular}

complex 29. In the ${ }^{13} \mathrm{C}\left\{{ }^{1} \mathrm{H}\right\}$ NMR spectrum, two strongly shifted signals arise at $\delta_{13} \mathrm{C}=$ 433.4 and $-9.6 \mathrm{ppm}$. Decoupling of the entire proton range was not possible, resulting in the signal at $\delta_{13} \mathrm{C}=433.4 \mathrm{ppm}$ to be split into a doublet, which collapsed to a singlet when the decoupler was set to the proton signal at $\delta_{1_{\mathrm{H}}}=-73.9 \mathrm{ppm}$. Finally, LIFDI mass spectrometry, independent synthesis by reaction of $\mathbf{2 9}$ with 2 eq TTBP and X-ray diffraction measurements on single crystals all confirmed the newly formed complex to be the backbone oxidized $\operatorname{Re}(\mathrm{III})$ complex $\left[\operatorname{ReCl}_{3}\left(\mathrm{P}=\mathrm{NP}^{i \mathrm{Pr}}\right)\right](40)$. In the $\mathrm{X}$-ray structure, the Re center is coordinated octahedrally by the $\mathrm{P}=\mathrm{NP}^{i \mathrm{Pr}}$ and three chloride ligands. Backbone oxidation to the imine is supported by one significantly shortened $\mathrm{N}=\mathrm{C}$ bond $(d(\mathrm{~N} 1-\mathrm{C} 9)=1.299(7) \AA$, $d(\mathrm{~N} 1-\mathrm{C} 1)=1.460(7) \AA)$, a planar coordination of the nitrogen atom $\left(\Sigma\left(\measuredangle_{\mathrm{N} 1}\right)=359.55^{\circ}\right)$ and a $\operatorname{Re}-\mathrm{N}$ bond distance which is too long for an amide ligand $(d(\operatorname{Re} 1-\mathrm{N} 1)=2.109(5) \AA)$. The electrochemical response of $\mathbf{4 0}$ in CV measurements is also quite similar to that of $\mathbf{2 9 .}$ A reversible $\operatorname{Re}(\mathrm{III} / \mathrm{IV})$ oxdiation wave at $E_{1 / 2}=-0.28 \mathrm{~V}$ vs. $\mathrm{Fc} / \mathrm{Fc}^{+}$and a quasi-reversible reduction wave at $E_{1 / 2}=-1.70 \mathrm{~V}$ are found, in line with the $\operatorname{Re}(\mathrm{III})$ formulation. Linear development of the forward peak current with $v^{1 / 2}$ shows both process to be induced by fast electron transfer to freely floating molecules in solution. The reversibility of the oxidation is 
reflected by a peak separation almost identical to that of ferrocene as well as an invariant ratio of the backward to the forward peak current close to unity, while for the reduction this ratio clearly decreases with lower scan rates, indicating an irreversible chemical follow-up process after reduction.

The strong shifts in NMR spectroscopy most likely arise from a comparable TIP resulting from SOC, just as in the amine complex 29 (compare Section 3.1.2). This is not surprising, since $\mathbf{4 0}$ is an octahedrally coordinated $d^{4}$ complex and the donor abilities of the imine vs. and amine ligand can be assumed to be comparable, so no big influence on the ligand field is to be expected.

The observed formation of $\mathbf{4 0}$ raises the question about the fate of the nitrido ligand. The formal $\operatorname{Re}(\mathrm{V} / \mathrm{III})$ reduction is accompanied by the backbone oxidation by two electrons and removal by two protons. Considering benzoyl chloride as the most likely source of the additional chloride ligand in $\mathbf{4 0}$, either the formation of benzamide or benzonitrile and water are suitable possiblities to balance the reaction. If the solvent of a neat reaction mixture is removed in vacuo and the products are analyzed in THF- $d_{8}$, the aromatic region of the ${ }^{1} \mathrm{H}$ NMR spectrum reveals signals for benzoic acid and benzamide $\left(\delta_{1_{\mathrm{H}}}=8.11\right.$ and $7.81 \mathrm{ppm}$, both in THF- $d_{8}$ ). If the reaction was performed directly in toluene- $d_{8}$ rather than 1,4 -dioxane, equimolar formation of benzonitrile (with respect to benzoic acid) was detectable. However, these latter reactions gave reduced overall yields. The occurance of all three products can be rationalized by assuming initial electrophilic attack of the benzoyl chloride at the nitride

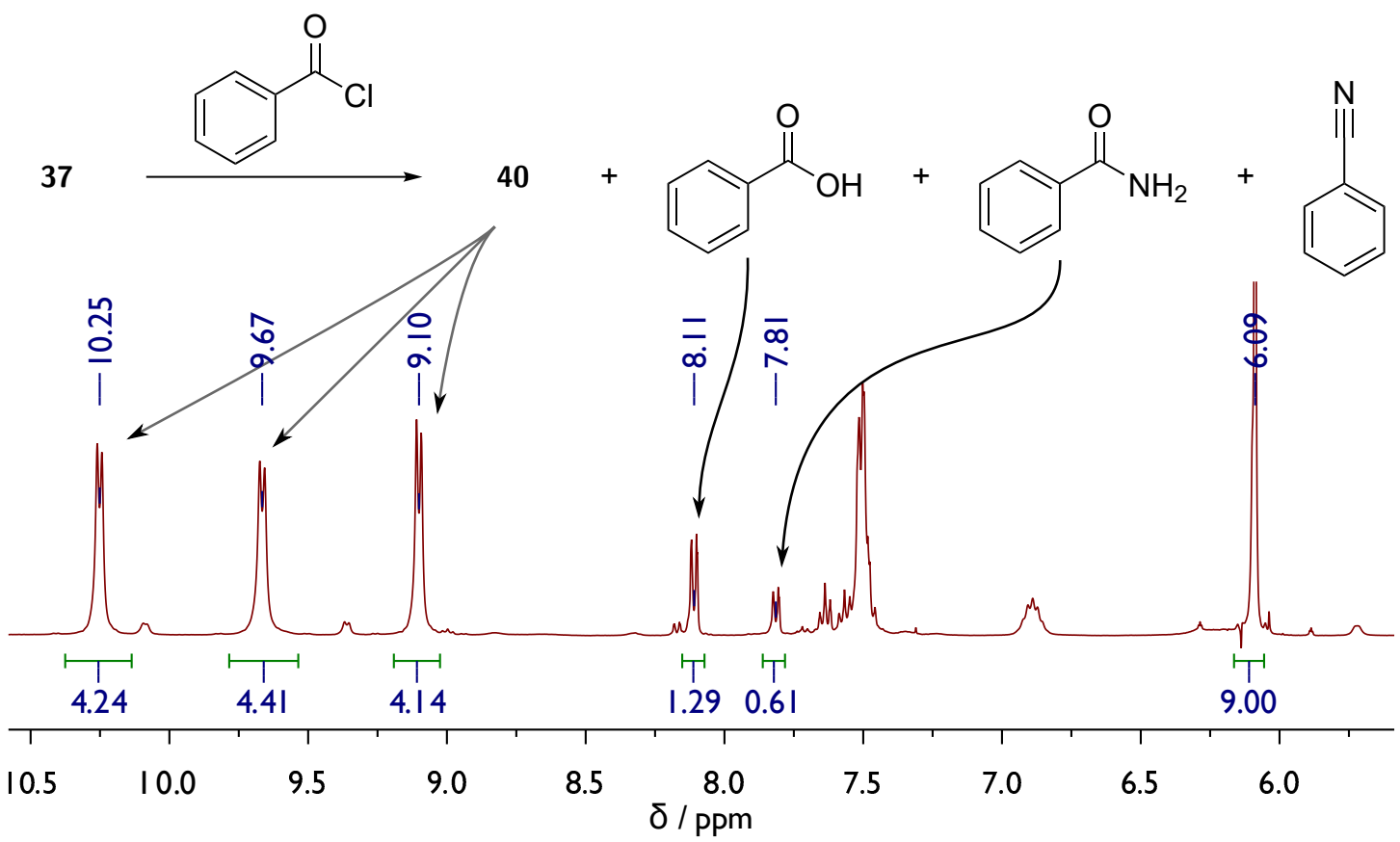

Fig. 3.28. ${ }^{1} \mathrm{H}$ NMR quantification of the formation of $\mathbf{4 0}(71 \%$ yield $)$, benzoic acid $\left(64.5 \%, \delta\left(\mathrm{H}_{\text {ortho }}\right)\right.$ $=8.11 \mathrm{ppm})$ and benzamide $\left(30.5 \%, \delta\left(\mathrm{H}_{\text {ortho }}\right)=7.81 \mathrm{ppm}\right)$ from the reaction of 37 with 2 eq benzoyl chloride. 1,3,5-trimethoxybenzene $\left(\delta\left(\mathrm{CH}_{\text {arom. }}\right)=6.09 \mathrm{ppm}\right)$ was used as internal standard (3 eq). 
and subsequent release of benzamide and formation of $\mathbf{4 0}$. Afterwards, benzamide itself can react with benzoyl chloride to form benzoic acid and benzonitrile while releasing $\mathrm{HCl}$. ${ }^{[222]}$ In fact, simply mixing commercial benzamide and benzoyl chloride and heating in 1,4-dioxane gave almost identical product distributions. If the reaction was performed at higher temperatures $\left(110^{\circ} \mathrm{C}\right)$, also tribenzamide was formed to minor degree. All organic products were identified by comparison to commercial samples.

In this reaction, the pincer ligand acts as a two electron, two proton reservoir enabling the one-pot synthesis of benzamide by PCET. This convenient reactivity of the PNP pincer ligand is not unprecedented. The group of $A$. J. M. Miller reported a quite analogous reaction (see Scheme 3.2). Starting from the square-planar Ru(IV) nitride complex $\left[\mathrm{Ru}(\mathrm{N})\left(\mathrm{PNP}^{t \mathrm{Bu}}\right)\right]$ originally reported by the Schneider group, ${ }^{[138]}$ monoprotonation with a luthidinium salt results in the amine-pincer complex $\left[R u(N)\left(H_{P N P^{t B u}}\right)\right]^{+}$. Subsequent treatment with carboxylic acids like benzoic acid leads to conversion of the nitrido ligand into an amine with concomitant oxidation of the amine pincer to the imine variant, resulting in $\left[\mathrm{Ru}\left(\mathrm{NH}_{3}\right)\left(\kappa^{2}-\mathrm{O}_{2} \mathrm{CAr}\right)\left(\mathrm{P}=\mathrm{NP}^{t \mathrm{Bu}}\right)\right]^{+}$. [223] A DFT derived mechanism of the reaction identified the possibility of the benzoic acid / benzoate anion to act as a chelate and thereby serving as a proton shuttle to be of significant importance. A comparable role could be imagined for the $\mathrm{C}=\mathrm{O}$ moiety of the benzoyl chloride after inital attack at the nitride. To further evaluate the importance of that functional group, additional experiments will be necessary. Especially, nitride methylation with e.g. MeOTf and subsequent heating in presence of a chloride salt could give further insights.

Both above discussed reactions reveal the PNP pincer ligands to have a certain potential as PCET agents in nitride functionalization. In applied systems, this reactivity could be quite valuable, but only if the pincer ligand could also be restored, making it act as a real proton and electron relay. While the group of Miller was unable to achieve re-reduction of the ligand, sequential addition of $\mathrm{Li}\left(\mathrm{HBEt}_{3}\right)$ and $\left(\mathrm{Ph}_{2} \mathrm{NH}_{2}\right) \mathrm{Cl}$ to 40 could restore amine complex 29 in $61.8 \%$ yield. In this reaction, the addition of $\mathrm{Li}\left(\mathrm{HBEt}_{3}\right)$ is accompanied by the formation of hydride-bearing side products as indicated by ${ }^{1} \mathrm{H}$ NMR spectroscopy. Upon acid addition, these hydride complexes then reform the imine complex 40, which limits the possible yields for the reaction via this route. Despite this limiation, a hydride and proton source are neither compatible with each other nor with the so far used reagents in this synthetic cylce (i.e. $\left(\mathrm{Ph}_{2} \mathrm{NH}_{2}\right) \mathrm{Cl}$ vs. $\mathrm{Co}\left(\mathrm{Cp}^{*}\right)_{2}$ and $\mathrm{Li}\left(\mathrm{HBEt}_{3}\right)$ vs. benzoyl chloride).

Therefore, possibilities to electrochemically re-reduce $\mathbf{4 0}$ in the presence of a proton source
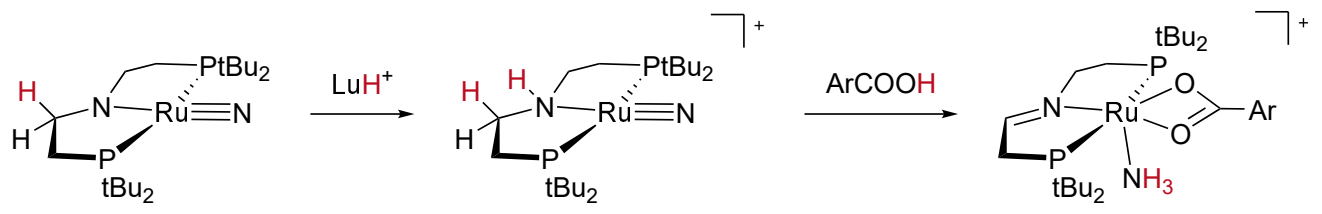

Scheme 3.2. Metal-ligand cooperative, PCET mediated ammonia formation at a Ru(IV) nitrido complex, reported by Miller and coworkers. 
were investigated. Initially, $(\mathrm{LuH}) \mathrm{BAr}_{24}^{\mathrm{F}}$ was used for this reaction, which resulted in very nice cyclic voltammograms clearly differing from those of the pure complex. However, it was found that the $\mathrm{N}_{2}$ bridged dimer $\mathbf{3 6}$, which would be the ultimate target in an electrolysis setup, is not stable towards acids with such high acidity $\left(\mathrm{p} K_{\mathrm{a}}{ }^{\mathrm{THF}}=7.2\right)$ or even weaker acids like $\left(\mathrm{HNEt}_{3}\right) \mathrm{BAr}_{24}^{\mathrm{F}}\left(\mathrm{p} K_{\mathrm{a}}{ }^{\mathrm{THF}}=12.5\right){ }^{[217]}$ On the other hand, too weak acids like phenol $\left(\mathrm{p} K_{\mathrm{a}}{ }^{\mathrm{THF}}=29.2\right)^{[224]}$ did not lead to any changes in the CV data. Finally, benzoic acid $\left(\mathrm{p} K_{\mathrm{a}}{ }^{\mathrm{THF}}=25.1\right)^{[225]}$ was found to be the sweet spot, which was weak enough to not react with $\mathbf{3 6}$ but at the same time resulted in the same electrochemical response as the stronger acids. Titration of $0-15$ eq of benzoic acid to $\mathbf{4 0}$ resulted in a strong increase of the initial reduction current up to doubling the starting value. This is associated with the appearance of a second, quasi reversible reduction wave at approximately the same potential as the reduction feature of $\mathbf{2 9}$, indicating the formation of that complex. The formation of 29 can be understood as an ECEC mechanism. After initial reduction, protonation leads to formation of a new complex, presumable the Re(IV) amide complex 31. This has a reduction potential which is anodically shifted with respect to 40 (i.e. $-1.16 \mathrm{~V}$ vs. $\mathrm{Fc} / \mathrm{Fc}^{+}$, see Figure 3.9) and can therefore be reduced a second time at the same potential as the first reduction, turning that response into a two-electron wave. Subsequent second protonation then results in formation of $\mathbf{2 9}$ and the appearance of the corresponding redox wave.

Even though these results were very promising, after an actual CPE of $\mathbf{4 0}$ in the presence

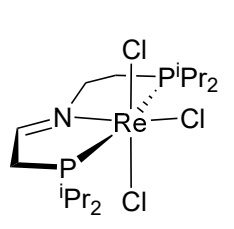

40

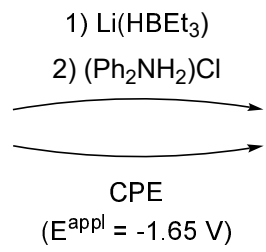

10 eq DCP

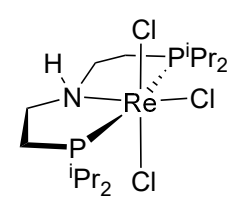

29

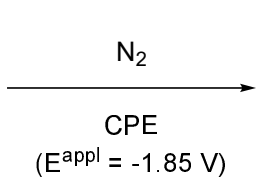

$\left(E^{\mathrm{appl}}=-1.85 \mathrm{~V}\right)$

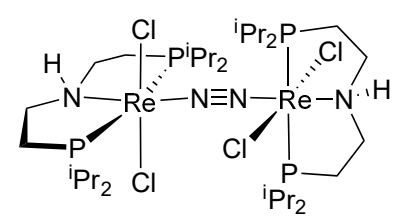

36
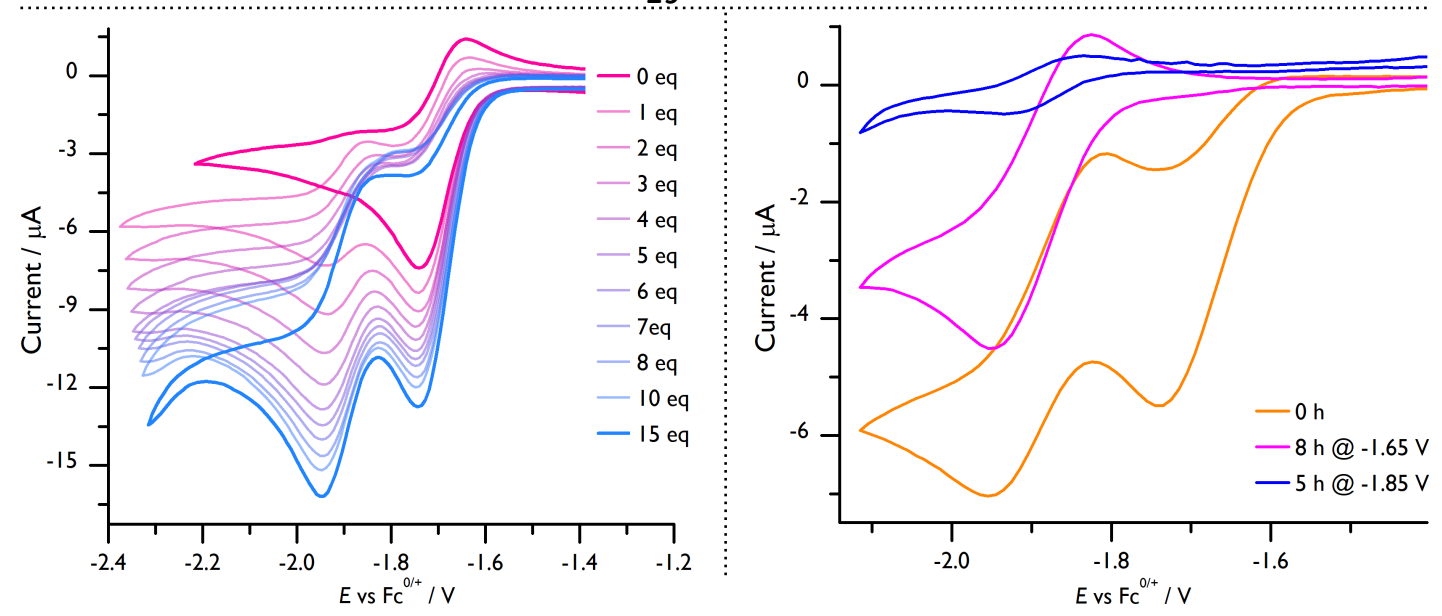

Fig. 3.29. Top: Regeneration of $\mathbf{2 9}$ from $\mathbf{4 0}$ via chemical reagents or electrolysis in presence of 2,6-dichlorophenol (DCP). Bottom left: CV measurements of 40 in presence of $0-15$ eq

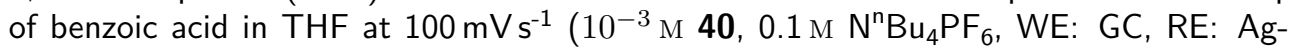
wire, $\mathrm{CE}$ : Pt-wire). Bottom right: $\mathrm{CV}$ of $\mathbf{4 0}$ under an $\mathrm{N}_{2}$ atmosphere in the presence of 10 eq DCP before electrolysis (orange), after $8 \mathrm{~h}$ controlled potential electrolysis (CPE) at $-1.65 \mathrm{~V}$ vs. $\mathrm{Fc} / \mathrm{Fc}^{+}$(pink) and after another $5 \mathrm{~h} \mathrm{CPE} \mathrm{at}-1.85 \mathrm{~V}$ (b/ue). 
of 10 eq of benzoic acid at the half-wave potential of the first reduction feature, $\mathbf{2 9}$ could not be identified spectroscopically. Instead, at least three other complexes with strongly shifted NMR signals are found, pointing to the formation of other $\operatorname{Re}(\mathrm{III})$ species. This was ascribed to the fact that the simultaneously formed benzoate anions can be very potent chelating ligands and might therefore interfere with the reaction. Exchanging benzoic acid with 2,6-dichlorophenol, which exhibits an almost identical $\mathrm{p} K_{\mathrm{a}}$ value $\left(\mathrm{p} K_{\mathrm{a}}{ }^{\mathrm{THF}}=25.1\right)^{[224]}$ but is a less potent ligand, results in identical CV measurements and if the CPE is performed with this acid, formation of target amine complex $\mathbf{2 9}$ can be observed in almost quantitative yields $(99 \%)$, determined by ${ }^{1} \mathrm{H}$ NMR integration vs. an internal standard. Thus, electrochemical regeneration of the PNP pincer ligand is not only feasible, but even outperforms the "chemical" pathway with $\mathrm{Li}\left(\mathrm{HBEt}_{3}\right)$ and $\left(\mathrm{Ph}_{2} \mathrm{NH}_{2}\right) \mathrm{Cl}$.

To further improve the so far obtained synthetic cycle, as last step the direct reduction of 40 all the way to the $\mu-N_{2}$ bridged dimer $\mathbf{3 6}$ was attempted, thus providing all reduction equivalents needed in this conversion from the electrochemical setup. Therefore, the electrolysis was repeated under a dinitrogen atmosphere in two sequential steps. First, the solution was electrolyzed at the half-wave potential of the first reduction wave to in situ generate 29 until in-between-measured control CVs showed no sign of the starting complex anymore. Afterwards, the electrolysis potential was changed to the half-wave potential of the newly formed reduction wave and the solution was again electrolyzed until that peak disappeared, leading to a characteristic, deep blue coloring of the solution, indicative of dimer formation. In these reactions, a total of $1.8 \mathrm{e}^{-}$in the first and $1.5 \mathrm{e}^{-}$in the second step are transferred per Re atom. The formation of dimer $\mathbf{3 6}$ was confirmed by NMR and quantified by UV/vis spectroscopy to $69.7 \%$, based on starting complex 40 . An attempt to directly photolyze the obtained solution with an $390 \mathrm{~nm}$ LED led to formation of nitride complex 37 as the only detectable product. However the yield dropped to $14 \%$. Similarly poor yields were also obtained when the entire electrolysis was performed under continuous irradiation. The fact that these yields are considerably lower than after photolysis of isolated $\mathbf{3 6}$ highlights the importance to better understand the photochemistry of this or comparable dimers.

\subsection{Summary}

The successful synthesis and characterization of a new rhenium trichloro complex supported by an iso-propyl based PNP pincer ligand (29) is reported. 29 exhibits unusually spectroscopic features and a large TIP from SOC. This is due to a rather complex electronic structure, dominated by a quasi-degenerate triplet ground state and resulting spin-orbit coupling effects, as rationalized by QDPT/NEVPT2/CASSCF $(14,10)$ calculations.

Starting from this new platform, a route to $\left[\mathrm{ReCl}_{2}\left(\mathrm{PNP}^{i \mathrm{Pr}}\right)\right] \mathbf{3 0}$ is developed and the ability of that complex towards $\mathrm{N}_{2}$ activation in analogy to the previously reported tert-butyl counterpart is investigated. Due to the reduced ligand sterics, $\mathbf{3 0}$ shows a high tendency to bind a 
sixth ligand, as exemplified by the reversible low temperature dimer formation with $\mathrm{N}_{2}$, without the need for any additional reagents. Unfortunately, $\mathrm{N}_{2}$ splitting was not achieved with this complex, despite several attempts. Instead, the $\operatorname{Re}(\mathrm{I})$ complex $\left[\operatorname{ReCl}\left(\mathrm{N}_{2}\right)_{2}\left(\mathrm{HPNP}^{i \operatorname{Pr}}\right)\right]$ could be identified as one of several reduction products, indicating overreduction to be a problem.

In contrast to this, direct reduction of the amine complex 29 under $\mathrm{N}_{2}$ yields a thermally stable, $\mu-\mathrm{N}_{2}$ bridged formal $\operatorname{Re}(\mathrm{II})$ dimer $\left.\left(\mu-\mathrm{N}_{2}\right)\left\{\operatorname{ReCl}_{2}\left(\mathrm{HPNP}^{\mathrm{IPr}}\right)\right\}_{2}\right]$, which exhibits a remarkable thermal stability, but does undergo clean photochemical splitting into the corresponding nitride complexes. The nitride complex $\mathbf{3 7}$ is then shown exhibit nitride centered, nucleophilic reactivity, resulting in borylation as well as protonation. Importantly, reaction with benzoyl chloride is shown to yield the imine complex $\left[\mathrm{ReCl}_{3}\left(\mathrm{P}=\mathrm{NP}{ }^{i \mathrm{Pr}}\right)\right]$ as well as benzamide. In this rare metal-ligand cooperative nitride functionalization, the pincer ligand serves as a $2 \mathrm{e}^{-} / 2 \mathrm{H}^{+}$donor. By controlled potential electrolysis in the presence of the weak acid 2,6-dicholorphenol, full regeneration of the ligand backbone could be achieved and eventually also dimer $\mathbf{3 6}$ could be obtained, closing the synthetic cycle without the need for chemical reductants. 



\section{Conclusion and outlook}

This thesis was devoted to further develop the available understanding of two main topics:

\section{A) Low-valent late transition metal nitrides and their reactivity \\ B) Dinitrogen activation and cleavage by transition metal complexes and their functionalization}

The targeted synthesis of $\left[\mathrm{Os}(\mathrm{N})\left(\mathrm{PNP}^{t \mathrm{Bu}}\right)\right](3)$ provides access to the second ever reported Os(IV) nitrido complex and thus to the first reactivity study on such a species. ${ }^{[174]}$ In line with the higher $d$ electron count in comparison with well established Os(VI) nitrides, 3 exhibits mainly nucleophilic reactivity. It was observed that small electrophiles, which are largely unaffected by the steric bulk of the pincer ligand, do react directly at the metal center, identifying the Os atom to be the most nucleophilic side, in accordance with the $d_{z^{2}}$ orbital being the HOMO. This is especially highlighted by the protonation of $\mathbf{3}$ to give fully characterized $\left[\mathrm{Os}(\mathrm{N})(\mathrm{H})\left(\mathrm{PNP}^{t \mathrm{Bu}}\right)\right]^{+}\left(2^{+}\right)$, as well as reactions with Mel, were NMR data suggest an osmium methylated complex to be one of several formed products. Using electrophiles with increased steric bulk like $\mathrm{Me}_{3} \mathrm{Si}^{+}$results in clean and selective formation of $\left[\mathrm{Os}\left(\mathrm{NSiMe}_{3}\right)\left(\mathrm{PNP}^{t \mathrm{Bu}}\right)\right]^{+}\left(\mathbf{6}^{+}\right)$.

In agreement with this mainly nucleophilic character of the nitride, no reactions were observed towards olefins, alkynes or Grignard reagents. However, slow reaction with $\mathrm{PMe}_{3}$ was observed, leading to an equilibrium mixture between the free reactants and the phosphoraneiminato complex $\left[\mathrm{Os}\left(\mathrm{N}=\mathrm{PMe}_{3}\right)\left(\mathrm{PNP}^{t \mathrm{Bu}}\right)\right](7)$. It was previously shown by Smith and coworkers that phosphines can be ambiphilic, with the possibility to act as $\sigma$-acceptor into their $\mathrm{P}_{-}^{\sigma^{*}} \mathrm{C}$ orbital. ${ }^{[159]}$ The same effect was identified for the reaction of $\mathbf{3}$ with $\mathrm{PMe}_{3}$ in a computational analysis, revealing two mutual donor-acceptor interactions between the nitride and the phosphine coming into play in the transition state. Population analyses of all involved species indicated a net electron transfer from the phosphine to the nitride in the transition state, justifying the formulation of $\mathbf{3}$ to be an ambiphile.

When 3 was heated in toluene under a hydrogen atmosphere, hydrogenolysis of the nitride moiety to form ammonia and the polyhydride Os(IV) complex $\left[\mathrm{Os}(\mathrm{H})_{4}\left(\mathrm{HPNP}^{t \mathrm{Bu}}\right)\right](8)$ occured. Such direct reactions with $\mathrm{H}_{2}$ are extremely rare (the selective formation of ammonia even more so) and emphasize the high reactivity of late transition metal nitrides.

Additionally, the $\mathrm{Os}(\mathrm{VI})$ nitride complex $\left.\left[\mathrm{Os}(\mathrm{N}) \mathrm{Cl}\left(\mathrm{PNP}^{t \mathrm{Bu}}\right)\right]^{+} \mathbf{1 0}^{+}\right)$was synthesized. This complex showed an interesting increase of nitride reactivity upon coordination of a strongly 


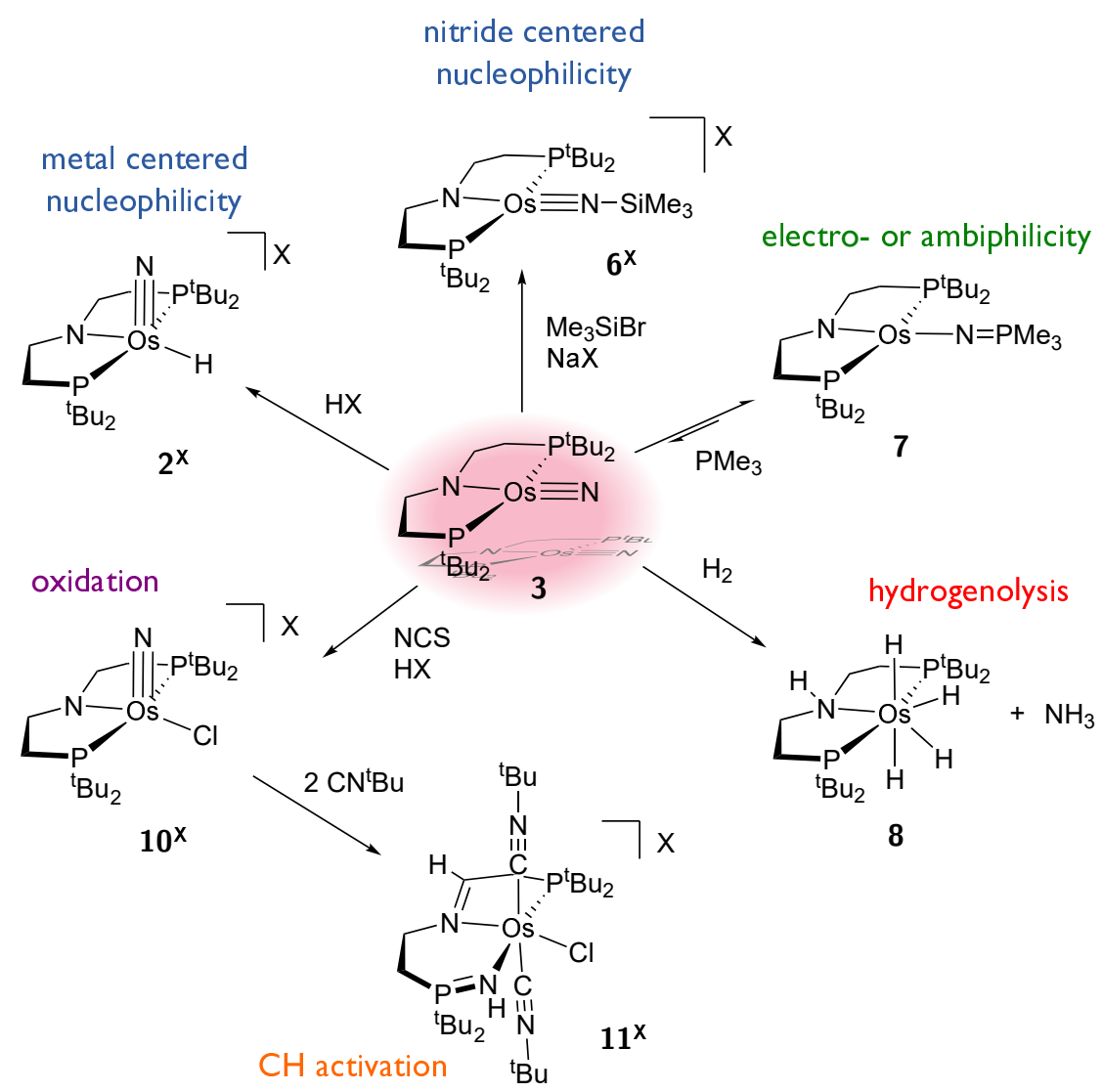

Scheme 4.1. Reactivity scheme of square planar Os(IV) nitrido complex 3.

$\sigma$-donating isonitrile ligand, leading to backbone $\mathrm{CH}$ activation and insertion of the formed imide into an $\mathrm{Os}-\mathrm{P}$ bond, yielding $\mathbf{1 1}^{\mathbf{X}}$.

Even though every effort has been made to generate one of the nitrides $3,2^{+}$or $\mathbf{1 0}^{+}$directly from dinitrogen, unfortunately no hint for successful splitting could be observed. Most reactions resulted in intractable mixtures of several compounds, with the selective formation of $\mathrm{Os}(\mathrm{II})$ complex $\left[\mathrm{OsH}\left(\mathrm{N}_{2}\right)\left(\mathrm{PNP}^{t \mathrm{Bu}}\right)\right]$ (13) under reductive conditions being among the only exceptions. In many reactions, especially those including bases for initial dehydrohalogenation, species were observed which most likely resulted from intramolecular $\mathrm{CH}$ activation, according to the NMR data. Interestingly, Josh Abbenseth prepared complementary osmium complexes with the fully oxidized ligand backbone which is inert towards $\beta$-hydride elimination from the backbone, i.e. $\left\{\mathrm{Os}\left(\mathrm{P}=\mathrm{N}=\mathrm{P}^{t \mathrm{Bu}}\right)\right\} \cdot{ }^{[195,226]}$ However, neither Os(II) complex $\left[\mathrm{OsCl}\left(\mathrm{P}=\mathrm{N}=\mathrm{P}^{t \mathrm{Bu}}\right)\right]$ nor $\mathrm{Os}(\mathrm{III})$ complex $\left[\mathrm{OsCl}_{2}\left(\mathrm{P}=\mathrm{N}=\mathrm{P}^{t \mathrm{Bu}}\right)\right]$ are reported to give any reactions with $\mathrm{N}_{2}$, which might be attributed to the reduced $\pi$-donor capabilities of the vinyl amide.

Since computational results indicate that $\mathrm{N}_{2}$ splitting should in principal be feasible on the osmium platform, these results strongly point towards the need to modify the ligand in order to meet the requirements and properties of the system. One possibility to obtain a complex 
which is unable to undergo $\beta$-hydride elimination and also features a strong amide donor would be to use a pincer ligand with $\mathrm{CMe}_{2}$ instead of $\mathrm{CH}_{2}$ groups next to the amide.

Synthesis of such a ligand can be envisioned by simply chlorination of literature known ${ }^{[227]}$ $\mathrm{HN}\left(\mathrm{CMe}_{2} \mathrm{CH}_{2} \mathrm{OH}\right)_{2}$ with thionyl chloride to form $\left(\mathrm{H}_{2} \mathrm{~N}\left(\mathrm{CMe}_{2} \mathrm{CH}_{2} \mathrm{Cl}\right)_{2}\right) \mathrm{Cl}$ and subsequent reaction with $\mathrm{LiP}^{\mathrm{t}} \mathrm{Bu}_{2}$ analog to the in here used procedures, with or without previous amine protection (see Scheme 4.2).

Another possibility to increase the reactivity towards $N_{2}$ would be to reduce the steric bulk of the ligand. The results obtained for the rhenium platform in Chapter 3 clearly show that the iso-propyl substituted pincer leads to significantly reduced shielding of the metal center and thus to an increased tendency to bind additional ligands. Investigations in connection with this proposal are already in progress, conducted by Lukas Alig. Also combinations of both approaches, i.e. smaller ligand substituents on pincer ligands which are inert towards $\mathrm{CH}$ activation (either the vinyl amido ligand or the $\mathrm{CMe}_{2}$ substituted analog) should lead to significantly different, probably enhanced dinitrogen chemistry.

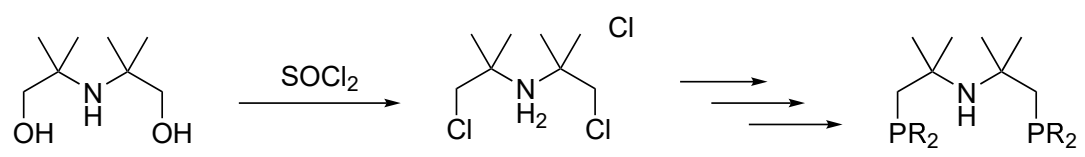

Scheme 4.2. Proposal for the synthesis of a new ligand designed to meet the problems which emerged during attempts to activate $\mathrm{N}_{2}$ on the osmium platform.

The previously reported dinitrogen activation, cleavage and incorporation of the resulting nitrides into organic nitriles by rhenium complex $\mathbf{X I X}$ marked the first extension of dinitrogen cleavage chemistry to a group 7 element and raised a number of interesting questions, of which some were addressed in this thesis.

Characterization of the previously postulated complex $\left[\left(\mu-\mathrm{N}_{2}\right)\left\{\operatorname{ReCl}\left(\mathrm{PNP}^{t \mathrm{Bu}}\right)\right\}\right](\mathbf{X X})$ was achieved by means of NMR data and X-ray diffraction. Monitoring the decay of the temperature labile complex by NMR spectroscopy revealed clean formation of the nitrido complex $\left[\operatorname{Re}(\mathrm{N}) \mathrm{Cl}\left(\mathrm{PNP}^{t \mathrm{Bu}}\right)\right](\mathbf{X X I})$. This way, $\mathbf{X X}$ was proven to be an actual intermediate in the reaction pathway to dinitrogen splitting. Repeating the experiments at various temperatures allowed for a first estimate of kinetic parameters and the activation barrier, which are in good agreement with the DFT calculations. ${ }^{[94]}$ This analysis proved the proposed dinitrogen cleavage mechanism to occur via an end-on bridged $\mathrm{N}_{2}$ dimer and once more underpinned the importance of a $10 \pi(4 \delta)$ electron count in the $\{$ MNNM $\}$ core for this reactivity, fully in line with the considerations made in Part I Section 3.2.1.

Additionally, the rhenium platform was varied in several ways, to evaluate the potential of the system. Following a reaction analog to the reported acetonitrile release (see Part I Section 4.2.1) by methylation, deprotonation and oxidation of the ketimido complex $\left[\mathrm{Re}\left(\mathrm{NCH}_{2}\right) \mathrm{Cl}\left(\mathrm{PNP}^{t \mathrm{Bu}}\right)\right]$ (18) resulted in $\mathrm{C}-\mathrm{C}$ coupling. This reaction was achieved in a clean manner and the complex $\left[\left(N, N-C_{2} \mathrm{H}_{4} \mathrm{~N}_{2}\right)\left\{\mathrm{ReCl}\left(\mathrm{PNP}^{t \mathrm{tBu}}\right)\right\}_{2}\right]^{(\mathrm{OTf})_{2}}\left(\mathbf{1 9}^{(\mathrm{OTf})_{2}}\right)$ was fully characterized. While follow-up chemistry of this complex was not developed, it offers the possibility to incorporate two nitrogen atoms into the same molecule and thus could broaden 

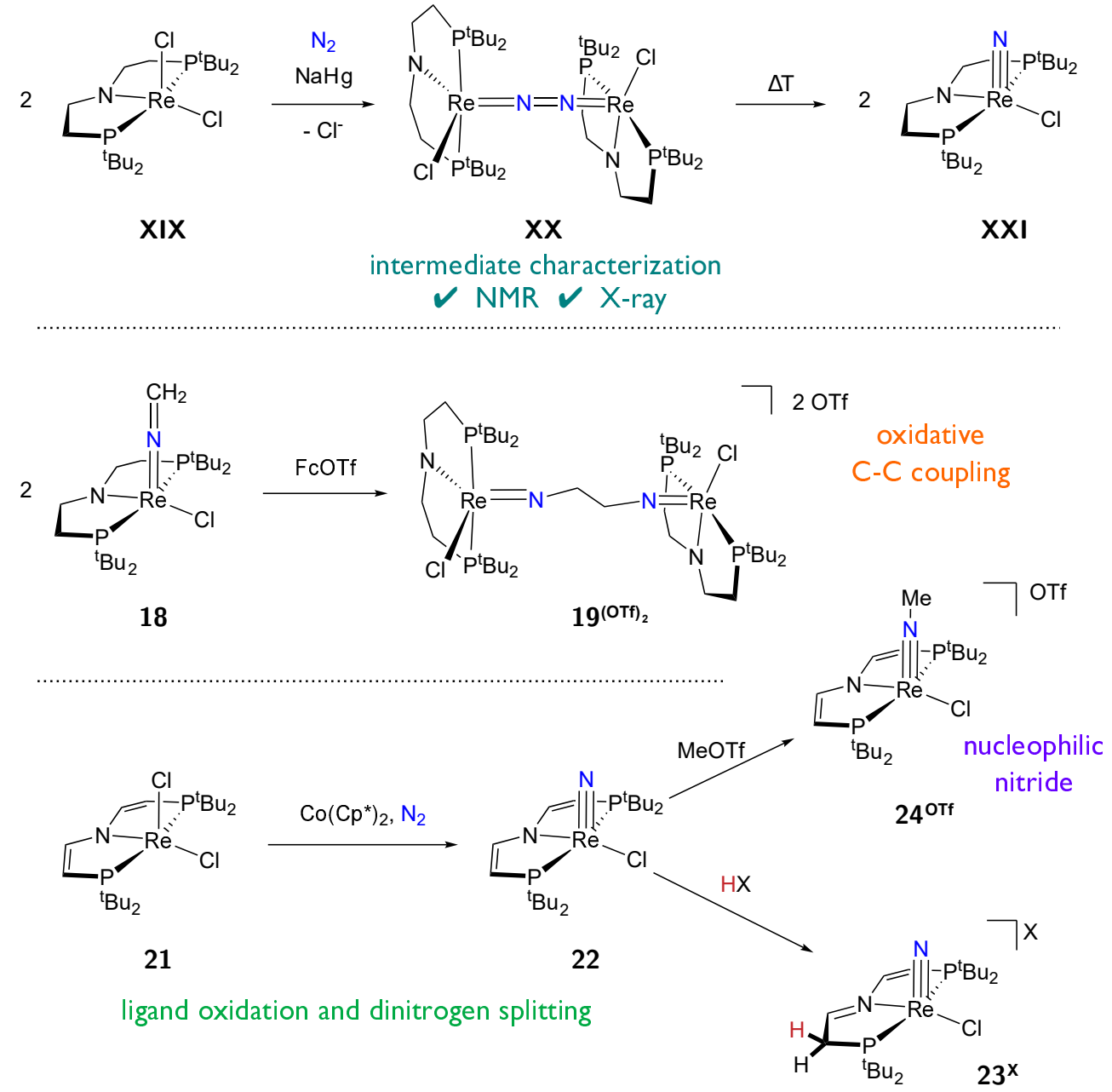

Scheme 4.3. Expansion of the understanding of rhenium mediated dinitrogen activaiton chemistry with tert-butyl substituted PNP pincer ligands.

the spectrum of accessible compounds. Therefore, further research on how to cleave off the obtained diimide would be of significant interest.

Overall, the development of a catalytic system capable of forming nitrogen containing organic molecules from $\mathrm{N}_{2}$ is a long-standing challenge which is so far unmet. With regard to the oxidative conditions needed for nitrile release from $\mathbf{X X I}$, a certain interest in improving the systems stability towards these conditions arose. Hence, the ligand backbone was oxidized to the vinyl amido pincer $\mathrm{P}=\mathrm{N}=\mathrm{P}$, which was previously found to have beneficial influence on stabilizing oxidized species. Preparation of $\left[\operatorname{ReCl}_{2}\left(\mathrm{P}=\mathrm{N}=\mathrm{P}^{t \mathrm{Bu}}\right)\right](\mathbf{2 1})$ by oxidation of XIX with TTBP was achieved in good yields. Investigation of the redox properties of this compound in comparison with parent $\mathbf{X I X}$ indicated a significant anodic shift of the first oxidation potential, but at the same time a reduced stability of the resulting species, thus only partially meeting the targeted properties. Nevertheless, the dinitrogen activation and functionalization capabilities of this platform were evaluated and cleavage of dinitrogen under reductive conditions was achieved in moderate yields $(\approx 60 \%)$. Followup investigation of the functionalization chemistry indicated the nitride $\left[\operatorname{Re}(N) \mathrm{Cl}\left(\mathrm{P}=\mathrm{N}=\mathrm{P}^{t \mathrm{Bu}}\right)\right]$ to exhibit a reactivity highly comparable to that of the alkyl amido congener $\mathbf{X X I}$. No reduction was 
achieved up to very low potentials and also protonation (which is centered at the pincer ligand) did not lead to a significant shift in the redox potentials. Nitride centered methylation with methyl triflate to $\left[\operatorname{Re}(\mathrm{NMe}) \mathrm{Cl}\left(\mathrm{P}=\mathrm{N}=\mathrm{P}^{t \mathrm{Bu}}\right)\right]^{\mathrm{OTf}}\left(\mathbf{2 4 ^ { \mathrm { OTf } }}\right)$ as well as subsequent deprotonation to the ketimidio complex $\left[\operatorname{Re}\left(\mathrm{NCH}_{2}\right) \mathrm{Cl}\left(\mathrm{P}=\mathrm{N}=\mathrm{P}^{t \mathrm{Bu}}\right)\right](25)$ occured fully analog to the parent system. Consequently, the new platform with the oxidized ligand performs comparably well in dinitrogen functionalization chemistry but does not lead to a significant improvement in terms of reactivity. This could become of importance in the future, given the possibility that functionalization schemes and potential catalytic cycles might be developed where backbone $\mathrm{CH}$ activation could become an actual issue.

In order to increase the reactivity of dinitrogen obtained nitrides, the steric shielding by the ligand framework is a highly important factor which needs to be controlled. This has already been demonstrated by Cummins and coworkers on their molybendum trisamide complexes, were significantly different reactivities were observed upon varying the sterics of the amide residues (see Part I Section 3.2.1). In general, tuning the steric bulk of the supporting ligands can be regarded as a tightrope walk between protection of the metal center from unwanted side-reactions and exposing the dinitrogen derived nitride towards reactants.

In this context, the synthesis of a complex analog to $\left[\operatorname{ReCl}_{2}\left(\mathrm{PNP}^{t \mathrm{Bu}}\right)\right](\mathbf{X I X})$ but with the iso-propyl substituted ligand was attempted. The amine complex $\left[\mathrm{ReCl}_{3}\left(\mathrm{HPNP}^{\mathrm{iPr}}\right)\right]$ (29) was prepared in good yields (70\%). Strongly shifted but sharp NMR signals with well resolved coupling constants indicated a non-trivial electronic structure, further substantiated by SQUID measurements, which revealed a large TIP. CASSCF calculations indeed showed 29 to feature a quasi-degenerated triplet ground state and multireference calculations by means of QDPT resulted in extensive mixing of the states by SOC.

29 provided a good starting platform for subsequent reactivity studies. Overall, the reduced steric shielding of the metal center resulted in a very high affinity towards even weak ligands. This became apparent during the attempts to dehydrohalogenate 29 in order to obtain $\left[\mathrm{ReCl}_{2}\left(\mathrm{PNP}^{\mathrm{iPr}}\right)\right](\mathbf{3 0})$. No isolable sample of $\mathbf{3 0}$ could be obtained with bases, and also the spectroscopically clean samples obtained from $\mathrm{H}$ atom abstraction from $\mathbf{2 9}$ to form $\left[\mathrm{ReCl}_{3}\left(\mathrm{PNP}^{\mathrm{iPr}}\right)\right](\mathbf{3 1})$ and subsequent reduction to $\mathbf{3 0}$ degraded upon solvent removal. $\mathbf{3 0}$ does form adducts with THF as well as with $\mathrm{N}_{2}$, highlighting the high tendency of the system to bind a sixth ligand. Especially the latter reaction was investigated in detail and 2D-NMR data strongly indicate reversible formation of an equilibrium mixture of two $\mathrm{N}_{2}$ containing dimers at low temperatures. A symmetric dimer $\left[\left(\mu-N_{2}\right)\left\{\operatorname{ReCl}_{2, \text { cis }}\left(P N P^{i \operatorname{Pr}}\right)\right\}_{2}\right]$ (33b) and a asymmetric complex $\left[\left\{\operatorname{ReCl}_{2, \text { cis }}\left(\mathrm{PNP}^{i \mathrm{Pr}}\right)\right\}\left(\mu-\mathrm{N}_{2}\right)\left\{\mathrm{ReCl}_{2, \text { trans }}\left(\mathrm{PNP}^{i \mathrm{Pr}}\right)\right\}\right]$ (33a) were identified as the most likely products of this reaction. While the formation of these dimers was a promissing starting point, all attempts for subsequent cleavage of the dinitrogen moieties by reduction failed and no sign for the expected nitride was observed. From the mostly intractable reaction mixtures, the $\operatorname{Re}(\mathrm{I})$ complex $\left[\mathrm{ReCl}\left(\mathrm{N}_{2}\right)_{2}\left(\mathrm{HPNP}^{i \mathrm{Pr}}\right)\right]$ (35) could be identified which pointed to overreduction as one unwanted side reaction in this system. Therefore, direct reduction of amine complex $\mathbf{2 9}$ was performed under $\mathrm{N}_{2}$, as here the 


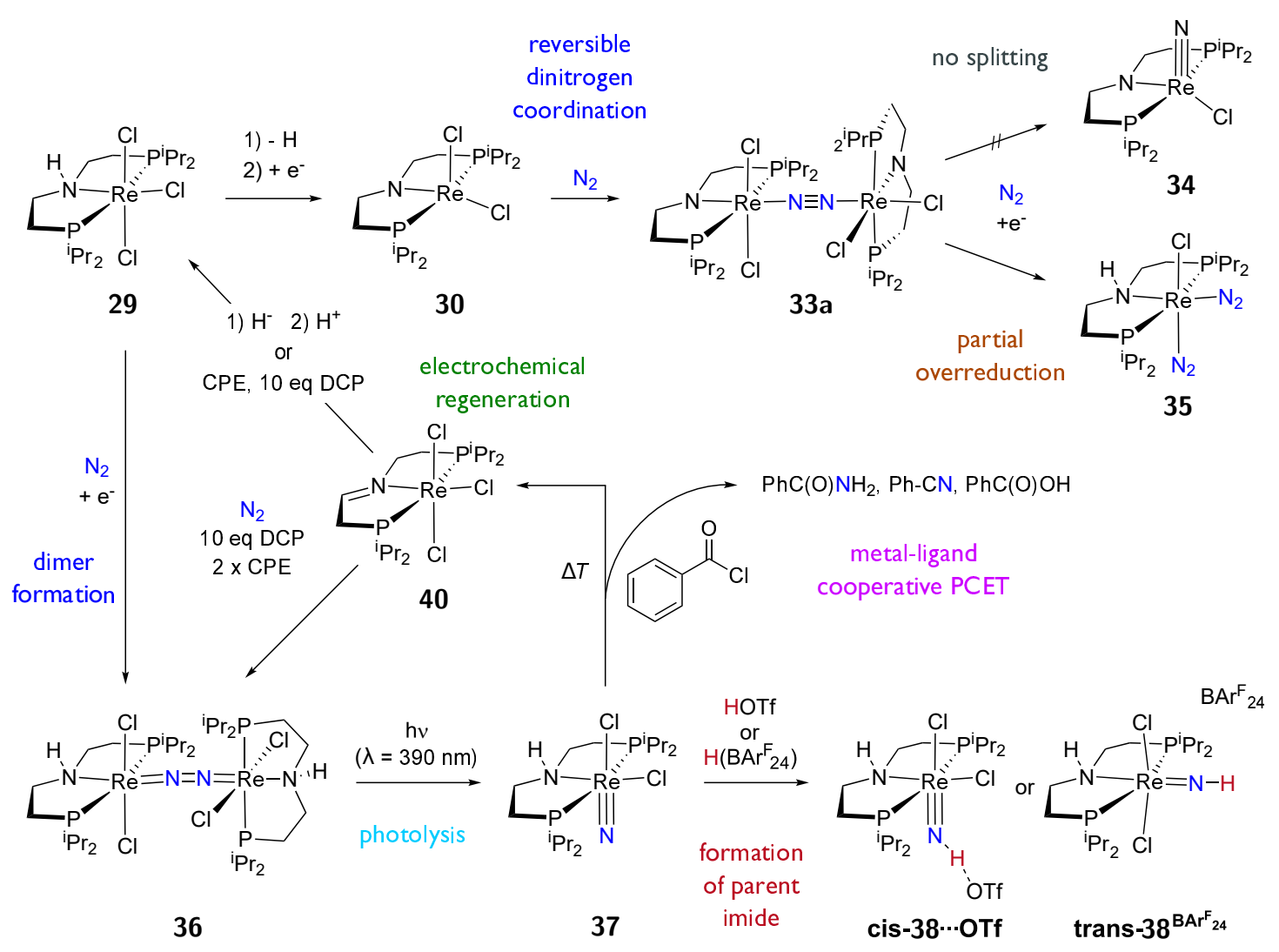

Scheme 4.4. Reaction scheme of a rhenium complex supported by a iso-propyl substituted PNP pincer ligand and it's dinitrogen chemistry developed within this thesis.

following chloride loss allows for binding of only one $\mathrm{N}_{2}$ ligand and thus potential inversion should be less of an issue. This reduction led to isolation and characterization of the temperature stable $\mu-\mathrm{N}_{2}$ bridged $\mathrm{N}_{2}$ dimer $\left[\left(\mu-\mathrm{N}_{2}\right)\left\{\operatorname{ReCl}_{2}\left(\mathrm{HPNP}^{i \operatorname{Pr}}\right)\right\}_{2}\right]$ (36). It's thermal stability is in stark contrast to the tert-butyl substitued dimer $\mathbf{X X}$ and can be explained by the octahedral coordination and thus the presence of a strong donor ligand trans to the $\mathrm{N}_{2}$ bridge. This ligand raises the $\mathrm{a}_{\mathrm{u}}$ symmetric $\mathrm{MO} \mathrm{M}^{-}-\mathrm{N}^{\sigma}-\mathrm{\sigma}^{*}-\stackrel{\sigma}{-} \mathrm{M}$ signficantly in energy, making it less available for mixing with the complex's HOMOs and thus increases the activation barrier. Population of the antibonding MO space was therefore sought by photoinduced electronic excitation and indeed, irradiation with a violet LED allowed for selective $\mathrm{N}_{2}$ cleavage into the corresponding nitrides $\left[\operatorname{Re}(\mathrm{N}) \mathrm{Cl}_{2}\left(\mathrm{HPNP}^{i \mathrm{Pr}}\right)\right]$ (37). TDDFT calculations point towards an MLCT from the metal centered, non-bonding $\delta$ orbitals into the $\operatorname{Re}^{\pi^{*}}-\mathrm{N}^{\pi^{*}}-\mathrm{N}^{\pi^{*}}$ Re MOs to be of importance for this reaction. There are only two system (by Cummins and Floriani) which do undergo photolytic $\mathrm{N}_{2}$ scission and do not feature filled $\delta$ symmetric orbitals. Given the similarities between both systems and the fact, that Cummins reported the splitting in his complex to occur from a vibrationally hot ground state rather than from an excited state, the question stands to reason if such populated $\delta$ orbitals are a general requirement for the observed reactivity. This particular question and as well as the entire excited state dynamics are still to be explored and should be addressed in future projects. 
The nitride complex 37 obtained from photochemical $N_{2}$ cleavage features a number of important properties. On the one hand, the smaller pincer ligand and the resulting affinity to form coordinatively saturated complexes leads to the trans chloride ligand being coordinated, which is in contrast to $\left[\operatorname{Re}(\mathrm{N}) \mathrm{Cl}\left(\mathrm{HPNP}^{t \mathrm{Bu}}\right)\right]^{\mathrm{Cl}}$, where the chloride was found to be non-coordinating. This renders the nitride the only nucleophilic side in the complex, allowing for $\mathrm{N}$ centered protonation to form a rare parent imido complex $\left[\mathrm{Re}(\mathrm{NH}) \mathrm{Cl}_{2}\left(\mathrm{HPNP}^{i \mathrm{Pr}}\right)\right]^{+}$ $\left(\mathbf{3 8}^{+}\right)$. Starting from an octahedral and thus uncharged complex was found to be essential for this reaction. Interestingly, DFT calculations indicated a strong influence of the anion on the preferred geometry and indeed, using HOTf and $\mathrm{H}\left(\mathrm{Et}_{2} \mathrm{O}\right)_{2}\left(\mathrm{BAr}_{24}{ }^{\mathrm{F}}\right)$ resulted in formation

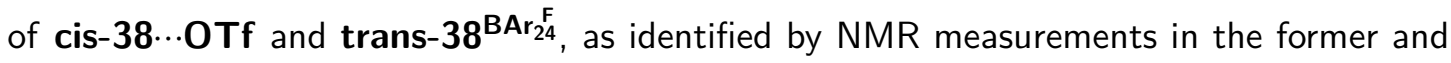
$\mathrm{X}$-ray diffraction in both cases. The formation of these imido complexes might provide an entry to ammonia formation reactions and closer investigation of followup reactions, also on the influence of the cis- vs. the trans- isomer are advisable.

Despite the protonation, reaction with benzoyl chloride resulted in selective formation of $\operatorname{Re}(\mathrm{III})$ imine complex $\left[\mathrm{ReCl}_{3}\left(\mathrm{P}=\mathrm{NP}^{i \mathrm{Pr}}\right)\right](40)$ accompanied by the formation of a mixture of benzamide, benzonitrile and benzoic acid. In this metal-ligand cooperative nitride functionalization, the amine pincer ligand acts as a $2 \mathrm{H}^{+} / 2 \mathrm{e}^{-}$reservoir for PCET reactivity. Regeneration of the original amine complex $\mathbf{2 9}$ was achieved either by stepwise addition of a hydride and a proton source in approximately $62 \%$ yield or almost quantitatively by CPE in the presence of weakly acidic 2,6-dichlorophenol. Furthermore, continued electrolysis under $\mathrm{N}_{2}$ without intermediate workup led to direct reformation of dimer 36 in $70 \%$ yield.

While this leads to a quite efficient three-step synthetic cycle for nitrogen incorporation into organic nitriles, still no catalytic turnover could be achieved. This is mainly due to two aspects: On the one hand, nitride complex 37 still requires harsh reaction conditions to be functionalized (i.e. $100^{\circ} \mathrm{C}$ overnight), which is not compatible with the other reaction conditions, on the other hand, photolysis of dimer $\mathbf{3 6}$ is only selective in pure solvent, but drops dramatically in yield when other substrates (electrolyte, benzoyl chloride, DCP) are present. The latter issue requires extensive studies of the excited state dynamics of the system to better understand the relevant processes while the former might be met by further increasing the nitrides reactivity, for example by introduction of stronger trans ligands, which was previously found to be beneficial (compare Part I Section 4.2.2 and Part II Section 1.3). A potentially promissing modification would be the introduction of an additional residue in the pincer ligand to form $\mathrm{N}(\mathrm{R})\left(\mathrm{CH}_{2} \mathrm{CH}_{2} \mathrm{P}^{\mathrm{i}} \mathrm{Pr}_{2}\right)_{2}$ where $\mathrm{R}$ can hold a number of anionic donor atoms (see Figure 4.1). The possible benifits of such a ligand are examplified by DFT calculations ${ }^{1}$ on the model complex $\left[\left(\mu-\mathrm{N}_{2}\right)\left\{\operatorname{ReCl}\left({ }^{\mathrm{Me}} \mathrm{NPNP}^{i \mathrm{Pr}}\right)\right\}_{2}\right]\left({ }^{\mathrm{Me} N P N P^{i P r}}=\right.$ $\left.\left(\mathrm{MeN}^{-} \mathrm{CH}_{2} \mathrm{CH}_{2}\right) \mathrm{N}\left(\mathrm{CH}_{2} \mathrm{CH}_{2} \mathrm{PiPr}_{2}\right)_{2}\right)$ :

- The strongly $\pi$-donating amide donor would raise the $\delta$ symmetric orbitals, which are predicted to be the donor orbitals in the photochemical process, significantly in

\footnotetext{
${ }^{1}$ M06/def2TZVP/SMD(THF)||PBE/D3BJ/RI/def2SVP, see Part III Section 3.8.
} 
energy, eventually becoming the HOMOs and thus leading to an overall $12 \pi 2 \delta$ electron count. The influence on thermal and photochemical splitting behavior could provide important insight into molecular dinitrogen activation.

- If the presumably high kinetic barrier for splitting $\left(\Delta G^{\ddagger} \mathrm{DFT}=+240 \mathrm{~kJ} \mathrm{~mol}^{-1}\right)$ can be overcome by photoexcitation, the tetradentate nature of the ligand would force an obtained nitride $\left(\Delta G^{0} \mathrm{DFT}=-68 \mathrm{~kJ} \mathrm{~mol}^{-1}\right)$ to be coordinated trans to the amine (comparable to $37^{\text {trans }}$, see Figure 3.20), which should greatly enhance the nitride centered reactivity, as expressed in the model nitride methylation with MeOTf $\left(\Delta G^{0}\right.$ DFT $=$ $\left.-99 \mathrm{~kJ} \mathrm{~mol}^{-1}, 37: \Delta G^{0}=-74 \mathrm{~kJ} \mathrm{~mol}^{-1}\right)$.

Further variation of the new ligand arm could then allow to fine tune the systems properties to optimize the behavior (e.g. by using $\mathrm{N}$-hetrocyclic donors like (substituted) pyrroles).

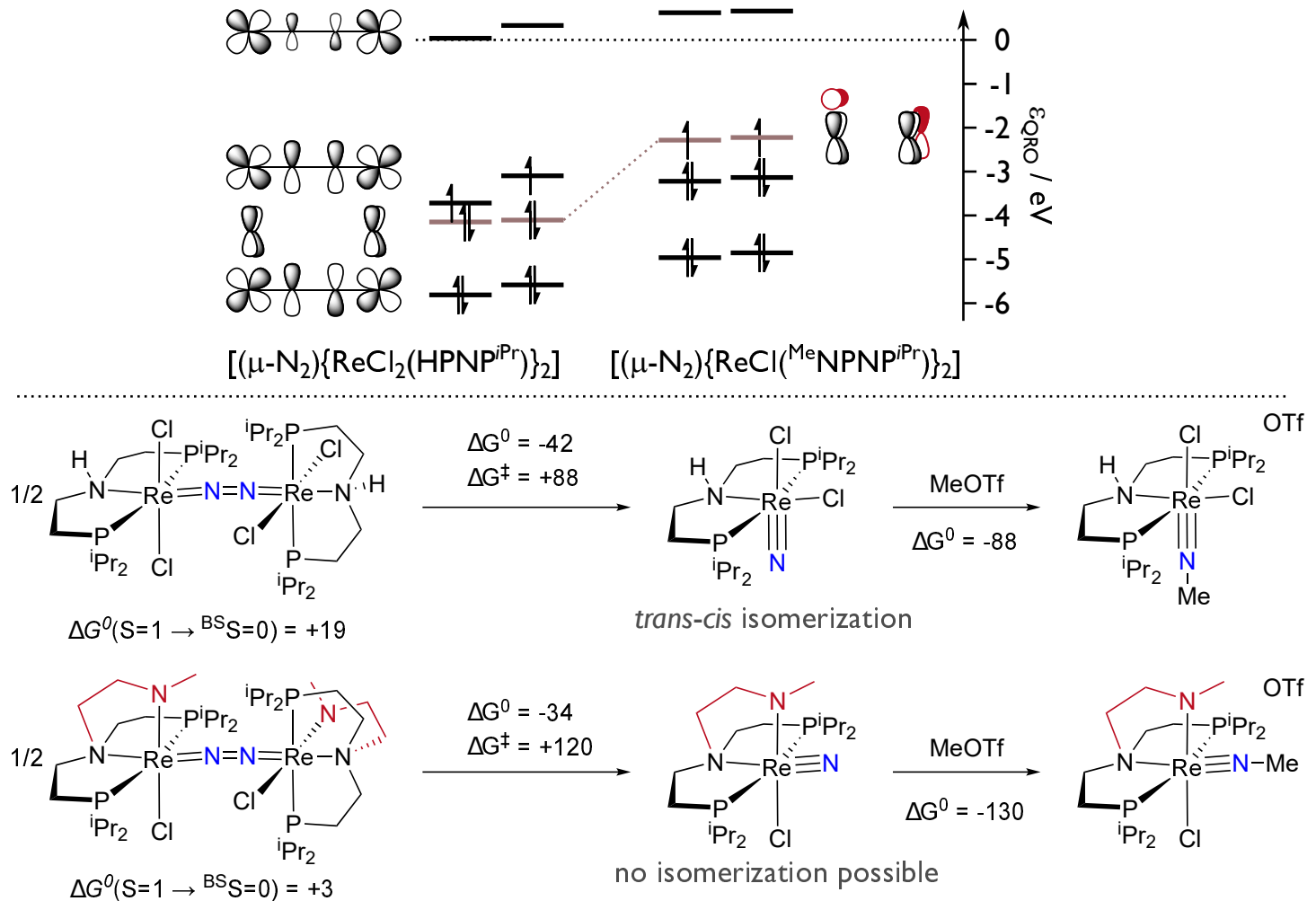

Fig. 4.1. Influence of an additional amide donor arm at the PNP pincer on the $\mathrm{N}_{2}$ activation of a rhenium system closely related to 36 . Top: quasi-restricted orbital (QRO) scheme of 36 (left) and $\left[\left(\mu-\mathrm{N}_{2}\right)\left\{\mathrm{ReCl}\left({ }^{\mathrm{Me}} \mathrm{NPNP}^{\mathrm{iPr}}\right)\right\}_{2}\right]$ (right), demonstrating the influence of an additional amide donor on the $\delta$ symmetric MOs. Bottom: DFT derived data (in $\mathrm{kJ} \mathrm{mol}^{-1}$ ) for $\mathrm{N}_{2}$ cleavage and functionalization in both systems. 


\section{Part III}

Experimental details

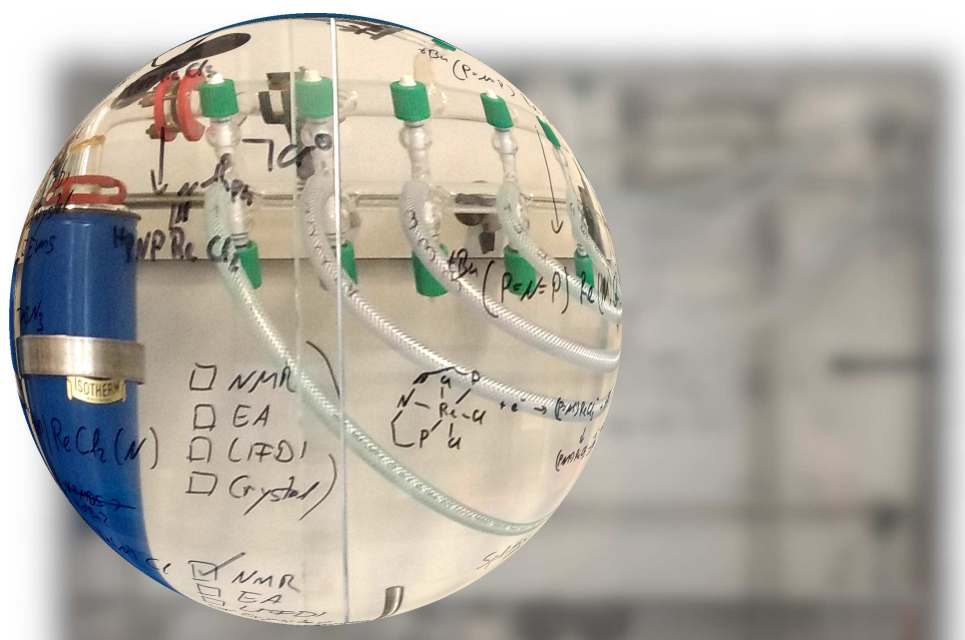





\section{Materials and Methods}

We walk this earth

With fire in our hands

- Arch Enemy

Doomsday Machine - Nemesis

\subsection{General synthesis and materials}

All synthetic procedures were performed under an inert atmosphere of $\mathrm{Ar}$ or $\mathrm{N}_{2}$ (both 5.0, LiNDE GAS) using standard Schlenk and glovebox techniques, unless otherwise noted. Purification of $\mathrm{H}_{2}$ gas (6.0, LINDE GAS) was obtained by passing the gas through a high capacity moisture filter (PURE GAS PRODUCTS) followed by a steel coil, cooled with liquid $\mathrm{N}_{2}$ to freeze out potential impurities.

All glasware was cleaned by $\mathrm{KOH} /$ iso-propanol baths, washed with deionized water and dried at $120^{\circ} \mathrm{C}$. Before usage, all vessels were evacuated at a Schlenk line with periodically applied heat. Small scale reactions $(\leq 10 \mathrm{mg})$ were typically performed in J-YOUNG NMR tubes.

All solvents were purchased in HPLC quality (MERCK) and used as obtained from an MBraun Solvent Purification System. THF and toluene were further purified by stirring over $\mathrm{Na} / \mathrm{K}$ alloy for serveral days. Deuterated solvents were obtained from EURISOTOP GmbH and dried by stirring over $\mathrm{Na} / \mathrm{K}\left(\mathrm{C}_{6} \mathrm{D}_{6}\right.$, toluene- $d_{8}$, THF- $\left.d_{8}\right)$ or $\mathrm{CaH}_{2}\left(\mathrm{CD}_{2} \mathrm{Cl}_{2}\right.$, $\left.\mathrm{CD}_{3} \mathrm{CN}\right) . \mathrm{D}_{2} \mathrm{O}$ was degassed. $\mathrm{CN}^{\mathrm{t}} \mathrm{Bu}, \mathrm{HOTf}, \mathrm{MeOTf}, \mathrm{Me}_{3} \mathrm{SiBr}, \mathrm{PhC}(\mathrm{O}) \mathrm{Cl}$ and $\mathrm{PMe}_{3}$ were distilled under an $\mathrm{Ar}$ atmosphere before usage, whereas ferrocene, $\mathrm{KO}^{t} \mathrm{Bu}, \mathrm{Me}_{3} \mathrm{NO}$, napthalene, $\mathrm{PPh}_{3}$ and 2,4,6-tris-tert-butylphenol were sublimed. $\mathrm{NEt}_{3}$ was dried over $\mathrm{KoH}$ and distilled. All other chemicals were used as purchased without further purification.

$\mathbf{X I X}, \mathbf{X X I}, \mathbf{1 8}, \mathbf{2 1},{ }^{[180]}, \mathbf{1}, \mathbf{2}^{\mathrm{Cl}},{ }^{[174]}$, the ligands HPNP${ }^{t \mathrm{Bu}}$ and $\mathrm{HPNP}^{i \operatorname{Pr}},{ }^{[228]}, \operatorname{ReCl}_{3}(\mathrm{MeCN})$ $\left(\mathrm{PPh}_{3}\right)_{2},{ }^{[229,230]}, \mathrm{NaHg},{ }^{[231]} \mathrm{KC}_{8},{ }^{[232]} 2,4,6$-tris-tert-butylphenoxy radical, ${ }^{[233]}, \mathrm{Na}\left(\mathrm{BAr}_{24}{ }^{\mathrm{F}}\right)$ and $\left.\mathrm{H}\left(\mathrm{Et}_{2} \mathrm{O}\right)_{2}\left(\mathrm{BAr}_{24}\right)^{\mathrm{F}}\right),{ }^{[234]}$, [PPN]N ${ }_{3},{ }^{[235]}\left[\mathrm{FC}^{\mathrm{OTf}},{ }^{[236]}\right.$ and $\mathrm{BAr}_{18}{ }^{\mathrm{F}}{ }^{[237]}$ have been synthesized according to published procedures. 


\subsection{Analytical methods}

\section{Crystallographic details}

Suitable single crystals for X-ray structure determination were selected from the mother liquor under an inert gas atmosphere and transferred in protective perfluoro polyether oil on a microscope slide. The selected and mounted crystals were transferred to the cold gas stream on the diffractometer. The diffraction data were obtained at $100 \mathrm{~K}$ on a BRUKER D8 threecircle diffractometer, equipped with a PHOTON 100 CMOS detector and an INCOATEC microfocus source with Quazar mirror optics (Mo-K $\alpha$ radiation, $\lambda=0.71073 \AA$ ).

The data obtained were integrated with SAINT and a semi-empirical absorption correction from equivalents with SADABS was applied. The structure was solved and refined using the BRUKER SHELX 2014 software package. ${ }^{[238-241]}$ All non-hydrogen atoms were refined with anisotropic displacement parameters. All $\mathrm{C}-\mathrm{H}$ hydrogen atoms were refined isotropically on calculated positions by using a riding model with their $\mathrm{U}_{\text {iso }}$ values constrained to $1.5 \mathrm{U}_{\text {eq }}$ of their pivot atoms for terminal sp $\mathrm{sp}^{3}$ carbon atoms and 1.2 times for all other atoms.

\section{Cyclic voltammetry}

Electrochemical data were recorded either on a Metrohm PGSTAT101 using the Nova 2.0 software (all data measured under argon) or a GAMRY 600 reference Potentiostat using the GAMRY software (all data measured under $\mathrm{N}_{2}$ ). The cyclic voltammetry (CV) data were recorded in a UV/vis cell equipped with a glassy carbon $(\varnothing=1.6 \mathrm{~mm})$ working electrode $(\mathrm{WE})$, a Pt wire as counter electrode (CE) and a $\mathrm{Ag}$ wire as pseudo-reference electrode (RE). Referencing was performed against the $\mathrm{Fc}^{+} / \mathrm{Fc}$ couple. All experiments were performed in $0.1 \mathrm{M} \mathrm{N}^{\mathrm{n}} \mathrm{Bu}_{4} \mathrm{PF}_{6}$ in THF.

During the second half of this thesis, CV data were compensated for internal resistance (iR). In these cases, the scan rate dependence of the peak currents $i_{p}$ was analyzed according to the Randles-Sevcik equation:

$$
i_{p}=0.446 n F A C^{0}\left(\frac{n F v D_{o}}{R T}\right)^{1 / 2}
$$

where $n$ is the number of transferred electrons, $F$ is Faraday's constant, $A$ is the electrode surface in $\mathrm{cm}^{2}, C^{0}$ is the bulk concentration of the analyte in $\mathrm{mol} \mathrm{cm}^{-3}, v$ is the scan rate in $\mathrm{V} \mathrm{s}^{-1}$ and $D_{o}$ is the diffusion coefficient of the oxidized species in $\mathrm{cm}^{2} \mathrm{~s}^{-1}$. [242] This equation is valid for freely diffusing (i.e. non-absorbed) analyte in solution which undergoes fast, electrochemically reversible electron transfer. Thus deviations from linearity of a plot of $i_{p}$ vs. $v^{1 / 2}$ can indicate quasi-reversible electron transfer (accompanied by a scan rate dependent 
peak-to-peak separation) or adsorption of the analyte on the electrode surface (which should have a constant peak-to-peak separation).

All controlled potential electrolysis (CPE) experiments were performed in a three-neck cell with reticulated vitreous carbon as $\mathrm{WE}$, a $\mathrm{Pt}$ wire as $\mathrm{CE}$ in a fritted sample holder compartment with $\mathrm{Fc}^{*+} / \mathrm{Fc}^{*}$ as sacrificial reductant and a $\mathrm{Ag}$ wire as $\mathrm{RE}$ in a compartment separated by a fritted sample holder from the main solution. The solution was stirred during electrolysis. All experiments were performed in $0.2 \mathrm{M} \mathrm{N}^{\mathrm{n}} \mathrm{Bu}_{4} \mathrm{PF}_{6}$ in $\mathrm{THF}$.

\section{Elemental analysis}

Elemental analyses were obtained from the Analytical Laboratories at the GeorgAugust University Göttingen using an ElEmentaR VARIO EL 3 analyzer.

\section{Indophenolic titration}

After reaction of $\mathbf{3}$ with $\mathrm{H}_{2}$ according to the procedure described in Section 2.1.7, all volatiles were transfered to a Schlenk tube containing $\mathrm{HCl}$ in $\mathrm{Et}_{2} \mathrm{O}$. This mixture was stirred for $10 \mathrm{~min}$, all volatiles were removed in vacuo and the precipiate was analyzed for $\mathrm{NH}_{4} \mathrm{Cl}$ according to a literature procedure. ${ }^{[184]}$
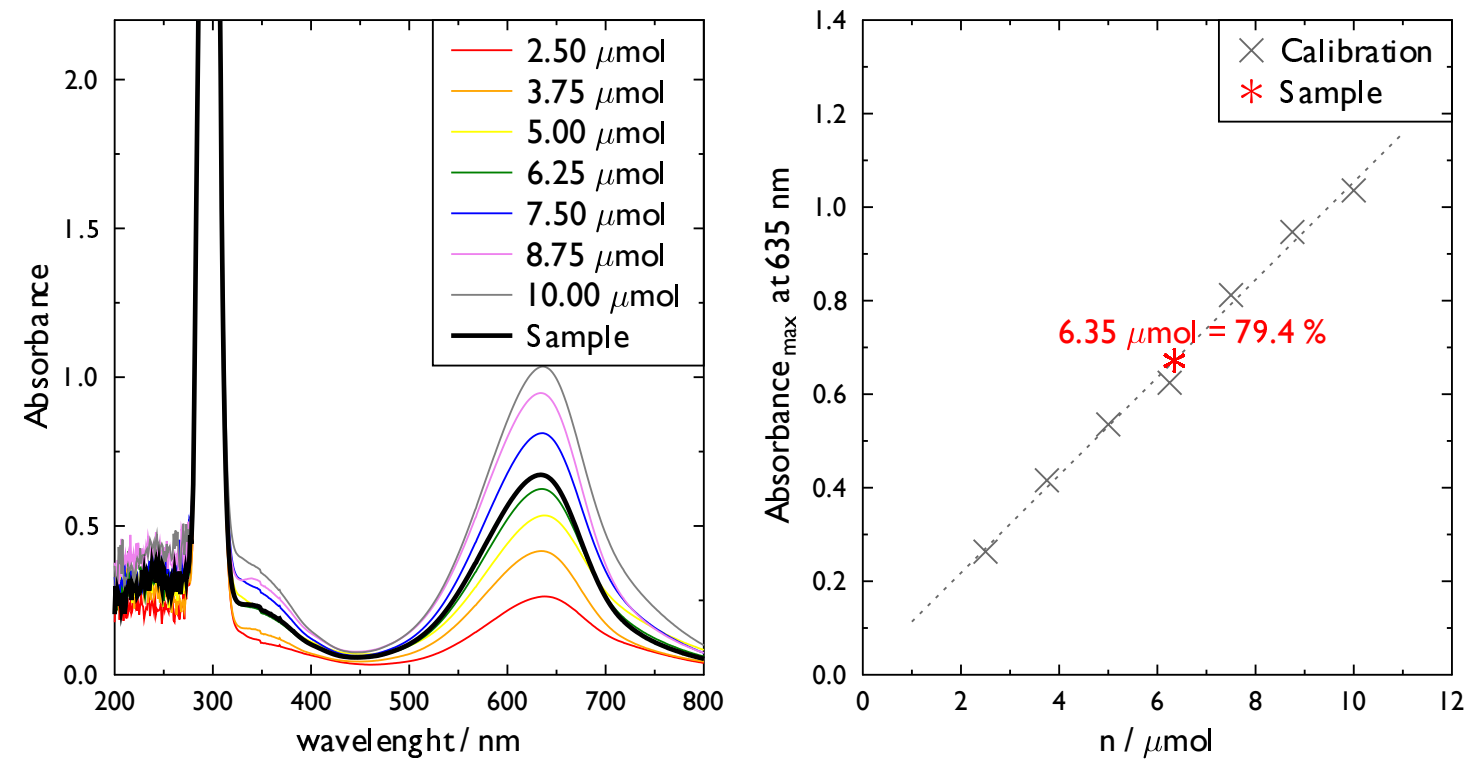

Fig. 1.1. Determination of the amount of $\mathrm{NH}_{3}$ from the reaction of 3 with $\mathrm{H}_{2}$ by indophenolic titration. Left: UV/vis spectra of the indophenol solutions of the calibration solutions (colored) and the actual sample (black). Right: Calibration curve and calculated yield of the reaction. 


\section{Irradiation and quantum yield determination}

All photolysis experiments were performed using either a KESSIL PR160-390 LED (390 nm) or a $150 \mathrm{~W} \mathrm{Hg}(\mathrm{Xe})$ arc lamp with lamp housing and arc lamp power supply from LOTQuAntum Design GmbH. For the latter, IR radiation was eliminated by use of a water filter and photolyzed samples were kept at room temperature by a water bath. Wavelength selection was achieved using filter with a cutoff of $305 \mathrm{~nm}$ and $420 \mathrm{~nm}$.

For the photolytic splitting of $\mathbf{3 6}$ into $\mathbf{3 7}$, the quantum yield was determined for the irradiation with a $390 \mathrm{~nm}$ LED lamp. The quantum yield of any photon-induced chemical reaction is defined as

$$
\Phi=\frac{n(\text { molecules reacted })}{n(\text { photons absorbed })}
$$

If the total amount of photons emitted by a monochromatic light source per time per sample area is known, the number of absorbed photons can be derived from the absorbance of the sample at the wavelength of interest. This initial amount of photons is termed photon flux and can be derived for any given light source by photolysis of an chemical actinometer with a known quantum yield. The workhorse system in this respect is the photolysis of potassium ferrioxalate $\left(\mathrm{K}_{3}\left[\mathrm{Fe}\left(\mathrm{C}_{2} \mathrm{O}_{4}\right)_{3}\right]\right)$, for which quantum yields are determined over a wide range of wavelengths and which proceeds according to the following equation:

$$
2\left[\mathrm{Fe}\left(\mathrm{C}_{2} \mathrm{O}_{4}\right)_{3}\right]^{3-} \stackrel{\mathrm{h} \nu}{\longrightarrow} 2\left[\mathrm{Fe}\left(\mathrm{C}_{2} \mathrm{O}_{4}\right)\right]^{2-}+\mathrm{CO}_{2}+\mathrm{C}_{2} \mathrm{O}_{4}^{2-}
$$

Formation of the $\mathrm{Fe}^{2+}$ compound can than be quantified by addition of phenanthroline which leads to the formation of an $\left[\mathrm{Fe}(\mathrm{phen}){ }_{3}^{2+}\right]$ complex which features a very pronounced absorption at $510 \mathrm{~nm}$.

In the actual experiment, three aliquots of an aqueous solution of potassium ferrioxalate $(12 \mathrm{mM}, 2 \mathrm{~mL})$ were placed in $20 \mathrm{~cm}$ distance of the lamp and photolyzed with $25 \%$ power output for $15 \mathrm{~s}$. Afterwards, to a portion of each photolyzed sample $(1 \mathrm{~mL})$ were added a sodium acetate buffer $(0.1 \mathrm{M}, \mathrm{pH}=3.5,2 \mathrm{~mL})$ and 1,10-phenanthroline solution $(5 \mathrm{mM}$, $2 \mathrm{~mL}$ ). The mixture was diluted to $10 \mathrm{~mL}$ and allowed to equilibrate for $1 \mathrm{~h}$. Afterwards, the concentration of the formed $\mathrm{Fe}^{2+}$ phenanthroline complex was determined by UV/vis spectroscopy. All reactions were performed with strict exclusion of ambient light.

The photonflux of a light source at a given distance can be determined using the following equation:

$$
I=\frac{k \cdot V \cdot A_{510 \mathrm{~nm}}}{\Phi \cdot \epsilon_{510 \mathrm{~nm}} \cdot d \cdot a \cdot t \cdot\left(1-10^{-A}\right)}
$$

where $I$ is the photon flux in mol cm${ }^{-2}, k$ is the dilution factor between photolyzed sample and analyzed solution (10), $V$ is the total volume of photolyzed actinometer solution $(0.002 \mathrm{~L})$, $A_{510 \mathrm{~nm}}$ is the absorbance of the formed $\mathrm{Fe}^{2+}$ phenanthroline complex at $\lambda=510 \mathrm{~nm}, \epsilon_{510 \mathrm{~nm}}$ is the corresponding molar attenuation coefficient in $\mathrm{M}^{-1} \mathrm{~cm}^{-2}\left(1.11 \cdot 10^{4}\right), \Phi$ is the quantum 
yield of the actinometer at $390 \mathrm{~nm}$ (1.18), $d$ is the pathlength of the used UV/vis cuvette in $\mathrm{cm}, a$ is the area of irradiation in $\mathrm{cm}^{2}$, and $A$ is the initial absorbance of the ferrioxalate solution at the wavelength of irradiation (9.6). For the three samples, $A_{510 \mathrm{~nm}}$ was 0.401 , 0.367 and 0.461 , respectively, resulting in a photon flux of

$$
I=1.1948 \pm 0.1391 \cdot 10^{-6} \mathrm{~mol} \mathrm{~min}^{-1} \mathrm{~cm}^{-2}
$$

For the quantum yield determination, a solution of complex 36 in THF $\left(2 \mathrm{~mL}, 4 \cdot 10^{-5} \mathrm{M}\right)$ was irradiated with the same setup (same cuvette dimension, same distance from the lightemitting diode (LED) lamp) for a total of $20 \mathrm{~min}$. After every $2 \mathrm{~min}$, the irradiation was stopped and the concentration of the dimer was determined by monitoring of the absorbance at $\lambda=578 \mathrm{~nm}$. The quantum yield was determined by

$$
\Phi=\frac{\Delta n_{t_{x}-t_{x-1}}}{n_{\text {Photons }, 2 \min } \cdot\left(1-10^{-A_{m} \text { athrm } 390 n m}\right)}
$$

where $\Delta n_{t_{x}-t_{x-1}}$ is the amount of $\mathbf{3 6}$ decomposed between two measurements, i.e. after irradiation for $2 \mathrm{~min}, n_{\text {Photons,2min }}$ is the amount of photons reaching the sample during $2 \mathrm{~min}\left(4.7792 \cdot 10^{-6} \mathrm{~mol}\right.$ in the above described setup, calculated by $\left.n=I \cdot t \cdot A\right)$ and $A_{390 \mathrm{~nm}}$ is the absorbance of the solution at $390 \mathrm{~nm}$ at the beginning of each photolysis step. To account for small absorption of the product at the same wavelength, this value is calculated from the initial absorbance, prior to the first irradiation, and the concentration of the dimer (i.e. referenced to the absorption at $\lambda=578 \mathrm{~nm}$ ). This results in a quantum yield of $0.36 \pm 0.03 \%$.
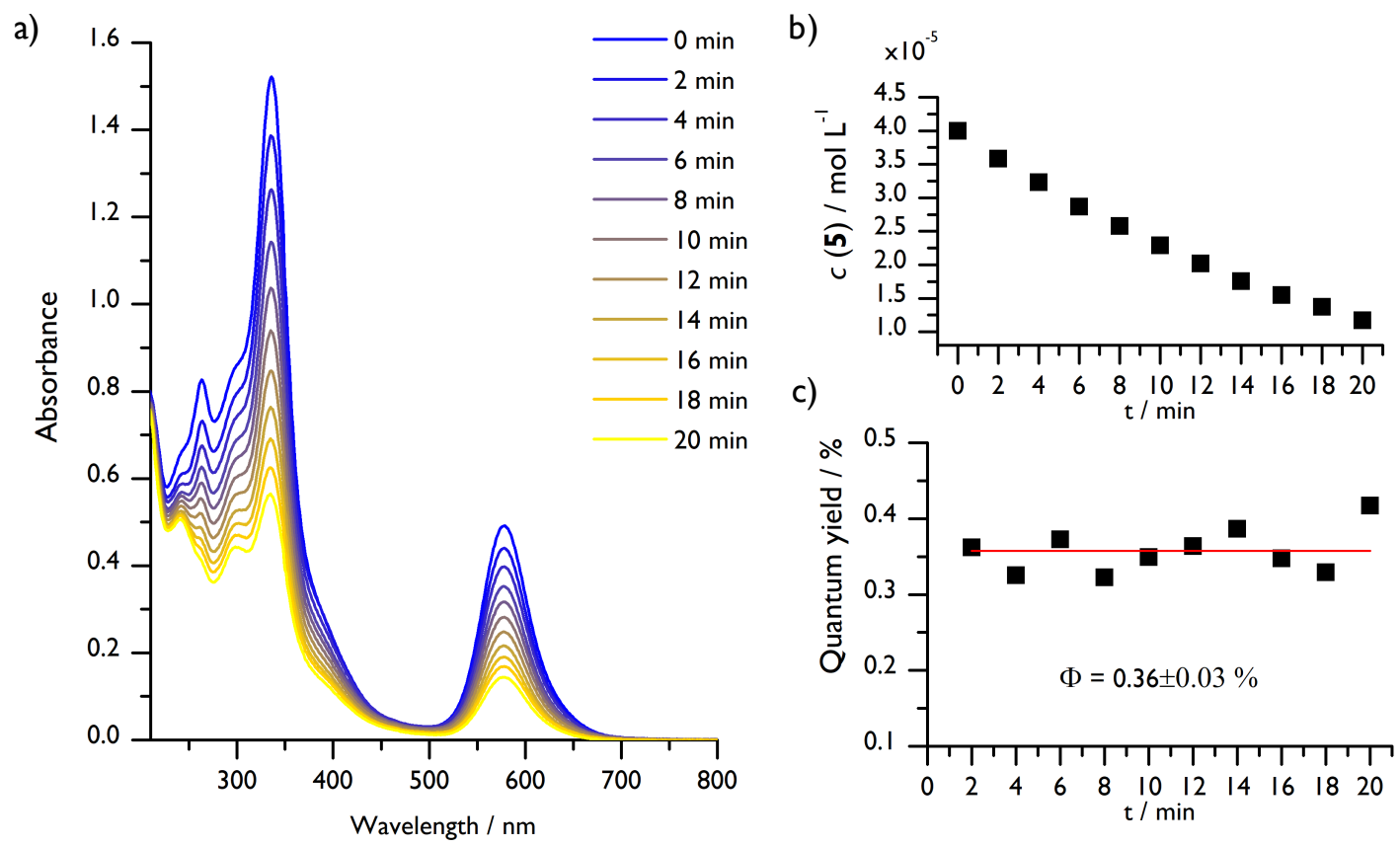

Fig. 1.2. Quantum yield determination of the photosplitting of $\mathbf{3 6}$ by $390 \mathrm{~nm}$ LED lamp. a) Decay of $\mathbf{3 6}$ during photolysis. b) Concentration profile of $\mathbf{3 6}$ during the reaction. c) Quantum yields between each irradiation interval. 


\section{Mass spectrometry}

ESI mass spectra (on a BRUker HCT ultra ESI-MS) and LIFDI (LINDEN CMS) mass spectra were measuerd by the Zentrale Massenabteilung, Fakultät für Chemie, Georg-AugustUniversität.

\section{Nuclear magnetic resonance}

NMR data were recorded on machines from Bruker (Avance III 300, Avance III 400 or Avance 500 with a Prodigy broadband CryoProbe). All spectra were referenced to residual solvent signals (THF- $d_{8}: \delta_{{ }_{1} \mathrm{H}}=3.58 \mathrm{ppm}, \delta_{13} \mathrm{C}=67.2 \mathrm{ppm} ; \mathrm{C}_{6} \mathrm{D}_{6}: \delta_{1_{\mathrm{H}}}=7.16 \mathrm{ppm}, \delta_{13} \mathrm{C}=$ $128.1 \mathrm{ppm}$; toluene- $d_{8}: \delta_{1_{\mathrm{H}}}=2.08 \mathrm{ppm}, \delta_{{ }^{13} \mathrm{C}}=20.4 \mathrm{ppm} ; \mathrm{CD}_{2} \mathrm{Cl}_{2}: \delta_{1_{\mathrm{H}}}=5.32 \mathrm{ppm}, \delta_{13} \mathrm{C}$ $=53.8 \mathrm{ppm}) .{ }^{15} \mathrm{~N}$ and ${ }^{31} \mathrm{P}$ chemical shifts are reported relative to external standards (nitromethane and phosphoric acid, respectively, both defining $\delta=0.0 \mathrm{ppm})$.

In general, signal multiplicity is denoted as broad (br), singlet $(\mathrm{s})$, doublet $(\mathrm{d})$, triplet $(\mathrm{t})$, virtual triplet (vt) or heptet (hept). In complexes where both phosphorous atoms are equivalent, deriving coupling constants in the ${ }^{1} \mathrm{H}$ NMR data was often not possible, due to higher order coupling effects. In these cases, the resonances are described either as multiplet $(\mathrm{m})$ or as spin system (spin-sys), if at least the ${ }^{3} J_{\mathrm{HP}}$ coupling constant could be determined (i.e. for the tert-butyl signals, where ${ }^{3} J_{\mathrm{HP}}$ corresponds to the separation of the outer peaks of a signal).

\section{SQUID measurement}

Temperature-dependent magnetic susceptibility measurements were carried out with a QUANTUM-DESIGN MPMS-XL-5 SQUID magnetometer equipped with a $5 \mathrm{~T}$ magnet in the range from 295 to $2.0 \mathrm{~K}$ at a magnetic field of $0.5 \mathrm{~T}$. The powdered sample was contained in a Teflon bucket and fixed in a non-magnetic sample holder. Each raw data file for the measured magnetic moment was corrected for the diamagnetic contribution of the Teflon bucket according to $M^{\text {dia }}$ (bucket) $=\mathcal{X}_{g} \cdot m \cdot H$, with an experimentally obtained gram susceptibility of the Teflon bucket. The molar susceptibility data were corrected for the diamagnetic contribution using the Pascal constants and the increment method according to Haberditzl.[243,244] Experimental data were modeled with the JULX program ${ }^{[245]}$ for $S=0$ including temperature independent paramagnetism (TIP) and paramagnetic impurities $(P I$, $\mathrm{S}=1 / 2)$ according to $\mathcal{X}_{\exp }=(1-P I) \cdot \mathcal{X}_{\text {sample }}+P I \cdot \mathcal{X}_{P I}+T I P$. 


\section{UV/vis spectroscopy}

UV/vis spectra were recorded in a cuvette equipped with a J-YOUnG cap at an AGILENT CARY 300 spectrometer using the SCAN software. Background measurements of the blank solvent were performed in an UV/vis cuvette identically constructed to the one of the actual measurement.

\section{Vibrational spectroscopy}

Standard IR spectra of pure compounds were recorded at a BRUKER AlPHA FT-IR spectrometer with the Platinum ATR module inside a glovebox. When a sample was prepared as $\mathrm{KBr}$ disk, it was diluted with solid $\mathrm{KBr}$, pestled until a homogeneous mixture was obtained an pressed into a pellet. The disk was then measured at a Thermo Science Nicolet iZ10 at ambient conditions.

For IR measurements in the magnetic field, the spectra were recorded using a commercial Fourier transform spectrometer (VERTEX 80v) coupled to a superconducting coil delivering the field up to $11 \mathrm{~T}$. To measure transmittance, the radiation of globar was delivered from the spectrometer using light-pipe optics to the sample (a mixture of the studied compound with $\mathrm{KBr}$ ) inside the magnet and detected by a silicon composite bolometer (IRLABS) placed just below the sample. Both the sample and bolometer were surrounded by the helium exchange gas and cooled down to $4.2 \mathrm{~K}$.

Resonance Raman ( $r R$ ) spectra were measured using a HoRIBA SCIEnTIFIC LabRAM HR 800 spectrometer with open-electrode CCD detector in combination with a free space optical microscope and a He:Ne-laser $(632.8 \mathrm{~nm})$. 



\section{Synthesis}

Ich bin meines Glückes Schmied

Ich bau mir einen Prototyp

Ich setz dich Stück für Stück zusammen

\section{- Eisbrecher}

"Prototyp" on "Die Hölle muss warten"

\subsection{Osmium compounds}

\subsection{1 $\left[\mathrm{Os}(\mathrm{H})(\mathrm{N})\left(\mathrm{PNP}^{\mathrm{tBu}}\right)\right]^{\mathrm{X}}\left(2^{\mathrm{X}}\right)$}

From reaction of 1 with $\mathrm{NaN}_{3}$ :

$\left[\mathrm{OsHCl}_{2}\left(\mathrm{PNP}^{\mathrm{tBu}}\right)\right](\mathbf{1})(150 \mathrm{mg}, 241 \mu \mathrm{mol}, 1.0 \mathrm{eq}), \mathrm{KPF}_{6}(57.6 \mathrm{mg}, 313 \mu \mathrm{mol}, 1.3 \mathrm{eq})$ and $\mathrm{NaN}_{3}(20 \mathrm{mg}, 313 \mu \mathrm{mol}, 1.3 \mathrm{eq})$ were diluted in $\mathrm{DCM}(10 \mathrm{~mL})$ and $\mathrm{EtOH}(20 \mathrm{~mL})$ and the solution was stirred for $1 \mathrm{~h}$. The solvent was removed in vacuo and the yellow residue was extracted with DCM. The solution was concentrated to 1-2 $\mathrm{mL}$, layered with pentane and stored in the freezer $\left(-35^{\circ} \mathrm{C}\right)$ overnight. $\left[\mathrm{Os}(\mathrm{H})(\mathrm{N})\left(\mathrm{PNP}^{t \mathrm{Bu}}\right)\right]^{\mathrm{PF} 6}\left(2^{\mathrm{PF}_{6}}\right)$ was obtained as yellow crystals, which were collected and dried (153 mg, $215 \mu \mathrm{mol}, 89.3 \%)$.

${ }^{1} \mathbf{H}-\mathrm{NMR}\left(400 \mathrm{MHz}, \mathrm{CD}_{2} \mathrm{Cl}_{2}, 25^{\circ} \mathrm{C}\right): \delta(\mathrm{ppm})=4.02-3.86(\mathrm{~m}, 2 \mathrm{H}, \mathrm{NCH}), 3.85-3.75(\mathrm{~m}$, $\left.2 \mathrm{H}, \mathrm{NCH}_{2}\right), 2.35-2.25\left(\mathrm{~m}, 2 \mathrm{H}, \mathrm{PCH} \mathrm{H}_{2}\right), 2.15-2.02\left(\mathrm{~m}, 2 \mathrm{H}, \mathrm{PCH} \mathrm{H}_{2}\right), 1.49$ (spin-sys, $3 J_{\mathrm{HP}}=$ $\left.15.6 \mathrm{~Hz}, 18 \mathrm{H}, \mathrm{CH}_{3}\right), 1.31$ (spin-sys, $\left.{ }^{3} J_{\mathrm{HP}}=15.6 \mathrm{~Hz}, 18 \mathrm{H}, \mathrm{CH}_{3}\right),-1.34\left(\mathrm{t},{ }^{2} J_{\mathrm{HP}}=16 \mathrm{~Hz}, 1 \mathrm{H}\right.$, Os $H) .{ }^{13} \mathrm{C}\left\{{ }^{1} \mathbf{H}\right\}-\mathrm{NMR}\left(100.7 \mathrm{MHz}, \mathrm{CD}_{2} \mathrm{Cl}_{2}, 25^{\circ} \mathrm{C}\right): \delta$ (ppm) $=70.0$ (virtual triplet (vt), $\left.\mathrm{AXX}^{\prime} \mathrm{A}^{\prime}, \mathrm{N}=\left|{ }^{2} J_{\mathrm{CP}}+{ }^{4} J_{\mathrm{CP}}\right|=6.2 \mathrm{~Hz}, \mathrm{NCH}_{2}\right), 38.5\left(\mathrm{vt}, \mathrm{AXX} \mathrm{A}^{\prime}, \mathrm{N}=\left|{ }^{1} J_{\mathrm{CP}}+{ }^{3} J_{\mathrm{CP}}\right|=\right.$ $\left.18.4 \mathrm{~Hz}, C\left(\mathrm{CH}_{3}\right)_{3}\right), 36.6\left(\mathrm{vt}, \mathrm{AXX} \mathrm{A}^{\prime}, \mathrm{N}=\left|{ }^{1} J_{\mathrm{CP}}+{ }^{3} J_{\mathrm{CP}}\right|=27.8 \mathrm{~Hz}, C\left(\mathrm{CH}_{3}\right)_{3}\right), 29.3(\mathrm{~m}$, $\left.\mathrm{CH}_{3}\right), 28.9\left(\mathrm{~m}, \mathrm{CH}_{3}\right), 23.7$ (vt, AXX'A', N $=\left|{ }^{1} J_{\mathrm{CP}}+{ }^{3} J_{\mathrm{CP}}\right|=27.2 \mathrm{~Hz}, \mathrm{PCH}$ ). ${ }^{31} \mathbf{P}\left\{{ }^{1} \mathbf{H}\right\}-$ NMR $\left(162.0 \mathrm{MHz}, \mathrm{CD}_{2} \mathrm{Cl}_{2}, 25^{\circ} \mathrm{C}\right): \delta(\mathrm{ppm})=107.6$ (s, POs), -144.5 (hept, ${ }^{1} J_{\mathrm{PF}}=$ $\left.711.8 \mathrm{~Hz}, P F_{6}\right)$. Elemental anal. Calc. for $\mathrm{C}_{20} \mathrm{H}_{45} \mathrm{~F}_{6} \mathrm{NOsP}_{3}: \mathrm{C}, 33.8 ; \mathrm{H}, 6.38 ; \mathrm{N}, 3.94$. Found: C, 33.27; $\mathrm{H}, 6.17 ; \mathrm{N}, 3.90 . \mathbf{I R}(\mathrm{KBr}): \tilde{\nu}\left(\mathrm{cm}^{-1}\right)=2215.9\left(\nu_{\mathrm{Os}-\mathrm{H}}\right)$

By protonation of $\mathbf{3}$ :

$\left[\mathrm{Os}(\mathrm{N})\left(\mathrm{PNP}^{t \mathrm{Bu}}\right)\right](3)(10.0 \mathrm{mg}, 17.7 \mu \mathrm{mol}, 1.0 \mathrm{eq})$ was dissolved in $\mathrm{THF}(2 \mathrm{~mL})$ and $\mathrm{H}$ 
$\left(\mathrm{Et}_{2} \mathrm{O}\right)_{2}\left(\mathrm{BAr}_{24}^{\mathrm{F}}\right)(17.9 \mathrm{mg}, 17.7 \mu \mathrm{mol}, 1.0 \mathrm{eq})$ was added. After stirring for $5 \mathrm{~min}$, the solvent was removed in vacuo and the residue was extracted with $\mathrm{Et}_{2} \mathrm{O}$. The solution was concentrated, layered with pentane and stored in the freezer at $-35{ }^{\circ} \mathrm{C}$ overnight. $[\mathrm{Os}(\mathrm{H})(\mathrm{N})$ $\left.\left(\mathrm{PNP}^{\mathrm{tBu}}\right)\right]^{\mathrm{BAr}_{24}^{\mathrm{F}}}\left(2^{\mathrm{BAr}_{24}^{\mathrm{F}}}\right)$ was collected as yellow crystals and dried $(19.7 \mathrm{mg}, 13.8 \mu \mathrm{mol}$, $78 \%)$.

The ${ }^{1} \mathrm{H},{ }^{13} \mathrm{C}\left\{{ }^{1} \mathrm{H}\right\}$ and ${ }^{31} \mathrm{P}\left\{{ }^{1} \mathrm{H}\right\}-N M R$ spectra of the cation are identical with those of $2^{\mathrm{PF}_{6}}$. Mass spect. (LIFDI), $m / z(\%)=567.3\left(\left[\mathrm{M}^{+}\right]\right)$. Elemental anal. Calc. for $\mathrm{C}_{52} \mathrm{H}_{57} \mathrm{BF}_{24} \mathrm{~N}_{2} \mathrm{OsP}_{2}$ : C,43.71; $\mathrm{H}, 4.02 ; \mathrm{N}, 1.96$. Found: C, 43.74; $\mathrm{H}, 4.27 ; \mathrm{N}, 1.93$.

\section{$2.1 .2\left[\mathrm{Os}(\mathrm{N})\left(\mathrm{PNP}^{t \mathrm{Bu}}\right)\right](3)$}

$\left[\mathrm{Os}(\mathrm{H})(\mathrm{N})\left(\mathrm{PNP}^{\mathrm{tBu}}\right)\right]^{\mathrm{PF} 6}\left(2^{\mathrm{PF}_{6}}\right)(103 \mathrm{mg}, 145 \mu \mathrm{mol}, 1.0 \mathrm{eq})$ was dissolved in THF $(12 \mathrm{~mL})$ andKO ${ }^{t} \mathrm{Bu}(16 \mathrm{mg}, 145 \mu \mathrm{mol}, 1.0 \mathrm{eq})$ in THF $(2 \mathrm{~mL})$ added dropwise. After stirring for $5 \mathrm{~min}$, the solvent was removed and the deep red residue was extracted with pentane. $\left[\mathrm{Os}(\mathrm{N})\left(\mathrm{PNP}^{t \mathrm{Bu}}\right)\right](3)$ was obtained as red powder $(73 \mathrm{mg}, 130 \mu \mathrm{mol}, 89.9 \%)$.

${ }^{1} \mathbf{H}-\mathrm{NMR}\left(400 \mathrm{MHz}, \mathrm{C}_{6} \mathrm{D}_{6}, 25^{\circ} \mathrm{C}\right): \delta(\mathrm{ppm})=2.72(\mathrm{~m}, 4 \mathrm{H}, \mathrm{NCH}), 1.92(\mathrm{~m}, 4 \mathrm{H}, \mathrm{PCH})$, 1.42 (spin-sys, $\left.{ }^{3} J_{\mathrm{HP}}=13.4 \mathrm{~Hz}, 36 \mathrm{H}, \mathrm{CH}_{3}\right) .{ }^{13} \mathrm{C}\left\{{ }^{1} \mathbf{H}\right\}-\mathrm{NMR}\left(100.7 \mathrm{MHz}, \mathrm{C}_{6} \mathrm{D}_{6}, 25^{\circ} \mathrm{C}\right.$ ): $\delta(\mathrm{ppm})=63.1\left(\mathrm{vt}, \mathrm{AXX} \mathrm{A}^{\prime}, \mathrm{N}=\left.\right|^{2} J_{\mathrm{CP}}+{ }^{4} J_{\mathrm{CP}} \mid=14.2 \mathrm{~Hz}, \mathrm{NCH}_{2}\right), 36.4\left(\mathrm{vt}, \mathrm{A}_{2} \mathrm{XX}^{\prime} \mathrm{A}^{\prime}{ }_{2}\right.$, $\left.\mathrm{N}=\left|{ }^{1} J_{\mathrm{CP}}+{ }^{3} J_{\mathrm{CP}}\right|=21.4 \mathrm{~Hz}, C\left(\mathrm{CH}_{3}\right)_{3}\right), 29.7\left(\mathrm{~A}_{6} \mathrm{XX}^{\prime} \mathrm{A}_{6}^{\prime}, \mathrm{N}=\left.\right|^{2} \mathrm{~J}_{\mathrm{CP}}+{ }^{4} \mathrm{~J}_{\mathrm{CP}} \mid=2.6 \mathrm{~Hz}, \mathrm{CH}_{3}\right)$, 25.3 (vt, AXX'A', N=| $\left.{ }^{1} J_{\mathrm{CP}}+{ }^{3} J_{\mathrm{CP}} \mid=24.2 \mathrm{~Hz}, \mathrm{PCH}_{2}\right) .{ }^{15} \mathbf{N}\left\{{ }^{1} \mathbf{H}\right\}-\mathbf{N M R}\left(50.7 \mathrm{MHz}, \mathrm{C}_{6} \mathrm{D}_{6}\right.$, $\left.25^{\circ} \mathrm{C}\right): \delta(\mathrm{ppm})=391.0(\mathrm{~s}) .{ }^{31} \mathbf{P}\left\{{ }^{1} \mathbf{H}\right\}-\mathbf{N M R}\left(162.0 \mathrm{MHz}, \mathrm{C}_{6} \mathrm{D}_{6}, 25^{\circ} \mathrm{C}\right): \delta(\mathrm{ppm})=100.1$ (s). Elemental anal. Calc. for $\mathrm{C}_{20} \mathrm{H}_{44} \mathrm{~N}_{2} \mathrm{OsP}_{2}$ : C, 42.53; $\mathrm{H}, 7.85 ; \mathrm{N}, 4.96$. Found: $\mathrm{C}$, 42.60; H, 7.99; N, 4.84. IR $(\mathrm{KBr}): \tilde{\nu}\left(\mathrm{cm}^{-1}\right)=999.2\left(\nu_{\mathrm{Os} \equiv \mathrm{N}}\right) .{ }^{15} \mathrm{~N}-3\left(\right.$ from $\left.\mathrm{Na}^{15} \mathrm{NN}_{2}\right)$ : $966.9\left(\nu_{\mathrm{Os} \equiv{ }^{15} \mathrm{~N}}\right)$. Mass spec. $\left(\mathrm{ESI}{ }^{+}, \mathrm{THF}\right): m / z=567.2673\left([\mathrm{M}+\mathrm{H}]^{+}\right.$, calc. 567.2668).

\subsection{3 $\left[\mathrm{Os}(\mathrm{H})(\mathrm{N}) \mathrm{Cl}\left(\mathrm{HPNP}^{t \mathrm{Bu}}\right)\right]^{\mathrm{Cl}}\left(4^{\mathrm{Cl}}\right)$}

By protonation of 3 :

$\left[\mathrm{Os}(\mathrm{N})\left(\mathrm{PNP}^{t \mathrm{Bu}}\right)\right](3)(6.0 \mathrm{mg}, 10.6 \mu \mathrm{mol}, 1.0 \mathrm{eq})$ in $\mathrm{THF}(0.6 \mathrm{~mL})$ was treated with 2 eq $\mathrm{HCl}\left(2.0 \mathrm{M}\right.$ in $\left.\mathrm{Et}_{2} \mathrm{O}, 10.6 \mu \mathrm{L}, 21.2 \mu \mathrm{mol}\right)$. After stirring for $15 \mathrm{~min}$, the solvent was removed in vacuo and the product was washed with $\mathrm{Et}_{2} \mathrm{O}(2 \times 0.5 \mathrm{~mL})$. The selective formation of one product was observed and assigned to $\left[\mathrm{Os}(\mathrm{H})(\mathrm{N}) \mathrm{Cl}\left(\mathrm{HPNP}^{t \mathrm{Bu}}\right)\right]^{\mathrm{Cl}}\left(4^{\mathrm{Cl}}\right)$. Reaction of 3 with 1 eq $\mathrm{HCl}$ gave the same product exclusively with $50 \%$ conversion.

By disproportionation of 3 :

$\left[\mathrm{Os}(\mathrm{H})(\mathrm{N})\left(\mathrm{PNP}^{t \mathrm{Bu}}\right)\right]^{\mathrm{Cl}}\left(2^{\mathrm{Cl}}\right)$ was prepared as described above in $\mathrm{DCM}$ without $\mathrm{KPF}_{6}$. After evaporation of the solvent the residue was dissolved in THF. Immediate reaction to $\mathbf{3}$ and $4^{\mathrm{Cl}}$ was detected by ${ }^{31} \mathrm{P}$ NMR spectroscopy. 
${ }^{1} \mathbf{H}-\mathrm{NMR}\left(300 \mathrm{MHz}, \mathrm{CD}_{2} \mathrm{Cl}_{2}, 25^{\circ} \mathrm{C}\right): \delta(\mathrm{ppm})=6.46-6.24$ (singlet $\left.(\mathrm{s})_{\mathrm{br}}, 1 \mathrm{H}, \mathrm{NH}\right), 3.04-2.81$ $\left(\mathrm{m}, 2 \mathrm{H}, \mathrm{CH}_{2}\right), 2.69-2.54\left(\mathrm{~m}, 2 \mathrm{H}, \mathrm{CH}_{2}\right), 2.47-2.23\left(\mathrm{~m}, 4 \mathrm{H}, \mathrm{CH}_{2}\right), 1.72$ (spin-sys, ${ }^{3} J_{\mathrm{HP}}=$ $\left.15.0 \mathrm{~Hz}, 18 \mathrm{H}, \mathrm{CH}_{3}\right), 1.60$ (spin-sys, $\left.{ }^{3} J_{\mathrm{HP}}=15.2 \mathrm{~Hz}, 18 \mathrm{H}, \mathrm{CH}_{3}\right),-2.52\left(\mathrm{t},{ }^{2} J_{\mathrm{HP}}=14.6 \mathrm{~Hz}\right.$, $1 \mathrm{H}, \mathrm{Os} H) .{ }^{31} \mathrm{P}\left\{{ }^{1} \mathbf{H}\right\}-\mathrm{NMR}\left(121.5 \mathrm{MHz}, \mathrm{CD}_{2} \mathrm{Cl}_{2}, 25^{\circ} \mathrm{C}\right): \delta(\mathrm{ppm})=65.0$ (s).

\subsection{4 $\left[\mathrm{Os}\left(\mathrm{NBAr}_{18}^{\mathrm{F}}\right)\left(\mathrm{PNP}^{t \mathrm{Bu}}\right)\right](5)$}

$\left[\mathrm{Os}(\mathrm{N})\left(\mathrm{PNP}^{\mathrm{tBu}}\right)\right](3)(5.0 \mathrm{mg}, 8.9 \mu \mathrm{mol}, 1.0 \mathrm{eq})$ and $\mathrm{BAr}_{18}^{\mathrm{F}}(5.8 \mathrm{mg}, 8.9 \mu \mathrm{mol}, 1.0 \mathrm{eq})$ were mixed in benzene $(0.5 \mathrm{~mL})$ and stirred for $30 \mathrm{~min}$. The solvent was removed in vacuo, the product was washed with pentane and $\mathrm{Et}_{2} \mathrm{O}$.

${ }^{1} \mathbf{H}-\mathrm{NMR}\left(300 \mathrm{MHz}, \mathrm{THF}-d_{8}, 25^{\circ} \mathrm{C}\right): \delta(\mathrm{ppm})=8.18\left(\mathrm{~s}, 6 \mathrm{H}, \mathrm{CH}_{\text {ortho }}\right), 7.69(\mathrm{~s}, 3 \mathrm{H}$, $\left.\mathrm{CH}_{\text {para }}\right), 2.75(\mathrm{~m}, 4 \mathrm{H}, \mathrm{NCH} 2), 2.32(\mathrm{~m}, 4 \mathrm{H}, \mathrm{PCH}), 1.17$ (spin-sys, ${ }^{3} J_{\mathrm{HP}}=14.1 \mathrm{~Hz}, 36 \mathrm{H}$, $\left.\mathrm{CH}_{3}\right) .{ }^{19} \mathbf{F}\left\{{ }^{1} \mathbf{H}\right\}-\mathbf{N M R}\left(282.4 \mathrm{MHz}, \mathrm{CD}_{2} \mathrm{Cl}_{2}, 2{ }^{\circ} \mathrm{C}\right): \delta(\mathrm{ppm})=-63.5(\mathrm{~s}) .{ }^{31} \mathbf{P}\left\{{ }^{1} \mathbf{H}\right\}-\mathbf{N M R}$ $\left(121.5 \mathrm{MHz}, \mathrm{CD}_{2} \mathrm{Cl}_{2}, 25^{\circ} \mathrm{C}\right): \delta(\mathrm{ppm})=98.2(\mathrm{~s})$.

\subsection{5 $\left[\mathrm{Os}\left(\mathrm{NSiMe}_{3}\right)\left(\mathrm{PNP}^{t \mathrm{Bu}}\right)\right]^{\mathrm{BAr}}{ }_{24}^{\mathrm{F}}\left(6^{\mathrm{BAr}_{24}^{\mathrm{F}}}\right)$}

[Os(N)(PNP $\left.\left.{ }^{t B u}\right)\right](3)(10 \mathrm{mg}, 17.7 \mu \mathrm{mol}, 1.0 \mathrm{eq})$ and $\mathrm{NaBAr}_{24}^{\mathrm{F}}(15.7 \mathrm{mg}, 17.7 \mu \mathrm{mol}, 1.0 \mathrm{eq})$ were stirred in THF $(2 \mathrm{~mL}) . \mathrm{Me}_{3} \mathrm{SiBr}(2.3 \mu \mathrm{L}, 2.7 \mathrm{mg}, 17.7 \mu \mathrm{mol}, 1.0 \mathrm{eq})$ in THF $(0.5 \mathrm{~mL})$ was added dropwise accompanied by a color change to deep green. After $15 \mathrm{~min}$ the solvent was removed in vacuo and the residue extracted with $\mathrm{Et}_{2} \mathrm{O}$. The solution was concentrated, layered with pentane and stored in the freezer at $-35^{\circ} \mathrm{C}$ overnight. The product was collected as green crystals and dried $(16.7 \mathrm{mg}, 11.2 \mu \mathrm{mol}, 63.0 \%)$.

${ }^{1} \mathbf{H}-\mathbf{N M R}\left(400 \mathrm{MHz}, \mathrm{CD}_{2} \mathrm{Cl}_{2}, 25^{\circ} \mathrm{C}\right): \delta(\mathrm{ppm})=7.72\left(\mathrm{~m}, 8 \mathrm{H}, H_{\text {ortho }}\right), 7.56\left(\mathrm{~m}, 4 \mathrm{H}, H_{\text {para }}\right)$, 2.42-2.33 (m, 4H, PCH $), 2.26-2.15\left(\mathrm{~m}, 4 \mathrm{H}, \mathrm{NCH}_{2}\right), 1.55$ (spin-sys, ${ }^{3} J_{\mathrm{HP}}=14.8 \mathrm{~Hz}, 36 \mathrm{H}$, $\left.\mathrm{C}\left(\mathrm{CH}_{3}\right)_{3}\right), 0.25\left(\mathrm{~s}, 9 \mathrm{H}, \mathrm{Si}\left(\mathrm{CH}_{3}\right)_{3}\right) \cdot{ }^{13} \mathrm{C}\left\{{ }^{1} \mathbf{H}\right\}-\mathrm{NMR}\left(100.7 \mathrm{MHz}, \mathrm{CD}_{2} \mathrm{Cl}_{2}, 25^{\circ} \mathrm{C}\right): \delta(\mathrm{ppm})$ $=161.7\left(\mathrm{q},{ }^{1} \mathrm{~J}_{\mathrm{CB}}=49.7 \mathrm{~Hz}, \mathrm{BC}\right), 134.8\left(\mathrm{~s}, \mathrm{C}_{\text {ortho }}\right), 128.8\left(\mathrm{qq},{ }^{2} \mathrm{~J}_{\mathrm{CF}}=31.5 \mathrm{~Hz},{ }^{3} \mathrm{~J}_{\mathrm{BC}}\right.$ $\left.=2.9 \mathrm{~Hz}, C_{\text {meta }}\right), 124.5\left(\mathrm{q},{ }^{1} \mathrm{~J}_{\mathrm{CF}}=272.8 \mathrm{~Hz}, C_{3}\right), 117.4\left(\mathrm{~m}, C_{\text {para }}\right), 67.9$ (vt, AXX'A', $\left.\mathrm{N}=\left.\right|^{2} \mathrm{~J}_{\mathrm{CP}}+{ }^{4} \mathrm{~J}_{\mathrm{CP}} \mid=13.8 \mathrm{~Hz}, \mathrm{NCH}_{2}\right), 39.0\left(\mathrm{vt}, \mathrm{AXX} \mathrm{A}^{\prime}, \mathrm{N}=\left|{ }^{1} \mathrm{~J}_{\mathrm{CP}}+{ }^{3} \mathrm{~J}_{\mathrm{CP}}\right|=21.0 \mathrm{~Hz}\right.$, $\left.\mathrm{PC}\left(\mathrm{CH}_{3}\right)_{3}\right), 30.0\left(\mathrm{~m}, \mathrm{C}\left(\mathrm{CH}_{3}\right)_{3}\right), 23.5\left(\mathrm{vt}, \mathrm{AXX} \mathrm{A}^{\prime}, \mathrm{N}=\left|{ }^{1} \mathrm{~J}_{\mathrm{CP}}+{ }^{3} \mathrm{~J}_{\mathrm{CP}}\right|=28.8 \mathrm{~Hz}, \mathrm{PCH}_{2}\right), 1.6$ $\left(\mathrm{s}, \mathrm{Si}\left(\mathrm{CH}_{3}\right)_{3}\right) .{ }^{11} \mathrm{~B}\left\{{ }^{1} \mathbf{H}\right\}-\mathbf{N M R}\left(93.6 \mathrm{MHz}, \mathrm{CD}_{2} \mathrm{Cl}_{2}, 25{ }^{\circ} \mathrm{C}\right): \delta(\mathrm{ppm})=-6.61$ (s). ${ }^{\mathbf{1 9}} \mathbf{F}\left\{{ }^{1} \mathbf{H}\right\}-$ $\operatorname{NMR}\left(282.3 \mathrm{MHz}, \mathrm{CD}_{2} \mathrm{Cl}_{2}, 25^{\circ} \mathrm{C}\right): \delta(\mathrm{ppm})=-62.9\left(\mathrm{~s}, \mathrm{C} F_{3}\right) .{ }^{31} \mathbf{P}\left\{{ }^{1} \mathbf{H}\right\}-\mathbf{N M R}(162.0 \mathrm{MHz}$, $\left.\mathrm{CD}_{2} \mathrm{Cl}_{2}, 25^{\circ} \mathrm{C}\right): \delta(\mathrm{ppm})=106.9$ (s). Elemental anal. Calc. for $\mathrm{C}_{55} \mathrm{H}_{65} \mathrm{BF}_{24} \mathrm{~N}_{2} \mathrm{OsP}_{2} \mathrm{Si}: \mathrm{C}$, 44.01; H, 4.36; N, 1.87. Found: C, 43.99; H, 4.30; N, 1.85 . 


\subsection{6 $\left[\mathrm{Os}\left(\mathrm{NPMe}_{3}\right)\left(\mathrm{PNP}^{\mathrm{tBu}}\right)\right](7)$}

$\left[\mathrm{Os}(\mathrm{N})\left(\mathrm{PNP}^{t \mathrm{Bu}}\right)\right](3)(20 \mathrm{mg}, 35 \mu \mathrm{mol}, 1.0 \mathrm{eq})$ was dissolved in $\mathrm{C}_{6} \mathrm{D}_{6}(0.6 \mathrm{~mL})$ and $\mathrm{PMe}_{3}$ $(4.0 \mu \mathrm{L}, 3.0 \mathrm{mg}, 39 \mu \mathrm{mol}, 1.1 \mathrm{eq})$ was added via variable volume pipette. The reaction solution was transferred into a NMR tube and shaken for $48 \mathrm{~h}$ for equilibrium formation. A mixture of the reactants and $\left[\mathrm{Os}\left(\mathrm{NPMe}_{3}\right)\left(\mathrm{PNP}^{t \mathrm{Bu}}\right)\right](7)$ was observed by NMR spectroscopy.

${ }^{1} \mathbf{H}-\mathrm{NMR}\left(400 \mathrm{MHz}, \mathrm{C}_{6} \mathrm{D}_{6}, 25^{\circ} \mathrm{C}\right): \delta(\mathrm{ppm})=1.62-1.54\left(\mathrm{~m}, 4 \mathrm{H}, \mathrm{P}-\mathrm{CH}_{2}\right), 1.57$ (spin-sys, $\left.{ }^{3} J_{\mathrm{HP}}=11.8 \mathrm{~Hz}, 18 \mathrm{H}, \mathrm{C}\left(-\mathrm{CH}_{3}\right)_{3}\right), 1.24-1.16\left(\mathrm{~m}, 4 \mathrm{H}, \mathrm{N}-\mathrm{CH}_{2}\right), 1.22\left(\mathrm{~d},{ }^{2} \mathrm{~J}_{\mathrm{PH}}=11.5 \mathrm{~Hz}, 9 \mathrm{H}\right.$, $\left.\mathrm{P}\left(-\mathrm{CH}_{3}\right)_{3}\right) .{ }^{13} \mathrm{C}\left\{{ }^{1} \mathbf{H}\right\}-\mathrm{NMR}\left(100.7 \mathrm{MHz}, \mathrm{C}_{6} \mathrm{D}_{6}, 25^{\circ} \mathrm{C}\right): \delta(\mathrm{ppm})=85.6$ (vt, AXX'A', N $\left.=\left|{ }^{2} \mathrm{~J}_{\mathrm{CP}}+{ }^{4} \mathrm{~J}_{\mathrm{CP}}\right|=8.4 \mathrm{~Hz}, 2 \mathrm{C}, \mathrm{N}-\mathrm{CH}_{2}\right), 38.3\left(\mathrm{vt}, \mathrm{AXX} \mathrm{A}^{\prime}, \mathrm{N}=\left|{ }^{1} \mathrm{~J}_{\mathrm{CP}}+{ }^{3} \mathrm{~J}_{\mathrm{CP}}\right|=8.4 \mathrm{~Hz}, 2\right.$ $\left.\mathrm{C}, \mathrm{C}\left(-\mathrm{CH}_{3}\right)_{3}\right), 32.7\left(\mathrm{~m}, 12 \mathrm{C}, \mathrm{C}\left(-\mathrm{CH}_{3}\right)_{3}\right), 29.6\left(\mathrm{vt}, \mathrm{AXX} \mathrm{A}^{\prime}, \mathrm{N}=\left|{ }^{1} \mathrm{~J}_{\mathrm{CP}}+{ }^{3} \mathrm{~J}_{\mathrm{CP}}\right|=17.6 \mathrm{~Hz}\right.$, $\left.2 \mathrm{C}, \mathrm{P}-\mathrm{CH}_{2}\right), 27.8\left(\mathrm{~d},{ }^{1} \mathrm{~J} \mathrm{PC}=64.7 \mathrm{~Hz}, 3 \mathrm{C}, \mathrm{P}-\left(\mathrm{CH}_{3}\right)_{3}\right) .{ }^{15} \mathrm{~N}\left\{{ }^{1} \mathrm{H}\right\}-\mathrm{NMR}\left(38.0 \mathrm{MHz}, \mathrm{C}_{6} \mathrm{D}_{6}\right.$, $\left.25^{\circ} \mathrm{C}\right): \delta(\mathrm{ppm})=-126.5\left(\mathrm{~d},{ }^{1} \mathrm{~J}_{15 \mathrm{~N}-31 \mathrm{P}}=22.6 \mathrm{~Hz}\right) .{ }^{31} \mathbf{P}\left\{{ }^{1} \mathbf{H}\right\}-\mathrm{NMR}\left(162.0 \mathrm{MHz}, \mathrm{C}_{6} \mathrm{D}_{6}\right.$, $\left.25^{\circ} \mathrm{C}\right): \delta(\mathrm{ppm})=43.9(\mathrm{~s}, 2 \mathrm{P}, \mathrm{Os}-\mathrm{P}), 22.1(\mathrm{~s}, 1 \mathrm{P}, \mathrm{N}=\mathrm{P})$. Using $50 \%{ }^{15} \mathrm{~N}$-labeled 7 the signal at $22.1 \mathrm{ppm}$ was reduced by $50 \%$ and an additional doublet arose: $22.1\left(\mathrm{~d},{ }^{1} \mathrm{~J}_{15 \mathrm{~N}-31 \mathrm{P}}\right.$ $=22.7 \mathrm{~Hz})$.). ATR-IR $\left(\mathrm{C}_{6} \mathrm{H}_{6}\right): \tilde{\nu}\left(\mathrm{cm}^{-1}\right)=1240\left(\nu_{\text {Os- }}\right)$.

\subsection{7 $\left[\mathrm{OsH}_{4}\left(\mathrm{HPNP}^{t \mathrm{Bu}}\right)\right](8)$}

$\left[\mathrm{Os}(\mathrm{N})\left(\mathrm{PNP}^{t \mathrm{Bu}}\right)\right](3)(46 \mathrm{mg}, 82 \mu \mathrm{mol}, 1.0 \mathrm{eq})$ was dissolved in toluene $(8 \mathrm{~mL})$ in a Teflonstoppered tube. The solution was degased by three successive freeze-pump-thaw cycles and the tube was backfilled with $\mathrm{H}_{2}$ gas (2 bar). The solution was stirred over $36 \mathrm{~h}$ at $110^{\circ} \mathrm{C}$. All volatiles were removed in vacuo and the remaining solid was washed with cold pentane $\left(-10^{\circ} \mathrm{C}\right)$ and lyophilized to obtain $\left[\mathrm{OsH}_{4}\left(\mathrm{HPNP}^{t \mathrm{Bu}}\right)\right](8)$ as a yellow powder $(34 \mathrm{mg}, 61 \mu \mathrm{mol}$, $74 \%)$.

${ }^{1} \mathbf{H}-\mathrm{NMR}\left(400 \mathrm{MHz}, \mathrm{THF}-d_{8}, 25^{\circ} \mathrm{C}\right): \delta(\mathrm{ppm})=4.88\left(\mathrm{~s}_{\mathrm{br}}, 1 \mathrm{H}, \mathrm{NH}\right), 3.29-3.12(\mathrm{~m}, 2 \mathrm{H}$, $\left.\mathrm{NCH}_{2}\right), 2.20-2.03\left(\mathrm{~m}, 4 \mathrm{H}, \mathrm{NCH}_{2}+\mathrm{PCH}_{2}\right), 1.53-1.40(\mathrm{~m}, 2 \mathrm{H}, \mathrm{PCH}), 1.33$ (spin-sys, ${ }^{3} J_{\mathrm{HP}}$ $\left.=11.8 \mathrm{~Hz}, 18 \mathrm{H}, \mathrm{CH}_{3}\right), 1.27$ (spin-sys, $\left.{ }^{3} J_{\mathrm{HP}}=12.0 \mathrm{~Hz}, 18 \mathrm{H}, \mathrm{CH}_{3}\right),-6.00--6.66\left(\mathrm{sbr}_{\mathrm{br}}\right.$, $1 \mathrm{H}, \mathrm{Os}-H),-6.28--7.48\left(\mathrm{~s}_{\mathrm{br}}, 1 \mathrm{H}, \mathrm{Os} H\right),-13.11--14.33\left(\mathrm{~s}_{\mathrm{br}}, 2 \mathrm{H}, \mathrm{Os}-\mathrm{H}\right) .{ }^{13} \mathrm{C}\left\{{ }^{1} \mathbf{H}\right\}-$ NMR $\left(100.7 \mathrm{MHz}\right.$, THF- $\left.d_{8}, 25^{\circ} \mathrm{C}\right): \delta(\mathrm{ppm})=59.4\left(\mathrm{vt}, \mathrm{AXX} \mathrm{A}^{\prime}, \mathrm{N}=\left|{ }^{2} \mathrm{~J}_{\mathrm{CP}}+{ }^{4} \mathrm{~J}_{\mathrm{CP}}\right|=\right.$ $8.0 \mathrm{~Hz}, \mathrm{NCH}_{2}$ ), 33.1 (vt, AXX'A', N=|1 $\left.\mathrm{J}_{\mathrm{CP}}+^{3} \mathrm{~J}_{\mathrm{CP}} \mid=19.0 \mathrm{~Hz}, \mathrm{PC}\left(\mathrm{CH}_{3}\right)_{3}\right), 31.2$ (vt, $\left.\mathrm{AXX}^{\prime} \mathrm{A}^{\prime}, \mathrm{N}=\left|{ }^{1} \mathrm{~J}_{\mathrm{CP}}+^{3} \mathrm{~J}_{\mathrm{CP}}\right|=18.0 \mathrm{~Hz}, \mathrm{PC}\left(\mathrm{CH}_{3}\right)_{3}\right), 30.0\left(\mathrm{~m}, \mathrm{CH}_{3}\right), 29.9$ (vt, AXX'A', N $\left.=\left|{ }^{1} \mathrm{~J}_{\mathrm{CP}}+{ }^{3} \mathrm{~J}_{\mathrm{CP}}\right|=14.2 \mathrm{~Hz}, \mathrm{PCH}_{2}\right), 29.5\left(\mathrm{~m}, \mathrm{CH}_{3}\right) .79 .4$ (s). Elemental anal. Calc. for $\left[\mathrm{C}_{20} \mathrm{H}_{49} \mathrm{NOsP}_{2}\right] \cdot\left(0.065 \times \mathrm{C}_{6} \mathrm{H}_{6}\right): \mathrm{C}, 43.66 ; \mathrm{H}, 8.88 ; \mathrm{N}, 2.50$. Found: $\mathrm{C}, 44.12 ; \mathrm{H}, 8.56 ; \mathrm{N}$, 2.59. Mass spec. (LIFDI, toluene): $m / z=555.2\left(\left[\mathrm{M}-\mathrm{H}_{2}\right]^{+}\right)$(558.2 from experiment with $\left.\mathrm{D}_{2}\right)$. 


\subsection{8 $\left[\mathrm{OsH}_{3}\left(\mathrm{PNP}^{t \mathrm{Bu}}\right)\right](9)$}

A solid sample of lyophilized $\left[\mathrm{OsH}_{4}\left(\mathrm{HPNP}^{t \mathrm{tBu}}\right)\right](8)(5.0 \mathrm{mg}, 9.0 \mu \mathrm{g}, 1.0 \mathrm{eq})$ was heated to $110^{\circ} \mathrm{C}$ under applied vacuum for $16 \mathrm{~h}$. The solid was redissolved in $\mathrm{C}_{6} \mathrm{D}_{6}$ and measured.

${ }^{1} \mathbf{H}-\mathrm{NMR}\left(300 \mathrm{MHz}, \mathrm{CD}_{2} \mathrm{Cl}_{2}, 25^{\circ} \mathrm{C}\right): \delta(\mathrm{ppm})=3.11-3.01\left(\mathrm{~m}, 4 \mathrm{H}, \mathrm{NCH}_{2}\right), 1.85-1.75$ $\left(\mathrm{m}, 4 \mathrm{H}, \mathrm{PCH}\right.$ ), 1.25 (spin-sys, $\left.{ }^{3} J_{\mathrm{HP}}=12.4 \mathrm{~Hz}, 36 \mathrm{H}, \mathrm{C}\left(\mathrm{CH}_{3}\right)_{3}\right),-13.64$ (br, 3H, OsH $\mathrm{O}_{3}$ ). ${ }^{31} \mathbf{P}\left\{{ }^{1} \mathbf{H}\right\}-N M R\left(121.5 \mathrm{MHz}, \mathrm{CD}_{2} \mathrm{Cl}_{2}, 25^{\circ} \mathrm{C}\right): \delta(\mathrm{ppm})=96.1$ (s).

\subsection{9 $\left[\mathrm{Os}(\mathrm{N}) \mathrm{Cl}\left(\mathrm{PNP}^{t \mathrm{Bu}}\right)\right]^{\mathrm{BAr} r_{24}^{\mathrm{F}}}\left(10^{\mathrm{BAr}} \mathrm{F}_{24}^{\mathrm{F}}\right)$ and $\left[\mathrm{Os}(\mathrm{N}) \mathrm{Cl}_{2}\left(\mathrm{PNP}^{t \mathrm{Bu}}\right)\right]$ $(10-\mathrm{Cl})$}

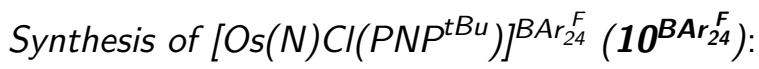

$\left[\mathrm{Os}(\mathrm{N})\left(\mathrm{PNP}^{t \mathrm{Bu}}\right]\right.$ (3) $(10.0 \mathrm{mg}, 17.8 \mu \mathrm{mol}, 1.0 \mathrm{eq})$ and $N$-chlorosuccinimide $(2.4 \mathrm{mg}, 17.8 \mu \mathrm{mol}$, $1.0 \mathrm{eq})$ are mixed in THF $(1 \mathrm{~mL})$ and stirred for $5 \mathrm{~min} . \mathrm{H}\left(\mathrm{Et}_{2} \mathrm{O}\right)_{2}\left\{\mathrm{BAr}_{24}^{\mathrm{F}}\right\}(18.0 \mathrm{mg}, 17.8 \mu \mathrm{mol}$, $1.0 \mathrm{eq})$ is added and the mixture is stirred for another $15 \mathrm{~min}$. The solvent is removed in vacuo, the product is extracted with $\mathrm{Et}_{2} \mathrm{O}$ and washed with pentanes.

${ }^{1} \mathbf{H}-N M R\left(400 \mathrm{MHz}, \mathrm{CD}_{2} \mathrm{Cl}_{2}, 25^{\circ} \mathrm{C}\right): \delta(\mathrm{ppm})=7.73\left(\mathrm{~m}, 8 \mathrm{H}, \mathrm{CH}_{\text {ortho }}\right), 7.57(\mathrm{~m}, 4 \mathrm{H}$, $\left.\mathrm{CH}_{\text {para }}\right), 4.18-4.01(\mathrm{~m}, 2 \mathrm{H}, \mathrm{N}-\mathrm{CHH}), 3.62-3.50(\mathrm{~m}, 2 \mathrm{H}, \mathrm{N}-\mathrm{CH}), 2.37-2.25(\mathrm{~m}, 2 \mathrm{H}$, $\mathrm{P}-\mathrm{CHH}), 2.18-2.08(\mathrm{~m}, 2 \mathrm{H}, \mathrm{P}-\mathrm{CH} H), 1.54$ (spin-sys, $\left.{ }^{3} \mathrm{~J}^{\mathrm{HP}}=16.0 \mathrm{~Hz}, 18 \mathrm{H}, \mathrm{C}\left(\mathrm{CH}_{3}\right)_{3}\right), 1.40$ (spin-sys, $\left.{ }^{3} \mathrm{JP}^{\mathrm{HP}}=15.8 \mathrm{~Hz}, 18 \mathrm{H}, \mathrm{C}\left(\mathrm{CH}_{3}\right)_{3}\right) .{ }^{13} \mathrm{C}\left\{{ }^{1} \mathbf{H}\right\}-\mathrm{NMR}\left(100.6 \mathrm{MHz}, \mathrm{CD}_{2} \mathrm{Cl}_{2}, 25^{\circ} \mathrm{C}\right): \delta$ $(\mathrm{ppm})=161.7\left(\mathrm{q},{ }^{1} J_{\mathrm{CB}}=49.8 \mathrm{~Hz}, C_{\text {ipso }}\right), 134.7\left(\mathrm{br}, C_{\text {ortho }} \mathrm{H}\right), 12 \mathrm{R} 8.8\left(\mathrm{qq},{ }^{2} J_{\mathrm{CF}}=31.2 \mathrm{~Hz}\right.$, ${ }^{3} J_{\mathrm{CB}}=2.9 \mathrm{~Hz}, C_{\text {meta }} \mathrm{CF}_{3}$ ), $124.5\left(\mathrm{q},{ }^{1} J_{\mathrm{CF}}=272.3 \mathrm{~Hz}, C_{3}\right.$ ), 117.4 (hept, ${ }^{3} J_{\mathrm{CF}}=4.1 \mathrm{~Hz}$, $\left.C_{\text {para }} \mathrm{H}\right), 72.0\left(\mathrm{~s}, \mathrm{~N}-\mathrm{CH}_{2}\right), 39.1\left(\mathrm{vt}, \mathrm{AXX} \mathrm{A}^{\prime}, \mathrm{N}=\left.\right|^{1} J_{\mathrm{CP}}+{ }^{2} J_{\mathrm{CP}} \mid=22.8 \mathrm{~Hz}, \mathrm{CMe}_{3}\right), 38.1$ (vt, $\left.\mathrm{AXX}^{\prime} \mathrm{A}^{\prime}, \mathrm{N}=\left.\right|^{1} J_{\mathrm{CP}}+{ }^{2} J_{\mathrm{CP}} \mid=16.1 \mathrm{~Hz}, \mathrm{CMe}_{3}\right), 28.7\left(\mathrm{~s}, \mathrm{C}\left(\mathrm{CH}_{3}\right)_{3}\right), 27.7\left(\mathrm{~s}, \mathrm{C}\left(\mathrm{CH}_{3}\right)_{3}\right), 23.1$ (vt, AXX'A', N=|1 $\left.J_{\mathrm{CP}}+{ }^{2} J_{\mathrm{CP}} \mid=26.9 \mathrm{~Hz}, \mathrm{P}-\mathrm{CH}_{2}\right) .{ }^{31} \mathrm{P}\left\{{ }^{1} \mathbf{H}\right\}-\mathrm{NMR}\left(162.0 \mathrm{MHz}, \mathrm{CD}_{2} \mathrm{Cl}_{2}\right.$, $\left.25^{\circ} \mathrm{C}\right): \delta(\mathrm{ppm})=96.2(\mathrm{~s})$. Elemental anal. Calc. for $\mathrm{C}_{52} \mathrm{H}_{56} \mathrm{BCIF}_{24} \mathrm{~N}_{2} \mathrm{OsP}_{2}: \mathrm{C}, 42.68 ; \mathrm{H}$, 3.86; N, 1.91. Found: C, 43.24; H, 3.95; N, 2.02. Mass spect. (LIFDI), $m / z(\%)=601.1$ $\left(100,[\mathrm{M}]^{+}\right)$.

Synthesis of $\left[\mathrm{Os}(\mathrm{N}) \mathrm{Cl}_{2}\left(P N P^{t B u}\right)\right](\mathbf{1 0}-\mathrm{Cl})$ :

Synthesis was done analogous to the above described procedure, but using $\mathrm{HCl}$ in $\mathrm{Et}_{2} \mathrm{O}$ instead of $\mathrm{H}\left(\mathrm{Et}_{2} \mathrm{O}\right)_{2}\left\{\mathrm{BAr}_{24}^{\mathrm{F}}\right\}$.

${ }^{1} \mathbf{H}-\mathbf{N M R}\left(300 \mathrm{MHz}, \mathrm{CD}_{2} \mathrm{Cl}_{2}, 25^{\circ} \mathrm{C}\right): \delta(\mathrm{ppm})=4.47-4.23(\mathrm{~m}, 2 \mathrm{H}, \mathrm{N}-\mathrm{CHH}), 3.74-3.57(\mathrm{~m}$, $2 \mathrm{H}, \mathrm{N}-\mathrm{CH} H), 2.59-2.42(\mathrm{~m}, 2 \mathrm{H}, \mathrm{P}-\mathrm{CHH}), 2.37-2.22(\mathrm{~m}, 2 \mathrm{H}, \mathrm{P}-\mathrm{CH}), 1.56$ (spin-sys, $\left.{ }^{3} J^{\mathrm{HP}}=15.9 \mathrm{~Hz}, 18 \mathrm{H}, \mathrm{C}\left(\mathrm{CH}_{3}\right)_{3}\right), 1.43\left(\right.$ spin-sys, $\left.{ }^{3} \mathrm{~J}^{\mathrm{HP}}=15.6 \mathrm{~Hz}, 18 \mathrm{H}, \mathrm{C}\left(\mathrm{CH}_{3}\right)_{3}\right) .{ }^{31} \mathbf{P}\left\{{ }^{1} \mathbf{H}\right\}-$ NMR $\left(121.5 \mathrm{MHz}, \mathrm{CD}_{2} \mathrm{Cl}_{2}, 25^{\circ} \mathrm{C}\right): \delta(\mathrm{ppm})=97.2(\mathrm{~s})$. Mass spect. (LIFDI), $\mathrm{m} / z(\%)$ $=601.2\left(100,[\mathrm{M}-\mathrm{Cl}]^{+}\right)$. 


\section{$\left[\mathrm{Os}\left(\mathrm{CN}^{\mathrm{t}} \mathrm{Bu}\right)_{2} \mathrm{Cl}\left\{\mathrm{N}\left(\mathrm{CH}_{2} \mathrm{CH}_{2} \mathrm{P}^{\mathrm{t}} \mathrm{Bu}_{2}\right)\left(=\mathrm{CHCH}_{2} \mathrm{P}\left({ }^{\mathrm{t}} \mathrm{Bu}\right)_{2}=\mathrm{NH}\right)\right\}\right]^{\mathrm{BAr}_{24}^{\mathrm{F}}}$ $\left(11 a^{\mathrm{BAr}_{24}^{\mathrm{F}}}\right.$ and $\left.11 \mathrm{~b}^{\mathrm{BAr}} \mathrm{r}_{24}^{\mathrm{F}}\right)$}

$\left[\mathrm{Os}(\mathrm{N}) \mathrm{Cl}\left(\mathrm{PNP}^{t \mathrm{Bu}}\right)\right]^{\mathrm{BAr}}{ }_{24}^{\mathrm{F}}\left(\mathbf{1 0}^{\mathrm{BAr}}{ }_{24}^{\mathrm{F}}\right)(5.0 \mathrm{mg}, 3.4 \mu \mathrm{mol}, 1.0 \mathrm{eq})$ is dissolved in THF $(0.5 \mathrm{~mL})$. ${ }^{\mathrm{t}} \mathrm{BuNC}(1.0 \mu, 8.5 \mu \mathrm{mol}, 2.5 \mathrm{eq})$ is added via Eppendorf pipette and the mixture is stirred overnight at RT. The solvent is removed in vacuo and the product is extracted with $\mathrm{Et}_{2} \mathrm{O}$.

${ }^{31} \mathbf{P}\left\{{ }^{1} \mathbf{H}\right\}$-NMR $\left(121.5 \mathrm{MHz}, \mathrm{CD}_{2} \mathrm{Cl}_{2}, 25^{\circ} \mathrm{C}\right): \delta(\mathrm{ppm})=62.5$ (doublet $(\mathrm{d}),{ }^{3} J_{\mathrm{PP}}=11.6 \mathrm{~Hz}$, A), $51.9\left(\mathrm{~d},{ }^{3} J_{\mathrm{PP}}=11.6 \mathrm{~Hz}, \mathbf{A}\right), 49.0\left(\mathrm{~d},{ }^{3} J_{\mathrm{PP}}=6.4 \mathrm{~Hz}, \mathbf{B}\right), 47.5\left(\mathrm{~d},{ }^{3} J_{\mathrm{PP}}=6.5 \mathrm{~Hz}, \mathbf{B}\right)$. Mass spect. (LIFDI), $m / z(\%)=767.2\left(100,[\mathrm{M}]^{+}\right), 731.2\left(25,[\mathrm{M}-\mathrm{HCl}]^{+}\right), 684.2(62$, $\left.\left[\mathrm{M}-\mathrm{CN}^{\mathrm{t}} \mathrm{Bu}\right]^{+}\right)$.

\subsubsection{1 $\left[\mathrm{OsH}\left(\mathrm{N}_{2}\right)\left(\mathrm{PNP}^{t \mathrm{Bu}}\right)\right]$}

$\left[\mathrm{OsHCl}_{2}\left(\mathrm{PNP}^{\mathrm{tBu}}\right)\right](\mathbf{1})(5.0 \mathrm{mg}, 8.0 \mu \mathrm{mol}, 1.0 \mathrm{eq})$ and $\mathrm{KC}_{8}(3.3 \mathrm{mg}, 24.1 \mu \mathrm{mol}, 3.0 \mathrm{eq})$ are suspended in THF $(\mathrm{Na} / \mathrm{K}, 0.7 \mathrm{~mL})$ under a dinitrogen atmosphere and stirred for $30 \mathrm{~min}$. All volatiles are removed in vacuo and the product is extracted with pentane. $\left[\mathrm{OsH}\left(\mathrm{N}_{2}\right)\left(\mathrm{PNP}^{t \mathrm{Bu}}\right)\right]$ (13) is obtained as orange solid.

${ }^{1} \mathbf{H}-\mathrm{NMR}\left(300 \mathrm{MHz}, \mathrm{C}_{6} \mathrm{D}_{6}, 25^{\circ} \mathrm{C}\right): \delta(\mathrm{ppm})=3.31-3.12(\mathrm{~m}, 2 \mathrm{H}, \mathrm{N}-\mathrm{CHH}), 2.56-2.44(\mathrm{~m}$, $2 \mathrm{H}, \mathrm{N}-\mathrm{CH} H), 1.91-1.77(\mathrm{~m}, 2 \mathrm{H}, \mathrm{P}-\mathrm{CHH}), 1.71-1.58(\mathrm{~m}, 2 \mathrm{H}, \mathrm{P}-\mathrm{CH}), 1.34$ (spin-sys, $\left.{ }^{3} J_{\mathrm{HP}}=12.6 \mathrm{~Hz}, 18 \mathrm{H}, \mathrm{C}\left(\mathrm{CH}_{3}\right)_{3}\right), 1.28$ (spin-sys, $\left.{ }^{3} J_{\mathrm{HP}}=12.6 \mathrm{~Hz}, 18 \mathrm{H}, \mathrm{C}\left(\mathrm{CH}_{3}\right)_{3}\right),-26.9$ (triplet (t), $\left.{ }^{2} J_{\mathrm{HP}}=12.2 \mathrm{~Hz}, 1 \mathrm{H}, \mathrm{OsH}\right) .{ }^{31} \mathrm{P}\left\{{ }^{1} \mathrm{H}\right\}-\mathrm{NMR}\left(121.5 \mathrm{MHz}, \mathrm{C}_{6} \mathrm{D}_{6}, 25^{\circ} \mathrm{C}\right): \delta(\mathrm{ppm})$ $=85.9(\mathrm{~s})$. ATR-IR: $\tilde{\nu}\left(\mathrm{cm}^{-1}\right)=2114.5\left(\nu_{\mathrm{OsH}}\right), 2007.7\left(\nu_{\mathrm{N} \equiv \mathrm{N}}\right)$.

\subsubsection{2 $\left[\mathrm{OsHCl}\left(\mathrm{PNP}^{t \mathrm{Bu}}\right)\right]^{\mathrm{BPh}_{4}}\left(12^{\mathrm{BPh}_{4}}\right)$}

$\left[\mathrm{OsHCl}_{2}\left(\mathrm{PNP}^{t \mathrm{Bu}}\right)\right](\mathbf{1})(33.0 \mathrm{mg}, 53.0 \mu \mathrm{mol}, 1.0 \mathrm{eq})$ and $\mathrm{NaBPh}_{4}(20.0 \mathrm{mg}, 58.3 \mu \mathrm{mol}, 1.1 \mathrm{eq})$ are stirred for $20 \mathrm{~min}$ in THF $(1 \mathrm{~mL})$. Afterwards, all volatiles are removed in vacuo, the product is extracted with $\mathrm{DCM}$, concentrated and crystallized by layering with $\mathrm{Et}_{2} \mathrm{O}$ at $-40^{\circ} \mathrm{C}$. $\left[\mathrm{OsHCl}\left(\mathrm{PNP}^{t \mathrm{Bu}}\right)\right]^{\mathrm{BPh}_{4}}\left(\mathbf{1 2}^{\mathrm{BPh}_{4}}\right)$ is obtained as green powder $(33.0 \mathrm{mg}, \approx 70 \%)$.

${ }^{1} \mathbf{H}-\mathbf{N M R}\left(300 \mathrm{MHz}, \mathrm{CD}_{2} \mathrm{Cl}_{2}, 25^{\circ} \mathrm{C}\right): \delta(\mathrm{ppm})=7.51-7.43\left(\mathrm{~m}, 8 \mathrm{H}, \mathrm{C}_{\text {ortho }} H\right), 7.07\left(\mathrm{t},{ }^{3} J_{\mathrm{HH}}=\right.$ $\left.7.3 \mathrm{~Hz}, 8 \mathrm{H}, \mathrm{C}_{\text {meta }} H\right), 6.88\left(\mathrm{t},{ }^{3} J_{\mathrm{HH}}=7.2 \mathrm{~Hz}, 4 \mathrm{H}, \mathrm{C}_{\text {para }} H\right), 2.45-2.28(\mathrm{~m}, 4 \mathrm{H}, \mathrm{N}-\mathrm{CHH}), 2.06-$ $1.81(\mathrm{~m}, 4 \mathrm{H}, \mathrm{P}-\mathrm{CHH}), 1.38$ (spin-sys, $\left.{ }^{3} J_{\mathrm{HP}}=14.5 \mathrm{~Hz}, 18 \mathrm{H}, \mathrm{C}\left(\mathrm{CH}_{3}\right)_{3}\right), 1.32$ (spin-sys, ${ }^{3} J_{\mathrm{HP}}$ $\left.=14.1 \mathrm{~Hz}, 18 \mathrm{H}, \mathrm{C}\left(\mathrm{CH}_{3}\right)_{3}\right),-20.79(\mathrm{br}, 1 \mathrm{H}, \mathrm{Os} H) .{ }^{31} \mathbf{P}\left\{{ }^{1} \mathbf{H}\right\}-\mathrm{NMR}\left(121.5 \mathrm{MHz}, \mathrm{CD}_{2} \mathrm{Cl}_{2}\right.$, $\left.25^{\circ} \mathrm{C}\right): \delta(\mathrm{ppm})=46.0\left(\mathrm{sbr}_{\mathrm{br}}\right.$. Mass spect. (LIFDI), $m / z(\%)=588.1\left(100,[\mathrm{M}]^{+}\right)$. 


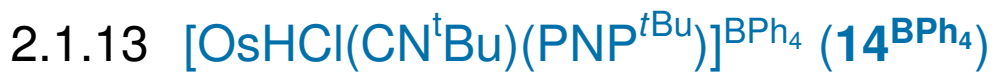

$\left[\mathrm{OsHCl}\left(\mathrm{PNP}^{t \mathrm{Bu}}\right)\right]^{\mathrm{BPh}_{4}}\left(\mathbf{1 2}^{\mathrm{BPh}_{4}}\right)(20.0 \mathrm{mg}, 22.1 \mu \mathrm{mol}, 1.0 \mathrm{eq})$ and tert-butylisocyanide $(2.5 \mu \mathrm{L}$, $1.8 \mathrm{mg}, 22.1 \mu \mathrm{mol}, 1.0 \mathrm{eq})$ are separately dissolved in DCM $(2 \mathrm{~mL}$ each $)$. The $\mathrm{CN}^{\mathrm{t}} \mathrm{Bu}$ solution is added dropwise to $12^{\mathrm{BPh}_{4}}$ at $-0^{\circ} \mathrm{C}$. The solution is stirred for $10 \mathrm{~min}$, the solvent is removed in vacuo and the product is washed with $\mathrm{Et}_{2} \mathrm{O}$ and dried. $\left[\mathrm{OsHCl}\left(\mathrm{CN}^{\mathrm{t}} \mathrm{Bu}\right)\left(\mathrm{PNP}^{t \mathrm{Bu}}\right)\right]^{\mathrm{BPh}_{4}}$ $\left(14^{\mathrm{BPh}_{4}}\right)$ is obtained as a spectroscopically clean yellow powder.

${ }^{1} \mathbf{H}-\mathrm{NMR}\left(300 \mathrm{MHz}, \mathrm{CD}_{2} \mathrm{Cl}_{2}, 25^{\circ} \mathrm{C}\right): \delta(\mathrm{ppm})=7.50-7.41\left(\mathrm{~m}, 8 \mathrm{H}, \mathrm{C}_{\text {ortho }} \mathrm{H}\right), 7.07\left(\mathrm{t},{ }^{3} \mathrm{~J}_{\mathrm{HH}}\right.$ $\left.=7.4 \mathrm{~Hz}, 8 \mathrm{H}, \mathrm{C}_{\text {meta }} H\right), 6.89\left(\mathrm{t},{ }^{3} J_{\mathrm{HH}}=7.2 \mathrm{~Hz}, 4 \mathrm{H}, \mathrm{C}_{\text {para }} H\right), 1.46$ (spin-sys, ${ }^{3} J_{\mathrm{HP}}=$ $\left.13.9 \mathrm{~Hz}, 18 \mathrm{H}, \mathrm{C}\left(\mathrm{CH}_{3}\right)_{3}\right), 1.47\left(\mathrm{~s}, 9 \mathrm{H}, \mathrm{C} \equiv \mathrm{N}-\mathrm{C}\left(\mathrm{CH}_{3}\right)_{3}\right), 1.10$ (spin-sys, ${ }^{3} J_{\mathrm{HP}}=12.9 \mathrm{~Hz}$, $\left.18 \mathrm{H}, \mathrm{C}\left(\mathrm{CH}_{3}\right)_{3}\right), 0.30(\mathrm{br}, 1 \mathrm{H}, \mathrm{OsH}) .{ }^{31} \mathbf{P}\left\{{ }^{1} \mathbf{H}\right\}-\mathrm{NMR}\left(121.5 \mathrm{MHz}, \mathrm{CD}_{2} \mathrm{Cl}_{2}, 25^{\circ} \mathrm{C}\right): \delta(\mathrm{ppm})$ $=48.2(\mathrm{~s})$. Mass spect. (LIFDI), $m / z(\%)=671.2\left(100,[\mathrm{M}]^{+}\right), 635.3\left(50,[\mathrm{M}-\mathrm{HCl}]^{+}\right)$.

\subsubsection{4 $\left[\mathrm{OsCl}\left(\mathrm{CN}^{\mathrm{t}} \mathrm{Bu}\right)\left(\mathrm{PNP}^{\mathrm{tB} u}\right)\right]$}

$\left[\mathrm{OsHCl}\left(\mathrm{CN}^{\mathrm{t}} \mathrm{Bu}\right)\left(\mathrm{PNP}^{t \mathrm{Bu}}\right)\right]^{\mathrm{BPh}_{4}}\left(\mathbf{1 4}^{\mathrm{BPh}_{4}}\right)(61.3 \mathrm{mg}, 61.9 \mu \mathrm{mol}, 1.0 \mathrm{eq})$ and $\mathrm{KO}^{t} \mathrm{Bu}(7.2 \mathrm{mg}$, $64.2 \mu \mathrm{mol}, 1.05 \mathrm{eq})$ are mixed in THF $(5 \mathrm{~mL})$ and stirred for $15 \mathrm{~min}$. All volatiles are removed in vacuo, the product is extracted with pentane, concentrated and stored at $-40^{\circ} \mathrm{C}$ overnight. The solution is filtered and the residue is taken up in $\mathrm{C}_{6} \mathrm{H}_{6}$ and lyophilized. $\left[\mathrm{OsCl}\left(\mathrm{CN}^{t} \mathrm{Bu}\right)\left(\mathrm{PNP}^{t \mathrm{Bu}}\right)\right](\mathbf{1 5}$ is obtained as a red-brownish powder.

${ }^{1} \mathbf{H}-\mathbf{N M R}\left(400 \mathrm{MHz}, \mathrm{THF}-d_{8}, 25^{\circ} \mathrm{C}\right): \delta(\mathrm{ppm})=2.95-2.81(\mathrm{~m}, 2 \mathrm{H}, \mathrm{N}-\mathrm{CHH}), 2.55-2.46(\mathrm{~m}$, $2 \mathrm{H}, \mathrm{N}-\mathrm{CHH}), 1.62-1.54\left(\mathrm{~m}, 4 \mathrm{H}, \mathrm{P}-\mathrm{CH}_{2}\right), 1.46$ (spin-sys, $\left.{ }^{3} J_{\mathrm{HP}}=12.8 \mathrm{~Hz}, 18 \mathrm{H}, \mathrm{C}\left(\mathrm{CH}_{3}\right)_{3}\right)$, 1.43 (spin-sys, $\left.{ }^{3} J_{\mathrm{HP}}=12.6 \mathrm{~Hz}, 18 \mathrm{H}, \mathrm{C}\left(\mathrm{CH}_{3}\right)_{3}\right), 1.40\left(\mathrm{~s}, 9 \mathrm{H}, \mathrm{C} \equiv \mathrm{N}-\mathrm{C}\left(\mathrm{CH}_{3}\right)_{3}\right) .{ }^{13} \mathrm{C}\left\{{ }^{1} \mathrm{H}\right\}-$ NMR $\left(100.6 \mathrm{MHz}, \mathrm{THF}-d_{8}, 25^{\circ} \mathrm{C}\right): \delta(\mathrm{ppm})=167.9\left(\mathrm{t},{ }^{2} J_{\mathrm{CP}}=6.3 \mathrm{~Hz}, C \equiv \mathrm{N}^{\mathrm{t}} \mathrm{Bu}\right), 69.8$

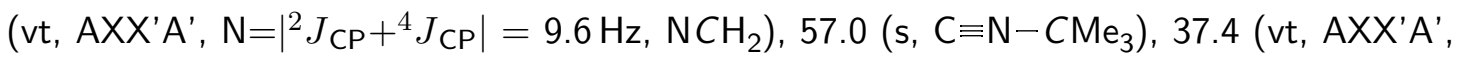
$\mathrm{N}=\left|{ }^{1} J_{\mathrm{CP}}+{ }^{3} J_{\mathrm{CP}}\right|=21.2 \mathrm{~Hz}, \mathrm{PCMe}$ ), $36.3\left(\mathrm{vt}, \mathrm{AXX} \mathrm{A}^{\prime}, \mathrm{N}=\left|{ }^{1} J_{\mathrm{CP}}+{ }^{3} J_{\mathrm{CP}}\right|=17.6 \mathrm{~Hz}, \mathrm{P}\right.$ $\left.\mathrm{CMe}_{3}\right), 33.2\left(\mathrm{~s}, \mathrm{C} \equiv \mathrm{N}-\mathrm{C}\left(\mathrm{CH}_{3}\right)_{3}\right), 29.8\left(\mathrm{~m}, \mathrm{PC}\left(\mathrm{CH}_{3}\right)_{3}\right), 29.8\left(\mathrm{~m}, \mathrm{PC}\left(\mathrm{CH}_{3}\right)_{3}\right), 25.7$ (vt, AXX'A', N=| $\left.{ }^{1} J_{\mathrm{CP}}+{ }^{3} J_{\mathrm{CP}} \mid=20.2 \mathrm{~Hz}, \mathrm{PCH}\right) .{ }^{31} \mathbf{P}\left\{{ }^{1} \mathbf{H}\right\}-\mathrm{NMR}\left(162.0 \mathrm{MHz}, \mathrm{THF}-d_{8}, 25^{\circ} \mathrm{C}\right)$ : $\delta(\mathrm{ppm})=66.8(\mathrm{~s})$. Mass spect. (LIFDI), $m / z(\%)=670.1\left(100,[\mathrm{M}]^{+}\right)$. 


\subsection{Rhenium compounds based on the PNP ${ }^{\text {BBu }}$ ligand}

\subsection{1 $\left[\left(\mu-\mathrm{N}_{2}\right)\left\{\operatorname{ReCl}\left(\mathrm{PNP}^{t \mathrm{Bu}}\right)\right\}_{2}\right](\mathbf{X X})$}

In a typical experiment, $\left[\operatorname{ReCl}_{2}\left(\mathrm{PNP}^{t \mathrm{Bu}}\right)\right](5.0 \mathrm{mg}, 8.1 \mu \mathrm{mol}, 1.0 \mathrm{eq})$ was mixed in solid state with $\mathrm{NaHg}(1.0 \mathrm{M}, 125 \mathrm{mg}, 9.2 \mu \mathrm{mol}, 1.15 \mathrm{eq})$ in a J-Young NMR tube. The tube was connected to a condensation bridge and THF- $d_{8}$ was degassed and vacuum-transferred to the tube. The NMR tube was backfilled with $\mathrm{N}_{2}$ gas, thawed and shaken in a $-35{ }^{\circ} \mathrm{C}$ cooling bath for $10 \mathrm{~min}$ before the tube was placed in a cooled spectrometer for analysis. If a kinetic study was performed, a capillary with $\mathrm{P}(\mathrm{OTMS})_{3}$ in toluene- $d_{8}$ was added at the very beginning of the reaction.

${ }^{1} \mathbf{H}-N M R\left(400 \mathrm{MHz}, \mathrm{THF}-d_{8},-50{ }^{\circ} \mathrm{C}\right): \delta(\mathrm{ppm})=12.23\left(\mathrm{~m}, 2 \mathrm{H}, \mathrm{CH}_{2}\right), 10.56(\mathrm{~m}, 2 \mathrm{H}$, $\left.\mathrm{CH}_{2}\right), 8.60\left(\mathrm{~m}, 2 \mathrm{H}, \mathrm{CH}_{2}\right), 4.00\left(\mathrm{~m}, 2 \mathrm{H}, \mathrm{CH}_{2}\right), 2.64\left(\mathrm{~m}, 2 \mathrm{H}, \mathrm{CH}_{2}\right),-0.22\left(\mathrm{~m}, 2 \mathrm{H}, \mathrm{CH}_{2}\right)$, $-11.75\left(\mathrm{~m}, 2 \mathrm{H}, \mathrm{CH}_{2}\right),-16.74\left(\mathrm{~m}, 2 \mathrm{H}, \mathrm{CH}_{2}\right)$ (signals for the tert-butyl groups could not be identified at this temperature, as they became very broad due to some dynamic effects). ${ }^{31} \mathbf{P}\left\{{ }^{1} \mathbf{H}\right\}-N M R\left(162.0 \mathrm{MHz}\right.$, THF- $\left.d_{8},-50{ }^{\circ} \mathrm{C}\right): \delta(\mathrm{ppm})=16.5\left(\mathrm{~d},{ }^{2} J_{\mathrm{PP}}=234.5 \mathrm{~Hz}\right)$, $-115.3\left(\mathrm{~d},{ }^{2} J_{\mathrm{PP}}=234.2 \mathrm{~Hz}\right)$.

\subsection{2 $\left[\mathrm{ReHCl}\left(\mathrm{PNP}^{t \mathrm{Bu}}\right)\right](17)$}

$\left[\operatorname{ReCl}_{2}\left(\mathrm{PNP}^{t \mathrm{Bu}}\right)\right](\mathbf{X I X})(16.1 \mathrm{mg}, 26.1 \mu \mathrm{mol}, 1.0 \mathrm{eq})$ and $\mathrm{Co}\left(\mathrm{Cp}^{*}\right)_{2}(17.2 \mathrm{mg}, 52.1 \mu \mathrm{mol}$, $2.0 \mathrm{eq})$ were mixed in THF $(\mathrm{Na} / \mathrm{K}, 1.0 \mathrm{~mL})$ and stirred for $1.5 \mathrm{~h}$. The solvent was removed in vacuo and the product is extracted with $\mathrm{Et}_{2} \mathrm{O}$. Afterwards the product was washed with cold pentane to yield rather clean $\left[\mathrm{ReHCl}\left(\mathrm{PNP}^{t \mathrm{Bu}}\right)\right](\mathbf{1 7})$.

${ }^{1} \mathbf{H}-N M R\left(300 \mathrm{MHz}, \mathrm{C}_{6} \mathrm{D}_{6}, 25^{\circ} \mathrm{C}\right): \delta(\mathrm{ppm})=3.15-2.87\left(\mathrm{~m}, 4 \mathrm{H}, \mathrm{CH}_{2}\right), 1.69-1.49(\mathrm{~m}, 2 \mathrm{H}$, $\mathrm{CH}_{2}$ ) 1.37 (spin-sys, $\left.{ }^{3} J_{\mathrm{HP}}=12.3 \mathrm{~Hz}, 18 \mathrm{H}, \mathrm{C}\left(\mathrm{CH}_{3}\right)_{3}\right), 1.32$ (spin-sys, ${ }^{3} J_{\mathrm{HP}}=12.0 \mathrm{~Hz}$, $\left.18 \mathrm{H}, \mathrm{C}\left(\mathrm{CH}_{3}\right)_{3}\right),-9.30\left(\mathrm{t},{ }^{2} J_{\mathrm{HP}}=13.1 \mathrm{~Hz}, 1 \mathrm{H}, \mathrm{Re}-\mathrm{H}\right)$. The last $\mathrm{CH}_{2}$ signal is superimposed by the tert-butyl signals and was thus not identified. ${ }^{31} \mathbf{P}\left\{{ }^{1} \mathbf{H}\right\}-\mathbf{N M R}\left(121.5 \mathrm{MHz}, \mathrm{C}_{6} \mathrm{D}_{6}\right.$, $\left.25^{\circ} \mathrm{C}\right): \delta(\mathrm{ppm})=29.8$.

\subsection{3 $\left[\left(\mathrm{N}, \mathrm{N}-\mathrm{C}_{2} \mathrm{H}_{4} \mathrm{~N}_{2}\right)\left\{\mathrm{ReCl}\left(\mathrm{PNP}^{t \mathrm{Bu}}\right)\right\}_{2}\right]^{(\mathrm{OTf})_{2}}\left(19^{(\mathrm{OTf})_{2}}\right)$}

$\left[\operatorname{Re}\left(\mathrm{NCH}_{2}\right) \mathrm{Cl}\left(\mathrm{PNP}^{t \mathrm{Bu}}\right)\right] \quad(\mathbf{1 8}) \quad(10.0 \mathrm{mg}, 16.4 \mu \mathrm{mol}, 1.0 \mathrm{eq})$ and $\left[\mathrm{Fe}(\mathrm{Cp})_{2}\right] \mathrm{OTf}(5.5 \mathrm{mg}$, $16.4 \mu \mathrm{mol}, 1.0 \mathrm{eq}$ ) are mixed in THF (dried over $\mathrm{Na} / \mathrm{K}, 0.5 \mathrm{~mL}$ ) and stirred for $1.5 \mathrm{~h}$. Afterwards the solvent is removed in vacuo, the product is washed with $\mathrm{Et}_{2} \mathrm{O}$ and recrys- 
tallized from THF $/ \mathrm{Et}_{2} \mathrm{O}$. $\left[\left(N, N-\mathrm{C}_{2} \mathrm{H}_{4} \mathrm{~N}_{2}\right)\left\{\mathrm{ReCl}\left(\mathrm{PNP}^{t \mathrm{Bu}}\right)\right\}_{2}\right]^{2 \mathrm{OTf}}\left(\mathbf{1 9}(\mathrm{OTf})_{2}\right)$ is obtained as deep green powder $(7.6 \mathrm{mg}, 5.0 \mu \mathrm{mol}, 61 \%$ yield $)$.

${ }^{1} \mathbf{H}-\mathrm{NMR}\left(400 \mathrm{MHz}, \mathrm{CD}_{2} \mathrm{Cl}_{2}, 25^{\circ} \mathrm{C}\right): \delta(\mathrm{ppm})=4.45-4.29\left(\mathrm{~m}, 4 \mathrm{H}, \mathrm{N}-\mathrm{CHH}-\mathrm{CH}_{2}\right), 3.93-$ $3.82\left(\mathrm{~m}, 4 \mathrm{H}, \mathrm{N}-\mathrm{CH} H-\mathrm{CH}_{2}\right), 3.31\left(\mathrm{~s}, 4 \mathrm{H}, \mathrm{Re}=\mathrm{N}-\mathrm{CH}_{2}\right)$ 2.55-2.42 (m, 4H, $\left.\mathrm{P}-\mathrm{CHH}-\mathrm{CH}_{2}\right)$, 2.23-2.11 (m, 4H, P-CHH- $\left.\mathrm{CH}_{2}\right), 1.47$ (spin-sys, $\left.{ }^{3} J_{\mathrm{HP}}=14.6 \mathrm{~Hz}, 32 \mathrm{H}, \mathrm{C}\left(\mathrm{CH}_{3}\right)_{3}\right), 1.29$ (spin-sys, $\left.{ }^{3} J_{\mathrm{HP}}=14.3 \mathrm{~Hz}, 32 \mathrm{H}, \mathrm{C}\left(\mathrm{CH}_{3}\right)_{3}\right) .{ }^{13} \mathrm{C}\left\{{ }^{1} \mathbf{H}\right\}-\mathrm{NMR}\left(100.6 \mathrm{MHz}, \mathrm{CD}_{2} \mathrm{Cl}_{2}, 25^{\circ} \mathrm{C}\right): \delta$ $(\mathrm{ppm})=76.6\left(\mathrm{~s}, \mathrm{~N}-\mathrm{CH}_{2}-\mathrm{CH}_{2}\right), 67.8\left(\mathrm{~s}, \mathrm{Re}=\mathrm{N}-\mathrm{CH}_{2}\right), 38.5\left(\mathrm{vt},{ }^{1} J_{\mathrm{CP}}=10.5 \mathrm{~Hz}, \mathrm{C}\left(\mathrm{CH}_{3}\right)_{3}\right)$, $38.0\left(\mathrm{vt},{ }^{1} J_{\mathrm{CP}}=8.7 \mathrm{~Hz}, \mathrm{C}\left(\mathrm{CH}_{3}\right)_{3}\right), 29.7\left(\mathrm{~m}\right.$, both $\left.\mathrm{C}\left(\mathrm{CH}_{3}\right)_{3}\right), 24.2\left(\mathrm{t},{ }^{1} J_{\mathrm{CP}}=11.2 \mathrm{~Hz}\right.$, $\left.\mathrm{P}-\mathrm{CH}_{2}-\mathrm{CH}_{2}\right) .{ }^{31} \mathrm{P}\left\{{ }^{1} \mathbf{H}\right\}-\mathrm{NMR}\left(162.0 \mathrm{MHz}, \mathrm{CD}_{2} \mathrm{Cl}_{2}, 25^{\circ} \mathrm{C}\right): \delta(\mathrm{ppm})=90.2$ (s). Elemental anal. Calcd. for $\mathrm{C}_{44} \mathrm{H}_{32} \mathrm{Cl}_{2} \mathrm{~F}_{6} \mathrm{~N}_{4} \mathrm{O}_{6} \mathrm{P}_{4} \mathrm{Re}_{2} \mathrm{~S}_{2}$ : C, 34.80; $\mathrm{H}, 6.11 ; \mathrm{N}, 3.69$. Found: $\mathrm{C}$, $35.09 ; \mathrm{H}, 6.04 ; \mathrm{N}, 3.46$.

2.2.4 $\left[\operatorname{Re}(\mathrm{O}) \mathrm{Cl}\left(\mathrm{PNP}^{t \mathrm{Bu}}\right)\right]^{\mathrm{BPh}_{4}}\left(20^{\mathrm{BPh}_{4}}\right)$

$\left[\operatorname{ReCl}_{2}\left(\mathrm{PNP}^{t \mathrm{Bu}}\right)\right](\mathbf{X I X})(8.0 \mathrm{mg}, 13.0 \mu \mathrm{mol}, 1.0 \mathrm{eq}), \mathrm{NaBPh}_{4}(4.4 \mathrm{mg}, 13.0 \mu \mathrm{mol}, 1.0 \mathrm{eq})$ and $\mathrm{Me}_{3} \mathrm{NO}(1.0 \mathrm{mg}, 13.0 \mu \mathrm{mol}, 1.0 \mathrm{eq})$ are mixed in THF (dried over $\mathrm{Na} / \mathrm{K}, 1 \mathrm{~mL}$ ) and stirred overnight. All volatiles are removed in vacuo, the product is washed with $\mathrm{Et}_{2} \mathrm{O}$ and extracted with $\mathrm{DCM}$. $\left[\mathrm{Re}(\mathrm{O}) \mathrm{Cl}\left(\mathrm{PNP}^{t \mathrm{Bu}}\right)\right]^{\mathrm{BPh}_{4}}\left(\mathbf{2 0}^{\mathrm{BPh}_{4}}\right)$ is obtained as a green powder (9.6 mg, $10.5 \mu \mathrm{mol}, 80.8 \%$ yield).

${ }^{1} \mathbf{H}-\mathbf{N M R}\left(500 \mathrm{MHz}, \mathrm{CD}_{2} \mathrm{Cl}_{2}, 25^{\circ} \mathrm{C}\right): \delta(\mathrm{ppm})=7.35\left(\mathrm{~m}, 8 \mathrm{H}, H_{\text {ortho }}\right), 7.04\left(\mathrm{t},{ }^{3} J_{\mathrm{HH}}=\right.$ $\left.7.0 \mathrm{~Hz}, 8 \mathrm{H}, H_{\text {meta }}\right), 6.89\left(\mathrm{t},{ }^{3} J_{\mathrm{HH}}=6.9 \mathrm{~Hz}, 4 \mathrm{H}, H_{\text {para }}\right), 3.99-3.88\left(\mathrm{~m}, 2 \mathrm{H}, \mathrm{N}-\mathrm{CHH}-\mathrm{CH}_{2}\right)$, 3.77-3.67 (m, 2H, N-CHH- $\left.\mathrm{CH}_{2}\right), 2.35-2.25\left(\mathrm{~m}, 2 \mathrm{H}, \mathrm{P}-\mathrm{CHH}-\mathrm{CH}_{2}\right), 2.03-1.93(\mathrm{~m}, 2 \mathrm{H}$, $\mathrm{P}-\mathrm{CH} H-\mathrm{CH}_{2}$ ), 1.50 (spin-sys, $\left.{ }^{3} J_{\mathrm{HP}}=15.2 \mathrm{~Hz}, 18 \mathrm{H}, \mathrm{C}\left(\mathrm{CH}_{3}\right)_{3}\right), 1.31$ (spin-sys, ${ }^{3} J_{\mathrm{HP}}=$ $\left.15.3 \mathrm{~Hz}, 18 \mathrm{H}, \mathrm{C}\left(\mathrm{CH}_{3}\right)_{3}\right) .{ }^{13} \mathrm{C}\left\{{ }^{1} \mathbf{H}\right\}-\mathbf{N M R}\left(125.8 \mathrm{MHz}, \mathrm{CD}_{2} \mathrm{Cl}_{2}, 25^{\circ} \mathrm{C}\right): \delta(\mathrm{ppm})=164.3$ $\left({ }^{1} J_{\mathrm{CB}}=49.4 \mathrm{~Hz}, C_{\text {ipso }}\right), 137.2\left({ }^{2} J_{\mathrm{CB}}=1.3 \mathrm{~Hz}, C_{\text {ortho }}\right), 126.1\left({ }^{3} J_{\mathrm{CB}}=2.7 \mathrm{~Hz}, C_{\text {meta }}\right), 122.2$ $\left(\mathrm{s}, C_{\text {para }}\right), 76.6\left(\mathrm{vt},{ }^{2} J_{\mathrm{CP}}=2.0 \mathrm{~Hz}, \mathrm{~N}-\mathrm{CH}_{2}-\mathrm{CH}_{2}\right), 39.8\left(\mathrm{vt},{ }^{1} J_{\mathrm{CP}}=11.1 \mathrm{~Hz}, \mathrm{P}-\mathrm{C}\left(\mathrm{CH}_{3}\right)_{3}\right)$,

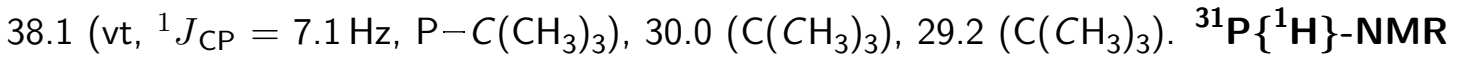
$\left(121.5 \mathrm{MHz}, \mathrm{CD}_{2} \mathrm{Cl}_{2}, 25^{\circ} \mathrm{C}\right): \delta(\mathrm{ppm})=97.7$ (s). Mass spect. (LIFDI), $m / z(\%)=598.3$ (100). 


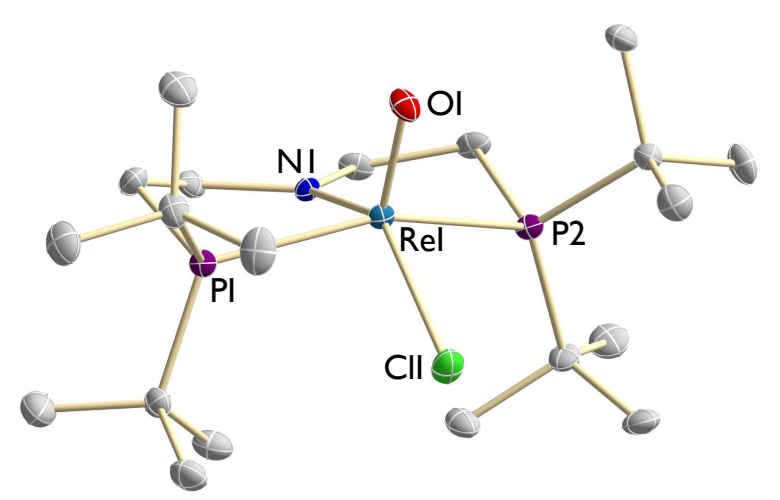

Fig. 2.1. Molecular structure of $\mathbf{3 7}$ obtained by single crystal $\mathrm{X}$-ray diffraction measurements. All $\mathrm{H}$ atoms, a cocrystallized $\mathrm{Et}_{2} \mathrm{O}$ molecule as well as the anion are omitted for clarity. Anisotropic displacement parameters are set to $50 \%$ probability. Selected bond length $[\AA]$ and angles [ $\left.{ }^{\circ}\right]$ : Re1-O1 1.681(3), Re1-N1 1.934(3), Re1-Cl1 2.3594(12), P1-Re1-P2 151.96(4), N1-Re1-Cl1 135.90(10), N1-Re1-O1 112.14(14).

\subsection{5 $\left[\operatorname{ReCl}_{2}\left(\mathrm{P}=\mathrm{N}=\mathrm{P}^{t \mathrm{Bu}}\right)\right](21)$}

$\left[\mathrm{ReCl}_{2}\left(\mathrm{PNP}^{t \mathrm{Bu}}\right)\right](\mathbf{X I X})(120 \mathrm{mg}, 194 \mu \mathrm{mol}, 1.0 \mathrm{eq})$ and the 2,4,6-tri-tert-butylphenoxy radical $(305 \mathrm{mg}, 1.17 \mathrm{mmol}, 6.0 \mathrm{eq})$ were mixed in dry benzene $(15 \mathrm{~mL})$ and stirred for $24 \mathrm{~h}$ at $50^{\circ} \mathrm{C}$. The solvent was removed in vacuo and the product was washed with excess pentane, until the washing solution was colorless. $\mathrm{Co}(\mathrm{Cp})_{2}(7 \mathrm{mg}, 37 \mu \mathrm{mol}, 0.2 \mathrm{eq})$ was added and the product was dissolved in benzene and stirred for $2 \mathrm{~h}$ at RT. The reaction mixture was filtered, the benzene phase was lyophilized and remaining $\mathrm{Co}(\mathrm{Cp})_{2}$ and 2,4,6-tri-tertbutylphenol were sublimed off overnight at $75^{\circ} \mathrm{C}$. $\left[\operatorname{ReCl}_{2}\left(\mathrm{P}=\mathrm{N}=\mathrm{P}^{t \mathrm{Bu}}\right)\right](\mathbf{2 1})$ was obtained as a brown powder $(75.1 \mathrm{mg}, 122.4 \mu \mathrm{mol}, 63 \%$ yield.

Spectroscopic characterization matched the previously published features of 21. ${ }^{180]}$

2.2.6 $\left[\operatorname{Re}(\mathrm{N}) \mathrm{Cl}\left(\mathrm{P}=\mathrm{N}=\mathrm{P}^{t \mathrm{Bu}}\right)\right]$

From $\mathrm{N}_{2}$ :

Degassed THF $(0.45 \mathrm{~mL})$ was vacuum-transferred to a mixture of $\left[\operatorname{ReCl}_{2}\left(\mathrm{P}=\mathrm{N}=\mathrm{P}^{t \mathrm{Bu}}\right)\right](\mathbf{2 1})$ $(5.0 \mathrm{mg}, 8.1 \mu \mathrm{mol}, 1.0 \mathrm{eq})$ and reductant $\left(\mathrm{Co}\left(\mathrm{Cp}^{*}\right)_{2}: 3.0 \mathrm{mg}, 9.0 \mu \mathrm{mol}, 1.1 \mathrm{eq} ; \mathrm{NaHg}(1 \mathrm{M})\right.$ : $121.3 \mathrm{mg}, 9.0 \mu \mathrm{mol}, 1.1 \mathrm{eq}$ or $\left.\mathrm{KC}_{8}: 1.1 \mathrm{mg} ; 8.1 \mu \mathrm{mol}, 1.0 \mathrm{eq}\right)$ in a J-Young NMR tube and placed under an $\mathrm{N}_{2}$ atmosphere. After thawing of the solvent the mixture was stirred vigorously upon which the color gradually changed from dark brown to light brown. After $30 \mathrm{~min}$ at RT the solvent was removed, hexamethylbenzene ( 1 eq via $0.08 \mathrm{M}$ stock solution in THF) was added and the solvent was removed again. The spectroscopic yield of the title compound was obtained by integration of the $\mathrm{P}=\mathrm{N}=\mathrm{P}^{t \mathrm{Bu}}$ ligand backbone ${ }^{1} \mathrm{H}$ NMR signals 
vs. the internal standard $\mathrm{C}_{6} \mathrm{Me}_{6}$ (maximum $60 \%$ for $\mathrm{Co}\left(\mathrm{Cp}^{*}\right)_{2}, 30 \%$ for $\mathrm{Na} / \mathrm{Hg}, 20 \%$ for $\left.\mathrm{KC}_{8}\right) \cdot\left[\operatorname{Re}(\mathrm{N}) \mathrm{Cl}\left(\mathrm{P}=\mathrm{N}=\mathrm{P}^{t \mathrm{Bu}}\right)\right](\mathbf{2 2})$ was not isolated via this route.

From azide:

$\left[\operatorname{ReCl}_{2}\left(\mathrm{P}=\mathrm{N}=\mathrm{P}^{t \mathrm{Bu}}\right)\right]$ (21) $(25.0 \mathrm{mg}, 40.7 \mu \mathrm{mol}, 1.0 \mathrm{eq})$ was dissolved in THF $(1 \mathrm{~mL})$ and was added dropwise over a period of $5 \mathrm{~min}$ to a stirring solution of $(\mathrm{TMS}) \mathrm{N}_{3}(26.78 \mu \mathrm{L}, 23.5 \mathrm{mg}$, $203 \mu \mathrm{mol}, 5.0 \mathrm{eq})$ in THF $(0.5 \mathrm{~mL})$. The solution was stirred at RT for $1.5 \mathrm{~h}$ after which the solvent was removed in vacuo. After extraction with pentane $(4 \times 5 \mathrm{~mL})$ and removal of the solvent, $\left[\operatorname{Re}(\mathrm{N}) \mathrm{Cl}\left(\mathrm{P}=\mathrm{N}=\mathrm{P}^{t \mathrm{Bu}}\right)\right]$ (22) was obtained as a light brown solid (17.4 mg, $29.3 \mu \mathrm{mol}, 72 \%$ yield).

${ }^{1} \mathbf{H}-\mathrm{NMR}\left(400 \mathrm{MHz}, \mathrm{C}_{6} \mathrm{D}_{6}, 25^{\circ} \mathrm{C}\right): \delta(\mathrm{ppm})=7.00\left(\mathrm{~A}_{9} \mathrm{~B}_{9} \mathrm{CDXX}^{\prime} \mathrm{D}^{\prime} \mathrm{C}^{\prime} \mathrm{B}^{\prime}{ }_{9} \mathrm{~A}^{\prime}{ }_{9}, N=\left.\right|^{3} J_{\mathrm{DX}}+{ }^{5}\right.$ $\left.J_{\mathrm{DX}} \mid=17.1 \mathrm{~Hz},{ }^{3} J_{\mathrm{DC}}=6.2 \mathrm{~Hz}, 2 \mathrm{H}, \mathrm{NCH}\right), 4.29\left(\mathrm{~A}_{9} \mathrm{~B}_{9} \mathrm{CDXX} \mathrm{D}^{\prime} \mathrm{C}^{\prime} \mathrm{B}^{\prime}{ }_{9} \mathrm{~A}^{\prime}{ }_{9},{ }^{3} J_{\mathrm{CD}}=6.3 \mathrm{~Hz}\right.$, $\left.N=\left|{ }^{2} J_{\mathrm{CX}}+{ }^{4} J_{\mathrm{CX}}\right|=2.2 \mathrm{~Hz}, 2 \mathrm{H}, \mathrm{PCH}\right), 1.49\left(\mathrm{~A}_{9} \mathrm{~B}_{9} \mathrm{CDXX} \mathrm{D}^{\prime} \mathrm{C}^{\prime} \mathrm{B}^{\prime}{ }_{9} \mathrm{~A}_{9}^{\prime},{ }^{3} J_{\mathrm{BX}}=7.0 \mathrm{~Hz}\right.$, $\left.18 \mathrm{H}, \mathrm{C}\left(\mathrm{CH}_{3}\right)_{3}\right), 1.18\left(\mathrm{~A}_{9} \mathrm{~B}_{9} \mathrm{CDXX} \mathrm{D}^{\prime} \mathrm{C}^{\prime} \mathrm{B}^{\prime}{ }_{9} \mathrm{~A}^{\prime}{ }_{9},{ }^{3} J_{\mathrm{AX}}=7.2 \mathrm{~Hz}, 18 \mathrm{H}, \mathrm{C}\left(\mathrm{CH}_{3}\right)_{3}\right) .{ }^{13} \mathrm{C}\left\{{ }^{1} \mathrm{H}\right\}-$ $\operatorname{NMR}\left(125.8 \mathrm{MHz}, \mathrm{C}_{6} \mathrm{D}_{6}, 25^{\circ} \mathrm{C}\right): \delta(\mathrm{ppm})=170.3\left(\mathrm{vt},{ }^{2} J_{\mathrm{CP}}=6.8 \mathrm{~Hz}, 2 \mathrm{C}, \mathrm{NCH}\right), 91.8(\mathrm{vt}$, $\left.{ }^{1} J_{\mathrm{CP}}=20.9 \mathrm{~Hz}, 2 \mathrm{C}, \mathrm{PCH}\right), 36.7\left(\mathrm{vt},{ }^{1} J_{\mathrm{CP}}=11.8 \mathrm{~Hz}, 2 \mathrm{C}, \mathrm{C}\left(\mathrm{CH}_{3}\right)_{3}\right), 34.9\left(\mathrm{vt},{ }^{1} J_{\mathrm{CP}}=\right.$ $\left.10.3 \mathrm{~Hz}, 2 \mathrm{C}, \mathrm{C}\left(\mathrm{CH}_{3}\right)_{3}\right), 29.4\left(\mathrm{~m}, 6 \mathrm{C}, \mathrm{C}\left(\mathrm{CH}_{3}\right)_{3}\right), 28.5\left(\mathrm{~m}, 6 \mathrm{C}, \mathrm{C}\left(\mathrm{CH}_{3}\right)_{3}\right) .{ }^{31} \mathbf{P}\left\{{ }^{1} \mathbf{H}\right\}-\mathbf{N M R}$ $\left(162.0 \mathrm{MHz}, \mathrm{C}_{6} \mathrm{D}_{6}, 25^{\circ} \mathrm{C}\right): \delta(\mathrm{ppm})=71.8(\mathrm{~s})$. Elemental anal. Calc. for $\mathrm{C}_{20} \mathrm{H}_{40} \mathrm{ClN}_{2} \mathrm{P}_{2} \mathrm{Re}$ : $\mathrm{C}, 40.57 ; \mathrm{H}, 6.81 ; \mathrm{N}, 4.73$. Found: $\mathrm{C}, 40.66 ; \mathrm{H}, 6.73 ; \mathrm{N}, 5.01$. Mass spect. (LIFDI), $\mathrm{m} / \mathrm{z}$ $(\%)=592.1(100)$.

\subsection{7 $\left[\operatorname{Re}(\mathrm{N}) \mathrm{Cl}\left(\mathrm{P}^{\mathrm{H}}=\mathrm{N}=\mathrm{P}^{t \mathrm{Bu}}\right)\right]^{\mathrm{OTf}}\left(\mathbf{2 3}^{\mathrm{OTf}}\right)$}

Compound $\left[\operatorname{Re}(\mathrm{N}) \mathrm{Cl}\left(\mathrm{P}=\mathrm{N}=\mathrm{P}^{\mathrm{tBu}}\right)\right](22)(5.0 \mathrm{mg}, 8.4 \mu \mathrm{mol}, 1.0 \mathrm{eq})$ was dissolved in $\mathrm{Et}_{2} \mathrm{O}$ $(1.0 \mathrm{~mL})$ and $\operatorname{HOTf}(0.74 \mu \mathrm{L}, 8.4 \mu \mathrm{mol}, 1.0 \mathrm{eq})$ was added via an Eppendorf pipette. Upon stirring for $1 \mathrm{~h}$ a red-brownish precipitate formed which was collected by filtration, washed with pentanes $(3 \times 2 \mathrm{~mL})$ and dried in vacuo to give $\left[\operatorname{Re}(\mathrm{N}) \mathrm{Cl}\left(\mathrm{P}^{\mathrm{H}}=\mathrm{N}=\mathrm{P}^{\mathrm{tBu}}\right)\right]^{\text {OTf }}\left(\mathbf{2 3}^{\text {OTf }}\right)$ (5.1 mg, $6.9 \mu \mathrm{mol}, 82 \%$ ).

${ }^{1} \mathbf{H}-\mathbf{N M R}\left(500 \mathrm{MHz}, \mathrm{CD}_{2} \mathrm{Cl}_{2}, 25^{\circ} \mathrm{C}\right): \delta(\mathrm{ppm})=9.35\left(\mathrm{~d},{ }^{3} \mathrm{~J}_{\mathrm{HP}}=20.9 \mathrm{~Hz}, 1 \mathrm{H}, \mathrm{N}=\mathrm{CH}-\mathrm{CH}_{2}\right)$, 8.02 (doublet of doublets (dd), $\left.{ }^{3} J_{\mathrm{HP}}=27.8 \mathrm{~Hz},{ }^{3} J_{\mathrm{HH}}=6.6 \mathrm{~Hz}, 1 \mathrm{H}, \mathrm{N}-\mathrm{CH}=\mathrm{CH}\right), 6.66(\mathrm{~d}$, $\left.{ }^{3} J_{\mathrm{HH}}=6.7 \mathrm{~Hz}, 1 \mathrm{H}, \mathrm{P}-\mathrm{CH}\right), 4.40\left(\mathrm{dd},{ }^{2} J_{\mathrm{HH}}=21.2 \mathrm{~Hz},{ }^{2} J_{\mathrm{HP}}=7.4 \mathrm{~Hz}, 1 \mathrm{H}, \mathrm{P}-\mathrm{CH}-\mathrm{CH}\right)$, $3.79\left(\mathrm{dd},{ }^{2} J_{\mathrm{HH}}=21.2 \mathrm{~Hz},{ }^{2} J_{\mathrm{HP}}=7.7 \mathrm{~Hz}, 1 \mathrm{H}, \mathrm{P}-\mathrm{CH}_{2}-\mathrm{CH}\right), 1.60\left(\mathrm{~d},{ }^{3} J_{\mathrm{HP}}=15.6 \mathrm{~Hz}, 9 \mathrm{H}\right.$, $\left.\mathrm{C}\left(\mathrm{CH}_{3}\right)_{3}\right), 1.57\left(\mathrm{~d},{ }^{3} J_{\mathrm{HP}}=15.9 \mathrm{~Hz}, 9 \mathrm{H}, \mathrm{C}\left(\mathrm{CH}_{3}\right)_{3}\right), 1.32\left(\mathrm{~d},{ }^{3} J_{\mathrm{HP}}=14.7 \mathrm{~Hz}, 9 \mathrm{H}, \mathrm{C}\left(\mathrm{CH}_{3}\right)_{3}\right)$, $1.28\left(\mathrm{~d},{ }^{3} J_{\mathrm{HP}}=14.8 \mathrm{~Hz}, 9 \mathrm{H}, \mathrm{C}\left(\mathrm{CH}_{3}\right)_{3}\right) .{ }^{13} \mathrm{C}\left\{{ }^{1} \mathrm{H}\right\}-\mathrm{NMR}\left(125.8 \mathrm{MHz}, \mathrm{CD}_{2} \mathrm{Cl}_{2}, 25^{\circ} \mathrm{C}\right): \delta$ $(\mathrm{ppm})=201.2\left(\mathrm{~s}, \mathrm{~N}=\mathrm{CH}-\mathrm{CH}_{2}\right), 163.9\left(\mathrm{~d},{ }^{2} J_{\mathrm{CP}}=3.8 \mathrm{~Hz}, \mathrm{~N}-\mathrm{CH}=\mathrm{CH}\right), 127.9\left(\mathrm{~d},{ }^{1} J_{\mathrm{CP}}\right.$ $=31.9 \mathrm{~Hz}, \mathrm{CH}-\mathrm{P}), 41.0\left(\mathrm{~d},{ }^{1} J_{\mathrm{CP}}=22.5 \mathrm{~Hz}, \mathrm{CH}_{2}-\mathrm{P}\right), 38.2\left(\mathrm{dd},{ }^{1} J_{\mathrm{CP}}=18.7 \mathrm{~Hz},{ }^{3} J_{\mathrm{CP}}\right.$ $\left.\left.\left.=3.7 \mathrm{~Hz}, C\left(\mathrm{CH}_{3}\right)_{3}\right)_{3}\right), 37.8\left(\mathrm{dd},{ }^{1} J_{\mathrm{CP}}=15.5 \mathrm{~Hz},{ }^{3} J_{\mathrm{CP}}=3.9 \mathrm{~Hz}, C\left(\mathrm{CH}_{3}\right)_{3}\right)_{3}\right), 36.3(\mathrm{~d}$, $\left.\left.{ }^{1} J_{\mathrm{CP}}=15.1 \mathrm{~Hz}, \mathrm{C}\left(\mathrm{CH}_{3}\right)_{3}\right)_{3}\right), 36.0\left(\mathrm{~d},{ }^{1} J_{\mathrm{CP}}=18.1 \mathrm{~Hz}, C\left(\mathrm{CH}_{3}\right)_{3}\right), 29.1\left(\mathrm{~d},{ }^{2} J_{\mathrm{CP}}=3.3 \mathrm{~Hz}\right.$, 
$\left.\mathrm{C}\left(\mathrm{CH}_{3}\right)_{3}\right), 29.0\left(\mathrm{~d},{ }^{2} J_{\mathrm{CP}}=3.9 \mathrm{~Hz}, \mathrm{C}\left(\mathrm{CH}_{3}\right)_{3}\right), 28.7\left(\mathrm{~d},{ }^{2} J_{\mathrm{CP}}=4.1 \mathrm{~Hz}, \mathrm{C}\left(\mathrm{CH}_{3}\right)_{3}\right), 28.6(\mathrm{~d}$, $\left.{ }^{2} J_{\mathrm{CP}}=3.5 \mathrm{~Hz}, \mathrm{C}\left(\mathrm{CH}_{3}\right)_{3}\right) .{ }^{31} \mathbf{P}\left\{{ }^{1} \mathbf{H}\right\}-\mathrm{NMR}\left(121.5 \mathrm{MHz}, \mathrm{CD}_{2} \mathrm{Cl}_{2}, 25^{\circ} \mathrm{C}\right): \delta(\mathrm{ppm})=73.0$ $\left(\mathrm{d},{ }^{2} J_{\mathrm{PP}}=148.1 \mathrm{~Hz}\right), 70.0\left(\mathrm{~d},{ }^{2} J_{\mathrm{PP}}=148.1 \mathrm{~Hz}\right)$.

\subsection{8 $\left[\mathrm{Re}(\mathrm{NMe}) \mathrm{Cl}\left(\mathrm{P}=\mathrm{N}=\mathrm{P}^{\mathrm{tBu}}\right)\right]^{\mathrm{OTf}}\left(\mathbf{2 4}^{\mathrm{OTf}}\right)$}

Compound $\left[\operatorname{Re}(\mathrm{N}) \mathrm{Cl}\left(\mathrm{P}=\mathrm{N}=\mathrm{P}^{t \mathrm{Bu}}\right)\right]$ (22) $(25.0 \mathrm{mg}, 44.2 \mu \mathrm{mol}, 1.0$ eq. $)$ and $\mathrm{MeOTf}(5.26 \mu \mathrm{L}$, $46.4 \mu \mathrm{mol}, 1.1 \mathrm{eq}$.) were dissolved in chlorobenzene and heated to $80^{\circ} \mathrm{C}$ for $12 \mathrm{~h}$. After removal of all volatiles in vacuo, the product was washed with $\mathrm{Et}_{2} \mathrm{O}$ and $[\mathrm{Re}(\mathrm{NMe}) \mathrm{Cl}$ $\left.\left(\mathrm{P}=\mathrm{N}=\mathrm{P}^{t \mathrm{Bu}}\right)\right]^{\mathrm{OTf}}\left(24^{\mathrm{OTf}}\right)$ was obtained as brown solid $(25.7 \mathrm{mg}, 34.0 \mu \mathrm{mol}, 80.5 \%)$.

${ }^{1} \mathbf{H}-\mathrm{NMR}\left(500 \mathrm{MHz}, \mathrm{CD}_{2} \mathrm{Cl}_{2}, 25^{\circ} \mathrm{C}\right): \delta(\mathrm{ppm})=7.99\left(\mathrm{~A}_{9} \mathrm{~B}_{9} \mathrm{CDXX}{ }^{\prime} \mathrm{D}^{\prime} \mathrm{C}^{\prime} \mathrm{B}^{\prime}{ }_{9} \mathrm{~A}^{\prime}{ }_{9}, N=\left.\right|^{3} J_{\mathrm{DX}}+{ }^{5}\right.$ $\left.J_{\mathrm{DX}^{\prime}} \mid=18.2 \mathrm{~Hz},{ }^{3} J_{\mathrm{DC}}=6.5 \mathrm{~Hz}, 2 \mathrm{H}, \mathrm{NCH}\right), 5.34$ (m, superimposed with solvent, $2 \mathrm{H}, \mathrm{PCH}$ ), $2.70\left(\mathrm{~s}_{\mathrm{br}}, 3 \mathrm{H}, \mathrm{N}-\mathrm{CH}_{3}\right), 1.49\left(\mathrm{~A}_{9} \mathrm{~B}_{9} \mathrm{CDXX} \mathrm{D}^{\prime} \mathrm{C}^{\prime} \mathrm{B}^{\prime}{ }_{9} \mathrm{~A}^{\prime}{ }_{9},{ }^{3} \mathrm{~J}_{\mathrm{AX}}=7.8 \mathrm{~Hz}, 18 \mathrm{H}, \mathrm{C}\left(\mathrm{CH}_{3}\right)_{3}\right), 1.29$ $\left(\mathrm{A}_{9} \mathrm{~B}_{9} \mathrm{CDXX} \mathrm{D}^{\prime} \mathrm{C}^{\prime} \mathrm{B}_{9}{ }_{9} \mathrm{~A}^{\prime}{ }_{9},{ }^{3} J_{\mathrm{AX}}=6.6 \mathrm{~Hz}, 18 \mathrm{H}, \mathrm{C}\left(\mathrm{CH}_{3}\right)_{3}\right) .{ }^{13} \mathrm{C}\left\{{ }^{1} \mathbf{H}\right\}-\mathrm{NMR}(125.8 \mathrm{MHz}$, $\left.\mathrm{CD}_{2} \mathrm{Cl}_{2}, 25^{\circ} \mathrm{C}\right): \delta(\mathrm{ppm})=172.9\left(\mathrm{vt},{ }^{2} J_{\mathrm{CP}}=5.3 \mathrm{~Hz}, 2 \mathrm{C}, \mathrm{NCH}\right), 99.1\left(\mathrm{AXX}^{\prime} \mathrm{A}^{\prime},{ }^{1} J_{\mathrm{CP}}\right.$ $\left.=22.6 \mathrm{~Hz},{ }^{3} J_{\mathrm{CP}}=20.4 \mathrm{~Hz}, \mathrm{PCH}\right), 61.9\left(\mathrm{~s}, \mathrm{NCH}_{3}\right), 40.2\left(\mathrm{vt},{ }^{1} J_{\mathrm{CP}}=9.7 \mathrm{~Hz}, \mathrm{C}\left(\mathrm{CH}_{3}\right)_{3}\right)$, $39.6\left(\mathrm{vt},{ }^{1} J_{\mathrm{CP}}=11.6 \mathrm{~Hz}, \mathrm{C}\left(\mathrm{CH}_{3}\right)_{3}\right), 30.2\left(\mathrm{~m}, \mathrm{C}\left(\mathrm{CH}_{3}\right)_{3}\right), 28.9\left(\mathrm{~m}, \mathrm{C}\left(\mathrm{CH}_{3}\right)_{3}\right) .{ }^{31} \mathbf{P}\left\{{ }^{1} \mathbf{H}\right\}-$ NMR $\left(202.4 \mathrm{MHz}, \mathrm{CD}_{2} \mathrm{Cl}_{2}, 25^{\circ} \mathrm{C}\right): \delta(\mathrm{ppm})=88.8(\mathrm{~s})$. Elemental anal. Calc. for $\mathrm{C}_{22} \mathrm{H}_{43} \mathrm{ClF}_{3} \mathrm{~N}_{2} \mathrm{O}_{3} \mathrm{P} 2 \mathrm{ReS}: \mathrm{C}, 34.94 ; \mathrm{H}, 5.71 ; \mathrm{N}, 3.70$. Found: C, 35.18; H, 5.71; N, 3.49. Mass spect. (LIFDI), $m / z(\%)=607.2(100)$.

\subsection{9 $\left[\mathrm{Re}\left(\mathrm{N}=\mathrm{CH}_{2}\right) \mathrm{Cl}\left(\mathrm{P}=\mathrm{N}=\mathrm{P}^{t \mathrm{Bu}}\right)\right]$}

$\left[\mathrm{Re}(\mathrm{NMe}) \mathrm{Cl}\left(\mathrm{P}=\mathrm{N}=\mathrm{P}^{t \mathrm{Bu}}\right)\right]^{\mathrm{OTf}}\left(\mathbf{2 4}{ }^{\mathrm{OTf}}\right)(20.0 \mathrm{mg}, 26.5 \mu \mathrm{mol}, 1.0 \mathrm{eq})$ was mixed with $\mathrm{NEt}_{3}$ $(5.5 \mu \mathrm{mL}, 39.7 \mu \mathrm{mol}, 1.5 \mathrm{eq})$ in THF $(5 \mathrm{~mL})$ and heated to $60^{\circ} \mathrm{C}$ over $16 \mathrm{~h}$. After evaporation of the solvent, the product was extracted with pentane and $\left[\mathrm{Re}\left(\mathrm{N}=\mathrm{CH}_{2}\right) \mathrm{Cl}\left(\mathrm{P}=\mathrm{N}=\mathrm{P}^{t \mathrm{Bu}}\right)\right]$ (25) could be obtained as dark brown solid ( $9.1 \mathrm{mg}, 15.0 \mu \mathrm{mol}, 56.7 \%)$.

${ }^{1} \mathrm{H}$-NMR $\left(500 \mathrm{MHz}, \mathrm{C}_{6} \mathrm{D}_{6}, 25^{\circ} \mathrm{C}\right): \delta(\mathrm{ppm})=7.16(\mathrm{~m}$, superimposed with solvent, $2 \mathrm{H}$, $\mathrm{NCH}), 4.71\left(\mathrm{~A}_{9} \mathrm{~B}_{9} \mathrm{CDXX} \mathrm{D}^{\prime} \mathrm{C}^{\prime} \mathrm{B}^{\prime}{ }_{9} \mathrm{~A}^{\prime}{ }_{9},{ }^{3} J_{\mathrm{CD}}=6.4 \mathrm{~Hz}, N=\left|{ }^{2} J_{\mathrm{CX}}+{ }^{4} J_{\mathrm{CX}^{\prime}}\right|=1.5 \mathrm{~Hz}, 2 \mathrm{H}\right.$, $\mathrm{PCH}), 1.74\left(\mathrm{~s}_{\mathrm{br}}, 1 \mathrm{H}, \mathrm{N}=\mathrm{CHH}\right), 1.63\left(\mathrm{~s}_{\mathrm{br}}, 1 \mathrm{H}, \mathrm{N}=\mathrm{CHH}\right), 1.30\left(\mathrm{~A}_{9} \mathrm{~B}_{9} \mathrm{CDXX} \mathrm{D}^{\prime} \mathrm{C}^{\prime} \mathrm{B}^{\prime}{ }_{9} \mathrm{~A}^{\prime}{ }_{9},{ }^{3} J_{\mathrm{AX}}\right.$ $\left.=6.8 \mathrm{~Hz}, 18 \mathrm{H}, \mathrm{C}\left(\mathrm{CH}_{3}\right)_{3}\right), 1.26\left(\mathrm{sbr}, 18 \mathrm{H}, \mathrm{C}\left(\mathrm{CH}_{3}\right)_{3}\right) .{ }^{13} \mathrm{C}\left\{{ }^{1} \mathrm{H}\right\}-\mathrm{NMR}\left(125.8 \mathrm{MHz}, \mathrm{C}_{6} \mathrm{D}_{6}\right.$, $\left.25^{\circ} \mathrm{C}\right): \delta(\mathrm{ppm})=170.3\left(\mathrm{vt},{ }^{2} J_{\mathrm{CP}}=7.7 \mathrm{~Hz}, \mathrm{NCH}\right), 141.7\left(\mathrm{t},{ }^{3} J_{\mathrm{CP}}=2.6 \mathrm{~Hz}, \mathrm{~N}=\mathrm{CH}_{2}\right)$, $102.6\left(\mathrm{vt},{ }^{1} J_{\mathrm{CP}}=17.0 \mathrm{~Hz}, \mathrm{PCH}\right), 38.6\left(\mathrm{vt},{ }^{1} J_{\mathrm{CP}}=10.0 \mathrm{~Hz}, \mathrm{C}\left(\mathrm{CH}_{3}\right)_{3}\right), 36.9\left(\mathrm{vt},{ }^{1} J_{\mathrm{CP}}=\right.$ $\left.8.6 \mathrm{~Hz}, \mathrm{C}\left(\mathrm{CH}_{3}\right)_{3}\right), 30.6\left(\mathrm{~m}, \mathrm{C}\left(\mathrm{CH}_{3}\right)_{3}\right), 28.9\left(\mathrm{~m}, \mathrm{C}\left(\mathrm{CH}_{3}\right)_{3}\right) .{ }^{31} \mathbf{P}\left\{{ }^{1} \mathbf{H}\right\}-\mathrm{NMR}(202.4 \mathrm{MHz}$, $\left.\mathrm{C}_{6} \mathrm{D}_{6}, 25^{\circ} \mathrm{C}\right): \delta(\mathrm{ppm})=38.9(\mathrm{~s})$. Mass spect. (LIFDI), $m / z(\%)=606.1(100)$. 


\subsubsection{0 $\left[\operatorname{Re}(\mathrm{N}) \mathrm{Cl}\left(\mathrm{P}=\mathrm{N}=\mathrm{P}^{t \mathrm{Bu}}\right)\right]^{\mathrm{SbF}_{6}}\left(26^{\mathrm{SbF}_{6}}\right)$}

$\left[\operatorname{Re}(\mathrm{N}) \mathrm{Cl}\left(\mathrm{P}=\mathrm{N}=\mathrm{P}^{t \mathrm{Bu}}\right)\right](22)(5.0 \mathrm{mg}, 8.4 \mu \mathrm{mol}, 1.0 \mathrm{eq})$ and $\mathrm{Ag}\left(\mathrm{SbF}_{6}\right)$ were dissolved in THF $(1 \mathrm{~mL})$ and stirred, upon which the solution turned red immediately. After stirring for $1.5 \mathrm{~h}$ the solution was filtered and the solvent was removed in vacuo. Despite the title compound $\left[\operatorname{Re}(\mathrm{N}) \mathrm{Cl}\left(\mathrm{P}=\mathrm{N}=\mathrm{P}^{t \mathrm{Bu}}\right)\right]^{\mathrm{SbF}_{6}}\left(\mathbf{2 6}^{\mathbf{S b F}_{6}}\right)$ the product always contained $\mathbf{2 3}^{\mathrm{SbF}_{6}}$ as a side product.

${ }^{1} \mathbf{H}-\mathrm{NMR}\left(300 \mathrm{MHz}, \mathrm{CD}_{2} \mathrm{Cl}_{2}, 25^{\circ} \mathrm{C}\right): \delta(\mathrm{ppm})=7-2\left(\mathrm{~s}_{\mathrm{br}}\right)$.

\subsubsection{1 $\left[\operatorname{Re}(\text { bipy }) \mathrm{Cl}\left(\mathrm{PNP}^{\mathrm{tBu}}\right)\right]^{\mathrm{BPh}_{4}}\left(27^{\mathrm{BPh}_{4}}\right)$}

$\left[\mathrm{ReCl}_{2}\left(\mathrm{PNP}^{t \mathrm{Bu}}\right)\right](\mathbf{X I X})(15.0 \mathrm{mg}, 24.3 \mu \mathrm{mol}, 1.0 \mathrm{eq}), 2,2^{\prime}$-bipyridine $(4.9 \mathrm{mg}, 31.6 \mu \mathrm{mol}$, $1.3 \mathrm{eq})$ and $\mathrm{NaBPh}_{4}(10.8 \mathrm{mg}$. $31.6 \mu \mathrm{mol}, 1.3 \mathrm{eq})$ are mixed in THF (dried over $\mathrm{Na} / \mathrm{K}$, $3 \mathrm{~mL}$ ) and the reaction mixture is heated to $60^{\circ} \mathrm{C}$ and stirred overnight. Afterwards the solvent is removed in vacuo and the product is extraced with DCM. Recrystallization from $\mathrm{DCM} / \mathrm{Et}_{2} \mathrm{O}$ at $-40^{\circ} \mathrm{C}$ afforded the target complex $27^{\mathrm{BPh}_{4}}$ as spectroscopically clean, deep red solid $(23.3 \mathrm{mg}, 22.3 \mu \mathrm{mol}, 91 \%$ yield).

${ }^{1} \mathbf{H}-\mathrm{NMR}\left(500 \mathrm{MHz}, \mathrm{CD}_{2} \mathrm{Cl}_{2}, 25^{\circ} \mathrm{C}\right): \delta(\mathrm{ppm})=9.99\left(\mathrm{~d},{ }^{3} J_{\mathrm{HH}}=6.2 \mathrm{~Hz}, 1 \mathrm{H}, 3-\mathrm{CH}_{\text {bipy }}\right), 8.26$ $\left(\mathrm{d},{ }^{3} J_{\mathrm{HH}}=7.9 \mathrm{~Hz}, 1 \mathrm{H}, 6-\mathrm{CH}_{\text {bipy }}\right), 8.20\left(\mathrm{~d},{ }^{3} J_{\mathrm{HH}}=8.3 \mathrm{~Hz}, 1 \mathrm{H}, 6{ }^{\prime}-\mathrm{CH}_{\text {bipy }}\right), 7.51$ (dd, ${ }^{3} J_{\mathrm{HH}}$ $\left.=8.3,7.3 \mathrm{~Hz}, 1 \mathrm{H}, 5-\mathrm{CH}_{\text {bipy }}\right), 7.37-7.33\left(\mathrm{~m}\right.$, superimposed, $\left.9 \mathrm{H}, \mathrm{CH}_{\mathrm{Ph}, \text { ortho }}, 4-\mathrm{CH}_{\text {bipy }}\right), 7.03$ $\left(\mathrm{t},{ }^{3} J_{\mathrm{HH}}=7.4 \mathrm{~Hz}, 8 \mathrm{H}, \mathrm{CH}_{\mathrm{Ph}, \text { meta }}\right), 6.91-6.84\left(\mathrm{~m}\right.$, superimposed, $5 \mathrm{H}, \mathrm{CH}_{\mathrm{Ph}, \text { para }}, 4^{\prime}-\mathrm{CH}_{\text {bipy }}$ ), $6.77\left(\mathrm{dd},{ }^{3} J_{\mathrm{HH}}=8.4,7.2 \mathrm{~Hz}, 1 \mathrm{H}, 5^{\prime}-\mathrm{CH}_{\text {bipy }}\right), 6.59\left(\mathrm{~d},{ }^{3} J_{\mathrm{HH}}=6.3 \mathrm{~Hz}, 1 \mathrm{H}, 3^{\prime}-\mathrm{CH}_{\text {bipy }}\right), 4.67-$ $4.55(\mathrm{~m}, 2 \mathrm{H}, \mathrm{N}-\mathrm{CHH}), 4.09-3.99(\mathrm{~m}, 2 \mathrm{H}, \mathrm{N}-\mathrm{CHH}), 2.22-2.13(\mathrm{~m}, 2 \mathrm{H}, \mathrm{P}-\mathrm{CHH}), 1.54-1.45$ $(\mathrm{m}, 2 \mathrm{H}, \mathrm{P}-\mathrm{CHH}), 1.38$ (spinsys, $\left.18 \mathrm{H}, \mathrm{C}\left(\mathrm{CH}_{3}\right)_{3}\right), 0.36$ (spinsys, $\left.18 \mathrm{H}, \mathrm{C}\left(\mathrm{CH}_{3}\right)_{3}\right) .{ }^{13} \mathrm{C}\left\{{ }^{1} \mathbf{H}\right\}-$ $\operatorname{NMR}\left(125.8 \mathrm{MHz}, \mathrm{CD}_{2} \mathrm{Cl}_{2}, 25^{\circ} \mathrm{C}\right): \delta(\mathrm{ppm})=164.6\left({ }^{1} \mathrm{~J}_{\mathrm{BC}}=49.3 \mathrm{~Hz}, 4 \mathrm{C}, \mathrm{B}-\mathrm{C}_{\text {ipso }}\right), 153.0$ (s, 2'- $\left.C_{\text {bipy }}\right), 150.5$ (s, 2- $C_{\text {bipy }}$ ), 149.8 (s, 3- $C_{\text {bipy }}$ ), 145.0 (s, 3'- $C_{\text {bipy }}$ ), 138.6 (s, 5- $C_{\text {bipy }}$ ),

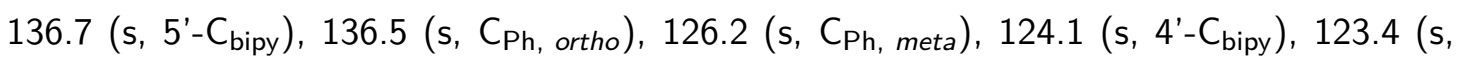
4- $\mathrm{C}_{\text {bipy }}$ ), 122.8 (s, 6'- $\mathrm{C}_{\text {bipy }}$ ), 122.3 (s, $\left.\mathrm{C}_{\mathrm{Ph}, \text { para }}\right), 121.9$ (s, 6- $\left.\mathrm{C}_{\text {bipy }}\right), 86.9$ (vt, ${ }^{2} J_{\mathrm{CP}}=3.9 \mathrm{~Hz}$, $\left.\mathrm{N}-\mathrm{CH}_{2}\right), 42.5\left(\mathrm{vt},{ }^{1} J_{\mathrm{CP}}=8.7 \mathrm{~Hz}, C\left(\mathrm{CH}_{3}\right)_{3}\right), 41.4\left(\mathrm{vt},{ }^{1} J_{\mathrm{CP}}=7.4 \mathrm{~Hz}, C\left(\mathrm{CH}_{3}\right)_{3}\right), 32.1$ $\left(\mathrm{m}, \mathrm{C}\left(\mathrm{CH}_{3}\right)_{3}\right), 29.6\left(\mathrm{~m}, \mathrm{C}\left(\mathrm{CH}_{3}\right)_{3}\right), 28.3\left(\mathrm{vt},{ }^{1} J_{\mathrm{CP}}=7.7 \mathrm{~Hz}, \mathrm{P}-\mathrm{CH}_{2}\right) .{ }^{31} \mathbf{P}\left\{{ }^{1} \mathbf{H}\right\}-\mathbf{N M R}$ $\left(162.0 \mathrm{MHz}, \mathrm{CD}_{2} \mathrm{Cl}_{2}, 25^{\circ} \mathrm{C}\right): \delta(\mathrm{ppm})=2.1(\mathrm{~s})$. Mass spect. (LIFDI), $\mathrm{m} / z(\%)=738.2$ (100). 


\subsection{Rhenium compounds based on the $\mathrm{PNP}^{i \mathrm{Pr}}$ ligand}

\subsection{1 $\left[\mathrm{ReCl}_{3}\left(\mathrm{HPNP}^{i \mathrm{Pr}}\right)\right](29)$}

$\left[\mathrm{ReCl}_{3}\left(\mathrm{PPh}_{3}\right)_{2}(\mathrm{NCMe})\right](600 \mathrm{mg}, 699 \mu \mathrm{mol}, 1.00 \mathrm{eq})$ and HPNP ${ }^{i \operatorname{Pr}}(224 \mathrm{mg}, 734 \mu \mathrm{mol}, 1.05 \mathrm{eq})$ are suspended in toluene (dried over $\mathrm{Na} / \mathrm{K}, 15 \mathrm{~mL}$ ), stirred vigorously and heated to $90^{\circ} \mathrm{C}$ overnight. After $16 \mathrm{~h}$, the solution is cooled to $-50^{\circ} \mathrm{C}$ and filtered. The residue is washed with $\mathrm{Et}_{2} \mathrm{O}$ until the washing solution is colorless and then extracted with $\mathrm{CH}_{2} \mathrm{Cl}_{2}$. The solvent is removed in vacuo and $\left[\mathrm{ReCl}_{3}\left(\mathrm{HPNP}^{\mathrm{iPr}}\right)\right](\mathbf{2 9})$ is obtained as a yellow powder $(292 \mathrm{mg}$, $488 \mu \mathrm{mol}, 70 \%)$.

${ }^{1} \mathbf{H}-\mathrm{NMR}\left(500 \mathrm{MHz}, \mathrm{CD}_{2} \mathrm{Cl}_{2}, 25^{\circ} \mathrm{C}\right): \delta(\mathrm{ppm})=154.45(\mathrm{~s}, \mathrm{NH}), 9.66\left(\mathrm{~m}, 6 \mathrm{H}, \mathrm{CH}\left(\mathrm{CH}_{3}\right)_{2}\right)$, $9.59\left(\mathrm{~m}, 6 \mathrm{H}, \mathrm{CH}\left(\mathrm{CH}_{3}\right)_{2}\right), 9.32\left(\mathrm{~m}, 2 \mathrm{H}, \mathrm{CH}\left(\mathrm{CH}_{3}\right)_{2}\right), 8.76\left(\mathrm{~m}, 6 \mathrm{H}, \mathrm{CH}\left(\mathrm{CH}_{3}\right)_{2}\right), 8.37(\mathrm{~m}$, $\left.6 \mathrm{H}, \mathrm{CH}\left(\mathrm{CH}_{3}\right)_{2}\right), 8.31\left(\mathrm{~m}, 2 \mathrm{H}, \mathrm{P}-\mathrm{CHH}-\mathrm{CH}_{2}\right), 5.46\left(\mathrm{~m}, 2 \mathrm{H}, \mathrm{CH}\left(\mathrm{CH}_{3}\right)_{2}\right), 0.54(\mathrm{~m}, 2 \mathrm{H}, \mathrm{P}-$ $\left.\mathrm{CHH}-\mathrm{CH}_{2}\right),-5.10\left(\mathrm{~m}, 2 \mathrm{H}, \mathrm{N}-\mathrm{CHH}-\mathrm{CH}_{2}\right),-10.44\left(\mathrm{~m}, 2 \mathrm{H}, \mathrm{N}-\mathrm{CH}-\mathrm{CH}_{2}\right) .{ }^{13} \mathrm{C}\left\{{ }^{1} \mathbf{H}\right\}-\mathrm{NMR}$ $\left(125.7 \mathrm{MHz}, \mathrm{CD}_{2} \mathrm{Cl}_{2}, 25^{\circ} \mathrm{C}\right): \delta(\mathrm{ppm})=160.9\left(\mathrm{~s}, \mathrm{~N}-\mathrm{CH}_{2}\right), 142.1\left(\mathrm{~s}, \mathrm{CHMe}_{2}\right), 132.1$ (s, $\left.\mathrm{CHMe}_{2}\right), 68.6(\mathrm{~s}, \mathrm{P}-\mathrm{CH} 2), 22.5\left(\mathrm{~s}, \mathrm{CH}_{3}\right), 22.2\left(\mathrm{~s}, \mathrm{CH}_{3}\right), 18.4\left(\mathrm{~s}, \mathrm{CH}_{3}\right), 16.8\left(\mathrm{~s}, \mathrm{CH}_{3}\right)$. ${ }^{15} \mathbf{N}\left\{{ }^{1} \mathbf{H}\right\}-\mathbf{N M R}\left(50.7 \mathrm{MHz}, \mathrm{CD}_{2} \mathrm{Cl}_{2}, 25{ }^{\circ} \mathrm{C}\right): \delta(\mathrm{ppm})=-1226.5(\mathrm{~s}, \mathrm{NH}) .{ }^{31} \mathbf{P}\left\{{ }^{\mathbf{1}} \mathbf{H}\right\}-\mathbf{N M R}$ $\left(202.6 \mathrm{MHz}, \mathrm{CD}_{2} \mathrm{Cl}_{2}, 25^{\circ} \mathrm{C}\right): \delta(\mathrm{ppm})=4-1525.9$ (s). CV: $E_{1 / 2}$ vs $\mathrm{Fc} / \mathrm{Fc}^{+}(\mathrm{V})=-1.84$ $(\operatorname{Re}(\mathrm{II} / \mathrm{III})),-0.24(\operatorname{Re}(\mathrm{III} / \mathrm{IV}))$. Elemental anal. Calc. for $\mathrm{C}_{16} \mathrm{H}_{37} \mathrm{Cl}_{3} \mathrm{NP}_{2} \operatorname{Re}(597.10): \mathrm{C}$, 32.14; H, 6.24; N, 2.34. Found: C, 32.16; H, 6.18; N, 2.37. ATR-IR: $\tilde{\nu}\left(\mathrm{cm}^{-1}\right)=3506.4(\mathrm{br}$, electronic absorption), $3181.0\left(\nu_{\mathrm{N}-\mathrm{H}}\right.$. Mass spect. (LIFDI), $m / z(\%)=596.9(100)$.

$H / D$ exchange to synthesize 29-d:

$\left[\mathrm{ReCl}_{3}\left(\mathrm{HPNP}^{i \mathrm{Pr}}\right)\right](\mathbf{2 9})(6.0 \mathrm{mg}, 10.0 \mu \mathrm{mol}, 1.0 \mathrm{eq})$ is suspended in a $\mathrm{DCM} / \mathrm{D}_{2} \mathrm{O}$ mixture $(0.4 \mathrm{~mL} / 0.1 \mathrm{~mL})$ and stirred overnight. Afterwards the solvent is removed in vacuo and the spectroscopically clean product $\left[\mathrm{ReCl}_{3}\left(\mathrm{DPNP}{ }^{i \mathrm{Pr}}\right)\right](\mathbf{2 9 - \boldsymbol { d }})$ is measured in $\mathrm{CD}_{2} \mathrm{Cl}_{2}$. The NMR spectra show basically identical signals to those of 29. However, the signal corresponding to the $\mathrm{NH}$ proton is largely gone $(\sim 2 \%$ remaining $)$ and the signals at $\delta_{1_{\mathrm{H}}}=-5.10$ and $-10.44 \mathrm{ppm}$ exhibit different coupling patterns.

${ }^{2} \mathbf{H}-\mathbf{N M R}\left(46.1 \mathrm{MHz}, \mathrm{CD}_{2} \mathrm{Cl}_{2}, 25^{\circ} \mathrm{C}\right): \delta(\mathrm{ppm})=152.6(\mathrm{~s}, \mathrm{ND})$. ATR-IR: $\tilde{\nu}\left(\mathrm{cm}^{-1}\right)=$ 3506.4 (br, electronic absorption), $2362.9\left(\nu_{\mathrm{N}-\mathrm{D}}\right)$.

\subsection{2 $\left[\operatorname{ReCl}_{3}\left(\mathrm{PNP}^{i \mathrm{Pr}}\right)\right](31)$}

$\left[\mathrm{ReCl}_{3}\left(\mathrm{HPNP}^{i \mathrm{Pr}}\right)\right](29)(30.0 \mathrm{mg}, 50.2 \mu \mathrm{mol}, 1.0 \mathrm{eq})$ and TTBP $(14.4 \mathrm{mg}, 55.2 \mu \mathrm{mol}, 1.1 \mathrm{eq})$ are suspended in benzene $(5 \mathrm{~mL})$ and stirred at RT for $1.5 \mathrm{~h}$, resulting in a deep red solution. All volatiles are removed in vacuo. The crude product is washed with pentane and 
extracted with benzene. Lyophilization gives $\left[\mathrm{ReCl}_{3}\left(\mathrm{PNP}^{i \mathrm{Pr}}\right)\right](\mathbf{3 1})$ as a red powder $(19.0 \mathrm{mg}$, $31.8 \mu \mathrm{mol}, 63 \%)$.

${ }^{1} \mathbf{H}-\mathrm{NMR}\left(400 \mathrm{MHz}, \mathrm{CD}_{2} \mathrm{Cl}_{2}, 25^{\circ} \mathrm{C}\right): \delta(\mathrm{ppm})=21.86(\mathrm{~b}, 4 \mathrm{H}), 19.20$ (br, $\left.12 \mathrm{H}\right), 16.00$ (br, $12 \mathrm{H}), 1.43(\mathrm{br}, 4 \mathrm{H}) . \mathbf{C V}: E_{1 / 2}$ vs $\mathrm{Fc} / \mathrm{Fc}^{+}(\mathrm{V})=-1.16(\mathrm{Re}(\mathrm{III} / \mathrm{IV}))$. Elemental anal. Calcd. for $\mathrm{C}_{16} \mathrm{H}_{36} \mathrm{Cl}_{3} \mathrm{NP}_{2} \operatorname{Re}$ (596.98): C, 32.19; $\mathrm{H}, 6.07 ; \mathrm{N}, 2.35$. Found: C, 32.55; H, 6.04; N, 2.18 .

\subsection{3 $\left[\operatorname{ReCl}_{2}(\right.$ thf $\left.)\left(\mathrm{PNP}^{\mathrm{iPr}}\right)\right](\mathbf{3 2})$ and $\left[\mathrm{ReCl}_{2}\left(\mathrm{PNP}^{\mathrm{iPr}}\right)\right](\mathbf{3 0})$}

In a typical experiment, $\left[\mathrm{ReCl}_{3}\left(\mathrm{PNP}^{i \mathrm{Pr}}\right)\right](31)(10 \mathrm{mg}, 16.8 \mu \mathrm{mol}, 1.0 \mathrm{eq})$ and $\mathrm{NaHg}(1.0 \mathrm{M}$, $250 \mathrm{mg}, 18.4 \mu \mathrm{mol}, 1.1 \mathrm{eq}$ ) are stirred in either THF (to form 32) or in toluene (to form 30 ) $(0.5 \mathrm{~mL})$ for $4 \mathrm{~h}$ at $\mathrm{RT}$. $\left[\mathrm{ReCl}_{2}(\mathrm{thf})\left(\mathrm{PNP}^{i \mathrm{Pr}}\right)\right]$ (32) becomes a deep orange solution while $\left[\mathrm{ReCl}_{2}\left(\mathrm{PNP}^{i \mathrm{Pr}}\right)\right](\mathbf{3 0})$ becomes deep violet. Formed $\mathrm{NaCl}$ and remaining $\mathrm{Hg}$ is removed by filtration. Both complexes could not be isolated to instability towards vacuum and were thus used in situ.

In THF- $d_{8}$ (32):

${ }^{1} \mathbf{H}-\mathbf{N M R}\left(400 \mathrm{MHz}, \mathrm{THF}-d_{8}, 25^{\circ} \mathrm{C}\right): \delta(\mathrm{ppm})=3.73-3.60\left(\mathrm{~m}, 4 \mathrm{H}, \mathrm{N}-\mathrm{CH}_{2}\right), 2.75-2.60(\mathrm{~m}$, $\left.4 \mathrm{HCH}\left(\mathrm{CH}_{3}\right)_{2}\right), 1.84-1.75\left(\mathrm{~m}, 4 \mathrm{H}, \mathrm{P}-\mathrm{CH}_{2}\right), 1.39-1.27\left(\mathrm{~m}, 12 \mathrm{H}, \mathrm{CH}\left(\mathrm{CH}_{3}\right)_{2}\right), 1.27-1.18(\mathrm{~m}$, $\left.12 \mathrm{H}, \mathrm{CH}\left(\mathrm{CH}_{3}\right)_{2}\right) .{ }^{31} \mathbf{P}\left\{{ }^{1} \mathbf{H}\right\}-\mathrm{NMR}\left(162.0 \mathrm{MHz}, \mathrm{THF}-d_{8}, 25^{\circ} \mathrm{C}\right): \delta(\mathrm{ppm})=-14.5(\mathrm{~s})$.

In toluene- $d_{8}(30)$ :

${ }^{1} \mathbf{H}-\mathrm{NMR}\left(400 \mathrm{MHz}\right.$, toluene- $\left.d_{8}, 25^{\circ} \mathrm{C}\right): \delta(\mathrm{ppm})=2.97-2.86\left(\mathrm{~m}, 4 \mathrm{H}, \mathrm{N}-\mathrm{CH}_{2}\right), 2.64-2.50$ $\left(\mathrm{m}, 4 \mathrm{HCH}\left(\mathrm{CH}_{3}\right)_{2}\right), 1.59-1.48\left(\mathrm{~m}, 4 \mathrm{H}, \mathrm{P}-\mathrm{CH}_{2}\right), 1.35-1.17\left(\mathrm{~m}, 24 \mathrm{H}, \mathrm{CH}\left(\mathrm{CH}_{3}\right)_{2}\right) .{ }^{1} \mathbf{H}\left\{{ }^{31} \mathrm{P}\right\}-$ NMR $\left(400 \mathrm{MHz}\right.$, toluene- $\left.d_{8}, 25^{\circ} \mathrm{C}\right): \delta(\mathrm{ppm})=2.97-2.86\left(\mathrm{~m}, 4 \mathrm{H}, \mathrm{N}-\mathrm{CH}_{2}\right), 2.57$ (heptet (hept), ${ }^{3} J_{\mathrm{HH}}=7.3 \mathrm{~Hz}, 1.59-1.48\left(\mathrm{~m}, 4 \mathrm{H}, \mathrm{P}-\mathrm{CH}_{2}\right), 1.28\left(\mathrm{~d},{ }^{3} J_{\mathrm{HH}}=7.2 \mathrm{~Hz}, 12 \mathrm{H}, \mathrm{CH}\left(\mathrm{CH}_{3}\right)_{2}\right)$, $1.23\left(\mathrm{~d},{ }^{3} \mathrm{~J}_{\mathrm{HH}}=7.2 \mathrm{~Hz}, 12 \mathrm{H}, \mathrm{CH}\left(\mathrm{CH}_{3}\right)_{2}\right) .{ }^{13} \mathrm{C}\left\{{ }^{1} \mathbf{H}\right\}-\mathrm{NMR}\left(100.6 \mathrm{MHz}\right.$, toluene-d, $\left.25^{\circ} \mathrm{C}\right)$ : $\delta(\mathrm{ppm})=84.8\left(\mathrm{vt},{ }^{2} J_{\mathrm{CP}}=5.4 \mathrm{~Hz}, 2 \mathrm{C}, \mathrm{N}-\mathrm{CH}_{2}\right), 33.5\left(\mathrm{vt},{ }^{1} J_{\mathrm{CP}}=8.5 \mathrm{~Hz}, 2 \mathrm{C}, \mathrm{P}-\mathrm{CH}_{2}\right)$, $30.2\left(\mathrm{vt},{ }^{1} J_{\mathrm{CP}}=11.1 \mathrm{~Hz}, 2 \mathrm{C}, \mathrm{CH}\left(\mathrm{CH}_{3}\right)_{2}\right), 19.0\left(\mathrm{~s}, 4 \mathrm{C}, \mathrm{C}\left(\mathrm{CH}_{3}\right)_{2}\right), 18.3\left(\mathrm{~s}, 4 \mathrm{C}, \mathrm{C}\left(\mathrm{CH}_{3}\right)_{2}\right)$. ${ }^{31} \mathbf{P}\left\{{ }^{1} \mathbf{H}\right\}-N M R\left(162.0 \mathrm{MHz}, \mathrm{CD}_{2} \mathrm{Cl}_{2}, 25^{\circ} \mathrm{C}\right): \delta(\mathrm{ppm})=10.0$ (s). Mass spect. (LIFDI), $m / z(\%)=561.1$ (100). Elemental anal. Calcd. for $\mathrm{C}_{16} \mathrm{H}_{36} \mathrm{Cl}_{2} \mathrm{NP}_{2} \operatorname{Re}$ (561.5): C, 34.22; $\mathrm{H}, 6.46 ; \mathrm{N}, 2.49$. Found: C, 33,63; H, 5.97; N, 2.25. NMR spectroscopy revealed the formerly spectroscopically clean sample to be partially decomposed after drying it for the elemental analysis. Since the analysis as well as the initial spectra were fine, it is assumed that $\mathbf{3 0}$ is present exclusively and cleanly in the in situ prepared solution but decomposes partially when the solvent is removed. 


\subsection{4 $\left[\left\{\operatorname{ReCl}_{2, \text { cis }}\left(\mathrm{PNP}^{\mathrm{iPr}}\right)\right\}\left(\mu-\mathrm{N}_{2}\right)\left\{\mathrm{ReCl}_{2, \text { trans }}\left(\mathrm{PNP}^{\mathrm{iPr}}\right)\right\}\right](33 \mathrm{a})$ and $\left[\left\{\operatorname{ReCl}_{2, c i s}\left(\mathrm{PNP}^{\mathrm{iPr}}\right)\left(\mu-\mathrm{N}_{2}\right) \operatorname{ReCl}_{2, \text { cis }}\left(\mathrm{PNP}^{\mathrm{iPr}}\right)\right\}\right](3 \mathbf{3 b})$}

Through a freshly prepared solution of $\left[\mathrm{ReCl}_{2}\left(\mathrm{PNP}^{i \mathrm{Pr}}\right)\right](30), \mathrm{N}_{2}$ gas is passed leading to an immediate color change from violet to deep brown. No isolation of the formed complexes was possible, but they could be identified by NMR spectroscopy at $-30^{\circ} \mathrm{C}$ (see Section 3.2.2 for a discussion).

$\left[\left\{\operatorname{ReCl}_{2, \text { cis }}\left(\mathrm{PNP}^{i \mathrm{Pr} r}\right)\right\}\left(\mu-\mathrm{N}_{2}\right)\left\{\mathrm{ReCl}_{2, \text { trans }}\left(\mathrm{PNP}^{i \mathrm{Pr}}\right)\right\}\right]$ (33a, composed of trans-Cl fragment $\mathbf{B}$ and cis- $\mathrm{Cl}$ fragment $\mathbf{C})$ :

${ }^{1} \mathbf{H}\left\{{ }^{31} \mathbf{P}\right\}$-NMR $\left(500 \mathrm{MHz}\right.$, toluene- $\left.d_{8},-30{ }^{\circ} \mathrm{C}\right): \delta(\mathrm{ppm})=2.72\left(\right.$ hept, ${ }^{3} \mathrm{~J}_{\mathrm{HH}}=7.1 \mathrm{~Hz}, 2 \mathrm{H}$, B, $\mathrm{CH}\left(\mathrm{CH}_{3}\right)_{2}$ ), 2.65 (hept, $\left.{ }^{3} \mathrm{~J}_{\mathrm{HH}}=7.2 \mathrm{~Hz}, 2 \mathrm{H}, \mathrm{C}, \mathrm{CH}\left(\mathrm{CH}_{3}\right)_{2}\right), 2.56$ (hept, ${ }^{3} \mathrm{~J}_{\mathrm{HH}}=7.2 \mathrm{~Hz}$, $\left.2 \mathrm{H}, \mathrm{B}, \mathrm{CH}\left(\mathrm{CH}_{3}\right)_{2}\right), 2.40$ (hept, $\left.{ }^{3} \mathrm{~J}_{\mathrm{HH}}=7.2 \mathrm{~Hz}, 2 \mathrm{H}, \mathrm{C}, \mathrm{CH}\left(\mathrm{CH}_{3}\right)_{2}\right), 1.85\left(\mathrm{~d},{ }^{3} J_{\mathrm{HH}}=7.5 \mathrm{~Hz}\right.$, C, $\left.\mathrm{CH}\left(\mathrm{CH}_{3}\right)\left(\mathrm{CH}_{3}\right)\right), 1.67\left(\mathrm{~d},{ }^{3} J_{\mathrm{HH}}=7.3 \mathrm{~Hz}, \mathrm{C}, \mathrm{CH}\left(\mathrm{CH}_{3}\right)\left(\mathrm{CH}_{3}\right)\right), 1.52\left(\mathrm{~d},{ }^{3} J_{\mathrm{HH}}=7.2 \mathrm{~Hz}, \mathbf{B}\right.$, $\left.\mathrm{CH}\left(\mathrm{CH}_{3}\right)\left(\mathrm{CH}_{3}\right)\right), 1.36\left(\mathrm{~d},{ }^{3} J_{\mathrm{HH}}=7.3 \mathrm{~Hz}, \mathbf{C}, \mathrm{CH}\left(\mathrm{CH}_{3}\right)\left(\mathrm{CH}_{3}\right)\right), 1.31\left(\mathrm{~d},{ }^{3} J_{\mathrm{HH}}=7.1 \mathrm{~Hz}, \mathbf{B}\right.$, $\left.\mathrm{CH}\left(\mathrm{CH}_{3}\right)\left(\mathrm{CH}_{3}\right)\right), 1.23\left(\mathrm{~d},{ }^{3} J_{\mathrm{HH}}=7.0 \mathrm{~Hz}, \mathbf{C}, \mathrm{CH}\left(\mathrm{CH}_{3}\right)\left(\mathrm{CH}_{3}\right)\right), 1.08\left(\mathrm{~d},{ }^{3} J_{\mathrm{HH}}=7.1 \mathrm{~Hz}, \mathbf{B}\right.$, $\left.\mathrm{CH}\left(\mathrm{CH}_{3}\right)\left(\mathrm{CH}_{3}\right)\right), 1.05\left(\mathrm{~d},{ }^{3} \mathrm{~J}_{\mathrm{HH}}=7.3 \mathrm{~Hz}, \mathrm{~B}, \mathrm{CH}\left(\mathrm{CH}_{3}\right)\left(\mathrm{CH}_{3}\right)\right) .{ }^{15} \mathrm{~N}\left\{{ }^{1} \mathbf{H}\right\}-\mathrm{NMR}(50.7 \mathrm{MHz}$, toluene- $\left.d_{8},-30^{\circ} \mathrm{C}\right): \delta(\mathrm{ppm})=-4.1\left(\mathrm{dt},{ }^{1} J_{\mathrm{NN}}=5.6 \mathrm{~Hz},{ }^{2} J_{\mathrm{NP}}=2.6 \mathrm{~Hz}, \operatorname{Re}_{\mathrm{C}}-\mathrm{N}\right),-18.8$ $\left(\mathrm{d},{ }^{1} J_{\mathrm{NN}}=5.6 \mathrm{~Hz}, \operatorname{Re}_{\mathrm{B}}-\mathrm{N}\right),{ }^{31} \mathbf{P}\left\{{ }^{1} \mathbf{H}\right\}-\mathrm{NMR}\left(202.5 \mathrm{MHz}\right.$, toluene- $\left.d_{8},-30^{\circ} \mathrm{C}\right): \delta(\mathrm{ppm})$ $=-10.8\left(\mathrm{~d},{ }^{2} J_{\mathrm{PP}}=253.0 \mathrm{~Hz}, \mathrm{P}_{\mathrm{B}}\right),-14.1\left(\mathrm{~d},{ }^{2} J_{\mathrm{PP}}=253.0 \mathrm{~Hz}, \mathrm{P}_{\mathrm{B}}\right),-16.4\left(\mathrm{~s}, 2 \mathrm{P}, \mathrm{P}_{\mathrm{C}}\right)$. Under a ${ }^{15} \mathrm{~N}\left\{{ }^{1} \mathrm{H}\right\}$ atmosphere, the singlet at $-16.4 \mathrm{ppm}$ is split into a doublet with ${ }^{2} J_{\mathrm{PN}}=$ $2.7 \mathrm{~Hz}$.

$\left[\left\{\operatorname{ReCl}_{2, \text { cis }}\left(\mathrm{PNP}^{i \mathrm{Pr}}\right)\right\}\left(\mu-\mathrm{N}_{2}\right)\left\{\mathrm{ReCl}_{2, \text { cis }}\left(\mathrm{PNP} \mathrm{Pr}^{\mathrm{Pr}}\right)\right\}\right]$ (33b, composed of two cis-Cl fragments A):

${ }^{15} \mathbf{N}\left\{{ }^{1} \mathbf{H}\right\}-\mathbf{N M R}\left(50.7 \mathrm{MHz}\right.$, toluene- $\left.d_{8},-30{ }^{\circ} \mathrm{C}\right): \delta(\mathrm{ppm})=-12.7(\mathrm{~s}),{ }^{31} \mathbf{P}\left\{{ }^{1} \mathbf{H}\right\}-\mathbf{N M R}$ $\left(202.5 \mathrm{MHz}\right.$, toluene- $\left.d_{8},-30^{\circ} \mathrm{C}\right): \delta(\mathrm{ppm})=1.3\left(\mathrm{~d},{ }^{2} J_{\mathrm{PP}}=276.2 \mathrm{~Hz}\right),-1.2\left(\mathrm{~d},{ }^{2} J_{\mathrm{PP}}=\right.$ $276.2 \mathrm{~Hz})$.

\subsection{5 $\left[\operatorname{Re}(\mathrm{N}) \mathrm{Cl}\left(\mathrm{PNP}^{i \mathrm{iPr}}\right)\right](34)$}

$\left[\operatorname{Re}(\mathrm{N}) \mathrm{Cl}_{2}\left(\mathrm{HPNP}^{i \mathrm{Pr}}\right)\right](37)(7.5 \mathrm{mg}, 12.9 \mu \mathrm{mol}, 1.0 \mathrm{eq})$ and $\mathrm{KO}^{t} \mathrm{Bu}(1.4 \mathrm{mg}, 12.9 \mu \mathrm{mol}, 1.0 \mathrm{eq})$ are mixed in benzene $(1 \mathrm{~mL})$ and stirred overnight. All volatiles are removed in vacuo and the product is extracted with $\mathrm{Et}_{2} \mathrm{O}$. After recrystallization from a minimal amount of pentane at $-80^{\circ} \mathrm{C},\left[\operatorname{Re}(\mathrm{N}) \mathrm{Cl}\left(\mathrm{PNP}^{i \mathrm{Pr}}\right)\right](34)$ can be isolated as orange solid.

${ }^{1} \mathbf{H}-\mathbf{N M R}\left(500 \mathrm{MHz}, \mathrm{C}_{6} \mathrm{D}_{6}, 25^{\circ} \mathrm{C}\right): \delta(\mathrm{ppm})=3.51-3.39(\mathrm{~m}, 2 \mathrm{H}, \mathrm{N}-\mathrm{CHH}), 3.20-3.12$ $(\mathrm{m}, 2 \mathrm{H}, \mathrm{N}-\mathrm{CHH}), 2.70-2.61\left(\mathrm{~m}, 2 \mathrm{H}, \mathrm{CH}\left(\mathrm{CH}_{3}\right)_{2}\right), 1.90-1.80\left(\mathrm{~m}, 2 \mathrm{H}, \mathrm{CH}\left(\mathrm{CH}_{3}\right)_{2}\right), 1.65-$ $1.55(\mathrm{~m}, 2 \mathrm{H}, \mathrm{P}-\mathrm{CHH}), 1.37-1.30\left(\mathrm{~m}, 6 \mathrm{H}, \mathrm{CH}\left(\mathrm{CH}_{3}\right)\left(\mathrm{CH}_{3}\right)\right), 1.29-1.20(\mathrm{~m}, 2 \mathrm{H}, \mathrm{P}-\mathrm{CH} H)$, 1.19-1.13 (m, 6H, CH(CH $\left.\left(\mathrm{CH}_{3}\right)\right), 1.04-0.98\left(\mathrm{~m}, 6 \mathrm{H}, \mathrm{CH}\left(\mathrm{CH}_{3}\right)\left(\mathrm{CH}_{3}\right)\right), 0.90-0.84(\mathrm{~m}, 6 \mathrm{H}$, 
$\left.\mathrm{CH}\left(\mathrm{CH}_{3}\right)\left(\mathrm{CH}_{3}\right)\right) .{ }^{1} \mathbf{H}\left\{{ }^{31} \mathbf{P}\right\}-N M R\left(500 \mathrm{MHz}, \mathrm{C}_{6} \mathrm{D}_{6}, 25^{\circ} \mathrm{C}\right): \delta(\mathrm{ppm})=3.45\left(\mathrm{ddd}, J_{\mathrm{HH}}=\right.$ $10.9,7.9,2.8 \mathrm{~Hz}, 2 \mathrm{H}, \mathrm{N}-\mathrm{CHH}), 3.16\left(\mathrm{ddd}, J_{\mathrm{HH}}=11.2,9.4,6.3 \mathrm{~Hz}, 2 \mathrm{H}, \mathrm{N}-\mathrm{CHH}\right), 2.66$ (hept, $\left.{ }^{3} J_{\mathrm{HH}}=7.1 \mathrm{~Hz}, 2 \mathrm{H}, \mathrm{CH}\left(\mathrm{CH}_{3}\right)_{2}\right), 1.85$ (hept, $\left.{ }^{3} J_{\mathrm{HH}}=6.9 \mathrm{~Hz}, 2 \mathrm{H}, \mathrm{CH}\left(\mathrm{CH}_{3}\right)_{2}\right), 1.60$ (ddd, $\left.J_{\mathrm{HH}}=14.3,9.4,7.9 \mathrm{~Hz}, 2 \mathrm{H}, \mathrm{P}-\mathrm{CHH}\right), 1.33\left(\mathrm{~d},{ }^{3} J_{\mathrm{HH}}=6.9 \mathrm{~Hz}, 6 \mathrm{H}, \mathrm{CH}\left(\mathrm{CH}_{3}\right)\left(\mathrm{CH}_{3}\right)\right.$ ), $1.23\left(\mathrm{ddd}, J_{\mathrm{HH}}=14.3,6.3,2.7 \mathrm{~Hz}, 2 \mathrm{H}, \mathrm{P}-\mathrm{CHH}\right), 1.16\left(\mathrm{~d},{ }^{3} J_{\mathrm{HH}}=6.9 \mathrm{~Hz}, 6 \mathrm{H}, \mathrm{CH}\left(\mathrm{CH}_{3}\right)\right.$ $\left.\left(\mathrm{CH}_{3}\right)\right), 1.01\left(\mathrm{~d},{ }^{3} J_{\mathrm{HH}}=7.1 \mathrm{~Hz}, 6 \mathrm{H}, \mathrm{CH}\left(\mathrm{CH}_{3}\right)\left(\mathrm{CH}_{3}\right)\right), 0.87\left(\mathrm{~d},{ }^{3} J_{\mathrm{HH}}=7.2 \mathrm{~Hz}, 6 \mathrm{H}\right.$, $\left.\mathrm{CH}\left(\mathrm{CH}_{3}\right)\left(\mathrm{CH}_{3}\right)\right) \cdot{ }^{13} \mathrm{C}\left\{{ }^{1} \mathrm{H}\right\}-\mathrm{NMR}\left(125.8 \mathrm{MHz}, \mathrm{C}_{6} \mathrm{D}_{6}, 25^{\circ} \mathrm{C}\right): \delta(\mathrm{ppm})=70.4$ (vt, ${ }^{2} J_{\mathrm{CP}}=$ $\left.4.3 \mathrm{~Hz}, 2 \mathrm{C}, \mathrm{N}-\mathrm{CH}_{2}\right), 25.1\left(\mathrm{vt},{ }^{1} J_{\mathrm{CP}}=12.1 \mathrm{~Hz}, 2 \mathrm{C}, \mathrm{CH}\left(\mathrm{CH}_{3}\right)_{2}\right), 23.8\left(\mathrm{vt},{ }^{1} J_{\mathrm{CP}}=12.3 \mathrm{~Hz}\right.$, $\left.2 \mathrm{C}, \mathrm{P}-\mathrm{CH}_{2}\right), 21.8\left(\mathrm{vt},{ }^{1} J_{\mathrm{CP}}=13.6 \mathrm{~Hz}, 2 \mathrm{C}, \mathrm{CH}\left(\mathrm{CH}_{3}\right)_{2}\right), 18.7\left(\mathrm{~s}, 2 \mathrm{C}, \mathrm{CH}\left(\mathrm{CH}_{3}\right)\left(\mathrm{CH}_{3}\right)\right.$ ), $18.4\left(\mathrm{~s}, 2 \mathrm{C}, \mathrm{CH}\left(\mathrm{CH}_{3}\right)\left(\mathrm{CH}_{3}\right)\right), 18.0\left(\mathrm{~s}, 2 \mathrm{C}, \mathrm{CH}\left(\mathrm{CH}_{3}\right)\left(\mathrm{CH}_{3}\right)\right), 16.6\left(\mathrm{~s}, 2 \mathrm{C}, \mathrm{CH}\left(\mathrm{CH}_{3}\right)\left(\mathrm{CH}_{3}\right)\right)$. ${ }^{31} \mathbf{P}\left\{{ }^{1} \mathbf{H}\right\}$-NMR $\left(202.5 \mathrm{MHz}, \mathrm{C}_{6} \mathrm{D}_{6}, 25^{\circ} \mathrm{C}\right): \delta(\mathrm{ppm})=74.7$ (s). Mass spect. (LIFDI), $m / z(\%)=540.6(100)$.

\subsection{6 $\left[\mathrm{ReCl}\left(\mathrm{N}_{2}\right)_{2}\left(\mathrm{HPNP}^{\mathrm{iPr}}\right)\right]$}

$\left[\mathrm{ReCl}_{3}\left(\mathrm{HPNP}^{i \mathrm{Pr}}\right)\right]$ (29) $(5.0 \mathrm{mg}, 8.4 \mu \mathrm{mol}, 1.0 \mathrm{eq})$ and $\mathrm{NaHg}(1.0 \mathrm{M}, \mathrm{xxmg}, 17.6 \mu \mathrm{mol}$, $2.1 \mathrm{eq}$ ) mixed in THF (dried over $\mathrm{Na} / \mathrm{K}, 1 \mathrm{~mL}$ ) under an $\mathrm{N}_{2}$ atmosphere and stirred for $2 \mathrm{~h}$. The solution always contained at least one side product, the NMR signature of which pointed to a THF double $\mathrm{CH}$ activation product comparable to a previous published $\mathrm{Ir}$ compound. ${ }^{[228]}$ Separation of this product was not achieved with a practial procedure and only once a rather clean batch of $\mathbf{3 5}$ was obtained by several recrystallizations but with overall yields below $15 \%$.

${ }^{1} \mathbf{H}-\mathbf{N M R}\left(400 \mathrm{MHz}, \mathrm{C}_{6} \mathrm{D}_{6}, 25^{\circ} \mathrm{C}\right): \delta(\mathrm{ppm})=3.08-2.95\left(\mathrm{~m}, 2 \mathrm{H}, \mathrm{CH}\left(\mathrm{CH}_{3}\right)_{2}\right), 2.89\left(\mathrm{~m}_{\mathrm{br}}, 1 \mathrm{H}\right.$, $\mathrm{NH})$, 2.57-2.43 (m, 2H, XXX), 2.43-2.31 (m, 2H, CH( $\left.\left.\mathrm{CH}_{3}\right)_{2}\right), 2.02-1.87(\mathrm{~m}, 2 \mathrm{H}, \mathrm{XXX}), 1.75-$ $1.65(\mathrm{~m}, 2 \mathrm{H}, \mathrm{XXX}), 1.51-1.40\left(\mathrm{~m}, 6 \mathrm{H}, \mathrm{CH}\left(\mathrm{CH}_{3}\right)\left(\mathrm{CH}_{3}\right)\right), 1.36-1.21(\mathrm{~m}, 12 \mathrm{H}$, superimposed 2 $\left.\times \mathrm{CH}\left(\mathrm{CH}_{3}\right)\left(\mathrm{CH}_{3}\right)\right), 1.14-1.04\left(\mathrm{~m}, 6 \mathrm{H}, \mathrm{CH}\left(\mathrm{CH}_{3}\right)\left(\mathrm{CH}_{3}\right)\right) .{ }^{1} \mathbf{H}\left\{{ }^{31} \mathbf{P}\right\}-\mathrm{NMR}\left(400 \mathrm{MHz}, \mathrm{C}_{6} \mathrm{D}_{6}\right.$, $\left.25^{\circ} \mathrm{C}\right): \delta(\mathrm{ppm})=3.01\left(\right.$ hept, $\left.2 \mathrm{H},{ }^{3} J_{\mathrm{HH}}=7.3 \mathrm{~Hz}, \mathrm{CH}\left(\mathrm{CH}_{3}\right)_{2}\right), 2.89\left(\mathrm{~m}_{\mathrm{br}}, 1 \mathrm{H}, \mathrm{NH}\right), 2.57-$ $2.43(\mathrm{~m}, 2 \mathrm{H}, \mathrm{XXX}), 2.38$ (hept, $\left.2 \mathrm{H},{ }^{3} J_{\mathrm{HH}}=7.2 \mathrm{~Hz}, 2 \mathrm{H}, \mathrm{CH}\left(\mathrm{CH}_{3}\right)_{2}\right), 2.02-1.87(\mathrm{~m}, 2 \mathrm{H}$, $\mathrm{XXX}), 1.75-1.65(\mathrm{~m}, 2 \mathrm{H}, \mathrm{XXX}), 1.45\left(\mathrm{~d},{ }^{3} \mathrm{~J}_{\mathrm{HH}}=7.4 \mathrm{~Hz}, 6 \mathrm{H}, \mathrm{CH}\left(\mathrm{CH}_{3}\right)\left(\mathrm{CH}_{3}\right)\right), 1.30(\mathrm{~d}$, $\left.{ }^{3} J_{\mathrm{HH}}=7.1 \mathrm{~Hz}, 6 \mathrm{H}, \mathrm{CH}\left(\mathrm{CH}_{3}\right)\left(\mathrm{CH}_{3}\right)\right), 1.26\left(\mathrm{~d},{ }^{3} J_{\mathrm{HH}}=7.4 \mathrm{~Hz}, 6 \mathrm{H}, \mathrm{CH}\left(\mathrm{CH}_{3}\right)\left(\mathrm{CH}_{3}\right)\right), 1.09$ $\left(\mathrm{d},{ }^{3} J_{\mathrm{HH}}=7.1 \mathrm{~Hz}, 6 \mathrm{H}, \mathrm{CH}\left(\mathrm{CH}_{3}\right)\left(\mathrm{CH}_{3}\right)\right) .{ }^{31} \mathrm{P}\left\{{ }^{1} \mathbf{H}\right\}-\mathrm{NMR}\left(162.0 \mathrm{MHz}, \mathrm{C}_{6} \mathrm{D}_{6}, 25^{\circ} \mathrm{C}\right): \delta$ $(\mathrm{ppm})=34.5(\mathrm{~s}) . \mathbf{C V}: E_{1 / 2}$ vs $\mathrm{Fc} / \mathrm{Fc}^{+}(\mathrm{V})=-0.23(\operatorname{Re}(\mathrm{I} / \mathrm{II})),-3.34\left(E_{\mathrm{p}, \mathrm{a}}\right.$, irreversible). ATR-IR: $\tilde{\nu}\left(\mathrm{cm}^{-1}\right)=3152.6\left(\nu_{\mathrm{N}-\mathrm{H}}\right), 2038.8\left(\nu_{\mathrm{N} \equiv \mathrm{N}}\right), 1935.1\left(\nu_{\mathrm{N} \equiv \mathrm{N}}\right)$. Mass spect. (LIFDI), $m / z(\%)=583.2(100)$.

\subsection{7 $\left[\left(\mu-\mathrm{N}_{2}\right)\left\{\mathrm{ReCl}_{2}\left(\mathrm{HPNP}^{\mathrm{iPr}}\right)\right\}_{2}\right](36)$}

$\left[\mathrm{ReCl}_{3}\left(\mathrm{HPNP}^{i \mathrm{Pr}}\right)\right]$ (29) $(20.0 \mathrm{mg}, 33.5 \mu \mathrm{mol}, 1.0 \mathrm{eq})$ and $\mathrm{Co}\left(\mathrm{Cp}^{*}\right)_{2}(15.4 \mathrm{mg}, 46.8 \mu \mathrm{mol}$, $1.4 \mathrm{eq})$ are placed in a Schlenk tube. Degassed THF $(2 \mathrm{~mL})$ is vacuum-transferred to the 
tube and the reaction is thawed under $\mathrm{N}_{2}$ atmosphere in a $-60^{\circ} \mathrm{C}$ cooling bath. The reaction is stirred, slowly warmed to RT over $3 \mathrm{~h}$ and finally stirred at RT for an additional hour. After that, all volatiles are removed in vacuo and the product is washed with $\mathrm{Et}_{2} \mathrm{O}$ and extracted with toluene. The solution is concentrated in vacuo, layered with $\mathrm{Et}_{2} \mathrm{O}$ and stored at $-80^{\circ} \mathrm{C}$ for four days. [( $\left.\left.\mu-\mathrm{N}_{2}\right)\left\{\mathrm{ReCl}_{2}\left(\mathrm{HPNP}^{\mathrm{iPr}}\right)\right\}_{2}\right](36)$ was isolated as a spectroscopically pure, deep blue powder $(10.8 \mathrm{mg}, 9.4 \mu \mathrm{mol}, 56.0 \%)$. A ${ }^{15} \mathrm{~N}_{2}$-labeled sample was prepared analogously under a ${ }^{15} \mathrm{~N}_{2}$ atmosphere.

${ }^{1} \mathbf{H}-\mathrm{NMR}\left(500 \mathrm{MHz}, \mathrm{THF}-d_{8}, 25^{\circ} \mathrm{C}\right): \delta(\mathrm{ppm})=93.23\left(\mathrm{t},{ }^{1} J_{\mathrm{NH}}=11.4 \mathrm{~Hz}, 2 \mathrm{H}, \mathrm{NH}\right), 6.28$ $\left(\mathrm{m},{ }^{3} J_{\mathrm{HH}}=7.4 \mathrm{~Hz}, 2 \mathrm{H}, \mathrm{CHMe}\right), 5.63\left(\mathrm{~m},{ }^{3} J_{\mathrm{HH}}=7.4 \mathrm{~Hz}, 2 \mathrm{H}, \mathrm{CHMe}\right), 4.28\left(\mathrm{~d},{ }^{3} J_{\mathrm{HP}}=\right.$ $\left.13.6 \mathrm{~Hz},{ }^{3} J_{\mathrm{HH}}=7.6 \mathrm{~Hz}, 6 \mathrm{H}, \mathrm{CH}\left(\mathrm{CH}_{3}\right)_{2}\right), 3.66\left(\mathrm{~m},{ }^{3} J_{\mathrm{HH}}=7.6 \mathrm{~Hz}, 2 \mathrm{H}, \mathrm{CHMe}\right), 3.57(\mathrm{~m}$, overlapping with THF $\left.-d_{8}, 6 \mathrm{H}, \mathrm{CH}\left(\mathrm{CH}_{3}\right)_{2}\right), 3.13\left(\mathrm{~m},{ }^{3} J_{\mathrm{HH}}=7.3 \mathrm{~Hz}, 2 \mathrm{H}, \mathrm{CHMe} 2\right), 2.88(\mathrm{dd}$, $\left.{ }^{3} J_{\mathrm{HP}}=14.2 \mathrm{~Hz},{ }^{3} J_{\mathrm{HH}}=7.6 \mathrm{~Hz}, 6 \mathrm{H}, \mathrm{CH}\left(\mathrm{CH}_{3}\right)_{2}\right), 2.76\left(\mathrm{~m},{ }^{3} J_{\mathrm{HH}}=7.2 \mathrm{~Hz}, 6 \mathrm{H}, \mathrm{CH}\left(\mathrm{CH}_{3}\right)_{2}\right)$, $2.75\left(\mathrm{~m},{ }^{3} J_{\mathrm{HH}}=7.1 \mathrm{~Hz}, 6 \mathrm{H}, \mathrm{CH}\left(\mathrm{CH}_{3}\right)_{2}\right), 2.30\left(\mathrm{dd},{ }^{3} J_{\mathrm{HP}}=11.7 \mathrm{~Hz},{ }^{3} J_{\mathrm{HH}}=7.1 \mathrm{~Hz}, 6 \mathrm{H}\right.$, $\left.\mathrm{CH}\left(\mathrm{CH}_{3}\right)_{2}\right), 2.14\left(\mathrm{dd},{ }^{3} J_{\mathrm{HP}}=14.7 \mathrm{~Hz},{ }^{3} J_{\mathrm{HH}}=7.6 \mathrm{~Hz}, 6 \mathrm{H}, \mathrm{CH}\left(\mathrm{CH}_{3}\right)_{2}\right), 1.49\left(\mathrm{dd},{ }^{3} J_{\mathrm{HP}}\right.$ $\left.=14.7 \mathrm{~Hz},{ }^{3} J_{\mathrm{HH}}=7.6 \mathrm{~Hz}, 6 \mathrm{H}, \mathrm{CH}\left(\mathrm{CH}_{3}\right)_{2}\right),-0.33\left(\mathrm{~m}, 2 \mathrm{H}, \mathrm{P}-\mathrm{CHH}-\mathrm{CH}_{2}\right),-1.34(\mathrm{~m}, 2 \mathrm{H}$, $\left.\mathrm{P}-\mathrm{CHH}-\mathrm{CH}_{2}\right),-5.51\left(\mathrm{~m}, 2 \mathrm{H}, \mathrm{P}-\mathrm{CH} H-\mathrm{CH}_{2}\right),-7.65\left(\mathrm{~m}, 2 \mathrm{H}, \mathrm{P}-\mathrm{CH}-\mathrm{CH}_{2}\right),-8.57(\mathrm{~m}, 2 \mathrm{H}$, $\left.\mathrm{N}-\mathrm{CHH}-\mathrm{CH}_{2}\right),-11.21\left(\mathrm{~m}, 2 \mathrm{H}, \mathrm{N}-\mathrm{CH} H-\mathrm{CH}_{2}\right),-12.30\left(\mathrm{~m}, 2 \mathrm{H}, \mathrm{N}-\mathrm{CHH}-\mathrm{CH}_{2}\right),-12.77(\mathrm{~m}$, $\left.2 \mathrm{H}, \mathrm{N}-\mathrm{CH} H-\mathrm{CH}_{2}\right)$. For the assignment of $J_{\mathrm{HH}}$ coupling constants, a ${ }^{1} \mathrm{H}\left\{{ }^{31} \mathrm{P}\right\}$ spectrum was measured. ${ }^{13} \mathbf{C}\left\{{ }^{1} \mathbf{H}\right\}-N M R\left(125.8 \mathrm{MHz}, \mathrm{THF}-d_{8}, 25^{\circ} \mathrm{C}\right): \delta(\mathrm{ppm})=162.0(\mathrm{~s}, \mathrm{~N}-$ $\left.\mathrm{CH}_{2}\right), 161.6\left(\mathrm{~s}, \mathrm{~N}-\mathrm{CH}_{2}\right), 147.7\left(\mathrm{~d},{ }^{1} J_{\mathrm{CP}}=18.9 \mathrm{~Hz}, \mathrm{CHMe}\right), 136.0\left(\mathrm{~d},{ }^{1} J_{\mathrm{CP}}=19.4\right.$ $\left.\mathrm{Hz}, \mathrm{CHMe}_{2}\right), 84.0\left(\mathrm{~d},{ }^{1} J_{\mathrm{CP}}=19.6 \mathrm{~Hz}, \mathrm{P}-\mathrm{CH}_{2}\right), 82.4\left(\mathrm{~d},{ }^{1} J_{\mathrm{CP}}=20.1 \mathrm{~Hz}, \mathrm{P}-\mathrm{CH}_{2}\right), 65.4$ $\left.\left(\mathrm{d},{ }^{1} J_{\mathrm{CP}}=17.3 \mathrm{~Hz}, \mathrm{CHMe}\right), 49.7\left(\mathrm{~d},{ }^{1} J_{\mathrm{CP}}=17.6 \mathrm{~Hz}, \mathrm{CHMe}\right)_{2}\right), 36.2\left(\mathrm{~s}, \mathrm{CH}_{3}\right), 35.5$ $\left(\mathrm{s}, \mathrm{CH}_{3}\right), 21.9\left(\mathrm{~s}, \mathrm{CH}_{3}\right), 21.4\left(\mathrm{~s}, \mathrm{CH}_{3}\right), 20.9\left(\mathrm{~s}, \mathrm{CH}_{3}\right), 20.8\left(\mathrm{~s}, \mathrm{CH}_{3}\right), 18.8\left(\mathrm{~s}, \mathrm{CH}_{3}\right)$, $18.5\left(\mathrm{~s}, \mathrm{CH}_{3}\right) .{ }^{15} \mathrm{~N}\left\{{ }^{1} \mathbf{H}\right\}-\mathrm{NMR}\left(50.7 \mathrm{MHz}, \mathrm{THF}-d_{8}, 25^{\circ} \mathrm{C}\right): \delta(\mathrm{ppm})=-999.6(\mathrm{~s}, \mathrm{NH})$, $-1113.0(\mathrm{~s}, \mathrm{Re}=\mathrm{N}=\mathrm{N}=\mathrm{Re}) .{ }^{31} \mathbf{P}\left\{{ }^{1} \mathbf{H}\right\}-\mathrm{NMR}\left(202.5 \mathrm{MHz}, \mathrm{THF}-d_{8}, 25^{\circ} \mathrm{C}\right): \delta(\mathrm{ppm})=$ $-370.6\left(\mathrm{~d},{ }^{2} J_{\mathrm{PP}}=236.9 \mathrm{~Hz}\right),-380.4\left(\mathrm{~d},{ }^{2} \mathrm{JPP}_{\mathrm{PP}}=237.2 \mathrm{~Hz}\right) . \mathbf{C V}: E_{1 / 2} \mathrm{vs} \mathrm{Fc} / \mathrm{Fc}^{+}(\mathrm{V})$ $=-1.03\left(\operatorname{Re}^{\mathrm{II}} \operatorname{Re}^{\mathrm{II}} / \operatorname{Re}^{\mathrm{II}} \operatorname{Re}^{\mathrm{III}}\right), \quad-0.37\left(\operatorname{Re}^{\mathrm{II}} \operatorname{Re}^{\mathrm{III}} / \mathrm{Re}^{\mathrm{III}} \operatorname{Re}^{\mathrm{III}}\right)$. ATR-IR: $\tilde{\nu}\left(\mathrm{cm}^{-1}\right)=3146.4$ $\left(\nu_{\mathrm{N}-\mathrm{H}}\right)$. Mass spect. (LIFDI), $m / z(\%)=1152.2\left(100,[\mathrm{M}]^{+}\right)$. rRaman (frozen THF solution, $\left.\mathrm{cm}^{-1}\right)$ : $1733.3(36) / 1675.2\left({ }^{15} \mathrm{~N}_{2}-36\right)$. UV/Vis $(T H F, R T): \lambda / \mathrm{nm}\left(\epsilon / \mathrm{M}^{-1}\right.$ $\left.\mathrm{cm}^{-1}\right)=578$ (12293), 336 (38027).

\subsection{8 $\left[\operatorname{Re}(\mathrm{N}) \mathrm{Cl}_{2}\left(\mathrm{HPNP}^{\mathrm{iPr}}\right)\right](37)$}

Route $A$ - from azide as nitrogen source, as published: $\left[\operatorname{ReCl}_{3}\left(\mathrm{HPNP}^{\mathrm{iPr}}\right)\right](\mathbf{2 9})(30.0 \mathrm{mg}$, $50.2 \mu \mathrm{mol}, 1.0 \mathrm{eq})$ and $\left[\mathrm{N}\left(\mathrm{PPh}_{3}\right)_{2}\right] \mathrm{N}_{3}(29.1 \mathrm{mg}, 50.2 \mu \mathrm{mol}, 1.0 \mathrm{eq})$ are mixed in THF $(6 \mathrm{~mL})$ and stirred at $60^{\circ} \mathrm{C}$ for $3 \mathrm{~h}$. After that, the solvent is removed in vacuo and the product is extracted with toluene. The solution is concentrated and stored at $-70^{\circ} \mathrm{C}$ overnight. $\left[\operatorname{Re}(\mathrm{N}) \mathrm{Cl}_{2}\left(\mathrm{HPNP}^{i \mathrm{Pr}}\right)\right](37)$ is obtained as orange crystals by filtration and washed with a minimal amount of cold toluene $(24.5 \mathrm{mg}, 42.5 \mu \mathrm{mol}, 85 \%)$. 
${ }^{1} \mathbf{H}-\mathrm{NMR}\left(500 \mathrm{MHz}, \mathrm{CD}_{2} \mathrm{Cl}_{2}, 25^{\circ} \mathrm{C}\right): \delta(\mathrm{ppm})=4.93\left(\mathrm{t},{ }^{1} \mathrm{~J}_{\mathrm{NH}}=12.5 \mathrm{~Hz}, 1 \mathrm{H}, \mathrm{NH}\right), 3.64$ $\left(\mathrm{m}, 2 \mathrm{H}, \mathrm{N}-\mathrm{CHH}-\mathrm{CH}_{2}\right), 2.93\left(\mathrm{~m},{ }^{3} \mathrm{~J}_{\mathrm{HH}}=7.3 \mathrm{~Hz}, 2 \mathrm{H}, \mathrm{CHMe} 2\right), 2.71\left(\mathrm{~m}, 2 \mathrm{H}, \mathrm{N}-\mathrm{CH}-\mathrm{CH}_{2}\right)$, $2.52\left(\mathrm{~m},{ }^{3} \mathrm{~J}_{\mathrm{HH}}=6.9 \mathrm{~Hz}, 2 \mathrm{H}, \mathrm{CHMe}\right), 2.32\left(\mathrm{~m}, 2 \mathrm{H}, \mathrm{P}-\mathrm{CHH}-\mathrm{CH}_{2}\right), 1.91(\mathrm{~m}, 2 \mathrm{H}, \mathrm{P}-\mathrm{CH}-$ $\left.\mathrm{CH}_{2}\right), 1.55\left(\mathrm{~m},{ }^{3} J_{\mathrm{HH}}=7.4 \mathrm{~Hz}, 6 \mathrm{H}, \mathrm{CH}\left(\mathrm{CH}_{3}\right)_{2}\right), 1.53\left(\mathrm{~m},{ }^{3} \mathrm{~J}_{\mathrm{HH}}=6.9 \mathrm{~Hz}, 6 \mathrm{H}, \mathrm{CH}\left(\mathrm{CH}_{3}\right)_{2}\right)$, $1.33\left(\mathrm{~m},{ }^{3} \mathrm{~J}_{\mathrm{HH}}=7.1 \mathrm{~Hz}, 6 \mathrm{H}, \mathrm{CH}\left(\mathrm{CH}_{3}\right)_{2}\right), 1.32\left(\mathrm{~m},{ }^{3} \mathrm{~J}_{\mathrm{HH}}=7.2 \mathrm{~Hz}, 6 \mathrm{H}, \mathrm{CH}\left(\mathrm{CH}_{3}\right)_{2}\right)$. For the assignment of $J_{\mathrm{HH}}$ coupling constants, a ${ }^{1} \mathrm{H}\left\{{ }^{31} \mathrm{P}\right\}$ spectrum was measured. ${ }^{13} \mathrm{C}\left\{{ }^{1} \mathbf{H}\right\}$-NMR $\left(125.8 \mathrm{MHz}, \mathrm{CD}_{2} \mathrm{Cl}_{2}, 25^{\circ} \mathrm{C}\right): \delta(\mathrm{ppm})=62.7\left(\mathrm{~s}, \mathrm{~N}-\mathrm{CH}_{2}\right), 31.4\left(\mathrm{AXY}, \mathrm{N}=\left|{ }^{1} J_{\mathrm{AX}}+{ }^{3} J_{\mathrm{AY}}\right|\right.$ $\left.=24.6 \mathrm{~Hz}, \mathrm{P}-\mathrm{CH}_{2}\right), 27.7\left(\mathrm{AXY}, \mathrm{N}=\left|{ }^{1} J_{\mathrm{AX}}+{ }^{3} J_{\mathrm{AY}}\right|=27.2 \mathrm{~Hz}, \mathrm{CHMe}_{2}\right), 25.0(\mathrm{AXY}, \mathrm{N}$ $\left.=\left|{ }^{1} J_{\mathrm{AX}}+{ }^{3} J_{\mathrm{AY}}\right|=20.2 \mathrm{~Hz}, C \mathrm{HMe}_{2}\right), 21.3\left(\mathrm{~s}, \mathrm{CH}_{3}\right), 19.2\left(\mathrm{~s}, \mathrm{CH}_{3}\right), 18.3\left(\mathrm{~s}, \mathrm{CH}_{3}\right), 18.0$ $\left(\mathrm{s}, \mathrm{CH}_{3}\right) \cdot{ }^{15} \mathrm{~N}\left\{{ }^{1} \mathrm{H}\right\}-\mathrm{NMR}\left(50.7 \mathrm{MHz}, \mathrm{CD}_{2} \mathrm{Cl}_{2}, 25^{\circ} \mathrm{C}\right): \delta(\mathrm{ppm})=-331.7(\mathrm{~s}, \mathrm{NH})$; the ${ }^{15} \mathrm{~N}$ chemical shift of the nitride ligand was obtained from ${ }^{15} \mathrm{~N}-37$ obtained on route $B$ (see below). ${ }^{31} \mathbf{P}\left\{{ }^{1} \mathbf{H}\right\}$-NMR $\left(202.5 \mathrm{MHz}, \mathrm{CD}_{2} \mathrm{Cl}_{2}, 25^{\circ} \mathrm{C}\right): \delta(\mathrm{ppm})=36.1$ (s). $\mathbf{C V}: E_{1 / 2}$ vs $\mathrm{Fc} / \mathrm{Fc}^{+}(\mathrm{V})=+0.01\left(\mathrm{Re}^{\mathrm{V}} / \mathrm{Re}^{\mathrm{vI}}\right)$. Elemental anal. Calc. for $\mathrm{C}_{16} \mathrm{H}_{37} \mathrm{Cl}_{2} \mathrm{~N}_{2} \mathrm{P}_{2} \mathrm{Re}(576.54)$ : $\mathrm{C}, 33.33 ; \mathrm{H}, 6.47 ; \mathrm{N}, 4.86$. Found: $\mathrm{C}, 33.27 ; \mathrm{H}, 6.34 ; \mathrm{N}, 4.24$. Mass spect. (LIFDI), $\mathrm{m} / \mathrm{z}$ $(\%)=576.1\left(100,[\mathrm{M}]^{+}\right), 540.1\left(60,[\mathrm{M}-\mathrm{Cl}]^{+}\right) . \mathbf{U V} / \mathrm{Vis}(\mathrm{THF}, \mathrm{RT}): \lambda / \mathrm{nm}\left(\epsilon / \mathrm{M}^{-1} \mathrm{~cm}^{-1}\right)$ $=\sim 484$ ( 100), 405 ( 180), 325 (1540), 285 (2030), 237 (6194).

Route $A$ - from azide as nitrogen source, improved: $\left[\mathrm{ReCl}_{3}\left(\mathrm{HPNP}{ }^{i \mathrm{Pr}}\right)\right]$ (29) (250.0 mg, $418 \mu \mathrm{mol}, 1.0 \mathrm{eq})$ and $\mathrm{NaN}_{3}(31.3 \mathrm{mg}, 481 \mu \mathrm{mol}, 1.15 \mathrm{eq})$ are mixed in DCM $(5 \mathrm{~mL})$ and $\mathrm{EtOH}(7.5 \mathrm{~mL})$ and stirred at $60^{\circ} \mathrm{C}$ for $16 \mathrm{~h}$. The solvent is removed in vacuo, the product is washed with $\mathrm{Et}_{2} \mathrm{O}$ and extracted with hot benzene $\left(60^{\circ} \mathrm{C}\right) .\left[\operatorname{Re}(\mathrm{N}) \mathrm{Cl}_{2}\left(\mathrm{HPNP}^{\mathrm{iPr}}\right)\right](37)$ is obtained as orange solid (186 mg, $323 \mu \mathrm{mol}, 77 \%$ ).

Route $B$ - photolysis of 36: A solution of $\left[\left(\mu-\mathrm{N}_{2}\right)\left\{\mathrm{ReCl}_{2}\left(\mathrm{HPNP}^{\mathrm{iPr}}\right)\right\}_{2}\right](\mathbf{3 6})(5 \mathrm{mg})$ in THF $(0.5 \mathrm{~mL})$ is irradiated with either a $\mathrm{Xe}(\mathrm{Hg})$ lamp equipped with a water filter and a long wave pass filter $(\lambda>305 \mathrm{~nm})$ or with a $390 \mathrm{~nm}$ LED lamp for $2 \mathrm{~h}$. $\left[\operatorname{Re}(\mathrm{N}) \mathrm{Cl}_{2}\left(\mathrm{HPNP}^{\mathrm{iPr}}\right)\right](37)$ is obtained in both cases in $95 \%$ spectroscopic yield relative to an internal standard. Labeled ${ }^{15} \mathrm{~N}_{2}$-36 gives the corresponding ${ }^{15} \mathrm{~N}$ nitride complex ${ }^{15} \mathrm{~N}-37$, which was used for ${ }^{15} \mathrm{~N} N M R$ spectroscopic characterization. Using the $\mathrm{Xe}(\mathrm{Hg})$ lamp with a long wave pass filter $(\lambda>$ $420 \mathrm{~nm})$ gave no detectable reaction. With a band gap filter $(\lambda=337 \pm 10 \mathrm{~nm})$ very slow formation to minor splitting products were observed over several hours. NMR spectroscopic features as well as the LIFDI mass spectrum are identical with those of $\mathbf{3 7}$ obtained from route $A$.

${ }^{15} \mathbf{N}\left\{{ }^{1} \mathbf{H}\right\}-\operatorname{NMR}\left(50.7 \mathrm{MHz}, \mathrm{CD}_{2} \mathrm{Cl}_{2}, 25^{\circ} \mathrm{C}\right): \delta(\mathrm{ppm})=383.8(\mathrm{~s}, \mathrm{Re} \equiv N)$.

\subsection{9 $\left.\operatorname{Re}(\mathrm{NH}) \mathrm{Cl}_{2}\left(\mathrm{HPNP}^{i \mathrm{Pr}}\right)\right]^{\mathrm{OTf}}\left(38^{\mathrm{OTf}}\right)$}

$\left[\operatorname{Re}(\mathrm{N}) \mathrm{Cl}_{2}\left(\mathrm{HPNP}^{i \mathrm{Pr}}\right)\right](37)(20.0 \mathrm{mg}, 34.7 \mu \mathrm{mol}, 1.0 \mathrm{eq})$ is supsended in toluene and HOTf $(3.65 \mu \mathrm{L}, 6.3 \mathrm{mg}, 41.6 \mu \mathrm{mol}, 1.2 \mathrm{eq})$ is added with an Eppendorf pipette. The solution is 
stirred for $16 \mathrm{~h}$ at RT. Afterwards all volatiles are removed in vacuo, the product is extracted with $\mathrm{DCM}$, concentrated, layered with $\mathrm{Et}_{2} \mathrm{O}$ and stored at $-40{ }^{\circ} \mathrm{C}$ overnight. After filtration and removal of residual solvent, $\left.\left[\mathrm{Re}(\mathrm{NH}) \mathrm{Cl}_{2}\left(\mathrm{HPNP}^{i \mathrm{Pr}}\right)\right]^{\mathrm{OTf}}\right]\left(\mathbf{3 8}^{\mathrm{OTf}}\right)$ is obtained as analytically pure complex. However, due to the broad nature of the peaks, judging the spectroscopic purity of the complex is tricky. Additionally the linewidth of the peaks prevented measurement of a ${ }^{1} \mathrm{H}-{ }^{1} \mathrm{H}$ correlation spectroscopy (COSY) or a ${ }^{1} \mathrm{H}-{ }^{13} \mathrm{C} \mathrm{HMBC}$, therefore some peaks are assigned based on empirism (i.e. by comparsion with other spectra).

${ }^{1} \mathbf{H}-\mathrm{NMR}\left(500 \mathrm{MHz}, \mathrm{CD}_{2} \mathrm{Cl}_{2},-35^{\circ} \mathrm{C}\right): \delta(\mathrm{ppm})=14.25(\mathrm{br}, 1 \mathrm{H}, \mathrm{Re} \equiv \mathrm{N}-\mathrm{H}), 4.96(\mathrm{br}$, $1 \mathrm{H}, \mathrm{N}_{\mathrm{PNP}}-\mathrm{H}$ ), 3.98-3.80 (br, $\left.2 \mathrm{H}, \mathrm{N}-\mathrm{HH}\right), 2.98-2.87$ (br, 2H, N-HH), 2.87-2.78 (br, $2 \mathrm{H}$, $\left.\mathrm{CH}\left(\mathrm{CH}_{3}\right)_{2}\right), 2.65-2.55$ (br, 2H, CH$\left.\left(\mathrm{CH}_{3}\right)_{2}\right), 2.55-2.45$ (br, 2H, $\left.\mathrm{P}-\mathrm{HH}\right), 2.08-1.93(\mathrm{br}, 2 \mathrm{H}$, $\mathrm{P}-\mathrm{HH}), 1.56-1.48\left(\mathrm{br}, 6 \mathrm{H}, \mathrm{CH}\left(\mathrm{CH}_{3}\right)\left(\mathrm{CH}_{3}\right)\right), 1.47-1.40\left(\mathrm{br}, 6 \mathrm{H}, \mathrm{CH}\left(\mathrm{CH}_{3}\right)\left(\mathrm{CH}_{3}\right)\right), 1.40-1.33$ (br, 6H, CH( $\left.\left.\mathrm{CH}_{3}\right)\left(\mathrm{CH}_{3}\right)\right), 1.27-1.20\left(\mathrm{br}, 6 \mathrm{H}, \mathrm{CH}\left(\mathrm{CH}_{3}\right)\left(\mathrm{CH}_{3}\right)\right) .{ }^{13} \mathrm{C}\left\{{ }^{1} \mathbf{H}\right\}-\mathrm{NMR}(125.8 \mathrm{MHz}$, $\left.\mathrm{CD}_{2} \mathrm{Cl}_{2},-35^{\circ} \mathrm{C}\right): \delta(\mathrm{ppm})=63.3\left(\mathrm{br}, \mathrm{N}-\mathrm{CH}_{2}\right), 30.9\left(\mathrm{br}, \mathrm{P}-\mathrm{CH}_{2}\right), 26.9\left(\mathrm{vt}, \mathrm{ABB}^{\prime} \mathrm{A}^{\prime}, \mathrm{N}\right.$ $\left.=\left|{ }^{1} J_{\mathrm{AB}}+{ }^{3} J_{\mathrm{AB}}\right|=28.9 \mathrm{~Hz}, C \mathrm{CHM}_{2}\right), 25.2\left(\mathrm{vt}, \mathrm{ABB}^{\prime} \mathrm{A}^{\prime}, \mathrm{N}=\left|{ }^{1} J_{\mathrm{AB}}+{ }^{3} J_{\mathrm{AB}^{\prime}}\right|=19.7 \mathrm{~Hz}\right.$, $\left.\mathrm{CHMe}_{2}\right), 20.8$ (br, $\left.\mathrm{CH}\left(\mathrm{CH}_{3}\right)\left(\mathrm{CH}_{3}\right)\right), 17.9$ (br, $\left.\mathrm{CH}\left(\mathrm{CH}_{3}\right)\left(\mathrm{CH}_{3}\right)\right), 17.7$ (br, $\mathrm{CH}\left(\mathrm{CH}_{3}\right)\left(\mathrm{CH}_{3}\right)$ ), $17.5\left(\mathrm{br}, \mathrm{CH}\left(\mathrm{CH}_{3}\right)\left(\mathrm{CH}_{3}\right)\right) .{ }^{15} \mathrm{~N}\left\{{ }^{1} \mathbf{H}\right\}-\mathrm{NMR}\left(50.7 \mathrm{MHz} \mathrm{CD}_{2} \mathrm{Cl}_{2},-35^{\circ} \mathrm{C}\right): \delta(\mathrm{ppm})=18.1$ $(\mathrm{Re} \equiv \mathrm{N}-\mathrm{H}),-329.8\left(N_{P N P}-H\right) .{ }^{31} \mathbf{P}\left\{{ }^{1} \mathbf{H}\right\}-\mathrm{NMR}\left(202.5 \mathrm{MHz} \mathrm{CD}_{2} \mathrm{Cl}_{2},-35^{\circ} \mathrm{C}\right): \delta(\mathrm{ppm})$ $=30.2$ (br). Elemental anal. Calc. for $\mathrm{C}_{17} \mathrm{H}_{38} \mathrm{Cl}_{2} \mathrm{~F}_{3} \mathrm{~N}_{2} \mathrm{O}_{3} \mathrm{P}_{2} \mathrm{ReS}$ (726.61): C, 28.10; $\mathrm{H}$, 5.27; N, 3.86. Found: C, 27.68; H, 5.03; N, 3.77.

\subsubsection{0 $\left[\operatorname{Re}\left(\mathrm{NBAr}_{18}^{\mathrm{F}}\right) \mathrm{Cl}_{2}\left(\mathrm{HPNP}^{\mathrm{iPr}}\right)\right]$}

$\left[\operatorname{Re}(\mathrm{N}) \mathrm{Cl}_{2}\left(\mathrm{HPNP}^{i \mathrm{Pr}}\right)\right](37)(5.0 \mathrm{mg}, 8.7 \mu \mathrm{mol}, 1.0 \mathrm{eq})$ and tris $\{3,5$-bis(trifluoromethyl)phenyl $\}$ borane $\left(\operatorname{BAr}_{18}^{\mathrm{F}}\right)(5.6 \mathrm{mg}, 8.7 \mu \mathrm{mol}, 1.0 \mathrm{eq})$ are mixed in DCM and stirred for $5 \mathrm{~min}$, during which the color of the solution changes to light blue. The solvent is removed in vacuo, the product is washed with pentane, extracted with $\mathrm{Et}_{2} \mathrm{O}$ and dried under reduced pressure. cis- $\left[\operatorname{Re}\left(\mathrm{NBAr}_{18}^{\mathrm{F}}\right) \mathrm{Cl}_{2}\left(\mathrm{HPNP}^{i \mathrm{Pr}}\right)\right]$ (cis-39) is obtained, which slowly converts into trans-[Re $\left.\left(\mathrm{NBAr}_{18}{ }^{\mathrm{F}}\right) \mathrm{Cl}_{2}\left(\mathrm{HPNP}^{i \mathrm{Pr}}\right)\right]$ (trans-39) overnight in solution.

cis-[Re( $\left.\left.\mathrm{NBAr}_{18}^{\mathrm{F}}\right) \mathrm{Cl}_{2}\left(\mathrm{HPNP}^{i \mathrm{Pr}}\right)\right]($ cis-39):

${ }^{1} \mathbf{H}-\mathbf{N M R}\left(400 \mathrm{MHz}, \mathrm{CD}_{2} \mathrm{Cl}_{2}, 25^{\circ} \mathrm{C}\right): \delta(\mathrm{ppm})=8.00\left(\mathrm{~s}, 6 \mathrm{H}, \mathrm{C}_{\text {ortho }} \mathrm{H}\right), 7.68\left(\mathrm{~s}, 3 \mathrm{H}, \mathrm{C}_{\text {para }} \mathrm{H}\right)$, $4.98(\mathrm{br}, 1 \mathrm{H}, \mathrm{NH}), 3.42-3.34(\mathrm{~m}, 2 \mathrm{H}, \mathrm{N}-\mathrm{CHH}), 2.95\left(\mathrm{~m},{ }^{3} J_{\mathrm{HH}}=7.4 \mathrm{~Hz}, 2 \mathrm{H}, \mathrm{CHMe} 2\right), 2.38-$ $2.27(\mathrm{~m}, 2 \mathrm{H}, \mathrm{P}-\mathrm{CHH}), 2.11\left(\mathrm{~m},{ }^{3} \mathrm{~J}_{\mathrm{HH}}=7.2 \mathrm{~Hz}, 2 \mathrm{H}, \mathrm{CHMe} 2\right), 2.05-1.93(\mathrm{~m}, 2 \mathrm{H}, \mathrm{N}-\mathrm{CH} H)$, 1.93-1.81 (m, 2H, P-CHH), $1.60\left(\mathrm{~m},{ }^{3} J_{\mathrm{HH}}=7.4 \mathrm{~Hz}, 6 \mathrm{H}, \mathrm{CH}\left(\mathrm{CH}_{3}\right)\left(\mathrm{CH}_{3}\right)\right), 1.34\left(\mathrm{~m},{ }^{3} J_{\mathrm{HH}}\right.$ $\left.=7.4 \mathrm{~Hz}, 6 \mathrm{H}, \mathrm{CH}\left(\mathrm{CH}_{3}\right)\left(\mathrm{CH}_{3}\right)\right), 1.09\left(\mathrm{~m},{ }^{3} J_{\mathrm{HH}}=7.2 \mathrm{~Hz}, 6 \mathrm{H}, \mathrm{CH}\left(\mathrm{CH}_{3}\right)\left(\mathrm{CH}_{3}\right)\right), 0.96(\mathrm{~m}$, $\left.{ }^{3} J_{\mathrm{HH}}=7.3 \mathrm{~Hz}, 6 \mathrm{H}, \mathrm{CH}\left(\mathrm{CH}_{3}\right)\left(\mathrm{CH}_{3}\right)\right) .{ }^{31} \mathbf{P}\left\{{ }^{1} \mathbf{H}\right\}-\mathrm{NMR}\left(162.0 \mathrm{MHz}, \mathrm{CD}_{2} \mathrm{Cl}_{2}, 25^{\circ} \mathrm{C}\right): \delta(\mathrm{ppm})$ $=23.9(\mathrm{~s})$. 
trans- $\left[\operatorname{Re}\left(\mathrm{NBAr}_{18}^{\mathrm{F}}\right) \mathrm{Cl}_{2}\left(\mathrm{HPNP}^{i \mathrm{Pr}}\right)\right]$ (trans-39):

${ }^{1} \mathbf{H}-\mathbf{N M R}\left(400 \mathrm{MHz}, \mathrm{CD}_{2} \mathrm{Cl}_{2}, 25^{\circ} \mathrm{C}\right): \delta(\mathrm{ppm})=7.86\left(\mathrm{~s}, 6 \mathrm{H}, \mathrm{C}_{\text {ortho }} \mathrm{H}\right), 7.68\left(\mathrm{~s}, 3 \mathrm{H}, \mathrm{C}_{\text {para }} \mathrm{H}\right)$, 3.03-2.96 (m, 2H, N-CHH), $2.96\left(\mathrm{~m},{ }^{3} J_{\mathrm{HH}}=7.3 \mathrm{~Hz}, 2 \mathrm{H}, \mathrm{CHMe}\right), 2.84\left(\mathrm{~m},{ }^{3} J_{\mathrm{HH}}=\right.$ $7.3 \mathrm{~Hz}, 2 \mathrm{H}, \mathrm{CHMe}$ ) , 2.56-2.51 (m, 2H, N-CHH), $2.51(\mathrm{br}, 1 \mathrm{H}, \mathrm{NH}), 2.37-2.30(\mathrm{~m}, 2 \mathrm{H}$, $\mathrm{P}-\mathrm{CH} H), 1.93-1.80(\mathrm{~m}, 2 \mathrm{H}, \mathrm{P}-\mathrm{CHH}), 1.29\left(\mathrm{~m},{ }^{3} J_{\mathrm{HH}}=7.3 \mathrm{~Hz}, 6 \mathrm{H}, \mathrm{CH}\left(\mathrm{CH}_{3}\right)\left(\mathrm{CH}_{3}\right)\right), 1.16$ $\left(\mathrm{m},{ }^{3} J_{\mathrm{HH}}=7.1 \mathrm{~Hz}, 6 \mathrm{H}, \mathrm{CH}\left(\mathrm{CH}_{3}\right)\left(\mathrm{CH}_{3}\right)\right), 0.87\left(\mathrm{~m},{ }^{3} J_{\mathrm{HH}}=7.0 \mathrm{~Hz}, 6 \mathrm{H}, \mathrm{CH}\left(\mathrm{CH}_{3}\right)\left(\mathrm{CH}_{3}\right)\right)$, $0.75\left(\mathrm{~m},{ }^{3} J_{\mathrm{HH}}=7.2 \mathrm{~Hz}, 6 \mathrm{H}, \mathrm{CH}\left(\mathrm{CH}_{3}\right)\left(\mathrm{CH}_{3}\right)\right) .{ }^{13} \mathrm{C}\left\{{ }^{1} \mathrm{H}\right\}-\mathrm{NMR}\left(100.7 \mathrm{MHz}, \mathrm{CD}_{2} \mathrm{Cl}_{2}, 25^{\circ} \mathrm{C}\right)$ : $\delta(\mathrm{ppm})=152.2\left(\mathrm{br}, C_{\mathrm{ipso}}\right), 134.8\left(\mathrm{br}, \mathrm{C}_{\text {ortho }} \mathrm{H}\right), 129.4\left(\right.$ quart, $\left.{ }^{2} J_{\mathrm{CF}}=32.2 \mathrm{~Hz}, C_{\text {meta }} \mathrm{CF}_{3}\right)$, 124.2 (quart, ${ }^{1} J_{\mathrm{CF}}=272.5 \mathrm{~Hz}, C_{3}$ ), 119.6 (hept, ${ }^{3} J_{\mathrm{CF}}=4.0 \mathrm{~Hz}, C_{\text {para }} \mathrm{H}$ ), 47.2 (br, $\left.\mathrm{N}-\mathrm{CH}_{2}\right), 24.9\left(\mathrm{vt}, \mathrm{ABB}^{\prime} \mathrm{A}^{\prime}, \mathrm{N}=\left|{ }^{1} J_{\mathrm{AB}}+{ }^{3} J_{\mathrm{AB}}\right|=27.1 \mathrm{~Hz}, \mathrm{P}-\mathrm{CH}_{2}\right), 24.6$ (vt, ABB'A', $\left.\mathrm{N}=\left|{ }^{1} J_{\mathrm{AB}}+{ }^{3} J_{\mathrm{AB}^{\prime}}\right|=25.1 \mathrm{~Hz}, \mathrm{CHMe}_{2}\right), 20.8\left(\mathrm{vt}, \mathrm{ABB}^{\prime} \mathrm{A}^{\prime}, \mathrm{N}=\left|{ }^{1} J_{\mathrm{AB}}+{ }^{3} J_{\mathrm{AB}^{\prime}}\right|=24.6 \mathrm{~Hz}\right.$, $\left.\mathrm{CHMe}_{2}\right), 19.4$ (br, $\left.\mathrm{CH}\left(\mathrm{CH}_{3}\right)\left(\mathrm{CH}_{3}\right)\right), 19.0$ (br, $\left.\mathrm{CH}\left(\mathrm{CH}_{3}\right)\left(\mathrm{CH}_{3}\right)\right), 18.2\left(\mathrm{br}, \mathrm{CH}\left(\mathrm{CH}_{3}\right)\left(\mathrm{CH}_{3}\right)\right.$ ), $18.2\left(\mathrm{br}, \mathrm{CH}\left(\mathrm{CH}_{3}\right)\left(\mathrm{CH}_{3}\right)\right) .{ }^{15} \mathrm{~N}\left\{{ }^{1} \mathbf{H}\right\}-\mathbf{N M R}\left(50.7 \mathrm{MHz}, \mathrm{CD}_{2} \mathrm{Cl}_{2}, 25^{\circ} \mathrm{C}\right): \delta(\mathrm{ppm})=-316.5$ (s). ${ }^{31} \mathbf{P}\left\{{ }^{1} \mathbf{H}\right\}-N M R\left(162.0 \mathrm{MHz}, \mathrm{CD}_{2} \mathrm{Cl}_{2}, 25^{\circ} \mathrm{C}\right): \delta(\mathrm{ppm})=21.2$ (s). Elemental anal. Calc. for $\mathrm{C}_{40} \mathrm{H}_{46} \mathrm{BCl}_{2} \mathrm{~F}_{18} \mathrm{~N}_{2} \mathrm{P}_{2} \operatorname{Re}$ (1226.66): C, 39.17; $\mathrm{H}, 3.78 ; \mathrm{N}, 2.28$. Found: C, 39.37; H, 3.88; N, 2.49 .

\subsubsection{1 $\left[\mathrm{ReCl}_{3}\left(\mathrm{P}=\mathrm{NP} \mathrm{PPr}^{\mathrm{PP}}\right)\right](40)$}

Route $A$ - oxidation of 29: $\left[\operatorname{ReCl}_{3}\left(\mathrm{HPNP}^{\mathrm{iPr}}\right)\right](29)(40 \mathrm{mg}, 66.9 \mu \mathrm{mol}, 1.0 \mathrm{eq})$ and 2,4,6-tristert-butylphenoxy radical $(35.0 \mathrm{mg}, 133.8 \mu \mathrm{mol}, 2.0 \mathrm{eq})$ are mixed in chlorobenzene $(5 \mathrm{~mL})$ and stirred at $60^{\circ} \mathrm{C}$ overnight. After $16 \mathrm{~h}$ the solvent is removed in vacuo. The product is washed with $\mathrm{Et}_{2} \mathrm{O}$ and benzene and subsequently extracted with $\mathrm{DCM}$. The product $\left[\operatorname{ReCl}_{3}\left(\mathrm{P}=\mathrm{NP}^{i \mathrm{Pr}}\right)\right](40)$ is obtained as an orange powder $(36.4 \mathrm{mg}, 61.1 \mu \mathrm{mol}, 91 \%)$.

${ }^{1} \mathbf{H}-\mathrm{NMR}\left(500 \mathrm{MHz}, \mathrm{CD}_{2} \mathrm{Cl}_{2}, 25^{\circ} \mathrm{C}\right): \delta(\mathrm{ppm})=62.96\left(\mathrm{~s}, 2 \mathrm{H}, \mathrm{P}-\mathrm{CH}_{2}-\mathrm{CH}\right), 12.60\left(\mathrm{~m},{ }^{3} \mathrm{~J}_{\mathrm{HH}}\right.$ $=7.3 \mathrm{~Hz}, 2 \mathrm{H}, \mathrm{CHMe}), 10.25\left(\mathrm{dd},{ }^{3} J_{\mathrm{HH}}=6.8 \mathrm{~Hz},{ }^{3} J_{\mathrm{HP}}=10.0 \mathrm{~Hz}, 6 \mathrm{H}, \mathrm{CH}\left(\mathrm{CH}_{3}\right)_{2}\right), 9.67$ $\left(\mathrm{dd},{ }^{3} J_{\mathrm{HH}}=7.2 \mathrm{~Hz},{ }^{3} J_{\mathrm{HP}}=13.3 \mathrm{~Hz}, 6 \mathrm{H}, \mathrm{CH}\left(\mathrm{CH}_{3}\right)_{2}\right), 9.09\left(\mathrm{dd},{ }^{3} J_{\mathrm{HH}}=6.8 \mathrm{~Hz},{ }^{3} J_{\mathrm{HP}}\right.$ $\left.=12.7 \mathrm{~Hz}, 6 \mathrm{H}, \mathrm{CH}\left(\mathrm{CH}_{3}\right)_{2}\right), 7.49\left(\mathrm{dd},{ }^{3} J_{\mathrm{HH}}=7.2 \mathrm{~Hz},{ }^{3} J_{\mathrm{HP}}=15.1 \mathrm{~Hz}, 6 \mathrm{H}, \mathrm{CH}\left(\mathrm{CH}_{3}\right)_{2}\right)$, $6.87\left(\mathrm{~m},{ }^{3} \mathrm{~J}_{\mathrm{HH}}=7.1 \mathrm{~Hz}, 2 \mathrm{H}, \mathrm{CHMe}_{2}\right), 4.20\left(\mathrm{~m},{ }^{3} \mathrm{~J}_{\mathrm{HH}}=6.2 \mathrm{~Hz}, 2 \mathrm{H}, \mathrm{P}-\mathrm{CH}_{2}-\mathrm{CH}_{2}\right),-16.27$ $\left(\mathrm{m},{ }^{3} J_{\mathrm{HH}}=6.2 \mathrm{~Hz}, 2 \mathrm{H}, \mathrm{N}-\mathrm{CH}_{2}-\mathrm{CH}_{2}\right),-73.85\left(\mathrm{~d},{ }^{3} J_{\mathrm{HP}}=14.6 \mathrm{~Hz}, 1 \mathrm{H}, \mathrm{N}-\mathrm{CH}-\mathrm{CH}_{2}\right) . J_{\mathrm{HH}}$ coupling constants were assigned by ${ }^{1} \mathrm{H}\left\{{ }^{31} \mathrm{P}\right\}$ spectroscopy. ${ }^{13} \mathrm{C}\left\{{ }^{1} \mathbf{H}\right\}-\mathrm{NMR}(125.8 \mathrm{MHz}$, $\left.\mathrm{CD}_{2} \mathrm{Cl}_{2}, 25^{\circ} \mathrm{C}\right): \delta(\mathrm{ppm})=433.4\left(\mathrm{~s}, \mathrm{~N}-\mathrm{CH}-\mathrm{CH}_{2}\right), 172.4\left(\mathrm{~d},{ }^{1} \mathrm{~J}_{\mathrm{CP}}=16.2 \mathrm{~Hz}, \mathrm{CHMe}_{2}\right)$, $143.0\left(\mathrm{~d},{ }^{1} J_{\mathrm{CP}}=16.6 \mathrm{~Hz}, \mathrm{CHMe}\right), 135.8\left(\mathrm{~d},{ }^{2} J_{\mathrm{CP}}=7.0 \mathrm{~Hz}, \mathrm{~N}-\mathrm{CH}_{2}-\mathrm{CH}_{2}\right), 21.7\left(\mathrm{~d},{ }^{1} J_{\mathrm{CP}}\right.$ $\left.=22.2 \mathrm{~Hz}, \mathrm{P}-\mathrm{CH}_{2}-\mathrm{CH}_{2}\right), 21.1\left(\mathrm{~s}, \mathrm{CH}\left(\mathrm{CH}_{3}\right)_{2}\right), 20.8\left(\mathrm{~s}, \mathrm{CH}\left(\mathrm{CH}_{3}\right)_{2}\right), 18.3\left(\mathrm{~s}, \mathrm{CH}\left(\mathrm{CH}_{3}\right)_{2}\right)$, $16.4\left(\mathrm{~s}, \mathrm{CH}\left(\mathrm{CH}_{3}\right)_{2}\right),-9.6\left(\mathrm{~s}, \mathrm{P}-\mathrm{CH}_{2}-\mathrm{CH}\right) .{ }^{31} \mathbf{P}\left\{{ }^{1} \mathbf{H}\right\}-\mathrm{NMR}\left(202.5 \mathrm{MHz}, \mathrm{CD}_{2} \mathrm{Cl}_{2}, 25^{\circ} \mathrm{C}\right): \delta$ $(\mathrm{ppm})=-1592.6\left(\mathrm{~d},{ }^{2} J_{\mathrm{PP}}=247.6 \mathrm{~Hz}\right),-1616.5\left(\mathrm{~d},{ }^{2} \mathrm{~J}_{\mathrm{PP}}=248.1 \mathrm{~Hz}\right) . \mathbf{C V}: E_{1 / 2} \mathrm{vs} \mathrm{Fc} / \mathrm{Fc}^{+}$ $(\mathrm{V})=-1.70\left(\operatorname{Re}^{\mathrm{II}} / \mathrm{Re}^{\mathrm{III}}\right),-0.28\left(\mathrm{Re}^{\mathrm{III}} / \mathrm{Re}^{\mathrm{IV}}\right)$. Elemental anal. Calc. for $\mathrm{C}_{16} \mathrm{H}_{35} \mathrm{Cl}_{3} \mathrm{NP}_{2} \mathrm{Re}$ (595.97): C, 32.25; H, 5.92; N, 2.35. Found: C, 32.56; H, 6.18; N, 2.39. Mass spect. (LIFDI), $m / z(\%)=95.0\left(100,[\mathrm{M}]^{+}\right)$. 
Route $B$ - reaction of 37 with benzoyl chloride: In a typical experiment, $\left[\mathrm{Re}(\mathrm{N}) \mathrm{Cl}_{2}\left(\mathrm{HPNP}^{i \mathrm{Pr}}\right)\right]$ (37) $(5.0 \mathrm{mg}, 8.7 \mu \mathrm{mol}, 1.0 \mathrm{eq})$ and benzoyl chloride $(2.01 \mu \mathrm{L}, 17.3 \mu \mathrm{mol}, 2.0 \mathrm{eq})$ are mixed in 1,4 -dioxane and heated to $80^{\circ} \mathrm{C}$ for $15 \mathrm{~h}$. All volatiles are subsequently removed in vacuo and 1,3,5-trimethoxybenzene $(4.4 \mathrm{mg}, 26.1 \mu \mathrm{mol}, 3 \mathrm{eq})$ is added as internal standard. NMR spectroscopic examination in $\mathrm{CD}_{2} \mathrm{Cl}_{2}$ reveals the formation of $\left[\mathrm{ReCl}_{3}\left(\mathrm{P}=\mathrm{NP} \mathrm{Pr}^{\mathrm{Pr}}\right)\right](\mathbf{4 0})$ in $71.0 \%$ yield. NMR spectroscopic features as well as the LIFDI mass spectrum are identical with those of $\mathbf{4 0}$ obtained from route $A$.

The reaction is accompanied by the formation of benzamide ( $30.5 \%$ spectroscopic yield) and equimolar amounts of benzoic acid and benzonitrile ( $64.5 \%$ spectroscopic yield each), respectively. Benzonitrile can be detected when the reaction is carried out in toluene- $d_{8}$. Use of 1 eq benzoyl chloride resultes in incomplete conversion of 37 . Tribenzamide is observed in minor quantities as additional product if the reaction is carried out at $100^{\circ} \mathrm{C}$ over $24 \mathrm{~h}$ or in THF for $65 \mathrm{~h}$ at $60^{\circ} \mathrm{C}$, respectively. 


\section{Computational details}

I love my computer

For all you give to me

Predictable errors and no identity

\section{- Bad Religion}

"I Love My Computer" on "The New America"

\subsection{General procedure}

For all independently carried out theoretical calculations, either the ORCA 3.0 or ORCA 4.0 program packages were used. ${ }^{[246,247]}$ DFT calculations were performed utilizing the PBE or PBE0 functionals. ${ }^{[248,249]}$ Ahlrich's double- $\zeta$ def2-SVP basis set (used in geometry optimizations and frequency calculations) as well as the triple- $\zeta$ def2-TZVP/def2-TZVPP (for single point calculations) were used for all elements but Os or Re, for which effective core potentials with the Stuttgart-Dresden 60 parameter sets replaced the inner shell $1 s-4 f$ orbitals. ${ }^{[250,251]}$ In all DFT calculations, the RI- $J(\mathrm{PBE})^{[252-255]}$ and RIJCOSX (PBE0) ${ }^{[256]}$ approximations together with the corresponding def2/J auxiliary basis sets ${ }^{[257]}$ were used, and the obtained energies were corrected for dispersion with Grimme's D3 model[258] with Becke-Johnson damping. ${ }^{[259]}$ In the ORCA nomenclature, the TightOpt and TightSCF convergence criteria were employed, the former only during geometry optimizations.

Frequency calculations were performed at the same level of theory as the geometry optimizations, verifying the obtained structures to be local minima or transition states (no or only one imaginary vibrational frequency, respectively). These calculations incorporated Grimme's quasi-RRHO aproach for low energy frequencies below $35 \mathrm{~cm}^{-1}$ in all calculations performed with ORCA 4.0. ${ }^{[260]}$ If solution data were simulated, solvent effects were accounted for in the single point calculations with the COSMO (ORCA 3.0) or the CPCM continuum solvation models. ${ }^{[261,262]}$ In these cases, the obtained Gibb's free energies were corrected for the difference between ideal gas standard conditions ( $1 \mathrm{~atm}, 298.15 \mathrm{~K}$ ) used by ORCA and standard solution conditions $\left(1 \mathrm{~mol} \mathrm{~L}^{-1}, 298.15 \mathrm{~K}\right)$ : 


$$
G_{\text {sol }}=G_{\text {gas }}+R T \ln \frac{R T}{p} G_{\text {sol }}=G_{\text {gas }}+R T \ln (24.47) G_{\text {sol }}=G_{\text {gas }}+7.93 \mathrm{~kJ} \mathrm{~mol}^{-1}
$$

Final free energies were obtained by combining the higher level single point energies (triple- $\zeta$ PBE0) with the obtained free energies according to:

$$
G=G_{\text {double } \zeta}-E_{\text {double } \zeta}^{\mathrm{el}}+E_{\text {triple } \zeta}^{\mathrm{el}}
$$

The results obtained from this approach are reported in the main text in the form "SinglePointMethod||FrequencyCalculationMethod". While the protonated HPNP pincer has only one possible conformation, the amide based, monoanionic PNP pincer ligand can adopt three different conformers, which exhibit slightly different energies. However, these were only compared when exact energies were needed, while during simple estimations, this effect was neglected. For the iso-propyl based PNP ligand it was found that rotation of the isopropyl groups did not lead to a significant variation of the energy, therefore conformations were derived from X-ray structures of the compounds (if available) or from closely related complexes, rather than comparing all 81 additionally possible rotation conformers (three possible orientations for each $i \operatorname{Pr}$ group).

The output files were analyzed using the CHEMCRAFT software package.[263]

\subsection{Model of $\left[\mathrm{Os}(\mathrm{N})\left(\mathrm{PNP}^{\mathrm{tBu}}\right)\right](3)$ and revised scan of the $\mathrm{N}-\mathrm{Os}=\mathrm{N}$ bending mode}

All calculations were performed with ORCA 3.0.3 according to the procedure described in Section 3.1.

Tab. 3.1. XZY geometry data for the DFT-derived structures of $\mathbf{3}$.

\begin{tabular}{llll|llll}
\hline & \multicolumn{1}{c}{$\mathrm{X}$} & \multicolumn{1}{c}{$\mathrm{Y}$} & \multicolumn{1}{c}{$\mathrm{Z}$} & & \multicolumn{1}{c}{$\mathrm{X}$} & \multicolumn{1}{c}{$\mathrm{Y}$} & \multicolumn{1}{c}{$\mathrm{Z}$} \\
\hline $\mathrm{Os}$ & -0.006854006 & 0.205414989 & 0.52005072 & $\mathrm{H}$ & 2.592067054 & 2.130772755 & -2.255401385 \\
$\mathrm{P}$ & -2.312891097 & 0.580036775 & 0.424485232 & $\mathrm{H}$ & -2.753875543 & 2.129887616 & -2.092107754 \\
$\mathrm{P}$ & 2.288611584 & 0.588687811 & 0.286122017 & $\mathrm{H}$ & -2.669420515 & 0.803222424 & -3.25405206 \\
$\mathrm{~N}$ & -0.012607445 & 2.283855916 & 0.342958922 & $\mathrm{H}$ & -1.315204363 & 1.093899165 & -2.144545362 \\
$\mathrm{~N}$ & 0.002746125 & -1.459589805 & 0.701208738 & $\mathrm{H}$ & -1.52147959 & -0.545922447 & 2.99263108 \\
$\mathrm{C}$ & -1.193376841 & 3.10149957 & 0.183092865 & $\mathrm{H}$ & -3.009270158 & -0.359550347 & 3.954141307 \\
$\mathrm{C}$ & -2.469222426 & 2.391767363 & 0.619826917 & $\mathrm{H}$ & -2.178686079 & 1.057155868 & 3.292694483 \\
$\mathrm{C}$ & 1.151459279 & 3.108076311 & 0.113052804 & $\mathrm{H}$ & 2.345716359 & 1.096529818 & 3.160000952 \\
$\mathrm{C}$ & 2.45678748 & 2.401937279 & 0.462184018 & $\mathrm{H}$ & 3.179500857 & -0.337394746 & 3.776479973 \\
$\mathrm{C}$ & 3.360787137 & -0.129477791 & 1.648788945 & $\mathrm{H}$ & 1.630573897 & -0.491542355 & 2.911483089 \\
$\mathrm{C}$ & 2.926684723 & 0.135317447 & -1.419500745 & $\mathrm{H}$ & 2.580138865 & -2.113620617 & 1.223529468 \\
$\mathrm{C}$ & -3.313312592 & -0.137597981 & 1.841228981 & $\mathrm{H}$ & 3.98363582 & -2.073780506 & 2.301265528 \\
$\mathrm{C}$ & -3.043219469 & 0.134791454 & -1.244537931 & $\mathrm{H}$ & 4.208938378 & -1.833199176 & 0.575049361 \\
$\mathrm{C}$ & 2.238377522 & 1.105969399 & -2.381402282 & $\mathrm{H}$ & 4.648219874 & 1.586469091 & 2.095020352
\end{tabular}




\begin{tabular}{llll|llll}
$\mathrm{C}$ & -2.403695994 & 1.10650989 & -2.237770937 & $\mathrm{H}$ & 5.322407336 & 0.482682341 & 0.888216137 \\
$\mathrm{C}$ & -2.448696434 & 0.01806729 & 3.093437625 & $\mathrm{H}$ & 5.282548125 & 0.022124218 & 2.585789156 \\
$\mathrm{C}$ & 2.575684542 & 0.049891154 & 2.950214535 & $\mathrm{H}$ & 1.400966089 & -1.396763231 & -1.645925309 \\
$\mathrm{C}$ & 3.538602613 & -1.624668168 & 1.409036077 & $\mathrm{H}$ & 2.9664351 & -2.032937288 & -1.147672176 \\
$\mathrm{C}$ & 4.726889616 & 0.53877522 & 1.797719665 & $\mathrm{H}$ & 2.748544987 & -1.488927321 & -2.809791925 \\
$\mathrm{C}$ & 2.479001937 & -1.284447595 & -1.768754105 & $\mathrm{H}$ & 4.816080605 & 1.234401774 & -1.348379349 \\
$\mathrm{C}$ & 4.436243236 & 0.237286753 & -1.583443982 & $\mathrm{H}$ & 4.695933299 & 0.013765212 & -2.623267565 \\
$\mathrm{C}$ & -3.533318622 & -1.628187316 & 1.609450616 & $\mathrm{H}$ & 4.961596195 & -0.487876604 & -0.962727751 \\
$\mathrm{C}$ & -4.656478115 & 0.555205398 & 2.0689134 & $\mathrm{H}$ & -2.594375851 & -2.13694092 & 1.382261992 \\
$\mathrm{C}$ & -2.617677778 & -1.285144817 & -1.619493754 & $\mathrm{H}$ & -4.244589558 & -1.823901582 & 0.807036364 \\
$\mathrm{C}$ & -4.55884438 & 0.243734706 & -1.328729684 & $\mathrm{H}$ & -3.947701736 & -2.067438239 & 2.521383943 \\
$\mathrm{H}$ & -1.29158506 & 3.433340822 & -0.863727688 & $\mathrm{H}$ & -4.541630138 & 1.599311175 & 2.365936862 \\
$\mathrm{H}$ & -1.091191895 & 4.026384388 & 0.773977732 & $\mathrm{H}$ & -5.178536822 & 0.044808874 & 2.883693101 \\
$\mathrm{H}$ & 1.08296729 & 4.029331642 & 0.714361901 & $\mathrm{H}$ & -5.301763227 & 0.515566175 & 1.193062346 \\
$\mathrm{H}$ & 1.181052293 & 3.445181517 & -0.936353279 & $\mathrm{H}$ & -1.535392561 & -1.400973194 & -1.549437224 \\
$\mathrm{H}$ & -3.349872214 & 2.785028827 & 0.107285685 & $\mathrm{H}$ & -2.935837218 & -1.485411536 & -2.647371075 \\
$\mathrm{H}$ & -2.619453231 & 2.552323469 & 1.688649928 & $\mathrm{H}$ & -3.076684004 & -2.034395105 & -0.977813833 \\
$\mathrm{H}$ & 2.684706181 & 2.568516576 & 1.516090591 & $\mathrm{H}$ & -4.920771228 & 1.243019635 & -1.075616052 \\
$\mathrm{H}$ & 3.298057128 & 2.792008455 & -0.114538905 & $\mathrm{H}$ & -5.055225636 & -0.478131684 & -0.680860376 \\
$\mathrm{H}$ & 1.15563665 & 1.092309581 & -2.235729422 & $\mathrm{H}$ & -4.873951187 & 0.020709413 & -2.353097625 \\
$\mathrm{H}$ & 2.454544841 & 0.801964813 & -3.409193871 & & & & \\
\hline
\end{tabular}
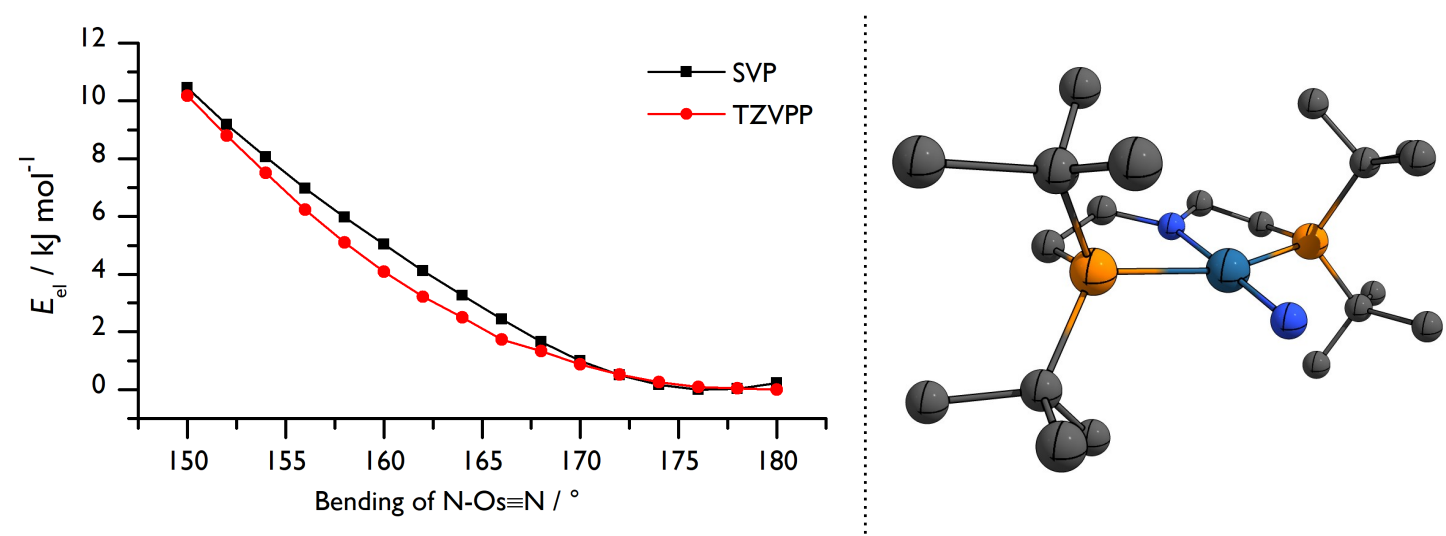

Fig. 3.1. Left: Relaxed surface scan of the $\mathrm{N}-\mathrm{Os} \equiv \mathrm{N}$ bending mode of 3. Right: DFT-derived geometry of 3 .

\subsection{Computational analysis of the reaction between 3 and $\mathrm{PMe}_{3}$ to form 7}

All calculations were performed using ORCA 3.0.3 according to the procedure described in Section 3.1. Geometry optimization and single point energy calculations at the double- and triple-zeta basis set quality, respectively, were performed with three functionals: PBE-D3BJ, PBE0-D3BJ and M06. The obtained results were benchmarked against the experimentally available Gibbs free energy of the reaction as well as known stretching vibrations of $\mathbf{3}$ and 
7 (see Table XX). PBE and M06 performed equally well with only $\approx 1 \mathrm{~kJ} \mathrm{~mol}^{-1}$ deviation in $\Delta G^{0}$ and rather good agreement with the vibrational frequencies while PBE0 exhibited less satisfying agreement. Due to lower computational costs and less problems with small imaginary frequencies, PBE was chosen for all subsequent computations and the presented data was obtained with this functional. The $\mathrm{N}$ analysis was performed by Dr. Markus Finger (see Tables 3.3 and 3.4 as well as Figure 1.3).

Tab. 3.2. Benchmarking of the DFT calculations.

\begin{tabular}{l|c|c|c|c}
\hline & PBE & PBE0 & M06 & Experimental \\
\hline$\Delta G^{0}\left(\mathbf{3}+\mathrm{PMe}_{3} \rightarrow \mathbf{7} / \mathrm{kJ} \mathrm{mol}^{-1}\right.$ & -13.2 & -32.6 & -11.3 & -12.4 \\
$\nu_{\mathrm{Os}=\mathrm{N}}(\mathbf{3}) / \mathrm{cm}^{-1}$ & 1040.3 & 1093.2 & 1973.4 & 999 \\
$\nu_{\mathrm{Os}-\mathrm{N}=\mathrm{P}}(\mathbf{7}) / \mathrm{cm}^{-1}$ & 1259.5 & 1317.0 & 1304.2 & 1240 \\
\hline
\end{tabular}

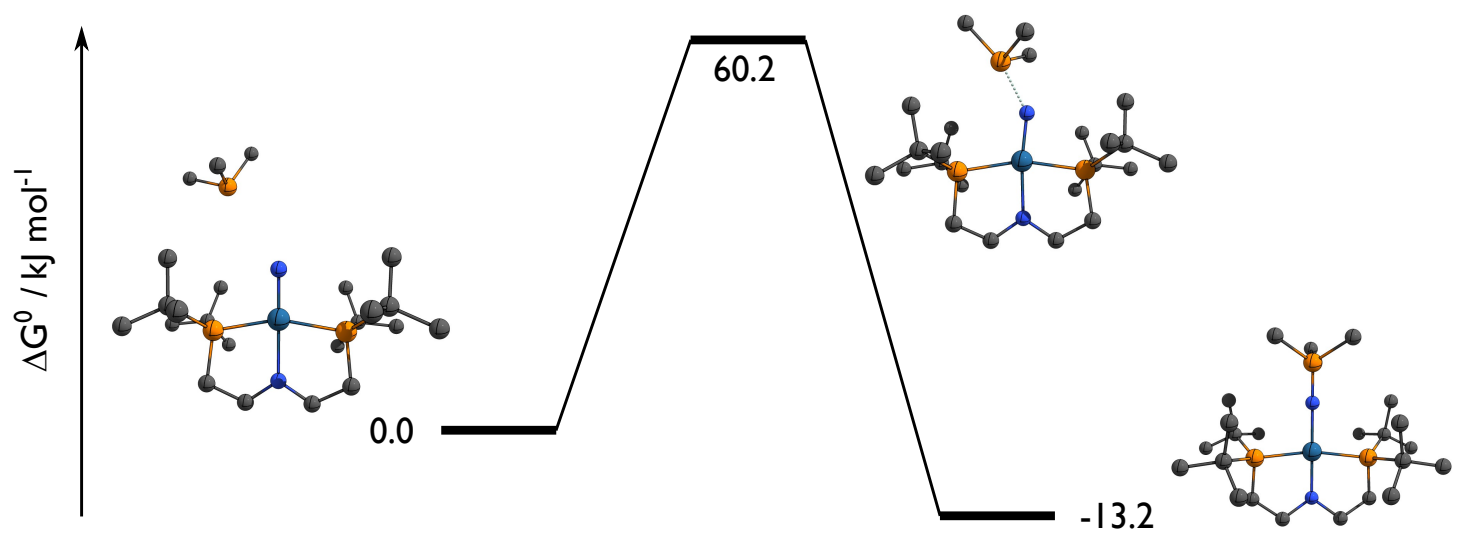

Fig. 3.2. Free Gibbs energy diagramm for the formation of $\mathbf{7}$ from $\mathbf{3}$ and $\mathrm{PMe}_{3}(\mathrm{PBE} / \mathrm{D} 3 \mathrm{BJ} / \mathrm{RI} / \mathrm{def} 2-$ TZVPP ||def2-SVP).

Tab. 3.3. Selected donor-acceptor interactions according to an NBO analysis of the transition state of the formation of 7 (PBE/D3BJ/RI/def2-TZVPP).

\begin{tabular}{c|c|c|c|c}
\hline Bond & Polarization (NLMOs) & Hybridization & LMO-Bond orders & Energy $\mathrm{kJ} \mathrm{mol}^{-1}$ \\
\hline $\mathrm{P} \longrightarrow \mathrm{N}$ & $78.5 \%(\mathrm{P} 3)$ & $\mathrm{sp} 0.71(\mathrm{P} 3)$ & $0.22(\mathrm{~N} 2-\mathrm{P} 3)$ & 168.2 \\
& $11.2 \%(\mathrm{~N} 2)$ & $\mathrm{p}(\mathrm{N} 2)$ & $-0.13(\mathrm{Os} 1-\mathrm{N} 2)$ & \\
& $6.7 \%(\mathrm{Os} 1)$ & $\mathrm{d}(\mathrm{Os} 1)$ & & \\
\hline $\mathrm{N} \longrightarrow \mathrm{P}$ & $91.2 \%(\mathrm{~N} 2)$ & $\mathrm{sp}^{0.40}(\mathrm{~N} 2)$ & $0.02(\mathrm{Os} 1-\mathrm{N} 2)$ & 142.4 \\
& $4.7 \%(\mathrm{P} 3)$ & $\mathrm{p}(\mathrm{P} 3)$ & $0.09(\mathrm{~N} 2-\mathrm{P} 3)$ & \\
& $2.0 \%(\mathrm{C} 21)$ & $\mathrm{sp}^{2.34}(\mathrm{C} 21)$ & $-0.04(\mathrm{P} 3-\mathrm{C} 21)$ & \\
& $0.9 \%(\mathrm{Os} 1)$ & $\mathrm{sd}^{0.18}(\mathrm{Os} 1)$ & & \\
\hline
\end{tabular}


Tab. 3.4. Atomic charges obtained from NPA analysis of $3, \mathrm{PMe}_{3}, \mathrm{TS}$, and 7 , respectively, with atoms with minor changes in grey.

\begin{tabular}{|c|c|c|}
\hline & def2-SVP & def2-TZVPP \\
\hline \multicolumn{3}{|c|}{3} \\
\hline Os & 76.03 & 75.97 \\
\hline$P_{\text {PNP }}$ & 14.01 & 13.90 \\
\hline $\mathrm{N}_{\mathrm{PNP}}$ & 7.74 & 7.64 \\
\hline$N_{\text {nitride }}$ & 7.32 & 7.38 \\
\hline \multicolumn{3}{|c|}{$\mathrm{PMe}_{3}$} \\
\hline$P$ & 14.28 & 14.26 \\
\hline C & 6.97 & 6.94 \\
\hline \multicolumn{3}{|c|}{ TS } \\
\hline Os & 76.04 & 75.98 \\
\hline$P_{\text {PNP }}$ & 13.99/14.02 & $13.87 / 13.91$ \\
\hline $\mathrm{N}_{\mathrm{PNP}}$ & 7.69 & 7.59 \\
\hline$N_{\text {nitride }}$ & 7.53 & 7.64 \\
\hline $\mathrm{P}_{\mathrm{PMe}_{3}}$ & 14.07 & 13.96 \\
\hline C21 & 6.98 & 6.97 \\
\hline C22/C23 & 6.96 & 6.95 \\
\hline \multicolumn{3}{|c|}{7} \\
\hline Os & 76.16 & 76.09 \\
\hline$P_{\text {PNP }}$ & 13.00 & 13.90 \\
\hline $\mathrm{N}_{\mathrm{PNP}}$ & 6.53 & 7.43 \\
\hline$N_{\text {nitride }}$ & 8.17 & 8.21 \\
\hline $\mathrm{P}_{\mathrm{PMe}_{3}}$ & 13.34 & 13.29 \\
\hline
\end{tabular}

\subsubsection{XYZ-Coordinates}

Tab. 3.5. $\mathrm{XYZ}$ geometries of $\mathbf{3}, \mathrm{PMe}_{3}$ and $\mathbf{7}$ derived with the PBE0 functional.

\begin{tabular}{|c|c|c|c|c|c|c|c|}
\hline & $x$ & $Y$ & Z & & $x$ & $\mathrm{Y}$ & Z \\
\hline \multicolumn{8}{|c|}{3} \\
\hline Os & -0.009576 & 0.174007 & 0.409504 & $\mathrm{H}$ & 2.726588 & 2.061792 & -2.33299 \\
\hline$P$ & -2.340317 & 0.563283 & 0.411803 & $\mathrm{H}$ & -2.941195 & 2.227809 & -2.044579 \\
\hline$P$ & 2.318477 & 0.562476 & 0.259128 & $\mathrm{H}$ & -2.891951 & 0.931069 & -3.258599 \\
\hline$N$ & -0.011518 & 2.284852 & 0.261143 & $\mathrm{H}$ & -1.478639 & 1.209081 & -2.198294 \\
\hline $\mathrm{N}$ & -0.012147 & -1.497198 & 0.475145 & $\mathrm{H}$ & -1.447917 & -0.649242 & 2.901646 \\
\hline C & -1.2015 & 3.091644 & 0.14433 & $\mathrm{H}$ & -2.903877 & -0.525841 & 3.948282 \\
\hline
\end{tabular}




\begin{tabular}{|c|c|c|c|c|c|c|c|}
\hline C & -2.447862 & 2.382776 & 0.672074 & $\mathrm{H}$ & -2.108096 & 0.939582 & 3.318435 \\
\hline C & 1.156717 & 3.084279 & -0.016086 & $\mathrm{H}$ & 2.283191 & 1.154249 & 3.149347 \\
\hline C & 2.454517 & 2.393773 & 0.399144 & $\mathrm{H}$ & 3.062579 & -0.299353 & 3.823985 \\
\hline C & 3.353129 & -0.102827 & 1.694191 & $\mathrm{H}$ & 1.543554 & -0.430785 & 2.869694 \\
\hline C & 3.038634 & 0.082876 & -1.421222 & $\mathrm{H}$ & 2.632305 & -2.123747 & 1.284872 \\
\hline C & -3.304894 & -0.189805 & 1.853509 & $\mathrm{H}$ & 4.014833 & -2.029797 & 2.407139 \\
\hline C & -3.157644 & 0.189404 & -1.250398 & $\mathrm{H}$ & 4.282595 & -1.810317 & 0.672113 \\
\hline C & 2.3759 & 1.023818 & -2.432058 & $\mathrm{H}$ & 4.56009 & 1.682398 & 2.128363 \\
\hline C & -2.579838 & 1.207085 & -2.237798 & $\mathrm{H}$ & 5.3328 & 0.556786 & 0.98603 \\
\hline C & -2.38333 & -0.098107 & 3.074702 & $\mathrm{H}$ & 5.237557 & 0.152336 & 2.709717 \\
\hline C & 2.50613 & 0.093575 & 2.956188 & $\mathrm{H}$ & 1.520621 & -1.456052 & -1.700182 \\
\hline C & 3.579108 & -1.600661 & 1.490124 & $\mathrm{H}$ & 3.061181 & -2.091727 & -1.086893 \\
\hline C & 4.690873 & 0.619707 & 1.873582 & $\mathrm{H}$ & 2.942017 & -1.58636 & -2.785048 \\
\hline C & 2.613541 & -1.349212 & -1.758923 & $\mathrm{H}$ & 4.909576 & 1.223969 & -1.264578 \\
\hline C & 4.555317 & 0.211734 & -1.515107 & $\mathrm{H}$ & 4.872136 & -0.006232 & -2.54919 \\
\hline C & -3.571086 & -1.667889 & 1.566263 & $\mathrm{H}$ & 5.073482 & -0.507064 & -0.866245 \\
\hline $\mathrm{C}$ & -4.614895 & 0.541974 & 2.157341 & $\mathrm{H}$ & -2.644657 & -2.192828 & 1.286361 \\
\hline C & -2.729258 & -1.208029 & -1.707198 & $\mathrm{H}$ & -4.31523 & -1.817649 & 0.771722 \\
\hline C & -4.678913 & 0.297775 & -1.237615 & $\mathrm{H}$ & -3.971362 & -2.145489 & 2.475598 \\
\hline $\mathrm{H}$ & -1.368597 & 3.412218 & -0.907189 & $\mathrm{H}$ & -4.447598 & 1.588831 & 2.453205 \\
\hline $\mathrm{H}$ & -1.082129 & 4.041124 & 0.709623 & $\mathrm{H}$ & -5.110934 & 0.043151 & 3.006773 \\
\hline $\mathrm{H}$ & 1.100899 & 4.053729 & 0.524108 & $\mathrm{H}$ & -5.317524 & 0.530688 & 1.314676 \\
\hline $\mathrm{H}$ & 1.206208 & 3.363375 & -1.091644 & $\mathrm{H}$ & -1.632813 & -1.279232 & -1.75316 \\
\hline $\mathrm{H}$ & -3.373895 & 2.805252 & 0.250409 & $\mathrm{H}$ & -3.14464 & -1.399108 & -2.711009 \\
\hline $\mathrm{H}$ & -2.501578 & 2.509619 & 1.763965 & $\mathrm{H}$ & -3.088277 & -2.000865 & -1.039254 \\
\hline $\mathrm{H}$ & 2.639301 & 2.585752 & 1.4665 & $\mathrm{H}$ & -5.026321 & 1.291868 & -0.914913 \\
\hline $\mathrm{H}$ & 3.325792 & 2.78171 & -0.151837 & $\mathrm{H}$ & -5.146056 & -0.457174 & -0.591705 \\
\hline $\mathrm{H}$ & 1.279722 & 1.010328 & -2.316978 & $\mathrm{H}$ & -5.061082 & 0.125823 & -2.257905 \\
\hline $\mathrm{H}$ & 2.623223 & 0.684734 & -3.451567 & & & & \\
\hline \multicolumn{8}{|c|}{$\mathrm{PMe}_{3}$} \\
\hline $\mathrm{P}$ & 0.159556158 & -0.432751689 & -0.388611653 & $\mathrm{H}$ & 2.427822791 & 0.458942186 & -0.602755251 \\
\hline C & 1.98700487 & -0.418691449 & -0.106525875 & $\mathrm{H}$ & 2.438587528 & -1.313306851 & -0.560913824 \\
\hline C & -0.302087626 & 1.015348468 & 0.664290179 & $\mathrm{H}$ & 2.251208267 & -0.390926714 & 0.964298085 \\
\hline C & -0.302848265 & -1.818399708 & 0.744236338 & $\mathrm{H}$ & 0.057761211 & -2.766098913 & 0.314962209 \\
\hline $\mathrm{H}$ & 0.078670718 & 1.935807295 & 0.195270406 & $\mathrm{H}$ & -1.398528738 & -1.885110459 & 0.822033394 \\
\hline $\mathrm{H}$ & 0.10700201 & 0.94594711 & 1.686735291 & $\mathrm{H}$ & 0.122839172 & -1.704267435 & 1.755599137 \\
\hline $\mathrm{H}$ & -1.397668096 & 1.100848157 & 0.722351565 & & & & \\
\hline
\end{tabular}

transition state

\begin{tabular}{llll|llll}
\hline Os & -0.07779724 & 0.361703503 & 0.387523649 & $\mathrm{H}$ & -2.165753044 & 1.325548505 & 3.285235877 \\
P & -2.405918612 & 0.733018986 & 0.402771766 & $\mathrm{H}$ & 2.082207937 & 1.120050398 & 3.160552722 \\
$\mathrm{P}$ & 2.256416282 & 0.643593665 & 0.279055274 & $\mathrm{H}$ & 3.023427034 & -0.239178141 & 3.836761423 \\
$\mathrm{~N}$ & -0.070720883 & 2.392048884 & 0.182341972 & $\mathrm{H}$ & 1.553522796 & -0.537347973 & 2.845819145 \\
$\mathrm{~N}$ & -0.270866354 & -1.364740421 & 0.504400298 & $\mathrm{H}$ & 2.82597942 & -2.050691736 & 1.278077668 \\
C & -1.245548255 & 3.220295651 & 0.007871238 & $\mathrm{H}$ & 4.183987908 & -1.826805931 & 2.417443047 \\
C & -2.508643728 & 2.566996439 & 0.557184871 & $\mathrm{H}$ & 4.442750236 & -1.57271072 & 0.684892917 \\
C & 1.121947359 & 3.173609458 & -0.068166347 & $\mathrm{H}$ & 4.356054672 & 1.8995574 & 2.229348168 \\
C & 2.392354796 & 2.482192532 & 0.410506763 & $\mathrm{H}$ & 5.235112602 & 0.89986679 & 1.046996339 \\
$\mathrm{C}$ & 3.329048537 & 0.023832441 & 1.714049413 & $\mathrm{H}$ & 5.175105182 & 0.418582144 & 2.753092472 \\
$\mathrm{C}$ & 3.020300938 & 0.179886268 & -1.386354589 & $\mathrm{H}$ & 1.567973632 & -1.419961409 & -1.611759409
\end{tabular}




\begin{tabular}{|c|c|c|c|c|c|c|c|}
\hline C & -3.358498966 & 0.091955012 & 1.908548656 & $\mathrm{H}$ & 3.142034342 & -1.985129783 & -1.015063931 \\
\hline C & -3.293706323 & 0.300291798 & -1.21158194 & $\mathrm{H}$ & 2.974071846 & -1.519248485 & -2.722083829 \\
\hline C & 2.326778918 & 1.068651794 & -2.423264291 & $\mathrm{H}$ & 4.826224013 & 1.419946637 & -1.258717423 \\
\hline C & -2.732757704 & 1.252693154 & -2.271103357 & $\mathrm{H}$ & 4.863719698 & 0.149434145 & -2.501631852 \\
\hline C & -2.410231775 & 0.26822088 & 3.099720227 & $\mathrm{H}$ & 5.083041968 & -0.283811542 & -0.80136097 \\
\hline C & 2.442648637 & 0.098766085 & 2.961241731 & $\mathrm{H}$ & -2.709851988 & -1.93138732 & 1.420960434 \\
\hline C & 3.715186224 & -1.43946172 & 1.497437218 & $\mathrm{H}$ & -4.40549602 & -1.596784365 & 0.969476219 \\
\hline C & 4.588133723 & 0.865881892 & 1.933192898 & $\mathrm{H}$ & -3.978262512 & -1.83373803 & 2.671441419 \\
\hline C & 2.6555559 & -1.274402865 & -1.694271691 & $\mathrm{H}$ & -4.497213313 & 1.903235214 & 2.412269242 \\
\hline C & 4.529133802 & 0.381897892 & -1.47607012 & $\mathrm{H}$ & -5.125624424 & 0.397674018 & 3.103496942 \\
\hline C & -3.626498919 & -1.401354571 & 1.720014509 & $\mathrm{H}$ & -5.395648986 & 0.756691769 & 1.388899779 \\
\hline C & -4.663722587 & 0.835765664 & 2.202196526 & $\mathrm{H}$ & -1.811606228 & -1.188524441 & -1.740169395 \\
\hline C & -2.901997681 & -1.120584657 & -1.620537311 & $\mathrm{H}$ & -3.384677181 & -1.370502747 & -2.580404463 \\
\hline C & -4.811473032 & 0.430273806 & -1.15408304 & $\mathrm{H}$ & -3.209557864 & -1.874580322 & -0.884701997 \\
\hline $\mathrm{H}$ & -1.385229223 & 3.48142282 & -1.06228689 & $\mathrm{H}$ & -5.136214579 & 1.436540988 & -0.847017722 \\
\hline $\mathrm{H}$ & -1.101375955 & 4.1928754 & 0.522363568 & $\mathrm{H}$ & -5.264488317 & -0.301558809 & -0.471992278 \\
\hline $\mathrm{H}$ & 1.047349445 & 4.152986424 & 0.448636528 & $\mathrm{H}$ & -5.22968572 & 0.235163868 & -2.156095761 \\
\hline $\mathrm{H}$ & 1.204235734 & 3.425320106 & -1.146564844 & $\mathrm{P}$ & 0.587826864 & -3.193568185 & 0.593713239 \\
\hline $\mathrm{H}$ & -3.420582308 & 2.974968886 & 0.093562544 & $\mathrm{C}$ & 1.770615181 & -4.681053516 & 0.647534806 \\
\hline $\mathrm{H}$ & -2.579109088 & 2.76625284 & 1.636897773 & $\mathrm{H}$ & 2.418850863 & -4.612645024 & 1.535071391 \\
\hline $\mathrm{H}$ & 2.519060066 & 2.680238331 & 1.485214083 & $\mathrm{H}$ & 2.415717188 & -4.663942921 & -0.244916006 \\
\hline $\mathrm{H}$ & 3.289537485 & 2.871303463 & -0.096597749 & $\mathrm{H}$ & 1.231840379 & -5.645181235 & 0.674352887 \\
\hline $\mathrm{H}$ & 1.231051483 & 1.013122499 & -2.314992585 & $\mathrm{C}$ & -0.345228481 & -3.782190646 & -0.875980081 \\
\hline $\mathrm{H}$ & 2.59880078 & 0.719056133 & -3.433149457 & $\mathrm{H}$ & 0.357016306 & -3.880399536 & -1.717945371 \\
\hline $\mathrm{H}$ & 2.634822154 & 2.121763075 & -2.343289068 & $\mathrm{H}$ & -1.119436786 & -3.054619689 & -1.140632968 \\
\hline $\mathrm{H}$ & -3.073127221 & 2.288864103 & -2.127974803 & $\mathrm{H}$ & -0.807869003 & -4.76570814 & -0.696075992 \\
\hline $\mathrm{H}$ & -3.078832315 & 0.923330939 & -3.265025344 & $\mathrm{C}$ & -0.383601706 & -3.641682009 & 2.091174504 \\
\hline $\mathrm{H}$ & -1.63078925 & 1.237556247 & -2.263868906 & $\mathrm{H}$ & 0.287311525 & -4.088488145 & 2.839064031 \\
\hline $\mathrm{H}$ & -1.458739956 & -0.255990604 & 2.928062032 & $\mathrm{H}$ & -1.179042394 & -4.369098275 & 1.864741311 \\
\hline $\mathrm{H}$ & -2.893949273 & -0.130513194 & 4.007709956 & $\mathrm{H}$ & -0.842068661 & -2.735881836 & 2.508922306 \\
\hline
\end{tabular}

\begin{tabular}{|c|c|c|c|c|c|c|c|}
\hline \multicolumn{8}{|c|}{7} \\
\hline Os & -0.008247756 & 0.399379046 & 0.320762425 & $\mathrm{H}$ & -2.083574554 & 1.226232955 & 3.184811917 \\
\hline$P$ & -2.311515854 & 0.65238043 & 0.312697481 & $\mathrm{H}$ & 2.063375344 & 1.233877724 & 3.188125713 \\
\hline$P$ & 2.29488371 & 0.650771474 & 0.317689059 & $\mathrm{H}$ & 2.864903495 & -0.180526691 & 3.92219625 \\
\hline$N$ & -0.007268199 & 2.322992312 & 0.164881754 & $\mathrm{H}$ & 1.426735802 & -0.381000646 & 2.857281618 \\
\hline $\mathrm{N}$ & -0.010897579 & -1.570597931 & 0.405745382 & $\mathrm{H}$ & 2.65252709 & -1.980904187 & 1.364814416 \\
\hline C & -1.193296648 & 3.160536156 & -0.011247476 & $\mathrm{H}$ & 3.965123014 & -1.878478197 & 2.574043348 \\
\hline C & -2.470963774 & 2.492095144 & 0.466554933 & $\mathrm{H}$ & 4.332926113 & -1.650783449 & 0.854988805 \\
\hline C & 1.179655094 & 3.159414992 & -0.010952066 & $\mathrm{H}$ & 4.414904762 & 1.853115913 & 2.324086991 \\
\hline C & 2.456005138 & 2.490537745 & 0.469659152 & $\mathrm{H}$ & 5.299741213 & 0.7453894 & 1.249536093 \\
\hline C & 3.29323695 & 0.033625637 & 1.811822333 & $\mathrm{H}$ & 5.0898228 & 0.345866874 & 2.964898937 \\
\hline C & 3.180640877 & 0.225320606 & -1.305629746 & $\mathrm{H}$ & 1.73069452 & -1.359216345 & -1.617696666 \\
\hline C & -3.314870524 & 0.033947151 & 1.802798999 & $\mathrm{H}$ & 3.29606366 & -1.944264247 & -1.012159612 \\
\hline C & -3.193467888 & 0.229778303 & -1.313450298 & $\mathrm{H}$ & 3.162559773 & -1.427004462 & -2.704629667 \\
\hline C & 2.563564363 & 1.14789458 & -2.361362684 & $\mathrm{H}$ & 4.997331747 & 1.423702095 & -1.027641319 \\
\hline C & -2.572548022 & 1.152994138 & -2.366395228 & $\mathrm{H}$ & 5.091000128 & 0.190412691 & -2.306827743 \\
\hline C & -2.379740925 & 0.180766663 & 3.007736321 & $\mathrm{H}$ & 5.189492528 & -0.298459953 & -0.6103288 \\
\hline C & 2.35564425 & 0.186823574 & 3.014054269 & $\mathrm{H}$ & -2.679807866 & -1.981573007 & 1.352353383 \\
\hline C & 3.579620411 & -1.454651543 & 1.631308774 & $\mathrm{H}$ & -4.35792715 & -1.64465704 & 0.839260204 \\
\hline C & 4.592191133 & 0.793281299 & 2.086955065 & $\mathrm{H}$ & -3.995007777 & -1.877591931 & 2.558605612 \\
\hline
\end{tabular}




\begin{tabular}{llll|llll}
$\mathrm{C}$ & 2.819757652 & -1.213502469 & -1.67724586 & $\mathrm{H}$ & -4.431651064 & 1.855655139 & 2.317523366 \\
$\mathrm{C}$ & 4.694096231 & 0.400082085 & -1.298272068 & $\mathrm{H}$ & -5.11392924 & 0.348833563 & 2.951425858 \\
$\mathrm{C}$ & -3.605794409 & -1.452893058 & 1.617797018 & $\mathrm{H}$ & -5.317045749 & 0.754474283 & 1.236637398 \\
$\mathrm{C}$ & -4.611984123 & 0.797215897 & 2.076453632 & $\mathrm{H}$ & -1.744818239 & -1.356444954 & -1.623643106 \\
$\mathrm{C}$ & -2.833534928 & -1.209166096 & -1.685791969 & $\mathrm{H}$ & -3.174049704 & -1.420803149 & -2.714327836 \\
$\mathrm{C}$ & -4.706716033 & 0.406745633 & -1.310138909 & $\mathrm{H}$ & -3.312515849 & -1.940226999 & -1.022928459 \\
$\mathrm{H}$ & -1.278732719 & 3.4510848 & -1.077220016 & $\mathrm{H}$ & -5.009180796 & 1.430643131 & -1.039601458 \\
$\mathrm{H}$ & -1.052208779 & 4.110441365 & 0.539465492 & $\mathrm{H}$ & -5.205239173 & -0.291546408 & -0.624210801 \\
$\mathrm{H}$ & 1.038636399 & 4.110271155 & 0.538119127 & $\mathrm{H}$ & -5.101024347 & 0.198564013 & -2.320032114 \\
$\mathrm{H}$ & 1.26677347 & 3.448143372 & -1.077285534 & $\mathrm{P}$ & -0.008059966 & -3.114369159 & 0.544046468 \\
$\mathrm{H}$ & -3.360162753 & 2.889899422 & -0.046859006 & $\mathrm{C}$ & 1.399121233 & -4.056943729 & -0.181777844 \\
$\mathrm{H}$ & -2.603503636 & 2.693854863 & 1.53963848 & $\mathrm{H}$ & 2.340549589 & -3.738109297 & 0.284318259 \\
$\mathrm{H}$ & 2.587223186 & 2.693632762 & 1.542640454 & $\mathrm{H}$ & 1.454785994 & -3.843611294 & -1.258853616 \\
$\mathrm{H}$ & 3.346334267 & 2.886696692 & -0.043031626 & $\mathrm{H}$ & 1.272282387 & -5.140539801 & -0.036951754 \\
$\mathrm{H}$ & 1.463079067 & 1.090786246 & -2.3320667 & $\mathrm{C}$ & -1.413893036 & -4.05696504 & -0.1838218 \\
$\mathrm{H}$ & 2.907035538 & 0.83220862 & -3.36106568 & $\mathrm{H}$ & -2.355840782 & -3.737454958 & 0.280781052 \\
$\mathrm{H}$ & 2.863261257 & 2.198156247 & -2.226307414 & $\mathrm{H}$ & -1.288249855 & -5.140762405 & -0.03945037 \\
$\mathrm{H}$ & -2.871878852 & 2.203318542 & -2.230973911 & $\mathrm{H}$ & -1.467702872 & -3.842805473 & -1.260861422 \\
$\mathrm{H}$ & -2.913401226 & 0.838795355 & -3.367464018 & $\mathrm{C}$ & -0.008009166 & -3.627117861 & 2.294871935 \\
$\mathrm{H}$ & -1.472186517 & 1.095067705 & -2.334025941 & $\mathrm{H}$ & 0.878652296 & -3.196913337 & 2.780817614 \\
$\mathrm{H}$ & -1.452866382 & -0.390589414 & 2.851877022 & $\mathrm{H}$ & -0.001257252 & -4.721033569 & 2.410808559 \\
$\mathrm{H}$ & -2.892755246 & -0.186624018 & 3.913749349 & $\mathrm{H}$ & -0.901595271 & -3.208115675 & 2.778000214 \\
\hline & & & & & & &
\end{tabular}

Tab. 3.6. $\mathrm{XYZ}$ geometries of $\mathbf{3}, \mathrm{PMe}_{3}$, the transition state and $\mathbf{7}$ derived with the PBE functional.

\begin{tabular}{|c|c|c|c|c|c|c|c|}
\hline & $x$ & $\mathrm{Y}$ & Z & & $x$ & $\mathrm{Y}$ & Z \\
\hline \multicolumn{8}{|c|}{3} \\
\hline Os & -0.008659244 & 0.16211101 & 0.51891145 & $\mathrm{H}$ & 2.643342433 & 2.163961747 & -2.281992082 \\
\hline$P$ & -2.341719665 & 0.558707662 & 0.420463175 & $\mathrm{H}$ & -2.812346351 & 2.175520132 & -2.121440057 \\
\hline$P$ & 2.315513411 & 0.555754803 & 0.286833154 & $\mathrm{H}$ & -2.72627516 & 0.836281689 & -3.304583592 \\
\hline N & -0.013176707 & 2.289081065 & 0.319750065 & $\mathrm{H}$ & -1.356078791 & 1.111872221 & -2.168723375 \\
\hline$N$ & -0.003098279 & -1.512488596 & 0.770740099 & $\mathrm{H}$ & -1.480569754 & -0.344270408 & 3.024093356 \\
\hline C & -1.206390662 & 3.101989236 & 0.156207222 & $\mathrm{H}$ & -3.001653971 & -0.377897102 & 4.008600682 \\
\hline C & -2.479262016 & 2.394710571 & 0.641643097 & $\mathrm{H}$ & -2.37945532 & 1.165658804 & 3.347052452 \\
\hline C & 1.169076259 & 3.099912903 & 0.082325519 & $\mathrm{H}$ & 2.525097489 & 1.176929717 & 3.20391014 \\
\hline C & 2.468018375 & 2.392446383 & 0.492599313 & $\mathrm{H}$ & 3.179781794 & -0.365719458 & 3.835367423 \\
\hline C & 3.400140509 & -0.172800789 & 1.681512411 & $\mathrm{H}$ & 1.604097932 & -0.331120411 & 2.940970762 \\
\hline C & 2.990630402 & 0.139953601 & -1.452575676 & $\mathrm{H}$ & 2.517836565 & -2.1573202 & 1.338646546 \\
\hline C & -3.345585122 & -0.174420016 & 1.871946998 & $\mathrm{H}$ & 3.980735125 & -2.146673762 & 2.378713925 \\
\hline C & -3.115632603 & 0.149932564 & -1.278989526 & $\mathrm{H}$ & 4.154412783 & -1.950870054 & 0.611277163 \\
\hline C & 2.283311128 & 1.123629767 & -2.405495548 & $\mathrm{H}$ & 4.731693857 & 1.575680191 & 1.903280919 \\
\hline C & -2.461868949 & 1.134947033 & -2.268105172 & $\mathrm{H}$ & 5.423204979 & 0.266622854 & 0.892201407 \\
\hline C & -2.496877269 & 0.084027892 & 3.133176117 & $\mathrm{H}$ & 5.320330755 & 0.072364051 & 2.663733641 \\
\hline C & 2.626549107 & 0.09396753 & 2.98850852 & $\mathrm{H}$ & 1.443114121 & -1.384430435 & -1.696552573 \\
\hline C & 3.515471392 & -1.692251425 & 1.478299407 & $\mathrm{H}$ & 3.023317912 & -2.0592453 & -1.192183158 \\
\hline C & 4.791397854 & 0.47639931 & 1.773962987 & $\mathrm{H}$ & 2.80647396 & -1.491835987 & -2.877196314 \\
\hline C & 2.541000277 & -1.285813468 & -1.818242895 & $\mathrm{H}$ & 4.870887155 & 1.286359123 & -1.268312516 \\
\hline C & 4.511495053 & 0.287589285 & -1.590257478 & $\mathrm{H}$ & 4.795862721 & 0.163524022 & -2.657323386 \\
\hline C & -3.475782555 & -1.692722748 & 1.668918469 & $\mathrm{H}$ & 5.060058948 & -0.483508785 & -1.015412473 \\
\hline
\end{tabular}




\begin{tabular}{llll|llll}
$\mathrm{C}$ & -4.727496746 & 0.477197066 & 2.048253138 & $\mathrm{H}$ & -2.488715376 & -2.159006263 & 1.470600313 \\
$\mathrm{C}$ & -2.691328581 & -1.275700069 & -1.674424302 & $\mathrm{H}$ & -4.1636601 & -1.946337722 & 0.838692004 \\
$\mathrm{C}$ & -4.641566111 & 0.301956127 & -1.329617473 & $\mathrm{H}$ & -3.889724641 & -2.150209581 & 2.592540677 \\
$\mathrm{H}$ & -1.331030793 & 3.411738567 & -0.913253434 & $\mathrm{H}$ & -4.657446174 & 1.575434881 & 2.181104583 \\
$\mathrm{H}$ & -1.10549247 & 4.066251458 & 0.718578341 & $\mathrm{H}$ & -5.205441302 & 0.068431295 & 2.964318032 \\
$\mathrm{H}$ & 1.103333748 & 4.065998122 & 0.646725162 & $\mathrm{H}$ & -5.409495205 & 0.274634944 & 1.20303294 \\
$\mathrm{H}$ & 1.229589728 & 3.406096462 & -0.993693649 & $\mathrm{H}$ & -1.588593445 & -1.377924819 & -1.615510862 \\
$\mathrm{H}$ & -3.392091124 & 2.803101162 & 0.160922167 & $\mathrm{H}$ & -3.016773636 & -1.476903951 & -2.717444325 \\
$\mathrm{H}$ & -2.588952403 & 2.540474108 & 1.734762354 & $\mathrm{H}$ & -3.139901261 & -2.050092388 & -1.024979783 \\
$\mathrm{H}$ & 2.644095886 & 2.542237989 & 1.576400397 & $\mathrm{H}$ & -4.97916915 & 1.300565998 & -0.984447691 \\
$\mathrm{H}$ & 3.350545677 & 2.797470593 & -0.044233065 & $\mathrm{H}$ & -5.159376225 & -0.469632456 & -0.727537334 \\
$\mathrm{H}$ & 1.184997717 & 1.102977099 & -2.242597504 & $\mathrm{H}$ & -4.986438728 & 0.18274486 & -2.379259426 \\
$\mathrm{H}$ & 2.487157839 & 0.821671299 & -3.454621858 & & & & \\
\hline
\end{tabular}

$\mathrm{PMe} 3$

\begin{tabular}{llll|llll}
\hline $\mathrm{P}$ & 0.164564039 & -0.429104852 & -0.41167768 & $\mathrm{H}$ & 2.457002125 & 0.460809275 & -0.611774057 \\
$\mathrm{C}$ & 2.006130075 & -0.42115242 & -0.113747865 & $\mathrm{H}$ & 2.457126834 & -1.328019419 & -0.564711557 \\
$\mathrm{C}$ & -0.308839517 & 1.017537344 & 0.666888697 & $\mathrm{H}$ & 2.2628954 & -0.392711361 & 0.966974117 \\
$\mathrm{C}$ & -0.308705817 & -1.816825444 & 0.741756214 & $\mathrm{H}$ & 0.04768641 & -2.780661011 & 0.325602191 \\
$\mathrm{H}$ & 0.04777801 & 1.958043245 & 0.200569658 & $\mathrm{H}$ & -1.413303605 & -1.872708166 & 0.818699378 \\
$\mathrm{H}$ & 0.114989765 & 0.942252076 & 1.691297618 & $\mathrm{H}$ & 0.115448921 & -1.687635458 & 1.760634124 \\
$\mathrm{H}$ & -1.41345264 & 1.077516191 & 0.740459161 & & & & \\
\hline
\end{tabular}

transition state

\begin{tabular}{|c|c|c|c|c|c|c|c|}
\hline Os & -0.080792582 & 0.351482594 & 0.411343928 & $\mathrm{H}$ & -2.326206134 & 1.184940622 & 3.335823663 \\
\hline$P$ & -2.416199428 & 0.718483945 & 0.397041935 & $\mathrm{H}$ & 2.261271054 & 1.386480614 & 3.137006703 \\
\hline $\mathrm{P}$ & 2.256523446 & 0.668394587 & 0.258857514 & $\mathrm{H}$ & 3.065026926 & -0.039189243 & 3.870232565 \\
\hline $\mathrm{N}$ & -0.08973921 & 2.408930419 & 0.228062905 & $\mathrm{H}$ & 1.536761012 & -0.225689511 & 2.910480671 \\
\hline $\mathrm{N}$ & -0.298063006 & -1.383037026 & 0.566207696 & $\mathrm{H}$ & 2.681906219 & -1.972274375 & 1.382927548 \\
\hline C & -1.277525465 & 3.248704297 & 0.142749074 & $\mathrm{H}$ & 4.080312347 & -1.805623446 & 2.509316249 \\
\hline C & -2.534817965 & 2.551125285 & 0.671996515 & $\mathrm{H}$ & 4.32876378 & -1.642434522 & 0.746021583 \\
\hline C & 1.095255642 & 3.19688742 & -0.094184332 & $\mathrm{H}$ & 4.517961632 & 1.948425839 & 1.99450004 \\
\hline C & 2.391588213 & 2.52246156 & 0.360994392 & $\mathrm{H}$ & 5.34293861 & 0.724959844 & 0.976716612 \\
\hline C & 3.355400551 & 0.087652681 & 1.717124246 & $\mathrm{H}$ & 5.224021872 & 0.491318362 & 2.742962576 \\
\hline C & 3.014789871 & 0.183402683 & -1.429820837 & $\mathrm{H}$ & 1.552912734 & -1.435143371 & -1.590650911 \\
\hline C & -3.421129978 & -0.02797673 & 1.842966639 & $\mathrm{H}$ & 3.164438292 & -1.993958713 & -1.050587377 \\
\hline C & -3.262324487 & 0.39161947 & -1.290538868 & $\mathrm{H}$ & 2.921565492 & -1.532352361 & -2.764903029 \\
\hline C & 2.290861801 & 1.069621031 & -2.462733162 & $\mathrm{H}$ & 4.827829295 & 1.436564192 & -1.289274757 \\
\hline C & -2.610607776 & 1.384526975 & -2.273127758 & $\mathrm{H}$ & 4.855538608 & 0.20204912 & -2.581256194 \\
\hline C & -2.518086034 & 0.122146404 & 3.083856716 & $\mathrm{H}$ & 5.098462116 & -0.29351397 & -0.883806552 \\
\hline C & 2.501507142 & 0.315286074 & 2.980052195 & $\mathrm{H}$ & -2.681818577 & -2.01410251 & 1.281732541 \\
\hline C & 3.626094952 & -1.417724583 & 1.572198025 & $\mathrm{H}$ & -4.38030041 & -1.702161644 & 0.767920357 \\
\hline C & 4.677671792 & 0.861765372 & 1.848654057 & $\mathrm{H}$ & -4.014661388 & -2.020139326 & 2.488356287 \\
\hline C & 2.644212832 & -1.282360751 & -1.718162558 & $\mathrm{H}$ & -4.629532109 & 1.758330792 & 2.314920656 \\
\hline C & 4.52999914 & 0.397026539 & -1.536483627 & $\mathrm{H}$ & -5.23875643 & 0.229038582 & 3.003714458 \\
\hline C & -3.635486956 & -1.525655087 & 1.568508453 & $\mathrm{H}$ & -5.474322085 & 0.574442792 & 1.267753296 \\
\hline C & -4.761596241 & 0.677962156 & 2.105750676 & $\mathrm{H}$ & -1.799845437 & -1.172100251 & -1.729440803 \\
\hline C & -2.899097463 & -1.031218055 & -1.750465411 & $\mathrm{H}$ & -3.260790056 & -1.183464052 & -2.790005233 \\
\hline C & -4.783284291 & 0.592407013 & -1.28940048 & $\mathrm{H}$ & -3.358114102 & -1.81376773 & -1.117997866 \\
\hline $\mathrm{H}$ & -1.441604799 & 3.584106299 & -0.911907254 & $\mathrm{H}$ & -5.076608153 & 1.592264679 & -0.909125654 \\
\hline $\mathrm{H}$ & -1.11894509 & 4.193643689 & 0.721081796 & $\mathrm{H}$ & -5.307456688 & -0.176507091 & -0.689275182 \\
\hline
\end{tabular}




\begin{tabular}{|c|c|c|c|c|c|c|c|}
\hline $\mathrm{H}$ & 1.031034875 & 4.202382782 & 0.392607852 & $\mathrm{H}$ & -5.165791642 & 0.51151731 & 32996169 \\
\hline r & 1.134479076 & 3.410254002 & -1.191598739 & $\mathrm{P}$ & 0.620056737 & -3.274717899 & 0.649137095 \\
\hline $\mathrm{H}$ & -3.46234833 & 2.98391388 & 0.243963427 & C & 1.873205505 & -4.721737414 & 0.617481525 \\
\hline $\mathrm{H}$ & -2.590827336 & 2.673414988 & 1.772261834 & $\mathrm{H}$ & 2.651425209 & -4.54878907 & 1.388423821 \\
\hline $\mathrm{H}$ & 2.554182991 & 2.738611744 & 1.435561791 & $\mathrm{H}$ & 2.372726731 & -4.741699799 & -0.372728837 \\
\hline$r$ & 3.276635658 & 2.904098062 & -0.188556887 & $\mathrm{H}$ & 1.404466421 & -5.713733087 & 0.800020858 \\
\hline $\mathrm{H}$ & 1.190017533 & 1.000903076 & -2.328587311 & C & -0.575259032 & -3.997035992 & -0.567370351 \\
\hline $\mathrm{H}$ & 2.544319302 & 0.718701527 & -3.485803691 & $\mathrm{H}$ & -0.095256805 & -4.037717677 & -1.565890114 \\
\hline $\mathrm{H}$ & 2.593631479 & 2.132979932 & -2.390921638 & $\mathrm{H}$ & -1.468944779 & -3.350560009 & -0.631079913 \\
\hline $\mathrm{H}$ & -2.926178481 & 2.429762631 & -2.086382052 & $\mathrm{H}$ & -0.873474616 & -5.025486383 & -0.277782524 \\
\hline $\mathrm{H}$ & -2.916724557 & 1.124675466 & -3.308819241 & C & -0.12917804 & -3.66142663 & 2.299308047 \\
\hline $\mathrm{H}$ & -1.503350856 & 1.327304519 & -2.209578572 & $\mathrm{H}$ & -0.557383272 & -4.684790691 & 2.323294739 \\
\hline $\mathrm{H}$ & -1.532703499 & -0.357691868 & 2.919817445 & $\mathrm{H}$ & -0.918733644 & -2.922148119 & 2.529079708 \\
\hline r & -3.020082936 & -0.345321588 & 3.958391502 & $\mathrm{H}$ & 0.659463277 & -3.592261276 & 3.075137191 \\
\hline \multicolumn{8}{|c|}{7} \\
\hline Os & -0.008198029 & 0.385220732 & 0.328967592 & $\mathrm{H}$ & -2.268167191 & 1.369160946 & 3.220008721 \\
\hline$P$ & -2.317275148 & 0.64427089 & 0.317971029 & $\mathrm{H}$ & 2.245347935 & 1.367726329 & 3.225513341 \\
\hline $\mathrm{P}$ & 2.301033697 & 0.642561689 & 0.323645614 & $\mathrm{H}$ & 2.888670272 & -0.132047848 & 3.965385646 \\
\hline $\mathrm{N}$ & -0.00728192 & 2.321311655 & 0.171791122 & $\mathrm{H}$ & 1.410776378 & -0.17645063 & 2.911336995 \\
\hline $\mathrm{N}$ & -0.00939362 & -1.588832843 & 0.432976416 & $\mathrm{H}$ & 2.519440042 & -1.970154677 & 1.458603171 \\
\hline C & -1.204585472 & 3.166668305 & -0.009120929 & $\mathrm{H}$ & 3.876925111 & -1.927523026 & 2.643772558 \\
\hline C & -2.486152059 & 2.499343603 & 0.480764939 & $\mathrm{H}$ & 4.216088251 & -1.765630898 & 0.894100114 \\
\hline C & 1.19107724 & 3.165749094 & -0.006428697 & $\mathrm{H}$ & 4.566079962 & 1.812428582 & 2.137688063 \\
\hline C & 2.470924434 & 2.497529161 & 0.486702846 & $\mathrm{H}$ & 5.391316133 & 0.501851841 & 1.23801642 \\
\hline C & 3.31518903 & 0.019167843 & 1.833195292 & $\mathrm{H}$ & 5.12139497 & 0.350118266 & 2.996566616 \\
\hline C & 3.204225946 & 0.242182001 & -1.326828212 & $\mathrm{H}$ & 1.785146649 & -1.391204114 & -1.621874679 \\
\hline C & -3.335506475 & 0.021429326 & 1.824974297 & $\mathrm{H}$ & 3.392669374 & -1.940778249 & -1.050204118 \\
\hline C & -3.216773577 & 0.244654395 & -1.334695055 & $\mathrm{H}$ & 3.199869221 & -1.410796936 & -2.747577435 \\
\hline C & 2.540911077 & 1.160283071 & -2.372938234 & $\mathrm{H}$ & 4.990726369 & 1.495505541 & -0.990616119 \\
\hline C & -2.550287626 & 1.162304954 & -2.379180956 & $\mathrm{H}$ & 5.115729363 & 0.350268198 & -2.357910012 \\
\hline C & -2.432451494 & 0.286316276 & 3.04651404 & $\mathrm{H}$ & 5.253180463 & -0.254942509 & -0.684382424 \\
\hline C & 2.409361472 & 0.284759851 & 3.052524273 & $\mathrm{H}$ & -2.54014507 & -1.968319674 & 1.451949089 \\
\hline C & 3.494784931 & -1.499090898 & 1.6917135 & $\mathrm{H}$ & -4.23535239 & -1.762646467 & 0.883529483 \\
\hline C & 4.669063639 & 0.712563629 & 2.047308923 & $\mathrm{H}$ & -3.900395151 & -1.925011261 & 2.633970447 \\
\hline C & 2.876475963 & -1.212155708 & -1.70263955 & $\mathrm{H}$ & -4.585968795 & 1.815512662 & 2.126390221 \\
\hline C & 4.719633084 & 0.473412276 & -1.325918378 & $\mathrm{H}$ & -5.144231423 & 0.353621924 & 2.984079124 \\
\hline C & -3.515743273 & -1.496699312 & 1.682869235 & $\mathrm{H}$ & -5.409925186 & 0.505384643 & 1.224889328 \\
\hline C & -4.689438211 & 0.715704049 & 2.035859558 & $\mathrm{H}$ & -1.798220455 & -1.389752421 & -1.626666908 \\
\hline C & -2.889237392 & -1.209910942 & -1.709822696 & $\mathrm{H}$ & -3.210476764 & -1.408282122 & -2.755476212 \\
\hline C & -4.732010053 & 0.477024085 & -1.337384187 & $\mathrm{H}$ & -3.407378552 & -1.938189225 & -1.058545751 \\
\hline $\mathrm{H}$ & -1.289566332 & 3.441219804 & -1.087422441 & $\mathrm{H}$ & -5.003153741 & 1.499274102 & -1.002599262 \\
\hline $\mathrm{H}$ & -1.054616437 & 4.13106483 & 0.531114789 & $\mathrm{H}$ & -5.267642844 & -0.251015499 & -0.69722836 \\
\hline $\mathrm{H}$ & 1.040545626 & 4.130376914 & 0.533235918 & $\mathrm{H}$ & -5.125727315 & 0.35431134 & -2.370337324 \\
\hline $\mathrm{H}$ & 1.278862596 & 3.439987446 & -1.084585566 & P & -0.009611692 & -3.150977838 & 0.55597802 \\
\hline $\mathrm{H}$ & -3.383661824 & 2.894375771 & -0.037247273 & C & 1.405431131 & -4.098077824 & -0.197180296 \\
\hline $\mathrm{H}$ & -2.617122444 & 2.70003243 & 1.562498475 & $\mathrm{H}$ & 2.359561867 & -3.775032522 & 0.258634258 \\
\hline $\mathrm{H}$ & 2.599333445 & 2.698211669 & 1.568744548 & $\mathrm{H}$ & 1.442568917 & -3.876527155 & -1.281322064 \\
\hline $\mathrm{H}$ & 3.370022051 & 2.891847145 & -0.029095963 & $\mathrm{H}$ & 1.277440135 & -5.190139943 & -0.054449631 \\
\hline $\mathrm{H}$ & 1.434503246 & 1.075151678 & -2.318409528 & C & -1.423082573 & -4.09697186 & -0.201341722 \\
\hline $\mathrm{H}$ & 2.869795928 & 0.856442998 & -3.390127738 & $\mathrm{H}$ & -2.378222949 & -3.773424712 & 0.25200175 \\
\hline
\end{tabular}




\begin{tabular}{llll|llll}
$\mathrm{H}$ & 2.822484409 & 2.223628807 & -2.23863829 & $\mathrm{H}$ & -1.296220613 & -5.189172967 & -0.058693725 \\
$\mathrm{H}$ & -2.831365495 & 2.225857562 & -2.245493542 & $\mathrm{H}$ & -1.457130054 & -3.874959679 & -1.285492742 \\
$\mathrm{H}$ & -2.877004247 & 0.858765377 & -3.397157689 & $\mathrm{C}$ & -0.012219634 & -3.690586007 & 2.314890999 \\
$\mathrm{H}$ & -1.444079675 & 1.076320861 & -2.322056514 & $\mathrm{H}$ & 0.885699334 & -3.277823568 & 2.813138798 \\
$\mathrm{H}$ & -1.43382334 & -0.175511743 & 2.90766883 & $\mathrm{H}$ & -0.012676556 & -4.795264552 & 2.400860009 \\
$\mathrm{H}$ & -2.91422625 & -0.130284189 & 3.958171036 & $\mathrm{H}$ & -0.91123035 & -3.277254729 & 2.810684782 \\
\hline
\end{tabular}

Tab. 3.7. $\mathrm{XYZ}$ geometries of $\mathbf{3}, \mathrm{PMe}_{3}$, the transition state and $\mathbf{7}$ derived with the M06 functional.

\begin{tabular}{|c|c|c|c|c|c|c|c|}
\hline & $x$ & $\mathrm{Y}$ & Z & & $x$ & $\mathrm{Y}$ & Z \\
\hline \multicolumn{8}{|l|}{3} \\
\hline Os & -0.010775244 & 0.196459748 & 0.456484918 & $\mathrm{H}$ & 2.74625922 & 2.059626408 & -2.337111194 \\
\hline$P$ & -2.373807283 & 0.578130031 & 0.423676115 & $\mathrm{H}$ & -3.001013802 & 2.225879446 & -2.050768303 \\
\hline$P$ & 2.348856911 & 0.561749875 & 0.27173653 & $\mathrm{H}$ & -2.957173141 & 0.921203912 & -3.259164743 \\
\hline $\mathrm{N}$ & -0.010986206 & 2.321675522 & 0.299546381 & $\mathrm{H}$ & -1.527377745 & 1.222093395 & -2.228843784 \\
\hline N & -0.014039871 & -1.472250233 & 0.525994898 & $\mathrm{H}$ & -1.551683141 & -0.631104496 & 2.983690292 \\
\hline C & -1.205898062 & 3.115344705 & 0.150013805 & $\mathrm{H}$ & -3.044282596 & -0.476561277 & 3.967218325 \\
\hline C & -2.463674478 & 2.408529471 & 0.663206855 & $\mathrm{H}$ & -2.211950791 & 0.975444367 & 3.348657421 \\
\hline C & 1.166517995 & 3.099877338 & 0.004172441 & $\mathrm{H}$ & 2.326483839 & 1.090917888 & 3.177130993 \\
\hline C & 2.46572979 & 2.401239385 & 0.414115323 & $\mathrm{H}$ & 3.205036867 & -0.322711572 & 3.822069491 \\
\hline C & 3.417861234 & -0.114768823 & 1.686410619 & $\mathrm{H}$ & 1.664993922 & -0.536457328 & 2.925507896 \\
\hline C & 3.062863823 & 0.082660453 & -1.419940497 & $\mathrm{H}$ & 2.726060285 & -2.15080613 & 1.267335476 \\
\hline C & -3.367922155 & -0.16919344 & 1.857150985 & $\mathrm{H}$ & 4.113871038 & -2.035249789 & 2.380002029 \\
\hline C & -3.185898311 & 0.186984719 & -1.246219849 & $\mathrm{H}$ & 4.371232113 & -1.80618176 & 0.645512538 \\
\hline C & 2.404710608 & 1.015459842 & -2.436307634 & $\mathrm{H}$ & 4.622784139 & 1.669531657 & 2.146895998 \\
\hline C & -2.631484307 & 1.204513953 & -2.241941876 & $\mathrm{H}$ & 5.380237153 & 0.576473639 & 0.956472925 \\
\hline C & -2.488938144 & -0.064293835 & 3.104755432 & $\mathrm{H}$ & 5.318847665 & 0.125205921 & 2.670899378 \\
\hline C & 2.601462958 & 0.042503271 & 2.970737586 & $\mathrm{H}$ & 1.555846714 & -1.47236967 & -1.730946127 \\
\hline C & 3.664733585 & -1.605684464 & 1.466787145 & $\mathrm{H}$ & 3.090995315 & -2.094305725 & -1.081069145 \\
\hline C & 4.750683085 & 0.612819391 & 1.857666767 & $\mathrm{H}$ & 3.001901775 & -1.589871819 & -2.781493069 \\
\hline C & 2.650815501 & -1.350278806 & -1.760843861 & $\mathrm{H}$ & 4.942527034 & 1.220244453 & -1.276189696 \\
\hline C & 4.576826513 & 0.210426959 & -1.53133898 & $\mathrm{H}$ & 4.877456583 & 0.004012444 & -2.575324267 \\
\hline C & -3.615670282 & -1.651935354 & 1.584383965 & $\mathrm{H}$ & 5.108814607 & -0.521245789 & -0.903971617 \\
\hline C & -4.693644817 & 0.541867526 & 2.126140681 & $\mathrm{H}$ & -2.680406422 & -2.182022679 & 1.334567413 \\
\hline C & -2.74858265 & -1.205281663 & -1.70365756 & $\mathrm{H}$ & -4.345981633 & -1.827487885 & 0.778584615 \\
\hline C & -4.707237338 & 0.268821657 & -1.248520526 & $\mathrm{H}$ & -4.032638736 & -2.12034336 & 2.493934441 \\
\hline $\mathrm{H}$ & -1.357530955 & 3.422798731 & -0.913257148 & $\mathrm{H}$ & -4.555982178 & 1.594419581 & 2.426622828 \\
\hline $\mathrm{H}$ & -1.110005371 & 4.079924887 & 0.700523157 & $\mathrm{H}$ & -5.204322622 & 0.035056508 & 2.965415643 \\
\hline $\mathrm{H}$ & 1.133570952 & 4.081884279 & 0.530390726 & $\mathrm{H}$ & -5.379465117 & 0.51489607 & 1.266762181 \\
\hline $\mathrm{H}$ & 1.211175144 & 3.370189715 & -1.079060751 & $\mathrm{H}$ & -1.651887809 & -1.268094963 & -1.79387977 \\
\hline $\mathrm{H}$ & -3.3849545 & 2.841460722 & 0.233245144 & $\mathrm{H}$ & -3.195959812 & -1.414308282 & -2.692696991 \\
\hline $\mathrm{H}$ & -2.53264022 & 2.542141441 & 1.756904302 & $\mathrm{H}$ & -3.071302119 & -2.003657812 & -1.019059249 \\
\hline $\mathrm{H}$ & 2.647511867 & 2.584505383 & 1.487254723 & $\mathrm{H}$ & -5.083179874 & 1.252905989 & -0.918389372 \\
\hline $\mathrm{H}$ & 3.341327379 & 2.805195642 & -0.125077904 & $\mathrm{H}$ & -5.174576193 & -0.507998398 & -0.624330868 \\
\hline $\mathrm{H}$ & 1.303857465 & 0.997626539 & -2.345427218 & $\mathrm{H}$ & -5.07287483 & 0.104129353 & -2.278773882 \\
\hline $\mathrm{H}$ & 2.669342676 & 0.677354138 & -3.4544025 & & & & \\
\hline \multicolumn{8}{|c|}{$\mathrm{PMe}_{3}$} \\
\hline$P$ & 0.144036134 & -0.435898055 & -0.42111999 & $\mathrm{H}$ & 2.424268235 & 0.464970621 & -0.5878414 \\
\hline C & 1.971986474 & -0.418372004 & -0.109338128 & $\mathrm{H}$ & 2.442124792 & -1.309392746 & -0.554785187 \\
\hline
\end{tabular}




\begin{tabular}{llll|llll}
$\mathrm{C}$ & -0.298271819 & 1.006017339 & 0.656582869 & $\mathrm{H}$ & 2.209607418 & -0.394613895 & 0.969991173 \\
$\mathrm{C}$ & -0.299998363 & -1.811148122 & 0.738741696 & $\mathrm{H}$ & 0.070168945 & -2.765426258 & 0.329223248 \\
$\mathrm{H}$ & 0.097489606 & 1.931658407 & 0.20719046 & $\mathrm{H}$ & -1.394547737 & -1.891939221 & 0.833806845 \\
$\mathrm{H}$ & 0.118459511 & 0.907964468 & 1.675890991 & $\mathrm{H}$ & 0.136387018 & -1.668517522 & 1.744157223 \\
$\mathrm{H}$ & -1.392390214 & 1.112036987 & 0.7284702 & & & & \\
\hline
\end{tabular}

\begin{tabular}{|c|c|c|c|c|c|c|c|}
\hline \multicolumn{8}{|c|}{ transition state } \\
\hline Os & -0.073840674 & 0.369449331 & 0.417357479 & $\mathrm{H}$ & -2.26698353 & 1.286468507 & 3.322588094 \\
\hline$P$ & -2.434166127 & 0.726239224 & 0.413086833 & $\mathrm{H}$ & 2.1295663 & 0.954994125 & 3.199478525 \\
\hline$P$ & 2.289207987 & 0.64697198 & 0.270902671 & $\mathrm{H}$ & 3.160051594 & -0.384336401 & 3.784234673 \\
\hline $\mathrm{N}$ & -0.079286093 & 2.412072606 & 0.225351448 & $\mathrm{H}$ & 1.689987762 & -0.71855693 & 2.809621783 \\
\hline$N$ & -0.267882346 & -1.364747112 & 0.560270991 & $\mathrm{H}$ & 3.024377896 & -2.088943964 & 1.169903372 \\
\hline C & -1.261741616 & 3.231432702 & 0.056622059 & $\mathrm{H}$ & 4.368136024 & -1.8164928 & 2.309842259 \\
\hline C & -2.528363276 & 2.565181636 & 0.587624631 & $\mathrm{H}$ & 4.612845826 & -1.496557533 & 0.587215378 \\
\hline C & 1.116182324 & 3.184165933 & -0.040616004 & $\mathrm{H}$ & 4.358385851 & 1.894716047 & 2.284504879 \\
\hline C & 2.391613495 & 2.492588008 & 0.430413455 & $\mathrm{H}$ & 5.267079577 & 1.004815777 & 1.033271075 \\
\hline C & 3.410460341 & 0.004186352 & 1.674045033 & $\mathrm{H}$ & 5.250780432 & 0.423682431 & 2.710943266 \\
\hline C & 3.058082254 & 0.226869192 & -1.413605615 & $\mathrm{H}$ & 1.690692686 & -1.461356073 & -1.625626661 \\
\hline C & -3.415147807 & 0.057782392 & 1.897252203 & $\mathrm{H}$ & 3.317054429 & -1.932166272 & -1.072020219 \\
\hline C & -3.319096527 & 0.316361743 & -1.220145705 & $\mathrm{H}$ & 3.076805042 & -1.459591348 & -2.76748248 \\
\hline C & 2.331763342 & 1.083209752 & -2.451310221 & $\mathrm{H}$ & 4.811594696 & 1.552050742 & -1.327791843 \\
\hline C & -2.773808845 & 1.289859961 & -2.264057607 & $\mathrm{H}$ & 4.881888884 & 0.268632814 & -2.556330689 \\
\hline C & -2.514703902 & 0.230049003 & 3.122172236 & $\mathrm{H}$ & 5.156649417 & -0.135735885 & -0.856537341 \\
\hline C & 2.545048907 & -0.032619472 & 2.934947602 & $\mathrm{H}$ & -2.727829027 & -1.95959428 & 1.401117991 \\
\hline C & 3.874676962 & -1.425900526 & 1.401363455 & $\mathrm{H}$ & -4.424588065 & -1.640358651 & 0.924641139 \\
\hline C & 4.629954201 & 0.889361318 & 1.923688045 & $\mathrm{H}$ & -4.020686946 & -1.883911858 & 2.631404347 \\
\hline C & 2.768346649 & -1.240934061 & -1.728371587 & $\mathrm{H}$ & -4.61000564 & 1.836384128 & 2.409389209 \\
\hline C & 4.553913592 & 0.496946574 & -1.524979247 & $\mathrm{H}$ & -5.218672691 & 0.299553482 & 3.050787846 \\
\hline C & -3.656813225 & -1.436236566 & 1.688613591 & $\mathrm{H}$ & -5.454228789 & 0.686663296 & 1.336627562 \\
\hline C & -4.741277 & 0.767470027 & 2.169716329 & $\mathrm{H}$ & -1.835309747 & -1.165903836 & -1.798495244 \\
\hline C & -2.926836276 & -1.092281564 & -1.661598608 & $\mathrm{H}$ & -3.418587357 & -1.322150163 & -2.624627353 \\
\hline C & -4.837374149 & 0.42578988 & -1.179792531 & $\mathrm{H}$ & -3.232539057 & -1.867128152 & -0.94202626 \\
\hline $\mathrm{H}$ & -1.398764082 & 3.507560069 & -1.015222744 & $\mathrm{H}$ & -5.187926364 & 1.416471194 & -0.842377967 \\
\hline $\mathrm{H}$ & -1.131554973 & 4.205043985 & 0.57950054 & $\mathrm{H}$ & -5.297696691 & -0.342269289 & -0.539090748 \\
\hline $\mathrm{H}$ & 1.055973956 & 4.172867517 & 0.467077752 & $\mathrm{H}$ & -5.235684627 & 0.262859357 & -2.198308994 \\
\hline $\mathrm{H}$ & 1.190594677 & 3.431380048 & -1.125507969 & $\mathrm{P}$ & 0.55094604 & -3.167132614 & 0.649723341 \\
\hline $\mathrm{H}$ & -3.440490782 & 2.987731122 & 0.129638117 & $\mathrm{C}$ & 1.72319193 & -4.684692637 & 0.677440158 \\
\hline $\mathrm{H}$ & -2.607111769 & 2.747992866 & 1.673197612 & $\mathrm{H}$ & 2.40667228 & -4.622910932 & 1.541098304 \\
\hline $\mathrm{H}$ & 2.506483565 & 2.670744872 & 1.513850642 & $\mathrm{H}$ & 2.334097878 & -4.696154583 & -0.241558067 \\
\hline $\mathrm{H}$ & 3.290438148 & 2.910465686 & -0.058088167 & $\mathrm{H}$ & 1.160811999 & -5.636529275 & 0.738137694 \\
\hline $\mathrm{H}$ & 1.235669312 & 0.969466568 & -2.376551324 & $\mathrm{C}$ & -0.427576928 & -3.793216083 & -0.77731375 \\
\hline $\mathrm{H}$ & 2.645164076 & 0.760622186 & -3.460800587 & $\mathrm{H}$ & -0.896430988 & -4.765973696 & -0.548211486 \\
\hline $\mathrm{H}$ & 2.57720057 & 2.154781553 & -2.360639272 & $\mathrm{H}$ & 0.256542226 & -3.940127173 & -1.629937949 \\
\hline $\mathrm{H}$ & -3.127642855 & 2.322481626 & -2.107662621 & $\mathrm{H}$ & -1.204127584 & -3.072458975 & -1.065873448 \\
\hline $\mathrm{H}$ & -3.122801024 & 0.971213687 & -3.263103014 & $\mathrm{C}$ & -0.360641134 & -3.613539296 & 2.191206989 \\
\hline $\mathrm{H}$ & -1.669295862 & 1.291989477 & -2.273593535 & $\mathrm{H}$ & 0.327734612 & -4.109299179 & 2.893646547 \\
\hline $\mathrm{H}$ & -1.560384413 & -0.308839659 & 3.004232018 & $\mathrm{H}$ & -1.194547566 & -4.307959429 & 1.988723727 \\
\hline $\mathrm{H}$ & -3.040598584 & -0.163587498 & 4.011586876 & $\mathrm{H}$ & -0.768312801 & -2.70796701 & 2.665307079 \\
\hline
\end{tabular}

7

\begin{tabular}{llll|llll}
\hline Os & -0.006148493 & 0.377652456 & 0.362921846 & H & -2.142884015 & 1.28623873 & 3.184016814
\end{tabular} 


\begin{tabular}{|c|c|c|c|c|c|c|c|}
\hline$P$ & -2.332157079 & 0.645512204 & 0.31332203 & $\mathrm{H}$ & 2.157099509 & 1.192532164 & 3.23536785 \\
\hline$P$ & 2.324991656 & 0.642391912 & 0.342578639 & $\mathrm{H}$ & 3.041687951 & -0.190508316 & 3.936542574 \\
\hline $\mathrm{N}$ & -0.003014972 & 2.320542823 & 0.199931838 & $\mathrm{H}$ & 1.566795225 & -0.452243711 & 2.941132569 \\
\hline N & -0.009645924 & -1.602916244 & 0.441216132 & $\mathrm{H}$ & 2.767090068 & -2.010248217 & 1.378506961 \\
\hline C & -1.185112772 & 3.151617033 & -0.028683896 & $\mathrm{H}$ & 4.103654012 & -1.867610719 & 2.559402486 \\
\hline C & -2.478441519 & 2.496025676 & 0.422327358 & $\mathrm{H}$ & 4.434815121 & -1.638009345 & 0.833379321 \\
\hline C & 1.185492701 & 3.157676929 & 0.039063684 & $\mathrm{H}$ & 4.492022155 & 1.854965619 & 2.327146817 \\
\hline C & 2.464169511 & 2.488453121 & 0.510576245 & $\mathrm{H}$ & 5.355515994 & 0.777063781 & 1.199167323 \\
\hline C & 3.375624417 & 0.027753486 & 1.812029513 & $\mathrm{H}$ & 5.204240007 & 0.341323272 & 2.913081918 \\
\hline C & 3.210257941 & 0.257354802 & -1.302390737 & $\mathrm{H}$ & 1.80228552 & -1.361207745 & -1.676316592 \\
\hline C & -3.367529558 & 0.069659944 & 1.809671373 & $\mathrm{H}$ & 3.360809261 & -1.913325688 & -1.011995065 \\
\hline C & -3.223694773 & 0.208450208 & -1.315194249 & $\mathrm{H}$ & 3.276036031 & -1.38746142 & -2.708271313 \\
\hline C & 2.577595105 & 1.176157727 & -2.348414032 & $\mathrm{H}$ & 5.015184777 & 1.486745726 & -1.032095466 \\
\hline C & -2.597621062 & 1.094060609 & -2.393487508 & $\mathrm{H}$ & 5.092267348 & 0.291364969 & -2.348546272 \\
\hline C & -2.464115345 & 0.241260513 & 3.032505523 & $\mathrm{H}$ & 5.252725247 & -0.248109536 & -0.672423663 \\
\hline C & 2.481663186 & 0.154692065 & 3.046935472 & $\mathrm{H}$ & -2.743737253 & -1.972356301 & 1.422567015 \\
\hline C & 3.687571393 & -1.453000415 & 1.62269772 & $\mathrm{H}$ & -4.422113511 & -1.632596857 & 0.891843018 \\
\hline C & 4.670366586 & 0.799913012 & 2.059457748 & $\mathrm{H}$ & -4.064503973 & -1.812747787 & 2.619260293 \\
\hline C & 2.891438582 & -1.184532827 & -1.690990797 & $\mathrm{H}$ & -4.496946952 & 1.899755289 & 2.284764054 \\
\hline C & 4.718647118 & 0.463337446 & -1.321340887 & $\mathrm{H}$ & -5.187574262 & 0.395617876 & 2.919405644 \\
\hline C & -3.666444002 & -1.418960095 & 1.665788049 & $\mathrm{H}$ & -5.360693155 & 0.782224035 & 1.195676609 \\
\hline C & -4.667235931 & 0.836016902 & 2.047926003 & $\mathrm{H}$ & -1.812425362 & -1.410007049 & -1.662351877 \\
\hline C & -2.90272179 & -1.242059332 & -1.667264831 & $\mathrm{H}$ & -3.298391922 & -1.47537633 & -2.673714436 \\
\hline C & -4.73249911 & 0.410204526 & -1.326479878 & $\mathrm{H}$ & -3.357484171 & -1.955919451 & -0.96309431 \\
\hline H & -1.236191807 & 3.424936305 & -1.106386332 & $\mathrm{H}$ & -5.029833095 & 1.439038938 & -1.058601804 \\
\hline $\mathrm{H}$ & -1.064359987 & 4.118615538 & 0.503041686 & $\mathrm{H}$ & -5.257095517 & -0.287345174 & -0.654877547 \\
\hline $\mathrm{H}$ & 1.04386915 & 4.106769726 & 0.597324357 & $\mathrm{H}$ & -5.115520955 & 0.212167584 & -2.345446553 \\
\hline $\mathrm{H}$ & 1.272642924 & 3.46827276 & -1.026111313 & P & -0.02347681 & -3.149210323 & 0.551152015 \\
\hline $\mathrm{H}$ & -3.35376301 & 2.891585542 & -0.122492007 & C & 1.376873732 & -4.072625258 & -0.207784663 \\
\hline $\mathrm{H}$ & -2.644481786 & 2.724592774 & 1.488443063 & $\mathrm{H}$ & 2.324826707 & -3.753652149 & 0.252891554 \\
\hline $\mathrm{H}$ & 2.594039658 & 2.680462286 & 1.589037845 & $\mathrm{H}$ & 1.420291503 & -3.836900046 & -1.283553481 \\
\hline $\mathrm{H}$ & 3.356323458 & 2.903413041 & 0.008738372 & $\mathrm{H}$ & 1.264156064 & -5.162505282 & -0.08450334 \\
\hline $\mathrm{H}$ & 1.475260942 & 1.103723045 & -2.336770195 & C & -1.442905051 & -4.076012954 & -0.173836556 \\
\hline $\mathrm{H}$ & 2.934105815 & 0.878794161 & -3.351810189 & $\mathrm{H}$ & -2.378481323 & -3.774943347 & 0.322534781 \\
\hline $\mathrm{H}$ & 2.857879031 & 2.233793708 & -2.205088698 & $\mathrm{H}$ & -1.312001186 & -5.16522925 & -0.062400153 \\
\hline $\mathrm{H}$ & -2.873809047 & 2.156772863 & -2.284658866 & $\mathrm{H}$ & -1.525051502 & -3.834109628 & -1.245813295 \\
\hline $\mathrm{H}$ & -2.960857435 & 0.763723237 & -3.38402848 & C & -0.018671202 & -3.709276767 & 2.288083765 \\
\hline $\mathrm{H}$ & -1.495478724 & 1.018680867 & -2.386246287 & $\mathrm{H}$ & 0.896447444 & -3.341436423 & 2.777809212 \\
\hline $\mathrm{H}$ & -1.546511144 & -0.363929838 & 2.939896999 & $\mathrm{H}$ & -0.067350635 & -4.807204195 & 2.373114661 \\
\hline $\mathrm{H}$ & -3.015867537 & -0.076957857 & 3.937303165 & $\mathrm{H}$ & -0.88398819 & -3.261061354 & 2.800433652 \\
\hline
\end{tabular}

\subsection{QDPT/NEVPT2/CASSCF $(14,10)$ on 29}

Starting from the X-ray structure (see Part II Section 3.1.1), the $S=0$ and $S=1$ spin states were optimized and verified according to the procedure described in Section 3.1 with the following exceptions: 
- To account for relativistic effects, the ZORA approximation was employed and the corresponding recontracted ZORA-def2 basis sets were used for all elements, which included the segmented all-electron relativistically contracted (SARC) basis set for Rhenium. ${ }^{[264]}$

- The RI and RIJCOSX approximations were used in combination with the decontracted SARC/J auxiliary basis sets.

Two principal geometries ( $C_{1}$ symmetry, derived from $\mathrm{X}$-ray structre and $C_{s}$ symmetry, obtained by rotation of one $i \operatorname{Pr}$ group and symmetrized using the ChemCraft software package) were compared for both spin states:

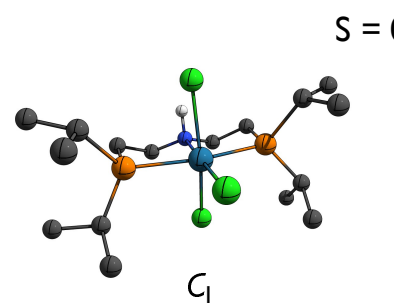

$$
S=0
$$
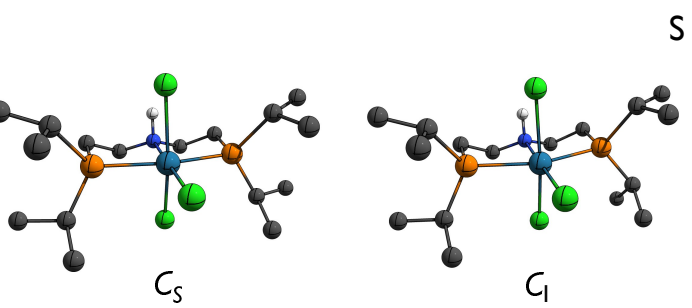

$S=1$

Fig. 3.3. DFT-optimized geometries of $\mathbf{2 9}$ for $C_{1}$ and $C_{S}$ symmetric singlet and triplet states.

Tab. 3.8. Geometry parameters and relative Gibb's free energy of the obtained isomers of $\mathbf{2 9}$.

\begin{tabular}{l|cc|cc}
\hline & \multicolumn{2}{|c|}{$S=0$} & \multicolumn{2}{c}{$S=1$} \\
& $C_{1}$ & $C_{S}$ & $C_{1}$ & $C_{S}$ \\
\hline$d(\mathrm{Re}-\mathrm{N}) / \AA$ & 2.219 & 2.2218 & 2.215 & 2.212 \\
$d(\mathrm{Re}-\mathrm{Cl} 1) / \AA$ & 2.341 & 2.344 & 2.373 & 2.384 \\
$d(\mathrm{Re}-\mathrm{Cl} 2) / \AA$ & 2.408 & 2.405 & 2.426 & 2.418 \\
$d \mathrm{Re}-\mathrm{Cl} 3) / \AA$ & 2.381 & 2.379 & 2.390 & 2.38 \\
$\measuredangle(\mathrm{N} 1-\mathrm{Re}-\mathrm{Cl} 1) /^{\circ}$ & 178.1 & 178.8 & 179.2 & 179.6 \\
$\measuredangle(\mathrm{Cl} 2-\mathrm{Re}-\mathrm{Cl}) /{ }^{\circ}$ & 161.4 & 161.6 & 168.3 & 168.4 \\
\hline$\Delta G_{\text {rel }} / \mathrm{kJ} \mathrm{mol}^{-1}$ & 36.5 & 36.9 & 0.0 & 0.5 \\
\hline
\end{tabular}

While the coordination geometry and energy is basically invariant between $C_{1}$ and $C_{S}$ symmetry, comparison of the singlet vs. triplet states reveals the singlet to be significant higher in energy and feature a slightly different coordination environment. Hence, the $S=1, C_{1}$ symmetric geometry was chosen for higher-level electron structure calculations.

For the QDPT based calculation of spin-orbit eigenstates described in Section 3.1.2, the ZORA-def2-TZVP basis sets (SARC-ZORA-TZVP for Re) and the RI-JK and RIJCOSX approximations were used along with the def2-TZVP/C auxiliary basis sets and a fine grid (GridX6 in ORCA nomenclature). The relativistic options to account for picture change effects was enabled (i.e. \%ref picturechange true end). No frozen core approximation was employed and the convergence criteria were tightened by the ORCA keyword VeryTightSCF. 
Several attempts were made to converge the calculations on the $C_{S}$ symmetric triplet using symmetric constrains, however, all attempts of basis set projection from the def2-SVP to def2-TZVP failed with extreme changes in the active space.

Starting orbitals were obtained from QROs derived from the geometry optimizations at the PBE/D3BJ/RI/ZORA-def2-SVP level of theory. After an initial $\operatorname{CASSCF}(4,5)$ calculation including only the $d$-orbitals, the occupied space was inspected to identify the ligand orbitals balancing the active space and they were included in the calculation as well.

The final evaluated CASSCF $(14,10)$ active space was corrected by strongly contracted NEVPT2 calculations ${ }^{[265-267]}$ for dynamic correlation and these energies entered QDPT treatment via a mean-field operator ${ }^{[268]}$ and are thus corrected also for second order effects.

\subsubsection{DFT-optimized geometries}

Tab. 3.9. XZY geometry data for the DFT-derived structures of $\mathbf{2 9}$ for the $C_{1}$ and $C_{S}$ symmetry, optimized as singlet and triplet each.

\begin{tabular}{|c|c|c|c|c|c|c|c|}
\hline & $x$ & $\mathrm{Y}$ & Z & & $x$ & $\mathrm{Y}$ & Z \\
\hline \multicolumn{8}{|c|}{$C_{1}, S=0$} \\
\hline $\operatorname{Re}$ & 2.527578799 & 8.66342235 & 9.317310602 & $\mathrm{H}$ & 0.342204683 & 4.90696198 & 6.813963567 \\
\hline $\mathrm{Cl}$ & 0.193805405 & 8.741058271 & 9.479827063 & $\mathrm{H}$ & 0.863646734 & 6.61133455 & 7.001520683 \\
\hline $\mathrm{P}$ & 2.750457286 & 6.309157811 & 9.386863735 & $\mathrm{H}$ & 0.058776385 & 5.783963005 & 8.348060642 \\
\hline $\mathrm{N}$ & 4.743754073 & 8.593639643 & 9.236730327 & C & 5.359979292 & 9.812840423 & 8.64386167 \\
\hline C & 5.284897002 & 7.308783335 & 8.712592381 & $\mathrm{H}$ & 5.136589075 & 9.793746951 & 7.561955178 \\
\hline $\mathrm{H}$ & 6.387066205 & 7.291377771 & 8.838187423 & $\mathrm{H}$ & 6.46184916 & 9.769256691 & 8.764950667 \\
\hline $\mathrm{H}$ & 5.064441496 & 7.283201823 & 7.630752879 & C & 4.785559628 & 11.06896544 & 9.300860602 \\
\hline$P$ & 2.898180697 & 11.00860807 & 9.2591073 & $\mathrm{H}$ & 5.194439597 & 11.97521041 & 8.8198394 \\
\hline $\mathrm{Cl}$ & 3.030434381 & 8.710864202 & 11.67214347 & $\mathrm{H}$ & 5.07743055 & 11.10490825 & 10.36812653 \\
\hline $\mathrm{C}$ & 4.631221296 & 6.129463521 & 9.433700131 & C & 2.481325264 & 11.93030541 & 7.668759833 \\
\hline $\mathrm{H}$ & 4.909286676 & 6.143215808 & 10.50559171 & $\mathrm{H}$ & 3.058314069 & 11.32757355 & 6.937663479 \\
\hline $\mathrm{H}$ & 4.989319754 & 5.172479249 & 9.016810916 & $\mathrm{C}$ & 2.932176613 & 13.39106897 & 7.578208417 \\
\hline C & 2.250431913 & 5.391706578 & 10.95506741 & $\mathrm{H}$ & 2.304368273 & 14.04541322 & 8.208226835 \\
\hline $\mathrm{H}$ & 2.649513579 & 6.070354815 & 11.73565838 & $\mathrm{H}$ & 2.835287735 & 13.75288611 & 6.536880039 \\
\hline $\mathrm{Cl}$ & 2.799143833 & 8.630161831 & 6.952208244 & $\mathrm{H}$ & 3.984102877 & 13.53936948 & 7.880605953 \\
\hline$C$ & 2.872570441 & 4.001791954 & 11.12249991 & $\mathrm{H}$ & 4.920502447 & 8.616990545 & 10.25222412 \\
\hline $\mathrm{H}$ & 2.58296644 & 3.572835898 & 12.1005337 & $\mathrm{C}$ & 0.998327714 & 11.75947797 & 7.306635132 \\
\hline $\mathrm{H}$ & 3.975911245 & 4.020253055 & 11.086694 & $\mathrm{H}$ & 0.667817267 & 10.71508111 & 7.432698956 \\
\hline $\mathrm{H}$ & 2.519838689 & 3.297347456 & 10.3460788 & $\mathrm{H}$ & 0.832546094 & 12.05060124 & 6.252812242 \\
\hline C & 0.722500792 & 5.364021007 & 11.0932451 & $\mathrm{H}$ & 0.350085344 & 12.39584595 & 7.933571286 \\
\hline $\mathrm{H}$ & 0.439213741 & 5.02628471 & 12.10747302 & C & 2.413755356 & 12.0384822 & 10.75646899 \\
\hline $\mathrm{H}$ & 0.263887042 & 4.659760971 & 10.37459676 & $\mathrm{H}$ & 2.669862216 & 11.31930897 & 11.56209621 \\
\hline $\mathrm{H}$ & 0.280016438 & 6.361731366 & 10.92533869 & $\mathrm{C}$ & 0.892567495 & 12.24455609 & 10.7854497 \\
\hline C & 2.152920388 & 5.165451828 & 7.999536031 & $\mathrm{H}$ & 0.349156535 & 11.31021225 & 10.55694723 \\
\hline $\mathrm{H}$ & 2.047775783 & 4.166659857 & 8.468872867 & $\mathrm{H}$ & 0.576863801 & 13.01299288 & 10.05686454 \\
\hline C & 3.130050304 & 5.04703926 & 6.822182015 & $\mathrm{H}$ & 0.575501264 & 12.59065891 & 11.78673389 \\
\hline $\mathrm{H}$ & 4.112503041 & 4.640950464 & 7.119499228 & C & 3.185966705 & 13.33989465 & 11.00446561 \\
\hline $\mathrm{H}$ & 3.283115077 & 6.029270482 & 6.342360512 & $\mathrm{H}$ & 2.931676309 & 13.73604877 & 12.00611057 \\
\hline
\end{tabular}




\begin{tabular}{|c|c|c|c|c|c|c|c|}
\hline $\mathrm{H}$ & 2.711796331 & 4.362619018 & 6.060093521 & $\mathrm{H}$ & 2.930853179 & 14.12233922 & 10.27007373 \\
\hline C & 0.772482145 & 5.641707746 & 7.520092728 & $\mathrm{H}$ & 4.280587051 & 13.19813265 & 10.98020444 \\
\hline \multicolumn{8}{|c|}{$C_{1}, S=1$} \\
\hline $\operatorname{Re}$ & -0.078958927 & -0.019850918 & 0.065044195 & $\mathrm{H}$ & -2.190804712 & -3.859668751 & -2.320467812 \\
\hline $\mathrm{Cl}$ & -2.450334257 & 0.052742069 & 0.101005993 & $\mathrm{H}$ & -1.741781797 & -2.1321891 & -2.143908745 \\
\hline$P$ & 0.19449035 & -2.396544045 & 0.243679378 & $\mathrm{H}$ & -2.501537146 & -2.986189898 & -0.789452946 \\
\hline$N$ & 2.134879177 & -0.086252502 & 0.06063405 & $\mathrm{C}$ & 2.754900167 & 1.121765502 & -0.542317038 \\
\hline C & 2.689036254 & -1.366915827 & -0.451201957 & $\mathrm{H}$ & 2.494021111 & 1.112916048 & -1.616446803 \\
\hline $\mathrm{H}$ & 3.794279053 & -1.369659338 & -0.346142369 & $\mathrm{H}$ & 3.860235364 & 1.064649038 & -0.456562953 \\
\hline $\mathrm{H}$ & 2.447108925 & -1.413496326 & -1.527827039 & C & 2.222378181 & 2.380595381 & 0.143624735 \\
\hline$P$ & 0.342717301 & 2.352371707 & 0.107095423 & $\mathrm{H}$ & 2.640973473 & 3.288003013 & -0.326000051 \\
\hline $\mathrm{Cl}$ & 0.280481593 & 0.033698119 & 2.464157723 & $\mathrm{H}$ & 2.52586605 & 2.392025386 & 1.208517806 \\
\hline $\mathrm{C}$ & 2.069305892 & -2.543512015 & 0.303776995 & $\mathrm{C}$ & -0.091993556 & 3.257953232 & -1.48401213 \\
\hline $\mathrm{H}$ & 2.351098732 & -2.498132403 & 1.37403047 & $\mathrm{H}$ & 0.452717946 & 2.6283 & 63061 \\
\hline $\mathrm{H}$ & 2.438813155 & -3.505073483 & -0.091950765 & C & 0.401008611 & 4.704517943 & -1.586970048 \\
\hline C & -0.332489349 & -3.279914097 & 1.816179494 & $\mathrm{H}$ & -0.195076397 & 5.378860363 & -0.947815457 \\
\hline $\mathrm{H}$ & 0.073392151 & -2.596340086 & 2.588697674 & $\mathrm{H}$ & 0.290390552 & 5.0611 & 27717 \\
\hline $\mathrm{Cl}$ & 0.052895505 & -0.03919481 & -2.321509524 & $\mathrm{H}$ & 1.463316388 & 4.824976652 & -1.309833356 \\
\hline $\mathrm{C}$ & 0.271785621 & -4.675560874 & 1.999323555 & $\mathrm{H}$ & 2.300302911 & -0.054009343 & \\
\hline $\mathrm{H}$ & -0.022709672 & -5.087160015 & 2.98304258 & $\mathrm{C}$ & -1.586936243 & 3.133023787 & -1.813930854 \\
\hline $\mathrm{H}$ & 1.375464601 & -4.671083878 & 1.962977498 & $\mathrm{H}$ & & & 3906678 \\
\hline $\mathrm{H}$ & -0.090866654 & -5.385613976 & 1.232673069 & $\mathrm{H}$ & & & \\
\hline C & -1.861129546 & -3.274777598 & 1.94747538 & $\mathrm{H}$ & -2.19610481 & 3.812616124 & -1.193298308 \\
\hline $\mathrm{H}$ & -2.151953047 & -3.578740683 & 2.969935067 & $\mathrm{C}$ & -0.151802762 & 3.349674809 & 1.61603128 \\
\hline $\mathrm{H}$ & -2.330708052 & -3.989667586 & 1.247035156 & $\mathrm{H}$ & 0.126391725 & 2.626445784 & 2.410665538 \\
\hline $\mathrm{H}$ & -2.281454682 & -2.272188701 & 1.751742491 & $\mathrm{C}$ & -1.676938924 & 3.517419759 & 1.662828243 \\
\hline C & -0.386474434 & -3.524574797 & -1.156139561 & $\mathrm{H}$ & -2.198112155 & 2.572949317 & 200736 \\
\hline $\mathrm{H}$ & -0.441550565 & -4.530465732 & -0.692963559 & $\mathrm{H}$ & -2.019118515 & 4.291407711 & 0.952637198 \\
\hline C & 0.588304141 & -3.580152138 & -2.338572547 & $\mathrm{H}$ & -1.989416521 & 3.835765348 & 2.674391774 \\
\hline $\mathrm{H}$ & 1.586676737 & -3.957555001 & -2.056964584 & $\mathrm{C}$ & 0.600499629 & 4.66291773 & 1.860669068 \\
\hline $\mathrm{H}$ & 0.696957398 & -2.581868485 & -2.798793112 & $\mathrm{H}$ & 0.358089208 & 5.04501565 & 2.870384422 \\
\hline $\mathrm{H}$ & 0.187060588 & -4.261832509 & -3.111551375 & $\mathrm{H}$ & & & 1.138259148 \\
\hline C & -1.788811661 & -3.102776264 & -1.621452368 & $\mathrm{H}$ & 1.697104437 & & 1.813400174 \\
\hline \multicolumn{8}{|c|}{$C_{S}, S=0$} \\
\hline $\operatorname{Re}$ & 0.045039728 & -0.093309332 & 9.69147E-05 & $\mathrm{H}$ & 0.166078445 & 2.585640278 & 5.146032363 \\
\hline $\mathrm{Cl}$ & 2.360636885 & -0.456916628 & $1.84859 \mathrm{E}-05$ & $\mathrm{H}$ & 0.523604676 & 0.861543749 & 5.391788772 \\
\hline $\mathrm{P}$ & -0.249834795 & -0.056037523 & 2.352269964 & $\mathrm{H}$ & -1.124518613 & 1.376020432 & 4.940877405 \\
\hline$N$ & -2.153216433 & 0.203266311 & 0.000140099 & $\mathrm{C}$ & -2.673847478 & 0.820778111 & -1.250998798 \\
\hline C & -2.673939444 & 0.820669633 & 1.251296798 & $\mathrm{H}$ & -2.346941801 & 1.876343335 & -1.24744745 \\
\hline $\mathrm{H}$ & -3.783168416 & 0.807858594 & 1.238913105 & $\mathrm{H}$ & -3.783082557 & 0.807761828 & -1.238962244 \\
\hline $\mathrm{H}$ & -2.346861299 & 1.876189754 & 1.247922331 & C & -2.129299673 & 0.081693314 & -2.47360951 \\
\hline$P$ & -0.249680525 & -0.055521255 & -2.352211859 & $\mathrm{H}$ & -2.463407179 & 0.577221306 & -3.402305222 \\
\hline $\mathrm{Cl}$ & -0.669819488 & -2.39002047 & $-4.23609 \mathrm{E}-05$ & $\mathrm{H}$ & -2.52152714 & -0.953381775 & -2.495265277 \\
\hline C & -2.129565245 & 0.081456343 & 2.473895242 & $\mathrm{C}$ & 0.349883284 & 1.463551621 & -3.292752229 \\
\hline $\mathrm{H}$ & -2.522185542 & -0.953463935 & 2.495707361 & $\mathrm{H}$ & -0.162366944 & 2.262789087 & -2.718439794 \\
\hline $\mathrm{H}$ & -2.463325269 & 0.577210096 & 3.40258274 & C & -0.05163231 & 1.570108764 & -4.766887048 \\
\hline C & 0.113945104 & -1.619236902 & 3.333329102 & $\mathrm{H}$ & 0.522134507 & 0.863262024 & -5.391707999 \\
\hline $\mathrm{H}$ & -0.248710515 & -2.375761774 & 2.60652123 & $\mathrm{H}$ & 0.165290205 & 2.587343778 & -5.144816124 \\
\hline $\mathrm{Cl}$ & -0.001324297 & 2.28505433 & 0.000668425 & $\mathrm{H}$ & -1.125520206 & 1.377982368 & -4.939312387 \\
\hline
\end{tabular}




\begin{tabular}{|c|c|c|c|c|c|c|c|}
\hline C & -0.634635432 & -1.819932546 & 4.656457808 & $\mathrm{H}$ & -2.429219441 & -0.790045257 & \\
\hline $\mathrm{H}$ & -0.473902928 & -2.852829492 & 5.020117122 & C & 1.858099893 & 1.672898281 & -3.090010128 \\
\hline $\mathrm{H}$ & -1.724895875 & -1.677419702 & 4.556655891 & $\mathrm{H}$ & 2.148758807 & 1.537693116 & -2.034947244 \\
\hline $\mathrm{H}$ & -0.276011686 & -1.137511973 & 5.44528451 & $\mathrm{H}$ & 2.141484183 & 2.696124597 & -3.399045878 \\
\hline C & 1.629942861 & -1.811925492 & 3.482074241 & $\mathrm{H}$ & 2.451288795 & 0.96613227 & -3.695743565 \\
\hline $\mathrm{H}$ & 1.852083933 & -2.85168185 & 3.786208385 & C & 0.114187119 & -1.618342198 & -3.333684549 \\
\hline $\mathrm{H}$ & 2.046828719 & -1.146323865 & 4.259601976 & $\mathrm{H}$ & -0.247875542 & -2.375214537 & -2.606947671 \\
\hline $\mathrm{H}$ & 2.161914409 & -1.610029811 & 2.535143663 & C & 1.63016612 & -1.810516077 & -3.483233596 \\
\hline C & 0.349729075 & 1.462842091 & 3.293299732 & $\mathrm{H}$ & 2.16246769 & -1.608900135 & -2.536436628 \\
\hline $\mathrm{H}$ & -0.163146331 & 2.262211917 & 2.719730975 & $\mathrm{H}$ & 2.046534019 & -1.144427071 & -4.260631592 \\
\hline C & 1.857777464 & 1.672585769 & 3.089632857 & $\mathrm{H}$ & 1.852422192 & -2.850065764 & -3.787985479 \\
\hline $\mathrm{H}$ & 2.147802395 & 1.537673731 & 2.034349933 & C & -0.634977409 & -1.818853062 & -4.656525583 \\
\hline $\mathrm{H}$ & 2.451457899 & 0.965761539 & 3.694801289 & $\mathrm{H}$ & -0.473406629 & -2.851329177 & -5.021006827 \\
\hline $\mathrm{H}$ & 2.141238161 & 2.695779481 & 3.398711848 & $\mathrm{H}$ & -0.277545226 & -1.135502211 & -5.4450961 \\
\hline C & -0.050814232 & 1.568532111 & 4.767739018 & $\mathrm{H}$ & -1.725319642 & -1.677545169 & -4.555897749 \\
\hline \multicolumn{8}{|c|}{$C_{S}, S=1$} \\
\hline $\operatorname{Re}$ & 0.071712539 & -0.015441077 & $-2.21098 \mathrm{E}-06$ & $\mathrm{H}$ & 0.120549687 & 2.600175732 & 5.131964844 \\
\hline $\mathrm{Cl}$ & 2.440421232 & -0.281203505 & $-6.26882 \mathrm{E}-05$ & $\mathrm{H}$ & 0.447246785 & 0.874254863 & 5.4057981 \\
\hline$P$ & -0.273726017 & -0.065183363 & 2.380757448 & $\mathrm{H}$ & -1.183212138 & 1.407950263 & 4.910424725 \\
\hline $\mathrm{N}$ & -2.127892727 & 0.216441201 & 0.000113528 & C & -2.651405248 & 0.837332301 & -1.244582414 \\
\hline C & -2.651198948 & 0.837101218 & 1.244910142 & $\mathrm{H}$ & -2.291189639 & 1.882392581 & -1.254404816 \\
\hline $\mathrm{H}$ & -3.760928121 & 0.857364042 & 1.222128794 & $\mathrm{H}$ & -3.761135473 & 0.857559742 & -1.221747524 \\
\hline $\mathrm{H}$ & -2.290918658 & 1.882141549 & 1.254820359 & C & -2.148188725 & 0.070113873 & -2.468034446 \\
\hline$P$ & -0.274193196 & -0.064355713 & -2.380743278 & $\mathrm{H}$ & -2.497812836 & 0.549038525 & -3.399430832 \\
\hline $\mathrm{Cl}$ & -0.51344415 & -2.361685496 & $-8.099 \mathrm{E}-05$ & $\mathrm{H}$ & -2.546804672 & -0.963065041 & -2.459884795 \\
\hline C & -2.147812887 & 0.069604734 & 2.468220634 & C & 0.334330052 & 1.461514364 & -3.298345835 \\
\hline $\mathrm{H}$ & -2.546399152 & -0.963587102 & 2.460045287 & $\mathrm{H}$ & -0.15572114 & 2.255495747 & -2.698214275 \\
\hline $\mathrm{H}$ & -2.497418398 & 0.548493283 & 3.399638317 & C & -0.103856781 & 1.583428074 & -4.761216205 \\
\hline C & 0.10830975 & -1.634313922 & 3.333635897 & $\mathrm{H}$ & 0.446247137 & 0.875693473 & -5.405495739 \\
\hline $\mathrm{H}$ & -0.265817112 & -2.3830658 & 2.604641857 & $\mathrm{H}$ & 0.119470672 & 2.601607922 & -5.13163943 \\
\hline $\mathrm{Cl}$ & 0.171918964 & 2.366070944 & 3.94839E-05 & $\mathrm{H}$ & -1.184215145 & 1.409359714 & -4.910036854 \\
\hline C & -0.622337859 & -1.837769853 & 4.665835765 & $\mathrm{H}$ & -2.401201273 & -0.781580122 & 0.000101836 \\
\hline $\mathrm{H}$ & -0.459822049 & -2.872473717 & 5.02259977 & C & 1.85002071 & 1.648847513 & -3.133779378 \\
\hline $\mathrm{H}$ & -1.713452546 & -1.690787725 & 4.580930548 & $\mathrm{H}$ & 2.177248863 & 1.481145543 & -2.093700873 \\
\hline $\mathrm{H}$ & -0.249797827 & -1.159477723 & 5.452012689 & $\mathrm{H}$ & 2.12809543 & 2.678917911 & -3.423048111 \\
\hline C & 1.627198897 & -1.827201679 & 3.448054346 & $\mathrm{H}$ & 2.414319296 & 0.956898122 & -3.782422585 \\
\hline $\mathrm{H}$ & 1.853425088 & -2.872194005 & 3.729191884 & C & 0.107588704 & -1.633136403 & -3.334018617 \\
\hline $\mathrm{H}$ & 2.059513236 & -1.174898677 & 4.228042862 & $\mathrm{H}$ & -0.266554576 & -2.381904762 & -2.605052915 \\
\hline $\mathrm{H}$ & 2.137719105 & -1.60920169 & 2.492500616 & C & 1.626445485 & -1.825982465 & -3.448578846 \\
\hline C & 0.335159021 & 1.460285228 & 3.298605531 & $\mathrm{H}$ & 2.136999392 & -1.608044195 & -2.493033577 \\
\hline $\mathrm{H}$ & -0.154891478 & 2.254319346 & 2.698524656 & $\mathrm{H}$ & 2.058771678 & -1.173665783 & -4.22856536 \\
\hline C & 1.850870018 & 1.647512891 & 3.133999159 & $\mathrm{H}$ & 1.852574966 & -2.870970123 & -3.729782783 \\
\hline $\mathrm{H}$ & 2.178047034 & 1.479844184 & 2.093890931 & C & -0.623180913 & -1.836399465 & -4.666220773 \\
\hline $\mathrm{H}$ & 2.415213038 & 0.955531339 & 3.782582706 & $\mathrm{H}$ & -0.460725508 & -2.871093056 & -5.022997 \\
\hline $\mathrm{H}$ & 2.128974657 & 2.67756524 & 3.423283607 & $\mathrm{H}$ & -0.250705426 & -1.158077758 & -5.452415939 \\
\hline C & -0.10286795 & 1.58202774 & 4.761539583 & $\mathrm{H}$ & -1.714291837 & -1.689334006 & -4.581296814 \\
\hline
\end{tabular}




\subsubsection{CASSCF state composition}

Tab. 3.10. CASSCF state wavefunctions of the first three triplets and singlets, listing the main contributing active space occupations and their weight.

\begin{tabular}{|c|c|}
\hline & Triplets \\
\hline \multicolumn{2}{|c|}{ ROOT 0: $E=-19942.24980286 \mathrm{Eh}$} \\
\hline Weight & Orbital occupation \\
\hline 0.87176 & [2222221100] \\
\hline 0.08309 & [2222212100] \\
\hline 0.00603 & [2212221110] \\
\hline 0.00472 & [2212221101] \\
\hline 0.00333 & [2122221110] \\
\hline 0.00322 & [2122221101] \\
\hline \multicolumn{2}{|c|}{ ROOT 1: $E=-19941.9972794320$ Eh $0.142 \mathrm{eV} 1145.3 \mathrm{~cm}^{-1}$} \\
\hline 0.86991 & [2222212100] \\
\hline 0.08256 & [2222221100] \\
\hline 0.00707 & [2212212110] \\
\hline 0.00538 & {$[2212212101]$} \\
\hline 0.00415 & [2122212110] \\
\hline 0.00365 & {$[2122212101]$} \\
\hline \multicolumn{2}{|c|}{ ROOT 2: $E=-19941.9759164604$ Eh $0.723 \mathrm{eV} 5834.0 \mathrm{~cm}^{-1}$} \\
\hline 0.95834 & [2222211200] \\
\hline 0.00511 & {$[2212211201]$} \\
\hline 0.00353 & [2212211210] \\
\hline 0.00324 & [2122211201] \\
\hline 0.00309 & [2222211002] \\
\hline
\end{tabular}

\begin{tabular}{lc}
\hline & Singlets \\
\hline & ROOT 0: $E=-19941.9768947318 \mathrm{Eh}$ \\
\hline 0.84551 & {$[222222000]$} \\
0.05281 & {$[222220200]$} \\
0.03468 & {$[2222202200]$} \\
0.01106 & {$[2222211110]$} \\
0.00852 & {$[2212222010]$} \\
0.00525 & {$[2122222010]$} \\
0.00472 & {$[222122100]$} \\
0.00471 & {$[222221100]$} \\
0.00309 & {$[2212122110]$} \\
0.00297 & {$[2212222001]$} \\
\hline ROOT $1: \mathrm{E}=-19941.9712235491 \mathrm{Eh} \mathrm{0.154} \mathrm{eV} 1244.7 \mathrm{~cm}^{-1}$ \\
\hline 0.83937 & {$[222221100]$} \\
0.09856 & {$[222212100]$} \\
0.00702 & {$[222210210]$} \\
0.00512 & {$[222212010]$} \\
0.00429 & {$[222222000]$} \\
0.00411 & {$[2212221110]$} \\
\end{tabular}




\begin{tabular}{lc}
0.00366 & {$[222221001]$} \\
0.00323 & {$[2212221101]$} \\
\hline ROOT 2: E = -19941.9641043707 Eh 0.348 eV $2807.2 \mathrm{~cm}^{-1}$ \\
\hline 0.84062 & {$[222212100]$} \\
0.09781 & {$[222221100]$} \\
0.0073 & {$[222221010]$} \\
0.00683 & {$[2222201210]$} \\
0.00515 & {$[2212212110]$} \\
0.00383 & {$[2212212101]$} \\
0.00327 & {$[222212001]$} \\
0.0029 & {$[2122212110]$} \\
\hline
\end{tabular}

\subsubsection{QDPT state eigenvectors}

Tab. 3.11. QDPT eigenvectors of the first 11 states, in the basis of the non-relativistic states.

\begin{tabular}{|c|c|c|c|c|c|}
\hline \multicolumn{6}{|c|}{ QDPT eigenvectors } \\
\hline & Energy & Weight & Spin & Root & $M_{S}$ \\
\hline \multirow[t]{11}{*}{ STATE 0: } & 0 & & & & \\
\hline & & 0.169538 & 1 & 0 & 1 \\
\hline & & 0.044763 & 1 & 1 & 1 \\
\hline & & 0.018389 & 1 & 2 & 1 \\
\hline & & 0.113456 & 1 & 0 & 0 \\
\hline & & 0.258238 & 1 & 1 & 0 \\
\hline & & 0.169538 & 1 & 0 & -1 \\
\hline & & 0.044763 & 1 & 1 & -1 \\
\hline & & 0.018389 & 1 & 2 & -1 \\
\hline & & 0.104686 & 0 & 0 & 0 \\
\hline & & 0.020309 & 0 & 5 & 0 \\
\hline \multirow[t]{7}{*}{ STATE 1: } & 1603.8502 & & & & \\
\hline & & 0.074715 & 1 & 0 & 1 \\
\hline & & 0.136609 & 1 & 1 & 1 \\
\hline & & 0.453287 & 1 & 0 & 0 \\
\hline & & 0.096849 & 1 & 1 & 0 \\
\hline & & 0.074715 & 1 & 0 & -1 \\
\hline & & 0.136609 & 1 & 1 & -1 \\
\hline \multirow[t]{4}{*}{ STATE 2: } & 2150.5038 & & & & \\
\hline & & 0.463774 & 1 & 0 & 1 \\
\hline & & 0.463774 & 1 & 0 & -1 \\
\hline & & 0.023146 & 0 & 2 & 0 \\
\hline \multirow[t]{5}{*}{ STATE 3: } & 2617.1434 & & & & \\
\hline & & 0.460602 & 1 & 1 & 1 \\
\hline & & 0.015706 & 1 & 2 & 0 \\
\hline & & 0.460602 & 1 & 1 & -1 \\
\hline & & 0.032307 & 0 & 1 & 0 \\
\hline
\end{tabular}

STATE 4: $\quad 3614.3358$ 


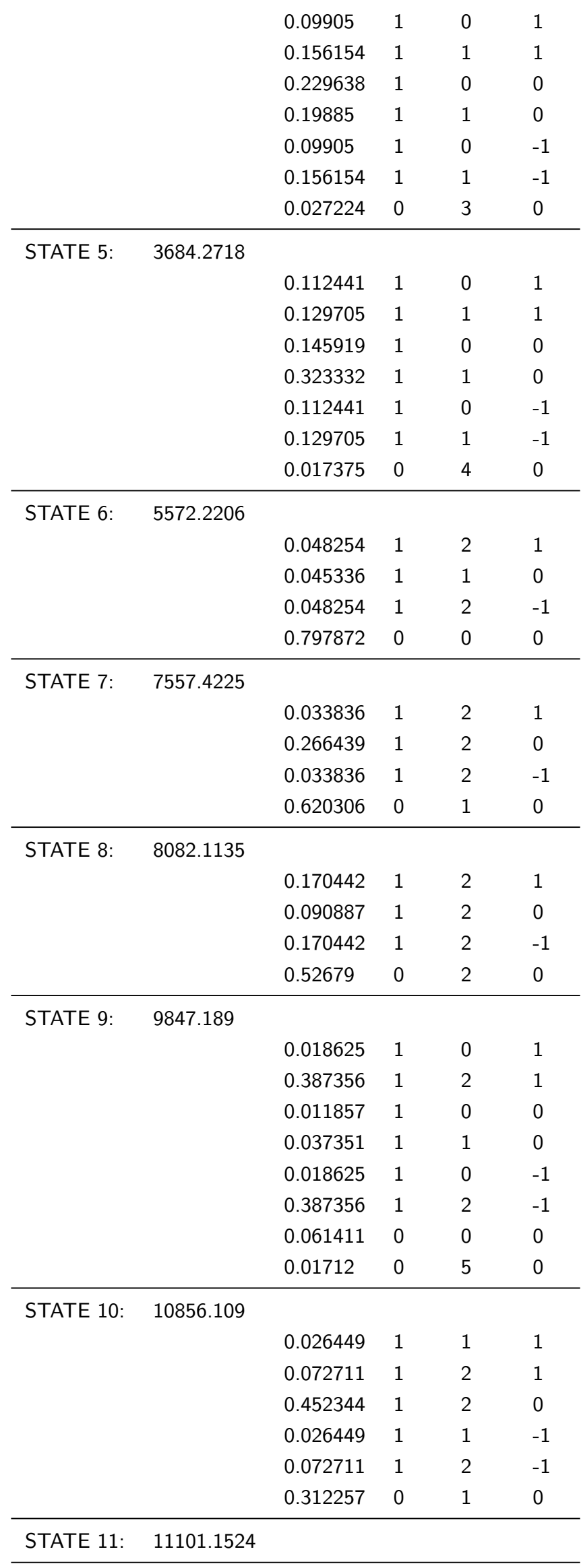




\begin{tabular}{llll}
0.024542 & 1 & 0 & 1 \\
0.188459 & 1 & 2 & 1 \\
0.121153 & 1 & 2 & 0 \\
0.024542 & 1 & 0 & -1 \\
0.188459 & 1 & 2 & -1 \\
0.416986 & 0 & 2 & 0 \\
\hline
\end{tabular}

\subsubsection{CASSCF, NEVPT2 and QDPT state energies}

Tab. 3.12. State energies of the first 40 states derived from CASSCF, NEVPT2 and QDPT calculations, given in $\mathrm{cm}^{-1}$

\begin{tabular}{|c|c|c|c|c|c|c|c|c|}
\hline \multirow[b]{2}{*}{ STATE } & \multicolumn{3}{|c|}{ CASSCF } & \multicolumn{3}{|c|}{ NEVPT2 } & \multicolumn{2}{|c|}{ QDPT } \\
\hline & ROOT & MULT & $\Delta E / \mathrm{cm}^{-1}$ & ROOT & MULT & $\Delta E / \mathrm{cm}^{-1}$ & $\Delta E / \mathrm{cm}^{-1}$ & oscillator strength \\
\hline $0:$ & 0 & 3 & 0 & 0 & 3 & 0 & 0 & \\
\hline 1: & 1 & 3 & 1145.3 & 1 & 3 & 460.6 & 1603.85 & 0.00075 \\
\hline 2: & 0 & 1 & 5619.3 & 0 & 1 & 2618.1 & 2150.5 & 0.00021 \\
\hline 3: & 2 & 3 & 5834 & 1 & 1 & 6058.8 & 2617.14 & 0.00031 \\
\hline 4: & 1 & 1 & 6864 & 2 & 1 & 6811.9 & 3614.34 & 0.0215 \\
\hline 5: & 2 & 1 & 8426.4 & 2 & 3 & 7202.3 & 3684.27 & 0.19496 \\
\hline 6: & 3 & 1 & 12058.6 & 3 & 1 & 12387.5 & 5572.22 & 0.00128 \\
\hline 7: & 4 & 1 & 13452.8 & 4 & 1 & 13840 & 7557.42 & 0.00049 \\
\hline 8: & 0 & 5 & 16770.1 & 5 & 1 & 18519.3 & 8082.11 & 0.00238 \\
\hline 9: & 5 & 1 & 19800.9 & 0 & 5 & 19890.9 & 9847.19 & 0.00004 \\
\hline 10: & 1 & 5 & 23404.6 & 1 & 5 & 24233 & 10856.11 & 0.00295 \\
\hline 11: & 3 & 3 & 24884.2 & 3 & 3 & 24775.3 & 11101.15 & 0.00465 \\
\hline $12:$ & 4 & 3 & 26011.5 & 4 & 3 & 26333.7 & 15018.53 & 0.0077 \\
\hline 13: & 5 & 3 & 28054.7 & 6 & 1 & 29646.1 & 16441.23 & 0.00208 \\
\hline 14: & 6 & 3 & 29488.3 & 5 & 3 & 29658.6 & 21568.53 & 0.02906 \\
\hline 15: & 7 & 3 & 30087.5 & 6 & 3 & 29895.9 & 22633.56 & 0.00023 \\
\hline 16: & 8 & 3 & 30596 & 7 & 3 & 31051.6 & 22648.37 & 0.00431 \\
\hline 17: & 6 & 1 & 30614.7 & 8 & 3 & 31383.9 & 22798.44 & 0.0002 \\
\hline 18: & 7 & 1 & 31973.5 & 7 & 1 & 31432.2 & 22877.06 & 0.00011 \\
\hline 19: & 9 & 3 & 32546.4 & 10 & 3 & 33135.4 & 22924.69 & 0.00008 \\
\hline 20: & 10 & 3 & 33649.3 & 9 & 3 & 33329.5 & 26843.64 & 0.02648 \\
\hline 21: & 8 & 1 & 34540.5 & 16 & 3 & 33365.4 & 26935.44 & 0.00001 \\
\hline $22:$ & 9 & 1 & 34879.8 & 12 & 1 & 33787.8 & 26953.3 & 0.00035 \\
\hline 23: & 11 & 3 & 36040.3 & 15 & 3 & 33788.3 & 27105.64 & 0.00051 \\
\hline $24:$ & 12 & 3 & 36201.1 & 8 & 1 & 34739 & 27159.22 & 0.00356 \\
\hline $25:$ & 10 & 1 & 37292.1 & 12 & 3 & 34766.2 & 27331.81 & 0.01788 \\
\hline 26: & 13 & 3 & 37479.1 & 11 & 3 & 34816.1 & 27600.38 & 0.00011 \\
\hline $27:$ & 14 & 3 & 38338.7 & 13 & 3 & 35405.7 & 27891.72 & 0.00099 \\
\hline 28: & 11 & 1 & 38522.8 & 9 & 1 & 35672 & 29268.85 & 0.0002 \\
\hline 29: & 15 & 3 & 39410.2 & 10 & 1 & 36484.6 & 30029.04 & 0.00295 \\
\hline 30: & 12 & 1 & 39755.5 & 14 & 3 & 36726.5 & 30117.37 & 0.00261 \\
\hline 31: & 16 & 3 & 39857.6 & 17 & 1 & 37837.8 & 30617.89 & 0.00301 \\
\hline $32:$ & 17 & 3 & 39919.5 & 22 & 3 & 37959 & 31769.08 & 0.0277 \\
\hline 33: & 13 & 1 & 40685.3 & 13 & 1 & 38277.1 & 32562.44 & 0.00052 \\
\hline 34: & 14 & 1 & 40868.8 & 11 & 1 & 38642.9 & 32797.66 & 0.04795 \\
\hline
\end{tabular}




\begin{tabular}{l|lll|lll|ll}
$35:$ & 18 & 3 & 41325.8 & 30 & 1 & 38787.4 & 33020.2 & 0.02169 \\
$36:$ & 19 & 3 & 42641.6 & 19 & 3 & 38814.4 & 33154.7 & 0.00398 \\
$37:$ & 15 & 1 & 42671.1 & 16 & 1 & 38880.6 & 33510.65 & 0.09476 \\
$38:$ & 20 & 3 & 42947.5 & 17 & 3 & 38975.7 & 33684.73 & 0.07304 \\
$39:$ & 16 & 1 & 43130.3 & 18 & 1 & 39160.3 & 34194.4 & 0.00426 \\
$\vdots$ & $\vdots$ & $\vdots$ & $\vdots$ & $\vdots$ & $\vdots$ & $\vdots$ & $\vdots$ & $\vdots$ \\
\hline
\end{tabular}

\subsubsection{Simulated influence of magnetic field on excitation} between QDPT states $|0\rangle \rightarrow|4\rangle$ and $|0\rangle \rightarrow|5\rangle$

Tab. 3.13. Simulated influence of an external magnetic field on the excitation energies of the $|0\rangle \rightarrow$ $|4\rangle$ and $|0\rangle \rightarrow|5\rangle$ excitations.

\begin{tabular}{|c|c|c|c|c|c|c|}
\hline \multicolumn{7}{|c|}{ Magnetic field along $\mathrm{x}$-axis } \\
\hline QDPT excitation energies & $B_{x}=0 \mathrm{~T}$ & $B_{x}=2 \mathrm{~T}$ & $B_{x}=4 \mathrm{~T}$ & $B_{x}=6 \mathrm{~T}$ & $B_{x}=8 \mathrm{~T}$ & $B_{x}=10 \mathrm{~T}$ \\
\hline$\Delta E_{|0\rangle} \rightarrow|4\rangle$ & 3614.3358 & 3614.3369 & 3614.3402 & 3614.3457 & 3614.3534 & 3614.3633 \\
\hline$\Delta E_{|0\rangle} \rightarrow|5\rangle$ & 3684.2718 & 3684.2730 & 3684.2768 & 3684.2832 & 3684.2921 & 3684.3035 \\
\hline \multicolumn{7}{|c|}{ Magnetic field along y-axis } \\
\hline & $B_{y}=0 \mathrm{~T}$ & $B_{y}=2 \mathrm{~T}$ & $B_{y}=4 \mathrm{~T}$ & $B_{y}=6 \mathrm{~T}$ & $B_{y}=8 \mathrm{~T}$ & $B_{y}=10 \mathrm{~T}$ \\
\hline$\Delta E_{|0\rangle} \rightarrow|4\rangle$ & 3614.3358 & 3614.3240 & 3614.2885 & 3614.2294 & 3614.1469 & 3614.0410 \\
\hline$\Delta E_{|0\rangle} \rightarrow|5\rangle$ & 3684.2718 & 3684.2834 & 3684.3184 & 3684.3766 & 3684.4580 & 3684.5623 \\
\hline \multicolumn{7}{|c|}{ Magnetic field along z-axis } \\
\hline & $B_{z}=0 \mathrm{~T}$ & $B_{z}=2 \mathrm{~T}$ & $B_{z}=4 \mathrm{~T}$ & $B_{z}=6 \mathrm{~T}$ & $B_{z}=8 \mathrm{~T}$ & $B_{z}=10 \mathrm{~T}$ \\
\hline$\Delta E_{|0\rangle} \rightarrow|4\rangle$ & 3614.3358 & 3614.3373 & 3614.3419 & 3614.3494 & 3614.3599 & 3614.3734 \\
\hline$\Delta E_{|0\rangle} \rightarrow|5\rangle$ & 3684.2718 & 3684.2731 & 3684.2772 & 3684.2840 & 3684.2935 & 3684.3057 \\
\hline
\end{tabular}

\subsection{DFT and TDDFT calculations on the $\mu-\mathrm{N}_{2}$ bridged Re-dimer 36}

The DFT and TDDFT calculations presented in Section 3.3.1 of this thesis were performed by Dr. Markus Finger and Prof. Dr. Vera Krewald, respectively. The details of these calculations might therefore differ from the above described general procedure and are explained in detail in the corresponding publication. ${ }^{[269]}$ 

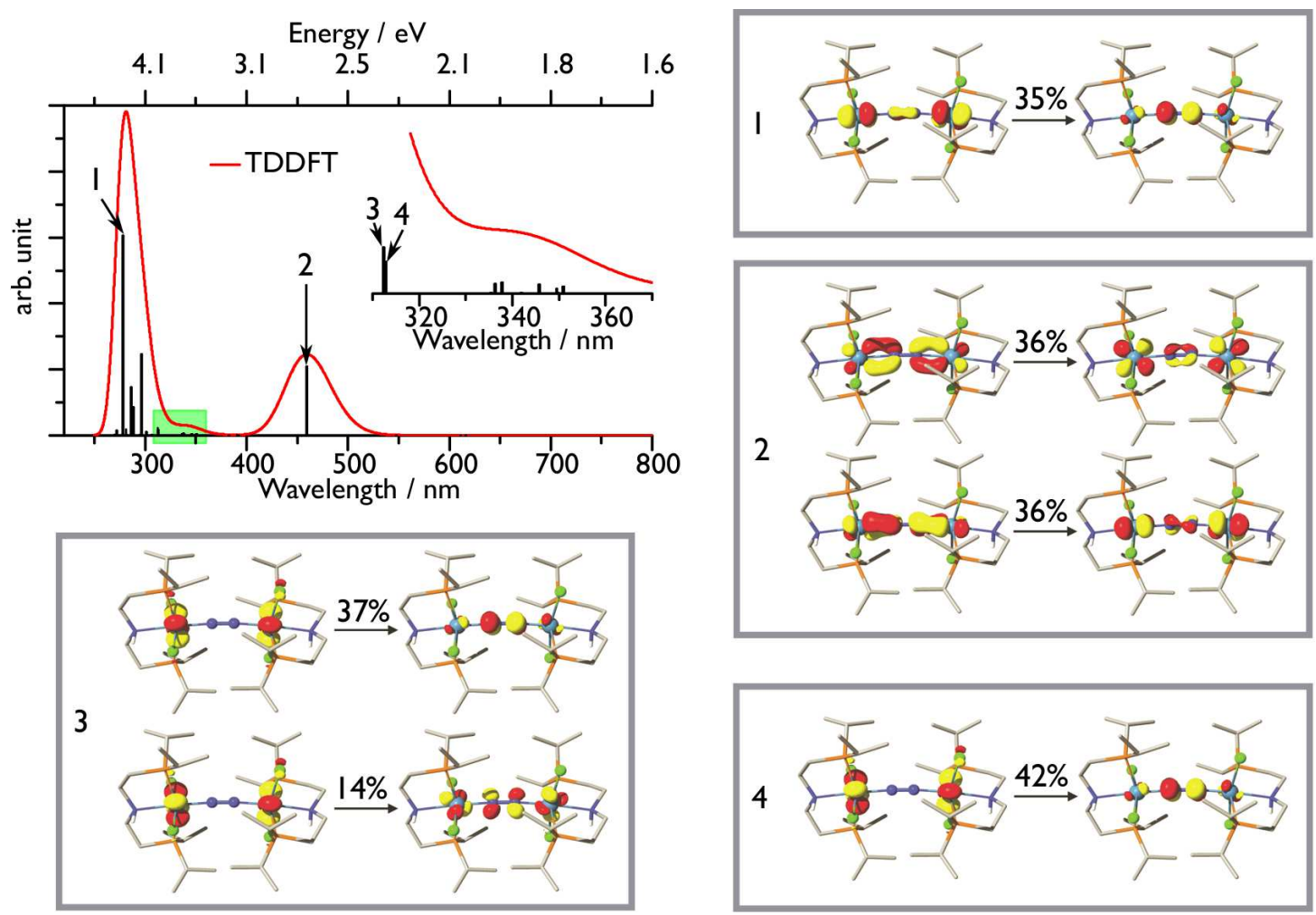

Fig. 3.4. TDDFT calculated electronic absorption spectrum of 36 with the most relevant transitions marked (1-4) and the corresponding natural transition orbitals of the most dominant contributions (weight in \% given on the arrows).

\subsection{Comparing the syn and anti isomers of $\left[\mathrm{Re}(\mathrm{N}) \mathrm{Cl}\left(\mathrm{HPNP}^{i \mathrm{Pr}}\right)\right]^{+}$}

DFT computations were performed with ORCA 4.0 according to the procedure described in Section 3.1. The two possible isomers which might be expected from chloride abstraction from 37, syn- $\left[\operatorname{Re}(\mathrm{N}) \mathrm{Cl}\left(\mathrm{HPNP}{ }^{i \mathrm{Pr}}\right)\right]^{+}$and anti-[Re(N)Cl(HPNP$\left.\left.{ }^{i \mathrm{Pr}}\right)\right]^{+}$, where syn / anti refers to the orientation of the $\mathrm{N}-\mathrm{H}$ bond to the $\mathrm{Re} \equiv \mathrm{N}$ bond, have been calculated and are found to be almost thermoneutral, well reflecting the appearance of two signals in the ${ }^{31} \mathrm{P}\left\{{ }^{1} \mathrm{H}\right\}$ NMR spectrum (see Section 3.3.2). 

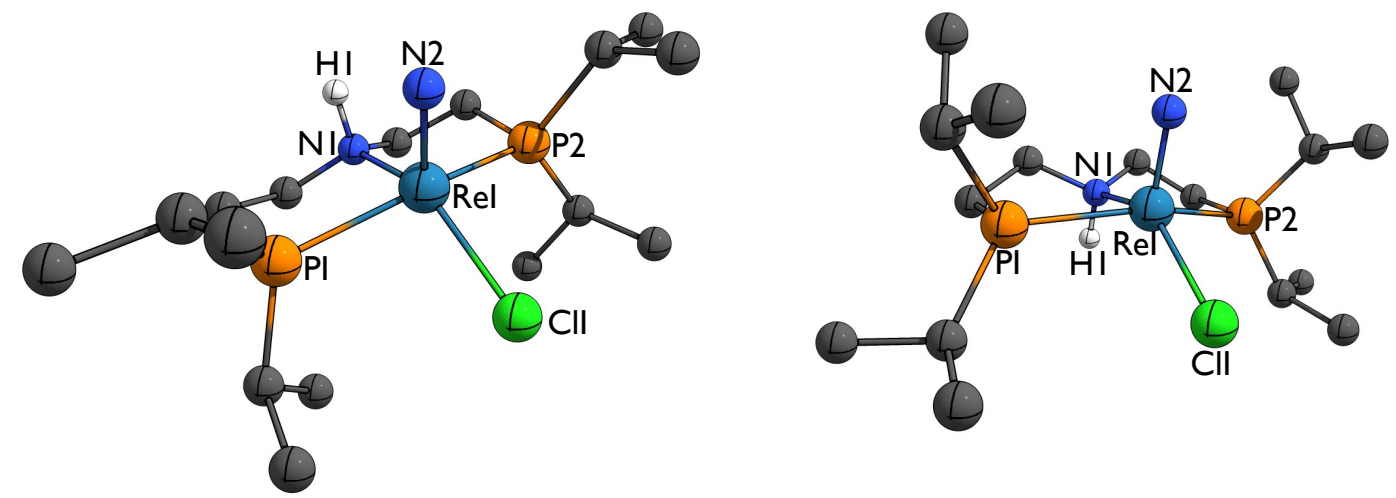

Fig. 3.5. DFT optimized structures of syn- $\left[\operatorname{Re}(\mathrm{N}) \mathrm{Cl}\left(\mathrm{HPNP}^{\mathrm{iPr}}\right)\right]^{+}$(left) and anti$\left[\mathrm{Re}(\mathrm{N}) \mathrm{Cl}\left(\mathrm{HPNP}^{\mathrm{iPr}}\right)\right]^{+}$(right) $\quad(\mathrm{PBE} / \mathrm{D} 3 \mathrm{BJ} / \mathrm{Rl} /$ def2-SVP). Carbon-bonded hydrogen atoms are omitted for clarity.

Tab. 3.14. Structural parameters and thermochemistry data for both isomers of $\left[\operatorname{Re}(\mathrm{N}) \mathrm{Cl}\left(\mathrm{HPNP}^{\mathrm{iPr}}\right)\right]^{+}$.

\begin{tabular}{|c|c|c|c|c|c|}
\hline \multicolumn{3}{|c|}{ Distances / $\AA$} & \multicolumn{3}{|c|}{ Angles / ${ }^{\circ}$} \\
\hline & syn & anti & & syn & anti \\
\hline Re1-N1 & 2.204 & 2.188 & N1-Re1-N2 & 103.5 & 102.0 \\
\hline Re1-N2 & 1.660 & 1.663 & N1-Re1-Cl1 & 144.4 & 144.2 \\
\hline $\mathrm{Re} 1-\mathrm{Cl} 1$ & 2.386 & 2.381 & P1-Re1-P2 & 160.1 & 159.5 \\
\hline $\mathrm{N} 1-\mathrm{H} 1$ & 1.034 & 1.037 & $\tau_{5}$ & 0.26 & 0.26 \\
\hline
\end{tabular}

\subsubsection{DFT-optimized geometries}

Tab. 3.15. XZY geometry data for the DFT-derived structures of syn- $\left[\operatorname{Re}(\mathrm{N}) \mathrm{Cl}\left(\mathrm{HPNP}^{i \mathrm{Pr}}\right)\right]^{+}$and anti-[Re(N)Cl(HPNPiPr$)]^{+}$.

\begin{tabular}{llllc|cccc}
\hline & \multicolumn{1}{c}{$\mathrm{X}$} & \multicolumn{1}{c}{$\mathrm{Y}$} & $\mathrm{Z}$ & $\mathrm{X}$ & $\mathrm{Y}$ & $\mathrm{Z}$ \\
\hline & \multicolumn{7}{c}{ anti-[Re(N)Cl(HPNP$\left.\left.{ }^{i \mathrm{Pr}}\right)\right]^{+}$} \\
\hline $\mathrm{Re}$ & 2.485173273 & 6.418611957 & 9.825514616 & $\mathrm{H}$ & 2.010374632 & 4.0055001 & 12.14627201 \\
$\mathrm{Cl}$ & 0.435023642 & 7.208042498 & 10.74234263 & $\mathrm{H}$ & 1.703833952 & 5.053437985 & 13.56259999 \\
$\mathrm{P}$ & 3.676016195 & 6.5961178 & 11.96739671 & $\mathrm{H}$ & 2.522114743 & 3.475246963 & 13.78024342 \\
$\mathrm{P}$ & 1.81456204 & 6.92578729 & 7.515597672 & $\mathrm{C}$ & 4.611759493 & 6.701899105 & 7.598669245 \\
$\mathrm{~N}$ & 4.437767162 & 7.034435024 & 9.053270228 & $\mathrm{H}$ & 4.600738084 & 5.596832929 & 7.522904712 \\
$\mathrm{~N}$ & 2.556951759 & 4.764795841 & 9.668802168 & $\mathrm{H}$ & 5.607018561 & 7.059904829 & 7.256170607 \\
$\mathrm{H}$ & 4.43271385 & 8.069715522 & 9.114790416 & $\mathrm{C}$ & 3.493443911 & 7.328380685 & 6.770532352 \\
$\mathrm{C}$ & 5.581612567 & 6.568803792 & 9.906994869 & $\mathrm{H}$ & 3.563500637 & 7.001276564 & 5.714368183 \\
$\mathrm{H}$ & 6.534588218 & 6.955879426 & 9.485281868 & $\mathrm{H}$ & 3.594425968 & 8.435635382 & 6.766189139 \\
$\mathrm{H}$ & 5.600954261 & 5.463827276 & 9.835613562 & $\mathrm{C}$ & 0.785355021 & 8.426807969 & 7.068177144
\end{tabular}




\begin{tabular}{lllll|lll}
$\mathrm{C}$ & 5.397319269 & 7.029889725 & 11.35029718 & $\mathrm{H}$ & 1.26270949 & 9.224078909 & 7.683343053 \\
$\mathrm{H}$ & 5.500253468 & 8.135050895 & 11.41750194 & $\mathrm{C}$ & 0.898863772 & 8.800448808 & 5.584507991 \\
$\mathrm{H}$ & 6.186946742 & 6.60205544 & 11.99856471 & $\mathrm{H}$ & 1.937536942 & 8.989800582 & 5.251268545 \\
$\mathrm{C}$ & 3.319673525 & 7.949366935 & 13.21327828 & $\mathrm{H}$ & 0.321512977 & 9.728539766 & 5.397289113 \\
$\mathrm{H}$ & 3.232261856 & 8.848202843 & 12.56024843 & $\mathrm{H}$ & 0.464391056 & 8.015805317 & 4.931899007 \\
$\mathrm{C}$ & 4.470353344 & 8.157715668 & 14.20715968 & $\mathrm{C}$ & -0.675308233 & 8.286356728 & 7.515337474 \\
$\mathrm{H}$ & 5.435457686 & 8.405770468 & 13.72475634 & $\mathrm{H}$ & -1.184434614 & 9.265277316 & 7.406290158 \\
$\mathrm{H}$ & 4.622471202 & 7.265822229 & 14.84995543 & $\mathrm{H}$ & -0.756775664 & 7.969655299 & 8.572774909 \\
$\mathrm{H}$ & 4.217568753 & 8.999347559 & 14.88368596 & $\mathrm{H}$ & -1.224063361 & 7.563166721 & 6.879712079 \\
$\mathrm{C}$ & 1.977240746 & 7.741747536 & 13.92564922 & $\mathrm{C}$ & 1.136033494 & 5.450631565 & 6.577350456 \\
$\mathrm{H}$ & 1.157383716 & 7.532231399 & 13.21263277 & $\mathrm{H}$ & 0.76768608 & 5.891529098 & 5.624300201 \\
$\mathrm{H}$ & 1.71958119 & 8.660634951 & 14.49003482 & $\mathrm{C}$ & 2.216314249 & 4.409816177 & 6.267064297 \\
$\mathrm{H}$ & 2.036799226 & 6.914735933 & 14.66108531 & $\mathrm{H}$ & 1.753943561 & 3.55935528 & 5.726328872 \\
$\mathrm{C}$ & 3.817963669 & 4.974273398 & 12.89861329 & $\mathrm{H}$ & 2.653891371 & 4.000886955 & 7.200945161 \\
$\mathrm{H}$ & 4.251108836 & 5.272616025 & 13.87968281 & $\mathrm{H}$ & 3.029582189 & 4.798183258 & 5.622657307 \\
$\mathrm{C}$ & 4.764558385 & 3.98335305 & 12.21455455 & $\mathrm{C}$ & -0.031969678 & 4.820181598 & 7.349227665 \\
$\mathrm{H}$ & 4.390848663 & 3.70810204 & 11.2073491 & $\mathrm{H}$ & -0.799946537 & 5.554692585 & 7.650528123 \\
$\mathrm{H}$ & 4.801934721 & 3.050199406 & 12.81172744 & $\mathrm{H}$ & 0.336054821 & 4.31940447 & 8.266268619 \\
$\mathrm{H}$ & 5.803975952 & 4.355432958 & 12.128364 & $\mathrm{H}$ & -0.520107775 & 4.054535637 & 6.712886187 \\
$\mathrm{C}$ & 2.430196944 & 4.353846534 & 13.11023796 & & & & \\
\hline & & & & & & \\
\hline
\end{tabular}

syn- $\left[\operatorname{Re}(\mathrm{N}) \mathrm{Cl}\left(\mathrm{HPNP}^{\mathrm{iPr}}\right)\right]^{+}$

\begin{tabular}{|c|c|c|c|c|c|c|c|}
\hline $\operatorname{Re}$ & 2.627379902 & 6.917532404 & 10.30184776 & $\mathrm{H}$ & 1.331647779 & 4.520651833 & 13.1117645 \\
\hline $\mathrm{Cl}$ & 0.869136257 & 5.333748865 & 10.58719245 & $\mathrm{H}$ & 1.79237069 & 5.117428163 & 14.74616341 \\
\hline $\mathrm{P}$ & 3.466818206 & 6.559324812 & 12.57973388 & $\mathrm{H}$ & 2.144118666 & 3.431325581 & 14.28346693 \\
\hline$P$ & 2.480381811 & 6.815121543 & 7.853504124 & C & 5.209995477 & 6.701210197 & 8.494666153 \\
\hline $\mathrm{N}$ & 4.774356089 & 7.15016212 & 9.86349151 & $\mathrm{H}$ & 5.250785365 & 5.593761306 & 8.52675611 \\
\hline $\mathrm{N}$ & 2.100843217 & 8.479104111 & 10.50311228 & $\mathrm{H}$ & 6.246496116 & 7.058023496 & 8.311760612 \\
\hline $\mathrm{H}$ & 4.828757466 & 8.182612567 & 9.881480766 & C & 4.264294109 & 7.175850108 & 7.396343197 \\
\hline C & 5.721277455 & 6.634345939 & 10.91375143 & $\mathrm{H}$ & 4.542961933 & 6.703811696 & 6.432641786 \\
\hline $\mathrm{H}$ & 6.740081608 & 7.026190212 & 10.70487289 & $\mathrm{H}$ & 4.3486866704 & 8.274496033 & 7.255924821 \\
\hline $\mathrm{H}$ & 5.763869478 & 5.534979732 & 10.78218743 & C & 1.534777558 & 8.229607072 & 7.077544734 \\
\hline C & 5.276230469 & 6.990124185 & 12.32895521 & $\mathrm{H}$ & 2.108631244 & 9.099600668 & 7.470693882 \\
\hline $\mathrm{H}$ & 5.374262311 & 8.08225937 & 12.50625888 & C & 1.622466556 & 8.211283834 & 5.547686825 \\
\hline $\mathrm{H}$ & 5.930725621 & 6.482639526 & 13.06596066 & $\mathrm{H}$ & 2.665106552 & 8.16435041 & 5.174962065 \\
\hline C & 2.875410046 & 7.820193749 & 13.82748252 & $\mathrm{H}$ & 1.16988949 & 9.137864673 & 5.139447352 \\
\hline $\mathrm{H}$ & 3.236428284 & 8.761554081 & 13.35475233 & $\mathrm{H}$ & 1.062430565 & 7.358219879 & 5.115718402 \\
\hline C & 3.55306791 & 7.645161813 & 15.19089443 & C & 0.099809232 & 8.347165182 & 7.603602555 \\
\hline $\mathrm{H}$ & 4.659284306 & 7.624216364 & 15.12114749 & $\mathrm{H}$ & -0.337717568 & 9.300631596 & 7.244667293 \\
\hline $\mathrm{H}$ & 3.220484415 & 6.716709487 & 15.69694402 & $\mathrm{H}$ & 0.065740635 & 8.350975023 & 8.710181392 \\
\hline $\mathrm{H}$ & 3.278553272 & 8.492321316 & 15.85212786 & $\mathrm{H}$ & -0.549047615 & 7.528491472 & 7.238662589 \\
\hline C & 1.346484265 & 7.900603819 & 13.9091038 & C & 2.084284537 & 5.215609987 & 6.971839101 \\
\hline $\mathrm{H}$ & 0.886985176 & 8.011468031 & 12.90874313 & $\mathrm{H}$ & 2.414590135 & 5.381760974 & 5.921791999 \\
\hline $\mathrm{H}$ & 1.063577478 & 8.78564959 & 14.51411342 & C & 2.909384271 & 4.072163936 & 7.581484936 \\
\hline $\mathrm{H}$ & 0.90449718 & 7.011515225 & 14.39644928 & $\mathrm{H}$ & 2.661703661 & 3.121923089 & 7.066951845 \\
\hline C & 3.484054934 & 4.857667445 & 13.35374386 & $\mathrm{H}$ & 2.665709309 & 3.9371111172 & 8.655669839 \\
\hline $\mathrm{H}$ & 4.212184239 & 4.938219631 & 14.19164601 & $\mathrm{H}$ & 4.002104329 & 4.221305429 & 7.470188984 \\
\hline C & 3.993904641 & 3.821432695 & 12.34107937 & C & 0.581801698 & 4.90905905 & 6.995022984 \\
\hline $\mathrm{H}$ & 3.317694341 & 3.762815635 & 11.46313216 & $\mathrm{H}$ & -0.007385197 & 5.64747427 & 6.420858752 \\
\hline $\mathrm{H}$ & 4.007650589 & 2.817778864 & 12.81231225 & $\mathrm{H}$ & 0.194254037 & 4.874988874 & 8.032018141 \\
\hline $\mathrm{H}$ & 5.026141455 & 4.024851759 & 11.99392841 & $\mathrm{H}$ & 0.40762243 & 3.916712172 & 6.531614933 \\
\hline
\end{tabular}




\subsection{Isomerization of $\left[\mathrm{Re}(\mathrm{NH}) \mathrm{Cl}_{2}\left(\mathrm{HPNP}^{i \mathrm{Pr}}\right)\right]^{\mathrm{OTf}}$}

\subsubsection{Method and results}

All calculations were performed with ORCA 4.0 according to the procedure described in Section 3.1. Single point energies were derived with the def2-TZVPP basis sets using both the PBE and the PBE0 functional and sovlent effects were included for both THF and DCM. Four possible products for the protonation of $\mathbf{3 7}$ with HOTf were investigated, i.e. the cisand trans-chloro isomers of $\left[\operatorname{Re}(\mathrm{NH}) \mathrm{Cl}_{2}\left(\mathrm{HPNP}^{i \mathrm{Pr}}\right)\right]^{+}$with either a hydrogen bonded or a

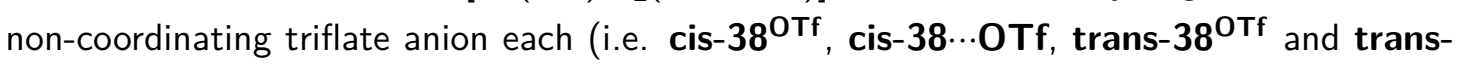

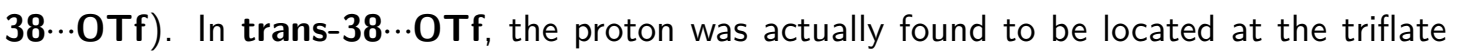
anion rather than at the nitride and no additional minimum was found upon decreasing the $\mathrm{N}$...H distance.
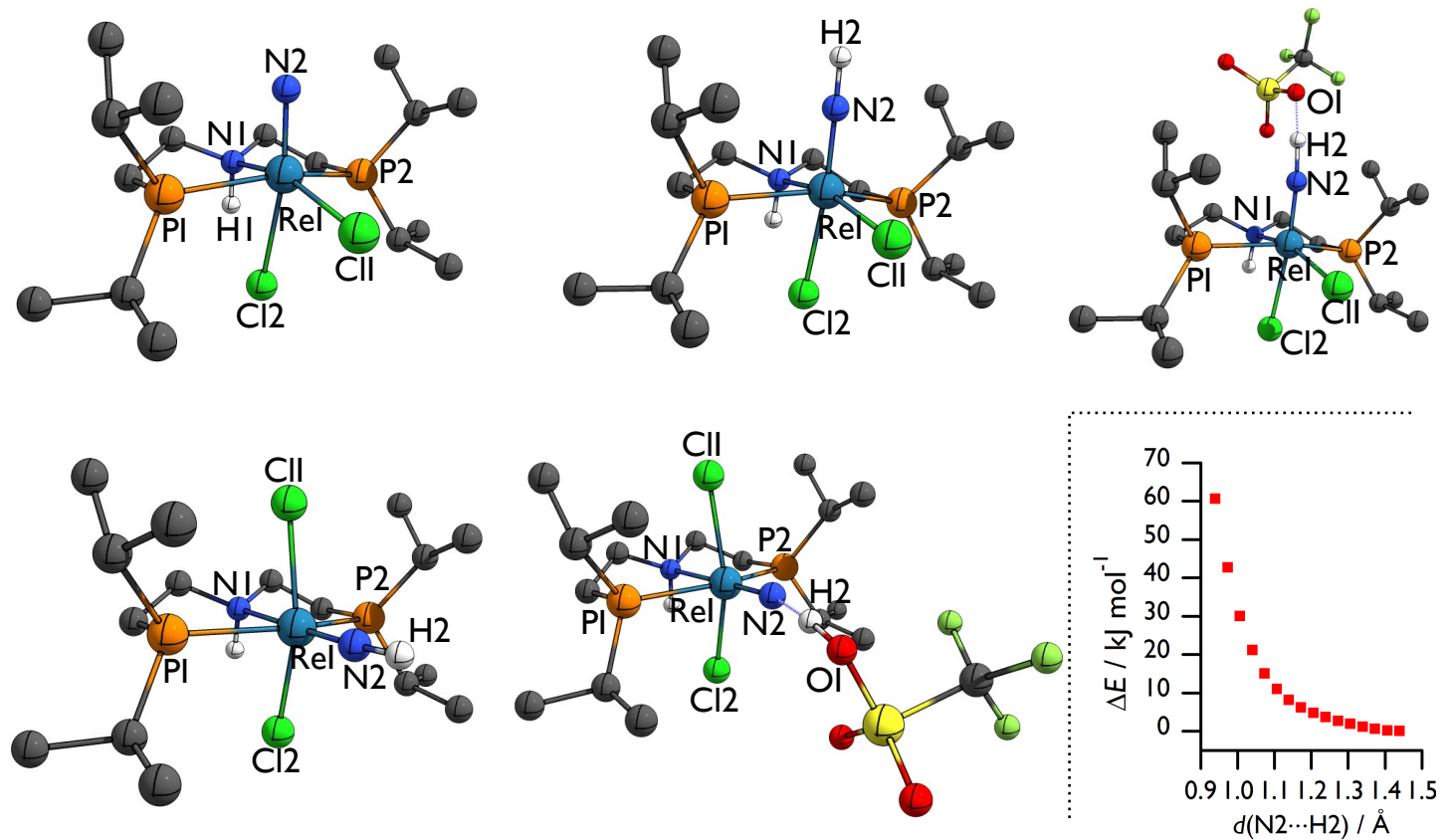

Fig. 3.6. DFT optimized structures of (top left to bottom right) 37 , cis-38 ${ }^{+}$, cis-38 $\cdots$ OTf, trans$38^{+}$and trans-38...0Tf. Carbon-bonded hydrogen atoms are omitted for clarity. Inset: Relaxed surface scan (PBE/D3BJ/RI/def2-SVP) for the $\mathrm{N} 2 \cdots \mathrm{H} 2$ bond distance in trans38...0Tf. 
Tab. 3.16. Structural parameters of the DFT optimized isomers. Bond lengths are given in $\AA$, bond angles in ${ }^{\circ}$.

\begin{tabular}{|c|c|c|c|c|c|c|c|}
\hline & 37 & $\exp$ & cis- $38^{+}$ & cis- $38 \cdots$ OTf & exp. & trans- $38^{+}$ & trans-38 $\cdots \mathrm{OTf}$ \\
\hline \multicolumn{8}{|l|}{ Distances } \\
\hline Re1-N1 & 2.224 & $2.159(2)$ & 2.222 & 2.217 & $2.164(5)$ & 2.408 & 2.482 \\
\hline Re1-N2 & 1.680 & $1.669(2)$ & 1.728 & 1.702 & $1.692(5)$ & 1.718 & 1.685 \\
\hline $\mathrm{N} 2-\mathrm{H} 2$ & & & 1.027 & 1.147 & $0.78(7)$ & 1.027 & 1.443 \\
\hline $\mathrm{H} 2-\mathrm{O} 1$ & & & & 1.378 & 2.166 & & 1.092 \\
\hline $\mathrm{Re} 1-\mathrm{Cl} 1$ & 2.423 & $2.4309(7)$ & 2.376 & 2.403 & $2.473(12)$ & 2.414 & 2.452 \\
\hline $\mathrm{Re} 1-\mathrm{Cl} 2$ & 2.771 & 2.4291(7) & 2.498 & 2.566 & $2.4600(14)$ & 2.425 & 2.452 \\
\hline \multicolumn{8}{|l|}{ Angles } \\
\hline N1-Re1-N2 & 91.9 & $92.65(11)$ & 92.8 & 92.5 & $90.8(2)$ & 179.4 & 177.8 \\
\hline $\mathrm{Cl} 1-\mathrm{Re} 1-\mathrm{Cl} 2$ & 91.7 & $88.23(2)$ & 88.8 & 88.7 & $87.7(3)$ & 157.0 & 155.7 \\
\hline P1-Re1-P2 & 159.6 & 159.92(2) & 158.8 & 161.2 & $164.3(3)$ & 154.7 & 156.2 \\
\hline Re1-N2-H2 & & & 178.0 & 170.7 & $167(5)$ & 176.9 & 166.5 \\
\hline
\end{tabular}

Tab. 3.17. Free Gibb's energies for the protonation reaction of $\mathbf{3 7}$ with HOTf. $\Delta G^{0}$ values are given in $\mathrm{kJ} \mathrm{mol}^{-1}$.

\begin{tabular}{|c|c|c|c|c|}
\hline & PBE(THF) & PBE0(THF) & $\operatorname{PBE}(\mathrm{DCM})$ & PBE0(DCM) \\
\hline 37 & 0.0 & 0.0 & 0.0 & 0.0 \\
\hline cis-38 0 Tf & -13.2 & -16.7 & -22.8 & -26.2 \\
\hline cis- $38 \cdots 0 T f$ & -29.7 & -31.6 & -29.0 & -31.0 \\
\hline trans-38 OTf & -22.2 & -28.9 & -30.9 & -37.5 \\
\hline 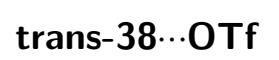 & -5.9 & -7.7 & -5.0 & -6.6 \\
\hline
\end{tabular}

\subsubsection{DFT-optimized geometries}

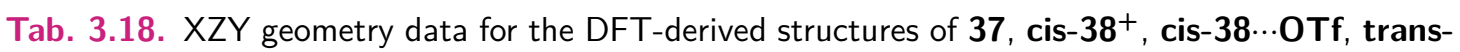

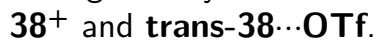

\begin{tabular}{|c|c|c|c|c|c|c|c|}
\hline & $x$ & $\mathrm{Y}$ & Z & & $X$ & $\mathrm{Y}$ & Z \\
\hline \multicolumn{8}{|c|}{37} \\
\hline C & 0.146027254 & 6.25104307 & 8.0351485 & $\mathrm{H}$ & -2.347494447 & 8.946066402 & 9.906482099 \\
\hline $\mathrm{H}$ & 1.157973703 & 6.132937912 & 8.472246676 & $\mathrm{H}$ & -1.065968072 & 9.804450602 & 10.81477063 \\
\hline $\mathrm{H}$ & 0.231710468 & 6.940152297 & 7.163748244 & $\mathrm{C}$ & -0.690031851 & 8.148267578 & 13.29256876 \\
\hline C & -0.423894967 & 4.901194615 & 7.586352154 & $\mathrm{H}$ & -1.204719369 & 9.041548611 & 13.71287233 \\
\hline $\mathrm{H}$ & 0.25853253 & 4.404490687 & 6.868401965 & $\mathrm{C}$ & 0.726028505 & 8.542016054 & 12.85889853 \\
\hline $\mathrm{H}$ & -1.394991687 & 5.059523324 & 7.071731602 & $\mathrm{H}$ & 1.235545381 & 7.685131716 & 12.37087797 \\
\hline C & 0.663668456 & 2.67483371 & 9.292442723 & $\mathrm{H}$ & 1.322881707 & 8.82080674 & 13.75195549 \\
\hline $\mathrm{H}$ & 0.555566554 & 1.898938799 & 8.50139472 & $\mathrm{H}$ & 0.741024147 & 9.409421363 & 12.16945989 \\
\hline C & 1.994459771 & 3.409146317 & 9.096682715 & $\mathrm{H}$ & -1.710022988 & 6.872201379 & 8.72031773 \\
\hline $\mathrm{H}$ & 2.83465069 & 2.706081446 & 9.273693148 & C & -0.644274721 & 7.028970018 & 14.34278283 \\
\hline
\end{tabular}




\begin{tabular}{lll} 
H & 2.093562441 & 4.237982941 \\
H & 2.117067538 & 3.816360408 \\
C & 0.604805088 & 2.018606396 \\
H & -0.356752198 & 1.50994052 \\
H & 0.733410808 & 2.784074266 \\
H & 1.420077214 & 1.271759839 \\
C & -2.247683199 & 2.782275835 \\
H & -3.010235067 & 3.582195929 \\
C & -1.98346715 & 2.072839452 \\
H & -2.908304674 & 1.560895462 \\
H & -1.199765188 & 1.292065907 \\
H & -1.678896605 & 2.76763225 \\
C & -2.757551599 & 1.829563546 \\
H & -3.75186758 & 1.434207907 \\
H & -2.859764893 & 2.34267356 \\
H & -2.08323093 & 0.957543636 \\
C & -0.340013822 & 8.230534089 \\
H & -0.244320085 & 8.879969498 \\
H & 0.657325976 & 8.171927199 \\
C & -1.383924216 & 8.810774692 \\
\hline
\end{tabular}

\begin{tabular}{l|llll}
9.828349196 & $\mathrm{H}$ & -1.648581247 & 6.690483188 & 14.65657695 \\
8.073371267 & $\mathrm{H}$ & -0.09877358 & 7.381452502 & 15.24274191 \\
10.67942655 & $\mathrm{H}$ & -0.116306147 & 6.144179589 & 13.93539804 \\
10.8749693 & $\mathrm{C}$ & -3.528834977 & 7.976608082 & 12.25944472 \\
11.47001673 & $\mathrm{H}$ & -4.015525873 & 7.669959359 & 11.30593968 \\
10.77563814 & $\mathrm{C}$ & -3.799924765 & 9.458232556 & 12.53628691 \\
8.473387969 & $\mathrm{H}$ & -3.48330544 & 10.11918378 & 11.70483079 \\
8.335859992 & $\mathrm{H}$ & -3.29435505 & 9.804136257 & 13.46236682 \\
7.142107792 & $\mathrm{H}$ & -4.888551421 & 9.615944877 & 12.68503286 \\
6.803392828 & $\mathrm{C}$ & -4.055136176 & 7.061169886 & 13.3707356 \\
7.238066615 & $\mathrm{H}$ & -5.162354704 & 7.124096621 & 13.40614914 \\
6.334089387 & $\mathrm{H}$ & -3.677500059 & 7.371197494 & 14.36698592 \\
9.560751742 & $\mathrm{H}$ & -3.769757741 & 6.003316835 & 13.19893733 \\
9.267121861 & $\mathrm{Re}$ & -1.10948082 & 5.504270421 & 10.79498931 \\
10.53864364 & $\mathrm{Cl}$ & -2.192478623 & 4.057783151 & 12.40889909 \\
9.688121462 & $\mathrm{Cl}$ & -3.489932378 & 5.911741119 & 9.436867735 \\
9.481209169 & $\mathrm{P}$ & -0.810832585 & 3.813878579 & 9.071915105 \\
8.58085822 & $\mathrm{P}$ & -1.747065014 & 7.606189553 & 11.84028278 \\
9.961883089 & $\mathrm{~N}$ & -0.722701924 & 6.85745323 & 9.072608757 \\
10.44140608 & $\mathrm{~N}$ & 0.501869602 & 5.55518395 & 11.2681288 \\
\hline
\end{tabular}

cis- $38^{+}$

\begin{tabular}{|c|c|c|c|c|c|c|c|}
\hline $\operatorname{Re}$ & 4.996523348 & 21.20182917 & 5.887995897 & $\mathrm{H}$ & 4.387499586 & 20.41247713 & 1.209905018 \\
\hline $\mathrm{Cl}$ & 2.516118746 & 21.41455044 & 5.684420279 & $\mathrm{H}$ & 3.880405541 & 21.64444464 & 2.420847923 \\
\hline P & 4.62765355 & 19.44004325 & 4.210055676 & $H$ & 2.675865393 & 20.90081793 & 1.316479546 \\
\hline $\mathrm{N}$ & 4.236221077 & 19.61690197 & 7.247131265 & & 5123 & 2599 & 111390367 \\
\hline $\mathrm{N}$ & 7594435 & 2309289 & 6.2313 & & & & 001 \\
\hline C & 4.534914845 & .89155541 & 8.680216401 & $P$ & 4.597016862 & 5691 & 7.873738738 \\
\hline $\mathrm{H}$ & 4.065114459 & 19.10911199 & 9.315747492 & C & 4.010630506 & 21.2772982 & 9.070125066 \\
\hline $\mathrm{H}$ & 5.63170803 & 19.81948586 & 8.819191676 & $\mathrm{H}$ & 4.289038286 & 21.51966937 & 10.11430088 \\
\hline C & 4.553291866 & 18.242436 & 6.769018 & $\mathrm{H}$ & & & \\
\hline $\mathrm{H}$ & 4.081934661 & 17.49497597 & 0618 & C & 3.252 & 588 & 9746 \\
\hline $\mathrm{H}$ & 5.650667362 & .10470099 & 6.834 & H & & 556 & 61576 \\
\hline C & 4.048649049 & .05397025 & 5.3346 & $c$ & 2.909 & 824 & 649069 \\
\hline $\mathrm{H}$ & 2.938568193 & 18.08677967 & 5.3162 & $\mathrm{H}$ & 3.746 & 3029 & 24318 \\
\hline $\mathrm{H}$ & 4.346412431 & 17.06 & 4.944 & $\mathrm{H}$ & 7395 & 594 & 39749 \\
\hline C & 6.186605506 & 18.85381 & 3.345 & $\mathrm{H}$ & 2.04 & & \\
\hline $\mathrm{H}$ & 5.798327377 & 18.23925777 & 2.5026 & C & 3.56572 & & 7165 \\
\hline C & 8661 & 941 & 2.784 & $\mathrm{H}$ & 3.870 & 867 & 5.959938225 \\
\hline $\mathrm{H}$ & 7.792221717 & 9171 & 4787 & $\pi$ & 4.365 & 25.7 & 40513 \\
\hline $\mathrm{H}$ & 7.426732927 & 20.63814725 & 3.604614362 & $\mathrm{H}$ & 2.6599 & 25.68580891 & 6.855610503 \\
\hline $\mathrm{H}$ & 6.349 & 5529 & 2.193 & C & 6.14 & 792 & 8.602 \\
\hline C & 7.066011724 & 17.96568044 & 4.22877 & $\mathrm{H}$ & 5.73778 & 24.1212 & 9.321911303 \\
\hline $\mathrm{H}$ & 6.559715942 & 17.03873639 & 4.560644792 & C & 7.012287454 & 22.37935822 & 9.373598126 \\
\hline $\mathrm{H}$ & 7.422840792 & 18.51202867 & 5.126506912 & $\mathrm{H}$ & 7.907658798 & 9282 & 9.767110017 \\
\hline $\mathrm{H}$ & 7.9678497 & 0523 & 3.660527676 & $\mathrm{H}$ & 7.380885435 & 21.56 & 8.715729447 \\
\hline C & & 19.58309683 & 2.922684229 & $\mathrm{H}$ & 6.494530126 & 21.92320517 & 10.23942508 \\
\hline $\mathrm{H}$ & 2.425059125 & & 3.549049323 & C & 6.946800218 & 24.10289341 & 7.516392311 \\
\hline C & 2.972446852 & 18.24395415 & 2.247090264 & $\mathrm{H}$ & 7.747377479 & 24.70564358 & 7.990500988 \\
\hline $\mathrm{H}$ & 2.109864534 & 18.37839789 & 1.563177321 & $\mathrm{H}$ & 6.328915047 & 24.77691284 & 6.895658848 \\
\hline $\mathrm{H}$ & 2.698248995 & 17.44558179 & 2.964607355 & $\mathrm{H}$ & 7.424994665 & 23.37879527 & 6.827443259 \\
\hline
\end{tabular}




\begin{tabular}{|c|c|c|c|c|c|c|c|}
\hline $\mathrm{H}$ & 3.818322469 & 17.88064854 & 1.627795292 & \multirow[t]{2}{*}{$\mathrm{Cl}$} & \multirow[t]{2}{*}{5.226857206} & \multirow[t]{2}{*}{22.99428286} & \multirow[t]{2}{*}{4.345852429} \\
\hline C & 3.585442321 & 20.70251297 & 1.918266215 & & & & \\
\hline \multicolumn{8}{|c|}{ cis- $38 \cdots 0 T f$} \\
\hline $\operatorname{Re}$ & 4.853355939 & 21.19133672 & 5.94643652 & $\mathrm{H}$ & 7.415800941 & 20.32826177 & 6.816045487 \\
\hline $\mathrm{Cl}$ & 2.327563323 & 21.46919051 & 5.586084296 & $P$ & 4.442170404 & 22.58454899 & 7.913745166 \\
\hline $\mathrm{P}$ & 4.636649078 & 19.40551053 & 4.282823489 & C & 3.637906264 & 21.32447581 & 9.041949879 \\
\hline $\mathrm{N}$ & 3.942996454 & 19.64333763 & 7.245747838 & $\mathrm{H}$ & 3.823001663 & 21.55170771 & 10.1102278 \\
\hline $\mathrm{N}$ & 6.433018979 & 20.75897877 & 6.409865606 & $\mathrm{H}$ & 2.544985599 & 21.42063886 & 8.866459131 \\
\hline C & 4.109648217 & 19.90843044 & 8.703070445 & C & 3.217690054 & 23.99022234 & 7.855872589 \\
\hline $\mathrm{H}$ & 3.517507563 & 19.16113931 & 9.277024119 & $\mathrm{H}$ & 2.370223324 & 23.50436321 & 7.322987336 \\
\hline $\mathrm{H}$ & 5.175789354 & 19.73901159 & 8.949154697 & C & 2.761259261 & 24.45378099 & 9.242956636 \\
\hline C & 4.274596712 & 18.25188028 & 6.827707266 & $\mathrm{H}$ & 3.595057807 & 24.90031162 & 9.824201639 \\
\hline $\mathrm{H}$ & 3.706258005 & 17.53585941 & 7.462211113 & $\mathrm{H}$ & 2.319155593 & 23.6373998 & 9.847555713 \\
\hline $\mathrm{H}$ & 5.348290331 & 18.08513788 & 7.040716001 & $\mathrm{H}$ & 1.986041968 & 25.24037795 & 9.134199235 \\
\hline C & 3.913996568 & 18.04691038 & 5.353957232 & C & 3.731233134 & 25.14330691 & 6.985603714 \\
\hline $\mathrm{H}$ & 2.812987749 & 18.11079262 & 5.218659057 & $\mathrm{H}$ & 4.111809172 & 24.78085015 & 6.009441056 \\
\hline $\mathrm{H}$ & 4.22917132 & 17.04213801 & 5.011020383 & $\mathrm{H}$ & 4.539955462 & 25.70717523 & 7.494147343 \\
\hline C & 6.241565394 & 18.75797505 & 3.555543948 & $\mathrm{H}$ & 2.904246861 & 25.85769895 & 6.794694402 \\
\hline $\mathrm{H}$ & 5.911600313 & 18.17781754 & 2.66426624 & $\mathrm{C}$ & 5.982544354 & 23.20899272 & 8.786217902 \\
\hline C & 7.12581866 & 19.92929789 & 3.103066415 & $\mathrm{H}$ & 5.599242377 & 23.99554722 & 9.474892253 \\
\hline $\mathrm{H}$ & 7.984540699 & 19.54145407 & 2.518020825 & $\mathrm{C}$ & 6.684031185 & 22.12621153 & 9.612270615 \\
\hline $\mathrm{H}$ & 7.527728927 & 20.47092754 & 3.981636451 & $\mathrm{H}$ & 7.589744137 & 22.557103 & 10.08566144 \\
\hline $\mathrm{H}$ & 6.585972761 & 20.67040148 & 2.485373727 & $\mathrm{H}$ & 7.030764789 & 21.2790145 & 8.989824061 \\
\hline C & 7.005567724 & 17.82359231 & 4.497592932 & $\mathrm{H}$ & 6.051469327 & 21.72036734 & 10.42517057 \\
\hline $\mathrm{H}$ & 6.422232335 & 16.93716525 & 4.812429266 & C & 6.948599575 & 23.84364812 & 7.775015363 \\
\hline $\mathrm{H}$ & 7.36550041 & 18.3428048 & 5.405515055 & $\mathrm{H}$ & 7.763059289 & 24.36565713 & 8.317620202 \\
\hline $\mathrm{H}$ & 7.913582432 & 17.44809282 & 3.98321181 & $\mathrm{H}$ & 6.458683247 & 24.56767571 & 7.098332308 \\
\hline C & 3.417217817 & 19.56614449 & 2.879554418 & $\mathrm{H}$ & 7.40861546 & 23.06260822 & 7.138568807 \\
\hline $\mathrm{H}$ & 2.520971959 & 19.93783746 & 3.424145689 & $\mathrm{Cl}$ & 5.216383494 & 22.98856474 & 4.392525047 \\
\hline C & 3.096124309 & 18.23186327 & 2.198748109 & $S$ & 8.266182233 & 18.49062454 & 8.152946314 \\
\hline $\mathrm{H}$ & 2.308059671 & 18.38732435 & 1.433033199 & $F$ & 9.280539366 & 17.7856748 & 10.49858961 \\
\hline $\mathrm{H}$ & 2.722157648 & 17.46415823 & 2.904780261 & $F$ & 9.086210745 & 19.94420745 & 10.23681528 \\
\hline $\mathrm{H}$ & 3.979541937 & 17.81408741 & 1.672242272 & $\mathrm{~F}$ & 10.68315681 & 18.85927466 & 9.216014741 \\
\hline C & 3.851873833 & 20.64862868 & 1.884793016 & $\mathrm{O}$ & 8.712604773 & 17.22383067 & 7.542942316 \\
\hline $\mathrm{H}$ & 4.707549502 & 20.31091636 & 1.26471286 & $\mathrm{O}$ & 8.562990997 & 19.73498732 & 7.295655019 \\
\hline $\mathrm{H}$ & 4.138658204 & 21.58714374 & 2.400358975 & $\mathrm{O}$ & 6.892777355 & 18.52572489 & 8.744466505 \\
\hline $\mathrm{H}$ & 3.013820099 & 20.87191169 & 1.193064162 & $\mathrm{C}$ & 9.408672992 & 18.79070214 & 9.619774641 \\
\hline $\mathrm{H}$ & 2.949051763 & 19.82259349 & 7.000856181 & & & & \\
\hline
\end{tabular}

\begin{tabular}{llll|llll}
\hline \multicolumn{7}{c}{ trans-38 $^{+}$} \\
\hline $\mathrm{Re}$ & 4.762616794 & 20.91557089 & 6.157410902 & $\mathrm{H}$ & 4.170850427 & 23.02463245 & 10.18144989 \\
$\mathrm{Cl}$ & 2.356627711 & 21.171214 & 5.997657916 & $\mathrm{H}$ & 3.479375509 & 21.63939777 & 9.26077791 \\
$\mathrm{P}$ & 4.781100725 & 23.19843956 & 7.097851345 & $\mathrm{H}$ & 2.402863453 & 22.83743469 & 10.0357302 \\
$\mathrm{~N}$ & 4.526900312 & 22.29169919 & 4.195730147 & $\mathrm{H}$ & 3.493918193 & 22.35965307 & 4.182042737 \\
$\mathrm{~N}$ & 4.949756328 & 19.93275592 & 7.55434046 & $\mathrm{H}$ & 5.116050811 & 19.3477929 & 8.382157361 \\
$\mathrm{C}$ & 4.966865696 & 21.6230917 & 2.948798068 & $\mathrm{P}$ & 4.544405713 & 19.24648965 & 4.350140836 \\
$\mathrm{H}$ & 4.73558007 & 22.26279164 & 2.06857482 & $\mathrm{C}$ & 4.277138924 & 20.26917598 & 2.820374467 \\
$\mathrm{H}$ & 6.067647383 & 21.50897653 & 3.006108765 & $\mathrm{H}$ & 4.621282782 & 19.72876998 & 1.917574226 \\
$\mathrm{C}$ & 5.081969036 & 23.65540086 & 4.361260229 & $\mathrm{H}$ & 3.177969871 & 20.39939291 & 2.727069814 \\
$\mathrm{H}$ & 4.8474186 & 24.2759419 & 3.468283508 & $\mathrm{C}$ & 3.005714748 & 18.19350025 & 4.469893767
\end{tabular}




\begin{tabular}{llll|llll}
$\mathrm{H}$ & 6.183821038 & 23.5574341 & 4.427170703 & $\mathrm{H}$ & 2.221907752 & 18.97131267 & 4.602027792 \\
$\mathrm{C}$ & 4.507911648 & 24.29449723 & 5.621215283 & $\mathrm{C}$ & 2.731984232 & 17.39669844 & 3.188976186 \\
$\mathrm{H}$ & 3.407560248 & 24.41576286 & 5.529864358 & $\mathrm{H}$ & 3.493973348 & 16.60971615 & 3.014148625 \\
$\mathrm{H}$ & 4.93599261 & 25.3012253 & 5.790703179 & $\mathrm{H}$ & 2.680074035 & 18.03426788 & 2.284668343 \\
$\mathrm{C}$ & 6.323866294 & 23.7676317 & 8.00219911 & $\mathrm{H}$ & 1.753927475 & 16.88216556 & 3.281581108 \\
$\mathrm{H}$ & 5.924889857 & 24.54972741 & 8.686399869 & $\mathrm{C}$ & 3.012395184 & 17.31070188 & 5.721743895 \\
$\mathrm{C}$ & 6.910669416 & 22.6246107 & 8.843566733 & $\mathrm{H}$ & 3.217419023 & 17.89763786 & 6.638610442 \\
$\mathrm{H}$ & 7.710024759 & 23.02337307 & 9.500015256 & $\mathrm{H}$ & 3.757135541 & 16.49226562 & 5.649727956 \\
$\mathrm{H}$ & 7.357407921 & 21.85165582 & 8.188177698 & $\mathrm{H}$ & 2.017563952 & 16.83734778 & 5.844083076 \\
$\mathrm{H}$ & 6.155711501 & 22.14092978 & 9.492501909 & $\mathrm{C}$ & 5.97974011 & 18.07403571 & 4.056285894 \\
$\mathrm{C}$ & 7.380960231 & 24.40665305 & 7.094486843 & $\mathrm{H}$ & 5.481470932 & 17.19832813 & 3.582971316 \\
$\mathrm{H}$ & 6.996334536 & 25.27827778 & 6.530133863 & $\mathrm{C}$ & 7.039097783 & 18.61500791 & 3.089313805 \\
$\mathrm{H}$ & 7.794004183 & 23.66717628 & 6.382094228 & $\mathrm{H}$ & 7.807916056 & 17.83287894 & 2.924562229 \\
$\mathrm{H}$ & 8.218061941 & 24.77431196 & 7.722279824 & $\mathrm{H}$ & 7.546845724 & 19.50318625 & 3.511300502 \\
$\mathrm{C}$ & 3.333916938 & 23.57124205 & 8.21957958 & $\mathrm{H}$ & 6.625034029 & 18.87263787 & 2.095152884 \\
$\mathrm{H}$ & 2.480882309 & 23.23023416 & 7.592853109 & $\mathrm{C}$ & 6.597559344 & 17.63155295 & 5.390902869 \\
$\mathrm{C}$ & 3.188204199 & 25.0677109 & 8.519981562 & $\mathrm{H}$ & 7.325407594 & 16.81586251 & 5.207096808 \\
$\mathrm{H}$ & 2.258976223 & 25.23395307 & 9.102040449 & $\mathrm{H}$ & 5.842138616 & 17.25110664 & 6.104856602 \\
$\mathrm{H}$ & 3.114845718 & 25.68775923 & 7.605070796 & $\mathrm{H}$ & 7.137789591 & 18.47402785 & 5.865317346 \\
$\mathrm{H}$ & 4.026462318 & 25.45508928 & 9.134631033 & $\mathrm{Cl}$ & 7.075186127 & 21.21195011 & 5.532514347 \\
$\mathrm{C}$ & 3.360336577 & 22.71654369 & 9.490435225 & & & & \\
\hline
\end{tabular}

\begin{tabular}{|c|c|c|c|c|c|c|c|}
\hline \multicolumn{8}{|c|}{ 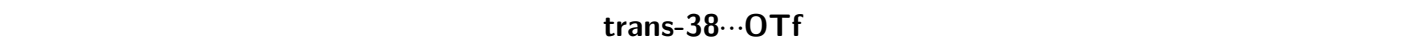 } \\
\hline $\operatorname{Re}$ & 4.727869925 & 21.45883878 & 5.724813403 & $\mathrm{H}$ & 5.635491313 & 23.40130434 & 3.47751213 \\
\hline $\mathrm{Cl}$ & 2.293229432 & 21.62692719 & 5.488974662 & $\mathrm{P}$ & 4.429479989 & 22.71473124 & 7.804182493 \\
\hline$P$ & 4.578168832 & 19.53194117 & 4.244685439 & C & 3.730515421 & 21.44637071 & 8.988689744 \\
\hline $\mathrm{N}$ & 3.876595173 & 19.70437239 & 7.260437966 & $\mathrm{H}$ & 3.968778044 & 21.70602963 & 10.03861628 \\
\hline N & 5.309281104 & 22.6055708 & 4.636026253 & $\mathrm{H}$ & 2.627578501 & 21.51988824 & 8.875564654 \\
\hline C & 4.19542193 & 20.02441352 & 8.65950415 & C & 3.099529018 & 24.02837534 & 7.745858193 \\
\hline $\mathrm{H}$ & 3.719963604 & 19.29877378 & 9.361414828 & $\mathrm{H}$ & 2.24751077 & 23.44204885 & 7.335977003 \\
\hline $\mathrm{H}$ & 5.295008658 & 19.93588355 & 8.774845883 & C & 2.729639096 & 24.58865694 & 9.122298917 \\
\hline C & 4.261979402 & 18.36308676 & 6.799416754 & $\mathrm{H}$ & 3.557086186 & 25.18040838 & 9.566283629 \\
\hline $\mathrm{H}$ & 3.785000325 & 17.56676103 & 7.418714992 & $\mathrm{H}$ & 2.445637774 & 23.79993274 & 9.847799984 \\
\hline $\mathrm{H}$ & 5.36064687 & 18.27538202 & 6.924129807 & $\mathrm{H}$ & 1.861891677 & 25.27346422 & 9.024107579 \\
\hline C & 3.868071471 & 18.18533419 & 5.329657307 & C & 3.439481984 & 25.12191769 & 6.729028247 \\
\hline $\mathrm{H}$ & 2.765782775 & 18.2724486 & 5.21973886 & $\mathrm{H}$ & 3.674871271 & 24.70321999 & 5.731788747 \\
\hline $\mathrm{H}$ & 4.154936824 & 17.18077418 & 4.961954096 & $\mathrm{H}$ & 4.292338518 & 25.74828906 & 7.061115153 \\
\hline C & 6.142696741 & 18.8873083 & 3.427921709 & $\mathrm{H}$ & 2.566891941 & 25.79562986 & 6.60529423 \\
\hline $\mathrm{H}$ & 5.776646473 & 18.38425842 & 2.504947679 & C & 5.924459022 & 23.52671077 & 8.601631894 \\
\hline C & 7.042977371 & 20.06895737 & 3.034375907 & $\mathrm{H}$ & 5.493650731 & 24.36905576 & 9.187428865 \\
\hline $\mathrm{H}$ & 7.883707033 & 19.7044111 & 2.408923826 & C & 6.68954518 & 22.60307245 & 9.556884904 \\
\hline $\mathrm{H}$ & 7.46160083 & 20.54703266 & 3.941586625 & $\mathrm{H}$ & 7.539856076 & 23.16026083 & 10.00231851 \\
\hline $\mathrm{H}$ & 6.506250888 & 20.84969331 & 2.462500386 & $\mathrm{H}$ & 7.102481467 & 21.73293356 & 9.009792836 \\
\hline C & 6.903317065 & 17.86332647 & 4.278822246 & $\mathrm{H}$ & 6.064585687 & 22.23775733 & 10.39578631 \\
\hline $\mathrm{H}$ & 6.296727456 & 16.96819538 & 4.520814917 & C & 6.844791276 & 24.0919561 & 7.50849899 \\
\hline $\mathrm{H}$ & 7.255445923 & 18.32575386 & 5.221772178 & $\mathrm{H}$ & 7.643915381 & 24.7043234 & 7.974872052 \\
\hline $\mathrm{H}$ & 7.794594541 & 17.51195025 & 3.718489248 & $\mathrm{H}$ & 6.307697688 & 24.72836341 & 6.78017936 \\
\hline C & 3.320082009 & 19.70301515 & 2.870958614 & $\mathrm{H}$ & 7.319686711 & 23.26485574 & 6.945238107 \\
\hline $\mathrm{H}$ & 2.432210591 & 20.05217429 & 3.443423631 & $\mathrm{Cl}$ & 6.830810291 & 20.57147165 & 6.619729036 \\
\hline C & 2.998808317 & 18.38064003 & 2.167839057 & S & 4.650544392 & 24.99286291 & 2.318907732 \\
\hline $\mathrm{H}$ & 2.177178824 & 18.53699992 & 1.438436283 & $\mathrm{~F}$ & 5.671454923 & 26.0870736 & 4.530710129 \\
\hline
\end{tabular}




\begin{tabular}{llll|llll}
$\mathrm{H}$ & 2.669410973 & 17.58802571 & 2.869182762 & $\mathrm{~F}$ & 4.445665369 & 27.44006273 & 3.330701276 \\
$\mathrm{H}$ & 3.867267501 & 17.99284467 & 1.595455028 & $\mathrm{~F}$ & 6.461970219 & 26.92310795 & 2.674335859 \\
$\mathrm{C}$ & 3.708199746 & 20.81272869 & 1.887853216 & $\mathrm{O}$ & 5.818916678 & 23.93848906 & 2.544636249 \\
$\mathrm{H}$ & 4.584346112 & 20.52919448 & 1.269429142 & $\mathrm{O}$ & 4.623859808 & 25.39803595 & 0.910931127 \\
$\mathrm{H}$ & 3.933426953 & 21.76760868 & 2.399633106 & $\mathrm{O}$ & 3.426276078 & 24.60181364 & 3.043952255 \\
$\mathrm{H}$ & 2.864327259 & 21.00254427 & 1.193615646 & $\mathrm{C}$ & 5.359031018 & 26.46268327 & 3.271960662 \\
$\mathrm{H}$ & 2.869621575 & 19.85941166 & 7.085621273 & & & & \\
\hline
\end{tabular}

\section{8 $\mathrm{N}_{2}$ splitting and functionalization by the $\left\{\operatorname{Re}\left({ }^{\mathrm{Me}} \mathrm{NPNP} \mathrm{P}^{\mathrm{iPr}}\right)\right\}$ platform}

All calculations were largely performed according to the in Section 3.1 described procedure. As the results were to be compared with those published for the splitting of $\mathbf{3 6}$ to $\mathbf{3 7},{ }^{[269]}$ the procedure was modified in some points: Single point calculations were performed using the M06 functional, no additional dispersion correction was applied and the RI approximation was switched off. Furthermore, solvent effects were modeled using the SMD solvation model and the ORCA grid size was changed to 5 .

The thermochemistry data for the existing iso-propyl substituted complexes $\mathbf{3 6}, \mathbf{3 7}$ and the connecting TS are given here for comparision, the corresponding xyz-data can be found in the above cited publication.

Tab. 3.19. Thermochemistry data calculated for the $\mathrm{N}_{2}$ splitting from the dimers 36 and $[(\mu-$ $\left.\left.\mathrm{N}_{2}\right)\left\{\mathrm{ReCl}\left({ }^{\mathrm{Me}} \mathrm{NPNP}{ }^{i \mathrm{Pr}}\right)\right\}_{2}\right]$ into the corresponding nitrides and subsequent methylation of the nitrides with MeOTf. All thermodynamic corrections to derive relative free energies $\Delta G$ are based on frequency calculations at the PBE/D3BJ/def2SVP level of theory. The data are given in $\mathrm{kJ} \mathrm{mol}^{-1}$.

\begin{tabular}{|c|c|}
\hline & $\Delta G$ \\
\hline $36(S=1)$ & 0 \\
\hline $36\left({ }^{\mathrm{BS} S}=0\right)$ & +19 \\
\hline $\mathrm{TS}(\mathrm{S}=0)$ & 175 \\
\hline $2 \times 37$ & -83 \\
\hline $2 \times\left[\operatorname{Re}(\mathrm{NMe}) \mathrm{Cl}_{2}\left(\mathrm{HPNP}^{i \mathrm{Pr}}\right)\right]^{\mathrm{OTf}}$ & -231 \\
\hline$\left[\left(\mu-\mathrm{N}_{2}\right)\left\{\operatorname{ReCl}\left({ }^{\mathrm{Me}} \mathrm{NPNP}^{i \mathrm{Pr}}\right)\right\}_{2}\right](\mathrm{S}=1)$ & 0 \\
\hline$\left[\left(\mu-\mathrm{N}_{2}\right)\left\{\mathrm{ReCl}\left({ }^{\mathrm{Me}} \mathrm{NPNP}^{i \mathrm{Pr}}\right)\right\}_{2}\right]\left({ }^{\mathrm{BS} S}=0\right)$ & +3 \\
\hline TS $(S=0)$ & 239 \\
\hline $2 \times\left[\operatorname{Re}(\mathrm{N}) \mathrm{Cl}\left({ }^{\mathrm{Me}} \mathrm{NPNP} \mathrm{P}^{\mathrm{Pr}}\right)\right]$ & -68 \\
\hline $2 \times\left[\operatorname{Re}(\mathrm{NMe}) \mathrm{Cl}\left({ }^{\mathrm{Me}} \mathrm{NPNP}^{i \operatorname{Pr}}\right)\right]^{\mathrm{OTf}}$ & -266 \\
\hline
\end{tabular}




\subsubsection{DFT-optimized geometries}

Tab. 3.20. XZY geometry data for the DFT-derived structures of $\left[\mathrm{Re}(\mathrm{NMe}) \mathrm{Cl}_{2}\left(\mathrm{HPNP}^{\mathrm{iPr}}\right)\right]^{+}$, $\left[\left(\mu-\mathrm{N}_{2}\right)\left\{\operatorname{ReCl}\left({ }^{\mathrm{Me}} \mathrm{NPNP} \mathrm{PPr}^{i \mathrm{r}}\right)\right\}_{2}\right] \quad(\mathrm{S}=1), \quad\left[\left(\mu-\mathrm{N}_{2}\right)\left\{\operatorname{ReCl}\left({ }^{\mathrm{Me}} \mathrm{NPNP}{ }^{i \mathrm{Pr}_{2}}\right)\right\}_{2}\right] \quad\left(\mathrm{BS}^{\mathrm{B}}=\right.$ $0), \quad\left[\left(\mu-\mathrm{N}_{2}\right)\left\{\operatorname{ReCl}\left({ }^{\mathrm{Me} N P N P}{ }^{i \mathrm{Pr}}\right)\right\}_{2}\right] \quad(\mathrm{TS}, \quad \mathrm{S}=0), \quad\left[\operatorname{Re}(\mathrm{N}) \mathrm{Cl}\left({ }^{\mathrm{Me}} \mathrm{NPNP}^{i \mathrm{Pr}}\right)\right] \quad$ and $\left[\mathrm{Re}(\mathrm{NMe}) \mathrm{Cl}\left({ }^{\mathrm{Me}} \mathrm{NPNP}^{\mathrm{iPr}}\right)\right]^{+}$.

\begin{tabular}{|c|c|c|c|c|c|c|c|}
\hline & $X$ & $\mathrm{Y}$ & Z & & $x$ & $\mathrm{Y}$ & Z \\
\hline \multicolumn{8}{|c|}{$\left[\mathrm{Re}(\mathrm{NMe}) \mathrm{Cl}_{2}\left(\mathrm{HPNP}^{\mathrm{iPr}}\right)\right]^{+}$} \\
\hline C & 0.193924736 & 6.244767527 & 8.039540742 & $\mathrm{C}$ & -0.76592672 & 8.203712506 & 13.32172106 \\
\hline $\mathrm{H}$ & 1.202618041 & 6.098432843 & 8.4751646 & $\mathrm{H}$ & -1.388718476 & 9.010598976 & 13.76743434 \\
\hline $\mathrm{H}$ & 0.300941541 & 6.939915647 & 7.177833516 & $\mathrm{C}$ & 0.590950874 & 8.804061623 & 12.94027458 \\
\hline C & -0.415721254 & 4.916845876 & 7.579235103 & $\mathrm{H}$ & 1.224955959 & 8.083529088 & 12.3866155 \\
\hline $\mathrm{H}$ & 0.247146568 & 4.415635997 & 6.847791669 & $\mathrm{H}$ & 1.143184094 & 9.082416583 & 13.86091657 \\
\hline $\mathrm{H}$ & -1.380726081 & 5.10483533 & 7.062372428 & $\mathrm{H}$ & 0.499275069 & 9.721689195 & 12.32792105 \\
\hline C & 0.608566002 & 2.600497627 & 9.238741327 & $\mathrm{H}$ & -1.634895109 & 6.906654875 & 8.694622172 \\
\hline $\mathrm{H}$ & 0.373705305 & 1.789946688 & 8.513943566 & $\mathrm{C}$ & -0.633331497 & 7.060714642 & 14.33763878 \\
\hline C & 1.964830388 & 3.202932254 & 8.858876589 & $\mathrm{H}$ & -1.60814753 & 6.619406689 & 14.61427201 \\
\hline $\mathrm{H}$ & 2.763106894 & 2.452799036 & 9.032522608 & $\mathrm{H}$ & -0.150416831 & 7.43652954 & 15.26227005 \\
\hline $\mathrm{H}$ & 2.214451957 & 4.090122246 & 9.474156046 & $\mathrm{H}$ & -0.0188534 & 6.231913673 & 13.93598648 \\
\hline $\mathrm{H}$ & 2.023281902 & 3.496509501 & 7.79314469 & $\mathrm{C}$ & -3.556724409 & 7.966757444 & 12.24017752 \\
\hline C & 0.608776768 & 2.011540095 & 10.65612925 & $\mathrm{H}$ & -4.050291734 & 7.646175914 & 11.29729026 \\
\hline $\mathrm{H}$ & -0.370683962 & 1.588162251 & 10.94382638 & $\mathrm{C}$ & -3.846601691 & 9.450358565 & 12.4924895 \\
\hline $\mathrm{H}$ & 0.850154788 & 2.781583946 & 11.41406384 & $\mathrm{H}$ & -3.540647203 & 10.10487887 & 11.65256616 \\
\hline $\mathrm{H}$ & 1.366946367 & 1.205482666 & 10.72523726 & $\mathrm{H}$ & -3.358385967 & 9.820082507 & 13.41775422 \\
\hline C & -2.281633768 & 2.801373715 & 8.458654687 & $\mathrm{H}$ & -4.938440798 & 9.586730035 & 12.63118715 \\
\hline $\mathrm{H}$ & -3.04998678 & 3.594968574 & 8.335227835 & $\mathrm{C}$ & -4.054656493 & 7.060394639 & 13.37196028 \\
\hline C & -2.03450439 & 2.112437834 & 7.112111271 & $\mathrm{H}$ & -5.159992254 & 7.1235186 & 13.42801752 \\
\hline $\mathrm{H}$ & -2.9690161 & 1.612667347 & 6.78515887 & $\mathrm{H}$ & -3.660854834 & 7.382867515 & 14.35711919 \\
\hline $\mathrm{H}$ & -1.257825242 & 1.323013993 & 7.181744928 & $\mathrm{H}$ & -3.776209175 & 6.000622172 & 13.20893181 \\
\hline $\mathrm{H}$ & -1.743321113 & 2.816085728 & 6.307425452 & $\mathrm{Re}$ & -1.062714075 & 5.514301659 & 10.7971606 \\
\hline C & -2.764558271 & 1.833182508 & 9.544827396 & $\mathrm{Cl}$ & -2.03055487 & 4.076431698 & 12.4356089 \\
\hline $\mathrm{H}$ & -3.752304186 & 1.423459574 & 9.252559389 & $\mathrm{Cl}$ & -3.315808759 & 5.738721546 & 9.730370932 \\
\hline $\mathrm{H}$ & -2.874388401 & 2.332286001 & 10.52772649 & $\mathrm{P}$ & -0.817691789 & 3.803385213 & 9.036589799 \\
\hline $\mathrm{H}$ & -2.076928703 & 0.970808186 & 9.659852644 & $\mathrm{P}$ & -1.764773592 & 7.638248846 & 11.83745679 \\
\hline C & -0.304172366 & 8.246406391 & 9.498547055 & $\mathrm{~N}$ & -0.670944348 & 6.865522825 & 9.080177879 \\
\hline $\mathrm{H}$ & -0.187543756 & 8.891446061 & 8.599942335 & $\mathrm{~N}$ & 0.579519182 & 5.518949593 & 11.34424854 \\
\hline $\mathrm{H}$ & 0.681017582 & 8.200620632 & 10.0044676 & $\mathrm{C}$ & 1.910511621 & 5.495053037 & 11.82385007 \\
\hline C & -1.383877199 & 8.814983636 & 10.42494321 & $\mathrm{H}$ & 2.294191432 & 4.452321398 & 11.82814389 \\
\hline $\mathrm{H}$ & -2.334333533 & 8.938680167 & 9.863670817 & $\mathrm{H}$ & 1.945241517 & 5.873954261 & 12.86776745 \\
\hline $\mathrm{H}$ & -1.095190843 & 9.818871638 & 10.79177938 & $\mathrm{H}$ & 2.584098912 & 6.118834764 & 11.19691 \\
\hline \multicolumn{8}{|c|}{$\left[\left(\mu-\mathrm{N}_{2}\right)\left\{\operatorname{ReCl}\left({ }^{\mathrm{Me}} \mathrm{NPNP}^{\mathrm{iPr}}\right)\right\}_{2}\right](\mathrm{S}=1)$} \\
\hline $\operatorname{Re}$ & 1.95554334 & 8.015991958 & 8.700877964 & $\mathrm{~N}$ & -1.017552715 & 7.027617705 & 8.543205125 \\
\hline $\mathrm{Cl}$ & 1.214019386 & 10.04779441 & 10.02806865 & $\mathrm{H}$ & -4.103179202 & 8.832062666 & 13.62636316 \\
\hline$P$ & 1.984302565 & 9.296596241 & 6.646135923 & $\mathrm{H}$ & -4.931647626 & 9.746818368 & 12.34036589 \\
\hline N & 4.115127203 & 8.732299046 & 8.773752602 & $\mathrm{C}$ & -4.49994813 & 8.754840021 & 12.59177804 \\
\hline C & 4.320827881 & 9.953048833 & 7.938246646 & $\mathrm{H}$ & -5.328126398 & 8.019306542 & 12.61267302 \\
\hline $\mathrm{H}$ & 5.407962336 & 10.20801093 & 7.935336484 & $\mathrm{H}$ & -1.771047013 & 9.256160529 & 12.81017759 \\
\hline $\mathrm{H}$ & 3.781412988 & 10.77235965 & 8.451480989 & $\mathrm{H}$ & -2.494409265 & 10.38153403 & 11.63695545 \\
\hline
\end{tabular}




\begin{tabular}{|c|c|c|c|c|c|c|c|}
\hline I & 2.591071687 & 7.141446045 & 10.88779394 & $\mathrm{H}$ & -4.465145552 & 10.52221773 & \\
\hline $\mathrm{C}$ & 3.792531009 & 9.793233187 & 6.522332689 & C & -2.181622476 & 9.32786096 & 11.781454 \\
\hline $\mathrm{r}$ & 4.350717486 & 9.011634648 & 5.970813886 & C & -3.370114307 & 8.372642555 & 11.62819025 \\
\hline H & 91870835 & 10.7374888 & 5.955764674 & C & -4.180689137 & 10.01314009 & 9.309631287 \\
\hline C & .681267369 & 8.422995713 & 4.993657689 & $\mathrm{H}$ & -3.029997316 & 388086 & 11.86224876 \\
\hline H & 2.063742603 & 7.399733085 & 5.204224263 & $\mathrm{H}$ & -5.391516402 & 11.29709498 & 8.052476762 \\
\hline N & 0.104439012 & 7.357779924 & 8.604985693 & $\mathrm{H}$ & -6.302532241 & 9.889823337 & 3.655156964 \\
\hline C & 2.449469028 & 9.014475073 & 3.80663187 & $\mathrm{H}$ & -6.373117274 & 8.398638019 & 10.17965337 \\
\hline H & 2.201340226 & 8.457677927 & 2.878057621 & $\mathrm{H}$ & -3.000555657 & 11.68684356 & 8.562962028 \\
\hline $\mathrm{H}$ & 3.548955378 & 8.969779095 & 3.933753015 & C & -5.308246001 & 556407 & 9479 \\
\hline $\mathrm{H}$ & 2.169082191 & 10.07437494 & 3.631881925 & $\mathrm{H}$ & -5.726711221 & 6.867694494 & 10.80865031 \\
\hline C & 0.182222181 & 8.332916228 & 4.689670305 & C & -2.87991898 & 10.60134677 & 8.76202312 \\
\hline $\mathrm{H}$ & 0.010454433 & 7.651527072 & 1807 & $\mathrm{H}$ & -1.3 & 7331 & 0031 \\
\hline $\mathrm{H}$ & -0.231666697 & 9.319322943 & 4.399953187 & $\mathrm{H}$ & -2.015043083 & 10.47477409 & 8131 \\
\hline $\mathrm{H}$ & -0.416334606 & .961629755 & 5.54342496 & C & -5.661901455 & 7.577060417 & 806988 \\
\hline C & 1.111176935 & 2732 & & $P$ & -3.899251793 & & 7994 \\
\hline $\mathrm{H}$ & 1.157509358 & 11.13284068 & 5.298579624 & $\mathrm{H}$ & -4.642375519 & 32468 & 20143155 \\
\hline C & 1.78733549 & 12.13647549 & 7.112230465 & $\mathrm{H}$ & -2.085963018 & 5.208697528 & 6879 \\
\hline $\mathrm{H}$ & 2.824972066 & 12.32494172 & 6.773038301 & $\mathrm{H}$ & -5.073816976 & 9.684654404 & 7.344532953 \\
\hline $\mathrm{H}$ & 1.783020268 & 11.97691638 & 8.210144653 & $\mathrm{H}$ & -2.635390601 & 10.0957724 & 7.810339692 \\
\hline$\vdash$ & 1.208892494 & 13.06219305 & 6.906773249 & C & -6.025008817 & 6.905658312 & 8.645663591 \\
\hline $\mathrm{C}$ & -0.341238538 & 10.84602378 & 6.858712987 & $\mathrm{H}$ & -7.05696736 & 6.481699521 & 8.68416922 \\
\hline $\mathrm{H}$ & -0.886970062 & 11.78917338 & 6.64243076 & C & -4.234817122 & 4.666132664 & 10.34195668 \\
\hline $\mathrm{H}$ & -0.355694519 & 10.67949511 & 7.951050904 & $\mathrm{H}$ & -5.995418475 & 7.648887254 & 90432 \\
\hline $\mathrm{H}$ & -0.898158047 & 10.01704983 & 1878 & C & -1.8 & 78561 & 01049 \\
\hline C & 4.47348 & 9.10 & 238 & $\mathrm{H}$ & -6.2 & 51 & 5499 \\
\hline $\mathrm{H}$ & 3.808095974 & 9.956034682 & 10.43339332 & $\mathrm{H}$ & -4.153333237 & 3.62253903 & 10.73843267 \\
\hline $\mathrm{H}$ & 5.532099332 & 9.46001125 & 10.21022172 & $\mathrm{~N}$ & -2.944711073 & 5.163063204 & 9.915102119 \\
\hline C & 4.25587929 & 7.982294888 & 11.18344233 & $\operatorname{Re}$ & -2.905897442 & 6.506864541 & 8.385971311 \\
\hline $\mathrm{H}$ & 4.301083658 & 8.407290267 & 12.20493032 & $\mathrm{H}$ & -0.936462849 & 5.223731648 & 10.49811964 \\
\hline $\mathrm{H}$ & 5.058730401 & 7.219105427 & & $\mathrm{~N}$ & -5.072276135 & 5.823075407 & 8.259007617 \\
\hline C & 1.617414086 & & & $\mathrm{H}$ & -1.789225958 & & \\
\hline $\mathrm{H}$ & 1.491252298 & 8.712936305 & 12.21070421 & C & -5.211610044 & 4.63775334 & 9.16749523 \\
\hline C & 2.28640202 & 7.440712676 & 13.78685191 & $\mathrm{Cl}$ & -3.209838909 & 7.988008365 & 6.352259861 \\
\hline $\mathrm{H}$ & 2.314231427 & 6.375969184 & 14.09187513 & $\mathrm{H}$ & -4.985376688 & & 8.565661793 \\
\hline $\mathrm{H}$ & 1.707638189 & 7.985998596 & 14.56205429 & C & -5.402029955 & 5.445656017 & 6.847331556 \\
\hline $\mathrm{H}$ & 3.32272083 & 7.829772356 & 13.82649997 & $\mathrm{H}$ & -5.280066695 & 6.378909275 & 6.262765118 \\
\hline C & 4.98050932 & 7.606774311 & 8.287219422 & $\mathrm{H}$ & -6.468243776 & 5.119125499 & 6.789598413 \\
\hline C & 0.230850194 & 6.984691738 & 12.39038827 & $\mathrm{H}$ & -0.62915038 & 3.989007153 & 8.627321937 \\
\hline $\mathrm{H}$ & -0.255154589 & 7.116681873 & 11.40576177 & $\mathrm{P}$ & -2.688442072 & 4.72145403 & 6.744859548 \\
\hline $\mathrm{H}$ & -0.423450325 & 7.444598136 & 13.15876292 & $\mathrm{H}$ & -2.900554437 & 2.920907326 & 8.232159731 \\
\hline $\mathrm{H}$ & 0.280390512 & 5.900454483 & 12.61039621 & C & -4.483272159 & 4.37645829 & 6.267988528 \\
\hline C & 3.078373603 & 5.316250239 & 10.97715147 & $\mathrm{H}$ & -0.043554438 & 5.686126345 & 6.161020499 \\
\hline $\mathrm{H}$ & 3.720446357 & 5.263707748 & 10.0685065 & $\mathrm{H}$ & -4.759316108 & 3.356365716 & 6.603929167 \\
\hline C & 1.868214029 & 4.410111657 & 10.73190283 & C & -0.774619329 & 3.062724576 & 8.042088611 \\
\hline $\mathrm{H}$ & 1.219980326 & 4.813559275 & 9.931252337 & C & -2.166288179 & 3.025606451 & 7.401766939 \\
\hline $\mathrm{H}$ & 1.252374012 & 4.298509032 & 11.64661996 & $\mathrm{H}$ & -0.637471555 & 2.198702743 & 8.726122894 \\
\hline $\mathrm{H}$ & 2.193961643 & 3.393976565 & 10.42701015 & C & -0.330428195 & 5.061685407 & 5.296038917 \\
\hline C & 3.895671457 & 4.857562801 & 12.18769272 & $\mathrm{H}$ & -2.183713131 & 6.102068347 & 4.931974009 \\
\hline $\mathrm{H}$ & 4.307199708 & 3.84171724 & 12.00607327 & $\mathrm{H}$ & -4.598227026 & 4.37444811 & 5.167173396 \\
\hline $\mathrm{H}$ & 3.270108802 & 4.794901831 & 13.09932808 & C & -1.845129273 & 5.052967831 & 5.094521118 \\
\hline
\end{tabular}




\begin{tabular}{|c|c|c|c|c|c|c|c|}
\hline $\mathrm{H}$ & 4.750937867 & 5.524105844 & 12.4128699 & $\mathrm{H}$ & 0.066015444 & 4.04015637 & 5.461322542 \\
\hline $\mathrm{H}$ & 5.911700406 & 8.013389458 & 7.832149991 & $\mathrm{H}$ & 0.026803979 & 3.008755931 & 7.279492974 \\
\hline C & 4.244544973 & 6.66447584 & 7.333097405 & $\mathrm{H}$ & 0.1793434 & 5.466322779 & 4.398223556 \\
\hline $\mathrm{H}$ & 5.28702792 & 7.017586476 & 9.17149088 & $\mathrm{C}$ & -2.321959006 & 1.839835043 & 6.446091364 \\
\hline $\mathrm{H}$ & 4.271175911 & 7.05270837 & 6.280010821 & $\mathrm{H}$ & -2.201559306 & 0.882961832 & 6.998727856 \\
\hline $\mathrm{N}$ & 2.891256413 & 6.447194405 & 7.798125791 & $\mathrm{H}$ & -3.311443277 & 1.809733194 & 5.948133535 \\
\hline $\mathrm{H}$ & 4.818178887 & 5.706204799 & 7.300855619 & C & -2.247045999 & 4.209855955 & 3.881587536 \\
\hline C & 2.371294083 & 5.161883706 & 7.41454193 & $\mathrm{H}$ & -1.545287957 & 1.85428819 & 5.654815042 \\
\hline $\mathrm{H}$ & 2.381451587 & 5.013154522 & 6.304050393 & $\mathrm{H}$ & -3.338820995 & 4.201802289 & 3.695107661 \\
\hline $\mathrm{H}$ & 1.326240654 & 5.061604303 & 7.75090774 & $\mathrm{H}$ & -1.908021768 & 3.158517758 & 3.964634546 \\
\hline $\mathrm{H}$ & 2.959863587 & 4.309506542 & 7.84538227 & $\mathrm{H}$ & -1.772096745 & 4.632856847 & 2.970704813 \\
\hline \multicolumn{8}{|c|}{$\left[\left(\mu-N_{2}\right)\left\{\operatorname{ReCl}\left({ }^{M e} N P N P^{i P r}\right)\right\}_{2}\right]\left({ }^{B S} S=0\right)$} \\
\hline $\operatorname{Re}$ & 1.95758955 & 8.014366822 & 8.706146463 & $\mathrm{~N}$ & -1.01582931 & 7.024085083 & 8.542279218 \\
\hline $\mathrm{Cl}$ & 1.211555025 & 10.04839491 & 10.026602 & $\mathrm{H}$ & -4.09584305 & 8.835985801 & 13.62333486 \\
\hline$P$ & 1.984290132 & 9.295122773 & 6.648482186 & $\mathrm{H}$ & -4.927990522 & 9.747688729 & 12.33762826 \\
\hline $\mathrm{N}$ & 4.116626726 & 8.729872818 & 8.778131542 & C & -4.49418585 & 8.756747509 & 12.58950767 \\
\hline $\mathrm{C}$ & 4.324281375 & 9.947295892 & 7.938245922 & $\mathrm{H}$ & -5.321051857 & 8.019812119 & 12.61274288 \\
\hline $\mathrm{H}$ & 5.411996851 & 10.19982323 & 7.933325809 & $\mathrm{H}$ & -1.765780611 & 9.263001603 & 12.80297127 \\
\hline $\mathrm{H}$ & 3.787532382 & 10.76954988 & 8.449523645 & $\mathrm{H}$ & -2.492512844 & 10.38564073 & 11.62921838 \\
\hline$P$ & 2.594182583 & 7.145758118 & 10.8931172 & $\mathrm{H}$ & -4.466564989 & 10.52037427 & 10.25519965 \\
\hline C & 3.794192345 & 9.784989307 & 6.523169578 & C & -2.178084335 & 9.332608637 & 11.77481035 \\
\hline $\mathrm{H}$ & 4.349202314 & 8.999402175 & 5.973950738 & $\mathrm{C}$ & -3.365212098 & 8.375300806 & 11.62465968 \\
\hline $\mathrm{H}$ & 3.923797132 & 10.72697792 & 5.953528569 & $\mathrm{C}$ & -4.182502241 & 10.01141084 & 9.305507558 \\
\hline C & 1.678153109 & 8.421069834 & 4.997278676 & $\mathrm{H}$ & -3.022981725 & 7.343992895 & 11.85965394 \\
\hline $\mathrm{H}$ & 2.060575493 & 7.397715975 & 5.207408232 & $\mathrm{H}$ & -5.398578415 & 11.29194971 & 8.049889449 \\
\hline $\mathrm{N}$ & 0.106041793 & 7.353787694 & 8.607519541 & $\mathrm{H}$ & -6.305179374 & 9.882901422 & 8.655057928 \\
\hline C & 2.445129259 & 9.011917306 & 3.809104094 & $\mathrm{H}$ & -6.369778367 & 8.393921194 & 10.18391785 \\
\hline $\mathrm{H}$ & 2.195562968 & 8.454732178 & 2.881177687 & $\mathrm{H}$ & -3.007607034 & 11.68795788 & 8.55697582 \\
\hline $\mathrm{H}$ & 3.544767106 & 8.96697249 & 3.934779965 & $\mathrm{C}$ & -5.312356725 & 10.21074169 & 8.287249385 \\
\hline $\mathrm{H}$ & 2.164718035 & 10.07177117 & 3.634182383 & $\mathrm{H}$ & -5.718787547 & 6.864036669 & 10.81090262 \\
\hline C & 0.178655348 & 8.332003045 & 4.695454222 & $\mathrm{C}$ & -2.884037371 & 10.60266937 & 8.755491119 \\
\hline $\mathrm{H}$ & 0.005340345 & 7.650266798 & 3.836925598 & $\mathrm{H}$ & -1.349636527 & 9.128755655 & 11.06940068 \\
\hline $\mathrm{H}$ & -0.234660521 & 9.318583063 & 4.405570736 & $\mathrm{H}$ & -2.017750727 & 10.47793113 & 9.429534489 \\
\hline $\mathrm{H}$ & -0.419296903 & 7.96182053 & 5.550132662 & $\mathrm{C}$ & -5.657827647 & 7.573537594 & 9.962861088 \\
\hline C & 1.11566712 & 10.95847076 & 6.401192721 & $\mathrm{P}$ & -3.896450616 & 8.210150104 & 9.812914406 \\
\hline $\mathrm{H}$ & 1.162617725 & 11.13262562 & 5.300893626 & $\mathrm{H}$ & -4.64001358 & 5.253733076 & 11.20019768 \\
\hline C & 1.79522938 & 12.13558563 & 7.113654457 & $\mathrm{H}$ & -2.091559462 & 5.221626354 & 11.87185563 \\
\hline $\mathrm{H}$ & 2.833271125 & 12.32094644 & 6.773877661 & $\mathrm{H}$ & -5.078429514 & 9.679774646 & 7.342290038 \\
\hline $\mathrm{H}$ & 1.79081919 & 11.97679326 & 8.211666147 & $\mathrm{H}$ & -2.640099673 & 10.09780223 & 7.803245454 \\
\hline $\mathrm{H}$ & 1.219264462 & 13.06272889 & 6.90771138 & $\mathrm{C}$ & -6.024237173 & 6.901508534 & 8.648939565 \\
\hline C & -0.337287012 & 10.85048329 & 6.860684514 & $\mathrm{H}$ & -7.055534168 & 6.47625164 & 8.690799486 \\
\hline $\mathrm{H}$ & -0.880541827 & 11.79472355 & 6.642924173 & $\mathrm{C}$ & -4.231157825 & 4.660373601 & 10.33896928 \\
\hline $\mathrm{H}$ & -0.352924161 & 10.68500698 & 7.95319136 & $\mathrm{H}$ & -5.998493227 & 7.644832135 & 7.828450279 \\
\hline $\mathrm{H}$ & -0.895731654 & 10.02238689 & 6.390081914 & $\mathrm{C}$ & -1.899182653 & 4.819330788 & 10.84317459 \\
\hline C & 4.474815112 & 9.109552442 & 10.18214993 & $\mathrm{H}$ & -6.262387603 & 4.533942381 & 9.50810148 \\
\hline $\mathrm{H}$ & 3.808654014 & 9.95888265 & 10.43364095 & $\mathrm{H}$ & -4.146872647 & 3.615895859 & 10.73278909 \\
\hline $\mathrm{H}$ & 5.533178029 & 9.463483507 & 10.21210193 & $\mathrm{~N}$ & -2.942462209 & 5.16275289 & 9.914345317 \\
\hline C & 4.257815123 & 7.987918118 & 11.19015612 & $\operatorname{Re}$ & -2.90505169 & 6.503831202 & 8.3829174 \\
\hline $\mathrm{H}$ & 4.299883929 & 8.416533755 & 12.21024527 & $\mathrm{H}$ & -0.936753563 & 5.235461077 & 10.50473598 \\
\hline $\mathrm{H}$ & 5.061958391 & 7.225770022 & 11.14364573 & $\mathrm{~N}$ & -5.071468238 & 5.82023918 & 8.258690318 \\
\hline
\end{tabular}




\begin{tabular}{|c|c|c|c|c|c|c|c|}
\hline C & 1.620578159 & 7.63064243 & 12.42797524 & $\mathrm{H}$ & -1.784661382 & 3.711265179 & 10.9616565 \\
\hline $\mathrm{H}$ & 1.497338502 & 8.717739014 & 12.21764318 & $\mathrm{C}$ & -5.208231188 & 4.63300449 & 9.164939279 \\
\hline C & 2.288603267 & 7.44105912 & 13.79212801 & $\mathrm{Cl}$ & -3.21118407 & 7.987590489 & 6.35111636 \\
\hline $\mathrm{H}$ & 2.313904 & 6.375730857 & 14.09529127 & $\mathrm{H}$ & -4.980775551 & 3.736434412 & 8.56097292 \\
\hline $\mathrm{H}$ & 1.710806626 & 7.986310364 & 14.5680675 & $\mathrm{C}$ & -5.404010134 & 5.444695497 & 6.847169576 \\
\hline $\mathrm{H}$ & 3.325842829 & 7.827596929 & 13.83281265 & $\mathrm{H}$ & -5.282553162 & 6.378554284 & 6.263436499 \\
\hline C & 4.981397623 & 7.601468575 & 8.297416875 & $\mathrm{H}$ & -6.47048058 & 5.118706067 & 6.790943828 \\
\hline C & 0.232536609 & 6.992494682 & 12.3934286 & $\mathrm{H}$ & -0.636380009 & 3.990154377 & 8.629905721 \\
\hline $\mathrm{H}$ & -0.25165614 & 7.128051819 & 11.4084295 & $\mathrm{P}$ & -2.691548962 & 4.720331302 & 6.74184038 \\
\hline $\mathrm{H}$ & -0.421395397 & 7.45229458 & 13.16215087 & $\mathrm{H}$ & -2.903510812 & 2.918276631 & 8.227956775 \\
\hline $\mathrm{H}$ & 0.279409509 & 5.907638571 & 12.61092909 & C & -4.48656516 & 4.375766755 & 6.265305299 \\
\hline C & 3.078659853 & 5.319950683 & 10.97923054 & $\mathrm{H}$ & -0.048270396 & 5.6844425 & 6.153189872 \\
\hline $\mathrm{H}$ & 3.722666685 & 5.268232941 & 10.07192496 & $\mathrm{H}$ & -4.76294242 & 3.355084189 & 6.599260519 \\
\hline C & 1.867084697 & 4.417241015 & 10.72794378 & C & -0.777736965 & 3.064697797 & 8.042192266 \\
\hline $\mathrm{H}$ & 1.224047589 & 4.823334663 & 9.924347856 & $\mathrm{C}$ & -2.168003936 & 3.024900928 & 7.398936572 \\
\hline $\mathrm{H}$ & 1.246663317 & 4.307004805 & 11.6397626 & $\mathrm{H}$ & -0.63936326 & 2.199333814 & 8.724315643 \\
\hline $\mathrm{H}$ & 2.191528411 & 3.400322801 & 10.42423475 & C & -0.335671457 & 5.059306806 & 5.289045255 \\
\hline C & 3.891856077 & 4.856890342 & 12.19073052 & $\mathrm{H}$ & -2.189190724 & 6.099643467 & 4.927197145 \\
\hline $\mathrm{H}$ & 4.302372275 & 3.840933423 & 12.00752903 & $\mathrm{H}$ & -4.601994767 & 4.376093464 & 5.164564115 \\
\hline $\mathrm{H}$ & 3.263696579 & 4.792703668 & 13.10047259 & C & -1.850492536 & 5.050566877 & 5.089718823 \\
\hline $\mathrm{H}$ & 4.747504117 & 5.521569267 & 12.42006034 & $\mathrm{H}$ & 0.061108195 & 4.037941231 & 5.454868626 \\
\hline $\mathrm{H}$ & 5.91461708 & 8.00470319 & 7.84360414 & $\mathrm{H}$ & 0.025321971 & 3.015072325 & 7.28100133 \\
\hline C & 4.247376556 & 6.657255612 & 7.344188198 & $\mathrm{H}$ & 0.173251719 & 5.463234932 & 4.390379001 \\
\hline $\mathrm{H}$ & 5.284498413 & 7.01487824 & 9.184494394 & C & -2.319851482 & 1.839423495 & 6.442387529 \\
\hline $\mathrm{H}$ & 4.280548066 & 7.040169527 & 6.289247646 & $\mathrm{H}$ & -2.198371414 & 0.882383331 & 6.99450584 \\
\hline $\mathrm{N}$ & 2.88988381 & 6.446434948 & 7.800712088 & $\mathrm{H}$ & -3.308590849 & 1.807859713 & 5.943001023 \\
\hline $\mathrm{H}$ & 4.817677754 & 5.696695315 & 7.31911016 & $\mathrm{C}$ & -2.253891851 & 4.206963469 & 3.877588285 \\
\hline C & 2.364117367 & 5.16815686 & 7.401201775 & $\mathrm{H}$ & -1.542077365 & 1.856049764 & 5.652229073 \\
\hline $\mathrm{H}$ & 2.37804514 & 5.030118921 & 6.289258709 & $\mathrm{H}$ & -3.345857489 & 4.198862652 & 3.692263557 \\
\hline $\mathrm{H}$ & 1.317679206 & 5.069119133 & 7.733498394 & $\mathrm{H}$ & -1.914788446 & 3.155669039 & 3.960698657 \\
\hline $\mathrm{H}$ & 2.947639908 & 4.309524849 & 7.82606291 & $\mathrm{H}$ & -1.779942395 & 4.629550808 & 2.965980094 \\
\hline \multicolumn{8}{|c|}{$\left[\left(\mu^{\prime}-{ }^{\prime} \mathrm{N}_{2}\right)\left\{\operatorname{ReCl}\left({ }^{\mathrm{Me}} \mathrm{NPNP}^{i \mathrm{Pr}}\right)\right\}_{2}\right](\mathrm{TS}, \mathrm{S}=0)$} \\
\hline $\operatorname{Re}$ & 1.860305104 & 7.719506314 & 8.758951738 & $\mathrm{~N}$ & -1.328942728 & 7.509197349 & 8.314507107 \\
\hline $\mathrm{Cl}$ & 2.159845367 & 9.52034558 & 10.46927057 & $\mathrm{H}$ & -4.732526864 & 10.22301347 & 12.47143724 \\
\hline$P$ & 1.797529897 & 9.590221599 & 7.231327481 & $\mathrm{H}$ & -4.868359046 & 11.06995777 & 10.90925739 \\
\hline $\mathrm{N}$ & 4.346330688 & 8.260033909 & 8.510723 & C & -4.937931344 & 10.07258651 & 11.39067065 \\
\hline C & 4.460228099 & 9.699632973 & 8.18819522 & $\mathrm{H}$ & -5.985431605 & 9.719685177 & 11.29837698 \\
\hline $\mathrm{H}$ & 5.529001385 & 9.958932523 & 7.985836378 & $\mathrm{H}$ & -2.21219135 & 9.656605564 & 12.02198229 \\
\hline $\mathrm{H}$ & 4.134552758 & 10.25644329 & 9.087382493 & $\mathrm{H}$ & -2.353515119 & 10.59722001 & 10.5131279 \\
\hline $\mathrm{P}$ & 2.806243954 & 6.302186625 & 10.59579238 & $\mathrm{H}$ & -5.271853842 & 10.71292409 & 8.86347495 \\
\hline C & 3.594278333 & 10.11631603 & 6.998897834 & $\mathrm{C}$ & -2.482353539 & 9.586398708 & 10.94812405 \\
\hline $\mathrm{H}$ & 3.969422961 & 9.677347359 & 6.05598921 & $\mathrm{C}$ & -3.923136912 & 9.089922942 & 10.79972004 \\
\hline $\mathrm{H}$ & 3.657769061 & 11.2136506 & 6.871343235 & $\mathrm{C}$ & -4.770320097 & 10.08203918 & 8.09574471 \\
\hline C & 1.150524701 & 9.30121695 & 5.481859456 & $\mathrm{H}$ & -4.003236431 & 8.121427478 & 11.34345735 \\
\hline $\mathrm{H}$ & 1.530961798 & 8.282149491 & 5.254341179 & $\mathrm{H}$ & -5.984059082 & 10.83925305 & 6.471394817 \\
\hline$N$ & 0.182063093 & 7.154092666 & 8.916953677 & $\mathrm{H}$ & -6.7245698 & 9.433965957 & 7.270528625 \\
\hline C & 1.669489061 & 10.28763957 & 4.432935568 & $\mathrm{H}$ & -6.78149103 & 8.51417408 & 9.187801905 \\
\hline $\mathrm{H}$ & 1.235025497 & 10.0461113 & 3.439625317 & $\mathrm{H}$ & -3.790763259 & 11.71635932 & 7.04178562 \\
\hline $\mathrm{H}$ & 2.772432359 & 10.26513198 & 4.322545935 & C & -5.756634137 & 9.859176982 & 6.942031208 \\
\hline $\mathrm{H}$ & 1.371208119 & 11.33102507 & 4.668677854 & $\mathrm{H}$ & -6.059347069 & 7.282512451 & 10.2578342 \\
\hline
\end{tabular}




\begin{tabular}{|c|c|c|c|c|c|c|}
\hline-0.380116374 & 9.229264159 & 5.496903513 & C & -3.516160847 & 10.79840361 & 7.602757079 \\
\hline-0.756916755 & 8.817442643 & 4.538990365 & $\mathrm{H}$ & -1.754586852 & 8.911388135 & 10.45723219 \\
\hline-0.838320339 & 10.23152914 & 5.621013102 & $\mathrm{H}$ & -2.859694242 & 11.09680888 & 8.437191591 \\
\hline-0.767088567 & 8.58137448 & 6.30692913 & C & -5.996526092 & 7.732861007 & 9.24854063 \\
\hline 0.923155573 & 11.20849939 & 7.699402784 & $\mathrm{P}$ & -4.302846093 & 8.503956906 & 9.045582754 \\
\hline 0.545531347 & 11.59033985 & 6.725259572 & $\mathrm{H}$ & -4.674878545 & 6.130577135 & 10.97309527 \\
\hline 1.801600278 & 12.29614799 & 8.326060293 & $\mathrm{H}$ & -2.247245632 & 6.660777279 & 186352 \\
\hline 2.590474861 & 12.67334784 & 7.645981552 & $\mathrm{H}$ & -5.31414437 & 9.200994888 & 6.167449648 \\
\hline 2.272930465 & 11.92997985 & 9.258160055 & $\mathrm{H}$ & -2.937634858 & 10.13694183 & 6.927312806 \\
\hline 1.163865898 & 13.16577412 & 8.591919257 & C & -6.217630539 & 6.695348236 & 8.154000229 \\
\hline-0.268733534 & 10.88628207 & 8.598472444 & $\mathrm{H}$ & -7.216466259 & 6.206311613 & 8.277107636 \\
\hline-0.923209088 & 11.7785212 & 8.683857622 & C & -4.240836061 & 5.247983011 & 10.41714048 \\
\hline 0.082582032 & 10.60578672 & 9.60786648 & $\mathrm{H}$ & -6.212844544 & 7.194912291 & 7.166211319 \\
\hline-0.864840618 & 10.03829187 & 8.216402507 & C & -1.97893755 & 5.772732543 & 10.91263918 \\
\hline 5.007431844 & 7.955656977 & 9.799405453 & $\mathrm{H}$ & -6.238644753 & 4.686348283 & 9.684832675 \\
\hline 4.67756847 & 8.739222402 & 10.51050679 & $\mathrm{H}$ & -4.200078449 & 4.434384753 & 11.18782515 \\
\hline 6.117576052 & 8.037516197 & 9.696427505 & $\mathrm{~N}$ & -2.93728605 & 5.489326546 & 9.875086919 \\
\hline 4.650483182 & 6.576504298 & 10.36029011 & $\operatorname{Re}$ & -2.894075803 & 6.748608129 & 8.133228142 \\
\hline 5.191984015 & 6.419071405 & 11.31555379 & $\mathrm{H}$ & -0.984181711 & 5.998023128 & 10.47780616 \\
\hline 4.997266648 & 5.774631026 & 9.677019801 & $\mathrm{~N}$ & -5.161201256 & 5.662714928 & 8.110571822 \\
\hline 2.493654681 & 6.986260911 & 12.32746161 & $\mathrm{H}$ & -1.87522409 & 4.906252288 & 15697 \\
\hline 2.91184721 & 8.007268715 & 12.20229006 & C & -5.192010675 & 4.78413151 & 9.314126586 \\
\hline 3.218977331 & 6.307826669 & 13.49301397 & $\mathrm{Cl}$ & -3.518369073 & 7.463280308 & 505305 \\
\hline 2.805449617 & 5.306039345 & 13.72494167 & $\mathrm{H}$ & -4.865104391 & 3.774518238 & 9.002435293 \\
\hline 3.095642094 & 6.924830185 & 14.40883008 & C & -5.293370228 & 4.897125194 & 6.846610979 \\
\hline 4.309557131 & 6.197032359 & 13.32310083 & $\mathrm{H}$ & -5.289263764 & 5.648756866 & 6.033490903 \\
\hline 4.834306695 & 7.420261929 & 7.391264375 & $\mathrm{H}$ & -6.273061086 & 4.359459626 & 6.825792942 \\
\hline 0.99397044 & 7.174144548 & 12.5991214 & $\mathrm{H}$ & -0.07512349 & 4.619638395 & 8.571730704 \\
\hline 0.47893724 & 7.602075732 & 1.71794279 & $\mathrm{P}$ & -2.452347151 & 4.590771473 & 166534 \\
\hline 0.856248986 & 7.878287507 & 13.44631495 & $\mathrm{H}$ & -2.270934969 & 3.510608418 & 9.023331528 \\
\hline 0.492122447 & 6.224942626 & 12.86901557 & C & -4.156099813 & 3.905149291 & 6.610036136 \\
\hline 2.692043353 & 4.410157982 & 10.75950213 & $\mathrm{H}$ & -0.419509088 & 6.408814307 & 3683 \\
\hline 2.603134139 & 4.091224094 & 9.704236366 & $\mathrm{H}$ & -4.290133208 & 2.989236807 & 7.215564914 \\
\hline 1.402934171 & 3.995908023 & 11.47656539 & C & -0.244007566 & 3.549321357 & 826821 \\
\hline 0.512704865 & 4.508315904 & 11.06827091 & C & -1.720530211 & 3.250331347 & 8.084955073 \\
\hline 1.451046539 & 4.20749165 & 12.56220377 & $\mathrm{H}$ & 0.134517683 & 2.942416672 & 9.176823041 \\
\hline 1.245457723 & 2.90260257 & 11.36214517 & C & -0.301747045 & 5.497767667 & 5.431780483 \\
\hline 3.920519612 & 3.704179018 & 11.34915759 & $\mathrm{H}$ & -2.346216508 & 5.327749516 & 4.802819713 \\
\hline 3.752504946 & 2.605574002 & 11.33786797 & $\mathrm{H}$ & -4.180130493 & 3.576802099 & 5.551782649 \\
\hline 4.114769321 & 3.994469857 & 12.39902265 & $\mathrm{C}$ & -1.605380917 & 4.696408968 & 5.342675468 \\
\hline 4.842657051 & 3.894381928 & 10.7663332 & $\mathrm{H}$ & 0.51915992 & 4.908411939 & 5.87884587 \\
\hline 5.646407969 & 7.94507795 & 6.834028616 & $\mathrm{H}$ & 0.370886152 & 3.292731383 & 7.443263917 \\
\hline 3.662740061 & 7.007165492 & 6.475681333 & $\mathrm{H}$ & 0.022257593 & 5.805123439 & 4.416076514 \\
\hline 5.28506238 & 6.501875556 & 7.813232699 & C & -1.949444076 & 1.787298391 & 7.702583122 \\
\hline 3.330893755 & 7.873274319 & 5.872284646 & $\mathrm{H}$ & -1.587768493 & 1.131802586 & 8.523801625 \\
\hline 2.58139076 & 6.47055048 & 7.261450403 & $\mathrm{H}$ & -3.015873548 & 1.534104003 & 7.539569673 \\
\hline 4.039582002 & 6.256451805 & 5.739641981 & C & -1.421241174 & 3.362388737 & 4.616296852 \\
\hline 2.769705555 & 5.05220157 & 7.45247173 & $\mathrm{H}$ & -1.393716942 & 1.499156854 & 6.787986348 \\
\hline 2.823723921 & 4.524639023 & 6.468492847 & $\mathrm{H}$ & -2.353683966 & 2.76417965 & 4.55964979 \\
\hline 1.91531488 & 4.627888015 & 8.000367138 & $\mathrm{H}$ & -0.645000213 & 2.740393575 & 5.107012947 \\
\hline
\end{tabular}




\begin{tabular}{|c|c|c|c|c|c|c|c|}
\hline $\mathrm{H}$ & 3.704916221 & 4.757591095 & 8.006888398 & $\mathrm{H}$ & -1.082666128 & 3.543657566 & 3.574272523 \\
\hline \multicolumn{8}{|c|}{$\left[\operatorname{Re}(\mathrm{N}) \mathrm{Cl}\left({ }^{\mathrm{Me}} \mathrm{NPNP}^{i \mathrm{Pr}}\right)\right]$} \\
\hline $\operatorname{Re}$ & 1.815958751 & 8.109020189 & 8.54029956 & C & 4.095846828 & 7.179524749 & 10.91552263 \\
\hline $\mathrm{Cl}$ & 1.680774185 & 10.06803811 & 10.10184221 & $\mathrm{H}$ & 4.311625671 & 7.175201374 & 12.00326263 \\
\hline$P$ & 2.228929629 & 9.695142532 & 6.764026676 & $\mathrm{H}$ & 4.624169239 & 6.306506503 & 10.48407014 \\
\hline $\mathrm{N}$ & 4.339111931 & 8.585891088 & 8.84542063 & $\mathrm{C}$ & 1.415878022 & 7.473602023 & 12.16496019 \\
\hline C & 4.560801606 & 9.957741878 & 8.347348748 & $\mathrm{H}$ & 1.805114987 & 8.514972314 & 12.20482125 \\
\hline $\mathrm{H}$ & 5.650004446 & 10.21191235 & 8.38068669 & C & 1.770604475 & 6.739594447 & 13.46068686 \\
\hline $\mathrm{H}$ & 4.026747028 & 10.63937631 & 9.038829463 & $\mathrm{H}$ & 1.303005205 & 5.736419623 & 13.5016835 \\
\hline $\mathrm{P}$ & 2.277286139 & 6.863698507 & 10.62026922 & $\mathrm{H}$ & 1.388179414 & 7.311413034 & 14.33236041 \\
\hline C & 4.035100563 & 10.16640065 & 6.923513708 & $\mathrm{H}$ & 2.862700347 & 6.611641576 & 13.60456674 \\
\hline $\mathrm{H}$ & 4.593348624 & 9.540198648 & 6.199230666 & $\mathrm{C}$ & 5.070508198 & 7.564215335 & 8.056778173 \\
\hline $\mathrm{H}$ & 4.194082432 & 11.21995957 & 6.619898737 & C & -0.098645821 & 7.573886833 & 11.94193724 \\
\hline C & 2.086923922 & 9.11563423 & 4.97653931 & $\mathrm{H}$ & -0.328490182 & 8.10655351 & 10.99886942 \\
\hline $\mathrm{H}$ & 2.725873479 & 8.206175126 & 4.986864646 & $\mathrm{H}$ & -0.562904313 & 8.139439638 & 12.77628929 \\
\hline$N$ & 0.244253007 & 7.703147222 & 8.119130465 & $\mathrm{H}$ & -0.579896455 & 6.576052788 & 11.90794595 \\
\hline $\mathrm{C}$ & 2.644607194 & 10.09290046 & 3.937203557 & C & 2.172355073 & 5.005820198 & 10.44907674 \\
\hline $\mathrm{H}$ & 2.617891061 & 9.627556023 & 2.92911461 & $\mathrm{H}$ & 2.706328221 & 4.928939589 & 9.47234649 \\
\hline $\mathrm{H}$ & 3.695526478 & 10.37994675 & 4.13912629 & C & 0.721257934 & 4.554979573 & 10.25027985 \\
\hline $\mathrm{H}$ & 2.042499747 & 11.02236634 & 3.878344485 & $\mathrm{H}$ & 0.184733176 & 5.207464003 & 9.532659569 \\
\hline C & 0.651958147 & 8.681870336 & 4.662666344 & $\mathrm{H}$ & 0.158251232 & 4.557546494 & 11.20564596 \\
\hline $\mathrm{H}$ & 0.62173598 & 8.142437466 & 3.693185887 & $\mathrm{H}$ & 0.698641541 & 3.517742089 & 9.856170277 \\
\hline $\mathrm{H}$ & -0.024968113 & 9.556243823 & 4.573314106 & $\mathrm{C}$ & 2.909087119 & 4.165555243 & 11.49672628 \\
\hline $\mathrm{H}$ & 0.250475548 & 8.011220222 & 5.448949224 & $\mathrm{H}$ & 2.968760442 & 3.111659236 & 11.15102231 \\
\hline C & 1.233655196 & 11.30754145 & 6.748365496 & $\mathrm{H}$ & 2.386030032 & 4.159330551 & 12.47271413 \\
\hline $\mathrm{H}$ & 1.202063423 & 11.59724132 & 5.674725537 & $\mathrm{H}$ & 3.94787025 & 4.509199467 & 11.67252851 \\
\hline C & 1.877983697 & 12.45518408 & 7.535035542 & $\mathrm{H}$ & 5.935795571 & 8.030160558 & 7.531667953 \\
\hline $\mathrm{H}$ & 2.881638434 & 12.72811056 & 7.153099518 & $\mathrm{C}$ & 4.160938354 & 6.820428973 & 7.07374477 \\
\hline $\mathrm{H}$ & 1.959499966 & 12.19458445 & 8.608188245 & $\mathrm{H}$ & 5.497477856 & 6.823409639 & 8.759462098 \\
\hline $\mathrm{H}$ & 1.241300735 & 13.36103755 & 7.448806221 & $\mathrm{H}$ & 4.03318269 & 7.427952032 & 6.132060297 \\
\hline C & -0.199482547 & 11.03881417 & 7.23174162 & $\mathrm{~N}$ & 2.923096264 & 6.464179193 & 7.709605732 \\
\hline $\mathrm{H}$ & -0.823829612 & 11.94287462 & 7.070250641 & $\mathrm{H}$ & 4.703402498 & 5.90021678 & 6.740170675 \\
\hline $\mathrm{H}$ & -0.197574477 & 10.79758513 & 8.312656713 & $\mathrm{C}$ & 2.164751416 & 5.538969784 & 6.895250847 \\
\hline $\mathrm{H}$ & -0.676183619 & 10.18979246 & 6.706035262 & $\mathrm{H}$ & 1.80713052 & 5.974699435 & 5.921761572 \\
\hline C & 4.60909436 & 8.485820737 & 10.2961182 & $\mathrm{H}$ & 1.261137594 & 5.191212371 & 7.432447214 \\
\hline $\mathrm{H}$ & 4.097961649 & 9.345161407 & 10.77368337 & $\mathrm{H}$ & 2.778812832 & 4.641681697 & 6.634863684 \\
\hline $\mathrm{H}$ & 5.70470478 & 8.58160358 & 10.50076901 & & & & \\
\hline \multicolumn{8}{|c|}{$\left[\mathrm{Re}(\mathrm{NMe}) \mathrm{Cl}\left({ }^{\mathrm{Me}} \mathrm{NPNP}^{i \mathrm{Pr}}\right)\right]^{+}$} \\
\hline $\operatorname{Re}$ & 1.803363692 & 8.113519966 & 8.512002239 & $\mathrm{H}$ & 4.623278877 & 6.287122418 & 10.43932328 \\
\hline $\mathrm{Cl}$ & 1.653391706 & 10.0030872 & 10.10470691 & $\mathrm{C}$ & 1.407779081 & 7.430952912 & 12.14951197 \\
\hline $\mathrm{P}$ & 2.251733113 & 9.735207772 & 6.720530484 & $\mathrm{H}$ & 1.798394754 & 8.470828362 & 12.19055287 \\
\hline N & 4.251960064 & 8.586486086 & 8.810649779 & $\mathrm{C}$ & 1.775590153 & 6.698232503 & 13.44382255 \\
\hline C & 4.519912791 & 9.97380039 & 8.345054048 & $\mathrm{H}$ & 1.303699713 & 5.698642288 & 13.50097754 \\
\hline $\mathrm{H}$ & 5.609390529 & 10.19187588 & 8.431051776 & $\mathrm{H}$ & 1.403010501 & 7.281773842 & 14.31058624 \\
\hline $\mathrm{H}$ & 3.977248999 & 10.65331782 & 9.030689379 & $\mathrm{H}$ & 2.867867203 & 6.570880248 & 13.58132467 \\
\hline $\mathrm{P}$ & 2.271476951 & 6.810357792 & 10.61007145 & $\mathrm{C}$ & 5.012857112 & 7.580701142 & 8.00067077 \\
\hline C & 4.049321836 & 10.19868168 & 6.910955326 & $\mathrm{C}$ & -0.109540365 & 7.528558521 & 11.94436904 \\
\hline $\mathrm{H}$ & 4.625479624 & 9.585478287 & 6.190067071 & $\mathrm{H}$ & -0.356732676 & 8.104312921 & 11.03175372 \\
\hline $\mathrm{H}$ & 4.204613001 & 11.25643616 & 6.623895345 & $\mathrm{H}$ & -0.563516448 & 8.060807909 & 12.80466556 \\
\hline
\end{tabular}




\begin{tabular}{|c|c|c|c|c|c|c|c|}
\hline C & 2.119930764 & 9.171476945 & 4.930488091 & $\mathrm{H}$ & -0.589429284 & 6.531890754 & 11.88485208 \\
\hline $\mathrm{H}$ & 2.810113006 & 8.300421126 & 4.909781352 & C & 2.18345665 & 4.948041256 & 10.46592579 \\
\hline $\mathrm{N}$ & 0.136918598 & 7.875077476 & 8.137893763 & $\mathrm{H}$ & 2.68891753 & 4.820556286 & 9.483930431 \\
\hline & 2.61493765 & 10.20711047 & 3.914229545 & C & 0.730391462 & 4.472419695 & 10.34449457 \\
\hline $\mathrm{H}$ & 2.654663249 & 9.745683253 & 2.906324281 & $\mathrm{H}$ & 0.147672412 & 5.086430911 & 9.628588214 \\
\hline $\mathrm{H}$ & 3.631441938 & 10.5831696 & 4.141771445 & $\mathrm{H}$ & 0.208970994 & 4.502601996 & 11.32139713 \\
\hline $\mathrm{H}$ & 1.934372435 & 11.07899596 & 3.840152038 & $\mathrm{H}$ & 0.705432486 & 3.422186179 & 9.990052158 \\
\hline C & 0.712672178 & 8.659480502 & 4.608256027 & C & 2.966383048 & 4.14875576 & 11.51561671 \\
\hline $\mathrm{H}$ & 0.699709963 & 8.203028039 & 3.597875345 & $\mathrm{H}$ & 3.004120229 & 3.084181653 & 11.20505442 \\
\hline $\mathrm{H}$ & -0.033896908 & 9.479695262 & 4.604273971 & $\mathrm{H}$ & 2.487291272 & 4.182500988 & 12.51173398 \\
\hline $\mathrm{H}$ & 0.387479023 & 7.886587625 & 5.333096466 & $\mathrm{H}$ & 4.013864023 & 4.489787687 & 11.62848231 \\
\hline C & 1.26212634 & 11.3440733 & 6.765844498 & $\mathrm{H}$ & 5.872860109 & 8.074210293 & 7.50020348 \\
\hline $\mathrm{H}$ & 1.268765833 & 11.66379647 & 5.701250806 & C & 4.109637001 & 6.851947271 & 7.003738616 \\
\hline C & 1.901002982 & 12.45757045 & 7.603780565 & $\mathrm{H}$ & 5.438325045 & 6.832860621 & 8.694112347 \\
\hline $\mathrm{H}$ & 2.914390466 & 12.73364332 & 7.254263634 & $\mathrm{H}$ & 3.988094142 & 7.452507782 & 6.065085136 \\
\hline $\mathrm{H}$ & 1.949984509 & 12.17595782 & 8.672575022 & $\mathrm{~N}$ & 2.869513256 & 6.532954499 & 7.668437893 \\
\hline $\mathrm{H}$ & 1.276354156 & 13.37052107 & 7.520421687 & $\mathrm{H}$ & 4.622448294 & 5.915361454 & 6.684375967 \\
\hline C & -0.19137082 & 11.09041643 & 7.188445587 & C & 2.135650587 & 5.443634345 & 7.055295937 \\
\hline $\mathrm{H}$ & -0.77671184 & 12.02477422 & 7.070945151 & $\mathrm{H}$ & 1.697735138 & 5.713855222 & 6.058860266 \\
\hline $\mathrm{H}$ & -0.238854632 & 10.78820356 & 8.252869186 & $\mathrm{H}$ & 1.291672763 & 5.123900601 & 7.698066997 \\
\hline $\mathrm{H}$ & -0.683600864 & 10.31115824 & 6.575395493 & $\mathrm{H}$ & 2.793384416 & 4.559665805 & 6.88970945 \\
\hline C & 4.567559294 & 8.467574593 & 10.26497705 & C & -1.247383999 & 7.710895631 & 7.896925158 \\
\hline $\mathrm{H}$ & 4.071893246 & 9.320738976 & 10.76675832 & $\mathrm{H}$ & -1.480417457 & 7.905679066 & 6.828495041 \\
\hline $\mathrm{H}$ & 5.666634473 & 8.573327608 & 10.41571336 & $\mathrm{H}$ & -1.83932637 & 8.412131859 & 8.523378241 \\
\hline C & 4.087464576 & 7.152119622 & 10.87726849 & $\mathrm{H}$ & -1.554773725 & 6.667918427 & 8.130268033 \\
\hline $\mathrm{H}$ & 4.311160154 & 7.145289906 & 11.96283647 & & & & \\
\hline \multicolumn{8}{|c|}{ MeOTf } \\
\hline c & 0.350555286 & -0.047655633 & -0.457374558 & $\mathrm{~F}$ & 1.251373231 & -0.128531451 & 0.521592685 \\
\hline S & -1.352872201 & 0.35798211 & 0.263301867 & C & -2.203043266 & -2.134404356 & 0.597781814 \\
\hline 0 & -1.175517672 & 1.469530085 & 1.192692684 & 0 & -2.29672535 & 0.361755492 & -0.862689695 \\
\hline O & -1.566083485 & -0.98385609 & 1.193680989 & $\mathrm{H}$ & -2.690516756 & -2.673001935 & 1.431393932 \\
\hline $\mathrm{F}$ & 0.696801426 & 0.913561092 & -1.314750184 & $\mathrm{H}$ & -1.447373406 & -2.786926806 & 0.115808004 \\
\hline $\mathrm{F}$ & 0.285065017 & -1.222693003 & -1.104349681 & $\mathrm{H}$ & -2.961832824 & -1.820479504 & -0.145017857 \\
\hline \multicolumn{8}{|c|}{ OTf } \\
\hline C & 0.262302783 & -0.195782365 & -0.396089987 & $\mathrm{~F}$ & 0.68161702 & 0.823396265 & -1.191394532 \\
\hline S & -1.502956737 & 0.078818457 & 0.244445258 & $\mathrm{~F}$ & 0.366753215 & -1.334389295 & -1.130643898 \\
\hline $\mathrm{O}$ & -1.366532668 & 1.365654438 & 0.990143175 & $\mathrm{~F}$ & 1.163606437 & -0.299234775 & 0.616370469 \\
\hline O & -1.722365422 & -1.138017068 & 1.082153459 & $\mathrm{O}$ & -2.287134627 & 0.143134343 & -1.025013945 \\
\hline
\end{tabular}




\section{Part IV}

\section{Literature}

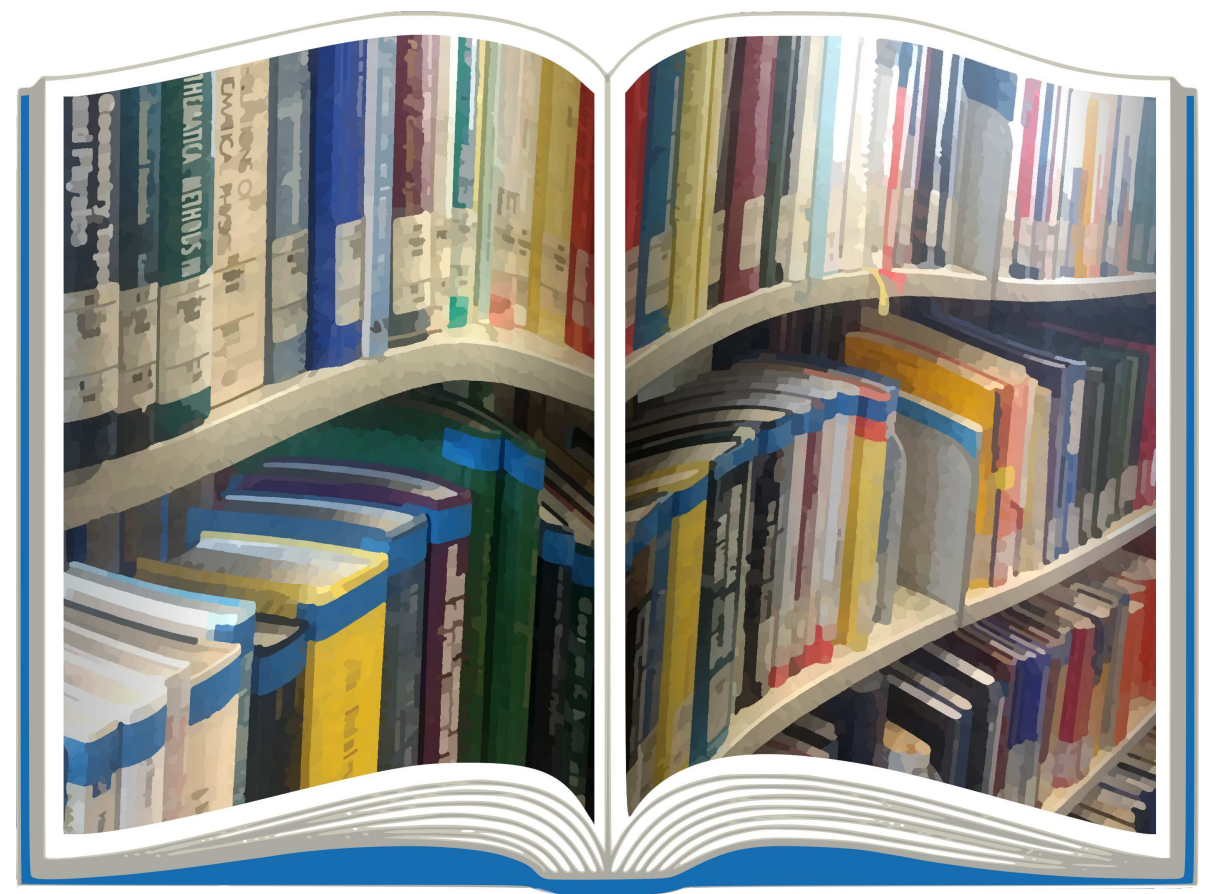



[1]B. A. Arndtsen, R. G. Bergman, T. A. Mobley, T. H. Peterson, „Selective Intermolecular CarbonHydrogen Bond Activation by Synthetic Metal Complexes in Homogeneous Solution", Acc. Chem. Res. 1995, 28, 154-162, DOI 10.1021/ar00051a009.

[2]B. Thamdrup, „New Pathways and Processes in the Global Nitrogen Cycle“, Annu. Rev. Ecol. Evol. Syst. 2012, 43, 407-428, DOI 10.1146/annurev-ecolsys-102710-145048.

[3]A. E. Shilov, "Catalytic Reduction of Molecular Nitrogen in Solutions", Russ Chem Bull 2003, 52, 2555-2562, DOI 10.1023/B:RUCB.0000019873.81002.60.

[4]H.-P. Jia, E. A. Quadrelli, „Mechanistic Aspects of Dinitrogen Cleavage and Hydrogenation to Produce Ammonia in Catalysis and Organometallic Chemistry: Relevance of Metal Hydride Bonds and Dihydrogen.", Chem Soc Rev 2014, 43, 547-564, DOI 10.1039/c3cs60206k.

[5]Q. Cheng, „Perspectives in Biological Nitrogen Fixation Research“, J. Integr. Plant Biol. 2008, 50, 786-798, DOI 10.1111/j.1744-7909.2008.00700.x.

[6]T. Spatzal, M. Aksoyoglu, L. Zhang, S. L. A. Andrade, E. Schleicher, S. Weber, D. C. Rees, O. Einsle, "Evidence for Interstitial Carbon in Nitrogenase FeMo Cofactor", Science 2011, 334, 940-940, DOI 10.1126/science.1214025.

[7]K. M. Lancaster, M. Roemelt, P. Ettenhuber, Y. Hu, M. W. Ribbe, F. Neese, U. Bergmann, S. DeBeer, „X-Ray Emission Spectroscopy Evidences a Central Carbon in the Nitrogenase IronMolybdenum Cofactor", Science 2011, 334, 974-977, DOI 10.1126/science.1206445.

[8]R. R. Eady, „Structure-Function Relationships of Alternative Nitrogenases“, Chem. Rev. 1996, 96, 3013-3030, DOI 10.1021/cr950057h.

[9]B. M. Hoffman, D. Lukoyanov, Z.-Y. Yang, D. R. Dean, L. C. Seefeldt, „Mechanism of Nitrogen Fixation by Nitrogenase: The Next Stage", Chem. Rev. 2014, 114, 4041-4062, DOI 10.1021/c r400641x.

[10]M. Appl in Ullmann's Encyclopedia of Industrial Chemistry, American Cancer Society, 2011, DOI 10.1002/14356007.002_o11.

[11]G. Ertl, „Surface Science and Catalysis-Studies on the Mechanism of Ammonia Synthesis: The P. H. Emmett Award Address", Catal. Rev. 1980, 21, 201-223, DOI 10.1080/036024580080 67533.

[12]Nitrogen Statistics and Information, https://www.usgs.gov/centers/nmic/nitrogen-sta tistics-and-information (visited on May 6, 2019).

[13]I. J. McPherson, T. Sudmeier, J. Fellowes, S. C. E. Tsang, „Materials for Electrochemical Ammonia Synthesis“, Dalton Trans. 2019, 48, 1562-1568, DOI 10.1039/C8DT04019B.

[14]A. J. Martín, T. Shinagawa, J. Pérez-Ramírez, „Electrocatalytic Reduction of Nitrogen: From Haber-Bosch to Ammonia Artificial Leaf", Chem 2019, 5, 263-283, DOI 10.1016/j.chempr .2018.10.010.

[15]J. M. Smith in Progress in Inorganic Chemistry Volume 58, (Ed.: K. D. Karlin), John Wiley \& Sons, Inc., 2014, pp. 417-470.

[16]I. Klopsch, E. Y. Yuzik-Klimova, S. Schneider in Nitrogen Fixation, Topics in Organometallic Chemistry, Springer Berlin Heidelberg, 2017, pp. 71-112, DOI 10.1007/3418_2016_12.

[17]A. D. Allen, C. V. Senoff, „Nitrogenopentammineruthenium(II) Complexes“, Chem. Commun. (London) 1965, 0, 621-622, DOI 10.1039/C19650000621. 
[18]M. D. Fryzuk, S. A. Johnson, „The Continuing Story of Dinitrogen Activation“, Coordination Chemistry Reviews 2000, 200-202, 379-409, DOI 10.1016/S0010-8545(00) 00264-2.

[19]B. A. MacKay, M. D. Fryzuk, „Dinitrogen Coordination Chemistry: On the Biomimetic Borderlands", Chem. Rev. 2004, 104, 385-402, DOI 10.1021/cr020610c.

[20]R. M. Badger, „A Relation Between Internuclear Distances and Bond Force Constants“, J. Chem. Phys. 1934, 2, 128-131, DOI 10.1063/1.1749433.

[21]A. Poveda, I. C. Perilla, C. R. Pérez, „Review: Some Considerations About Coordination Compounds with End-on Dinitrogen“, J. Coord. Chem. 2001, 54, 427-440, DOI 10.1080/009589 70108022654.

[22]D. F. Harrison, E. Weissberger, H. Taube, „Binuclear lon Containing Nitrogen as a Bridging Group", Science 1968, 159, 320-322, DOI 10.1126/science.159.3812.320.

[23]I. M. Treitel, M. T. Flood, R. E. Marsh, H. B. Gray, „Molecular and Electronic Structure of $\mu$-Nitrogen-Decaamminediruthenium(II)“, J. Am. Chem. Soc. 1969, 91, 6512-6513, DOI 10.1021/ja01051a070.

[24]J. Chatt, R. C. Fay, R. L. Richards, „Preparation and Characterisation of the Dinuclear Dinitrogen Complex, Trichloro- $\mu$-dinitrogen-bis(tetrahydrofuran)\{chlorotetrakis[dimethyl-(phenyl)phosphine]rhenium(I)\}chromium(III) [(PMe $\left.2 \mathrm{Ph}_{4} \mathrm{ClReN}_{2} \mathrm{CrCl}_{3}(\mathrm{thf})_{2}\right]^{\prime \prime}$, J. Chem. Soc. A 1971, 0, 702-704, DOI 10.1039/J19710000702.

[25]M. D. Fryzuk, T. S. Haddad, M. Mylvaganam, D. H. McConville, S. J. Rettig, „End-on versus Side-on Bonding of Dinitrogen to Dinuclear Early Transition-Metal Complexes", J. Am. Chem. Soc. 1993, 115, 2782-2792, DOI 10.1021/ja00060a028.

[26]R. D. Sanner, J. M. Manriquez, R. E. Marsh, J. E. Bercaw, „Structure of $\mu$-Dinitrogenbis(bis(pentamethylcyclopentadienyl)-dinitrogenzirconium(II)), $\left\{\left(\eta^{5}-\mathrm{C}_{5}\left(\mathrm{CH}_{3}\right)_{5}\right)_{2} \mathrm{ZrN}_{2}\right\}_{2} \mathrm{~N}_{2}$ “, J. Am. Chem. Soc. 1976, 98, 8351-8357, DOI 10.1021/ja00442a007.

[27]C. E. Laplaza, M. J. A. Johnson, J. C. Peters, A. L. Odom, E. Kim, C. C. Cummins, G. N. George, I. J. Pickering, „Dinitrogen Cleavage by Three-Coordinate Molybdenum(III) Complexes: Mechanistic and Structural Data", J. Am. Chem. Soc. 1996, 118, 8623-8638, DOI 10.1021/j a960574x.

[28]J. J. Curley, T. R. Cook, S. Y. Reece, P. Müller, C. C. Cummins, „Shining Light on Dinitrogen Cleavage: Structural Features, Redox Chemistry, and Photochemistry of the Key Intermediate Bridging Dinitrogen Complex", J. Am. Chem. Soc. 2008, 130, 9394-9405, DOI 10.1021/ja 8002638.

[29]C. B. Powell, M. B. Hall, „Molecular Orbital Calculations on Dinitrogen-Bridged TransitionMetal Dimers", Inorg. Chem. 1984, 23, 4619-4627, DOI 10.1021/ic00194a042.

[30]D. E. Richardson, J. P. Sen, J. D. Buhr, H. Taube, „Preparation and Properties of Mixed-Valence ( $\mu$-Dinitrogen)Bis(Pentaammine) Complexes of Osmium and Ruthenium", Inorg. Chem. 1982, 21, 3136-3140, DOI 10.1021/ic00138a043.

[31]M. Mercer, R. H. Crabtree, R. L. Richards, „A $\mu$-Dinitrogen Complex with a Long N-N Bond. X-Ray Crystal Structure of $\left[\left(\mathrm{PMe}_{2} \mathrm{Ph}\right)_{4} \mathrm{CIReN}_{2} \mathrm{MoCl}_{4}(\mathrm{OMe})\right]^{\prime}$, J. Chem. Soc. Chem. Commun. 1973, 0, 808-809, DOI 10.1039/C39730000808.

[32]M. J. Bezdek, S. Guo, P. J. Chirik, „Terpyridine Molybdenum Dinitrogen Chemistry: Synthesis of Dinitrogen Complexes That Vary by Five Oxidation States", Inorg. Chem. 2016, DOI 10.1 021/acs. inorgchem. 6b00053. 
[33]S. Rafiq, M. J. Bezdek, M. Koch, P. J. Chirik, G. D. Scholes, „Ultrafast Photophysics of a Dinitrogen-Bridged Molybdenum Complex“, J. Am. Chem. Soc. 2018, 140, 6298-6307, DOI 10.1021/jacs. 8b00890.

[34]S. Rafiq, M. J. Bezdek, P. J. Chirik, G. D. Scholes, „Dinitrogen Coupling to a TerpyridineMolybdenum Chromophore Is Switched on by Fermi Resonance", Chem 2019, 5, 402-416, DOI $10.1016 / j$. chempr.2018.11.003.

[35]P. P. Fontaine, B. L. Yonke, P. Y. Zavalij, L. R. Sita, „Dinitrogen Complexation and Extent of $\mathrm{N} \equiv \mathrm{N}$ Activation within the Group 6 "End-On-Bridged" Dinuclear Complexes, $\left\{\left(\eta^{5}-\mathrm{C}_{5} \mathrm{Me}_{5}\right)\right.$ $\mathrm{M}[\mathrm{N}(i-\operatorname{Pr}) \mathrm{C}(\mathrm{Me}) \mathrm{N}(i-\operatorname{Pr})]\}_{2}\left(\mu-\eta^{1}: \eta^{1}-\mathrm{N}_{2}\right)(\mathrm{M}=\mathrm{Mo}$ and $\mathrm{W}) “, J$. Am. Chem. Soc. 2010, 132, 12273-12285, DOI 10.1021/ja100469f.

[36]M. Hirotsu, P. P. Fontaine, P. Y. Zavalij, L. R. Sita, „Extreme N”N Bond Elongation and Facile N-Atom Functionalization Reactions within Two Structurally Versatile New Families of Group 4 Bimetallic "Side-on-Bridged" Dinitrogen Complexes for Zirconium and Hafnium", J. Am. Chem. Soc. 2007, 129, 12690-12692, DOI 10.1021/ja0752989.

[37]A. J. Keane, B. L. Yonke, M. Hirotsu, P. Y. Zavalij, L. R. Sita, „Fine-Tuning the Energy Barrier for Metal-Mediated Dinitrogen N=N Bond Cleavage", J. Am. Chem. Soc. 2014, 136, 99069909, DOI $10.1021 / \mathrm{ja} 505309 \mathrm{j}$.

[38]M. Hirotsu, P. P. Fontaine, A. Epshteyn, L. R. Sita, „Dinitrogen Activation at Ambient Temperatures: New Modes of $\mathrm{H}_{2}$ and $\mathrm{PhSiH}_{3}$ Additions for an "End-On-Bridged" [Ta(IV) $]_{2}\left(\mu-\eta^{1}: \eta^{1}-\mathrm{N}_{2}\right)$ Complex and for the Bis $\left(\mu\right.$-Nitrido $[\mathrm{Ta}(\mathrm{v})(\mu "-" \mathrm{~N})]_{2}$ Product Derived from Facile $\mathrm{N} \equiv \mathrm{N}$ Bond Cleavage", J. Am. Chem. Soc. 2007, 129, 9284-9285, DOI 10.1021/ja072248v.

[39]B. L. Tran, B. Pinter, A. J. Nichols, F. T. Konopka, R. Thompson, C.-H. Chen, J. Krzystek, A. Ozarowski, J. Telser, M.-H. Baik, K. Meyer, D. J. Mindiola, „A Planar Three-Coordinate Vanadium(II) Complex and the Study of Terminal Vanadium Nitrides from $\mathrm{N}_{2}$ : A Kinetic or Thermodynamic Impediment to N-N Bond Cleavage?", J. Am. Chem. Soc. 2012, 134, 1303513045, DOI 10.1021/ja303360v.

[40]J. Jeffrey, M. F. Lappert, P. I. Riley, "Organozirconium Dinitrogen Complexes $\left[\mathrm{Zr}\left(\eta-\mathrm{C}_{5} \mathrm{H}_{4} \mathrm{R}^{\prime}\right)_{2}\right.$ $\left.\left(\eta_{2}-\mathrm{N}_{2}\right) \mathrm{R}\right]$ and $\left[\left\{\mathrm{Zr}\left(\eta-\mathrm{C}_{5} \mathrm{H}_{5}\right)_{2} \mathrm{R}\right\}_{2} \mathrm{~N}_{2}\right]\left[\mathrm{R}=\left(\mathrm{Me} \mathrm{Si}_{2}\right)_{2} \mathrm{CH}, \mathrm{R}^{\prime}=\mathrm{H}\right.$ or Me] ", Journal of Organometallic Chemistry 1979, 181, 25-36, DOI 10.1016/S0022-328X(00)85734-4.

[41]G. A. Ozin, A. V. Voet, "Sideways" Bonded Dinitrogen in Matrix Isolated Cobalt Dinitrogen, $\mathrm{CoN}_{2}{ }^{\prime}$, Can. J. Chem. 1973, 51, 637-640, DOI 10.1139/v73-097.

[42]J. N. Armor, H. Taube, "Linkage Isomerization in Nitrogen-Labeled $\left[\mathrm{Ru}\left(\mathrm{NH}_{3}\right)_{5} \mathrm{~N}_{2}\right] \mathrm{Br}_{2}{ }$, J. Am. Chem. Soc. 1970, 92, 2560-2562, DOI 10.1021/ja00711a066.

[43]A. Cusanelli, D. Sutton, „End-to-End Rotation of Co-Ordinated Dinitrogen in $\left(\eta_{5}-\mathrm{C}_{5} \mathrm{Me}_{5}\right)$ $\operatorname{Re}(\mathrm{CO})_{2}\left(\mathrm{~N}_{2}\right)^{\prime \prime}$, J. Chem. Soc. Chem. Commun. 1989, 0, 1719-1720, DOI 10.1039/C3989 0001719.

[44]A. Cusanelli, D. Sutton, „End-to-End Rotation of Rhenium-Bound Dinitrogen“, Organometallics 1996, 15, 1457-1464, DOI 10.1021/om950792j.

[45]D. V. Fomitchev, K. A. Bagley, P. Coppens, „The First Crystallographic Evidence for Side-On Coordination of $\mathrm{N}_{2}$ to a Single Metal Center in a Photoinduced Metastable State", J. Am. Chem. Soc. 2000, 122, 532-533, DOI 10.1021/ja993623p.

[46]K. Jonas, „ $\pi$-Bonded Nitrogen in a Crystalline Nickel-Lithium Complex“, Angew. Chem. Int. Ed. Engl. 1973, 12, 997-998, DOI 10.1002/anie.197309971. 
[47]C. Krüger, Y.-H. Tsay, „Molecular Structure of a $\pi$-Dinitrogen-Nickel-Lithium Complex“, Angew. Chem. Int. Ed. Engl. 1973, 12, 998-999, DOI 10.1002/anie.197309981.

[48]K. Jonas, D. J. Brauer, C. Krueger, P. J. Roberts, Y. H. Tsay, " "Side-on" Dinitrogen-Transition Metal Complexes. The Molecular Structure of $\left\{\mathrm{C}_{6} \mathrm{H}_{5}\left[\mathrm{Na} \cdot \mathrm{O}\left(\mathrm{C}_{2} \mathrm{H}_{5}\right)_{2}\right]_{2}\left[\left(\mathrm{C}_{6} \mathrm{H}_{5}\right)_{2} \mathrm{Ni}_{2} \mathrm{~N}_{2} \mathrm{NaLi}_{6}(\mathrm{O}\right.\right.$ $\left.\left.\mathrm{C}_{2} \mathrm{H}_{5}\right)_{4} \cdot \mathrm{O}\left(\mathrm{C}_{2} \mathrm{H}_{5}\right)_{2}\right\}_{2}{ }^{\prime \prime}$, J. Am. Chem. Soc. 1976, 98, 74-81, DOI 10.1021/ja00417a013.

[49]W. J. Evans, T. A. Ulibarri, J. W. Ziller, "Isolation and X-Ray Crystal Structure of the First Dinitrogen Complex of an f-Element Metal, $\left[\left(\mathrm{C}_{5} \mathrm{Me}_{5}\right)_{2} \mathrm{Sm}\right]_{2} \mathrm{~N}_{2}{ }^{\prime \prime}, J$. Am. Chem. Soc. 1988, 110, 6877-6879, DOI 10.1021/ja00228a043.

[50]M. D. Fryzuk, T. S. Haddad, S. J. Rettig, „Reduction of Dinitrogen by a Zirconium Phosphine Complex to Form a Side-on-Bridging $\mathrm{N}_{2}$ Ligand. Crystal Structure of $\left\{\left[\left(\mathrm{Pr}_{2}^{i} \mathrm{PCH}_{2} \mathrm{SiMe}_{2}\right)_{2} \mathrm{~N}\right]\right.$ $\mathrm{ZrCl}_{2}\left(\mu-\eta^{2}: \eta^{2}-\mathrm{N}_{2}\right)^{\prime \prime}$, J. Am. Chem. Soc. 1990, 112, 8185-8186, DOI 10.1021/ja00178a 063.

[51]F. A. Cotton, D. G. Nocera, "The Whole Story of the Two-Electron Bond, with the $\delta$ Bond as a Paradigm", Acc. Chem. Res. 2000, 33, 483-490, DOI 10.1021/ar980116o.

[52]F. Studt, L. Morello, N. Lehnert, M. D. Fryzuk, F. Tuczek, „Side-On Bridging Coordination of $\mathrm{N}_{2}$ : Spectroscopic Characterization of the Planar $\mathrm{Zr}_{2} \mathrm{~N}_{2}$ Core and Theoretical Investigation of Its Butterfly Distortion“, Chem. - Eur. J. 2003, 9, 520-530, DOI 10.1002/chem. 200390055.

[53]J. M. Manriquez, J. E. Bercaw, „Preparation of a Dinitrogen Complex of Bis(pentamethylcyclopentadienyl)zirconium(II). Isolation and Protonation Leading to Stoichiometric Reduction of Dinitrogen to Hydrazine", J. Am. Chem. Soc. 1974, 96, 6229-6230, DOI 10.1021/ja00826a 071.

[54]J. A. Pool, W. H. Bernskoetter, P. J. Chirik, "On the Origin of Dinitrogen Hydrogenation Promoted by $\left[\left(\eta^{5}-\mathrm{C}_{5} \mathrm{Me}_{4} \mathrm{H}\right)_{2} \mathrm{Zr}\right]_{2}\left(\mu^{2}, \eta^{2}, \eta^{2}-\mathrm{N}_{2}\right)^{\prime \prime}$, J. Am. Chem. Soc. 2004, 126, 14326-14327, DOI $10.1021 / \mathrm{ja0} 04566 \mathrm{~s}$.

[55]J. A. Pool, E. Lobkovsky, P. J. Chirik, „Hydrogenation and Cleavage of Dinitrogen to Ammonia with a Zirconium Complex“, Nature 2004, 427, 527, DOI 10.1038/nature02274.

[56]R. J. Burford, M. D. Fryzuk, „Examining the Relationship between Coordination Mode and Reactivity of Dinitrogen“" Nat. Rev. Chem. 2017, 1, 0026, DOI 10.1038/s41570-017-0026.

[57]Y. Roux, C. Duboc, M. Gennari, „Molecular Catalysts for $\mathrm{N}_{2}$ Reduction: State of the Art, Mechanism, and Challenges", ChemPhysChem 2017, 18, 2606-2617, DOI 10.1002/cphc .201700665 .

[58]Nitrogen Fixation, (Ed.: Y. Nishibayashi), Springer International Publishing, 2017.

[59] Transition Metal-Dinitrogen Complexes: Preparation and Reactivity, (Ed.: Y. Nishibayashi), Wiley-VCH Verlag GmbH \& Co. KGaA, Weinheim, Germany, 2019, DOI 10.1002/978352 7344260 .

[60]J. Chatt, G. A. Heath, R. L. Richards, „The Reduction of Ligating Dinitrogen to Yield a Ligating $\mathrm{N}_{2} \mathrm{H}_{2}$ Moiety“, J. Chem. Soc. Chem. Commun. 1972, 1010-1011, DOI 10.1039/C397200010 10.

[61]J. Chatt, A. J. Pearman, R. L. Richards, „The Reduction of Mono-Coordinated Molecular Nitrogen to Ammonia in a Protic Environment", Nature 1975, 253, 39, DOI 10.1038/253039b 0 .

[62]J. Chatt, R. L. Richards, "The Reactions of Dinitrogen in Its Metal Complexes", Journal of Organometallic Chemistry 1982, 239, 65-77, DOI 10.1016/S0022-328X (00)94103-2. 
[63]G. C. Stephan, C. Sivasankar, F. Studt, F. Tuczek, „Energetics and Mechanism of Ammonia Synthesis through the Chatt Cycle: Conditions for a Catalytic Mode and Comparison with the Schrock Cycle", Chem. Eur. J. 2008, 14, 644-652, DOI 10.1002/chem. 200700849.

[64]J. Chatt, G. A. Heath, G. J. Leigh, „The Formation of a Nitrogen to Carbon Bond in a Reaction of a Dinitrogen Complex", J. Chem. Soc. Chem. Commun. 1972, 444-445, DOI 10.1039/C3 9720000444 .

[65]J. Chatt, G. A. Heath, N. E. Hooper, G. J. Leigh, „Direct Formation of Acylazo- and AroylazoRhenium(III) Complexes from Rhenium(I) Dinitrogen Complexes", Journal of Organometallic Chemistry 1973, 57, C67-C68, DOI 10.1016/S0022-328X (00)86587-0.

[66]D. V. Yandulov, R. R. Schrock, "Catalytic Reduction of Dinitrogen to Ammonia at a Single Molybdenum Center", Science 2003, 301, 76-78, DOI 10.1126/science.1085326.

[67]D. V. Yandulov, R. R. Schrock, „Studies Relevant to Catalytic Reduction of Dinitrogen to Ammonia by Molybdenum Triamidoamine Complexes", Inorg. Chem. 2005, 44, 1103-1117, DOI 10.1021/ic040095w.

[68]R. R. Schrock, "Catalytic Reduction of Dinitrogen to Ammonia by Molybdenum: Theory versus Experiment", Angew. Chem. Int. Ed. 2008, 47, 5512-5522, DOI 10.1002/anie. 200705246.

[69]F. Neese, „The Yandulov/Schrock Cycle and the Nitrogenase Reaction: Pathways of Nitrogen Fixation Studied by Density Functional Theory“, Angew. Chem. Int. Ed. 2006, 45, 196-199, DOI 10.1002/anie.200502667.

[70]W. Thimm, C. Gradert, H. Broda, F. Wennmohs, F. Neese, F. Tuczek, „Free Reaction Enthalpy Profile of the Schrock Cycle Derived from Density Functional Theory Calculations on the Full [MoHIPTN ${ }_{3}$ N] Catalyst", Inorg. Chem. 2015, 54, 9248-9255, DOI 10.1021/acs.inorgchem $.5 \mathrm{~b} 00787$.

[71]A. Sharma, M. Roemelt, M. Reithofer, R. R. Schrock, B. M. Hoffman, F. Neese, „EPR/ENDOR and Theoretical Study of the Jahn-Teller-Active $\left[\mathrm{HIPTN}_{3} \mathrm{~N}\right] \mathrm{Mo}(\mathrm{v}) \mathrm{L}$ Complexes $\left(\mathrm{L}=\mathrm{N}^{-}, \mathrm{NH}\right)$ ", Inorg. Chem. 2017, 56, 6906-6919, DOI 10.1021/acs.inorgchem.7b00364.

[72]J. S. Anderson, J. Rittle, J. C. Peters, „Catalytic Conversion of Nitrogen to Ammonia by an Iron Model Complex", Nature 2013, 501, 84-87, DOI 10.1038/nature12435.

[73]S. E. Creutz, J. C. Peters, "Catalytic Reduction of $\mathrm{N}_{2}$ to $\mathrm{NH}_{3}$ by an $\mathrm{Fe}-\mathrm{N}_{2}$ Complex Featuring a C-Atom Anchor", J. Am. Chem. Soc. 2014, 136, 1105-1115, DOI 10.1021/ja4114962.

[74]J. Rittle, J. C. Peters, „An Fe- $\mathrm{N}_{2}$ Complex That Generates Hydrazine and Ammonia via $\mathrm{Fe}=\mathrm{NNH}_{2}$ : Demonstrating a Hybrid Distal-to-Alternating Pathway for $\mathrm{N}_{2}$ Reduction", J. Am. Chem. Soc. 2016, 138, 4243-4248, DOI 10.1021/jacs.6b01230.

[75]N. B. Thompson, M. T. Green, J. C. Peters, „Nitrogen Fixation via a Terminal Fe(IV) Nitride“, J. Am. Chem. Soc. 2017, 139, 15312-15315, DOI 10.1021/jacs.7b09364.

[76]M. J. Chalkley, T. J. Del Castillo, B. D. Matson, J. P. Roddy, J. C. Peters, „Catalytic $\mathrm{N}_{2}$-to- $\mathrm{NH}_{3}$ Conversion by Fe at Lower Driving Force: A Proposed Role for Metallocene-Mediated PCET“" ACS Cent. Sci. 2017, DOI 10.1021/acscentsci.7b00014.

[77]K. Arashiba, Y. Miyake, Y. Nishibayashi, „A Molybdenum Complex Bearing PNP-Type Pincer Ligands Leads to the Catalytic Reduction of Dinitrogen into Ammonia“, Nat. Chem. 2011, 3, 120-125, DOI 10.1038/nchem.906. 
[78]H. Tanaka, Y. Nishibayashi, K. Yoshizawa, „Interplay between Theory and Experiment for Ammonia Synthesis Catalyzed by Transition Metal Complexes", Acc. Chem. Res. 2016, 49, 987995, DOI 10.1021/acs.accounts.6b00033.

[79]A. Eizawa, K. Arashiba, H. Tanaka, S. Kuriyama, Y. Matsuo, K. Nakajima, K. Yoshizawa, Y. Nishibayashi, „Remarkable Catalytic Activity of Dinitrogen-Bridged Dimolybdenum Complexes Bearing NHC-Based PCP-Pincer Ligands toward Nitrogen Fixation", Nat. Commun. 2017, 8, 14874, DOI 10.1038/ncomms14874.

[80]K. Arashiba, A. Eizawa, H. Tanaka, K. Nakajima, K. Yoshizawa, Y. Nishibayashi, „Catalytic Nitrogen Fixation via Direct Cleavage of Nitrogen-Nitrogen Triple Bond of Molecular Dinitrogen under Ambient Reaction Conditions", BCSJ 2017, 90, 1111-1118, DOI 10.1246/bcsj. 2017 0197.

[81]Y. Ashida, K. Arashiba, K. Nakajima, Y. Nishibayashi, „Molybdenum-Catalysed Ammonia Production with Samarium Diiodide and Alcohols or Water", Nature 2019, 568, 536, DOI 10.1038/s41586-019-1134-2.

[82]C. E. Laplaza, C. C. Cummins, „Dinitrogen Cleavage by a Three-Coordinate Molybdenum(III) Complex", Science 1995, 268, 861-863, DOI 10.1126/science.268.5212.861.

[83]Q. Cui, D. G. Musaev, M. Svensson, S. Sieber, K. Morokuma, „N $\mathrm{N}_{2}$ Cleavage by Three-Coordinate Group 6 Complexes. W(III) Complexes Would Be Better Than Mo(III) Complexes", J. Am. Chem. Soc. 1995, 117, 12366-12367, DOI 10.1021/ja00154a052.

[84]M. Kol, R. R. Schrock, R. Kempe, W. M. Davis, „Synthesis of Molybdenum and Tungsten Complexes That Contain Triamidoamine Ligands of the Type $\left(\mathrm{C}_{6} \mathrm{~F}_{5} \mathrm{NCH}_{2} \mathrm{CH}_{2}\right)_{3} \mathrm{~N}$ and Activation of Dinitrogen by Molybdenum“, J. Am. Chem. Soc. 1994, 116, 4382-4390, DOI 10.1021/ja 00089a028.

[85]K.-Y. Shih, R. R. Schrock, R. Kempe, "Synthesis of Molybdenum Complexes That Contain Silylated Triamidoamine Ligands. A $\mu$-Dinitrogen Complex, Methyl and Acetylide Complexes, and Coupling of Acetylides", J. Am. Chem. Soc. 1994, 116, 8804-8805, DOI 10.1021/ja $00098 \mathrm{a} 048$.

[86]E. Solari, C. D. Silva, B. lacono, J. Hesschenbrouck, C. Rizzoli, R. Scopelliti, C. Floriani, „Photochemical Activation of the $\mathrm{N} \equiv \mathrm{N}$ Bond in a Dimolybdenum-Dinitrogen Complex: Formation of a Molybdenum Nitride", Angew. Chem. Int. Ed. 2001, 40, 3907-3909, DOI 10.1002/1521 -3773(20011015) 40: 20<3907: : AID-ANIE3907>3.0.CO;2-\#.

[87]G. Christian, R. Stranger, B. F. Yates, D. C. Graham, "Ligand Rotation in $[\operatorname{Ar}(\mathrm{R}) \mathrm{N}]_{3} \mathrm{M}-\mathrm{N}_{2}-\mathrm{M}$ ' $\left[\mathrm{N}(\mathrm{R}) \mathrm{Ar}_{3}\left(\mathrm{M}, \mathrm{M}\right.\right.$ ' = Mo(iii), $\mathrm{Nb}\left(\mathrm{III} ; \mathrm{R}={ }^{\mathrm{i}} \mathrm{Pr}\right.$ and $\left.{ }^{\mathrm{t}} \mathrm{Bu}\right)$ Dimers", Dalton Trans. 2005, 962-968, DOI 10.1039/B413766C.

[88]N. J. Brookes, D. C. Graham, G. Christian, R. Stranger, B. F. Yates, „The Influence of Peripheral Ligand Bulk on Nitrogen Activation by Three-Coordinate Molybdenum Complexes-A Theoretical Study Using the ONIOM Method“, J. Comput. Chem. 2009, 30, 2146-2156, DOI $10.1002 /$ jcc. 21199.

[89]M. H. Chisholm, F. A. Cotton, B. A. Frenz, W. W. Reichert, L. W. Shive, B. R. Stults, „The Molybdenum-Molybdenum Triple Bond. 1. Hexakis(Dimethylamido)Dimolybdenum and Some Homologs: Preparation, Structure, and Properties", J. Am. Chem. Soc. 1976, 98, 4469-4476, DOI $10.1021 / \mathrm{ja} 00431 \mathrm{a} 024$. 
[90]Y.-C. Tsai, M. J. A. Johnson, D. J. Mindiola, C. C. Cummins, W. T. Klooster, T. F. Koetzle, „A Cyclometalated Resting State for a Reactive Molybdenum Amide: Favorable Consequences of $\beta$-Hydrogen Elimination Including Reductive Cleavage, Coupling, and Complexation", J. Am. Chem. Soc. 1999, 121, 10426-10427, DOI 10.1021/ja9917464.

[91]J. C. Peters, J.-P. F. Cherry, J. C. Thomas, L. Baraldo, D. J. Mindiola, W. M. Davis, C. C. Cummins, „Redox-Catalyzed Binding of Dinitrogen by Molybdenum N-Tert-Hydrocarbylanilide Complexes: Implications for Dinitrogen Functionalization and Reductive Cleavage", J. Am. Chem. Soc. 1999, 121, 10053-10067, DOI 10.1021/ja991435t.

[92]Y.-C. Tsai, C. C. Cummins, „Base-Catalyzed Dinitrogen Cleavage by Molybdenum Amides“, Inorganica Chimica Acta, Protagonists in Chemistry: Richard R. Schrock 2003, 345, 63-69, DOI 10.1016/S0020-1693(02)01344-0.

[93]M. Pucino, F. Allouche, C. P. Gordon, M. Wörle, V. Mougel, C. Coperet, „Reactive Coordinatively Saturated Mo(III) Complex: Exploiting the Adaptive Hapticity of Tris(Tert-Butoxy)Silanolate Ligands", Chem. Sci. 2019, 10.1039.C9SC01955C, DOI 10.1039/C9SC01955C.

[94]I. Klopsch, M. Finger, C. Würtele, B. Milde, D. B. Werz, S. Schneider, „Dinitrogen Splitting and Functionalization in the Coordination Sphere of Rhenium", J. Am. Chem. Soc. 2014, 136, 6881-6883, DOI 10.1021/ja502759d.

[95]B. M. Lindley, R. S. van Alten, M. Finger, F. Schendzielorz, C. Würtele, A. J. M. Miller, I. Siewert, S. Schneider, „Mechanism of Chemical and Electrochemical $\mathrm{N}_{2}$ Splitting by a Rhenium Pincer Complex", J. Am. Chem. Soc. 2018, 140, 7922-7935, DOI 10.1021/jacs.8b03755.

[96]G. A. Silantyev, M. Förster, B. Schluschaß, J. Abbenseth, C. Würtele, C. Volkmann, M. C. Holthausen, S. Schneider, „Dinitrogen Splitting Coupled to Protonation“, Angew. Chem. Int. Ed. 2017, 56, 5872-5876, DOI 10.1002/anie.201701504.

[97]J. M. Smith, R. J. Lachicotte, K. A. Pittard, T. R. Cundari, G. Lukat-Rodgers, K. R. Rodgers, P. L. Holland, „Stepwise Reduction of Dinitrogen Bond Order by a Low-Coordinate Iron Complex“, J. Am. Chem. Soc. 2001, 123, 9222-9223, DOI 10.1021/ja016094+.

[98]J. M. Smith, A. R. Sadique, T. R. Cundari, K. R. Rodgers, G. Lukat-Rodgers, R. J. Lachicotte, C. J. Flaschenriem, J. Vela, P. L. Holland, „Studies of Low-Coordinate Iron Dinitrogen Complexes", J. Am. Chem. Soc. 2006, 128, 756-769, DOI 10.1021/ja052707x.

[99]M. M. Rodriguez, E. Bill, W. W. Brennessel, P. L. Holland, „N $\mathrm{N}_{2}$ Reduction and Hydrogenation to Ammonia by a Molecular Iron-Potassium Complex“, Science 2011, 334, 780-783, DOI 10 $.1126 /$ science. 1211906 .

[100]T. M. Figg, P. L. Holland, T. R. Cundari, „Cooperativity Between Low-Valent Iron and Potassium Promoters in Dinitrogen Fixation", Inorg. Chem. 2012, 51, 7546-7550, DOI 10.1021/i c300150u.

[101]K. C. MacLeod, D. J. Vinyard, P. L. Holland, „A Multi-Iron System Capable of Rapid $\mathrm{N}_{2}$ Formation and $\mathrm{N}_{2}$ Cleavage", J. Am. Chem. Soc. 2014, 136, 10226-10229, DOI 10.1021/ja $505193 z$.

[102]G. K. B. Clentsmith, V. M. E. Bates, P. B. Hitchcock, F. G. N. Cloke, „Reductive Cleavage of Dinitrogen by a Vanadium Diamidoamine Complex: The Molecular Structures of [ $\mathrm{V}\left(\mathrm{Me}_{3} \mathrm{SiN}\right.$ $\left.\left.\left\{\mathrm{CH}_{2} \mathrm{CH}_{2} \mathrm{NSiMe}_{3}\right\}_{2}\right)(\mu-\mathrm{N})\right]_{2}$ and $\mathrm{K}\left[\mathrm{V}\left(\mathrm{Me}_{3} \mathrm{SiN}\left\{\mathrm{CH}_{2} \mathrm{CH}_{2} \mathrm{NSiMe}_{3}\right\}_{2}\right)(\mu-\mathrm{N})\right]_{2}{ }^{\prime \prime}$ J. Am. Chem. Soc. 1999, 121, 10444-10445, DOI 10.1021/ja9921219. 
[103]F. Studt, V. M. E. Lamarche, G. K. B. Clentsmith, F. G. N. Cloke, F. Tuczek, „Vibrational and Electronic Structure of the Dinuclear Bis $(\mu-N i t r i d o)$ Vanadium(v) Complex $\left[\mathrm{V}\left(\mathrm{N}\{\mathrm{N} "\}_{2}\right)\right.$ $(\mu-\mathrm{N})]_{2}$ : Spectroscopic Properties of the $\mathrm{M}_{2}(\mu-\mathrm{N})_{2}$ Diamond Core", Dalton Trans. 2005, 0 , 1052-1057, DOI 10.1039/B418856J.

[104]V. M. E. Bates, G. K. B. Clentsmith, F. G. N. Cloke, J. C. Green, H. D. LI. Jenkin, „Theoretical Investigation of the Pathway for Reductive Cleavage of Dinitrogen by a Vanadium Diamidoamine Complex", Chem. Commun. 2000, 0, 927-928, DOI 10.1039/B002534H.

[105]I. Fischler, E. K. von Gustorf, "Chemische und biologische Aspekte der Fixierung und Reduktion molekularen Stickstoffs", Naturwissenschaften 1975, 62, 63-70, DOI 10.1007/BF00592178.

[106]T. M. Buscagan, P. H. Oyala, J. C. Peters, „, $\mathrm{N}_{2}$-to- $\mathrm{NH}_{3}$ Conversion by a Triphos-Iron Catalyst and Enhanced Turnover under Photolysis“, Angew. Chem. Int. Ed. 2017, 56, 6921-6926, DOI 10.1002/anie. 201703244.

[107]M. J. A. Johnson, P. M. Lee, A. L. Odom, W. M. Davis, C. C. Cummins, „Atom-Bridged Intermediates in N- and P-Atom Transfer Reactions", Angew. Chem. Int. Ed. Engl. 1997, 36, 87-91, DOI 10.1002/anie.199700871.

[108]A. S. Huss, J. J. Curley, C. C. Cummins, D. A. Blank, „Relaxation and Dissociation Following Photoexcitation of the $\left(\mu-\mathrm{N}_{2}\right)\left[\mathrm{Mo}\left(\mathrm{N}\left[{ }^{\mathrm{t}} \mathrm{Bu}\right] \mathrm{Ar}\right)_{3}\right]_{2}$ Dinitrogen Cleavage Intermediate", J. Phys. Chem. B 2013, 117, 1429-1436, DOI 10.1021/jp310122x.

[109]H. Kunkely, A. Vogler, „Photolysis of Aqueous $\left[\left(\mathrm{NH}_{3}\right)_{5} \mathrm{Os}\left(\mu-\mathrm{N}_{2}\right) \mathrm{Os}\left(\mathrm{NH}_{3}\right)_{5}\right]^{5+}$ : Cleavage of Dinitrogen by an Intramolecular Photoredox Reaction", Angewandte Chemie International Edition 2010, 49, 1591-1593, DOI 10.1002/anie.200905026.

[110]H. Kunkely, A. Vogler, „Photolysis of Aqueous $\left[\mathrm{Os}\left(\mathrm{NH}_{3}\right)_{5}\left(\mathrm{~N}_{2}\right)\right]^{2+}$ : Dismutation of Coordinated Dinitrogen“, Inorganica Chimica Acta 2012, 391, 229-231, DOI 10.1016/j .ica.2012.04.0 21.

[111]A. Vogler, H. Kunkely, „Photooxidation of Coordinated Dinitrogen. Photolysis of the $\mathrm{ClO}_{2}$ Adduct of $\left[\left(\mathrm{NH}_{3}\right)_{5} \mathrm{Os}\left(\mu-\mathrm{N}_{2}\right) \mathrm{Os}\left(\mathrm{NH}_{3}\right)_{5}\right]^{5+\cdots}$, Inorganic Chemistry Communications 2012, 18, 7374, DOI 10.1016/j.inoche.2012.01.013.

[112]H. Kunkely, A. Vogler, „Photolysis of Aqueous $\left[\mathrm{Os}\left(\mathrm{NH}_{3}\right)_{5}\left(\mathrm{~N}_{2}\right)\right]^{2+}$. Photoreduction of Coordinated Dinitrogen to Hydrazine as a Model for a New Type of Artificial Photosynthesis?", Z. Für Naturforschung B 2014, 67, 488-490, DOI 10.5560/znb.2012-0080.

[113]V. Krewald, L. González, „A Valence-Delocalised Osmium Dimer Capable of Dinitrogen Photocleavage: Ab Initio Insights into Its Electronic Structure", Chem. - Eur. J. 2018, 24, 5112-5123, DOI 10.1002/chem. 201704651.

[114]T. Miyazaki, H. Tanaka, Y. Tanabe, M. Yuki, K. Nakajima, K. Yoshizawa, Y. Nishibayashi, "Cleavage and Formation of Molecular Dinitrogen in a Single System Assisted by Molybdenum Complexes Bearing Ferrocenyldiphosphine", Angew. Chem. Int. Ed. 2014, 53, 11488-11492, DOI 10.1002/anie.201405673.

[115]C. Rebreyend, B. de Bruin, „Photolytic $\mathrm{N}_{2}$ Splitting: A Road to Sustainable $\mathrm{NH}_{3}$ Production?“, Angew. Chem. Int. Ed. 2015, 54, 42-44, DOI 10.1002/anie.201409727.

[116]A. J. Keane, W. S. Farrell, B. L. Yonke, P. Y. Zavalij, L. R. Sita, „Metal-Mediated Production of Isocyanates, $\mathrm{R}_{3} \mathrm{EN}=\mathrm{C}=\mathrm{O}$ from Dinitrogen, Carbon Dioxide, and $\mathrm{R}_{3} \mathrm{ECl}^{\prime}$, Angew. Chem. Int. Ed. 2015, 54, 10220-10224, DOI 10.1002/anie. 201502293. 
[117]M. D. Fryzuk, J. B. Love, S. J. Rettig, V. G. Young, „Transformation of Coordinated Dinitrogen by Reaction with Dihydrogen and Primary Silanes", Science 1997, 275, 1445-1447, DOI 10.1 126/science. 275.5305.1445.

[118]L. Morello, J. B. Love, B. O. Patrick, M. D. Fryzuk, „Carbon-Nitrogen Bond Formation via the Reaction of Terminal Alkynes with a Dinuclear Side-on Dinitrogen Complex", J. Am. Chem. Soc. 2004, 126, 9480-9481, DOI 10.1021/ja049490b.

[119]W. H. Bernskoetter, E. Lobkovsky, P. J. Chirik, „Nitrogen-Carbon Bond Formation from $\mathrm{N}_{2}$ and $\mathrm{CO}_{2}$ Promoted by a Hafnocene Dinitrogen Complex Yields a Substituted Hydrazine", Angew. Chem. Int. Ed. 2007, 46, 2858-2861, DOI 10.1002/anie.200604099.

[120]J. Fritzsche, H. Struve, „Ueber Die Osman-Osmiumsäure“, J. Für Prakt. Chem. 1847, 41, 97113, DOI 10.1002/prac. 18470410113.

[121]R. A. Eikey, M. M. Abu-Omar, „Nitrido and Imido Transition Metal Complexes of Groups 6-8“, Coordination Chemistry Reviews 2003, 243, 83-124, DOI 10.1016/S0010-8545(03) 00048-1.

[122]C. J. Ballhausen, H. B. Gray, „The Electronic Structure of the Vanadyl lon“, Inorg. Chem. 1962, 1, 111-122, DOI 10.1021/ic50001a022.

[123]J. M. Mayer, „Why Are There No Terminal Oxo Complexes of the Late Transition Metals? Or The Importance of Metal-Ligand $\pi$ Antibonding Interactions", Comments Inorg. Chem. 1988, 8, 125-135, DOI 10.1080/02603598808035790.

[124]K. G. Caulton, „The Influence of $\pi$-Stabilized Unsaturation an Filled/Filled Repulsions in Transition Metal Chemistry", New J. Chem. 1994, 18, 25-41.

[125]J. F. Berry, „Terminal Nitrido and Imido Complexes of the Late Transition Metals“, Comments Inorg. Chem. 2009, 30, 28-66, DOI 10.1080/02603590902768875.

[126]W. D. Wagner, K. Nakamoto, „Formation of Nitridoiron(V) Porphyrins Detected by Resonance Raman Spectroscopy“, J. Am. Chem. Soc. 1988, 110, 4044-4045, DOI 10.1021/ja00220a057.

[127]W. D. Wagner, K. Nakamoto, „Resonance Raman Spectra of Nitridoiron(V) Porphyrin Intermediates Produced by Laser Photolysis", J. Am. Chem. Soc. 1989, 111, 1590-1598, DOI 10.1021/ja00187a010.

[128]K. Meyer, E. Bill, B. Mienert, T. Weyhermüller, K. Wieghardt, „Photolysis of cis- and trans$\left[\mathrm{Fe}\left(\mathrm{III}(\mathrm{Cyclam})\left(\mathrm{N}_{3}\right)_{2}\right]^{+}\right.$Complexes: Spectroscopic Characterization of a Nitridoiron(V) Species", J. Am. Chem. Soc. 1999, 121, 4859-4876, DOI 10.1021/ja983454t.

[129]N. Aliaga-Alcalde, S. D. George, B. Mienert, E. Bill, K. Wieghardt, F. Neese, „The Geometric and Electronic Structure of [(Cyclam-Acetato)Fe(N)]+: A Genuine Iron(v) Species with a Ground-State Spin S=1/2", Angew. Chem. Int. Ed. 2005, 44, 2908-2912, DOI 10.1002/ani e.200462368.

[130]J. F. Berry, E. Bill, E. Bothe, S. D. George, B. Mienert, F. Neese, K. Wieghardt, „An Octahedral Coordination Complex of Iron(VI)“, Science 2006, 312, 1937-1941, DOI 10.1126/science .1128506 .

[131], A Tetrahedrally Coordinated $\mathrm{L}_{3} \mathrm{Fe}-\mathrm{N}_{\mathrm{x}}$ Platform“.

[132]C. Vogel, F. W. Heinemann, J. Sutter, C. Anthon, K. Meyer, „An Iron Nitride Complex“, Angew. Chem. Int. Ed. 2008, 47, 2681-2684, DOI 10.1002/anie.200800600. 
[133]J. J. Scepaniak, M. D. Fulton, R. P. Bontchev, E. N. Duesler, M. L. Kirk, J. M. Smith, „Structural and Spectroscopic Characterization of an Electrophilic Iron Nitrido Complex", J. Am. Chem. Soc. 2008, 130, 10515-10517, DOI 10.1021/ja8027372.

[134]A. K. Maity, J. Murillo, A. J. Metta-Magaña, B. Pinter, S. Fortier, „A Terminal Iron(IV) Nitride Supported by a Super Bulky Guanidinate Ligand and Examination of Its Electronic Structure and Reactivity", J. Am. Chem. Soc. 2017, 139, 15691-15700, DOI 10.1021/jacs.7b06919.

[135]J.-U. Rohde, T. A. Betley, T. A. Jackson, C. T. Saouma, J. C. Peters, L. Que, „XAS Characterization of a Nitridoiron(IV) Complex with a Very Short Fe-N Bond“, Inorg. Chem. 2007, 46, 5720-5726, DOI 10.1021/ic700818q.

[136]R. Hoffmann, „Building Bridges Between Inorganic and Organic Chemistry (Nobel Lecture)“, Angew. Chem. Int. Ed. Engl. 1982, 21, 711-724, DOI 10.1002/anie.198207113.

[137]A. Walstrom, M. Pink, X. Yang, J. Tomaszewski, M.-H. Baik, K. G. Caulton, „A Facile Approach to a $d^{4} \mathrm{Ru}=\mathrm{N}$ : Moiety", J. Am. Chem. Soc. 2005, 127, 5330-5331, DOI 10.1021/ja050361k.

[138]B. Askevold, J. T. Nieto, S. Tussupbayev, M. Diefenbach, E. Herdtweck, M. C. Holthausen, S. Schneider, „Ammonia Formation by Metal-Ligand Cooperative Hydrogenolysis of a Nitrido Ligand“", Nat Chem 2011, 3, 532-537, DOI 10.1038/nchem.1051.

[139]J. Schöffel, A. Y. Rogachev, S. DeBeer George, P. Burger, „Isolation and Hydrogenation of a Complex with a Terminal Iridium-Nitrido Bond", Angew. Chem. Int. Ed. 2009, 48, 4734-4738, DOI 10.1002/anie.200901494.

[140]M. G. Scheibel, B. Askevold, F. W. Heinemann, E. J. Reijerse, B. de Bruin, S. Schneider, "Closed-Shell and Open-Shell Square-Planar Iridium Nitrido Complexes", Nat Chem 2012, 4, 552-558, DOI 10.1038/nchem.1368.

[141]M. G. Scheibel, Y. Wu, A. C. Stückl, L. Krause, E. Carl, D. Stalke, B. de Bruin, S. Schneider, "Synthesis and Reactivity of a Transient, Terminal Nitrido Complex of Rhodium.", J Am Chem Soc 2013, 135, 17719-17722, DOI 10.1021/ja409764j.

[142]W. A. Nugent, J. M. Mayer, Metal-Ligand Multiple Bonds: The Chemistry of Transition Metal Complexes Containing Oxo, Nitrido, Imido, Alkylidene, or Alkylidyne Ligands, Wiley-Interscience, 1988.

[143]E. D. Hedegård, J. Bendix, S. P. A. Sauer, „Partial Charges as Reactivity Descriptors for Nitrido Complexes", Journal of Molecular Structure: THEOCHEM 2009, 913, 1-7, DOI 10.1016/j.t heochem.2009.06.042.

[144]T. J. Crevier, J. M. Mayer, „Direct Attack of Phenyl Anion at an Electrophilic Osmium-Nitrido Ligand“, J. Am. Chem. Soc. 1998, 120, 5595-5596, DOI 10.1021/ja980548u.

[145]T. J. Crevier, J. M. Mayer, "Insertion of an Osmium Nitride into Boron-Carbon Bonds“, Angewandte Chemie International Edition 1998, 37, 1891-1893, DOI 10.1002/(SICI) 1521-3773 (19980803) $37: 13 / 14<1891$ : : AID-ANIE1891>3.0.CO;2-Y.

[146]T. J. Crevier, B. K. Bennett, J. D. Soper, J. A. Bowman, A. Dehestani, D. A. Hrovat, S. Lovell, W. Kaminsky, J. M. Mayer, "C-N Bond Formation on Addition of Aryl Carbanions to the Electrophilic Nitrido Ligand in $\mathrm{TpOs}(\mathrm{N}) \mathrm{Cl}_{2}{ }^{\prime \prime}, \mathrm{J}$. Am. Chem. Soc. 2001, 123, 1059-1071, DOI 10.1021/ja0028424. 
[147]E. L. Sceats, J. S. Figueroa, C. C. Cummins, N. M. Loening, P. Van der Wel, R. G. Griffin, "Complexes Obtained by Electrophilic Attack on a Dinitrogen-Derived Terminal Molybdenum Nitride: Electronic Structure Analysis by Solid State CP/MAS ${ }^{15}$ N NMR in Combination with DFT Calculations", Polyhedron, Special Issue in Honour of M.L.H. Green 2004, 23, 2751-2768, DOI 10.1016/j.poly.2004.08.010.

[148]J. J. Curley, A. F. Cozzolino, C. C. Cummins, „Nitrogen Fixation to Cyanide at a Molybdenum Center", Dalton Trans. 2011, 40, 2429-2432, DOI 10.1039/CODT01326A.

[149]H. Henderickx, G. Kwakkenbos, A. Peters, J. van der Spoel, K. de Vries, „Direct Formation of an Organonitrogen Compound from a Molybdenum Nitrido Species“, Chem. Commun. 2003, 2050-2051, DOI 10.1039/B305774G.

[150]Q. Liao, A. Cavaillé, N. Saffon-Merceron, N. Mézailles, „Direct Synthesis of Silylamine from $\mathrm{N}_{2}$ and a Silane: Mediated by a Tridentate Phosphine Molybdenum Fragment", Angew. Chem. Int. Ed. 2016, 55, 11212-11216, DOI 10.1002/anie. 201604812.

[151]Q. Liao, N. Saffon-Merceron, N. Mézailles, „N $N_{2}$ Reduction into Silylamine at Tridentate Phosphine/Mo Center: Catalysis and Mechanistic Study“, ACS Catal. 2015, 5, 6902-6906, DOI 10.1021/acscatal.5b01626.

[152]M. F. Espada, S. Bennaamane, Q. Liao, N. Saffon-Merceron, S. Massou, E. Clot, N. Nebra, M. Fustier-Boutignon, N. Mézailles, „Room-Temperature Functionalization of $\mathrm{N}_{2}$ to Borylamine at a Molybdenum Complex", Angew. Chem. 2018, 130, 13047-13050, DOI 10.1002/ange .201805915 .

[153]I. Klopsch, M. Kinauer, M. Finger, C. Würtele, S. Schneider, „Conversion of Dinitrogen into Acetonitrile under Ambient Conditions", Angew. Chem. Int. Ed. 2016, 55, 4786-4789, DOI 10.1002/anie. 201600790.

[154]I. Klopsch, F. Schendzielorz, C. Volkmann, C. Würtele, S. Schneider, „Synthesis of Benzonitrile from Dinitrogen“, Z. Für Anorg. Allg. Chem. 2018, 644, 916-919, DOI 10.1002/zaac. 2018 00181.

[155]G. P. Connor, B. Q. Mercado, H. M. C. Lant, J. M. Mayer, P. L. Holland, „Chemical Oxidation of a Coordinated PNP-Pincer Ligand Forms Unexpected Re-Nitroxide Complexes with Reversal of Nitride Reactivity", Inorg. Chem. 2019, DOI 10.1021/acs. inorgchem.9b01075.

[156]L. M. Duman, W. S. Farrell, P. Y. Zavalij, L. R. Sita, „Steric Switching from Photochemical to Thermal Reaction Pathways for Enhanced Efficiency in Metal-Mediated Nitrogen Fixation", J. Am. Chem. Soc. 2016, 138, 14856-14859, DOI 10.1021/jacs.6b09789.

[157]L. M. Duman, L. R. Sita, „Closing the Loop on Transition-Metal-Mediated Nitrogen Fixation: Chemoselective Production of $\mathrm{HN}\left(\mathrm{SiMe}_{3}\right)_{2}$ from $\mathrm{N}_{2}, \mathrm{Me}_{3} \mathrm{SiCl}$, and $\mathrm{X}-\mathrm{OH}\left(\mathrm{X}=\mathrm{R}, \mathrm{R}_{3} \mathrm{Si}\right.$, or Silica Gel)“, J. Am. Chem. Soc. 2017, 139, 17241-17244, DOI 10.1021/jacs.7b08859.

[158]M. P. Shaver, M. D. Fryzuk, „Activation of Molecular Nitrogen: Coordination, Cleavage and Functionalization of $\mathrm{N}_{2}$ Mediated By Metal Complexes", Adv. Synth. Catal. 2003, 345, 10611076, DOI 10.1002/adsc. 200303081.

[159]J. J. Scepaniak, C. G. Margarit, J. N. Harvey, J. M. Smith, „Nitrogen Atom Transfer from Iron(IV) Nitrido Complexes: A Dual-Nature Transition State for Atom Transfer", Inorg. Chem. 2011, 50, 9508-9517, DOI 10.1021/ic201190c.

[160]J. J. Scepaniak, R. P. Bontchev, D. L. Johnson, J. M. Smith, „Snapshots of Complete Nitrogen Atom Transfer from an Iron(IV) Nitrido Complex", Angew. Chem. Int. Ed. 2011, 50, 6630-6633, DOI 10.1002/anie.201102028. 
[161]S. B. Muñoz, W.-T. Lee, D. A. Dickie, J. J. Scepaniak, D. Subedi, M. Pink, M. D. Johnson, J. M. Smith, "Styrene Aziridination by Iron(IV) Nitrides", Angew. Chem. Int. Ed. 2015, 54, 10600-10603, DOI 10.1002/anie.201503773.

[162]D. W. Crandell, S. B. Muñoz, J. M. Smith, M.-H. Baik, „Mechanistic Study of Styrene Aziridination by Iron(IV) Nitrides", Chem. Sci. 2018, 9, 8542-8552, DOI 10.1039/C8SC03677B.

[163]W.-L. Man, W. W. Y. Lam, S.-M. Yiu, T.-C. Lau, S.-M. Peng, „Direct Aziridination of Alkenes by a Cationic (Salen)Ruthenium(vi) Nitrido Complex", J. Am. Chem. Soc. 2004, 126, 1533615337, DOI 10.1021/ja045845f.

[164]S. N. Brown, „Insertion of a Metal Nitride into Carbon-Carbon Double Bonds“, J. Am. Chem. Soc. 1999, 121, 9752-9753, DOI 10.1021/ja992385v.

[165]A. G. Maestri, S. D. Taylor, S. M. Schuck, S. N. Brown, „Cleavage of Conjugated Alkenes by Cationic Osmium Nitrides: Scope of the Reaction and Dynamics of the Azaallenium Products", Organometallics 2004, 23, 1932-1946, DOI 10.1021/om034404m.

[166]A. Walstrom, H. Fan, M. Pink, K. G. Caulton, „Unexpected Selectivity in Electrophilic Attack on (PNP)RuN", Inorganica Chimica Acta, Protagonist in Chemistry: Paul S. Pregosin 2010, 363, 633-636, DOI 10.1016/j.ica.2008.11.010.

[167]A. Walstrom, M. Pink, H. Fan, J. Tomaszewski, K. G. Caulton, „Radical (NO) and Nonradical $\left(\mathrm{N}_{2} \mathrm{O}\right)$ Reagents Convert a Ruthenium(IV) Nitride to the Same Nitrosyl Complex", Inorg. Chem. 2007, 46, 7704-7706, DOI 10.1021/ic700789y.

[168]N. Tsvetkov, M. Pink, H. Fan, J.-H. Lee, K. G. Caulton, „Redox and Lewis Acid Reactivity of Unsaturated OsiI", Eur. J. Inorg. Chem. 2010, 2010, 4790-4800, DOI 10.1002 / ejic .201000503 .

[169]A. Okuniewski, D. Rosiak, J. Chojnacki, B. Becker, "Coordination Polymers and Molecular Structures among Complexes of Mercury(II) Halides with Selected 1-Benzoylthioureas", Polyhedron 2015, 90, 47-57, DOI 10.1016/j.poly.2015.01.035.

[170]D. Sieh, J. Schöffel, P. Burger, "Synthesis of a Chloro Protected Iridium Nitrido Complex", Dalton Trans. 2011, 40, 9512-9524, DOI 10.1039/C1DT10886G.

[171]D. Sieh, P. Burger, „Si-H Activation in an Iridium Nitrido Complex-A Mechanistic and Theoretical Study“, J. Am. Chem. Soc. 2013, 135, 3971-3982, DOI 10.1021/ja311905h.

[172]J. Schöffel, N. Šušnjar, S. Nückel, D. Sieh, P. Burger, „4d vs. 5d - Reactivity and Fate of Terminal Nitrido Complexes of Rhodium and Iridium", Eur. J. Inorg. Chem. 2010, 2010, 49114915, DOI 10.1002/ejic.201000899.

[173]J. Abbenseth, M. Finger, C. Würtele, M. Kasanmascheff, S. Schneider, „Coupling of Terminal Iridium Nitrido Complexes", Inorg. Chem. Front. 2016, 3, 469-477, DOI 10.1039/C5QI00267B.

[174]F. Schendzielorz, master's thesis, Georg-August-Universität, Göttingen, 2014.

[175]M. M. Konnick, S. M. Bischof, D. H. Ess, R. A. Periana, B. G. Hashiguchi, „Base Accelerated Generation of $\mathrm{N}_{2}$ and $\mathrm{NH}_{3}$ from an Osmium Nitride", Journal of Molecular Catalysis A: Chemical 2014, 382, 1-7, DOI 10.1016/j.molcata.2013.10.019.

[176]J. T. Anhaus, T. P. Kee, M. H. Schofield, R. R. Schrock, „Planar "20-Electron" Osmium Imido Complexes. A Linear Imido Ligand Does Not Necessarily Donate Its Lone Pair of Electrons to the Metal", J. Am. Chem. Soc. 1990, 112, 1642-1643, DOI 10.1021/ja00160a061. 
[177]J. R. Wolf, G. C. Bazan, R. R. Schrock, „Exchange of Oxo Ligands in $\mathrm{OsO}_{4}$ with Imido Ligands in $\mathrm{Mo}(\mathrm{NAr})_{2}(\mathrm{O}-t \mathrm{Bu})_{2}$. A Facile Route to $\mathrm{Os}(\mathrm{NAr})_{2} \mathrm{O}_{2}$ and $\mathrm{Os}(\mathrm{NAr})_{3} \mathrm{O}$ and Osmium(IV) Complexes of the Type $\mathrm{Os}(\mathrm{NAr})_{2} \mathrm{~L}_{2}$ (NAr $=\mathrm{N}-2,6-\mathrm{C}_{6} \mathrm{H}_{3}-\mathrm{Iso}_{2}-\mathrm{Pr}_{2} ; \mathrm{L}=$ a Phosphine)", Inorg. Chem. 1993, 32, 4155-4156, DOI 10.1021/ic00071a032.

[178]M. H. V. Huynh, P. S. White, C. A. Carter, T. J. Meyer, „Formation and Reactivity of the Osmium(IV)-Cyanoimido Complex $\left[\mathrm{Os}\left(\mathrm{IV}(\mathrm{bpy})(\mathrm{Cl})_{3}(\mathrm{NCN})\right]^{-“}\right.$, Angew. Chem. Int. Ed. 2001, 40, 3037-3039, DOI 10.1002/1521-3773 (20010817) 40:16<3037: : AID-ANIE3037>3. 0 . CO ;2-0.

[179]M. H. V. Huynh, R. T. Baker, D. L. Jameson, A. Labouriau, T. J. Meyer, „Formation and Reactivity of the Os(IV)-Azidoimido Complex, PPN|[Os(IV)|(bpy)(Cl)3|(N4)]“, J. Am. Chem. Soc. 2002, 124, 4580-4582, DOI 10.1021/ja0122086.

[180]I. Klopsch, PhD thesis, Georg-August-Universität, Göttingen, 2016.

[181]U. Abram, A. Hagenbach, "Trans-[TcNCl $\left.2\left(\mathrm{Ph}_{3} \mathrm{PNH}\right)_{2}\right]$ - Synthesis and Structure", Z. Für Anorg. Allg. Chem. 2002, 628, 1719-1720, DOI 10.1002/1521-3749(200208) 628:8<1719 $::$ AID-ZAAC1719>3.0.CO;2-0.

[182]M. Bertoli, A. Choualeb, D. G. Gusev, A. J. Lough, Q. Major, B. Moore, „PNP Pincer Osmium Polyhydrides for Catalytic Dehydrogenation of Primary Alcohols", Dalton Trans. 2011, 40, 8941-8949, DOI 10.1039/C1DT10342C.

[183]N. Lease, E. M. Pelczar, T. Zhou, S. Malakar, T. J. Emge, F. Hasanayn, K. Krogh-Jespersen, A. S. Goldman, „PNP-Pincer Complexes of Osmium: Comparison with Isoelectronic (PCP)Ir and (PNP)Ir ${ }^{+}$Units“, Organometallics 2018, 37, 314-326, DOI 10.1021/acs . organomet.7b 00738.

[184]I. Ivančič, D. Degobbis, „An Optimal Manual Procedure for Ammonia Analysis in Natural Waters by the Indophenol Blue Method", Water Research 1984, 18, 1143-1147, DOI 10.1016/0043 -1354 (84) 90230-6.

[185]J. Abbenseth, S. C. Bete, M. Finger, C. Volkmann, C. Würtele, S. Schneider, „Four- and FiveCoordinate Osmium(IV) Nitrides and Imides: Circumventing the "Nitrido Wall"', Organometallics 2018, 37, 802-811, DOI 10.1021/acs. organomet.7b00707.

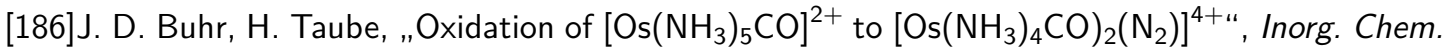
1979, 18, 2208-2212, DOI 10.1021/ic50198a032.

[187]D. C. Ware, H. Taube, „Substitution-Induced Nitrogen-Nitrogen Coupling for Nitride Coordinated to Osmium(VI)", Inorg. Chem. 1991, 30, 4605-4610, DOI 10.1021/ic00024a029.

[188]T. J. Meyer, M. H. V. Huynh, „The Remarkable Reactivity of High Oxidation State Ruthenium and Osmium Polypyridyl Complexes", Inorg. Chem. 2003, 42, 8140-8160, DOI 10.1021/ic 020731v.

[189]H.-W. Lam, C.-M. Che, K.-Y. Wong, „Photoredox Properties of $\left[\mathrm{OsN}\left(\mathrm{NH}_{3}\right)_{4}\right]^{3+}$ and Mechanism of Formation of $\left[\left\{\mathrm{Os}\left(\mathrm{NH}_{3}\right)_{4}\left(\mathrm{CH}_{3} \mathrm{CN}\right)\right\}_{2} \mathrm{~N}_{2}\right]^{5+}$ through a Nitrido-Coupling Reaction", J. Chem. Soc. Dalton Trans. 1992, 0, 1411-1416, DOI 10.1039/DT9920001411.

[190]K. D. Demadis, T. J. Meyer, P. S. White, „Localization in trans, trans-[(Tpy) $(\mathrm{Cl})_{2} \mathrm{Os}(\mathrm{III})\left(\mathrm{N}_{2}\right)$ $\left.\mathrm{Os}(\mathrm{II})(\mathrm{Cl})_{2}(\mathrm{Tpy})\right]^{+}\left(\mathrm{Tpy}=2,2^{4}: 6^{\prime}, 2^{\prime \prime} \text {-Terpyridine }\right)^{\prime \prime}$, Inorg. Chem. 1997, 36, 5678-5679, DOI 10.1021/ic970885o. 
[191]D. Adhikari, F. Basuli, H. Fan, J. C. Huffman, M. Pink, D. J. Mindiola, „P=N Bond Formation via Incomplete N-Atom Transfer from a Ferrous Amide Precursor", Inorg. Chem. 2008, 47, 4439-4441, DOI 10.1021/ic800182m.

[192]V. Vreeken, L. Baij, B. de Bruin, M. A. Siegler, J. I. van der Vlugt, „N-Atom Transfer via Thermal or Photolytic Activation of a Co-Azido Complex with a PNP Pincer Ligand", Dalton Trans. 2017, 46, 7145-7149, DOI 10.1039/C7DT01712J.

[193]Y. Zhang, P. Tong, D. Yang, J. Li, B. Wang, J. Qu, „Migratory Insertion and Hydrogenation of a Bridging Azide in a Thiolate-Bridged Dicobalt Reaction Platform", Chem. Commun. 2017, 53, 9854-9857, DOI 10.1039/C7CC05092E.

[194]V. Vreeken, M. A. Siegler, B. de Bruin, J. N. H. Reek, M. Lutz, J. I. van der Vlugt, „C-H Activation of Benzene by a Photoactivated Ni(II(Azide): Formation of a Transient Nickel Nitrido Complex", Angew. Chem. Int. Ed. 2015, 54, 7055-7059, DOI 10.1002/anie.201501437.

[195]J. Abbenseth, M. Diefenbach, S. C. Bete, C. Würtele, C. Volkmann, S. Demeshko, M. C. Holthausen, S. Schneider, „A Square-Planar Osmium(II) Complex“, Chem. Commun. 2017, DOI 10.1039/C7CC01569K.

[196]A. W. Addison, T. N. Rao, J. Reedijk, J. van Rijn, G. C. Verschoor, „Synthesis, Structure, and Spectroscopic Properties of Copper(II) Compounds Containing Nitrogen-Sulphur Donor Ligands; the Crystal and Molecular Structure of Aqua[1,7-Bis(N-Methylbenzimidazol-2-YI)-2,6Dithiaheptane]Copper(II) Perchlorate“, J. Chem. Soc. Dalton Trans. 1984, 0, 1349-1356, DOI 10.1039/DT9840001349.

[197]S. P. Semproni, W. S. McNeil, R. A. Baillie, B. O. Patrick, C. F. Campana, P. Legzdins, „GroundState Electronic Asymmetry in $\mathrm{Cp}^{*} \mathrm{~W}(\mathrm{NO})\left(\eta_{1} \text {-Isonitrile }\right)_{2}$ Complexes", Organometallics 2010 , 29, 867-875, DOI 10.1021/om901018n.

[198]J. Bauer, H. Braunschweig, C. Hörl, K. Radacki, J. Wahler, „Synthesis of Zwitterionic Cobaltocenium Borate and Borata-Alkene Derivatives from a Borole-Radical Anion“, Chem. - Eur. J. 2013, 19, 13396-13401, DOI 10.1002/chem.201302201.

[199]P. O. Lagaditis, B. Schluschaß, S. Demeshko, C. Würtele, S. Schneider, „Square-Planar Cobalt(iii) Pincer Complex", Inorg. Chem. 2016, 55, 4529-4536, DOI 10.1021 /acs . inorgchem.6b 00369.

[200]F. Schneck, M. Finger, M. Tromp, S. Schneider, „Chemical Non-Innocence of an Aliphatic PNP Pincer Ligand“, Chem. Eur. J. 2017, 23, 33-37, DOI 10.1002/chem. 201604407.

[201]W. E. Truce, D. G. Brady, „Stereochemistry of Amine Additions to Acetylenic Sulfones and Carboxylic Esters", J. Org. Chem. 1966, 31, 3543-3550, DOI 10.1021/jo01349a018.

[202]T. L. Brown, K. J. Lee, „Ligand Steric Properties“, Coordination Chemistry Reviews 1993, 128, 89-116, DOI 10.1016/0010-8545(93)80025-Z.

[203]B. N. Figgis, J. Lewis, „The Magnetic Properties of Transition Metal Complexes“, Prog. Inorg. Chem., Progress in Inorganic Chemistry 1964, DOI 10.1002/9780470166079.ch2.

[204]J. Chatt, G. J. Leigh, D. M. P. Mingos, „Configurations of Some Complexes of Rhenium, Ruthenium, Osmium, Rhodium, Iridium, and Platinum Halides with Mono(Tertiary Phosphines) and Mono(Tertiary Arsines)“, J. Chem. Soc. A 1969, 0, 1674-1680, DOI 10.1039/J1969000 1674 
[205]D. F. Evans, „400. The Determination of the Paramagnetic Susceptibility of Substances in Solution by Nuclear Magnetic Resonance“, J. Chem. Soc. 1959, 0, 2003-2005, DOI 10.1039 /JR9590002003.

[206]P. Zanello, Inorganic Electrochemistry: Theory, Practice and Application, The Royal Society of Chemistry, 2003, DOI 10.1039/9781847551146.

[207]C. Kiene, Bachelor's thesis, Georg-August-Universität, Göttingen, 2019.

[208]C. A. Tolman, „Steric Effects of Phosphorus Ligands in Organometallic Chemistry and Homogeneous Catalysis“, Chem. Rev. 1977, 77, 313-348, DOI 10.1021/cr60307a002.

[209]M. Käß, A. Friedrich, M. Drees, S. Schneider, „Ruthenium Complexes with Cooperative PNP Ligands: Bifunctional Catalysts for the Dehydrogenation of Ammonia-Borane", Angew. Chem. Int. Ed. 2009, 48, 905-907, DOI 10.1002/anie.200805108.

[210]B. Askevold, M. M. Khusniyarov, E. Herdtweck, K. Meyer, S. Schneider, „A Square-Planar Ruthenium(II) Complex with a Low-Spin Configuration“, Angew. Chem. Int. Ed. 2010, 49, 7566-7569, DOI 10.1002/anie.201002296.

[211]F. J. V. Santos, C. A. Nieto de Castro, J. H. Dymond, N. K. Dalaouti, M. J. Assael, A. Nagashima, „Standard Reference Data for the Viscosity of Toluene“, J. Phys. Chem. Ref. Data 2006, 35, 1-8, DOI 10.1063/1.1928233.

[212] Tetrahydrofuran - High Purity Solvents, https://www.sigmaaldrich.com/chemistry/solv ents/tetrahydrofuran-center.html (visited on Feb. 15, 2019).

[213]Nitrogen, https: //webbook.nist.gov/cgi/cbook. cgi?ID=C7727379\&Units=SI\&Mask=10 00\#Diatomic (visited on Feb. 19, 2019).

[214]J. Chatt, J. R. Dilworth, R. L. Richards, „Recent Advances in the Chemistry of Nitrogen Fixation", Chem. Rev. 1978, 78, 589-625, DOI 10.1021/cr60316a001.

[215]F. Neese, „Prediction of Molecular Properties and Molecular Spectroscopy with Density Functional Theory: From Fundamental Theory to Exchange-Coupling", Coordination Chemistry Reviews, Theory and Computing in Contemporary Coordination Chemistry 2009, 253, 526-563, DOI $10.1016 / j . c c r .2008 .05 .014$.

[216]G. Christian, R. Stranger, B. F. Yates, C. C. Cummins, „Rationalizing the Different Products in the Reaction of $\mathrm{N}_{2}$ with Three-Coordinate $\mathrm{MoL}_{3}$ Complexes“, Dalton Trans. 2007, 0, 19391947, DOI 10.1039/B701050H.

[217]T. Rodima, I. Kaljurand, A. Pihl, V. Mäemets, I. Leito, I. A. Koppel, „Acid-Base Equilibria in Nonpolar Media. 2. Self-Consistent Basicity Scale in THF Solution Ranging from 2Methoxypyridine to EtP 1 (Pyrr) Phosphazene“, J. Org. Chem. 2002, 67, 1873-1881, DOI 10.1 021/jo016185p.

[218]K. C. Mullane, H. Ryu, T. Cheisson, L. N. Grant, J. Y. Park, B. C. Manor, P. J. Carroll, M.-H. Baik, D. J. Mindiola, E. J. Schelter, „C-H Bond Addition across a Transient Uranium-Nitrido Moiety and Formation of a Parent Uranium Imido Complex", J. Am. Chem. Soc. 2018, 140, 11335-11340, DOI 10.1021/jacs.8b06090.

[219]J. R. Dilworth, J. S. Lewis, J. R. Miller, Y. Zheng, „Rhenium Complex of a Triply Deprotonated Chelated Thiosemicarbazide and Its Conversion to a Nitride Complex via a Hydrazide Intermediate. Crystal Structure of $\left[\mathrm{ReCl}_{2}(\mathrm{NH})\left(\mathrm{NHNH}_{2}\right)\left(\mathrm{PPh}_{3}\right)_{2}\right]^{\prime \prime}$, J. Chem. Soc. Dalton Trans. 1995, 0, 1357-1361, DOI 10.1039/DT9950001357. 
[220]A. Haug, J. Strähle, „Synthese und Struktur von $\left[\mathrm{Re}(\mathrm{NH}) \mathrm{Cl}_{2}\left(\mathrm{PMe}_{2} \mathrm{Ph}\right)_{3}\right]\left[\mathrm{TaCl}_{6}\right]$ und $\left[\left(\mathrm{Me}_{2} \mathrm{PhP}\right)_{3}\right.$ $\mathrm{Cl}_{2} \mathrm{Re}=\mathrm{N}_{2} \mathrm{VOCl}_{2}{ }^{\prime \prime}$, Z. Für Anorg. Allg. Chem. 1998, 624, 1746-1750, DOI 10.1002/(SICI) 1 521-3749(1998110)624:11<1746: :AID-ZAAC1746>3. 0. C0;2-2.

[221]W.-H. Leung, J. L. C. Chim, I. D. Williams, W.-T. Wong, „Reactions of Nitridorhenium(v) and -Osmium(VI) Complexes with Acylating Agents", Inorg. Chem. 1999, 38, 3000-3005, DOI 10.1021/ic981244b.

[222]D. Davidson, H. Skovronek, „The Acylation of Amides“, J. Am. Chem. Soc. 1958, 80, 376-379, DOI 10.1021/ja01535a032.

[223]B. M. Lindley, Q. J. Bruch, P. S. White, F. Hasanayn, A. J. M. Miller, „Ammonia Synthesis from a Pincer Ruthenium Nitride via Metal-Ligand Cooperative Proton-Coupled Electron Transfer", J. Am. Chem. Soc. 2017, 139, 5305-5308, DOI 10.1021/jacs.7b01323.

[224]D. Barrón, J. Barbosa, „Acid-Base Behaviour of Substituted Phenolic Substances and Resolution of Acid Strength in Tetrahydrofuran", Analytica Chimica Acta 2000, 403, 339-347, DOI 10.1016/S0003-2670 (99) 00635-2.

[225]J. Barbosa, D. Barrón, E. Bosch, M. Rosés, „Resolution of Acid Strength in Tetrahydrofuran of Substituted Benzoic Acids", Analytica Chimica Acta 1992, 265, 157-165, DOI 10.1016/0 003-2670 (92) 85165-3.

[226]J. Abbenseth, PhD thesis, Georg-August-Universität, Göttingen, 2019.

[227]C. He, M. J. Gaunt, „Ligand-Enabled Catalytic C-H Arylation of Aliphatic Amines by a FourMembered-Ring Cyclopalladation Pathway“, Angew. Chem. Int. Ed. 2015, 54, 15840-15844, DOI 10.1002/anie.201508912.

[228]J. Meiners, A. Friedrich, E. Herdtweck, S. Schneider, „Facile Double C-H Activation of Tetrahydrofuran by an Iridium PNP Pincer Complex“, Organometallics 2009, 28, 6331-6338, DOI 10.1021/om9006906.

[229]G. W. Parshall, L. W. Shive, F. A. Cotton in Inorganic Syntheses, Vol. XVII, John Wiley \& Sons, Ltd, 1977, pp. 110-112, DOI 10.1002/9780470132487.ch31.

[230]G. Rouschias, G. Wilkinson, „The Preparation and Reactions of Trihaolgeno(Alkanonitrile)Bis(Triphenylphosphine)Rhenium(III) Complexes", J. Chem. Soc. Inorg. Phys. Theor. 1967, 993, DOI 10.1039/j19670000993.

[231]S. H. Babcock, H. P. Lankelma, E. Vopicka in Inorganic Syntheses, Vol. I, John Wiley \& Sons, Ltd, 1939, pp. 10-11, DOI 10.1002/9780470132326.ch4.

[232]J.-M. Lalancette, G. Rollin, P. Dumas, „Metals Intercalated in Graphite. I. Reduction and Oxidation", Can. J. Chem. 1972, 50, 3058-3062, DOI 10.1139/v72-485.

[233]V. W. Manner, T. F. Markle, J. H. Freudenthal, J. P. Roth, J. M. Mayer, „The First Crystal Structure of a Monomeric Phenoxyl Radical: 2,4,6-Tri-Tert-Butylphenoxyl Radical“", Chem. Commun. 2007, 256-258, DOI 10.1039/B712872J.

[234]M. Brookhart, B. Grant, A. F. Volpe, ,[(3,5- $\left.\left.\left(\mathrm{CF}_{3}\right)_{2} \mathrm{C}_{6} \mathrm{H}_{3}\right)_{4} \mathrm{~B}\right]^{-}\left[\mathrm{H}\left(\mathrm{OEt}_{2}\right)_{2}\right]^{+}$: A Convenient Reagent for Generation and Stabilization of Cationic, Highly Electrophilic Organometallic Complexes", Organometallics 1992, 11, 3920-3922, DOI 10.1021/om00059a071.

[235]A. Martinsen, J. Songstad, R. Larsson, M. Pouchard, P. Hagenmuller, A. F. Andresen, „Preparation and Properties of Some Bis(Triphenylphosphine)Iminium Salts, $\left[\left(\mathrm{Ph}_{3} \mathrm{P}\right)_{2} \mathrm{~N}\right] \mathrm{X}$. ", Acta Chem. Scand. 1977, 31a, 645-650, DOI 10.3891/acta.chem.scand.31a-0645. 
[236]D. Adhikari, S. Mossin, F. Basuli, J. C. Huffman, R. K. Szilagyi, K. Meyer, D. J. Mindiola, „Structural, Spectroscopic, and Theoretical Elucidation of a Redox-Active Pincer-Type Ancillary Applied in Catalysis", J. Am. Chem. Soc. 2008, 130, 3676-3682, DOI 10.1021/ja7108486.

[237]T. J. Herrington, A. J. W. Thom, A. J. P. White, A. E. Ashley, „Novel $\mathrm{H}_{2}$ Activation by a Tris[3,5-Bis(Trifluoromethyl)Phenyl]Borane Frustrated Lewis Pair", Dalton Trans. 2012, 41, 9019-9022, DOI 10.1039/C2DT30384A.

[238]APEX3 V2016.9-0 (SAINT/SADABS/SHELXT/SHELXL), Bruker AXS Inc., 2016.

[239]G. M. Sheldrick, „SHELXT - Integrated Space-Group and Crystal-Structure Determination“, Acta Cryst A 2015, 71, 3-8, DOI 10.1107/S2053273314026370.

[240]G. M. Sheldrick, „Crystal Structure Refinement with SHELXL“, Acta Cryst C 2015, 71, 3-8, DOI $10.1107 /$ S2053229614024218.

[241]G. M. Sheldrick, „A Short History of SHELX“, Acta Cryst A 2008, 64, 112-122, DOI 10.110 7/S0108767307043930.

[242]N. Elgrishi, K. J. Rountree, B. D. McCarthy, E. S. Rountree, T. T. Eisenhart, J. L. Dempsey, "A Practical Beginner's Guide to Cyclic Voltammetry“, J. Chem. Educ. 2018, 95, 197-206, DOI 10.1021/acs. jchemed.7b00361.

[243]G. A. Bain, J. F. Berry, „Diamagnetic Corrections and Pascal's Constants“, J. Chem. Educ. 2008, 85, 532, DOI 10.1021/ed085p532.

[244]W. Haberditzl, „Advances in Molecular Diamagnetism“, Angew. Chem. Int. Ed. Engl. 1966, 5, 288-298, DOI 10.1002/anie.196602881.

[245]E. Bill, JULX, Program for Simulation of Molecular Magnetic Data, Max-Planck Institute for Chemical Energy Conversion, Mülheim/Ruhr, 2008.

[246]F. Neese, „The ORCA Program System“, Wiley Interdiscip. Rev. Comput. Mol. Sci. 2012, 2, 73-78, DOI 10.1002/wcms.81.

[247]F. Neese, "Software Update: The ORCA Program System, Version 4.0“, Wiley Interdiscip. Rev. Comput. Mol. Sci. 2018, 8, e1327, DOI 10.1002/wcms.1327.

[248]J. P. Perdew, K. Burke, M. Ernzerhof, "Generalized Gradient Approximation Made Simple“, Phys. Rev. Lett. 1996, 77, 3865-3868, DOI 10.1103/PhysRevLett.77.3865.

[249]J. P. Perdew, M. Ernzerhof, K. Burke, „Rationale for Mixing Exact Exchange with Density Functional Approximations", J. Chem. Phys. 1996, 105, 9982-9985, DOI 10.1063/1.472933.

[250]F. Weigend, R. Ahlrichs, „Balanced Basis Sets of Split Valence, Triple Zeta Valence and Quadruple Zeta Valence Quality for H to Rn: Design and Assessment of Accuracy“, Phys. Chem. Chem. Phys. 2005, 7, 3297-3305, DOI 10.1039/B508541A.

[251]D. Andrae, U. Häußermann, M. Dolg, H. Stoll, H. Preuß, „Energy-Adjusted Ab Initio Pseudopotentials for the Second and Third Row Transition Elements", Theoret. Chim. Acta 1990, 77, 123-141, DOI 10.1007/BF01114537.

[252]O. Treutler, R. Ahlrichs, „Efficient Molecular Numerical Integration Schemes“, J. Chem. Phys. 1995, 102, 346-354, DOI 10.1063/1.469408.

[253]K. Eichkorn, O. Treutler, H. Öhm, M. Häser, R. Ahlrichs, „Auxiliary Basis Sets to Approximate Coulomb Potentials", Chem. Phys. Letters 1995, 240, 283-290, DOI 10.1016/0009-2614 (9 5) 00621-A. 
[254]K. Eichkorn, O. Treutler, H. Öhm, M. Häser, R. Ahlrichs, „Auxiliary Basis Sets to Approximate Coulomb Potentials (Chem. Phys. Letters 240 (1995) 283-290)“, Chem. Phys. Letters 1995, 242, 652-660, DOI 10.1016/0009-2614(95)00838-U.

[255]K. Eichkorn, F. Weigend, O. Treutler, R. Ahlrichs, „Auxiliary Basis Sets for Main Row Atoms and Transition Metals and Their Use to Approximate Coulomb Potentials", Theor Chem Acta 1997, 97, 119-124, DOI 10.1007/s002140050244.

[256]F. Neese, F. Wennmohs, A. Hansen, U. Becker, „Efficient, Approximate and Parallel Hartree-Fock and Hybrid DFT Calculations. A 'Chain-of-Spheres' Algorithm for the Hartree-Fock Exchange", Chemical Physics, Moving Frontiers in Quantum Chemistry: 2009, 356, 98-109, DOI 10.101 6/j.chemphys.2008.10.036.

[257]F. Weigend, „Accurate Coulomb-Fitting Basis Sets for H to Rn“, Phys. Chem. Chem. Phys. 2006, 8, 1057-1065, DOI 10.1039/B515623H.

[258]S. Grimme, J. Antony, S. Ehrlich, H. Krieg, „A Consistent and Accurate Ab Initio Parametrization of Density Functional Dispersion Correction (DFT-D) for the 94 Elements H-Pu", J. Chem. Phys. 2010, 132, 154104, DOI 10.1063/1.3382344.

[259]S. Grimme, S. Ehrlich, L. Goerigk, „Effect of the Damping Function in Dispersion Corrected Density Functional Theory“, J. Comput. Chem. 2011, 32, 1456-1465, DOI 10.1002/ jcc .21759 .

[260]S. Grimme, „Supramolecular Binding Thermodynamics by Dispersion-Corrected Density Functional Theory", Chem. - Eur. J. 2012, 18, 9955-9964, DOI 10.1002/chem.201200497.

[261]A. Klamt, G. Schüürmann, „COSMO: A New Approach to Dielectric Screening in Solvents with Explicit Expressions for the Screening Energy and Its Gradient", J. Chem. Soc. Perkin Trans. 2 1993, 0, 799-805, DOI 10.1039/P29930000799.

[262]V. Barone, M. Cossi, „Quantum Calculation of Molecular Energies and Energy Gradients in Solution by a Conductor Solvent Model“", J. Phys. Chem. A 1998, 102, 1995-2001, DOI 10.1 021/jp9716997.

[263]Chemcraft - graphical software for visualization of quantum chemistry computations. https://www.chemcraftprog.com.

[264]D. A. Pantazis, X.-Y. Chen, C. R. Landis, F. Neese, „All-Electron Scalar Relativistic Basis Sets for Third-Row Transition Metal Atoms", J. Chem. Theory Comput. 2008, 4, 908-919, DOI 10.1021/ct800047t.

[265]C. Angeli, R. Cimiraglia, S. Evangelisti, T. Leininger, J.-P. Malrieu, „Introduction of N-Electron Valence States for Multireference Perturbation Theory“, J. Chem. Phys. 2001, 114, 1025210264, DOI 10.1063/1.1361246.

[266]C. Angeli, R. Cimiraglia, J.-P. Malrieu, „N-Electron Valence State Perturbation Theory: A Fast Implementation of the Strongly Contracted Variant" ${ }^{\prime \prime}$ Chemical Physics Letters 2001, 350, 297305, DOI 10.1016/S0009-2614(01) 01303-3.

[267]C. Angeli, R. Cimiraglia, J.-P. Malrieu, „N-Electron Valence State Perturbation Theory: A Spinless Formulation and an Efficient Implementation of the Strongly Contracted and of the Partially Contracted Variants", J. Chem. Phys. 2002, 117, 9138-9153, DOI 10.1063/1.1515 317. 
[268]B. A. HeB, C. M. Marian, U. Wahlgren, O. Gropen, „A Mean-Field Spin-Orbit Method Applicable to Correlated Wavefunctions", Chemical Physics Letters 1996, 251, 365-371, DOI 10.1016/0 009-2614 (96)00119-4.

[269]F. Schendzielorz, M. Finger, J. Abbenseth, C. Würtele, V. Krewald, S. Schneider, „Metal-Ligand Cooperative Synthesis of Benzonitrile by Electrochemical Reduction and Photolytic Splitting of Dinitrogen“, Angew. Chem. 2019, 131, 840-844, DOI 10.1002/ange. 201812125. 



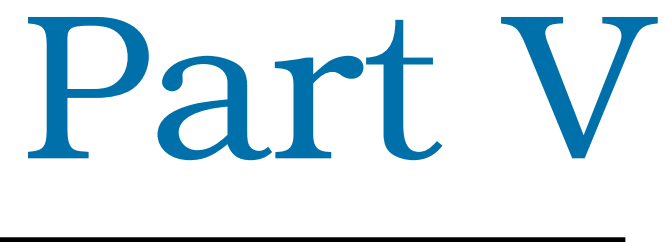

Appendix 



\section{Crystal structures}

\section{A. $1\left[\mathrm{Os}\left(\mathrm{NBAr}_{18}^{\mathrm{F}}\right)\left(\mathrm{PNP}^{\mathrm{tBu}}\right)\right](5)$}

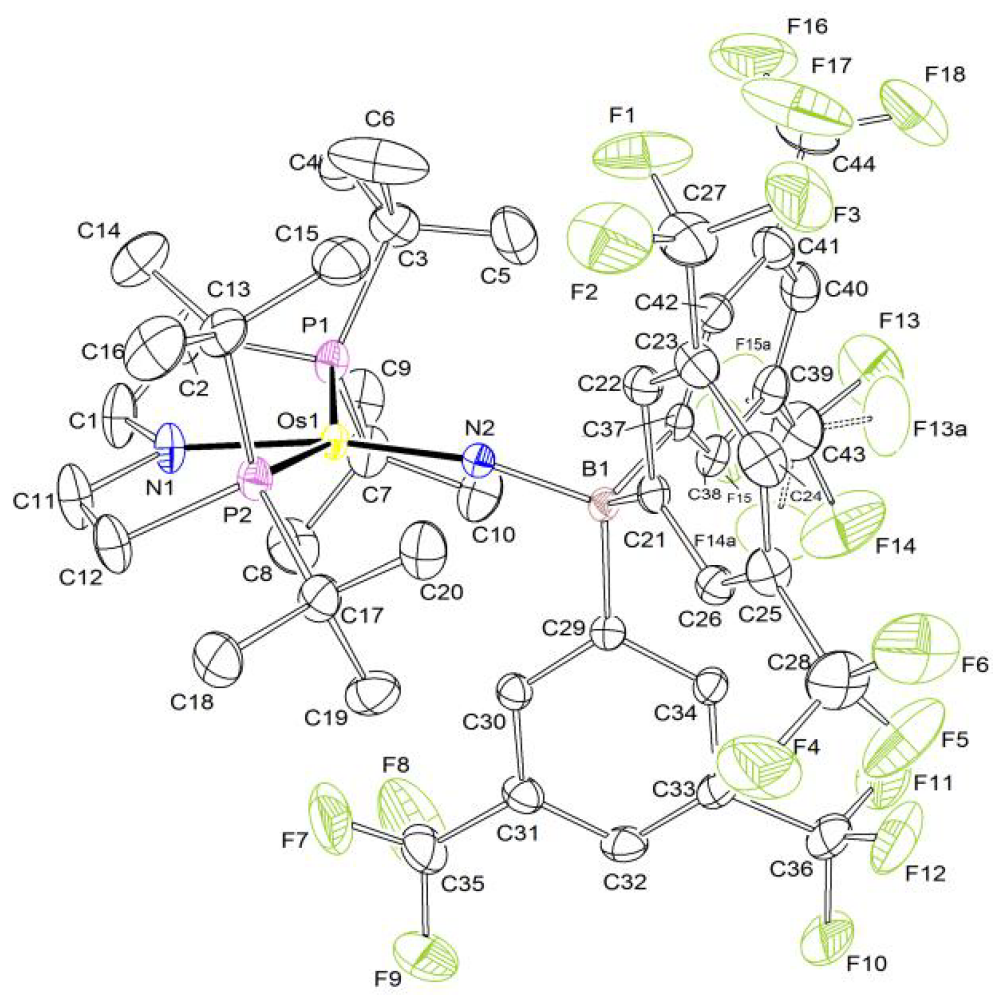

Fig. A.1. Thermal ellipsoid plot of $\mathbf{5}$ with the anisotropic displacement parameters drawn at the $50 \%$ probability level. The asymmetric unit contains one disordered complex molecule. The disordered complex molecule was refined with population of $0.67(2)$ on the main domain using some restraints (SADI, RIGU).

Tab. A.1. Crystal data and structure refinement of $\mathbf{5}$.

\begin{tabular}{l|l}
\hline Identification code & $\mathbf{5}$ \\
\hline Empirical formula & $\mathrm{C}_{44} \mathrm{H}_{53} \mathrm{BF}_{18} \mathrm{~N}_{2} \mathrm{OsP}_{2}$ \\
\hline Formula weight & 1214.83 \\
\hline Temperature & $173(2) \mathrm{K}$ \\
\hline Wavelength & $0.71073 \AA$ \\
\hline Crystal system & Monoclinic \\
\hline
\end{tabular}




\begin{tabular}{|c|c|c|}
\hline Space group & \multicolumn{2}{|l|}{$P 2_{1} / \mathrm{n}$} \\
\hline Unit cell dimensions & $\begin{array}{l}\mathrm{a}=14.2710(5) \AA \\
\mathrm{b}=22.0590(7) \AA \\
\mathrm{c}=15.7378(5) \AA\end{array}$ & $\begin{array}{l}\text { alpha }=90^{\circ} \\
\text { beta }=90.4440(10)^{\circ} \\
\text { gamma }=90^{\circ}\end{array}$ \\
\hline Volume & \multicolumn{2}{|l|}{$4954.2(3) \AA^{3}$} \\
\hline Z & \multicolumn{2}{|l|}{4} \\
\hline Density (calculated) & \multicolumn{2}{|l|}{$1.629 \mathrm{Mg} / \mathrm{m}^{3}$} \\
\hline Absorption coefficient & \multicolumn{2}{|l|}{$2.740 \mathrm{~mm}^{-1}$} \\
\hline$F(000)$ & \multicolumn{2}{|l|}{2416} \\
\hline Crystal size & \multicolumn{2}{|c|}{$0.241 \times 0.162 \times 0.153 \mathrm{~mm}^{3}$} \\
\hline Crystal shape and color & \multicolumn{2}{|c|}{ Block, dark red } \\
\hline Theta range for data collection & \multicolumn{2}{|l|}{2.255 to $30.605^{\circ}$} \\
\hline Index ranges & \multicolumn{2}{|c|}{$-20 \leq h \leq 20,-31 \leq k \leq 31,-22 \leq \mathrm{I} \leq 22$} \\
\hline Reflections collected & \multicolumn{2}{|l|}{216917} \\
\hline Independent reflections & \multicolumn{2}{|c|}{$15244[\mathrm{R}($ int $)=0.0544]$} \\
\hline Completeness to theta $=25.242^{\circ}$ & \multicolumn{2}{|c|}{$99.90 \%$} \\
\hline Refinement method & \multicolumn{2}{|c|}{ Full-matrix least-squares on $\mathrm{F}^{2}$} \\
\hline Data / restraints / parameters & \multicolumn{2}{|l|}{15244 / 366 / 653} \\
\hline Goodness-of-fit on $F^{2}$ & \multicolumn{2}{|l|}{1.066} \\
\hline Final $R$ indices $[I>2 \operatorname{sigma}(I)]$ & $\mathrm{R} 1=0.0265$ & $w R 2=0.0482$ \\
\hline $\mathrm{R}$ indices (all data) & $\mathrm{R} 1=0.0499$ & $\mathrm{wR} 2=0.0551$ \\
\hline Largest diff. peak and hole & \multicolumn{2}{|c|}{0.795 and $-0.746 \mathrm{e}^{-3}$} \\
\hline
\end{tabular}




\section{A.2 $\left[\mathrm{Os}\left(\mathrm{NSiMe}_{3}\right)\left(\mathrm{PNP}^{t \mathrm{Bu}}\right)\right]^{\mathrm{BPh}_{4}}\left(6^{\mathrm{BPh}_{4}}\right)$}

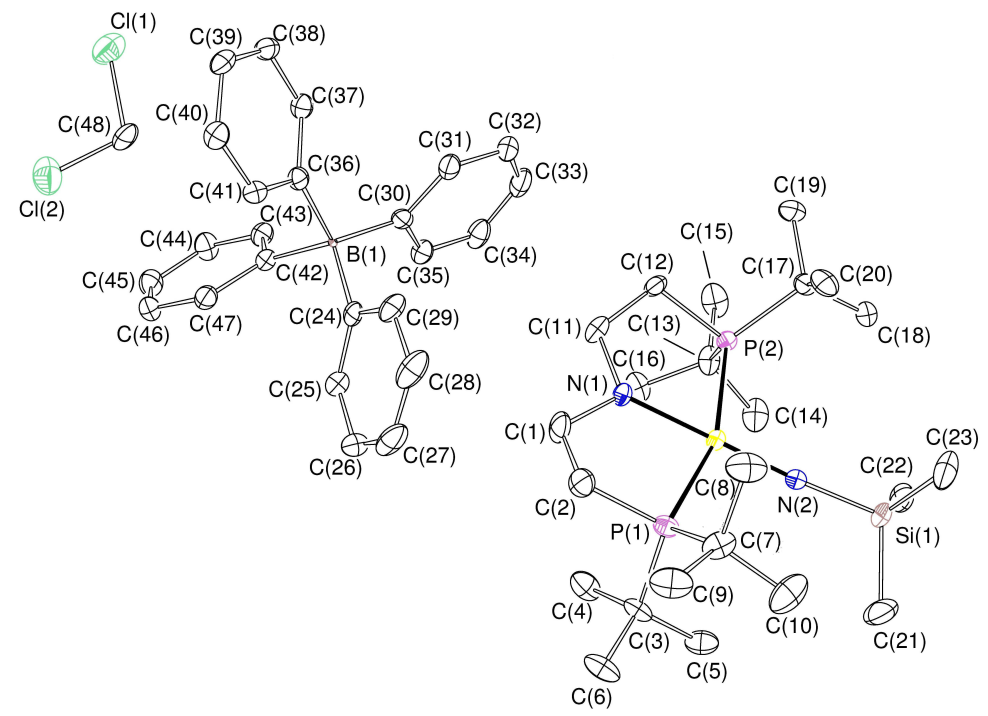

Fig. A.2. Thermal ellipsoid plot of $6^{\mathrm{BPh}_{4}}$ with the anisotropic displacement parameters drawn at the $50 \%$ probability level. All hydrogen atoms are omitted for clarity. The asymmetric unit contains one complex molecule, one $\mathrm{CH}_{2} \mathrm{Cl}_{2}$ solvent molecule and two different anions at the same position $\left(\mathrm{BPh}_{4}^{-}\right.$and $\left.\mathrm{Br}^{-}\right)$. The two alternative anions were refined with occupation factors of 0.95 for $\mathrm{BPh}_{4}^{-}$and 0.05 for $\mathrm{Br}^{-}$using EXYZ and EADP commands. Possible room filling solvent molecules next to $\mathrm{Br}^{-}$were not found due to overlay with phenyl groups of $\mathrm{BPh}_{4}^{-}$and low occupancy.

Tab. A.2. Crystal data and structure refinement of $6^{\mathrm{BPh}_{4}}$.

\begin{tabular}{|c|c|c|}
\hline Identification code & \multicolumn{2}{|l|}{$6^{\mathrm{BPh}_{4}}$} \\
\hline Empirical formula & \multicolumn{2}{|c|}{$\mathrm{C}_{24} \mathrm{H}_{55} \mathrm{Cl}_{2} \mathrm{~N}_{2} \mathrm{OsP}_{2} \mathrm{Si}\left(\mathrm{BC}_{24} \mathrm{H}_{20}\right)_{0.95}(\mathrm{Br})_{0.05}$} \\
\hline Formula weight & \multicolumn{2}{|l|}{1030.07} \\
\hline Temperature & \multicolumn{2}{|l|}{$100(2) \mathrm{K}$} \\
\hline Wavelength & \multicolumn{2}{|l|}{$0.71073 \AA$} \\
\hline Crystal system & \multicolumn{2}{|l|}{ Monoclinic } \\
\hline Space group & \multicolumn{2}{|l|}{$P 22_{1} / \mathrm{c}$} \\
\hline Unit cell dimensions & $\begin{array}{l}\mathrm{a}=17.7529(7) \AA \\
\mathrm{b}=21.2994(7) \AA \\
\mathrm{c}=13.7921(5) \AA\end{array}$ & $\begin{array}{l}\alpha=90^{\circ} \\
\beta=109.088(2)^{\circ} \\
\gamma=90^{\circ}\end{array}$ \\
\hline Volume & \multicolumn{2}{|l|}{$4928.4(3) \AA^{3}$} \\
\hline Z & \multicolumn{2}{|l|}{4} \\
\hline Density (calculated) & \multicolumn{2}{|l|}{$1.388 \mathrm{Mg} / \mathrm{m}^{3}$} \\
\hline
\end{tabular}




\begin{tabular}{l|l} 
Absorption coefficient & $2.858 \mathrm{~mm}^{-1}$ \\
\hline $\mathrm{F}(000)$ & 2117 \\
\hline Crystal size & $0.162 \times 0.103 \times 0.065 \mathrm{~mm}^{3}$ \\
\hline Crystal shape and color: & Block, green-brown \\
\hline Theta range for data collection & 2.265 to $30.508^{\circ}$ \\
\hline Index ranges & $-24 \leq \mathrm{h} \leq 25,-30 \leq \mathrm{k} \leq 30,-19 \leq \mathrm{I} \leq 19$ \\
\hline Reflections collected & 100115 \\
\hline Independent reflections & $14845[\mathrm{R}(\mathrm{int})=0.1174]$ \\
\hline Completeness to theta $=25.242^{\circ}$ & $99.90 \%$ \\
\hline Absorption correction & Numerical \\
\hline Max. and min. transmission & 0.9393 and 0.7241 \\
\hline Refinement method & Full-matrix least-squares on $\mathrm{F}^{2}$ \\
\hline Data / restraints / parameters & $14845 / 0 / 529$ \\
\hline Goodness-of-fit on $\mathrm{F}^{2}$ & 1.16 \\
\hline Final R indices [l $>2$ sigma(I)] & $\mathrm{R} 1=0.0493$ \\
\hline $\mathrm{R}$ indices (all data) & $\mathrm{R} 1=0.1099$ \\
\hline Largest diff. peak and hole & 2.098 and -1.723 eA ${ }^{-3}$ \\
\hline
\end{tabular}




\section{A.3 $\left[\mathrm{Os}\left(\mathrm{NPMe}_{3}\right)\left(\mathrm{PNP}^{\mathrm{tBu}}\right)\right](7)$}

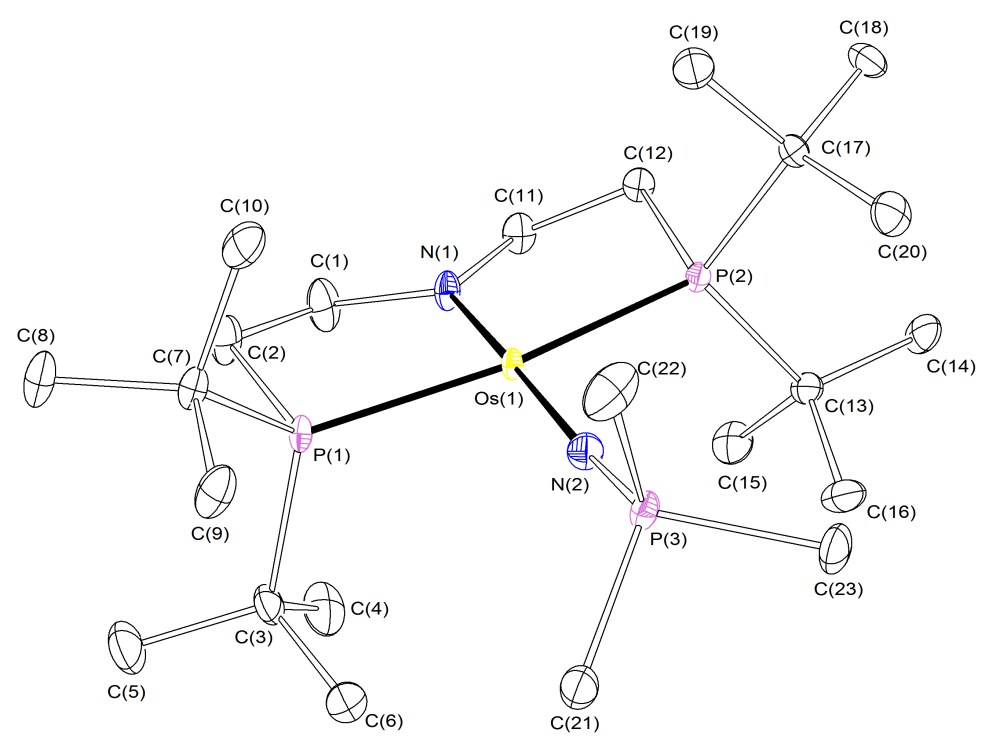

Fig. A.3. Thermal ellipsoid plot of $\mathbf{7}$ with the anisotropic displacement parameters drawn at the $50 \%$ probability level All hydrogen atoms are omitted for clarity.

Tab. A.3. Crystal data and structure refinement of 7 .

\begin{tabular}{|c|c|c|}
\hline Identification code & \multicolumn{2}{|l|}{7} \\
\hline Empirical formula & \multicolumn{2}{|l|}{$\mathrm{C}_{23} \mathrm{H}_{53} \mathrm{~N}_{2} \mathrm{OsP}_{3}$} \\
\hline Formula weight & \multicolumn{2}{|l|}{640.78} \\
\hline Temperature & \multicolumn{2}{|l|}{$100(2) \mathrm{K}$} \\
\hline Wavelength & \multicolumn{2}{|l|}{$0.71073 \AA$} \\
\hline Crystal system & \multicolumn{2}{|l|}{ Monoclinic } \\
\hline Space group & \multicolumn{2}{|l|}{$P 2_{1} / \mathrm{c}$} \\
\hline Unit cell dimensions & $\begin{array}{l}\mathrm{a}=11.2796(7) \AA \\
\mathrm{b}=16.1565(10) \AA \\
\mathrm{c}=15.9319(11) \AA\end{array}$ & $\begin{array}{l}\alpha=90^{\circ} \\
\beta=100.141(3)^{\circ} \\
\gamma=90^{\circ}\end{array}$ \\
\hline Volume & \multicolumn{2}{|l|}{$2858.1(3) \AA^{3}$} \\
\hline Z & \multicolumn{2}{|l|}{4} \\
\hline Density (calculated) & \multicolumn{2}{|l|}{$1.489 \mathrm{Mg} / \mathrm{m}^{3}$} \\
\hline Absorption coefficient & \multicolumn{2}{|l|}{$4.642 \mathrm{~mm}^{-1}$} \\
\hline$F(000)$ & \multicolumn{2}{|l|}{1304} \\
\hline Crystal size & \multicolumn{2}{|c|}{$0.228 \times 0.216 \times 0.138 \mathrm{~mm}^{3}$} \\
\hline
\end{tabular}




\begin{tabular}{|c|c|c|}
\hline Crystal shape and color & \multicolumn{2}{|c|}{ Block, clear light brown } \\
\hline Theta range for data collection & \multicolumn{2}{|c|}{2.226 to $28.372^{\circ}$} \\
\hline Index ranges & \multicolumn{2}{|c|}{$-15 \leq h \leq 15,-21 \leq k \leq 21,-21 \leq \mathrm{I} \leq 20$} \\
\hline Reflections collected & \multicolumn{2}{|l|}{41683} \\
\hline Independent reflections & \multicolumn{2}{|c|}{$7138[R($ int $)=0.0536]$} \\
\hline Completeness to theta $=25.242^{\circ}$ & \multicolumn{2}{|l|}{$100.00 \%$} \\
\hline Absorption correction & \multicolumn{2}{|c|}{ Semi-empirical from equivalents } \\
\hline Max. and min. transmission & \multicolumn{2}{|c|}{0.7457 and 0.5893} \\
\hline Refinement method & \multicolumn{2}{|c|}{ Full-matrix least-squares on $\mathrm{F}^{2}$} \\
\hline Data / restraints / parameters & \multicolumn{2}{|c|}{$7138 / 0 / 277$} \\
\hline Goodness-of-fit on $\mathrm{F}^{2}$ & \multicolumn{2}{|l|}{1.027} \\
\hline Final R indices $[I>2 \operatorname{sigma}(I)]$ & $\mathrm{R} 1=0.0265$ & $\mathrm{wR} 2=0.0458$ \\
\hline $\mathrm{R}$ indices (all data) & $\mathrm{R} 1=0.0404$ & $\mathrm{wR} 2=0.0491$ \\
\hline Largest diff. peak and hole & \multicolumn{2}{|c|}{1.080 and $-0.937 \mathrm{e}^{-3}$} \\
\hline
\end{tabular}




\section{A.4 $\left[\mathrm{OsH}_{4}\left(\mathrm{HPNP}^{t \mathrm{Bu}}\right)\right](8)$}

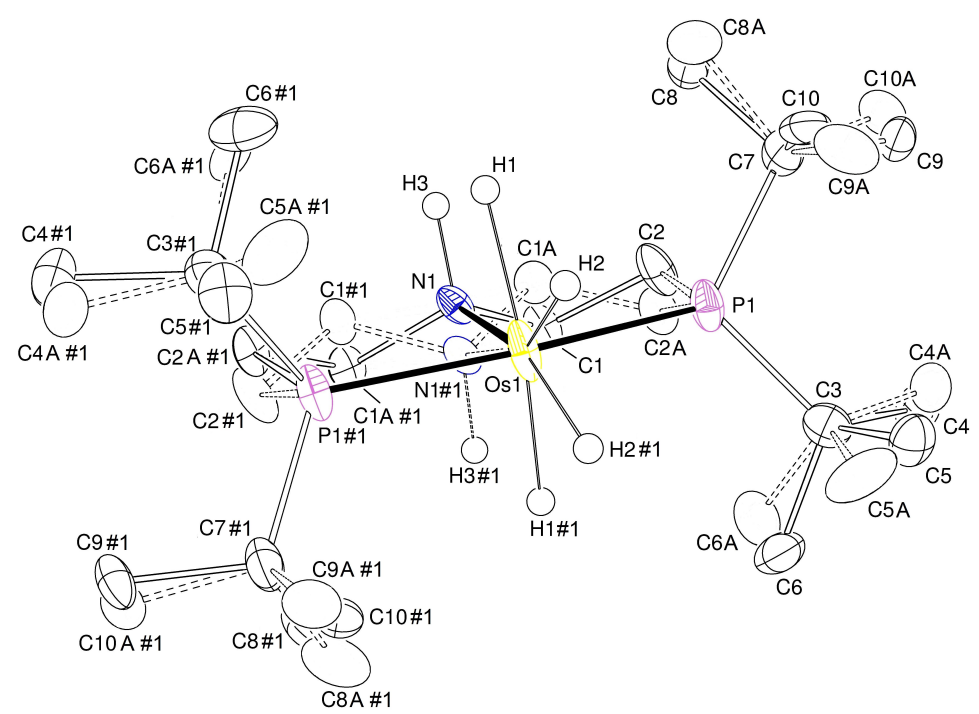

Fig. A.4. Thermal ellipsoid plot of 4 with the anisotropic displacement parameters drawn at the $50 \mathrm{i} \%$ probability level. All $\mathrm{C}-\mathrm{H}$ hydrogen atoms are omitted for clarity. The asymmetric unit contains a half complex molecule which is disordered in two positions. The disorder was refined with site occupation factors of 0.5 for both sites using PART commands and some restraints on the anisotropic displacement parameters (RIGU). All Os-H hydrogen atoms were found from the residual density map and isotropically refined. The isotropically refined $\mathrm{N}-\mathrm{H}$ hydrogen atom was found from the residual density map and constrained to $1.2 \mathrm{U}_{\text {eq }}$ of the connected atom.

Tab. A.4. Crystal data and structure refinement of $\mathbf{8}$.

\begin{tabular}{|c|c|c|}
\hline Identification code & \multicolumn{2}{|l|}{8} \\
\hline Empirical formula & \multicolumn{2}{|l|}{$\mathrm{C}_{20} \mathrm{H}_{49} \mathrm{NOsP}_{2}$} \\
\hline Formula weight & \multicolumn{2}{|l|}{555.74} \\
\hline Temperature & \multicolumn{2}{|l|}{$100(2) \mathrm{K}$} \\
\hline Wavelength & \multicolumn{2}{|l|}{$0.71073 \AA$} \\
\hline Crystal system & \multicolumn{2}{|l|}{ Monoclinic } \\
\hline Space group & \multicolumn{2}{|l|}{$C 2 / c$} \\
\hline Unit cell dimensions & $\begin{array}{l}\mathrm{a}=22.7190(17) \AA \\
\mathrm{b}=7.3382(6) \AA \\
\mathrm{c}=14.6831(13) \AA\end{array}$ & $\begin{array}{l}\alpha=90^{\circ} \\
\beta=104.993(4)^{\circ} \\
\gamma=90^{\circ}\end{array}$ \\
\hline Volume & \multicolumn{2}{|l|}{$2364.6(3) \AA^{3}$} \\
\hline Z & \multicolumn{2}{|l|}{4} \\
\hline Density (calculated) & \multicolumn{2}{|l|}{$1.561 \mathrm{Mg} / \mathrm{m}^{3}$} \\
\hline
\end{tabular}




\begin{tabular}{l|l} 
Absorption coefficient & $5.532 \mathrm{~mm}^{-1}$ \\
\hline $\mathrm{F}(000)$ & 1128 \\
\hline Crystal size & $0.279 \times 0.094 \times 0.071 \mathrm{~mm}^{3}$ \\
\hline Crystal shape and color: & Block, yellow-orange \\
\hline Theta range for data collection & 2.872 to $27.875^{\circ}$ \\
\hline Index ranges & $-29 \leq \mathrm{h} \leq 29,-9 \leq \mathrm{k} \leq 9,-19 \leq \mathrm{I} \leq 19$ \\
\hline Reflections collected & 64019 \\
\hline Independent reflections & $2827[\mathrm{R}($ int $)=0.0423]$ \\
\hline Completeness to theta $=25.242^{\circ}$ & $100.00 \%$ \\
\hline Absorption correction & Semi-empirical from equivalents \\
\hline Max. and min. transmission & 0.7458 and 0.6663 \\
\hline Refinement method & Full-matrix least-squares on $\mathrm{F}^{2}$ \\
\hline Data / restraints / parameters & $2827 / 36 / 210$ \\
\hline Goodness-of-fit on $\mathrm{F}^{2}$ & 1.213 \\
\hline Final R indices [l>2sigma(I)] & $\mathrm{R} 1=0.0255$ \\
\hline R indices (all data) & $\mathrm{R} 1=0.0334$ \\
\hline Largest diff. peak and hole & 1.157 and -2.106 eA $\AA^{-3}$ \\
\hline
\end{tabular}




\section{A.5 $\left[\mathrm{Os}\left(\mathrm{CN}^{\mathrm{t}} \mathrm{Bu}\right)_{2} \mathrm{Cl}\left\{\mathrm{N}\left(\mathrm{CH}_{2} \mathrm{CH}_{2} \mathrm{P}^{\mathrm{t}} \mathrm{Bu} 2\right)\left(=\mathrm{CHCH}_{2} \mathrm{P}\left({ }^{\mathrm{t}} \mathrm{Bu}\right)_{2}=\mathrm{NH}\right)\right\}\right]^{\mathrm{BAr}_{24}^{\mathrm{F}}}$ $\left(11^{\mathrm{BAr}}{ }^{\mathrm{F}}\right)$}

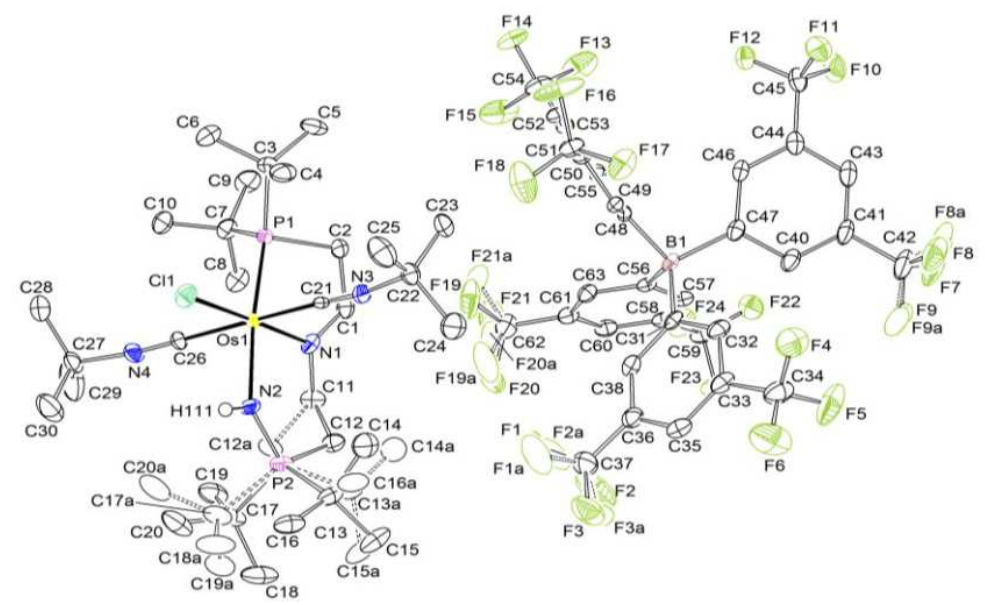

Fig. A.5. Thermal ellipsoid plot of $11^{\mathrm{BAr}_{24}^{\mathrm{F}}}$ with the anisotropic displacement parameters drawn at the $50 \%$ probability level. The asymmetric unit contains one disordered complex molecule and one $\mathrm{BAr}_{24}^{\mathrm{F}}$ anion with three disordered $\mathrm{CF}_{3}$ groups. The disordered complex molecule was refined with population of $0.869(2)$ on the main domain using some restraints and constrains (SADI, EADP). The disordered $\mathrm{CF}_{3}$ groups were refined with populations of $0.776(7), 0.53(2)$ and $0.60(4)$ on their main domains using some restraints (SADI, RIGU). The $\mathrm{N}-\mathrm{H}$ hydrogen atom was found from the residual density map and isotropically refined.

Tab. A.5. Crystal data and structure refinement of $11^{\mathrm{BAr}_{24}^{\mathrm{F}}}$.

\begin{tabular}{|c|c|c|}
\hline Identification code & \multicolumn{2}{|l|}{$11^{\mathrm{BAr}_{24}^{\mathrm{F}}}$} \\
\hline Empirical formula & \multicolumn{2}{|c|}{$\mathrm{C}_{62} \mathrm{H}_{74} \mathrm{BCIF}_{24} \mathrm{~N}_{4} \mathrm{OsP}_{2}$} \\
\hline Formula weight & \multicolumn{2}{|l|}{1629.65} \\
\hline Temperature & \multicolumn{2}{|l|}{$100(2) \mathrm{K}$} \\
\hline Wavelength & \multicolumn{2}{|l|}{$0.71073 \AA$} \\
\hline Crystal system & \multicolumn{2}{|l|}{ Monoclinic } \\
\hline Space group & \multicolumn{2}{|l|}{$P 2_{1} / \mathrm{c}$} \\
\hline Unit cell dimensions & $\begin{array}{l}\mathrm{a}=19.189(2) \AA \\
\mathrm{b}=18.6585(19) \AA \\
\mathrm{c}=19.091(2) \AA\end{array}$ & $\begin{array}{l}\alpha=90^{\circ} \\
\beta=90.631(5)^{\circ} \\
\gamma=90^{\circ}\end{array}$ \\
\hline Volume & \multicolumn{2}{|l|}{$6835.0(12) \AA^{3}$} \\
\hline Z & \multicolumn{2}{|l|}{4} \\
\hline Density (calculated) & \multicolumn{2}{|l|}{$1.584 \mathrm{Mg} / \mathrm{m}^{3}$} \\
\hline
\end{tabular}




\begin{tabular}{|c|c|c|}
\hline Absorption coefficient & \multicolumn{2}{|l|}{$2.059 \mathrm{~mm}^{-1}$} \\
\hline$F(000)$ & \multicolumn{2}{|l|}{3272} \\
\hline Crystal size & \multicolumn{2}{|c|}{$0.292 \times 0.158 \times 0.124 \mathrm{~mm}^{3}$} \\
\hline Crystal shape and color & \multicolumn{2}{|c|}{ Block, clear intense yellow } \\
\hline Theta range for data collection & \multicolumn{2}{|c|}{1.852 to $30.630^{\circ}$} \\
\hline Index ranges & \multicolumn{2}{|c|}{$-27 \leq \mathrm{h} \leq 26,-26 \leq \mathrm{k} \leq 26,-27 \leq \mathrm{l} \leq 27$} \\
\hline Reflections collected & \multicolumn{2}{|l|}{291617} \\
\hline Independent reflections & \multicolumn{2}{|c|}{$20995[R($ int $)=0.1089]$} \\
\hline Completeness to theta $=25.242^{\circ}$ & \multicolumn{2}{|l|}{$100.00 \%$} \\
\hline Max. and min. transmission & \multicolumn{2}{|c|}{0.7461 and 0.6423} \\
\hline Refinement method & \multicolumn{2}{|c|}{ Full-matrix least-squares on $\mathrm{F}^{2}$} \\
\hline Data / restraints / parameters & \multicolumn{2}{|c|}{20995 / 609 / 1002} \\
\hline Goodness-of-fit on $F^{2}$ & \multicolumn{2}{|l|}{1.037} \\
\hline Final $R$ indices $[I>2 \operatorname{sigma}(I)]$ & $\mathrm{R} 1=0.0351$ & $w R 2=0.0659$ \\
\hline $\mathrm{R}$ indices (all data) & $\mathrm{R} 1=0.0639$ & $w R 2=0.0759$ \\
\hline Largest diff. peak and hole & \multicolumn{2}{|c|}{2.766 and $-1.532 \mathrm{e}^{-3}$} \\
\hline
\end{tabular}

\section{A. $6\left[\mathrm{OsCl}\left(\mathrm{CN}^{\mathrm{t}} \mathrm{Bu}\right)\left(\mathrm{PNP}^{t \mathrm{Bu}}\right)\right](15)$}

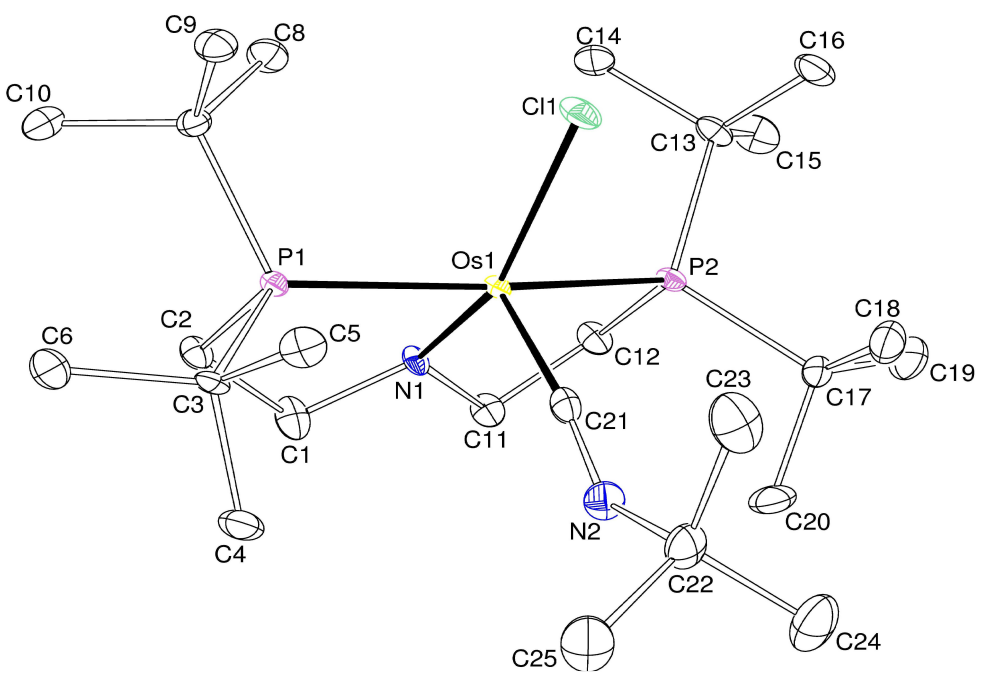

Fig. A.6. Thermal ellipsoid plot of $\mathbf{1 5}$ with the anisotropic displacement parameters drawn at the $50 \%$ probability level. The asymmetric unit contains one complex molecule. 
Tab. A.6. Crystal data and structure refinement of $\mathbf{1 5 .}$

\begin{tabular}{|c|c|c|}
\hline Identification code & \multicolumn{2}{|l|}{15} \\
\hline Empirical formula & \multicolumn{2}{|l|}{$\mathrm{C}_{25} \mathrm{H}_{53} \mathrm{ClN}_{2} \mathrm{OsP}_{2}$} \\
\hline Formula weight & \multicolumn{2}{|l|}{669.28} \\
\hline Temperature & \multicolumn{2}{|l|}{$100(2) \mathrm{K}$} \\
\hline Wavelength & \multicolumn{2}{|l|}{$0.71073 \AA$} \\
\hline Crystal system & \multicolumn{2}{|l|}{ Monoclinic } \\
\hline Space group & \multicolumn{2}{|l|}{$P 2_{1} / \mathrm{c}$} \\
\hline Unit cell dimensions & $\begin{array}{l}\mathrm{a}=19.7104(12) \AA \\
\mathrm{b}=9.7951(6) \AA \\
\mathrm{c}=16.4534(10) \AA\end{array}$ & $\begin{array}{l}\alpha=90^{\circ} \\
\beta=114.422(2)^{\circ} \\
\gamma=90^{\circ}\end{array}$ \\
\hline Volume & \multicolumn{2}{|l|}{$2892.4(3) \AA^{3}$} \\
\hline Z & \multicolumn{2}{|l|}{4} \\
\hline Density (calculated) & \multicolumn{2}{|l|}{$1.537 \mathrm{Mg} / \mathrm{m}^{3}$} \\
\hline Absorption coefficient & \multicolumn{2}{|l|}{$4.627 \mathrm{~mm}^{-1}$} \\
\hline$F(000)$ & \multicolumn{2}{|l|}{1360} \\
\hline Crystal size & \multicolumn{2}{|c|}{$0.214 \times 0.188 \times 0.036 \mathrm{~mm}^{3}$} \\
\hline Crystal shape and color & \multicolumn{2}{|c|}{ Plate, clear intense brown } \\
\hline Theta range for data collection & \multicolumn{2}{|l|}{2.270 to $28.402^{\circ}$} \\
\hline Index ranges & \multicolumn{2}{|c|}{$-26 \leq h \leq 26,-13 \leq k \leq 13,-22 \leq \mathrm{l} \leq 21$} \\
\hline Reflections collected & \multicolumn{2}{|l|}{129552} \\
\hline Independent reflections & \multicolumn{2}{|c|}{$7246[R($ int $)=0.0843]$} \\
\hline Completeness to theta $=25.242^{\circ}$ & \multicolumn{2}{|l|}{$100.00 \%$} \\
\hline Max. and min. transmission & \multicolumn{2}{|l|}{0.7457 and 0.6125} \\
\hline Refinement method & \multicolumn{2}{|c|}{ Full-matrix least-squares on $\mathrm{F}^{2}$} \\
\hline Data / restraints / parameters & \multicolumn{2}{|l|}{$7246 / 0 / 295$} \\
\hline Goodness-of-fit on $\mathrm{F}^{2}$ & \multicolumn{2}{|l|}{1.048} \\
\hline Final $R$ indices $[\mathrm{I}>2 \operatorname{sigma}(\mathrm{I})]$ & \multicolumn{2}{|c|}{$\mathrm{R} 1=0.0240, \quad \mathrm{wR} 2=0.0348$} \\
\hline $\mathrm{R}$ indices (all data) & \multicolumn{2}{|r|}{$w R 2=0.0387$} \\
\hline Largest diff. peak and hole & \multicolumn{2}{|l|}{0.623 and $-0.993 \mathrm{e}^{-3}$} \\
\hline
\end{tabular}




\section{A.7 $\left[\left(\mu-\mathrm{N}_{2}\right)\left\{\operatorname{ReCl}\left(\mathrm{PNP}^{t \mathrm{Bu}}\right)\right\}_{2}\right](\mathbf{X X})$}

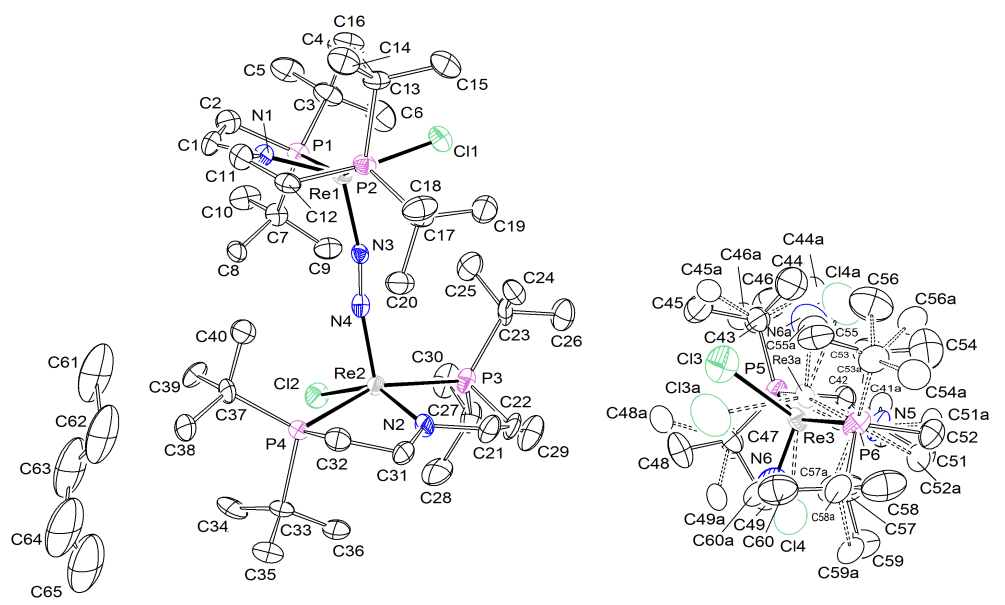

Fig. A.7. Thermal ellipsoid plot of $\mathbf{X X}$ with the anisotropic displacement parameters drawn at the $50 \%$ probability level. The asymmetric unit contains one dimeric species, a monomeric species and a half pentane solvent molecule. The monomeric species was refined as two different disordered complex molecules $\left(\left[\operatorname{ReCl}_{2}\left(\mathrm{PNP}^{t \mathrm{Bu}}\right)\right](\mathbf{X I X})\right.$ and $\left[\mathrm{ReNCI}\left(\mathrm{PNP}^{t \mathrm{Bu}}\right)\right](\mathbf{X X I})$ with occupation factors of 0.5 for both complexes) using some restraints and constraints (SADI, RIGU, EADP). The pentane solvent molecule was refined using some restraints and constraints (SADI, RIGU, DFIX).

Tab. A.7. Crystal data and structure refinement of $\mathbf{X X}$.

\begin{tabular}{|c|c|c|}
\hline Identification code & \multicolumn{2}{|l|}{$x x$} \\
\hline Empirical formula & \multicolumn{2}{|c|}{$\begin{array}{l}\mathrm{C}_{40} \mathrm{H}_{88} \mathrm{Cl}_{2} \mathrm{~N}_{4} \mathrm{P}_{4} \operatorname{Re}(100 \%) \\
\mathrm{C}_{20} \mathrm{H}_{44} \mathrm{Cl}_{2} \mathrm{NP}_{2} \operatorname{Re}(50 \%) \\
\mathrm{C}_{20} \mathrm{H}_{44} \mathrm{CIN}_{2} \mathrm{P}_{2} \operatorname{Re}(50 \%) \\
\mathrm{C}_{5} \mathrm{H}_{12}(50 \%)\end{array}$} \\
\hline Formula weight & \multicolumn{2}{|l|}{1835.27} \\
\hline Temperature & \multicolumn{2}{|l|}{$100(2) \mathrm{K}$} \\
\hline Wavelength & \multicolumn{2}{|l|}{$0.71073 \AA$} \\
\hline Crystal system & \multicolumn{2}{|l|}{ Monoclinic } \\
\hline Space group & \multicolumn{2}{|l|}{$P 2_{1} / \mathrm{c}$} \\
\hline Unit cell dimensions & $\begin{array}{l}\mathrm{a}=12.4165(8) \AA \\
\mathrm{b}=33.602(2) \AA \\
\mathrm{c}=19.7243(14) \AA\end{array}$ & $\begin{array}{l}\alpha=90^{\circ} \\
\beta=101.313(2)^{\circ} \\
\gamma=90^{\circ}\end{array}$ \\
\hline Volume & \multicolumn{2}{|l|}{$8069.5(9) \AA^{3}$} \\
\hline Z & \multicolumn{2}{|l|}{4} \\
\hline Density (calculated) & \multicolumn{2}{|l|}{$1.511 \mathrm{Mg} / \mathrm{m}^{3}$} \\
\hline
\end{tabular}




\begin{tabular}{|c|c|c|}
\hline Absorption coefficient & \multicolumn{2}{|l|}{$4.761 \mathrm{~mm}^{-1}$} \\
\hline$F(000)$ & \multicolumn{2}{|l|}{3704} \\
\hline Crystal size & \multicolumn{2}{|c|}{$0.217 \times 0.085 \times 0.064 \mathrm{~mm}^{3}$} \\
\hline Crystal shape and color & \multicolumn{2}{|c|}{ Plate, clear dark brown } \\
\hline Theta range for data collection & \multicolumn{2}{|c|}{2.228 to $26.451^{\circ}$} \\
\hline Index ranges & \multicolumn{2}{|c|}{$-15 \leq h \leq 15,-39 \leq k \leq 42,-24 \leq 1 \leq 24$} \\
\hline Reflections collected & \multicolumn{2}{|l|}{143213} \\
\hline Independent reflections & \multicolumn{2}{|c|}{$16610[R($ int $)=0.1745]$} \\
\hline Completeness to theta $=25.242^{\circ}$ & \multicolumn{2}{|c|}{$99.90 \%$} \\
\hline Refinement method & \multicolumn{2}{|c|}{ Full-matrix least-squares on $\mathrm{F}^{2}$} \\
\hline Data / restraints / parameters & \multicolumn{2}{|c|}{16610 / 469 / 963} \\
\hline Goodness-of-fit on $\mathrm{F}^{2}$ & \multicolumn{2}{|l|}{1.077} \\
\hline Final R indices [I>2sigma $(\mathrm{I})]$ & $\mathrm{R} 1=0.0639$ & $\mathrm{wR} 2=0.1178$ \\
\hline $\mathrm{R}$ indices (all data) & $\mathrm{R} 1=0.1113$ & $\mathrm{wR} 2=0.1328$ \\
\hline Largest diff. peak and hole & \multicolumn{2}{|c|}{2.340 and $-1.787 \mathrm{e}^{-3}$} \\
\hline
\end{tabular}

\section{A.8 $\left[\mathrm{ReHCl}\left(\mathrm{PNP}^{t \mathrm{Bu}}\right)\right](17)$}

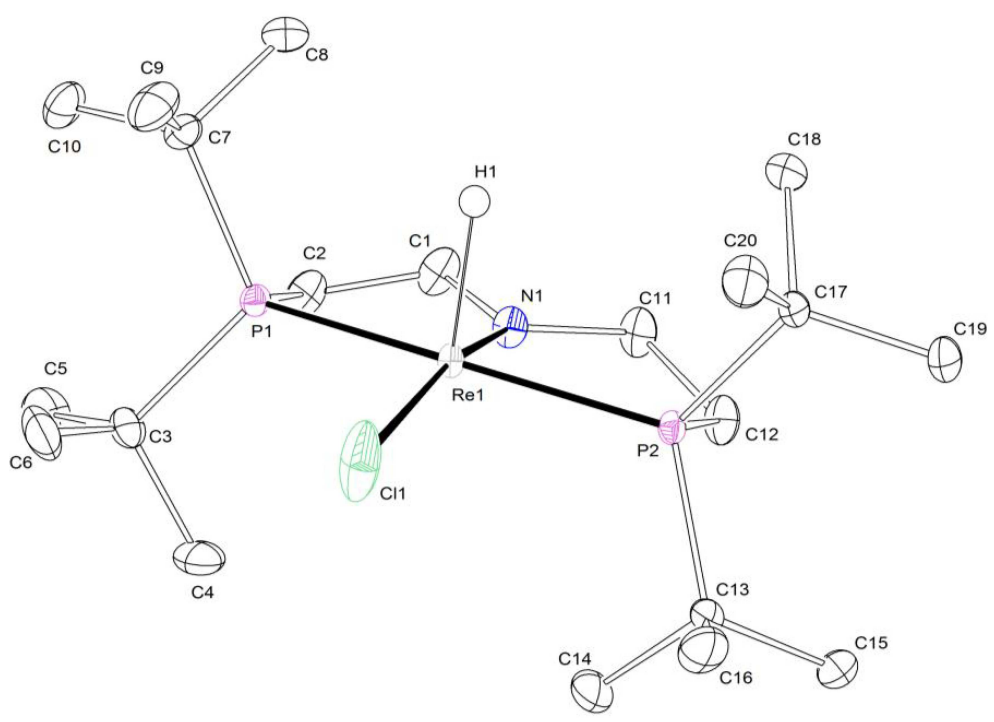

Fig. A.8. Thermal ellipsoid plot of $\mathbf{1 7}$ with the anisotropic displacement parameters drawn at the $50 \%$ probability level. The asymmetric unit contains one complex molecule. The isotropically refined Re-H hydrogen atom was found from the residual density map and constrained to $1.2 \mathrm{U}_{\text {eq }}$ of the connected rhenium atom. 
Tab. A.8. Crystal data and structure refinement of 17 .

\begin{tabular}{|c|c|c|}
\hline Identification code & \multicolumn{2}{|l|}{17} \\
\hline Empirical formula & \multicolumn{2}{|l|}{$\mathrm{C}_{20} \mathrm{H}_{45} \mathrm{CINP}_{2} \mathrm{Re}$} \\
\hline Formula weight & \multicolumn{2}{|l|}{583.16} \\
\hline Temperature & \multicolumn{2}{|l|}{$103(2) \mathrm{K}$} \\
\hline Wavelength & \multicolumn{2}{|l|}{$0.71073 \AA$} \\
\hline Crystal system & \multicolumn{2}{|l|}{ Monoclinic } \\
\hline Space group & \multicolumn{2}{|l|}{$P 2_{1} / \mathrm{c}$} \\
\hline Unit cell dimensions & $\begin{array}{l}\mathrm{a}=12.1666(5) \AA \\
\mathrm{b}=16.1172(7) \AA \\
\mathrm{c}=12.8698(6) \AA\end{array}$ & $\begin{array}{l}\alpha=90^{\circ} \\
\beta=104.311(2)^{\circ} \circ \\
\gamma=90^{\circ}\end{array}$ \\
\hline Volume & \multicolumn{2}{|l|}{$2445.35(19) \AA^{3}$} \\
\hline Z & \multicolumn{2}{|l|}{4} \\
\hline Density (calculated) & \multicolumn{2}{|l|}{$1.584 \mathrm{Mg} / \mathrm{m}^{3}$} \\
\hline Absorption coefficient & \multicolumn{2}{|l|}{$5.214 \mathrm{~mm}^{-1}$} \\
\hline$F(000)$ & \multicolumn{2}{|l|}{1176} \\
\hline Crystal size & \multicolumn{2}{|c|}{$0.204 \times 0.156 \times 0.078 \mathrm{~mm}^{3}$} \\
\hline Crystal shape and color & \multicolumn{2}{|c|}{ Block, clear dark brown } \\
\hline Theta range for data collection & \multicolumn{2}{|l|}{2.420 to $30.602^{\circ}$} \\
\hline Index ranges & \multicolumn{2}{|c|}{$-17 \leq h \leq 17,-23 \leq k \leq 23,-18 \leq \mathrm{l} \leq 18$} \\
\hline Reflections collected & \multicolumn{2}{|l|}{94866} \\
\hline Independent reflections & \multicolumn{2}{|c|}{$7511[R($ int $)=0.0618]$} \\
\hline Completeness to theta $=25.242^{\circ}$ & \multicolumn{2}{|l|}{$99.9 \%$} \\
\hline Max. and min. transmission & \multicolumn{2}{|l|}{0.7461 and 0.5615} \\
\hline Refinement method & \multicolumn{2}{|c|}{ Full-matrix least-squares on $\mathrm{F}^{2}$} \\
\hline Data / restraints / parameters & \multicolumn{2}{|l|}{$7511 / 0 / 241$} \\
\hline Goodness-of-fit on $F^{2}$ & \multicolumn{2}{|l|}{1.028} \\
\hline Final R indices [I>2sigma $(I)]$ & $\mathrm{R} 1=0.0227$ & $\mathrm{wR} 2=0.0371$ \\
\hline $\mathrm{R}$ indices (all data) & $\mathrm{R} 1=0.0358$ & $\mathrm{wR} 2=0.0399$ \\
\hline Largest diff. peak and hole & \multicolumn{2}{|c|}{1.819 and $-1.218 \mathrm{e}^{-3}$} \\
\hline
\end{tabular}




\section{A.9 $\left[\left(N, N-\mathrm{C}_{2} \mathrm{H}_{4} \mathrm{~N}_{2}\right)\left\{\mathrm{ReCl}\left(\mathrm{PNP}^{t \mathrm{Bu}}\right)\right\}_{2}\right]^{(\mathrm{OTf})_{2}}\left(19^{(\mathrm{OTf})_{2}}\right)$}

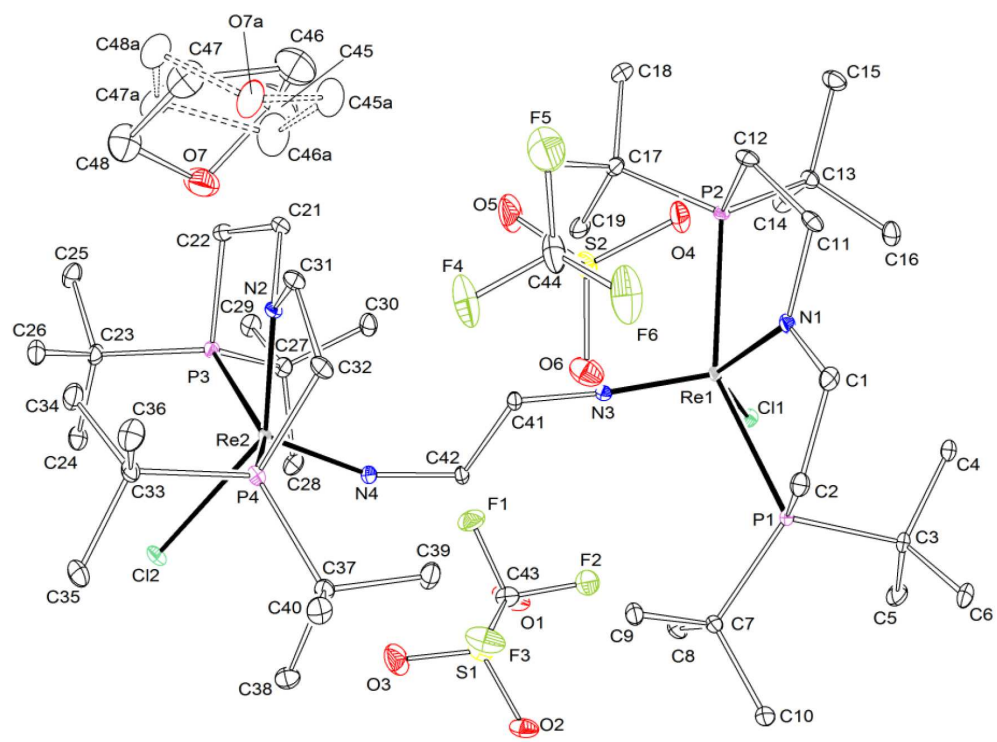

Fig. A.9. Thermal ellipsoid plot of $\mathbf{1 9}^{(\mathrm{OTf})_{2}}$ with the anisotropic displacement parameters drawn at the $50 \%$ probability level. The asymmetric unit contains one complex molecule, two $\mathrm{CF}_{3} \mathrm{SO}_{3}^{-}$anions and one disordered THF solvent molecule. The disordered solvent molecule was refined with population of $0.620(5)$ on the main domain.

Tab. A.9. Crystal data and structure refinement of $19^{(\mathrm{OTf})_{2}}$.

\begin{tabular}{|c|c|c|}
\hline Identification code & \multicolumn{2}{|l|}{$19^{(\mathrm{OTf})_{2}}$} \\
\hline Empirical formula & \multicolumn{2}{|c|}{$\mathrm{C}_{48} \mathrm{H}_{100} \mathrm{Cl}_{2} \mathrm{~F}_{6} \mathrm{~N}_{4} \mathrm{O}_{7} \mathrm{P}_{4} \mathrm{Re}_{2} \mathrm{~S}_{2}$} \\
\hline Formula weight & \multicolumn{2}{|l|}{1590.61} \\
\hline Temperature & \multicolumn{2}{|l|}{$101(2) \mathrm{K}$} \\
\hline Wavelength & \multicolumn{2}{|l|}{$0.71073 \AA$} \\
\hline Crystal system & \multicolumn{2}{|l|}{ Monoclinic } \\
\hline Space group & \multicolumn{2}{|l|}{$P 2_{1} / \mathrm{c}$} \\
\hline Unit cell dimensions & $\begin{array}{l}\mathrm{a}=15.8769(5) \AA \\
\mathrm{b}=27.6973(9) \AA \\
\mathrm{c}=16.3093(5) \AA\end{array}$ & $\begin{array}{l}\alpha=90^{\circ} \\
\beta=117.5340(10)^{\circ} \\
\gamma=90^{\circ}\end{array}$ \\
\hline Volume & \multicolumn{2}{|l|}{$6359.6(4) \AA^{3}$} \\
\hline Z & \multicolumn{2}{|l|}{4} \\
\hline Density (calculated) & \multicolumn{2}{|l|}{$1.661 \mathrm{Mg} / \mathrm{m}^{3}$} \\
\hline Absorption coefficient & \multicolumn{2}{|l|}{$4.119 \mathrm{~mm}^{-1}$} \\
\hline$F(000)$ & \multicolumn{2}{|l|}{3208} \\
\hline
\end{tabular}




\begin{tabular}{|c|c|c|}
\hline Crystal size & \multicolumn{2}{|c|}{$0.266 \times 0.237 \times 0.153 \mathrm{~mm}^{3}$} \\
\hline Crystal shape and color & \multicolumn{2}{|c|}{ Block, clear intense brown } \\
\hline Theta range for data collection & \multicolumn{2}{|c|}{2.502 to $30.589^{\circ}$} \\
\hline Index ranges & \multicolumn{2}{|c|}{$-22 \leq \mathrm{h} \leq 22,-39 \leq \mathrm{k} \leq 39,-23 \leq \mathrm{l} \leq 23$} \\
\hline Reflections collected & \multicolumn{2}{|l|}{263962} \\
\hline Independent reflections & \multicolumn{2}{|c|}{$19533[R($ int $)=0.0628]$} \\
\hline Completeness to theta $=25.242^{\circ}$ & \multicolumn{2}{|l|}{$99.9 \%$} \\
\hline Refinement method & \multicolumn{2}{|c|}{ Full-matrix least-squares on $\mathrm{F}^{2}$} \\
\hline Data / restraints / parameters & \multicolumn{2}{|c|}{$19533 / 0 / 746$} \\
\hline Goodness-of-fit on $F^{2}$ & \multicolumn{2}{|c|}{1.07} \\
\hline Final R indices [I>2sigma $(I)]$ & $\mathrm{R} 1=0.0274$ & $\mathrm{wR} 2=0.0470$ \\
\hline $\mathrm{R}$ indices (all data) & $\mathrm{R} 1=0.0390$ & $\mathrm{wR} 2=0.0494$ \\
\hline Largest diff. peak and hole & \multicolumn{2}{|c|}{1.955 and $-1.466 \mathrm{e}^{-3}$} \\
\hline
\end{tabular}

\section{A.10 $\left[\mathrm{Re}(\mathrm{O}) \mathrm{Cl}\left(\mathrm{PNP}^{t \mathrm{Bu}}\right)\right]^{\mathrm{BPh}_{4}}\left(20^{\mathrm{BPh}_{4}}\right)$}

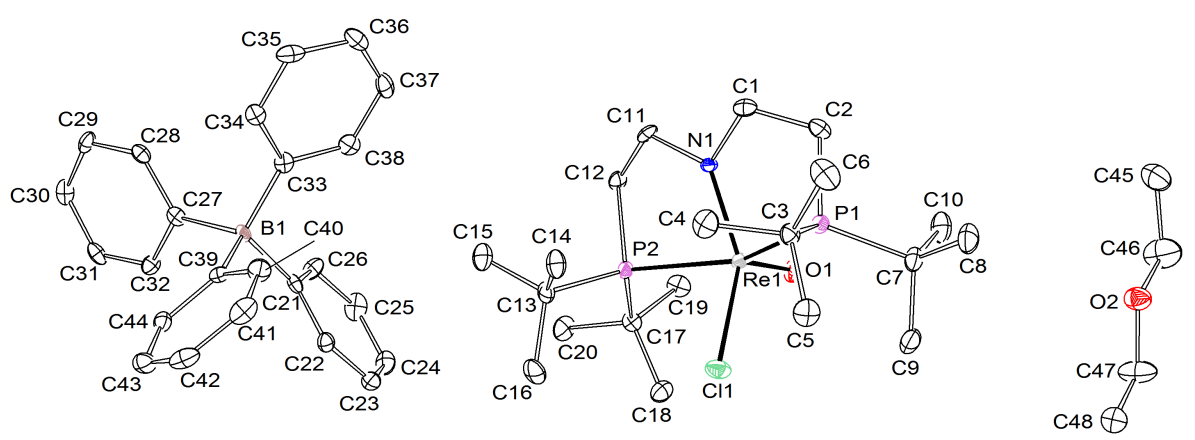

Fig. A.10. Thermal ellipsoid plot of $20^{\mathrm{BPh}_{4}}$ with the anisotropic displacement parameters drawn at the $50 \%$ probability level. The asymmetric unit contains one complex molecule, one $\mathrm{BPh}_{4}{ }^{-}$anion and one diethyl ether solvent molecule.

Tab. A.10. Crystal data and structure refinement of $\mathbf{2 0}^{\mathrm{BPh}_{4}}$.

\begin{tabular}{l|l}
\hline Identification code & $\mathbf{2 0}^{\mathrm{BPh}_{4}}$ \\
\hline Empirical formula & $\mathrm{C}_{48} \mathrm{H}_{74} \mathrm{BCINO}_{2} \mathrm{P}_{2} \mathrm{Re}$ \\
\hline Formula weight & 991.48 \\
\hline Temperature & $100(2) \mathrm{K}$ \\
\hline
\end{tabular}




\begin{tabular}{|c|c|c|}
\hline Wavelength & \multicolumn{2}{|l|}{$0.71073 \AA$} \\
\hline Crystal system & \multicolumn{2}{|l|}{ Triclinic } \\
\hline Space group & \multicolumn{2}{|l|}{$P \overline{1}$} \\
\hline Unit cell dimensions & $\begin{array}{l}\mathrm{a}=11.124(3) \AA \\
\mathrm{b}=14.432(4) \AA \\
\mathrm{c}=16.248(4) \AA\end{array}$ & $\begin{array}{l}\alpha=113.774(13)^{\circ} \\
\beta=92.511(8)^{\circ} \\
\gamma=90.987(9)^{\circ}\end{array}$ \\
\hline Volume & \multicolumn{2}{|l|}{$2383.3(11) \AA^{3}$} \\
\hline Z & \multicolumn{2}{|l|}{2} \\
\hline Density (calculated) & \multicolumn{2}{|l|}{$1.382 \mathrm{Mg} / \mathrm{m}^{3}$} \\
\hline Absorption coefficient & \multicolumn{2}{|l|}{$2.709 \mathrm{~mm}^{-1}$} \\
\hline$F(000)$ & \multicolumn{2}{|l|}{1024} \\
\hline Crystal size & \multicolumn{2}{|c|}{$0.084 \times 0.040 \times 0.035 \mathrm{~mm}^{3}$} \\
\hline Crystal shape and color & \multicolumn{2}{|c|}{ Block, clear pale yellow } \\
\hline Theta range for data collection & \multicolumn{2}{|l|}{2.228 to $26.628^{\circ}$} \\
\hline Index ranges & \multicolumn{2}{|c|}{$-13 \leq h \leq 13,-18 \leq k \leq 18,-20 \leq \mathrm{I} \leq 20$} \\
\hline Reflections collected & \multicolumn{2}{|l|}{93699} \\
\hline Independent reflections & \multicolumn{2}{|c|}{$9615[\mathrm{R}(\mathrm{int})=0.1652]$} \\
\hline Completeness to theta $=25.242^{\circ}$ & \multicolumn{2}{|l|}{$97.6 \%$} \\
\hline Refinement method & \multicolumn{2}{|c|}{ Full-matrix least-squares on $\mathrm{F}^{2}$} \\
\hline Data / restraints / parameters & \multicolumn{2}{|l|}{$9615 / 0 / 519$} \\
\hline Goodness-of-fit on $\mathrm{F}^{2}$ & \multicolumn{2}{|l|}{1.021} \\
\hline Final $R$ indices [I>2sigma $(I)]$ & $\mathrm{R} 1=0.0403$ & $\mathrm{wR} 2=0.0615$ \\
\hline $\mathrm{R}$ indices (all data) & $\mathrm{R} 1=0.0646$ & $\mathrm{wR} 2=0.0670$ \\
\hline Largest diff. peak and hole & \multicolumn{2}{|c|}{1.159 and $-1.339 \mathrm{e}^{-3}$} \\
\hline
\end{tabular}




\section{A.11 $\left[\operatorname{Re}(\mathrm{N}) \mathrm{Cl}\left(\mathrm{P}=\mathrm{N}=\mathrm{P}^{t \mathrm{Bu}}\right)\right](22)$}

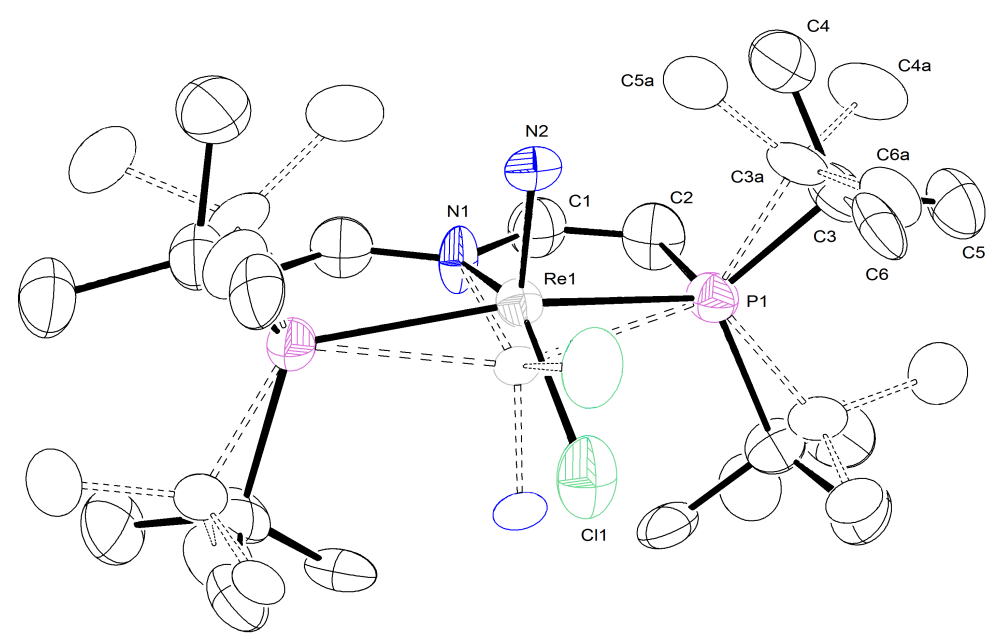

Fig. A.11. Thermal ellipsoid plot of 22 with the anisotropic displacement parameters drawn at the $50 \%$ probability level. The asymmetric unit contains a half disordered complex molecule. The disorder was refined with site occupation factors of 0.5 for both sites using PART commands and some restraints (RIGU). The structure was refined as an inversion twin using the twin law -100 0-10 00-1 (BASF: 0.50(3)).

Tab. A.11. Crystal data and structure refinement of 22 .

\begin{tabular}{|c|c|c|}
\hline Identification code & \multicolumn{2}{|l|}{22} \\
\hline Empirical formula & \multicolumn{2}{|l|}{$\mathrm{C}_{20} \mathrm{H}_{40} \mathrm{CIN}_{2} \mathrm{P}_{2} \mathrm{Re}$} \\
\hline Formula weight & \multicolumn{2}{|l|}{592.13} \\
\hline Temperature & \multicolumn{2}{|l|}{$100(2) \mathrm{K}$} \\
\hline Wavelength & \multicolumn{2}{|l|}{$0.71073 \AA$} \\
\hline Crystal system & \multicolumn{2}{|l|}{ Tetragonal } \\
\hline Space group & \multicolumn{2}{|l|}{$P \overline{4} 2{ }_{1} m$} \\
\hline Unit cell dimensions & $\begin{array}{l}\mathrm{a}=12.0771(7) \AA \\
\mathrm{b}=12.0771(7) \AA \\
\mathrm{c}=8.3696(6) \AA\end{array}$ & $\begin{array}{l}\alpha=90^{\circ} \\
\beta=90^{\circ} \\
\gamma=90^{\circ}\end{array}$ \\
\hline Volume & \multicolumn{2}{|l|}{$2383.3(11) \AA^{3}$} \\
\hline Z & \multicolumn{2}{|l|}{2} \\
\hline Density (calculated) & \multicolumn{2}{|l|}{$1.611 \mathrm{Mg} / \mathrm{m}^{3}$} \\
\hline Absorption coefficient & \multicolumn{2}{|l|}{$5.225 \mathrm{~mm}^{-1}$} \\
\hline$F(000)$ & \multicolumn{2}{|l|}{592} \\
\hline Crystal size & \multicolumn{2}{|c|}{$0.234 \times 0.229 \times 0.130 \mathrm{~mm}^{3}$} \\
\hline
\end{tabular}




\begin{tabular}{|c|c|c|}
\hline Crystal shape and color & \multicolumn{2}{|c|}{ Block, light brown } \\
\hline Theta range for data collection & \multicolumn{2}{|c|}{2.385 to $27.443^{\circ}$} \\
\hline Index ranges & \multicolumn{2}{|c|}{$-15 \leq h \leq 15,-15 \leq k \leq 15,-10 \leq \mathrm{l} \leq 10$} \\
\hline Reflections collected & \multicolumn{2}{|l|}{20488} \\
\hline Independent reflections & \multicolumn{2}{|c|}{$1492[R($ int $)=0.0883]$} \\
\hline Completeness to theta $=25.242^{\circ}$ & \multicolumn{2}{|l|}{$100 \%$} \\
\hline Max. and min. transmission & \multicolumn{2}{|c|}{0.7457 and 0.5234} \\
\hline Refinement method & \multicolumn{2}{|c|}{ Full-matrix least-squares on $\mathrm{F}^{2}$} \\
\hline Data / restraints / parameters & \multicolumn{2}{|c|}{1492 / 36 / 121} \\
\hline Goodness-of-fit on $\mathrm{F}^{2}$ & \multicolumn{2}{|l|}{1.151} \\
\hline Final $R$ indices [I>2sigma( $(I)]$ & $\mathrm{R} 1=0.0444$ & $\mathrm{wR} 2=0.0841$ \\
\hline $\mathrm{R}$ indices (all data) & $\mathrm{R} 1=0.0611$ & $w R 2=0.0925$ \\
\hline Largest diff. peak and hole & \multicolumn{2}{|c|}{1.888 and $-1.191 \mathrm{e}^{-3}$} \\
\hline
\end{tabular}

\section{A.12 $\left[\operatorname{Re}(\text { bipy }) \mathrm{Cl}\left(\mathrm{PNP}^{t \mathrm{Bu}}\right)\right]^{\mathrm{BPh}_{4}}\left(27^{\mathrm{BPh}_{4}}\right)$}

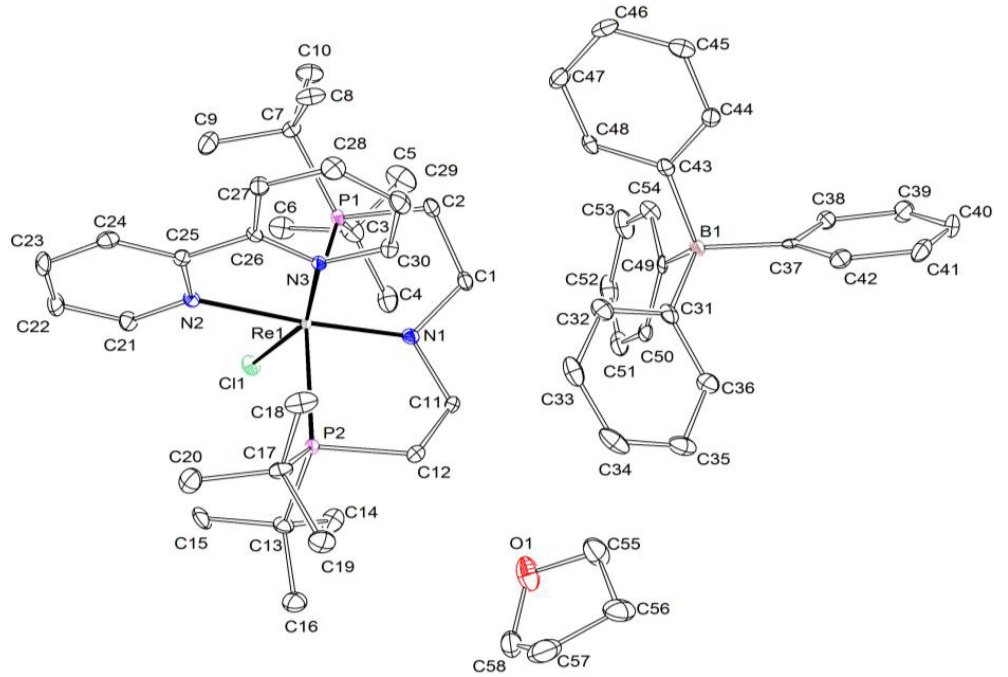

Fig. A.12. Thermal ellipsoid plot of $27^{\mathrm{BPh}_{4}}$ with the anisotropic displacement parameters drawn at the $50 \%$ probability level. The asymmetric unit contains one complex molecule, one THF molecule and one $\mathrm{BPh}_{4}^{-}$anion.

Tab. A.12. Crystal data and structure refinement of $27^{\mathrm{BPh}_{4}}$.

\begin{tabular}{l|l}
\hline Identification code & $27^{\mathrm{BPh}_{4}}$ \\
\hline
\end{tabular}




\begin{tabular}{|c|c|c|}
\hline Empirical formula & \multicolumn{2}{|l|}{$\mathrm{C}_{58} \mathrm{H}_{80} \mathrm{BCIN}_{3} \mathrm{OP}_{2} \mathrm{Re}$} \\
\hline Formula weight & \multicolumn{2}{|l|}{1129.65} \\
\hline Temperature & \multicolumn{2}{|l|}{$100(2) \mathrm{K}$} \\
\hline Wavelength & \multicolumn{2}{|l|}{$0.71073 \AA$} \\
\hline Crystal system & \multicolumn{2}{|l|}{ Monoclinic } \\
\hline Space group & \multicolumn{2}{|l|}{$P 2_{1} / \mathrm{n}$} \\
\hline Unit cell dimensions & $\begin{array}{l}\mathrm{a}=21.0255(10) \AA \\
\mathrm{b}=13.6445(7) \AA \\
\mathrm{c}=21.1205(11) \AA\end{array}$ & $\begin{array}{l}\alpha=90^{\circ} \\
\beta=117.775(2)^{\circ} \\
\gamma=90^{\circ}\end{array}$ \\
\hline Volume & \multicolumn{2}{|l|}{$5361.0(5) \AA^{3}$} \\
\hline Z & \multicolumn{2}{|l|}{4} \\
\hline Density (calculated) & \multicolumn{2}{|l|}{$1.611 \mathrm{Mg} / \mathrm{m}^{3}$} \\
\hline Absorption coefficient & \multicolumn{2}{|l|}{$1.400 \mathrm{~mm}^{-1}$} \\
\hline$F(000)$ & \multicolumn{2}{|l|}{2336} \\
\hline Crystal size & \multicolumn{2}{|c|}{$0.271 \times 0.122 \times 0.106 \mathrm{~mm}^{3}$} \\
\hline Crystal shape and color & \multicolumn{2}{|c|}{ Plate, dark red } \\
\hline Theta range for data collection & \multicolumn{2}{|l|}{2.190 to $29.218^{\circ}$} \\
\hline Index ranges & \multicolumn{2}{|c|}{$-28 \leq h \leq 27,-18 \leq k \leq 18,-29 \leq \mathrm{l} \leq 29$} \\
\hline Reflections collected & \multicolumn{2}{|l|}{240096} \\
\hline Independent reflections & \multicolumn{2}{|c|}{$14515[R($ int $)=0.1752]$} \\
\hline Completeness to theta $=25.242^{\circ}$ & \multicolumn{2}{|c|}{$99.9 \%$} \\
\hline Max. and min. transmission & \multicolumn{2}{|l|}{0.7458 and 0.6637} \\
\hline Refinement method & \multicolumn{2}{|c|}{ Full-matrix least-squares on $\mathrm{F}^{2}$} \\
\hline Data / restraints / parameters & \multicolumn{2}{|l|}{14515 / 0 / 616} \\
\hline Goodness-of-fit on $F^{2}$ & \multicolumn{2}{|l|}{1.054} \\
\hline Final $R$ indices $[I>2 \operatorname{sigma}(I)]$ & $\mathrm{R} 1=0.0454$ & $\mathrm{wR} 2=0.0571$ \\
\hline $\mathrm{R}$ indices (all data) & $\mathrm{R} 1=0.0777$ & $\mathrm{wR} 2=0.0636$ \\
\hline Largest diff. peak and hole & \multicolumn{2}{|c|}{1.622 and $-1.415 \mathrm{e}^{-3}$} \\
\hline
\end{tabular}




\section{A.13 $\left[\mathrm{ReCl}_{3}\left(\mathrm{HPNP}^{\mathrm{iPr}}\right)\right](29)$}

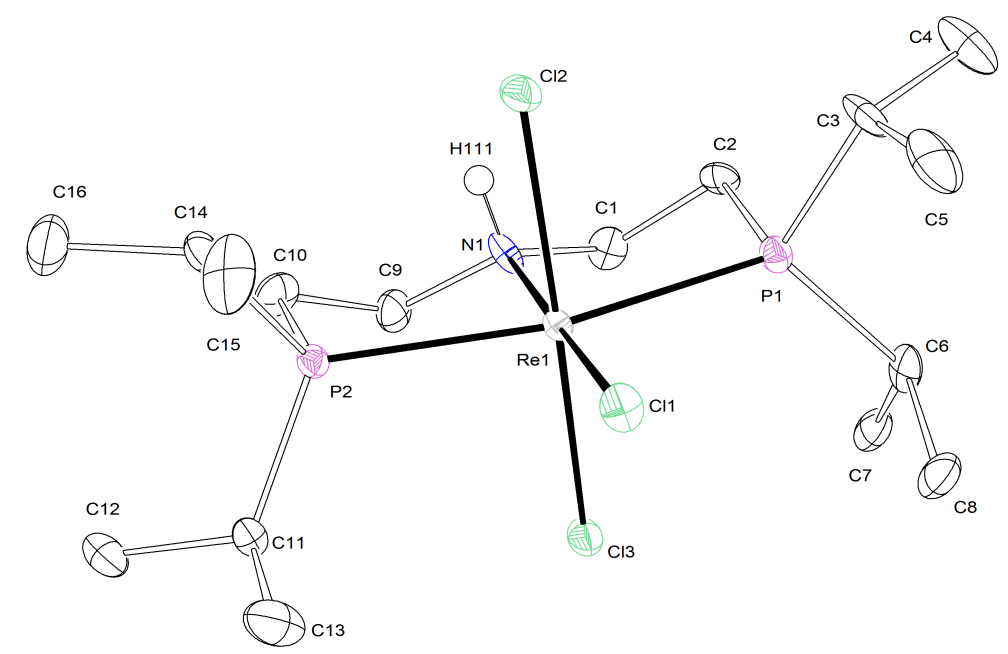

Fig. A.13. Thermal ellipsoid plot of $\mathbf{2 9}$ with the anisotropic displacement parameters drawn at the $50 \%$ probability level. The asymmetric unit contains one complex molecule. The $\mathrm{N}-\mathrm{H}$ hydrogen atom was found from the residual density map and isotropically refined. The structure was refined as an inversion twin using the twin law -100 0-10 00-1 (BASF: $0.015(9))$.

Tab. A.13. Crystal data and structure refinement of 29 .

\begin{tabular}{|c|c|c|}
\hline Identification code & \multicolumn{2}{|l|}{29} \\
\hline Empirical formula & \multicolumn{2}{|l|}{$\mathrm{C}_{16} \mathrm{H}_{37} \mathrm{Cl}_{3} \mathrm{NP}_{2} \mathrm{Re}$} \\
\hline Formula weight & \multicolumn{2}{|l|}{597.95} \\
\hline Temperature & \multicolumn{2}{|l|}{$100(2) \mathrm{K}$} \\
\hline Wavelength & \multicolumn{2}{|l|}{$0.71073 \AA$} \\
\hline Crystal system & \multicolumn{2}{|l|}{ Orthorhombic } \\
\hline Space group & \multicolumn{2}{|l|}{$P 2{ }_{1} 2_{1} 2_{1}$} \\
\hline Unit cell dimensions & $\begin{array}{l}\mathrm{a}=7.3805(3) \AA \\
\mathrm{b}=13.0720(6) \AA \\
\mathrm{c}=23.6017(11) \AA\end{array}$ & $\begin{aligned} \alpha & =90^{\circ} \\
\beta & =90^{\circ} \\
\gamma & =90^{\circ}\end{aligned}$ \\
\hline Volume & \multicolumn{2}{|l|}{$2277.04(18) \AA^{3}$} \\
\hline Z & \multicolumn{2}{|l|}{4} \\
\hline Density (calculated) & \multicolumn{2}{|l|}{$1.744 \mathrm{Mg} / \mathrm{m}^{3}$} \\
\hline Absorption coefficient & \multicolumn{2}{|l|}{$5.829 \mathrm{~mm}^{-1}$} \\
\hline$F(000)$ & \multicolumn{2}{|l|}{1184} \\
\hline
\end{tabular}




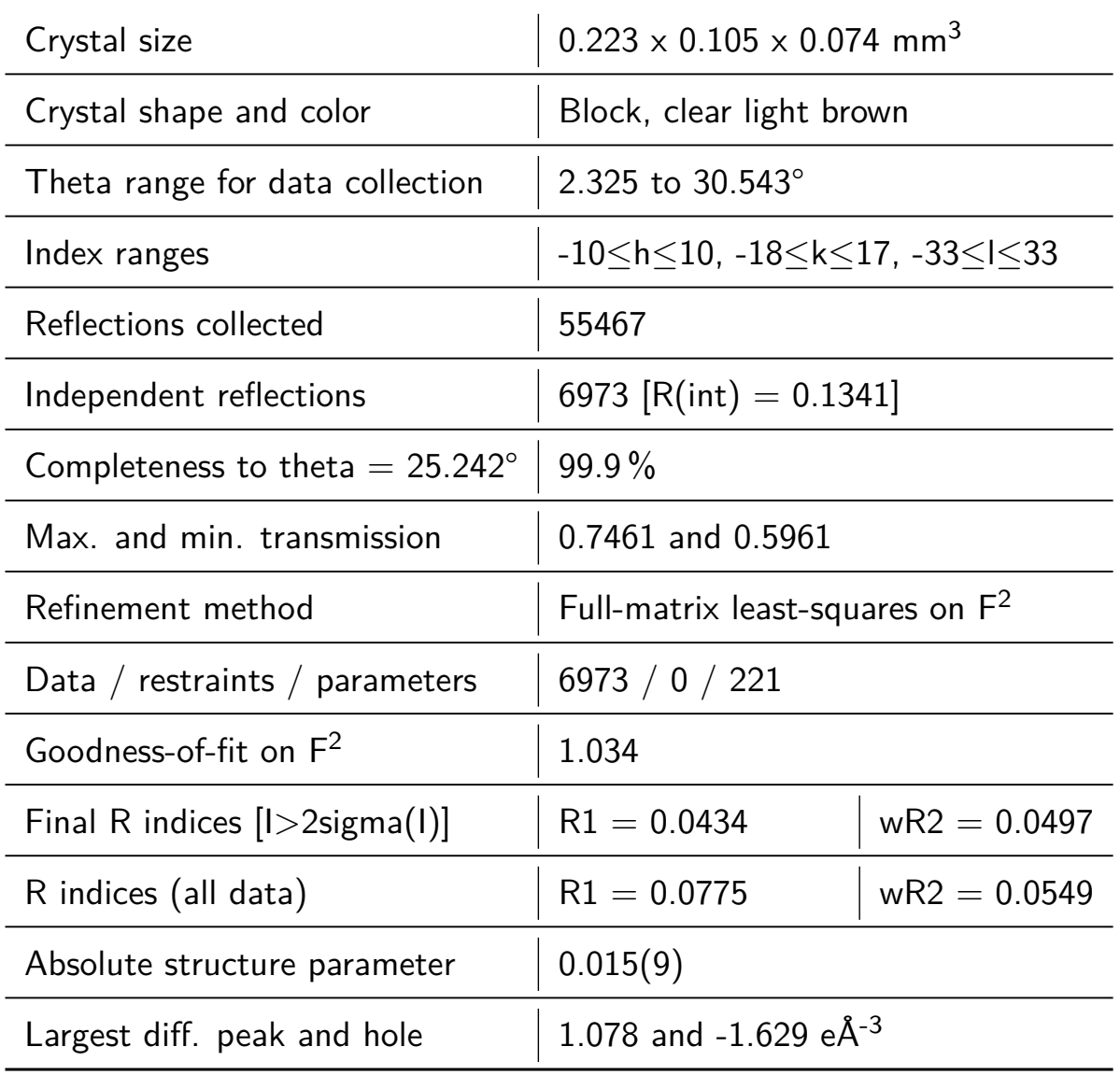

\section{A.14 $\left[\mathrm{ReCl}_{3}\left(\mathrm{PNP}^{\mathrm{iPr}}\right)\right](\mathbf{3 1})$}

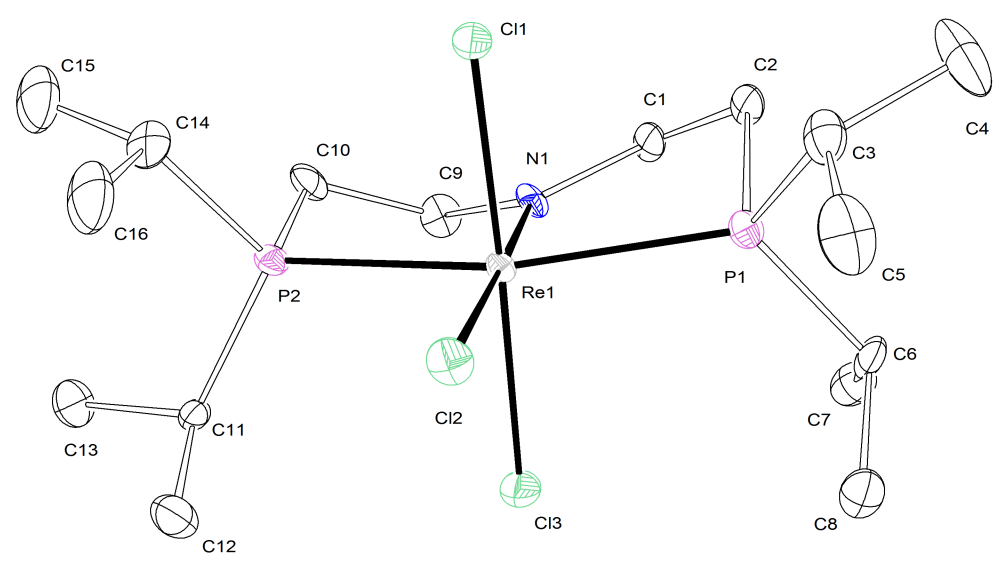

Fig. A.14. Thermal ellipsoid plot of $\mathbf{3 1}$ with the anisotropic displacement parameters drawn at the $50 \%$ probability level. The asymmetric unit contains one complex molecule. The structure was refined using some restraints and constraints (RIGU, EADP).

Tab. A.14. Crystal data and structure refinement of $\mathbf{3 1}$.

\begin{tabular}{l|l}
\hline Identification code & $\mathbf{3 1}$ \\
\hline
\end{tabular}




\begin{tabular}{|c|c|c|}
\hline Empirical formula & \multicolumn{2}{|l|}{$\mathrm{C}_{16} \mathrm{H}_{36} \mathrm{Cl}_{3} \mathrm{NP}_{2} \mathrm{Re}$} \\
\hline Formula weight & \multicolumn{2}{|l|}{596.95} \\
\hline Temperature & \multicolumn{2}{|l|}{$113(2) \mathrm{K}$} \\
\hline Wavelength & \multicolumn{2}{|l|}{$0.71073 \AA$} \\
\hline Crystal system & \multicolumn{2}{|l|}{ Orthorhombic } \\
\hline Space group & \multicolumn{2}{|l|}{$P 2{ }_{1} 2{ }_{1} 2_{1}$} \\
\hline Unit cell dimensions & $\begin{array}{l}\mathrm{a}=7.3389(5) \AA \\
\mathrm{b}=12.6285(7) \AA \\
\mathrm{c}=24.1756(14) \AA\end{array}$ & $\begin{array}{l}\alpha=90^{\circ} \\
\beta=90^{\circ} \\
\gamma=90^{\circ}\end{array}$ \\
\hline Volume & \multicolumn{2}{|l|}{$2240.6(2) \AA^{3}$} \\
\hline Z & \multicolumn{2}{|l|}{4} \\
\hline Density (calculated) & \multicolumn{2}{|l|}{$1.770 \mathrm{Mg} / \mathrm{m}^{3}$} \\
\hline Absorption coefficient & \multicolumn{2}{|l|}{$5.923 \mathrm{~mm}^{-1}$} \\
\hline$F(000)$ & \multicolumn{2}{|l|}{1180} \\
\hline Crystal size & \multicolumn{2}{|c|}{$0.099 \times 0.050 \times 0.038 \mathrm{~mm}^{3}$} \\
\hline Crystal shape and color & \multicolumn{2}{|c|}{ Block, clear dark red-orange } \\
\hline Theta range for data collection & \multicolumn{2}{|c|}{2.332 to $28.342^{\circ}$} \\
\hline Index ranges & \multicolumn{2}{|c|}{$-9 \leq \mathrm{h} \leq 9,-16 \leq \mathrm{k} \leq 16,-32 \leq \mathrm{I} \leq 32$} \\
\hline Reflections collected & \multicolumn{2}{|l|}{67072} \\
\hline Independent reflections & \multicolumn{2}{|c|}{$5593[\mathrm{R}($ int $)=0.1661]$} \\
\hline Completeness to theta $=25.242^{\circ}$ & \multicolumn{2}{|l|}{$99.9 \%$} \\
\hline Max. and min. transmission & \multicolumn{2}{|l|}{0.7410 and 0.6416} \\
\hline Refinement method & \multicolumn{2}{|c|}{ Full-matrix least-squares on $\mathrm{F}^{2}$} \\
\hline Data / restraints / parameters & \multicolumn{2}{|l|}{5593 / 24 / 204} \\
\hline Goodness-of-fit on $\mathrm{F}^{2}$ & \multicolumn{2}{|l|}{1.041} \\
\hline Final $R$ indices $[I>2 \operatorname{sigma}(I)]$ & $\mathrm{R} 1=0.0428$ & $w R 2=0.0594$ \\
\hline $\mathrm{R}$ indices (all data) & $\mathrm{R} 1=0.0601$ & $\mathrm{wR} 2=0.0631$ \\
\hline Absolute structure parameter & \multicolumn{2}{|l|}{$0.013(6)$} \\
\hline Largest diff. peak and hole & \multicolumn{2}{|c|}{1.024 and $-2.523 \mathrm{e}^{-3}$} \\
\hline
\end{tabular}




\section{A.15 $\left[\operatorname{ReCl}_{2}(\right.$ thf $\left.)\left(\mathrm{PNP}^{i \mathrm{Pr}}\right)\right](32)$}

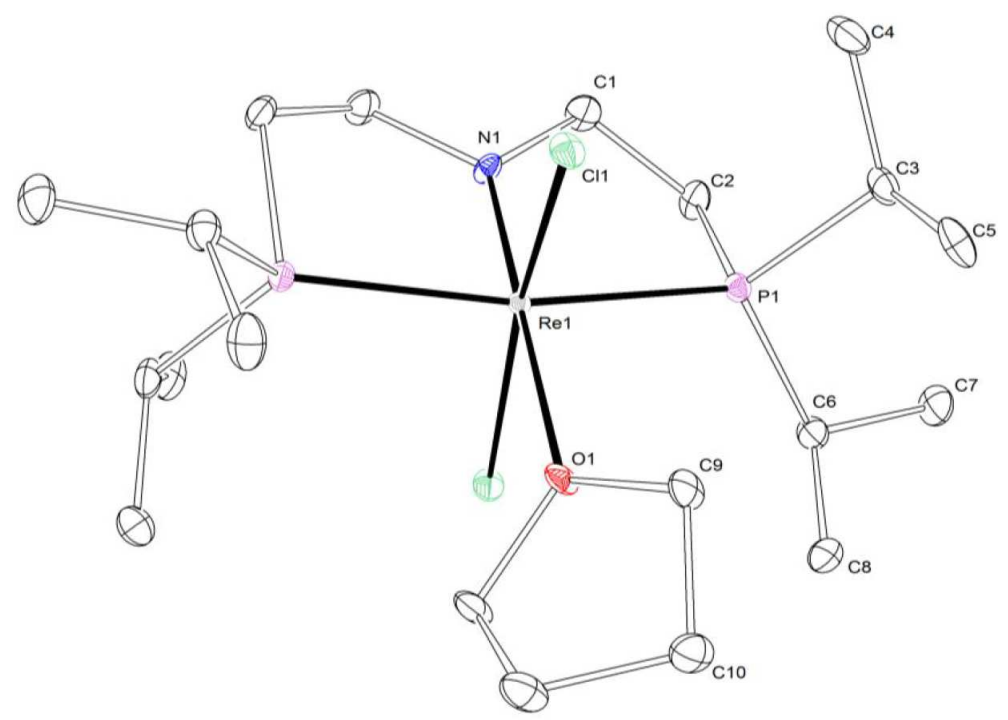

Fig. A.15. Thermal ellipsoid plot of $\mathbf{3 2}$ with the anisotropic displacement parameters drawn at the $50 \%$ probability level. The asymmetric unit contains a half complex molecule.

Tab. A.15. Crystal data and structure refinement of 32 .

\begin{tabular}{|c|c|c|}
\hline Identification code & \multicolumn{2}{|l|}{32} \\
\hline Empirical formula & \multicolumn{2}{|l|}{$\mathrm{C}_{20} \mathrm{H}_{44} \mathrm{Cl}_{2} \mathrm{NOP}_{2} \mathrm{Re}$} \\
\hline Formula weight & \multicolumn{2}{|l|}{633.6} \\
\hline Temperature & \multicolumn{2}{|l|}{$113(2) \mathrm{K}$} \\
\hline Wavelength & \multicolumn{2}{|l|}{$0.71073 \AA$} \\
\hline Crystal system & \multicolumn{2}{|l|}{ Monoclinic } \\
\hline Space group & \multicolumn{2}{|l|}{$\mathrm{C} 2 / \mathrm{c}$} \\
\hline Unit cell dimensions & $\begin{array}{l}\mathrm{a}=18.2122(7) \AA \\
\mathrm{b}=11.0408(4) \AA \\
\mathrm{c}=12.5053(6) \AA\end{array}$ & $\begin{array}{l}\alpha=90^{\circ} \\
\beta=101.887(2)^{\circ} \\
\gamma=90^{\circ}\end{array}$ \\
\hline Volume & \multicolumn{2}{|l|}{$2460.61(18) \AA^{3}$} \\
\hline Z & \multicolumn{2}{|l|}{4} \\
\hline Density (calculated) & \multicolumn{2}{|l|}{$1.710 \mathrm{Mg} / \mathrm{m}^{3}$} \\
\hline Absorption coefficient & \multicolumn{2}{|l|}{$5.297 \mathrm{~mm}^{-1}$} \\
\hline$F(000)$ & \multicolumn{2}{|l|}{1272} \\
\hline Crystal size & \multicolumn{2}{|c|}{$0.171 \times 0.128 \times 0.035 \mathrm{~mm}^{3}$} \\
\hline
\end{tabular}




\begin{tabular}{|c|c|}
\hline Crystal shape and color & Plate, clear light green-brown \\
\hline Theta range for data collection & 2.286 to $30.609^{\circ}$ \\
\hline Index ranges & $-26 \leq h \leq 26,-15 \leq k \leq 15,-17 \leq \mathrm{l} \leq 17$ \\
\hline Reflections collected & 46525 \\
\hline Independent reflections & $3777[\mathrm{R}(\mathrm{int})=0.0759]$ \\
\hline Completeness to theta $=25.242^{\circ}$ & $100 \%$ \\
\hline Max. and min. transmission & 0.7461 and 0.6072 \\
\hline Refinement method & Full-matrix least-squares on $\mathrm{F}^{2}$ \\
\hline Data / restraints / parameters & $3777 / 0 / 128$ \\
\hline Goodness-of-fit on $F^{2}$ & 1.07 \\
\hline Final R indices $[I>2 \operatorname{sigma}(I)]$ & $w R 2=0.0365$ \\
\hline $\mathrm{R}$ indices (all data) & $w R 2=0.0389$ \\
\hline Largest diff. peak and hole & 1.521 and $-1.126 \mathrm{e}^{-3}$ \\
\hline
\end{tabular}

\section{A.16 $\left[\operatorname{ReCl}_{2}\left(\mathrm{PNP}^{\mathrm{iPr}}\right)\right](30)$}

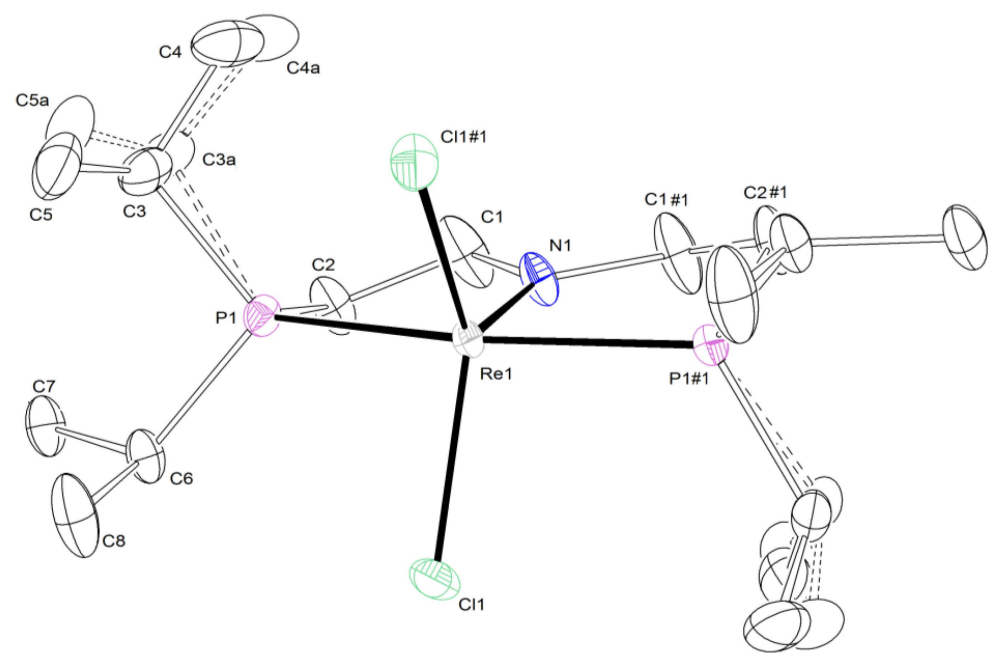

Fig. A.16. Thermal ellipsoid plot of $\mathbf{3 0}$ with the anisotropic displacement parameters drawn at the $50 \%$ probability level. The asymmetric unit contains a half disordered complex molecule. The disordered complex molecule was refined with population of $0.52(2)$ on the main domain using some constraints (EADP).

Tab. A.16. Crystal data and structure refinement of $\mathbf{3 0}$

\begin{tabular}{l|l}
\hline Identification code & $\mathbf{3 0}$ \\
\hline
\end{tabular}




\begin{tabular}{|c|c|c|}
\hline Empirical formula & \multicolumn{2}{|l|}{$\mathrm{C}_{16} \mathrm{H}_{36} \mathrm{Cl}_{2} \mathrm{NP}_{2} \mathrm{Re}$} \\
\hline Formula weight & \multicolumn{2}{|l|}{561.5} \\
\hline Temperature & \multicolumn{2}{|l|}{$113(2) \mathrm{K}$} \\
\hline Wavelength & \multicolumn{2}{|l|}{$0.71073 \AA$} \\
\hline Crystal system & \multicolumn{2}{|l|}{ Monoclinic } \\
\hline Space group & \multicolumn{2}{|l|}{$C 2 / c$} \\
\hline Unit cell dimensions & $\begin{array}{l}\mathrm{a}=14.7630(7) \AA \\
\mathrm{b}=7.4571(4) \AA \\
\mathrm{c}=20.3176(10) \AA\end{array}$ & $\begin{array}{l}\alpha=90^{\circ} \\
\beta=106.718(2)^{\circ} \\
\gamma=90^{\circ}\end{array}$ \\
\hline Volume & \multicolumn{2}{|l|}{$2142.20(19) \AA^{3}$} \\
\hline Z & \multicolumn{2}{|l|}{4} \\
\hline Density (calculated) & \multicolumn{2}{|l|}{$1.741 \mathrm{Mg} / \mathrm{m}^{3}$} \\
\hline Absorption coefficient & \multicolumn{2}{|l|}{$6.069 \mathrm{~mm}^{-1}$} \\
\hline$F(000)$ & \multicolumn{2}{|l|}{1112} \\
\hline Crystal size & \multicolumn{2}{|c|}{$0.604 \times 0.171 \times 0.160 \mathrm{~mm}^{3}$} \\
\hline Crystal shape and color & \multicolumn{2}{|c|}{ Plate, clear pale yellow-violet } \\
\hline Theta range for data collection & \multicolumn{2}{|l|}{2.881 to $28.348^{\circ}$} \\
\hline Index ranges & \multicolumn{2}{|c|}{$-19 \leq \mathrm{h} \leq 19,-9 \leq \mathrm{k} \leq 9,-27 \leq \mathrm{l} \leq 27$} \\
\hline Reflections collected & \multicolumn{2}{|c|}{36014} \\
\hline Independent reflections & \multicolumn{2}{|c|}{$2658[R($ int $)=0.0627]$} \\
\hline Completeness to theta $=25.242^{\circ}$ & \multicolumn{2}{|l|}{$99.9 \%$} \\
\hline Max. and min. transmission & \multicolumn{2}{|l|}{0.7457 and 0.4736} \\
\hline Refinement method & \multicolumn{2}{|c|}{ Full-matrix least-squares on $\mathrm{F}^{2}$} \\
\hline Data / restraints / parameters & \multicolumn{2}{|l|}{$2658 / 0 / 117$} \\
\hline Goodness-of-fit on $F^{2}$ & \multicolumn{2}{|l|}{1.102} \\
\hline Final $R$ indices $[I>2 \operatorname{sigma}(I)]$ & $\mathrm{R} 1=0.0216$ & $w R 2=0.0460$ \\
\hline $\mathrm{R}$ indices (all data) & $\mathrm{R} 1=0.0248$ & $\mathrm{wR} 2=0.0631$ \\
\hline Largest diff. peak and hole & \multicolumn{2}{|c|}{1.703 and $-1.240 \mathrm{e}^{-3}$} \\
\hline
\end{tabular}




\section{A.17 $\left[\operatorname{Re}(\mathrm{N}) \mathrm{Cl}\left(\mathrm{PNP}^{\mathrm{iPr}}\right)\right](34)$}

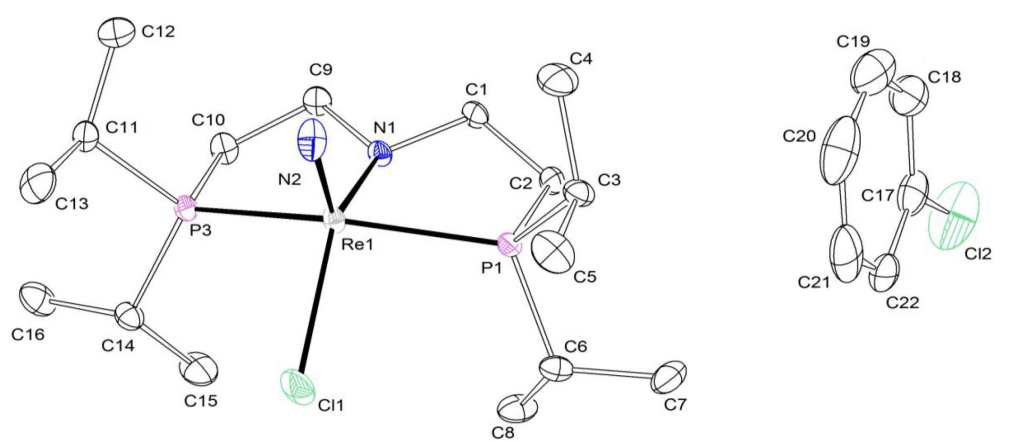

Fig. A.17. Thermal ellipsoid plot of $\mathbf{3 4}$ with the anisotropic displacement parameters drawn at the $50 \%$ probability level. The asymmetric unit contains one complex molecule and one chlorobenzene solvent molecule.

Tab. A.17. Crystal data and structure refinement of $\mathbf{3 4}$.

\begin{tabular}{|c|c|c|}
\hline Identification code & \multicolumn{2}{|l|}{34} \\
\hline Empirical formula & \multicolumn{2}{|l|}{$\mathrm{C}_{22} \mathrm{H}_{41} \mathrm{Cl}_{2} \mathrm{~N}_{2} \mathrm{P}_{2} \mathrm{Re}$} \\
\hline Formula weight & \multicolumn{2}{|l|}{652.61} \\
\hline Temperature & \multicolumn{2}{|l|}{$120(2) \mathrm{K}$} \\
\hline Wavelength & \multicolumn{2}{|l|}{$0.71073 \AA$} \\
\hline Crystal system & \multicolumn{2}{|l|}{ Monoclinic } \\
\hline Space group & \multicolumn{2}{|l|}{$P 2_{1} / \mathrm{c}$} \\
\hline Unit cell dimensions & $\begin{array}{l}\mathrm{a}=13.5319(7) \AA \\
\mathrm{b}=14.5498(7) \AA \\
\mathrm{c}=14.0355(7) \AA\end{array}$ & $\begin{array}{l}\alpha=90^{\circ} \\
\beta=102.555(2)^{\circ} \\
\gamma=90^{\circ}\end{array}$ \\
\hline Volume & \multicolumn{2}{|l|}{$2697.3(2) \AA^{3}$} \\
\hline Z & \multicolumn{2}{|l|}{4} \\
\hline Density (calculated) & \multicolumn{2}{|l|}{$1.607 \mathrm{Mg} / \mathrm{m}^{3}$} \\
\hline Absorption coefficient & \multicolumn{2}{|l|}{$4.834 \mathrm{~mm}^{-1}$} \\
\hline$F(000)$ & \multicolumn{2}{|l|}{1304} \\
\hline Crystal size & \multicolumn{2}{|c|}{$0.220 \times 0.114 \times 0.058 \mathrm{~mm}^{3}$} \\
\hline Crystal shape and color & \multicolumn{2}{|c|}{ Block, clear intense yellow } \\
\hline Theta range for data collection & \multicolumn{2}{|l|}{2.356 to $30.610^{\circ}$} \\
\hline Index ranges & \multicolumn{2}{|c|}{$-19 \leq h \leq 19,-20 \leq k \leq 20,-20 \leq \mathrm{l} \leq 20$} \\
\hline
\end{tabular}




\begin{tabular}{|c|c|c|}
\hline Reflections collected & \multicolumn{2}{|l|}{94562} \\
\hline Independent reflections & \multicolumn{2}{|c|}{$8294[\mathrm{R}(\mathrm{int})=0.0768]$} \\
\hline Completeness to theta $=25.242^{\circ}$ & \multicolumn{2}{|l|}{$99.9 \%$} \\
\hline Max. and min. transmission & \multicolumn{2}{|c|}{0.7461 and 0.5667} \\
\hline Refinement method & \multicolumn{2}{|c|}{ Full-matrix least-squares on $\mathrm{F}^{2}$} \\
\hline Data / restraints / parameters & \multicolumn{2}{|c|}{$8294 / 0 / 270$} \\
\hline Goodness-of-fit on $\mathrm{F}^{2}$ & \multicolumn{2}{|l|}{1.028} \\
\hline Final $R$ indices $[I>2 \operatorname{sigma}(I)]$ & $\mathrm{R} 1=0.0269$ & $\mathrm{wR} 2=0.0385$ \\
\hline $\mathrm{R}$ indices (all data) & $\mathrm{R} 1=0.0502$ & $w R 2=0.0430$ \\
\hline Largest diff. peak and hole & \multicolumn{2}{|c|}{0.930 and $-1.091 \mathrm{eA}^{-3}$} \\
\hline
\end{tabular}

\section{A.18 $\left[\mathrm{ReCl}\left(\mathrm{N}_{2}\right)_{2}\left(\mathrm{HPNP}^{\mathrm{iPr}}\right)\right](35)$}

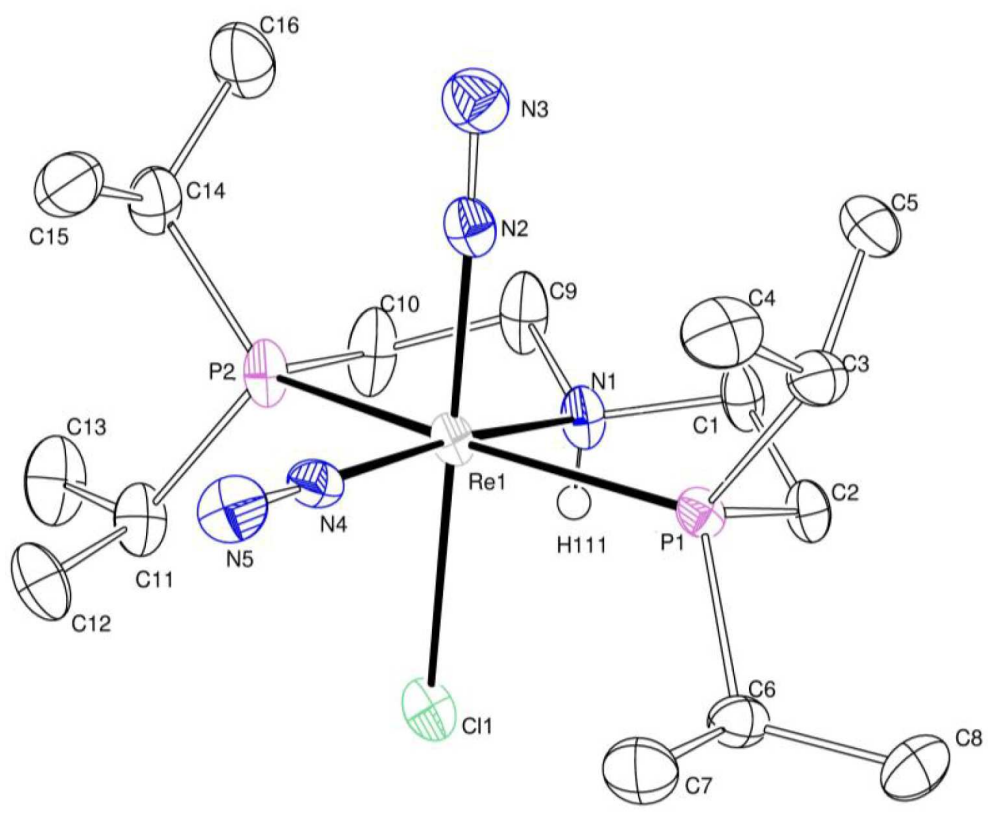

Fig. A.18. Thermal ellipsoid plot of 35 with the anisotropic displacement parameters drawn at the $50 \%$ probability level. The asymmetric unit contains one complex molecule. The $\mathrm{N}-\mathrm{H}$ hydrogen atom was found from the residual density map and isotropically refined.

Tab. A.18. Crystal data and structure refinement of $\mathbf{3 5}$.

\begin{tabular}{l|l}
\hline Identification code & 35 \\
\hline Empirical formula & $\mathrm{C}_{16} \mathrm{H}_{37} \mathrm{CIN}_{5} \mathrm{P}_{2} \mathrm{Re}$ \\
\hline
\end{tabular}




\begin{tabular}{|c|c|c|}
\hline Formula weight & \multicolumn{2}{|l|}{583.09} \\
\hline Temperature & \multicolumn{2}{|l|}{$100(2) \mathrm{K}$} \\
\hline Wavelength & \multicolumn{2}{|l|}{$0.71073 \AA$} \\
\hline Crystal system & \multicolumn{2}{|l|}{ Monoclinic } \\
\hline Space group & \multicolumn{2}{|l|}{$P 2_{1} / \mathrm{n}$} \\
\hline Unit cell dimensions & $\begin{array}{l}\mathrm{a}=8.4054(4) \AA \\
\mathrm{b}=12.8887(6) \AA \\
\mathrm{c}=21.2215(9) \AA\end{array}$ & $\begin{array}{l}\alpha=90^{\circ} \\
\beta=96.241(2)^{\circ} \\
\gamma=90^{\circ}\end{array}$ \\
\hline Volume & \multicolumn{2}{|l|}{$2285.40(18) \AA^{3}$} \\
\hline Z & \multicolumn{2}{|l|}{4} \\
\hline Density (calculated) & \multicolumn{2}{|l|}{$1.695 \mathrm{Mg} / \mathrm{m}^{3}$} \\
\hline Absorption coefficient & \multicolumn{2}{|l|}{$5.584 \mathrm{~mm}^{-1}$} \\
\hline$F(000)$ & \multicolumn{2}{|l|}{1160} \\
\hline Crystal size & \multicolumn{2}{|c|}{$0.255 \times 0.140 \times 0.133 \mathrm{~mm}^{3}$} \\
\hline Crystal shape and color & \multicolumn{2}{|c|}{ Block, clear pale yellow-green } \\
\hline Theta range for data collection & \multicolumn{2}{|c|}{2.495 to $30.597^{\circ}$} \\
\hline Index ranges & \multicolumn{2}{|c|}{$-12 \leq \mathrm{h} \leq 12,-18 \leq \mathrm{k} \leq 18,-29 \leq \mathrm{l} \leq 30$} \\
\hline Reflections collected & \multicolumn{2}{|c|}{89022} \\
\hline Independent reflections & \multicolumn{2}{|c|}{$7010[\mathrm{R}($ int $)=0.0572]$} \\
\hline Completeness to theta $=25.242^{\circ}$ & \multicolumn{2}{|l|}{$100 \%$} \\
\hline Max. and min. transmission & \multicolumn{2}{|c|}{0.7461 and 0.6204} \\
\hline Refinement method & \multicolumn{2}{|c|}{ Full-matrix least-squares on $\mathrm{F}^{2}$} \\
\hline Data / restraints / parameters & \multicolumn{2}{|l|}{$7010 / 0 / 238$} \\
\hline Goodness-of-fit on $F^{2}$ & \multicolumn{2}{|l|}{1.05} \\
\hline Final $R$ indices $[I>2 \operatorname{sigma}(I)]$ & $\mathrm{R} 1=0.0264$ & $w R 2=0.0502$ \\
\hline $\mathrm{R}$ indices (all data) & $\mathrm{R} 1=0.0423$ & $\mathrm{wR} 2=0.0557$ \\
\hline Largest diff. peak and hole & \multicolumn{2}{|c|}{1.740 and $-0.868 e^{-3} \AA^{-3}$} \\
\hline
\end{tabular}




\section{A.19 $\left[\left(\mu-\mathrm{N}_{2}\right)\left\{\operatorname{ReCl}_{2}\left(\mathrm{HPNP}^{i \mathrm{Pr}}\right)\right\}_{2}\right](36)$}

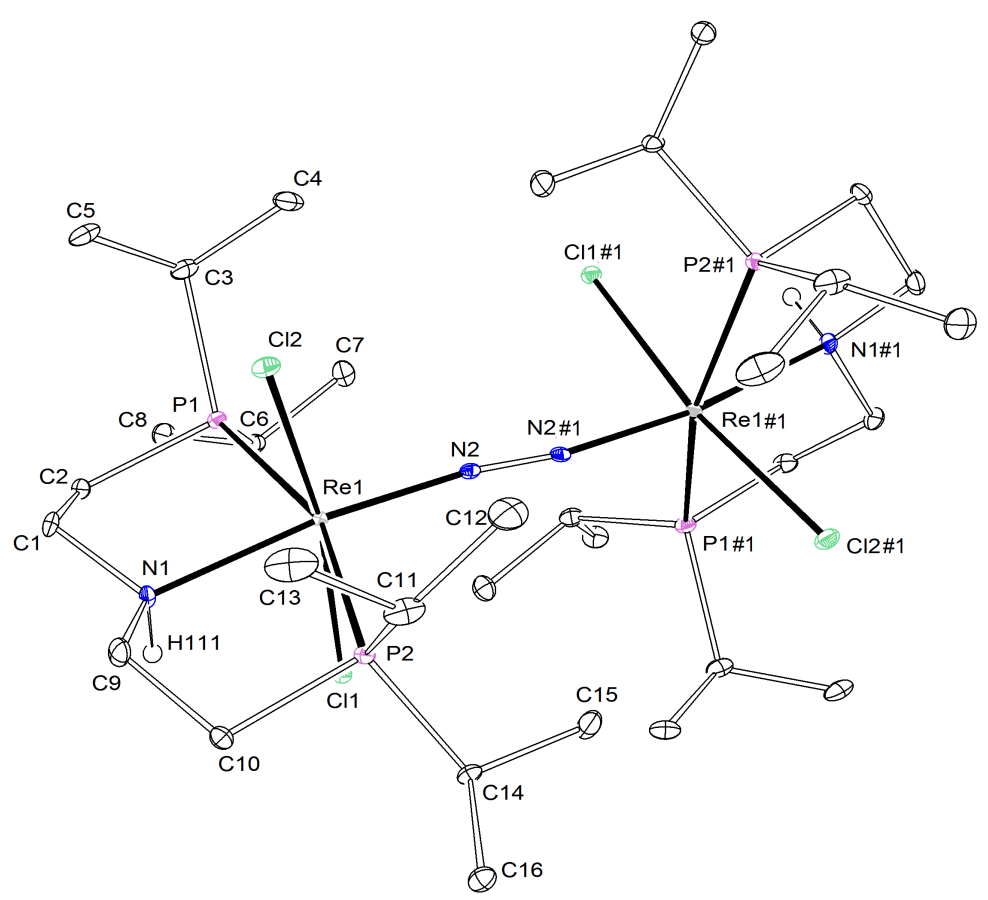

Fig. A.19. Thermal ellipsoid plot of $\mathbf{3 6}$ with the anisotropic displacement parameters drawn at the $50 \%$ probability level. The asymmetric unit contains one complex molecule. The $\mathrm{N}-\mathrm{H}$ hydrogen atom was found from the residual density map and isotropically refined.

Tab. A.19. Crystal data and structure refinement of $\mathbf{3 6}$.

\begin{tabular}{|c|c|c|}
\hline Identification code & \multicolumn{2}{|l|}{36} \\
\hline Empirical formula & \multicolumn{2}{|l|}{$\mathrm{C}_{32} \mathrm{H}_{74} \mathrm{Cl}_{4} \mathrm{~N}_{4} \mathrm{P}_{4} \mathrm{Re}_{2}$} \\
\hline Formula weight & \multicolumn{2}{|l|}{1153.03} \\
\hline Temperature & \multicolumn{2}{|l|}{$101(2) \mathrm{K}$} \\
\hline Wavelength & \multicolumn{2}{|l|}{$0.71073 \AA$} \\
\hline Crystal system & \multicolumn{2}{|l|}{ Monoclinic } \\
\hline Space group & \multicolumn{2}{|l|}{$C 2 / \mathrm{c}$} \\
\hline Unit cell dimensions & $\begin{array}{l}\mathrm{a}=16.7163(11) \AA \\
\mathrm{b}=14.5042(10) \AA \\
\mathrm{c}=18.2802(12) \AA\end{array}$ & $\begin{array}{l}\alpha=90^{\circ} \\
\beta=96.445(2)^{\circ} \\
\gamma=90^{\circ}\end{array}$ \\
\hline Volume & \multicolumn{2}{|l|}{$4404.1(5) \AA^{3}$} \\
\hline Z & \multicolumn{2}{|l|}{4} \\
\hline Density (calculated) & \multicolumn{2}{|l|}{$1.739 \mathrm{Mg} / \mathrm{m}^{3}$} \\
\hline
\end{tabular}




\begin{tabular}{|c|c|c|}
\hline Absorption coefficient & \multicolumn{2}{|l|}{$5.908 \mathrm{~mm}^{-1}$} \\
\hline$F(000)$ & \multicolumn{2}{|l|}{2288} \\
\hline Crystal size & \multicolumn{2}{|c|}{$0.085 \times 0.043 \times 0.036 \mathrm{~mm}^{3}$} \\
\hline Crystal shape and color & \multicolumn{2}{|c|}{ Block, clear intense blue-green } \\
\hline Theta range for data collection & \multicolumn{2}{|c|}{2.242 to $28.367^{\circ}$} \\
\hline Index ranges & \multicolumn{2}{|c|}{$-22 \leq \mathrm{h} \leq 22,-19 \leq \mathrm{k} \leq 19,-24 \leq \mathrm{I} \leq 24$} \\
\hline Reflections collected & \multicolumn{2}{|l|}{73468} \\
\hline Independent reflections & \multicolumn{2}{|c|}{$5510[\mathrm{R}$ (int) $=0.0773]$} \\
\hline Completeness to theta $=25.242^{\circ}$ & \multicolumn{2}{|l|}{$99.90 \%$} \\
\hline Refinement method & \multicolumn{2}{|c|}{ Full-matrix least-squares on $\mathrm{F}^{2}$} \\
\hline Data / restraints / parameters & \multicolumn{2}{|c|}{$5510 / 0 / 220$} \\
\hline Goodness-of-fit on $\mathrm{F}^{2}$ & \multicolumn{2}{|l|}{1.062} \\
\hline Final R indices $[\mathrm{I}>2 \operatorname{sigma}(\mathrm{I})]$ & $\mathrm{R} 1=0.0262$ & $\mathrm{wR} 2=0.0410$ \\
\hline $\mathrm{R}$ indices (all data) & $\mathrm{R} 1=0.0376$ & $\mathrm{wR} 2=0.0432$ \\
\hline Largest diff. peak and hole & \multicolumn{2}{|c|}{1.422 and $-1.299 \mathrm{e}^{-3}$} \\
\hline
\end{tabular}




\section{A.20 $\left[\operatorname{Re}(\mathrm{N}) \mathrm{Cl}_{2}\left(\mathrm{HPNP}^{\mathrm{iPr}}\right)\right](37)$}

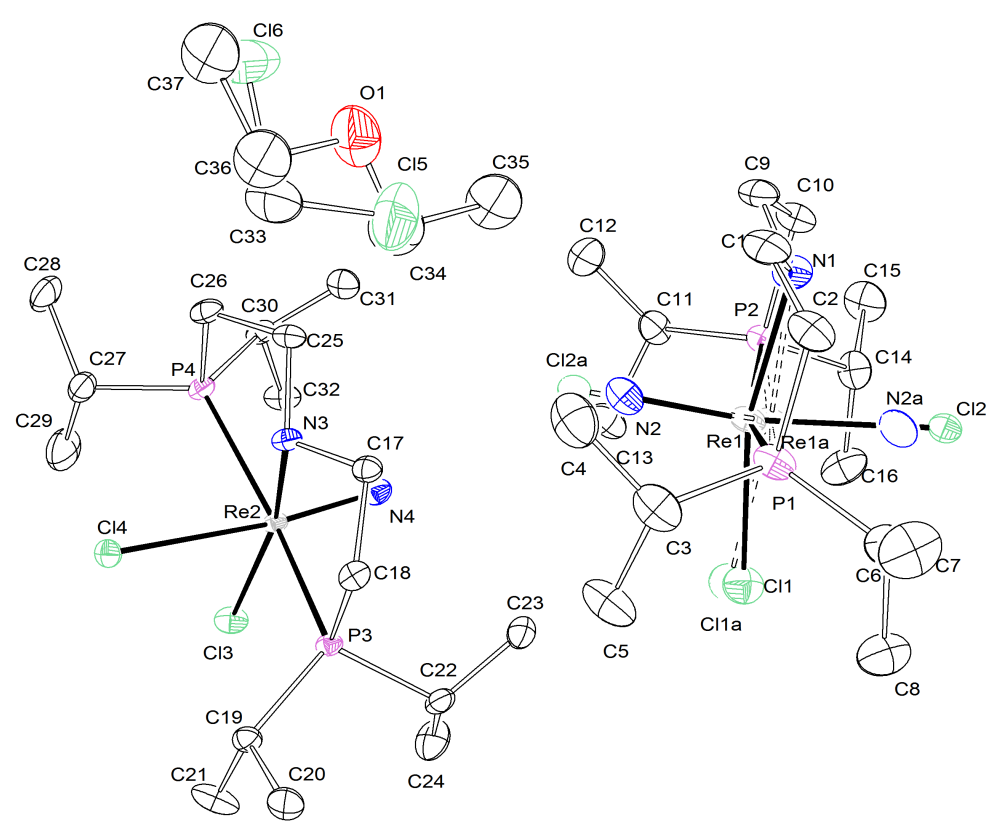

Fig. A.20. Thermal ellipsoid plot of $\mathbf{3 7}$ with the anisotropic displacement parameters drawn at the $50 \%$ probability level. The asymmetric unit contains one disordered complex molecule, one complex molecule without disorder and a dichloromethane and molecule which is one the same position as a diethylether molecule. The disordered complex molecule was refined with population of $0.962(2)$ on the main domain using some restraints and constraints (SADI, EADP). The solvent molecules were set to a population of 0.7 for the dichloromethane molecule and 0.3 for the diethylether molecule using some restraints and constrains (DFIX, EADP, RIGU, SADI).

Tab. A.20. Crystal data and structure refinement of 37 .

\begin{tabular}{|c|c|c|}
\hline Identification code & \multicolumn{2}{|l|}{37} \\
\hline Empirical formula & \multicolumn{2}{|c|}{$\mathrm{C}_{33.90} \mathrm{H}_{77.40} \mathrm{Cl}_{5.40} \mathrm{~N}_{4} \mathrm{O}_{0.30} \mathrm{P}_{4} \mathrm{Re}_{2}$} \\
\hline Formula weight & \multicolumn{2}{|l|}{1233.7} \\
\hline Temperature & \multicolumn{2}{|l|}{$100(2) \mathrm{K}$} \\
\hline Wavelength & \multicolumn{2}{|l|}{$0.71073 \AA$} \\
\hline Crystal system & \multicolumn{2}{|l|}{ Monoclinic } \\
\hline Space group & \multicolumn{2}{|l|}{$P 2_{1} / \mathrm{n}$} \\
\hline Unit cell dimensions & $\begin{array}{l}\mathrm{a}=14.1911(7) \AA \\
\mathrm{b}=18.7893(10) \AA \\
\mathrm{c}=19.6013(10) \AA\end{array}$ & $\begin{array}{l}\alpha=90^{\circ} \\
\beta=106.477(2)^{\circ} \\
\gamma=90^{\circ}\end{array}$ \\
\hline Volume & \multicolumn{2}{|l|}{$5011.9(4) \AA^{3}$} \\
\hline Z & \multicolumn{2}{|l|}{4} \\
\hline
\end{tabular}




\begin{tabular}{|c|c|c|}
\hline Density (calculated) & \multicolumn{2}{|l|}{$1.635 \mathrm{Mg} / \mathrm{m}^{3}$} \\
\hline Absorption coefficient & \multicolumn{2}{|l|}{$5.270 \mathrm{~mm}^{-1}$} \\
\hline$F(000)$ & \multicolumn{2}{|l|}{2452} \\
\hline Crystal size & \multicolumn{2}{|c|}{$0.314 \times 0.266 \times 0.103 \mathrm{~mm}^{3}$} \\
\hline Crystal shape and color & \multicolumn{2}{|c|}{ Block, clear intense brown } \\
\hline Theta range for data collection & \multicolumn{2}{|c|}{2.168 to $28.381^{\circ}$} \\
\hline Index ranges & \multicolumn{2}{|c|}{$-18 \leq h \leq 18,-25 \leq k \leq 25,-26 \leq \mathrm{I} \leq 26$} \\
\hline Reflections collected & \multicolumn{2}{|l|}{230643} \\
\hline Independent reflections & \multicolumn{2}{|c|}{$12540[R($ int $)=0.0407]$} \\
\hline Completeness to theta $=25.242^{\circ}$ & \multicolumn{2}{|l|}{$99.9 \%$} \\
\hline Max. and min. transmission & \multicolumn{2}{|c|}{0.7461 and 0.6204} \\
\hline Refinement method & \multicolumn{2}{|c|}{ Full-matrix least-squares on $\mathrm{F}^{2}$} \\
\hline Data / restraints / parameters & \multicolumn{2}{|c|}{$12540 / 83 / 507$} \\
\hline Goodness-of-fit on $F^{2}$ & \multicolumn{2}{|c|}{1.178} \\
\hline Final $R$ indices $[I>2 \operatorname{sigma}(I)]$ & $\mathrm{R} 1=0.0228$ & $\mathrm{wR} 2=0.0467$ \\
\hline $\mathrm{R}$ indices (all data) & $\mathrm{R} 1=0.0289$ & $\mathrm{wR} 2=0.0504$ \\
\hline Largest diff. peak and hole & \multicolumn{2}{|c|}{1.177 and $-0.974 \mathrm{e}^{-3}$} \\
\hline
\end{tabular}




\section{A.21 cis- $\left[\mathrm{Re}(\mathrm{NH}) \mathrm{Cl}_{2}\left(\mathrm{HPNP}^{\mathrm{iPr}}\right)\right]^{\mathrm{OTf}}\left(\right.$ cis-38 $\left.{ }^{\mathrm{OTf}}\right)$}

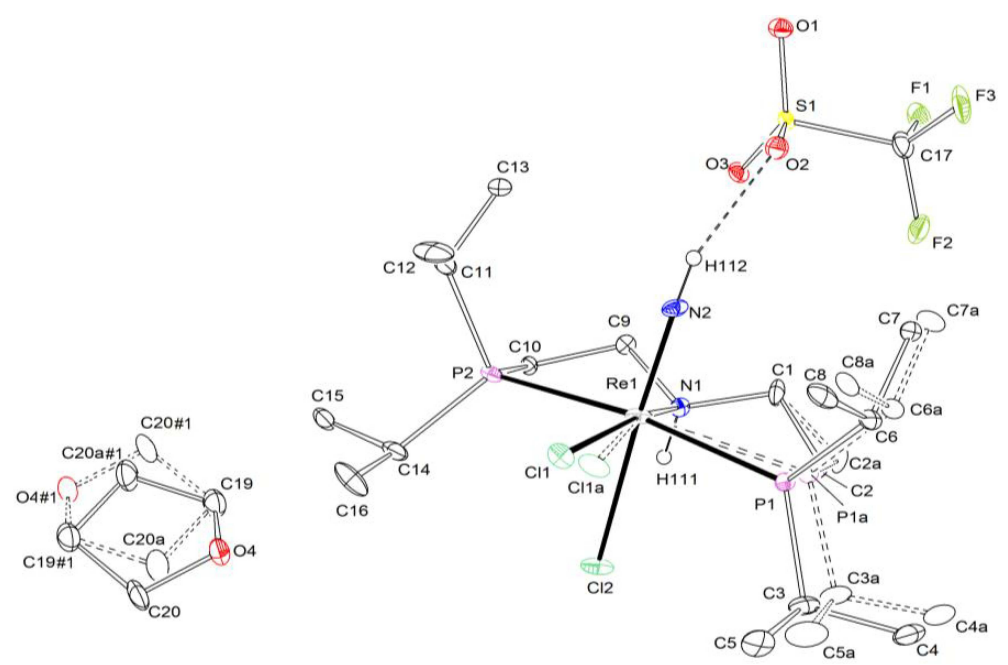

Fig. A.21. Thermal ellipsoid plot of cis-380Tf with the anisotropic displacement parameters drawn at the $50 \%$ probability level. The asymmetric unit contains one disordered complex molecule, one $\mathrm{CF}_{3} \mathrm{SO}_{3}^{-}$anion and a half disordered THF solvent molecule. The disordered complex molecule and the disordered THF solvent molecule were refined with site occupation factors of 0.5 for both sites using PART commands and some restraints and constraints (SADI, RIGU, EADP). All N-H hydrogen atoms were found from the residual density map and isotropically refined.

Tab. A.21. Crystal data and structure refinement of cis-38 ${ }^{\text {OTf }}$.

\begin{tabular}{|c|c|c|}
\hline Identification code & \multicolumn{2}{|l|}{ cis-380Tf } \\
\hline Empirical formula & \multicolumn{2}{|c|}{$\mathrm{C}_{16} \mathrm{H}_{38} \mathrm{Cl}_{2} \mathrm{~N}_{2} \mathrm{P}_{2} \mathrm{Re} \cdot \mathrm{CF}_{3} \mathrm{SO}_{3} \cdot \frac{1}{2} \mathrm{C}_{4} \mathrm{H}_{8} \mathrm{O}$} \\
\hline Formula weight & \multicolumn{2}{|l|}{762.64} \\
\hline Temperature & \multicolumn{2}{|l|}{$101(2) \mathrm{K}$} \\
\hline Wavelength & \multicolumn{2}{|l|}{$0.71073 \AA$} \\
\hline Crystal system & \multicolumn{2}{|l|}{ Monoclinic } \\
\hline Space group & \multicolumn{2}{|l|}{$P 2_{1} / \mathrm{n}$} \\
\hline Unit cell dimensions & $\begin{array}{l}\mathrm{a}=7.4700(4) \AA \\
\mathrm{b}=32.5017(18) \AA \\
\mathrm{c}=12.4358(8) \AA\end{array}$ & $\begin{array}{l}\alpha=90^{\circ} \\
\beta=102.681(2)^{\circ} \\
\gamma=90^{\circ}\end{array}$ \\
\hline Volume & \multicolumn{2}{|l|}{$2945.6(3) \AA^{3}$} \\
\hline Z & \multicolumn{2}{|l|}{4} \\
\hline Density (calculated) & \multicolumn{2}{|l|}{$1.720 \mathrm{Mg} / \mathrm{m}^{3}$} \\
\hline Absorption coefficient & \multicolumn{2}{|l|}{$4.530 \mathrm{~mm}^{-1}$} \\
\hline
\end{tabular}




\begin{tabular}{|c|c|c|}
\hline $\mathrm{F}(000)$ & \multicolumn{2}{|l|}{1520} \\
\hline Crystal size & \multicolumn{2}{|c|}{$0.162 \times 0.060 \times 0.054 \mathrm{~mm}^{3}$} \\
\hline Crystal shape and color & \multicolumn{2}{|c|}{ Plate, clear light blue } \\
\hline Theta range for data collection & \multicolumn{2}{|c|}{2.507 to $28.384^{\circ}$} \\
\hline Index ranges & \multicolumn{2}{|c|}{$-9 \leq h \leq 9,-43 \leq k \leq 43,-16 \leq 1 \leq 16$} \\
\hline Reflections collected & \multicolumn{2}{|l|}{98467} \\
\hline Independent reflections & \multicolumn{2}{|c|}{$7353[R($ int $)=0.1698]$} \\
\hline Completeness to theta $=25.242^{\circ}$ & \multicolumn{2}{|l|}{$99.9 \%$} \\
\hline Refinement method & \multicolumn{2}{|c|}{ Full-matrix least-squares on $\mathrm{F}^{2}$} \\
\hline Data / restraints / parameters & \multicolumn{2}{|c|}{7353 / 136 / 393} \\
\hline Goodness-of-fit on $F^{2}$ & \multicolumn{2}{|l|}{1.079} \\
\hline Final $R$ indices $[I>2 \operatorname{sigma}(I)]$ & $\mathrm{R} 1=0.0514$ & $\mathrm{wR} 2=0.0724$ \\
\hline $\mathrm{R}$ indices (all data) & $\mathrm{R} 1=0.0908$ & $\mathrm{wR} 2=0.0805$ \\
\hline Largest diff. peak and hole & \multicolumn{2}{|c|}{1.498 and $-1.862 \mathrm{e}^{-3}$} \\
\hline
\end{tabular}




\section{A.22 trans- $\left[\mathrm{Re}(\mathrm{NH}) \mathrm{Cl}_{2}\left(\mathrm{HPNP}^{\mathrm{iPr}}\right)\right]^{\mathrm{BAr} \mathrm{F}_{24}^{\mathrm{F}}}\left(\right.$ trans-38 $\left.^{\mathrm{BAr}}{ }_{24}^{\mathrm{F}}\right)$}

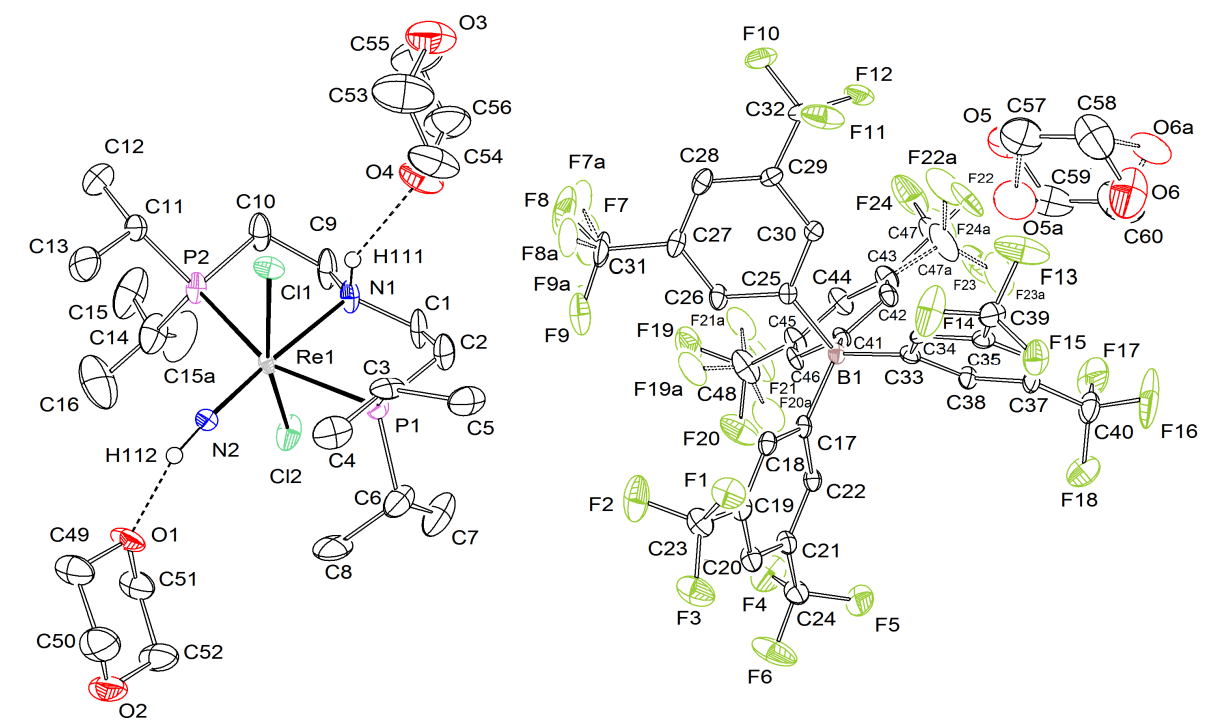

Fig. A.22. Thermal ellipsoid plot of trans-38 ${ }^{\mathrm{BAr}}{ }_{24}^{\mathrm{F}}$ with the anisotropic displacement parameters drawn at the $50 \%$ probability level. The asymmetric unit contains one complex molecule, three dioxane solvent molecules and one $\mathrm{BAr}_{24} \mathrm{~F}_{-}$anion. The disordered complex molecule was refined with population of $0.876(9)$ on the main domain using some restraints (RIGU, $\mathrm{SADI})$. One disordered dioxane solvent molecule was refined with population of $0.67(1)$ on the main domain using some restraints (RIGU, SADI). The disordered $\mathrm{BAr}_{24}^{\mathrm{F}-}$ anion was refined with population of $0.70(1), 0.73(2)$ and $0.876(9)$ on their main domains using some restraints (RIGU, SADI). Both $\mathrm{N}-\mathrm{H}$ hydrogen atoms were found from the residual density map and isotropically refined.

Tab. A.22. Crystal data and structure refinement of trans-38 $8^{\mathrm{BAr}}{ }_{24}^{\mathrm{F}}$.

\begin{tabular}{|c|c|c|}
\hline Identification code & \multicolumn{2}{|l|}{ trans-38 $8^{\mathrm{BAr}}{ }_{24}^{\mathrm{F}}$} \\
\hline Empirical formula & \multicolumn{2}{|c|}{$\mathrm{C}_{60} \mathrm{H}_{74} \mathrm{BCl}_{2} \mathrm{~F}_{24} \mathrm{~N}_{2} \mathrm{O}_{6} \mathrm{P}_{2} \mathrm{Re}$} \\
\hline Formula weight & \multicolumn{2}{|c|}{1705.06} \\
\hline Temperature & \multicolumn{2}{|l|}{$100(2) \mathrm{K}$} \\
\hline Wavelength & \multicolumn{2}{|l|}{$0.71073 \AA$} \\
\hline Crystal system & \multicolumn{2}{|l|}{ Monoclinic } \\
\hline Space group & \multicolumn{2}{|l|}{$P 2_{1} / \mathrm{c}$} \\
\hline Unit cell dimensions & $\begin{array}{l}\mathrm{a}=14.6803(12) \AA \\
\mathrm{b}=30.215(2) \AA \\
\mathrm{c}=17.4634(15) \AA\end{array}$ & $\begin{array}{l}\alpha=90^{\circ} \\
\beta=114.467(3)^{\circ} \\
\gamma=90^{\circ}\end{array}$ \\
\hline Volume & \multicolumn{2}{|l|}{$7050.5(10) \AA^{3}$} \\
\hline Z & \multicolumn{2}{|l|}{4} \\
\hline
\end{tabular}




\begin{tabular}{|c|c|c|}
\hline Density (calculated) & \multicolumn{2}{|l|}{$1.606 \mathrm{Mg} / \mathrm{m}^{3}$} \\
\hline Absorption coefficient & \multicolumn{2}{|l|}{$1.957 \mathrm{~mm}^{-1}$} \\
\hline$F(000)$ & \multicolumn{2}{|l|}{3424} \\
\hline Crystal size & \multicolumn{2}{|c|}{$0.100 \times 0.086 \times 0.038 \mathrm{~mm}^{3}$} \\
\hline Crystal shape and color & \multicolumn{2}{|c|}{ Plate, clear light blue } \\
\hline Theta range for data collection & \multicolumn{2}{|c|}{2.378 to $25.360^{\circ}$} \\
\hline Index ranges & \multicolumn{2}{|c|}{$-17 \leq h \leq 17,-36 \leq k \leq 36,-20 \leq \mathrm{l} \leq 21$} \\
\hline Reflections collected & \multicolumn{2}{|l|}{143381} \\
\hline Independent reflections & \multicolumn{2}{|c|}{$12865[R($ int $)=0.1675]$} \\
\hline Completeness to theta $=25.242^{\circ}$ & \multicolumn{2}{|l|}{$99.9 \%$} \\
\hline Refinement method & \multicolumn{2}{|c|}{ Full-matrix least-squares on $\mathrm{F}^{2}$} \\
\hline Data / restraints / parameters & \multicolumn{2}{|c|}{12865 / 947 / 1021} \\
\hline Goodness-of-fit on $\mathrm{F}^{2}$ & \multicolumn{2}{|l|}{1.139} \\
\hline Final R indices [I>2sigma $(I)]$ & $\mathrm{R} 1=0.0572$ & $\mathrm{wR} 2=0.0914$ \\
\hline $\mathrm{R}$ indices (all data) & $\mathrm{R} 1=0.0849$ & $w R 2=0.0984$ \\
\hline Largest diff. peak and hole & \multicolumn{2}{|c|}{0.771 and $-2.023 \mathrm{e}^{-3}$} \\
\hline
\end{tabular}




\section{A.23 $\left[\operatorname{Re}\left(\mathrm{NBAr}_{18}{ }_{18}^{\mathrm{F}}\right) \mathrm{Cl}_{2}\left(\mathrm{HPNP}^{\mathrm{iPr}}\right)\right](39)$}

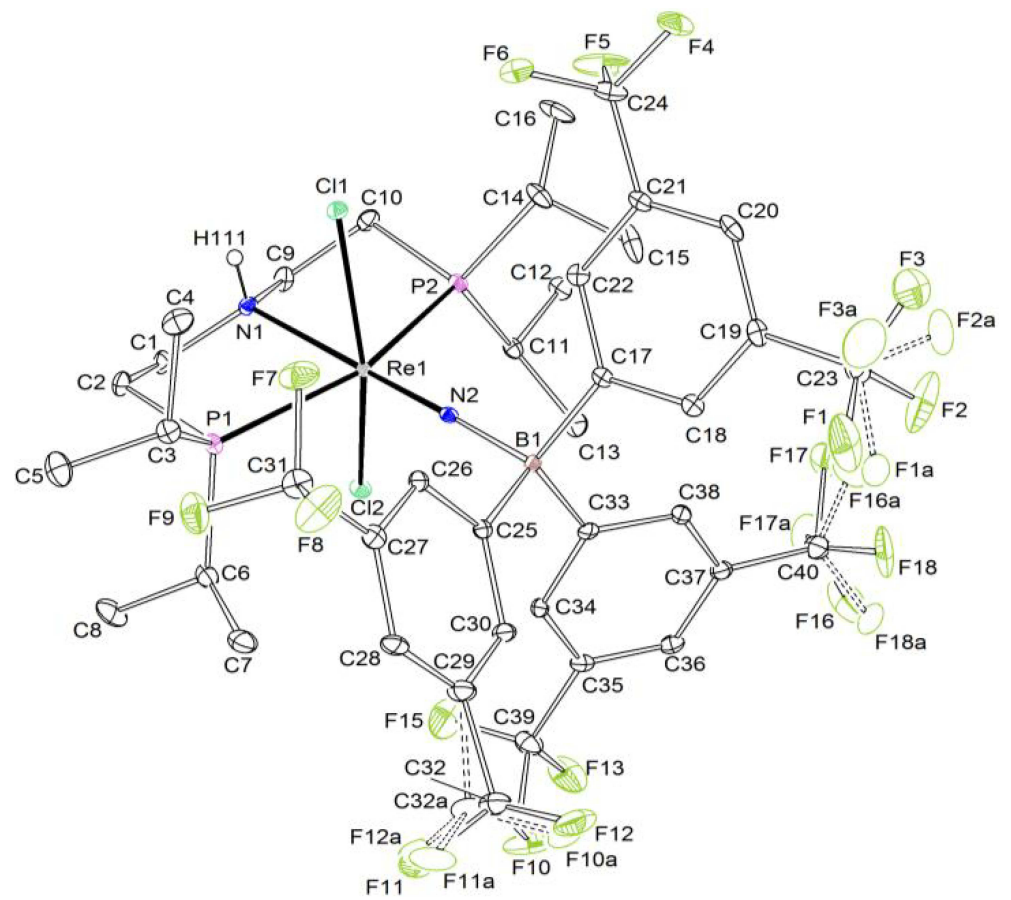

Fig. A.23. Thermal ellipsoid plot of $\mathbf{3 9}$ with the anisotropic displacement parameters drawn at the $50 \%$ probability level. The asymmetric unit contains one disordered complex molecule. The three disordered $\mathrm{CF}_{3}$ groups were refined in two different positions with population of $0.640(4), 0.793(8)$ and $0.731(7)$ on their main domains using PART commands and some restraints and constrains (RIGU, EADP). The $\mathrm{N}-\mathrm{H}$ hydrogen atom was found from the residual density map and isotropically refined.

Tab. A.23. Crystal data and structure refinement of 39 .

\begin{tabular}{|c|c|c|}
\hline Identification code & \multicolumn{2}{|l|}{39} \\
\hline Empirical formula & \multicolumn{2}{|c|}{$\mathrm{C}_{40} \mathrm{H}_{46} \mathrm{BCl}_{2} \mathrm{~F}_{18} \mathrm{~N}_{2} \mathrm{P}_{2} \mathrm{Re}$} \\
\hline Formula weight & \multicolumn{2}{|l|}{1226.64} \\
\hline Temperature & \multicolumn{2}{|l|}{$103(2) \mathrm{K}$} \\
\hline Wavelength & \multicolumn{2}{|l|}{$0.71073 \AA$} \\
\hline Crystal system & \multicolumn{2}{|l|}{ Monoclinic } \\
\hline Space group & \multicolumn{2}{|l|}{$C 2 / \mathrm{c}$} \\
\hline Unit cell dimensions & $\begin{array}{l}\mathrm{a}=25.0171(15) \AA \\
\mathrm{b}=12.5947(7) \AA \\
\mathrm{c}=32.3189(19) \AA\end{array}$ & $\begin{array}{l}\alpha=90^{\circ} \\
\beta=108.879(2)^{\circ} \\
\gamma=90^{\circ}\end{array}$ \\
\hline Volume & \multicolumn{2}{|l|}{$9635.3(10) \AA^{3}$} \\
\hline Z & \multicolumn{2}{|l|}{8} \\
\hline
\end{tabular}




\begin{tabular}{|c|c|c|}
\hline Density (calculated) & \multicolumn{2}{|l|}{$1.691 \mathrm{Mg} / \mathrm{m}^{3}$} \\
\hline Absorption coefficient & \multicolumn{2}{|l|}{$2.801 \mathrm{~mm}^{-1}$} \\
\hline $\mathrm{F}(000)$ & \multicolumn{2}{|l|}{4848} \\
\hline Crystal size & \multicolumn{2}{|c|}{$0.339 \times 0.240 \times 0.151 \mathrm{~mm}^{3}$} \\
\hline Crystal shape and color & \multicolumn{2}{|c|}{ Block, clear intense blue-green } \\
\hline Theta range for data collection & \multicolumn{2}{|c|}{2.423 to $28.370^{\circ}$} \\
\hline Index ranges & \multicolumn{2}{|c|}{$-33 \leq h \leq 33,-16 \leq k \leq 16,-43 \leq 1 \leq 43$} \\
\hline Reflections collected & \multicolumn{2}{|l|}{133858} \\
\hline Independent reflections & \multicolumn{2}{|c|}{$12043[R($ int $)=0.1225]$} \\
\hline Completeness to theta $=25.242^{\circ}$ & \multicolumn{2}{|l|}{$99.9 \%$} \\
\hline Refinement method & \multicolumn{2}{|c|}{ Full-matrix least-squares on $\mathrm{F}^{2}$} \\
\hline Data / restraints / parameters & \multicolumn{2}{|c|}{12043 / 450 / 694} \\
\hline Goodness-of-fit on $\mathrm{F}^{2}$ & \multicolumn{2}{|l|}{1.059} \\
\hline Final $R$ indices [I>2sigma $(I)]$ & $\mathrm{R} 1=0.0445$ & $w R 2=0.0676$ \\
\hline $\mathrm{R}$ indices (all data) & $\mathrm{R} 1=0.0758$ & $w R 2=0.0741$ \\
\hline Largest diff. peak and hole & \multicolumn{2}{|c|}{1.038 and $-1.278 \mathrm{e}^{-3}$} \\
\hline
\end{tabular}




\section{A.24 $\left[\mathrm{ReCl}_{3}\left(\mathrm{P}=\mathrm{NP}{ }^{i \mathrm{Pr}}\right)\right](40)$}

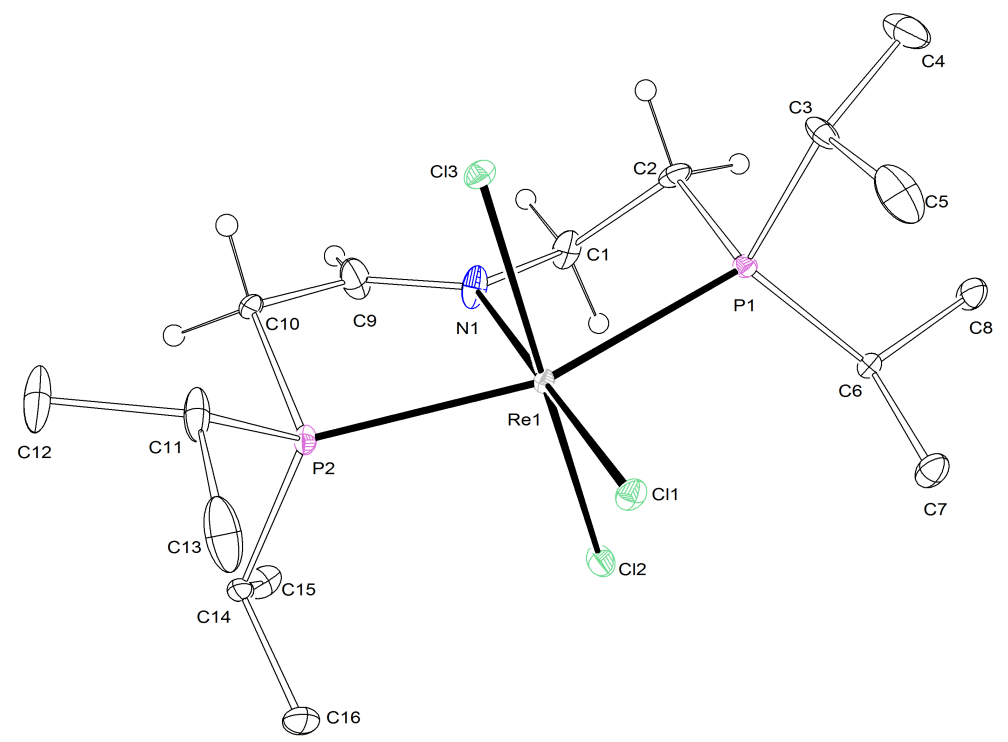

Fig. A.24. Thermal ellipsoid plot of $\mathbf{4 0}$ with the anisotropic displacement parameters drawn at the $50 \%$ probability level. The asymmetric unit contains one complex molecule. The structure was refined as an inversion twin using the twin law -100 0-10 00-1 (BASF: $0.016(7))$.

Tab. A.24. Crystal data and structure refinement of $\mathbf{4 0 .}$

\begin{tabular}{|c|c|c|}
\hline Identification code & \multicolumn{2}{|l|}{40} \\
\hline Empirical formula & \multicolumn{2}{|l|}{$\mathrm{C}_{16} \mathrm{H}_{35} \mathrm{Cl}_{3} \mathrm{NP}_{2} \mathrm{Re}$} \\
\hline Formula weight & \multicolumn{2}{|l|}{595.94} \\
\hline Temperature & \multicolumn{2}{|l|}{$102(2) \mathrm{K}$} \\
\hline Wavelength & \multicolumn{2}{|l|}{$0.71073 \AA$} \\
\hline Crystal system & \multicolumn{2}{|l|}{ Orthorhombic } \\
\hline Space group & \multicolumn{2}{|l|}{$P 2{ }_{1}{ }_{1}{ }_{2}{ }_{1}$} \\
\hline Unit cell dimensions & $\begin{array}{l}\mathrm{a}=7.2793(2) \AA \\
\mathrm{b}=12.8079(4) \AA \\
\mathrm{c}=23.9040(7) \AA\end{array}$ & $\begin{array}{l}\alpha=90^{\circ} \\
\beta=90^{\circ} \\
\gamma=90^{\circ}\end{array}$ \\
\hline Volume & \multicolumn{2}{|l|}{$2228.63(11) \AA^{3}$} \\
\hline Z & \multicolumn{2}{|l|}{4} \\
\hline Density (calculated) & \multicolumn{2}{|l|}{$1.776 \mathrm{Mg} / \mathrm{m}^{3}$} \\
\hline Absorption coefficient & \multicolumn{2}{|l|}{$5.955 \mathrm{~mm}^{-1}$} \\
\hline$F(000)$ & \multicolumn{2}{|l|}{1176} \\
\hline
\end{tabular}




\begin{tabular}{|c|c|c|}
\hline Crystal size & \multicolumn{2}{|c|}{$0.217 \times 0.122 \times 0.089 \mathrm{~mm}^{3}$} \\
\hline Crystal shape and color & \multicolumn{2}{|c|}{ Block, clear intense orange } \\
\hline Theta range for data collection & \multicolumn{2}{|c|}{2.331 to $28.353^{\circ}$} \\
\hline Index ranges & \multicolumn{2}{|c|}{$-9 \leq \mathrm{h} \leq 9,-17 \leq \mathrm{k} \leq 16,-31 \leq \mathrm{I} \leq 31$} \\
\hline Reflections collected & \multicolumn{2}{|l|}{42851} \\
\hline Independent reflections & \multicolumn{2}{|c|}{$5555[\mathrm{R}(\mathrm{int})=0.0666]$} \\
\hline Completeness to theta $=25.242^{\circ}$ & \multicolumn{2}{|l|}{$99.9 \%$} \\
\hline Refinement method & \multicolumn{2}{|c|}{ Full-matrix least-squares on $\mathrm{F}^{2}$} \\
\hline Data / restraints / parameters & \multicolumn{2}{|c|}{$5555 / 0 / 217$} \\
\hline Goodness-of-fit on $\mathrm{F}^{2}$ & \multicolumn{2}{|l|}{1.033} \\
\hline Final R indices [I $>2 \operatorname{sigma}(I)]$ & $\mathrm{R} 1=0.0245$ & $w R 2=0.0423$ \\
\hline $\mathrm{R}$ indices (all data) & $\mathrm{R} 1=0.0301$ & $w R 2=0.0435$ \\
\hline Absolute structure parameter & \multicolumn{2}{|l|}{$0.016(7)$} \\
\hline Largest diff. peak and hole & \multicolumn{2}{|c|}{0.791 and $-1.120 \mathrm{e}^{-3}$} \\
\hline
\end{tabular}





\section{List of chemical compounds}

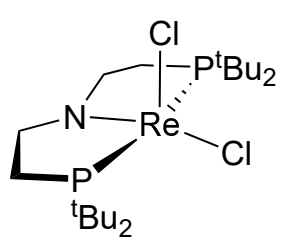

XIX

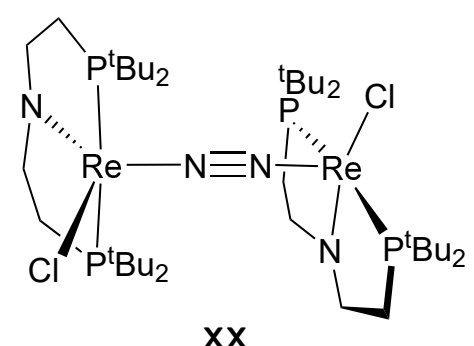

XX

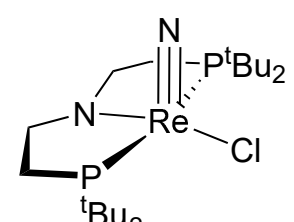

${ }^{\mathrm{t}} \mathrm{Bu}_{2}$

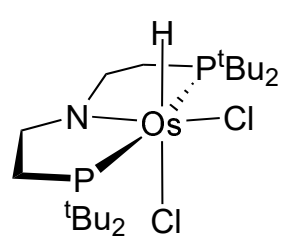

$\mathbf{X X I}$

1

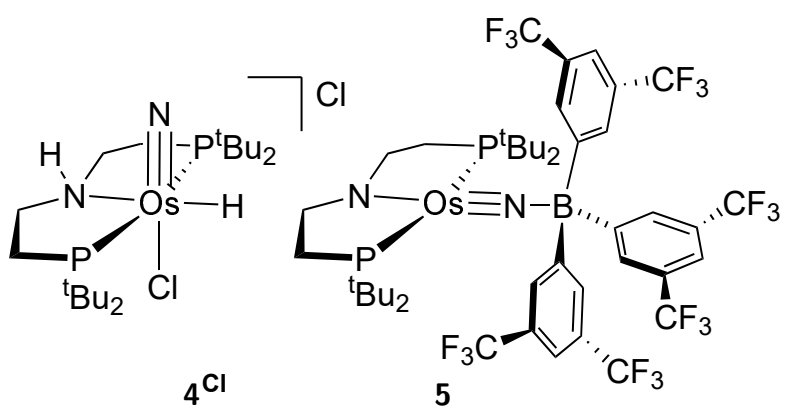

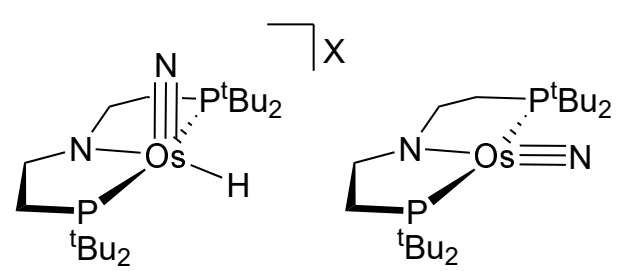

${ }^{\mathrm{B}} \mathrm{Bu}_{2}$

$2^{x}$

3

$4^{\mathrm{Cl}}$

5

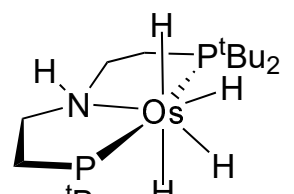

${ }^{\mathrm{B}} \mathrm{Bu}_{2} \mathrm{H}$

8

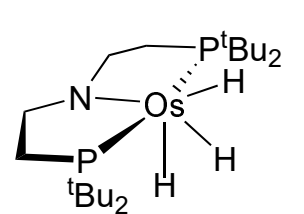

7

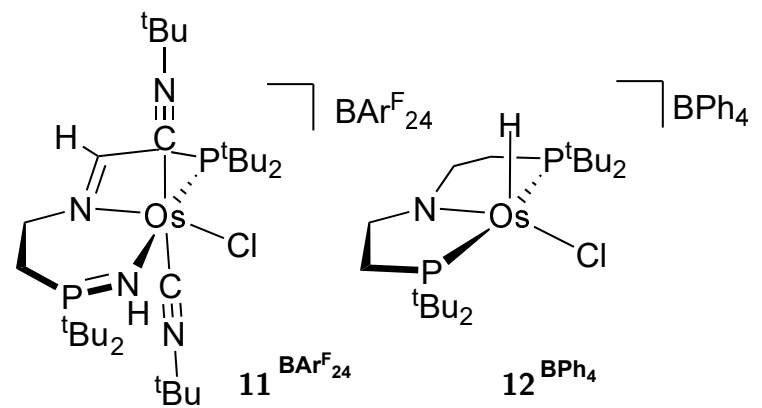

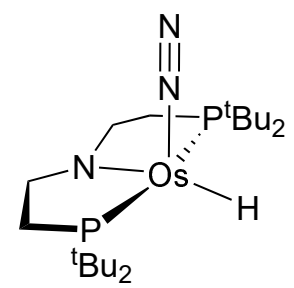

13

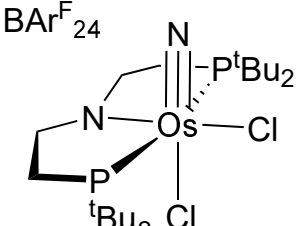

$12^{\mathrm{BPh}_{4}}$

$$
10^{\text {BArF }_{24}} \quad 10-\mathrm{Cl}
$$

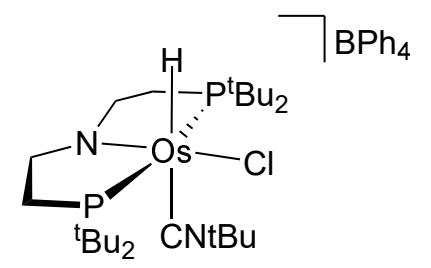

$14^{\mathrm{BPh}_{4}}$

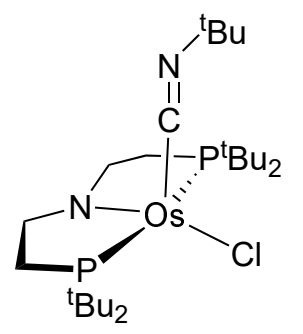

15 


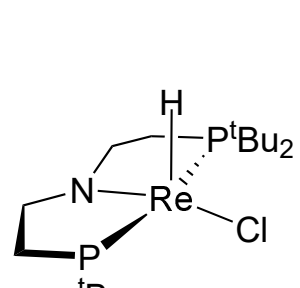

${ }^{\mathrm{t}} \mathrm{Bu}_{2}$

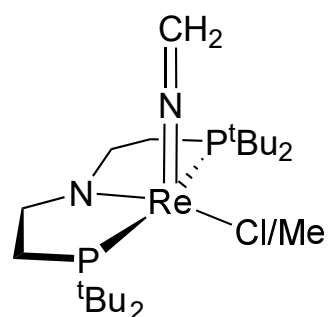

${ }_{18} \mathrm{Cl} / \mathrm{Me}$

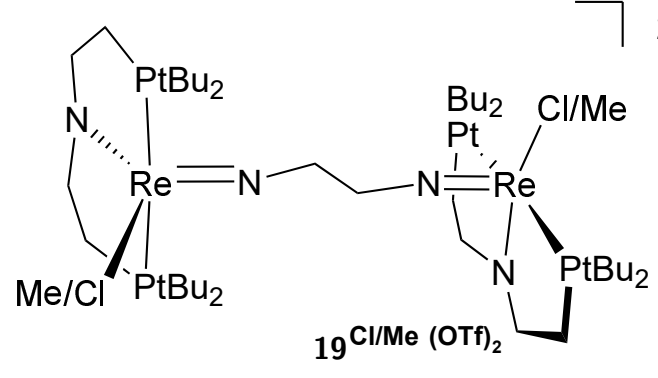

2 OTf

17
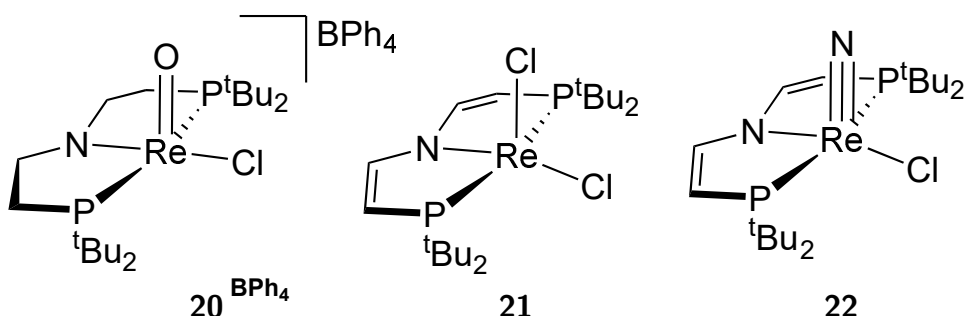

22

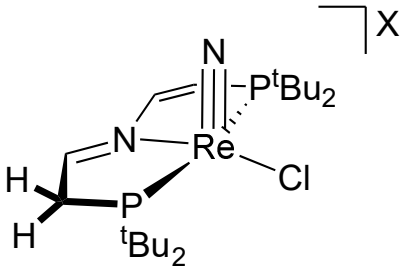

$23^{x}$

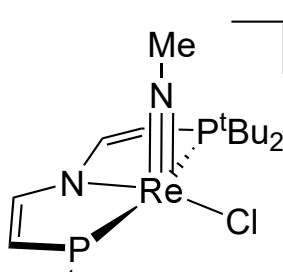

${ }^{\mathrm{t}} \mathrm{Bu}_{2}$ $24^{\text {OTf }}$

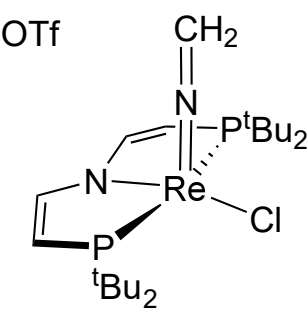

25

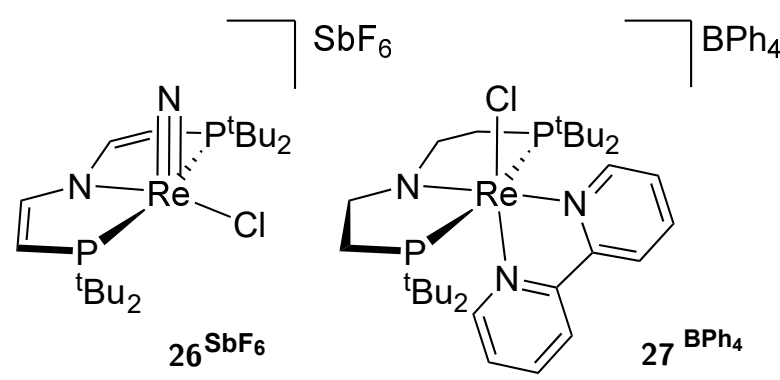

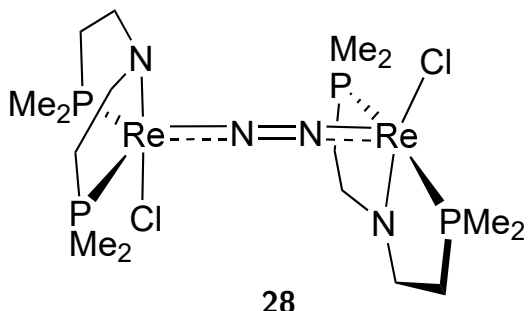

28

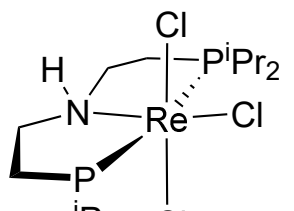

${ }^{\mathrm{i}} \mathrm{Pr}_{2} \mathrm{C}$

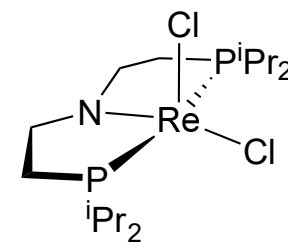

30

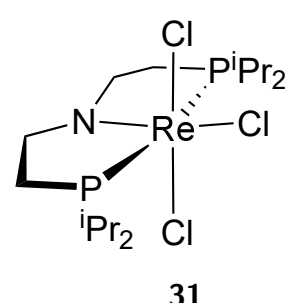

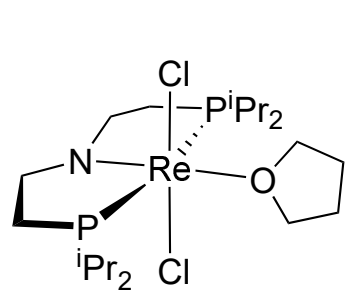

32

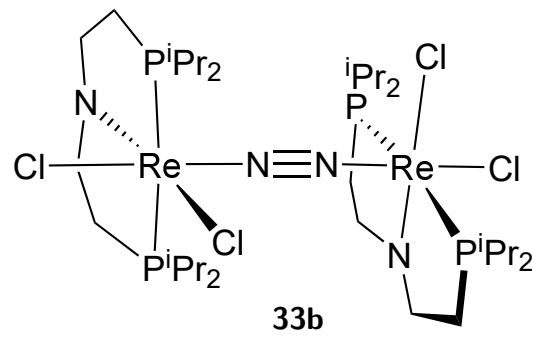

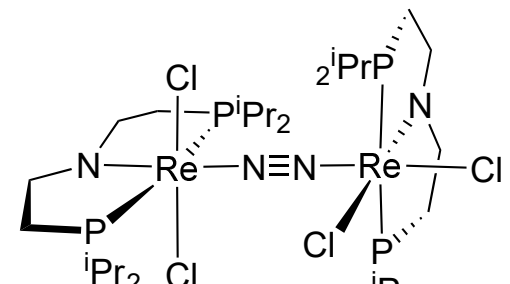

$33 a$

${ }^{\mathrm{i}} \mathrm{Pr}_{2}$
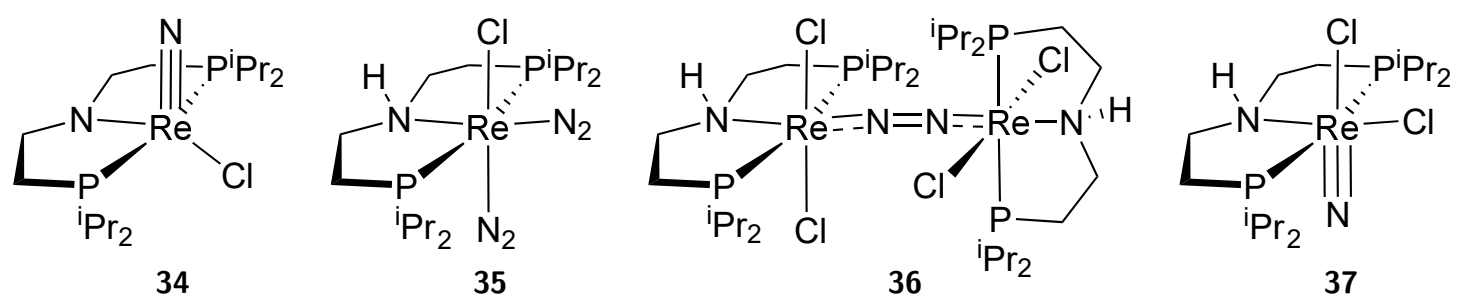

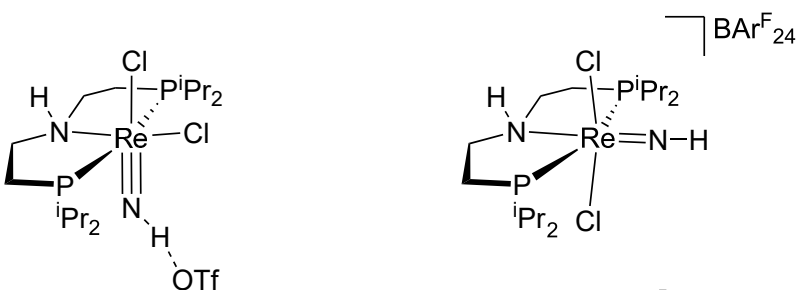

cis $38 \cdots$ OTf

$$
\text { trans } 38^{\mathrm{BAr}_{24}}
$$

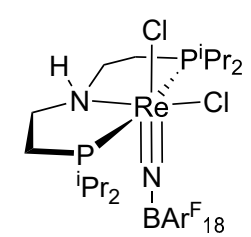

cis-39
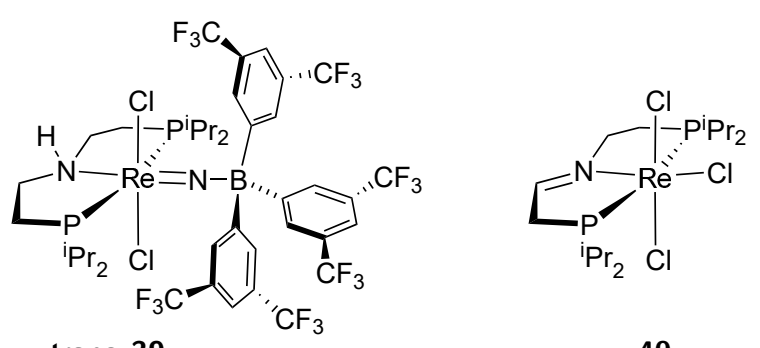

trans-39

40 



\section{List of abbreviations}

ATR-IR attenuated total reflection infrared $\ldots \ldots \ldots \ldots \ldots \ldots \ldots \ldots \ldots \ldots \ldots \ldots \ldots \ldots \ldots$

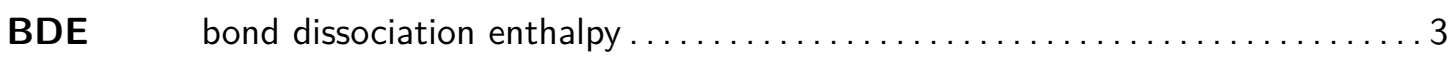

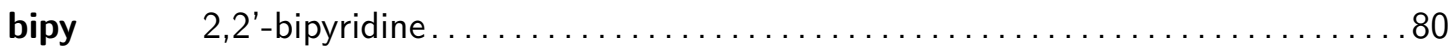

CASSCF complete active space self consistent field ....................... 88

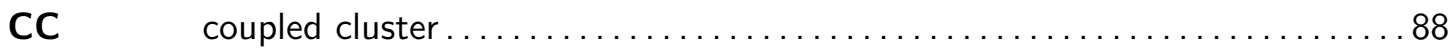

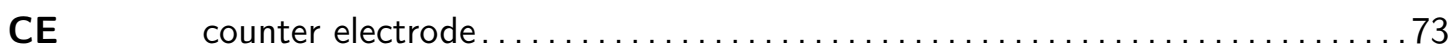

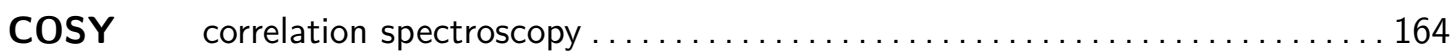

CPE controlled potential electrolysis............................. 123

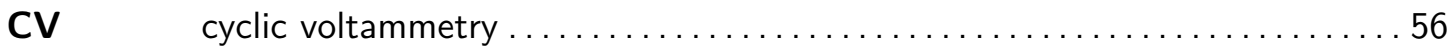

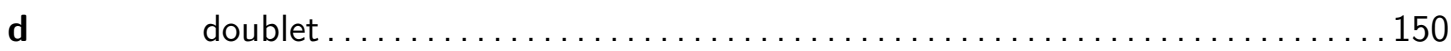

DBU 1,8-diazabicyclo[5.4.0]undec-7-ene

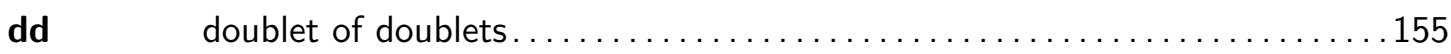

DCM dichloromethane......................................... 49

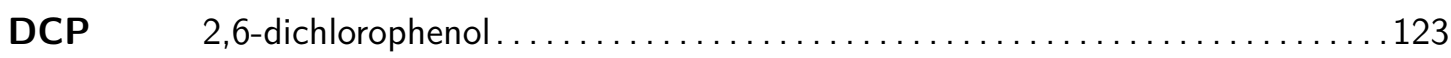

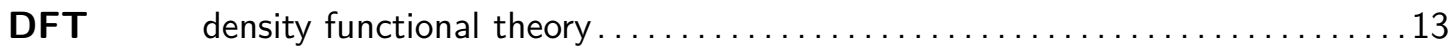

DOSY diffusion ordered spectroscopy. . . . . . . . . . . . . . . . . . . . 66

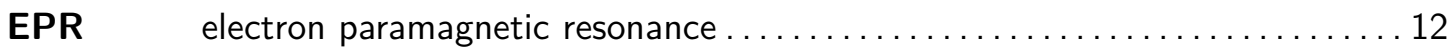

ESI electron spray ionization

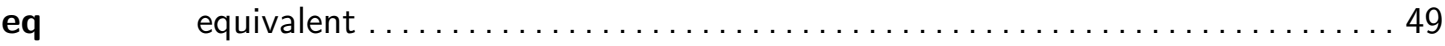

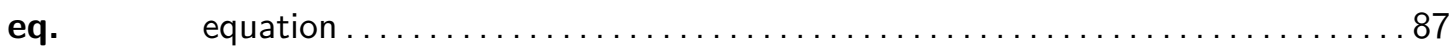

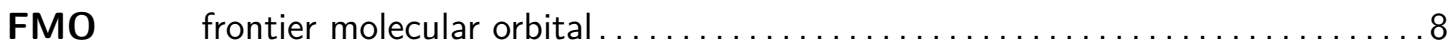

FWHM full width at half maximum

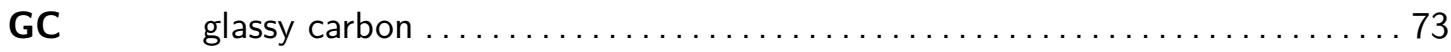

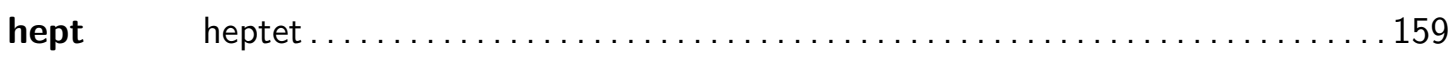

HMBC heteronuclear multiple bond correlation ....................... 97

HMDSO hexamethyldisiloxane ................................... 70

HOMO highest occupied molecular orbital ............................. 3

HSQC heteronuclear single quantum coherence ...................... 77

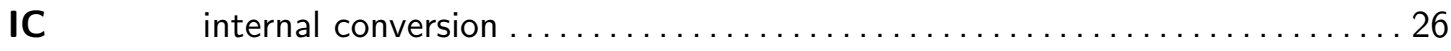

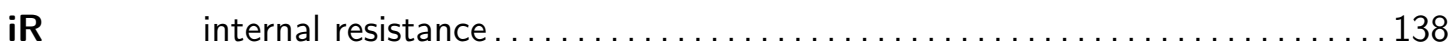

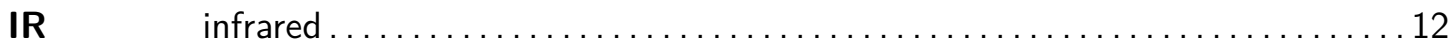

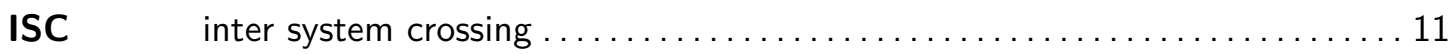

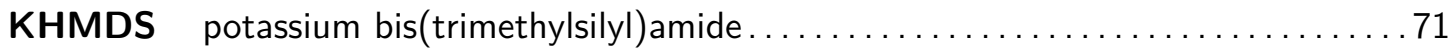

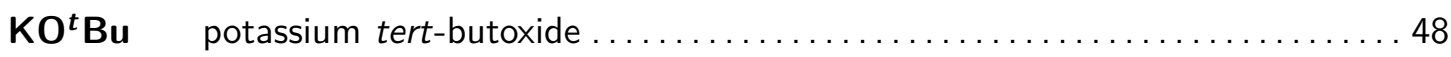




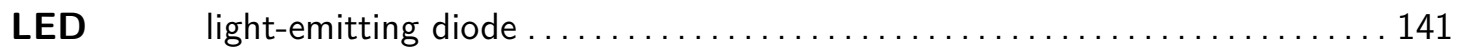

LIFDI liquid injection field desorption ionization ....................... 62

LUMO lowest unoccupied molecular orbital ........................... 3

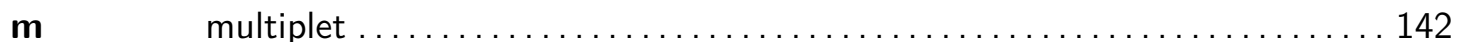

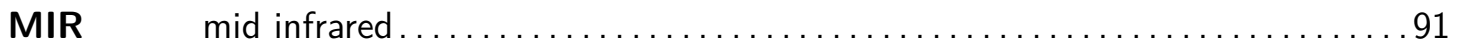

MLCT metal-ligand charge transfer...............................

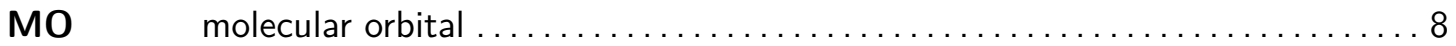

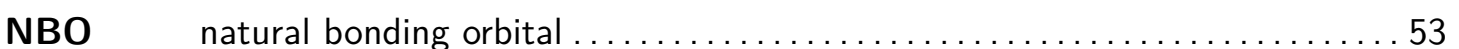

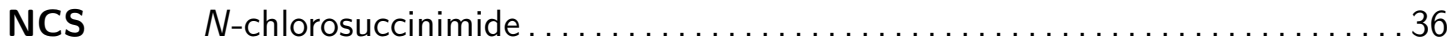

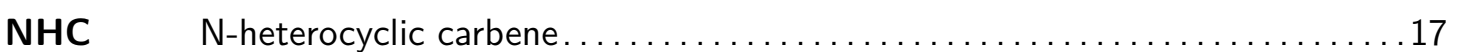

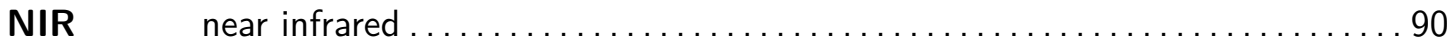

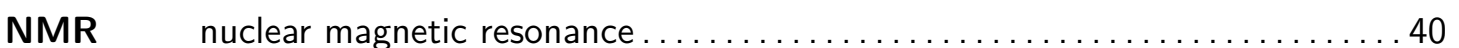

NEVPT2 N-electron valence state perturbation theory $\ldots \ldots \ldots \ldots \ldots \ldots \ldots$

NOESY nuclear Overhauser effect spectroscopy ........................... 50

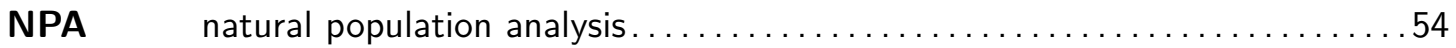

PCET proton coupled electron transfer . . . . . . . . . . . . . . . . . . . . . . . . 17

QDPT quasi-degenerate perturbation theory $\ldots \ldots \ldots \ldots \ldots \ldots \ldots \ldots \ldots$

QRO quasi-restricted orbital . . . . . . . . . . . . . . . . . . . . . . . . . . . . 134

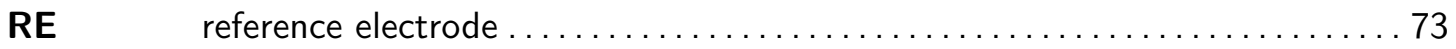

RMSD root-mean-square deviation (of atomic positions) . . . . . . . . . . . . . . . 77

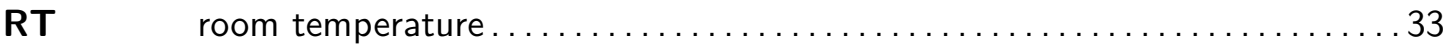

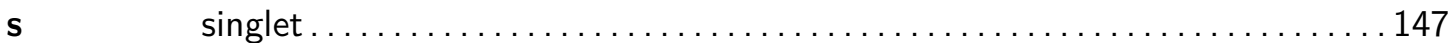

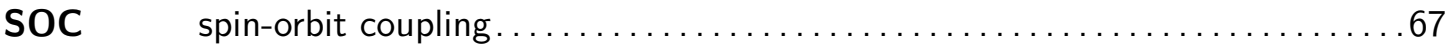

SOMO singly occupied molecular orbital ............................ 41

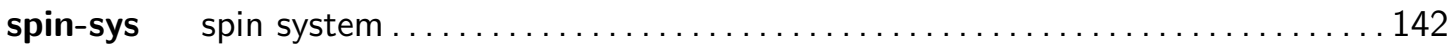

SQUID superconducting quantum interference device .................... 10

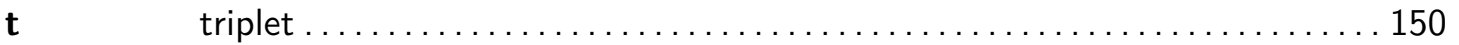

TDDFT time-dependent density functional theory $\ldots \ldots \ldots \ldots \ldots \ldots \ldots \ldots \ldots \ldots \ldots \ldots$

TEMPOH 1-hydroxyl-2,2,6,6-tetramethylpiperidine ........................ 39

TIP temperature independent paramagnetism . . . . . . . . . . . . . . . . . 67

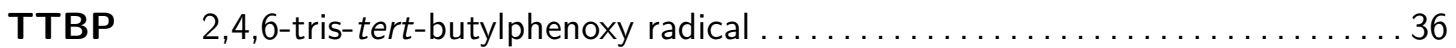

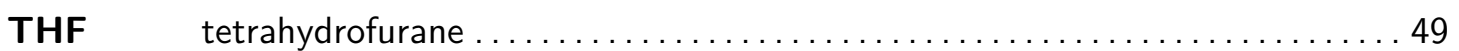

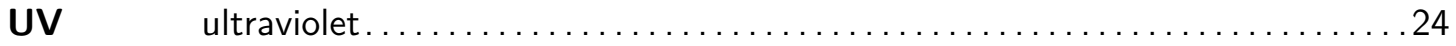

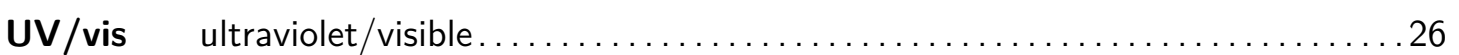

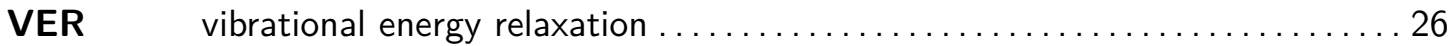

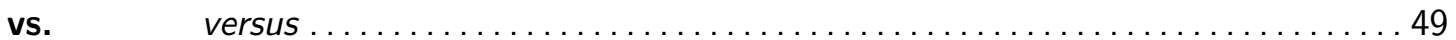

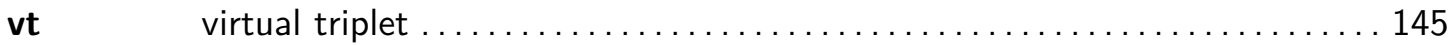

VT-NMR variable temperature nuclear magnetic resonance ..................6 68

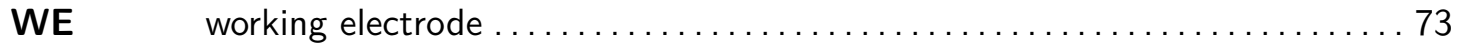

